\title{
ELUCIDATION OF UNCONVENTIONAL BONDING IN LithIUM ORganic COMPOUNDS
}

\author{
Dissertation zur Erlangung des \\ mathematisch-naturwissenschaftlichen Doktorgrades \\ der Georg-August-Universität Göttingen
}

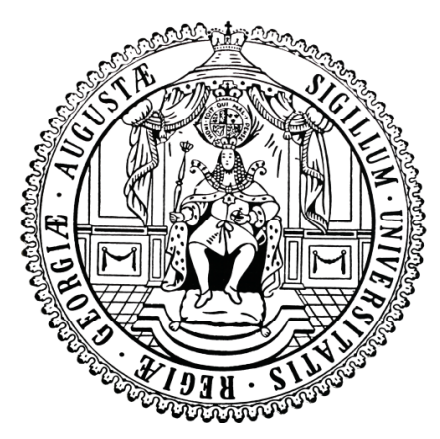

vorgelegt von

Holger Ott aus München

Göttingen 2009 





\title{
ELUCIDATION OF UNCONVENTIONAL BONDING IN LithIUM ORganic COMPOUNDS
}

\author{
Dissertation zur Erlangung des \\ mathematisch-naturwissenschaftlichen Doktorgrades \\ der Georg-August-Universität Göttingen
}

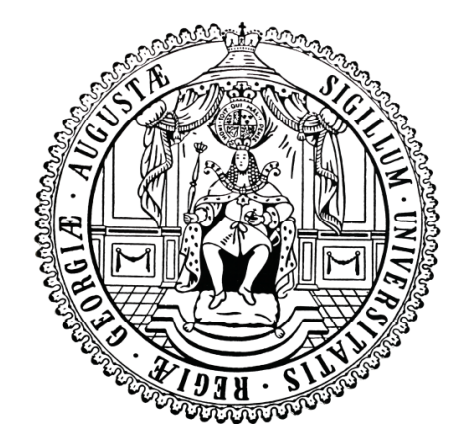

vorgelegt von

Holger Ott aus München

Göttingen 2009 

Eingereicht am:

18.03.2009

D7

Referent:

Prof. Dr. D. Stalke

Korreferent:

Prof. Dr. H. W. Roesky

Tag der mündlichen Prüfung: $\quad$ 28.04.2009

Prüfer Anorganische Chemie: Prof. Dr. D. Stalke

Prüfer Analytische Chemie: Prof. Dr. U. Klingebiel

Prüfer Kristallographie: $\quad$ Prof. Dr. W. Kuhs

Erweiterte Prüfungskommission: Prof. Dr. U. Diederichsen

Prof. Dr. G. Eckold 

„I love it when a plan comes together." Col. John "Hannibal" Smith 



\section{DaNkSAgUng}

Die vorliegende Arbeit wurde in der Zeit von Juni 2006 bis März 2009 im Arbeitskreis Stalke am Institut für Anorganische Chemie der Universität Göttingen angefertigt.

Der größte Dank gebührt meinem Chef Herrn Professor Dietmar Stalke. Er stellte mir als Alkalimetallorganiker eine hochwertige Laborausrüstung sowie die weltbesten beiden Maschinen zur Verfügung, die man sich als Kristallograph wünschen kann. Ihm war im Bezug auf Gase und Kühlmittel kaum etwas zu teuer, auch wenn sich leider die Installation der Heliumkristallkühlung soweit verzögerte, dass keine Ergebnisse mehr ihren Weg in diese Arbeit gefunden haben. Sein in mich gesetztes Vertrauen, sei es im Bezug auf Projektvergaben, Kompetenzen, Reisen und Vorträge, ehrte mich ganz besonders.

Dem Arbeitskreis Stalke darf ich für die hervorragende Stimmung danken, ohne die diese Arbeit kaum vollbracht worden wäre. Im Besonderen auch den Göttinger Rekruten, die unseren anfangs bayerischen Arbeitskreis im südschwedischen Ausland grandios verstärkten, den Charakter des AKs mitbestimmten und die aussterbende Spezies der Würzburger Dinosaurier gekonnt ersetzten bzw. noch ersetzen werden. Viele infrastrukturelle und gesellschaftliche Vorzüge des AK Stalkes machten die Promotion eigentlich fast zum "Kinderspiel". Dazu gehörten der Bierkühlschrank, der Turnier-Kicker, viele AK-Grillen, das gemeinsame Kochen um der Mensa zu entfliehen und natürlich die zahlreichen DoDs, denen wir so fundamentale Erfindungen wie den noch nicht patentierten Tischspringbrunnen zu verdanken haben.

Mein spezieller Dank gilt Herrn Dr. Dirk Leusser, meinem Mentor, temporären WGGenossen und Freund, der fast uneingeschränkt die Verantwortung für die hier behandelten Themen übernehmen muss. Denn ohne inn wäre ich niemals in das Gebiet der experimentellen Elektronendichtebestimmung eingetaucht. Seine tatkräftige Unterstützung beim Montieren von Kristallen, sein unglaubliches Kristallographie- und EDWissen waren immer Gold wert. Viel Glück in Südamerika und auf Wiedersehen zu gegebener Zeit in Deutschland!

Meinem ehemaligen F-Praktikumsleiter, späteren Laborpartner und lieben Freund Herrn Dr. Stephan Deuerlein möchte ich herzlich danken. Sowohl in Würzburg, in Göttingen, als auch nunmehr über weitere Stecken hinweg besteht ein enger Kontakt, der hoffentlich auch Jahrzehnte überdauern wird. Von einer detaillierten Aufzählung deiner Verdienste muss ich leider absehen, da sonst außer dir - und deiner guten Seele keine weiteren Personen mehr genannt werden könnten.

Meiner Mitleidensgenossin und Langzeit-Schreibtischnachbarin Frau Ulrike Flierler sei gedankt für die fruchtbare Zusammenarbeit, die stete Unterstützung und Organisation kleiner und großer Veranstaltungen. Es war mir eine Ehre mit dir zu promovieren und ich freue mich, dass es mit der gemeinsamen Punktlandung nun doch geklappt hat.

Meinem Salinen- und Saunakumpel Herrn Dr. Gerald "Schnucki" Schwab gilt mein Dank für die gemeinsame Zeit in Ruhe- und sonstigen Räumen mit all der schönen Musik und den Likören. Auch deine federführende Gestaltung meines Junggesellenabschieds macht dich wirklich unsterblich. Auf baldiges Wiedersehen im Juni!

Der Familie Hagermeister, namentlich dem fast immer gut gelaunten und entspannten Herrn Dipl. Chem. Thomas Schulz genauso wie Frau Mandy Hartrampf und Klein-Alana sei gedankt, ohne die wohl niemals die Institution des DoDs entstanden wäre. Halt das Würzburg-Fähnchen hoch, Thomas! 
Frau Dipl. Chem. Ina Objartel, seines Zeichens Hutbastelbeauftragte (Wir zählen auf dich!) und Hüterin der Heißklebepistole, darf ich großen Dank aussprechen. Mit keiner Eisprinzessin hat das Paper-Schreiben und das Cocktail/Sekt-Trinken soviel Spaß gemacht wie mit dir. Als moralische Stütze und praktische Hilfe in der letzten Zeit warst du unersetzlich.

Dem Foto- und Grafikbeauftragten Herrn Dipl. Chem. Christian Kling muss u. a. für das unvergessliche Kristallabfackeln, das Design des hoffentlich zukünftigen Titelbilds der Angewandten Chemie und die netten Fahrten nach Amsterdam zu K1-Wettkämpfen gedankt werden.

Bei meinem Lieblings-Computerinder und ED-Nachfolger Herrn Dipl. Chem. Daniel Kratzert, meinem NMR Lexikon Herrn Dipl. Chem. Daniel Stern und meinem Kickertrainer und leider niemals im Turniereinsatz gewesenen Teampartner Herr Dipl. Chem. Nils Finkelmeier will ich mich für die vielen netten Stunden bedanken. Diese Zeit teilte ich dankenswerterweise auch mit meiner Laborpartnerin und "Fast-Ehebrecherin" Frau Dipl. Chem. Magret Meinholz, die mich im Labor allerdings kaum angetroffen hat, und Herrn Dipl. Chem. Markus "Granickel" Granitzka, ein inzwischen überzeugter Genießer mittelfränkischen Bieres, der mir noch einen Wettkampf zwischen Tarantel und Venusfliegenfalle schuldet.

Den "Maschinenschraubern" Daniel Stern und Thomas Schulz, die mich nach langer eigener Amtszeit abgelöst haben und die beiden Diffraktometer in der Folgezeit grandios in Schuss hielten, sowie Herrn Helmut Dehnhard, der uns tatkräftig dabei unterstützte, bin ich für eine mehr oder weniger ungestörte Zeit zum Zusammenschreiben zu Dank verpflichtet.

All den lieben und gnädigen Korrektoren zolle ich tiefsten Respekt, da sie Teile oder das Gesamtwerk durchgesehen haben, das ich selbst schon kaum mehr sehen konnte. Danke dafür namentlich Herrn Michael Witt, der gerade rechtzeitig zu diesem Spektakel zurückkam.

Den F-Praktikanten darf für das Warmhalten des Labors und ihre Ergebnisse auf dem Gebiet der Picolylverbindungen gedankt werden, vor allem Herrn Jacob Hey, der leider zu spät in den AK kam, um hier mit großen Lobeshymnen überschüttet zu werden, auch wenn er sie sich noch verdienen wird.

Den Doktoren Henn und Meindl, die den AK theoretisch bereicherten und nützliche Programme zur Verfügung stellten, sowie Frau Dipl. Chem. Tanja Tatić für die stete $\mathrm{TMSCH}_{2} \mathrm{Li}$-Kristallzucht danke ich hiermit.

Dem gesamten Serviceteam des Instituts für Anorganische Chemie, darunter "Ha-Jo" Heymel und der Werkstatt, der NMR Abteilung mit Dr. Michael John und Wolfgang Zolke, Thomas Schuchardt von der Masse, Herrn H. G. Schmidt, dessen Büro ich glücklicherweise okkupieren durfte, und Martin Schlote, unserem Chemikalienwart, bin ich überaus dankbar, denn ohne sie wären Teile dieser Arbeit nicht möglich gewesen. Im Besonderen darf Frau Heike Tappe, unsere stete moralische Unterstützung, nicht vergessen werden.

Bei den vielen Kooperationspartner, vor allen den Herrn Professoren Carsten Strohmann und Uwe Klingebiel mit samt ihren Leuten, die meinen wissenschaftlichen Horizont positiv erweiterten, bedanke ich mich herzlich.

Dem Fonds der chemischen Industrie verdanke ich die finanzielle Unterstützung.

Meiner Familie, die ich durch geschickte Heiratspolitik um viele nette Menschen vergrößern konnte, danke ich für den steten moralischen Rückhalt. Ganz besonders natürlich meiner Frau Katrin, die konstant auf Verlagerung meines Lebensmittelpunkts vertraut und hoffentlich bald belohnt wird. Danke für alles! 


\section{CONTENTS}

1 LITHIUM ORGANICS IN CHEMISTRY ......................................... 1

2 ANIONIC PICOLYL METAL COMPOUNDS $\ldots \ldots \ldots \ldots \ldots \ldots \ldots \ldots \ldots \ldots \ldots \ldots \ldots$

2.1 Introduction .................................................................... 13

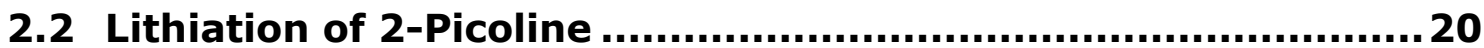

2.2.1 $\quad \eta^{1}$-Carbanionic 2-Picolyllithium Aggregates....................... 20

2.2.2 $\eta^{1}$-Amidic 2-Picolyllithium Aggregates .............................. 26

2.2.3 Monomeric 2-Picolyllithium ....................................... 33

\subsection{Experimental Charge Density Study on Unsubstituted}

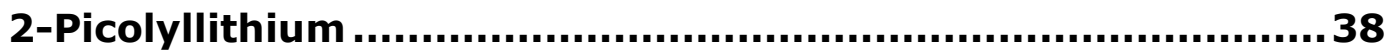

2.3.1 Principles of X-Ray Diffraction ................................. 39

2.3.2 Independent Atom Model .......................................... 43

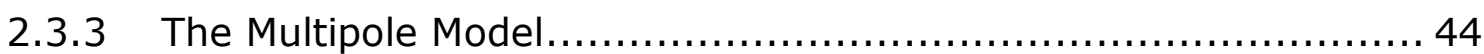

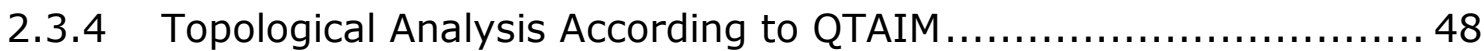

2.3.5 Experimental Details and Refinement Strategy..................... 52

2.3.6 Molecular Structure of the 2-Picolyllithium - 2-Picoline Dimer........ 56

2.3.7 Topological Analysis of the EDD in the 2-Picolyllithium-2-Picoline Dimer

2.4 On the Quest of the Coordination Pattern in 2-Picolyllithium Compounds

2.52 2-Picolyllithium Adducts in Solution ................................... 84

$2.62-P i c o l y l$ Complexes of Sodium ........................................... 95

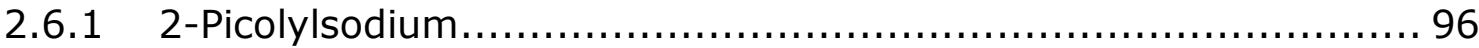

2.6.2 Attempted One-pot Synthesis of 2-Picolylsodium/-potassium..... 102

2.7 Unsubstituted 4-Picolyllithium .......................................... 108

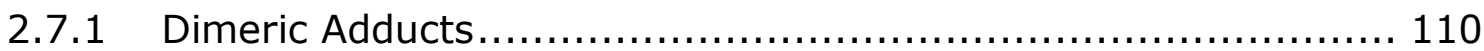

2.7.2 4-Picolyllithium Monomer and Polymer........................... 117

2.8 Conclusion....................................................................... 123

3 TRIMETHYLSILYLMETHYLLITHIUM $\ldots \ldots \ldots \ldots \ldots \ldots \ldots \ldots \ldots \ldots \ldots \ldots \ldots \ldots \ldots$

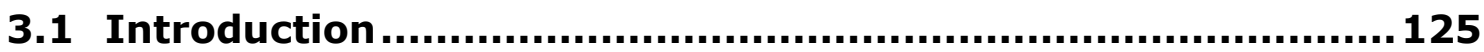


3.2 Charge Density Study of a Hexameric Lithium Organic

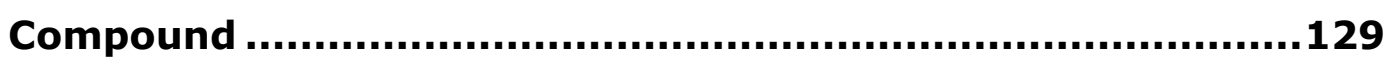

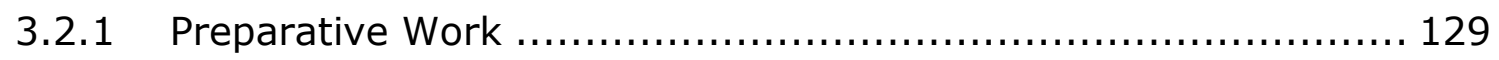

3.2.2 Experimental and Refinement Details................................ 133

3.2.3 Molecular Structure Considerations.................................. 137

3.2.4 Analysis of the Electron Density Distribution ....................... 142

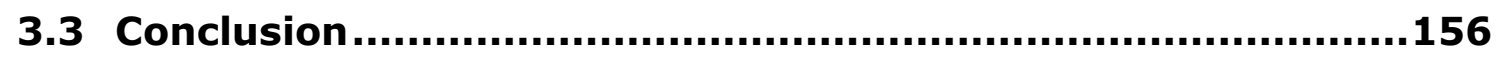

4 A CHIRAL BENZYLLITHIUM DERIVATIVE .....................................157

4.1 Asymmetric Lithium Organic Compounds ...........................157

4.2 Preliminary Work .............................................................161

4.3 Experiment and Refinement ...............................................165

4.4 Multipole Model and the Electron Density Distribution in 17.169

4.5 Theoretical EDD of the Benzyllithium Derivative ...................183

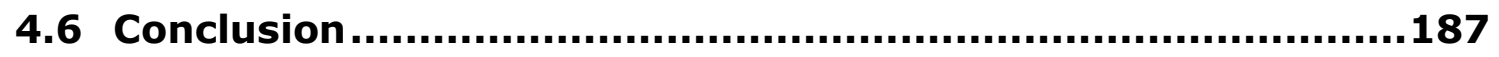

5 CONCLUSIONS AND FUTURE OBJECTIVES....................................189

6 ZUSAMMENFASSUNG UND AUSBLICK.............................................

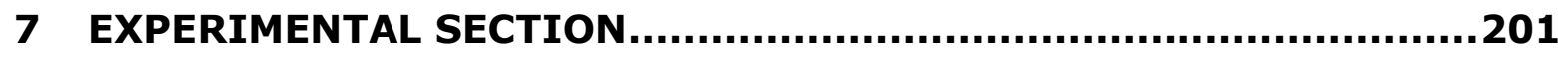

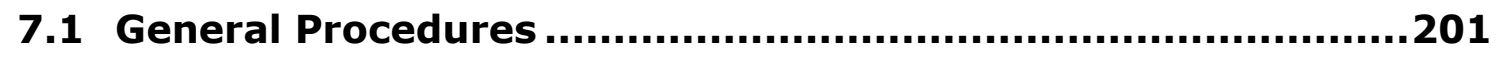

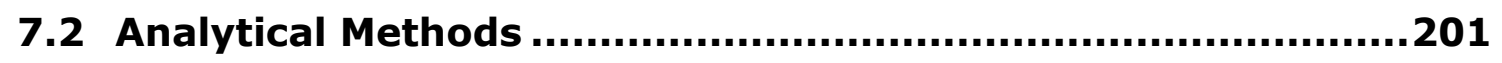

7.3 Syntheses and Characterisations............................................203

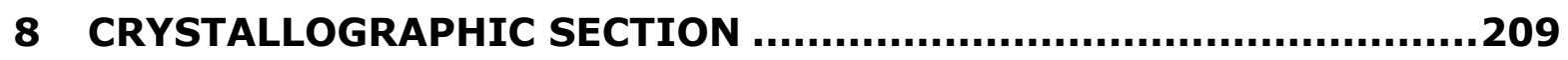

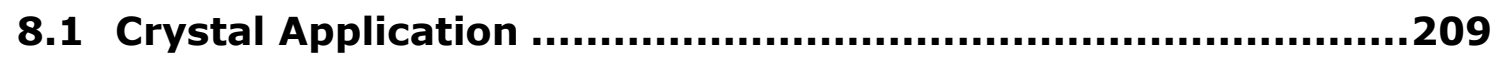

8.2 Data Collection, Integration, and Reduction .........................210

8.3 Structure Solution and Refinement ..................................212

8.4 Crystallographic Details for 1-5, 8-15, and 17....................213

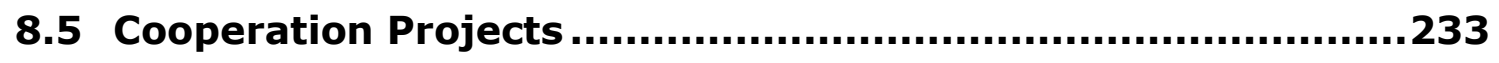

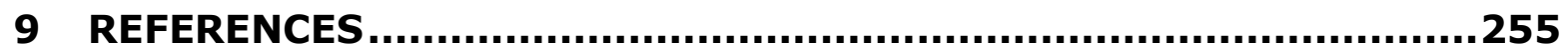




\section{Abbreviations}

\begin{tabular}{|c|c|c|c|}
\hline$\AA$ & Angström & $\mathrm{dr}$ & diastereomeric ratio \\
\hline \multirow[t]{2}{*}{ ADP } & anisotropic displacement & e & electron(s) \\
\hline & parameters & e.g. & for example \\
\hline AP & atomic position & ED & electron density \\
\hline au & atomic units & EDD & electron density distribution \\
\hline av. & average & EPR & electron paramagnetic \\
\hline BCC & bonding charge concentration & & resonance \\
\hline $\mathrm{BCP}$ & bond critical point & Eq. & equation \\
\hline $\mathrm{BP}$ & bond path & eq. & equivalent(s) \\
\hline $\mathrm{Bu}$ & butyl & ESP & electrostatic potential \\
\hline $\mathrm{Bz}$ & benzyl & Et & ethyl \\
\hline C & cyclo & et al. & and others \\
\hline calc. & calculated & exp. & experiment \\
\hline $\mathrm{CC}$ & charge concentration & GoF & goodness of fit \\
\hline CCD & charge coupled device & $\mathrm{h}$ & hour(s) \\
\hline $\mathrm{CCP}$ & cage critical point & $\mathrm{Hal}$ & halogen atom \\
\hline CIPE & complex induced proximity effect & Hex & hexyl \\
\hline COD & cycloocta-1,5-diene & HMBC & heteronuclear multiple bond \\
\hline $\mathrm{CP}$ & critical point & & correlation \\
\hline CSD & Cambridge structural database & hmpa & hexamethylphosphoramide \\
\hline $\mathrm{D}$ & donor atom & HOESY & heteronuclear Overhauser \\
\hline dabco & 1,4-diazabicyclo[2.2.2]octane & & enhancement spectroscopy \\
\hline decomp. & decomposition & HOMO & highest occupied molecular orbital \\
\hline DFG & Deutsche Forschungsgemeinschaft & $i$ & iso \\
\hline & (German research foundation) & i.e. & that is \\
\hline diff. & difference & IAM & independent atom model \\
\hline DMAE & dimethylaminoethoxide & IPP & integrated projected \\
\hline DMAP & 4-dimethylaminopyridine & & populations \\
\hline dme & dimethoxyethane & IR & infrared \\
\hline DMSDA & difference of mean square & LICKOR & super basic mixtures of $\mathbf{R} \mathbf{L i}$ \\
\hline & displacement amplitudes & & and potassium alkoxides \\
\hline DOSY & diffusion-ordered spectroscopy & LP & lone pair \\
\hline
\end{tabular}




\begin{tabular}{|c|c|c|c|}
\hline LUMO & $\begin{array}{l}\text { lowest unoccupied molecular } \\
\text { orbital }\end{array}$ & $\mathrm{R}, \mathrm{R}^{\prime}$ & $\begin{array}{l}\text { hydrogen atom or alkyl or ary } \\
\text { group }\end{array}$ \\
\hline M & molar & $\mathrm{RCP}$ & ring critical point \\
\hline M & metal atom & $\mathbf{R L i}$ & lithium organic compounds \\
\hline $\max$ & maximal & restr. & restraint(s) \\
\hline Me & methyl & RT & room temperature \\
\hline $\min$. & minimal & sof & site occupation factor \\
\hline MM & multipole model & SOMO & singly occupied molecular \\
\hline MO & molecular orbital & & orbital \\
\hline M.p. & melting point & $\mathrm{sp}$ & (-)-sparteine \\
\hline MS & mass spectrometry & $t$ & tertiary \\
\hline$n$ & normal & $\operatorname{tg}$ & tetraglyme \\
\hline NBCC & non-bonding charge concentration & thf & tetrahydrofuran \\
\hline NMR & nuclear magnetic resonance & tmcda & $(R, R)-N, N, N^{\prime}, N^{\prime}$-tetramethyl- \\
\hline NOESY & nuclear Overhauser effect & & 1,2-diaminocyclohexane \\
\hline & spectroscopy & tmeda & tetramethylethylenediamine \\
\hline param. & parameter(s) & TMS & trimethylsilyl \\
\hline $\mathrm{Ph}$ & phenyl & tol & toluene \\
\hline Pic & picolyl & vs. & versus \\
\hline pmdeta & $\begin{array}{l}N, N, N^{\prime}, N^{\prime}, N^{\prime \prime} \text {-pentamethyl } \\
\text { diethylenetriamine }\end{array}$ & VSCC & $\begin{array}{l}\text { valence shell charge } \\
\text { concentration }\end{array}$ \\
\hline ppm & parts per million & VSEPR & valence shell electron pair \\
\hline $\mathrm{Pr}$ & propyl & & repulsion \\
\hline Py & pyridyl & & \\
\hline QTAIM & $\begin{array}{l}\text { quantum theory of atoms in } \\
\text { molecules }\end{array}$ & & \\
\hline
\end{tabular}




\section{LIST OF COMPOUNDS}

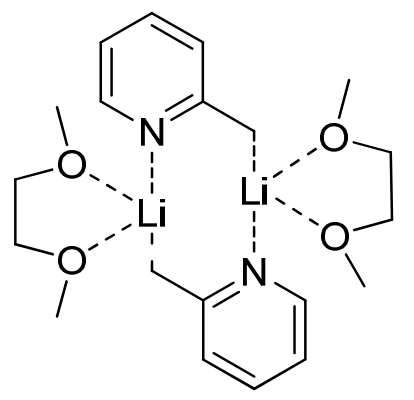

$[2-\mathrm{PiCLi} \cdot \mathrm{dme}]_{2}(\mathbf{1})$

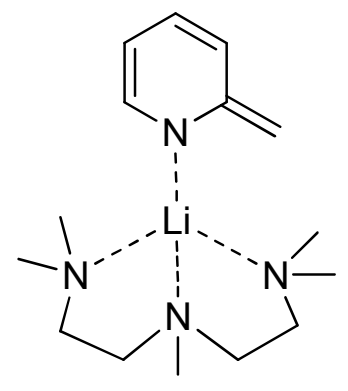

[2-PicLi·pmdeta] (3)

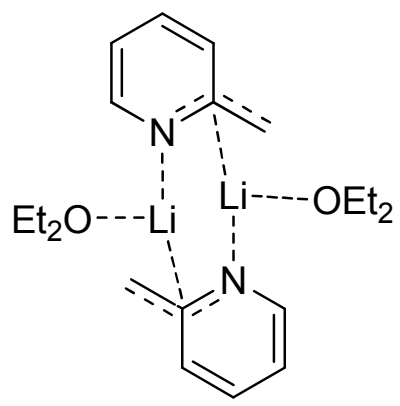

$\left[2-\mathrm{PiCLi} \cdot \mathrm{OEt}_{2}\right]_{2}(\mathbf{5})$

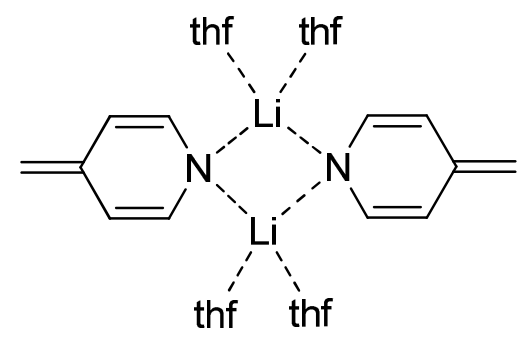

$\left[4-\mathrm{PicLi} \cdot(\text { thf })_{2}\right]_{2}(\mathbf{1 0})$

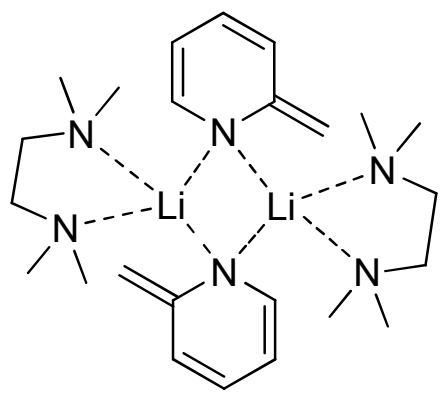

$[2-\text { PicLi·tmeda }]_{2}(\mathbf{2})$

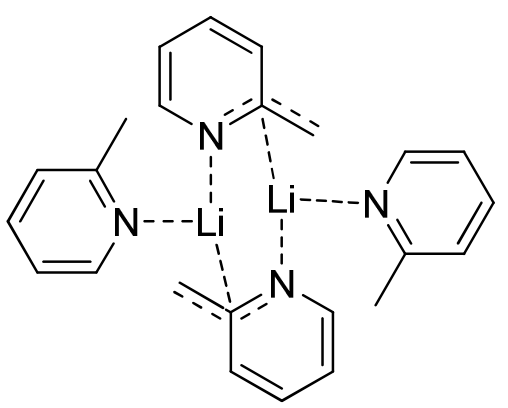

$[2-\mathrm{PiCLi} \cdot 2-\mathrm{PiCH}]_{2}(4)$

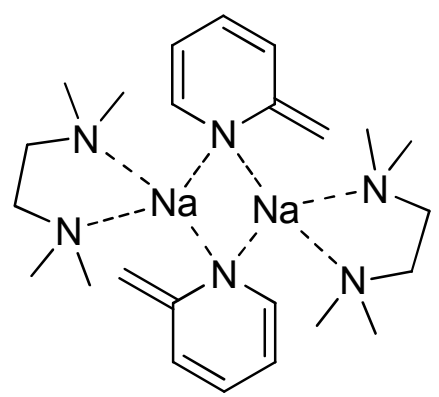

$[2-\mathrm{PicNa} \cdot \mathrm{tmeda}]_{2}(\mathbf{8})$

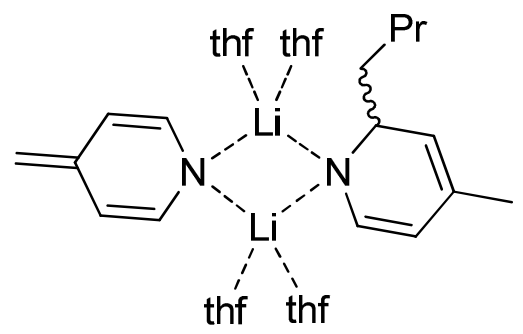

[4-PicLi $\cdot(\text { thf })_{2} \cdot 2-n B u-4-M e-$ $\left.2-\left(\mathrm{H}_{4} \mathrm{C}_{5} \mathrm{~N}\right) \mathrm{Li} \cdot(\text { thf })_{2}\right](\mathbf{1 1})$ 


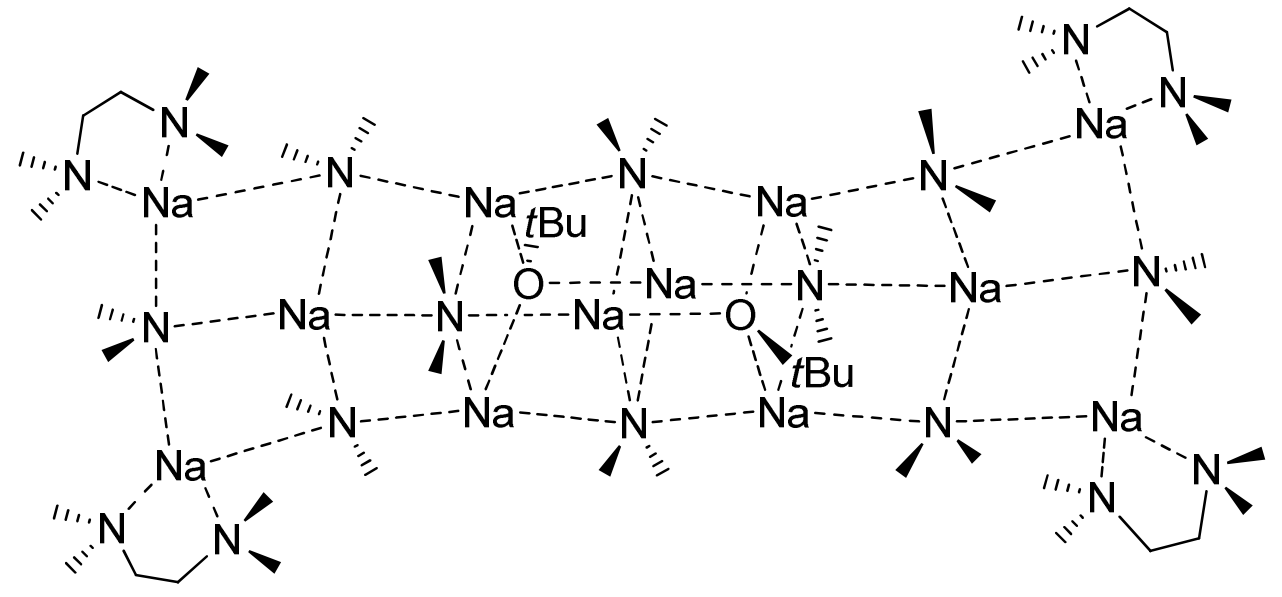

$\left[\mathrm{Na}_{6}\left(\mathrm{NMe}_{2}\right)_{5}(\mathrm{OtBu}) \cdot(\text { tmeda })_{2}\right]_{2}(\mathbf{9})$

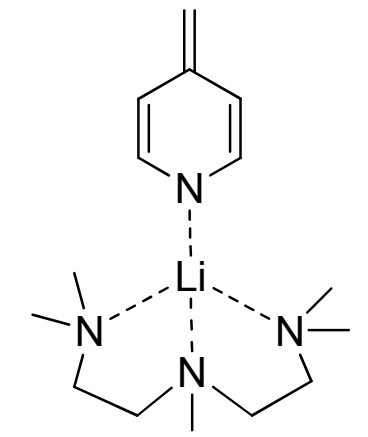

[4-PicLi·pmdeta] (12)

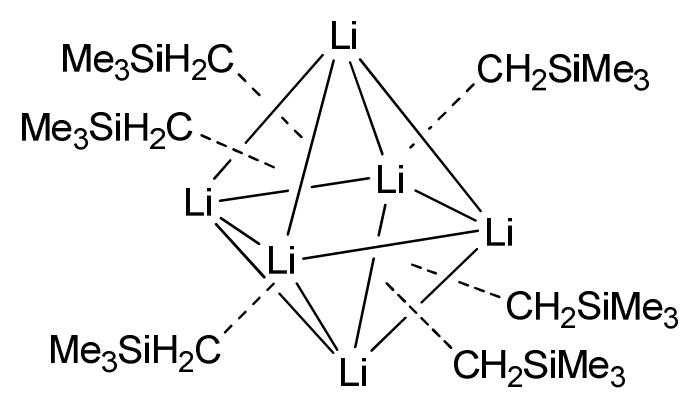

$\left[\mathrm{TMSCH}_{2} \mathrm{Li}\right]_{6}(\mathbf{1 4}, \mathbf{1 5})$

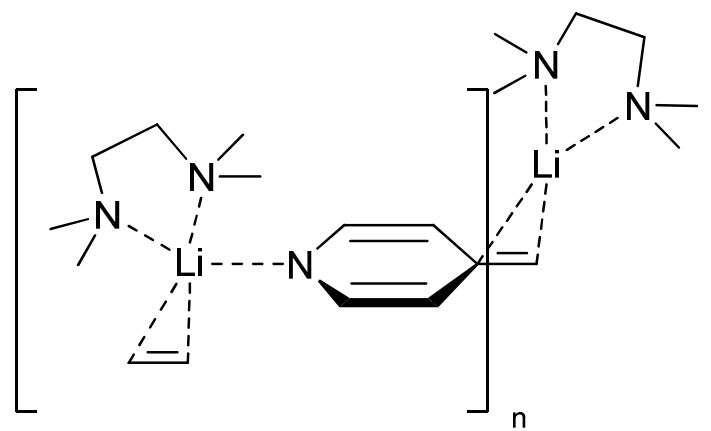

$[4-P i c L i \cdot t m e d a]_{n}(\mathbf{1 3})$

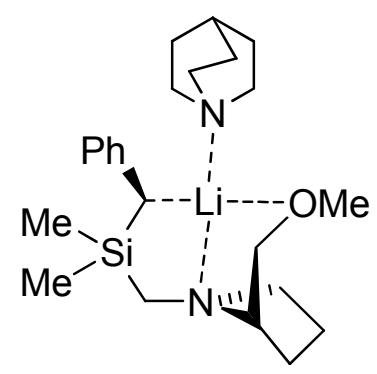

$\left[\mathrm{PhCH}(\mathrm{Li}) \mathrm{Si}(\mathrm{Me})_{2} \mathrm{CH}_{2}\left\{\mathrm{NC}_{4} \mathrm{H}_{7}\left(\mathrm{CH}_{2} \mathrm{OMe}\right)\right\}\right.$. (N $\left.\left.\left\{\left(\mathrm{CH}_{2}\right)_{2}\right\}_{3} \mathrm{CH}\right)\right](\mathbf{1 7})$ 


\section{Lithium Organics in Chemistry}

The discovery of lithium in 1817 is owed to J. A. Arfvedson, who found significant discrepancies in the elemental analysis of the minerals spodumene $\left(\mathrm{LiAlSi}_{2} \mathrm{O}_{6}\right)$ and mica lepidolite $\left(\mathrm{K}(\mathrm{Li}, \mathrm{Al})_{3}\left[\mathrm{AlSi}_{3} \mathrm{O}_{10}\right](\mathrm{F}, \mathrm{OH})_{2}\right)$ and claimed a missing element. He named it after the Greek word $\lambda_{\imath} \theta 0 \varsigma$ (lithos = stone) to emphasise the fact that sodium and potassium were known, by contrast, from vegetable ashes. One year later H. Davy isolated the metal by electrolysis of molten $\mathrm{Li}_{2} \mathrm{O} \cdot{ }^{[1,2]}$

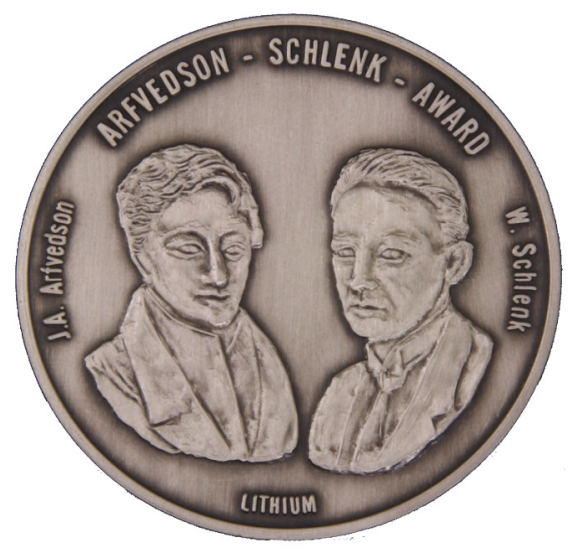

Figure 1-1. Arfvedson-Schlenk silver medal, which is awarded by the GERMAN CHEMICAL SOCIETY (GDCh) and the CHEMETALL AG to outstanding researchers in the field of lithium chemistry.

It then took 100 years until the first organometallic compounds containing lithium were synthesised by $W$. Schlenk and J. Holtz. ${ }^{[3,4]}$ In the following period several properties of lithium organic compounds ( $\mathbf{R} \mathbf{L i})$ were discovered. Due to the high charge-to-ion-radius ratio, the metal cation shows a strong tendency towards solvation, a high Pearson hardness, ${ }^{[5,6]}$ and $\mathbf{R L i}$ an exceptionally high solubility in ethers and hydrocarbons. Moreover, lithium organic compounds are relatively volatile (they show much lower melting and boiling points than expected for alkaline salt compounds) and possess a low conductivity when molten. They are strong bases and readily react with protic compounds, oxygen, and carbon dioxide. Therefore, they need to be handled under inert gas techniques, which were reported for the first time by $W$. Schlenk and $A$. Thal, ${ }^{[7]}$ to prevent the formation of the corresponding hydrocarbon compounds, alkoxides, and carboxylates. In addition, lithium organic compounds tend to 
decompose thermally via a $\beta$-hydrogen elimination pathway, if accessible, while the more reactive ones even cleave ethers ${ }^{[8,9]}$ at room temperature (RT).

The quantitative analysis of organic solutions of RLi with titrimetric methods is rather demanding because of the inherent high basicity of their decomposition products (e.g. LiH, LiOR). H. Gilman and F. K. Cartledge solved this problem with a double-titration procedure. ${ }^{[10]}$ First, they hydrolysed a certain amount of the solution and afterwards determined the total amount of base by titration with a strong acid. In a second run, he removed the lithium organic compound with an electrophile, e.g. 1,2-dibromoethane. The subsequent analysis of the remaining base yielded the concentration of the decomposition products and had to be subtracted from the total base concentration. Alternatively, $\mathrm{N}$-pivaloyl-o-toluidine can be used as an organic indicator. ${ }^{[11]}$ The addition of more than one equivalent of $\mathbf{R L i}$ results in an intense yellow colour of the generated dianion (Scheme 1-1). Alkoxides do not perturb the titration in this case.

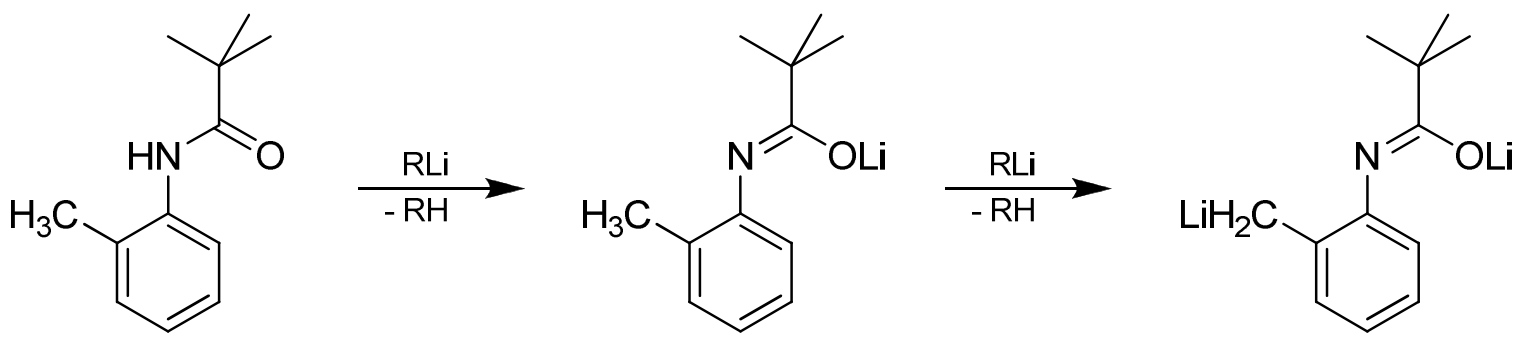

Scheme 1-1. Reaction of $N$-pivaloyl-o-toluidine with lithium organic compounds.

The latest development in the field of RLi concentration determination was published in 2004. ${ }^{[12]}$ A defined mixture of cycloocta-1,5-diene (COD) and the lithium organic compound is filled in an NMR tube and a ${ }^{1} \mathrm{H}$ spectrum is recorded without using a deuterated solvent. By integration and comparison of the hydrogen atom signals of the internal COD standard and a chosen signal of $\mathbf{R L i}$, the concentration can easily be calculated.

Several synthetic routes to lithium organic compounds are well-established (Scheme 1-2). ${ }^{[13,14]}$ The direct synthesis (A) with elemental lithium and a halocarbon is the preferred industrial procedure to synthesise standard lithium organic compounds, i.e. hexyl-, butyl-, ethyl-, methyl-, and phenyllithium. ${ }^{[1]}$ In metallation reactions (B) the basicity of metallic lithium or $\mathbf{R L i}$ is used to deprotonate $\mathrm{C}-\mathrm{H}$ acidic substrates. The metal halide exchange reaction $(\mathbf{C})$, first reported by $G$. Wittig et al. in $1938^{[15]}$ is mainly applied to aryl halides while $n$-butyllithium normally acts as the lithium donor. Moreover, a metal metal 
exchange (D) can yield the desired $\mathbf{R} \mathbf{L i}$, e.g. in the reaction of a tin organic with a lithium organic compound. Historically important, but nowadays far less applied due to the high toxicity, is the transmetallation route (E) of elemental lithium and a mercury organic compound. ${ }^{[4]}$ Indeed, this reaction type was used by W. Schlenk and J. Holtz to synthesise the first lithium organic compounds, phenyl- and ethyllithium. ${ }^{[3]}$

\begin{tabular}{|c|c|c|c|c|c|c|}
\hline $2 \mathrm{Li}$ & + & RHal & $\longrightarrow$ & RLi & + & LiHal \\
\hline RLi & + & R'H & $\longrightarrow$ & R'Li & + & $\mathrm{RH}$ \\
\hline RLi & + & R'Hal & & R'Li & + & RHal \\
\hline RLi & + & MR' & $\longrightarrow$ & R'Li & + & MR \\
\hline $2 \mathrm{Li}$ & + & $\mathrm{HgR}_{2}$ & $\longrightarrow$ & $2 \mathrm{RLi}$ & + & $\mathrm{Hg}$ \\
\hline
\end{tabular}

Scheme 1-2. Synthetic routes to lithium organic compounds.

Soon after their discovery, lithium organic compounds gained in importance. By now, they are well-established key substances in organic as well as inorganic synthesis. ${ }^{[16-19]}$ Prominent examples in organic chemistry are substitution reactions with halocarbons, aromatic ring metallations, ${ }^{[20,21]}$ additions to $\mathrm{C}-\mathrm{C}$ double ${ }^{[22]}$ or triple bonds and ketonic functions. Among those, the addition to $\mathrm{CO}_{2}$ is the most important $C_{1}$ chain-extension reaction in chemistry. ${ }^{[19]}$ Furthermore, nitriles are converted to lithium imines (ketones after hydrolysis) ${ }^{[14]}$ and epoxides $^{[23]}$ to lithium alkoxides. Moreover, the deprotonation capacity of $\mathbf{R L i}$ is used in Wittig-reactions, ${ }^{[24]}$ and geminal carbolithium halides can even serve as carbene precursors. Even in the field of asymmetric synthesis, lithium organic compounds are more and more applied to synthesise natural products and active pharmaceutical drugs. ${ }^{[25]}$ In combination with chiral auxiliaries such as (-)-sparteine, they can induce the asymmetric deprotonation of a substrate ${ }^{[26,27]}$, whereas sterically demanding groups can serve as the directing force in substitution reactions of pro-chiral anions (e.g. Schöllkopf amino acid synthesis $\left.{ }^{[28]}\right)$. Besides, lithium organic compounds are valuable precursors for cuprates which are synthetically interesting on their own. ${ }^{[29]} \mathbf{R L i}$ is thereby converted into a soft nucleophile on the Pearson hardness scale. The addition to a Michael system then proceeds in a 1,4- and not in a 1,2-manner. ${ }^{[30]}$

Inorganic chemistry substitution reactions can be undertaken with main group halides (e.g. chlorophosphanes) as well as transition metal halides. Furthermore, 
RLi can be added to transition metal complexes to yield the corresponding lithium salts of the anionic at-complex (e.g. $\left.\mathrm{Li}_{3}\left[\mathrm{CrMe}_{6}\right]\right)^{[31]}$ or in the case of carbonyl compounds the precursor to Fischer-carbene complexes. ${ }^{[32]}$ The reaction with elemental oxygen and sulfur offers straight-forward access to alkoxides and thiolates. ${ }^{[33]}$

It is noteworthy that RLi can also serve as initiators in anionic polymerisation reactions. $n$-Butyllithium ( $n \mathrm{BuLi}$ ), for example, is routinely applied in the fabrication of synthetic rubber and plastics (i.e. polyisoprene, -butadiene, and butadiene/styrene copolymers). ${ }^{[1,34,35]}$ This is actually the reason for the large annual $n B u L i$ production of about $1000 t$ worldwide. ${ }^{[2]}$ All basic reagents mentioned above are nowadays commercially available and affordable, so that their application spectrum will steadily increase in the forthcoming years.

In all synthetic reactions given in the short summary above, these highly reactive compounds are employed in solution. Even though the abbreviation RLi suggests the existence of defined monomers, this is hardly the case. Quite the contrary is commonly observed in solution as well as in the solid state. A cornucopia of different aggregates has been identified by molecular weight determination, NMR, MS, IR, EPR and X-ray diffraction experiments. The first systematic studies were published in the 1960 s. $^{[36,37]}$
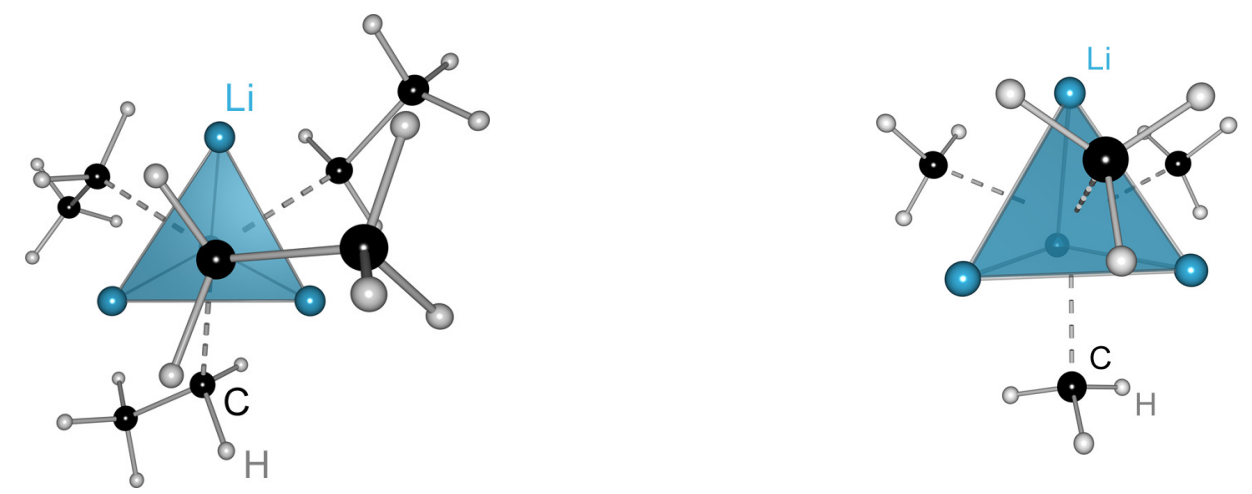

Figure 1-2. First determined molecular structures of lithium organic compounds (left:

$[\mathrm{EtLi}]_{4}$; right: $\left.[\mathrm{MeLi}]_{4}\right)$.

Cluster formations of RLi were proven in the gas and liquid phase while the first visualisations of $\mathbf{R L i}$ aggregates were accomplished in the pioneering molecular structure determinations by $\mathrm{H}$. Dietrich (EtLi) ${ }^{[38]}$ and E. Weiss and E. A. C. Lucken (MeLi) ${ }^{[39]}$ in 1963 and 1964, respectively (Figure 1-2). Since then the lithium structural chemistry has developed tremendously. Several basic trends were discovered: 
Lithium tends to form multiple interactions to anions, donor atoms and regions of $\pi$-electron density. The aggregation is mainly determined by the donating capabilities of the solvents applied as well as the steric demand of the organic substituents. The highest aggregation of RLi can be found with sterically less demanding organic groups in hydrocarbons, while polar solvents reduce the degree of aggregation. ${ }^{[36,37,40]}$

Interestingly, the structure in the solid state normally represents the upper aggregation limit. A prominent example of that statement is tert-butyllithium $(t B u L i)$. It crystallises from hydrocarbons as a tetramer ${ }^{[41]}$ and the same degree of aggregation is adopted in hydrocarbon solutions. After adding ether, dimers and monomers coexist. ${ }^{[42]}$ The only exception among the standard $\mathbf{R L i}$ is ethyllithium, which exists as a hexamer in hydrocarbons and as a tetramer in ethereal solutions as well as in the crystal. ${ }^{[40]}$

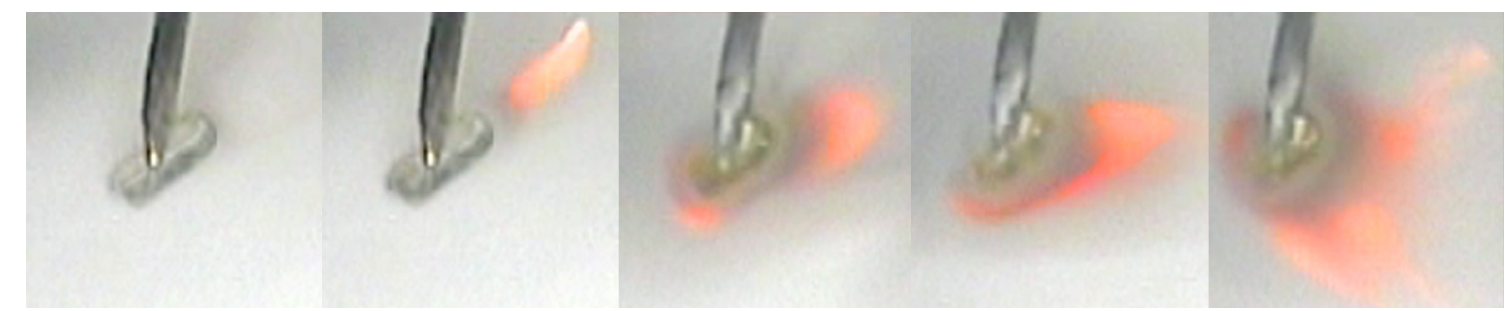

Figure 1-3. Snapshot series of a tert-butyllithium crystal catching fire during the mounting procedure without using XTEMP 2.

Nowadays, the method of choice to determine the aggregation state of lithium organic compounds is the cryo-X-ray structure determination, as it is the only source providing detailed geometrical information of a compound in the solid state. Since the development of area detectors (i.e. CCD cameras) and the description of appropriate crystal handling and mounting procedures for highly reactive and labile compounds (Figure 1-3 displays the potential results of an inappropriate crystal mounting procedure), ${ }^{[43-45]}$ the number of published RLi solid state structures increased enormously. More than $1000 \mathbf{R L i}$ structures can be found in the latest Cambridge Crystallographic Database distribution, ${ }^{[46]}$ thus outnumbering all other alkaline and even alkaline earth metal compounds (Figure $1-4)$.

A huge variety of possible structure types have been found in known molecules. Nonetheless, some lead structural motifs of lithium organic compounds are evident. $^{[42,47,48]}$ Alkyllithium compounds without external donor molecules or internal donor sites form deltahedral aggregates, e.g. tetrahedra and octahedra. 


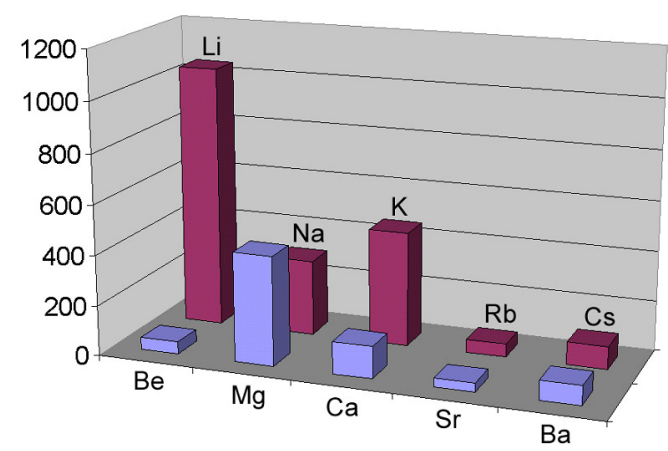

Figure 1-4. Number of molecular structures in the CSD containing an alkaline or alkaline earth metal carbon bond.

Well-known examples are the tetrameric $[\mathrm{MeLi}]_{4}{ }^{[39,49]}[\text { EtLi }]_{4}$ (Figure 1-2), ${ }^{[38]}$ and $[t \mathrm{BuLi}]_{4}{ }^{[41]}$ solid state structures as well as the hexameric $[\mathrm{nBuLi}]_{6}$ (Figure 1-5, left $),{ }^{[41]}[\mathrm{iPrLi}]_{6},{ }^{[50]}$ and $[\mathrm{CHexLi}]_{6} \cdot{ }^{[51]}$ Dimeric ring structures are far less formed and can be found e.g. in $\left[\mathrm{LiC}\left(\mathrm{SiMe}_{3}\right)_{3}\right]_{2}$ (Figure 1-5, right). ${ }^{[52]}$
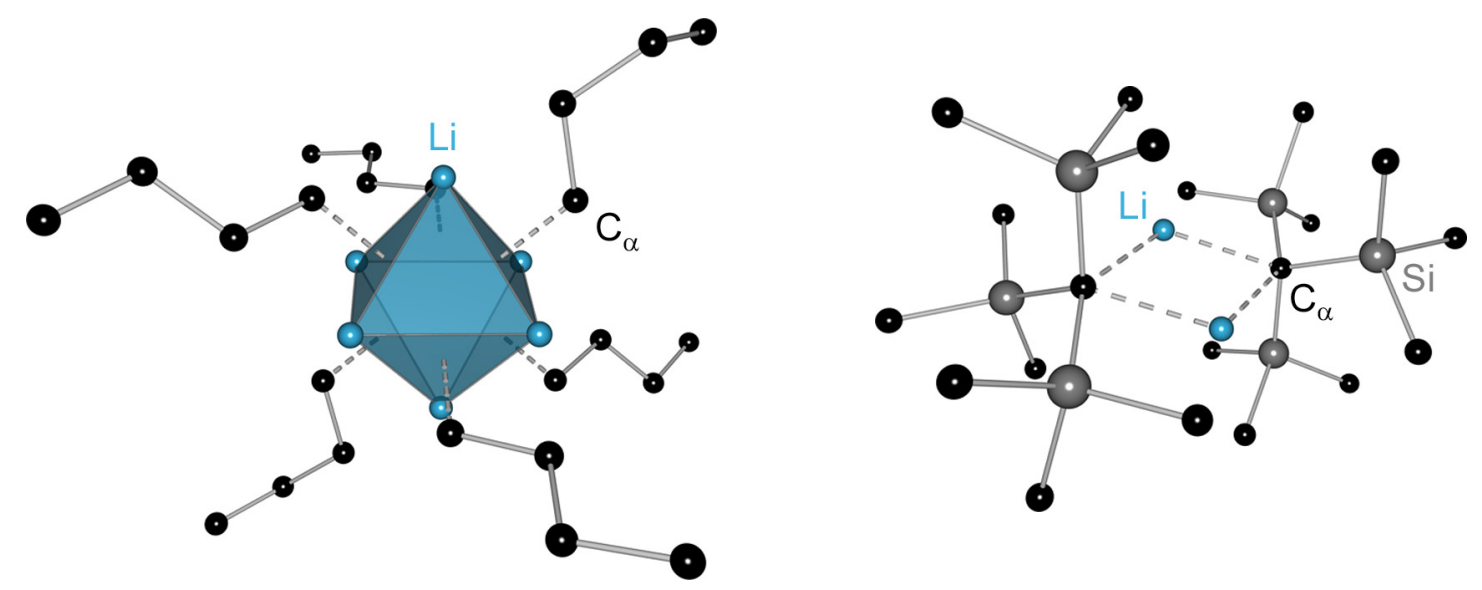

Figure 1-5. Molecular structures of a hexameric $\left([n B u L i]_{6}\right.$, left) and a dimeric lithium organic compound $\left(\left[\mathrm{LiC}\left(\mathrm{SiMe}_{3}\right)_{3}\right]_{2}\right.$, right).

Thus, knowledge of the solid state structure and the aggregation degree of lithium organic compounds is essential for the understanding of their reactivity. ${ }^{[53]}$ In order to tune the reactivity, a great variety of donor bases can be applied to disaggregate the lithium clusters. It is believed that only the monomeric RLi species are involved in reactions. ${ }^{[54]}$ Accordingly, complete disaggregation will give the most reactive mixtures. This can be illustrated by the deprotonation of toluene. If commercially available standard RLi solutions are used, hardly any metallated toluene can be isolated, but a 1:1 nBuLi/tmeda mixture gives the benzyllithium compound in excellent yields. ${ }^{[55]}$ However, the stability of $\mathbf{R L i}$ in solution is reduced by the disaggregation procedure. tert-Butyllithium is commercially available as pentane solution and can be further 
activated by adding diethyl ether. But as soon as more than two equivalents of ether are added, the solution decomposes violently under cleavage of the ether even at $-78{ }^{\circ} \mathrm{C} .{ }^{[56]}$ Hence, special care has to be taken so that the reactivity enhancement is not overdone.

Yet, the knowledge of the molecular structure of lithium organic compounds in the crystal can also serve as the starting point for theoretical calculations. This enables the elucidation of possible reaction pathways for substrates to understand mechanistic details or observed stereoselectivities. ${ }^{[57,58]}$ Additionally, several molecular properties are provided by theory. Orbital populations, HOMO/LUMO diagrams, and charges may be interpreted. ${ }^{[59,60]}$ Even the existence of so far unknown compounds can be predicted and stimulate the synthetic chemist to realise them.

Moreover, the electron density distribution derived from the corresponding wave functions can be analysed according to R. F. W. Bader's Quantum Theory of Atoms in Molecules (QTAIM). ${ }^{[61]}$ This separates a given molecule into single atomic units, which enables an atom-centred interpretation of molecular interactions. The origin of the investigated electron density distribution (EDD) is indeed irrelevant under the heading of QTAIM. The EDD can also be derived from experimental data. These originate from high resolution $X$-ray diffraction experiments and permit the accurate determination of the molecular electron density distribution in the crystal. Thus, a more detailed inspection of molecular interactions beyond bond lengths and angles is facilitated. Even the determination of real atomic connectivities in a molecule can be accomplished, independent from ion or van-der-Waals radii.

In recent years it was stated that the experimental charge density determination is not in the fledgling stages anymore. ${ }^{[62,63]}$ In the $1990 \mathrm{~s}$, the method was limited to a selected group of people who had access to powerful large X-ray facilities (i.e. synchrotron beam lines) with accurate detectors, could afford the extraordinary long measuring times per compound (including the expensive crystal coolant), and had fast computers to do the calculations. Moreover, the application was limited to small organic molecules. Nowadays, novel brilliant and intense X-ray sources, X-ray mirror optics and improved CCD detectors facilitate the collection of high-resolution data even with in-house facilities (Figure 1-6). This allows the examination of highly reactive, labile compounds with interesting bonding situations at a known workplace with established equipment to handle these crystals. 


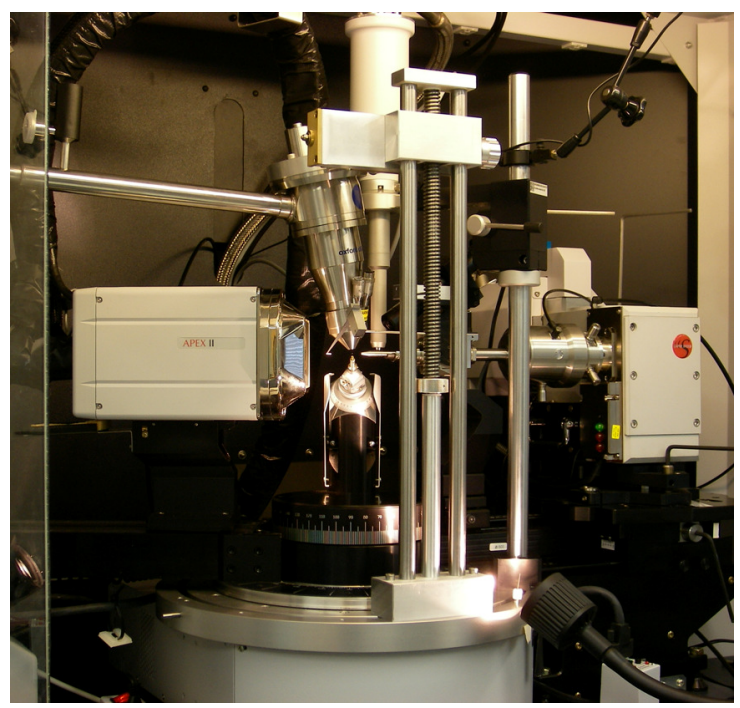

Figure 1-6. BRUKER TXS Mo-rotating anode equipped with INCOATEC HELIOS mirror optics, APEX II CCD detector, D8 three-cycle goniometer, and an OXFORD DIFFRACTION HELIJET, for helium open stream crystal cooling at the research group of $D$. Stalke in Göttingen.

Remarkable discoveries have been made using experimental charge density studies. Among those were studies focussing on the multi-centre bonds in borane clusters, ${ }^{[64,65]}$ banana bonds in strained ring systems, ${ }^{[66-69]}$ compounds long believed to be hypervalent ${ }^{[70-72]}$ and metal-metal bonding, ${ }^{[73,74]}$ just to mention a few examples. Consequently, this method is now being employed to answer several questions of fundamental and applied chemical interest as well as in the revision of various established chemical models. This development was and is still funded since 2005 by the Deutsche Forschungsgemeinschaft (DFG - German research association) with the priority programme 1178 "Experimental charge density as the key to understand chemical interactions" under the coordination of $D$. Stalke. A network of experts cooperate in this context to elaborate topics in life science, functional materials, catalysis, and basic chemical concepts. ${ }^{[75]}$

The method of experimental charge density determination is a well suited tool to investigate the nature of bonding in lithium organic compounds. The discussion about the interactions in $\mathbf{R L i}$ aggregates is as old as the substance class itself and no ultimate consensus has been found yet. The $\mathrm{Li}-\mathrm{C}$ bond characteristics are under constant debate, even though a highly polarised character is commonly accepted. Several high-profile standard textbooks ${ }^{[2,76,77]}$ go even further and state the prevailing ionic character of lithium-carbon bonds; others also use molecular orbital or hybridisation models to explain the interactions. ${ }^{[14,78]}$ Textbooks mainly focussing on synthetic applications even seem to avoid this topic and hardly spend pages on it, $^{[17,18]}$ albeit the understanding of these interactions is essential to estimate the reactivity. 
Unfortunately, it is impossible to exactly quantify the degree of covalent or ionic character and the polarisation of a bond in a given substance, because neither attribute is a real observable. These characteristics are properties, which can only be deduced from the electron density distribution within the framework of a given theory and do therefore always depend on the applied approximations, simplifications, and basic definitions (e.g. the definition of an atom in QTAIM or the separation of a molecular wave function into atomic contributions).

Experimental evidence already stated above actually point to a covalent description of the $\mathrm{Li}-\mathrm{C}$ bonds, such as the good solubility in hydrocarbons and quite pronounced lithium-carbon NMR coupling constants. This interpretation was supported for a long time by early quantum chemical calculations, in which the Mulliken population analyses showed large overlap populations and just small opposite charges on lithium and carbon. ${ }^{[79]}$ Contrarily, lithium aggregates were theoretically reproduced by simple electrostatic models and, as a consequence, the ionic character of these compounds was stated. ${ }^{[60,80,81]}$ Other theoretical studies analysed the electron density in the bonding region, which is tending towards zero, or determined integrated charges of around +0.8 to +0.9 e at the lithium atom from two-dimensional atomic basins (integrated projected populations, IPP), thus strengthening the ionic description of the $\mathrm{Li}-\mathrm{C}$ bonds. ${ }^{[79]}$ Also the energy comparison of an enforced ionic structure with the ground state of the molecule results in the conclusion of highly ionic bonds. ${ }^{[82]}$ The calculation of ${ }^{1} J(\mathrm{C}, \mathrm{Li})$ NMR coupling constants by the same procedure came to a similar result. ${ }^{\left[{ }^{83]}\right.}$ As a consequence of various studies, A. Streitwieser, P. v. R. Schleyer et al. suggested in 1995 that "the carbon lithium bond in theory and in chemical properties can be modelled as an essentially ionic bond". $\left.{ }^{79}\right]$

Nevertheless, it is doubtful that carbanions, possessing pronounced electron density accumulated in the lone pair region, interact without a back donation of electron density to positively charged lithium atoms with vacant second shell orbitals. Thus, minor covalent contributions are most probably involved in the $\mathrm{Li}-\mathrm{C}$ bonding as well. But a molecular orbital representation of e.g. a tetrameric lithium organic compound showing $\mathrm{sp}^{3}$ hybrid orbitals forming $\mathrm{Li}_{4} \mathrm{MOs}$ is equally doubtful, since Raman, IR, and NMR results show diminishing Li-Li bonding. ${ }^{[79]}$ Yet a related $\mathrm{MO}$ model of radical $\mathrm{Me}_{4}$ and $\mathrm{Li}_{4}$ SOMO interactions in methyllithium results in an increase of covalency of $\mathbf{R L i}$ upon tetramerisation. ${ }^{[84]}$ F. M. Bickelhaupt et al. thus emphasised the covalent character of $\mathrm{Li}-\mathrm{C}$ compounds in recent years $^{[84,85]}$ and the discussion of ionic vs. covalent character of these bonds was fuelled again. 
Hence, experimental results from charge density studies already have contributed to this debate. Yet solely two investigations of lithium organic compounds are published in that field so far. W. Scherer et al. studied lithium hydrogen agostic interactions and negative hyperconjugation effects in a silylsubstituted lithium organic compound with close $\mathrm{Li}-\mathrm{H}$ contacts. In this joint neutron and X-ray diffraction project, they were able to show that the $\mathrm{Li}-\mathrm{H}$ orientation under investigation does not originate from agostic interactions. ${ }^{[86,87]}$ But almost no attention was paid to the Li-C bond. S. Deuerlein in the research group of $D$. Stalke has selected a lithium sulfur ylide (Figure 1-7) to investigate the bonding situation in one of the dominant structural motifs in lithium organic chemistry - the interaction of a formal carbanion with a lithium triangle. ${ }^{[88]}$

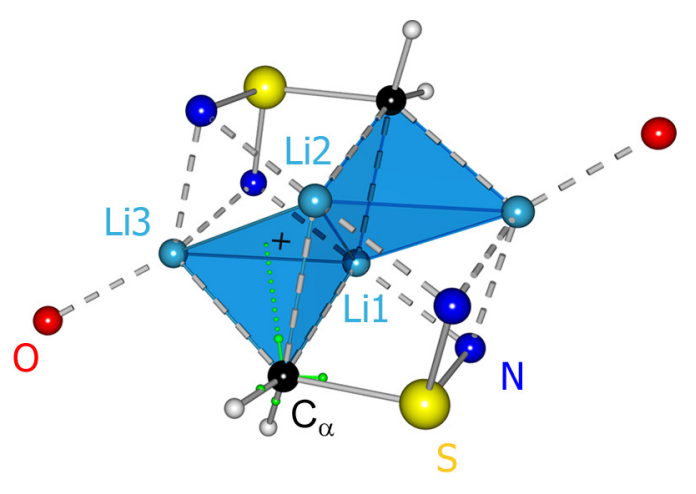

a)

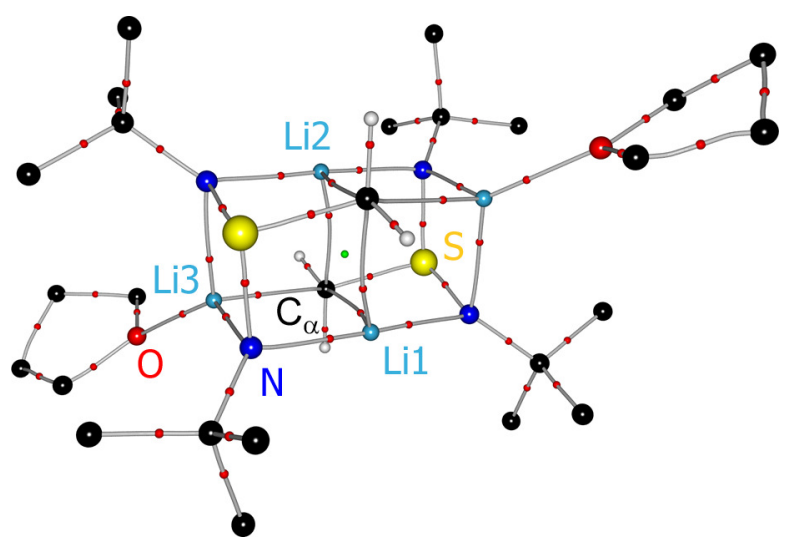

c)

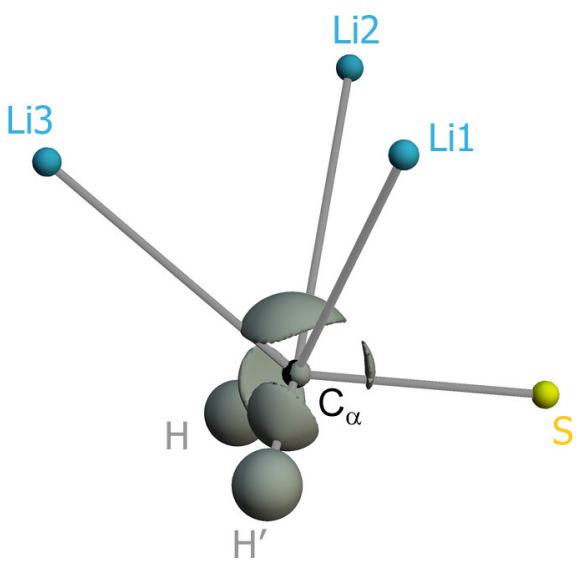

b)

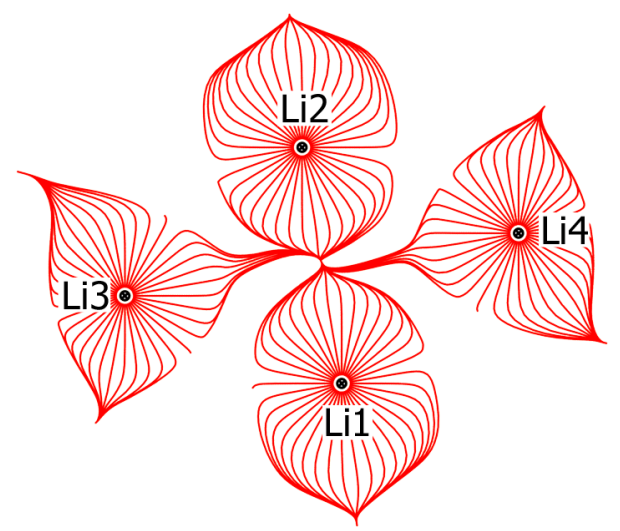

d)

Figure 1-7. Results of the charge density study on [(thf) $\left.\mathrm{Li}_{2}\left\{\mathrm{H}_{2} \mathrm{CS}(\mathrm{NtBu})_{2}\right\}\right]_{2}:$ a) VSCC orientation (green) at one ylidic carbon atom; b) isosurface representation of $\nabla^{2} \rho(\mathbf{r})$ with a lone pair facing a lithium triangle; c) molecular graph with determined chemical bonds; d) lithium basins showing no attractive metal-metal interactions. 
It was shown that a distinct charge accumulation associated with a lone pair at the negatively charged deprotonated carbon atom $\left(\mathrm{C}_{\alpha}\right)$ faces the centre of the lithium triangle (Figure 1-7, $a+b$ ) and that three bonds of the carbon atom to all lithium atoms are present, which were interpreted as strongly polarised (Figure 1-7, c). Moreover, no attractive $\mathrm{Li}-\mathrm{Li}$ interactions were found (Figure 1-7, d). Hence, first experimental charge density contributions to lithium organic chemistry have been made, but more studies are definitely necessary to gain further insights into this fascinating substance class. This was one major intention of this work. The application of low-temperature high-resolution X-ray experiments to the fascinating and challenging class of lithium organic compounds should give direct, experimental insight into the structure- as well as reactivity-determining distribution of the molecular electron density. The second aim was to find reasonable synthetic routes to picolyl metal compounds and their structural characterisation. 



\section{Anionic Picolyl Metal Compounds}

\subsection{Introduction}

While the general introduction summarised major applications of lithium organic compounds, this chapter focuses mainly on picolyl metal species and related topics. This class of substances is highly useful in substitution and metal exchange reactions. Especially in ligand design and complex formation, picolyllithium compounds are in use. Minor attention has been paid to magnesium and the higher alkaline metal compounds.

Picoline (also known as methyl pyridine) exists in three isomers (2-, 3-, and 4-PicH) which differ in their proton acidity at the methyl side chain. A study on the reaction of 2 - and 4 -picoline $(2-/ 4-\mathrm{PicH})$ with potassium amide in liquid ammonia proved that 4-picoline is more acidic at the methyl group than the 2-isomer. $p K_{a}$ values of about $29(4-\mathrm{PicH})$ and $31(2-\mathrm{PicH})$ were calculated for these substances. ${ }^{[89]}$ A redetermination of these values in thf solutions by R. R. Fraser et al. showed the same tendency (2-PicH: 34; 4-PicH: 32.2) and completed the series with the $p K_{a}$ value of 37.7 for 3 -picoline. ${ }^{[90]}$ Thus, it was stated that even the "low" basicity of lithium di-iso-propylamide should lead to the complete deprotonation of 2- and 4-picoline. The acidity of 2-/4-picoline is thus higher than that of toluene, for which values between 35 and 41 were measured (depending on the method of carbon acidity determination). ${ }^{[91]}$ A sidechain deprotonation with standard lithium organic compounds is thus feasible and enables the functionalisation of this valuable $N$-heterocycle.

Indeed, the deprotonation was described as early as in the first half of the $20^{\text {th }}$ century. K. Ziegler and $H$. Zeiser used phenyllithium and methyllithium in 1931 to generate 2-picolyllithium (2-PicLi; $\mathbf{A}$ in Scheme 2-1) which readily reacts with halocarbons under alkylation. ${ }^{[92]}$ F. W. Bergstrom reported on the reaction of 2-pi-
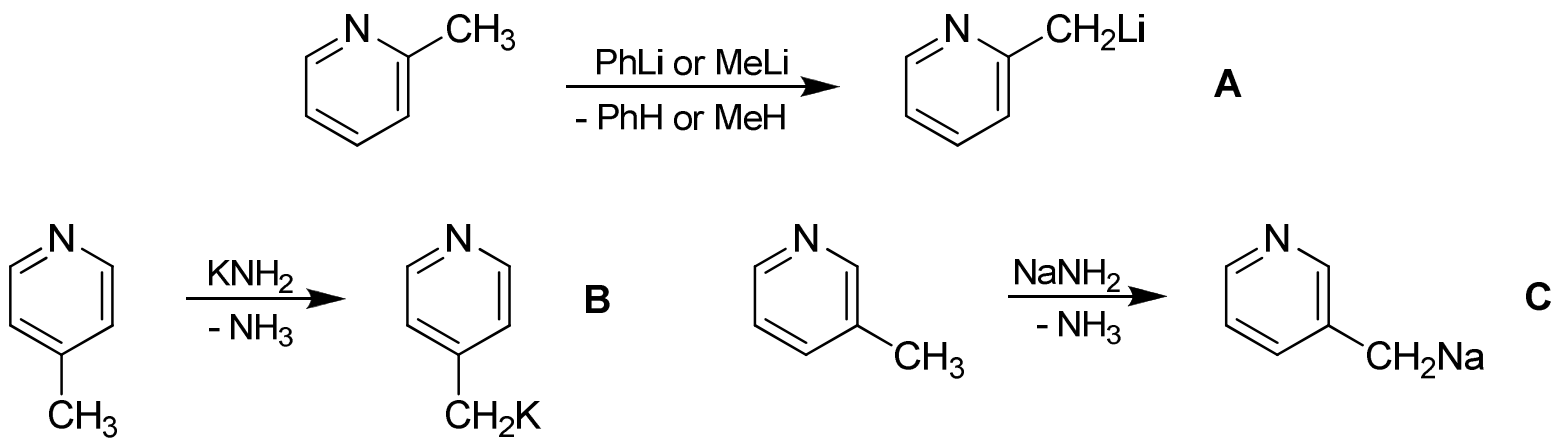

Scheme 2-1. Synthesis of the first picolyl alkaline metal organic compounds. 
coline with potassium amide in the same year, ${ }^{[93]}$ while $A$. E. Chichibabin made use of sodium amide to generate the 2-picolylsodium compound 5 years later. ${ }^{[94]}$ Subsequently, the research was expanded to metallated 4-picolyl compounds, first mentioned by F. W. Bergstrom et al. in 1945. ${ }^{[95]}$ Again, they applied potassium amide for the deprotonation ( $B$ in Scheme 2-1) and substituted the potassium organic compound successfully, sometimes even in excellent yields. In 1951 H. C. Brown and W. A. Murphey reported the first 3-picolyl metal compound (3-PicM) generated from sodium amide (C in Scheme 2-1). ${ }^{\text {966] }}$

Among the metallated picoline derivatives, again the lithium compounds are the most important ones (cf. chapter 1) and in the middle of the 1970s, two publications established standard synthetic routes. $n$-Butyllithium was used to deprotonate 2-/4-picoline in a thf solution, ${ }^{[97]}$ whereas a $1: 1$ mixture of lithium di-iso-propylamide and hexamethylphosphoric triamide in thf was applied for 3-picoline. ${ }^{[98]}$<smiles>CCc1ccccn1</smiles>

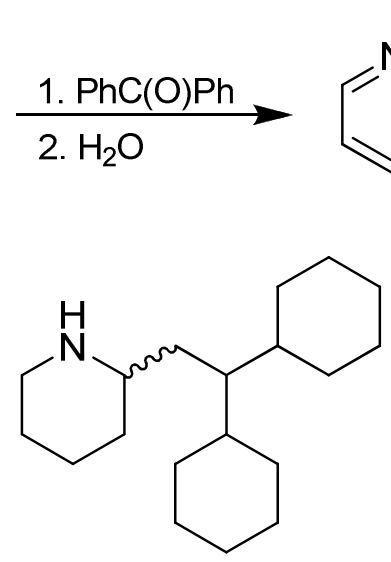<smiles>OC(Cc1ccccn1)(c1ccccc1)c1ccccc1</smiles><smiles>C[CH]C</smiles><smiles>C(=C(c1ccccc1)c1ccccn1)c1ccccn1</smiles>

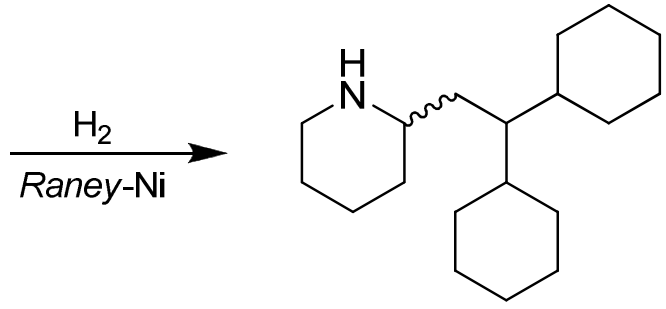

Perhexilin

Scheme 2-2. Synthetic route to Perhexilin.

The synthetic potential of PicM compounds in organic chemistry was established in numerous studies have been well documented. ${ }^{[99-102]}$ Therefore, only one example of a pharmaceutical product will be presented here. The synthesis of the anti-arrhythmic drug Perhexilin starts with the addition of 2-picolyllithium to benzophenone. After hydrolysis, the tertiary alcohol is dehydrated with hydrogen chloride and completely hydrogenated using Raney nickel (Scheme $2-2) .^{[103]}$

It is noteworthy to mention, that also picoline derivatives with additional ring or side-chain substitution can be readily metallated and further functionalised. ${ }^{[99-102]}$ Interestingly, 3-picoline is far less applied in synthesis compared to its two congeners. A reason might be the fact that it is the least acidic picoline and 
the metallation is thus less favoured. In contrast, 2-picolyllithium is the most widely employed picolyl metal compound. It is a valuable building block in organic as well as inorganic ligand synthesis. ${ }^{[104-106]}$ The application of 2-PicLi is an elegant way to introduce pyridyl substituents into a given molecule of electrophilic character.

Alternative synthetic routes are the ring metallation of pure pyridine, either by a metal halide exchang $e^{[17,100]}$ or by the direct deprotonation, ${ }^{[107,108]}$ and an introduction by electrophiles like picolinaldehyde, vinylpyridine, and picolyl halide compounds. Unfortunately, pyridyllithium is a quite labile species and the halide compounds are unstable and can only be stored as hydrogen halide salts. This normally causes problems in the synthesis as the compatibility of many functional groups with strong acids is not given. Even if the picolyl halide is liberated, it strongly tends to polymerise. ${ }^{[109,110]}$ If inorganic atoms such as phosphorus are be incorporated in ligands, other synthetic options than picolyllithium are even more limited, since those atoms are weak nucleophiles.<smiles>c1ccc(P(c2ccccc2)P(c2ccccc2)c2ccccn2)cc1</smiles><smiles>c1ccc(P(Cc2ccccn2)Cc2ccccn2)cc1</smiles><smiles>CN(C)P(=P)(Cc1ccccn1)c1ccccc1</smiles>

Scheme 2-3. Inorganic ligands containing the picolyl fragment.

Prominent inorganic ligand systems containing picolyl groups are phenyldipicolylphosphane $\left(\mathrm{PhPPic}_{2}\right)^{[111-114]}$ and diphenylpicolylphosphane $\left(\mathrm{Ph}_{2} \mathrm{PPic}\right){ }^{[115-117]}$ which can be further functionalised at phosphorus by a Staudinger reaction ${ }^{[118]}$ with trimethylsilyazide to give $\mathrm{Ph}_{2} \mathrm{PicPNSiMe}_{3}{ }^{[71,119,120]}$ (Scheme 2-3). Thus, multidentate ligands with donor sites differing in their donor strength and Pearson hardness ${ }^{[5,6]}$ are accessible by addition of picolyllithium to the corresponding chlorophosphanes. These ligands can even have hemilabile character, ${ }^{[121,122]}$ which is of importance in catalysis. A weakly bound side chain can provide a reaction site for a given substrate and stabilise the catalytically active metal centre if no reaction at the metal atom takes place. Hence, several metal complexes with a phosphorus based picolyl backbone with catalytic properties are known. ${ }^{[123-125]}$ A recent example of such a potent catalyst is a bis(2-picolyl)phenylphosphane nickel dichloride reported by $P$. Braunstein et al., which showed a reasonable activity in ethene oligomerisation reactions. ${ }^{[111]} \mathrm{Ph}_{2} \mathrm{PPic}$ and $\mathrm{PhPPic}_{2}$ also have the ability to act as Janus Head ligands. ${ }^{[126-128]}$ Their donor sites can 
bind two different metal atoms. The softer metal is complexed by the phosphorus atom and the second harder one by the nitrogen atom. Thereby, it may be possible to incorporate the catalytic metal and a co-catalyst in close proximity in the same complex. The activity is dramatically enhanced in a synergetic way.

A direct linkage to other metal or main group atoms via the methylene group can also be realised with picolyl metal compounds. The picolyl unit was transferred via the lithium as well as the potassium precursor to zirconium tetrachloride to yield a catalyst complex ( $\left.\mathrm{Pic}_{2} \mathrm{ZrR}\right)$ for ethylene polymerisation after an additional ligand exchange. ${ }^{[129]}$ Further functionalisations at the methyl side chain are reported for a picolyl yttrium complex which readily inserts nitriles in the $\mathrm{Y}-\mathrm{C}$ bond. ${ }^{[130]}$ Moreover, several group 11-15 complexes were prepared with lithiated trimethylsilylmethylpyridine or bis(trimethylsilyl)methylpyridine and are well elaborated as well as reviewed by C. L. Raston ${ }^{[131]}$. These ligands, synthesised from unsubstituted PicLi, proved to have benevolent properties in the kinetic stabilisation of main group and transition metal alkyl complexes. ${ }^{[131,132]}$
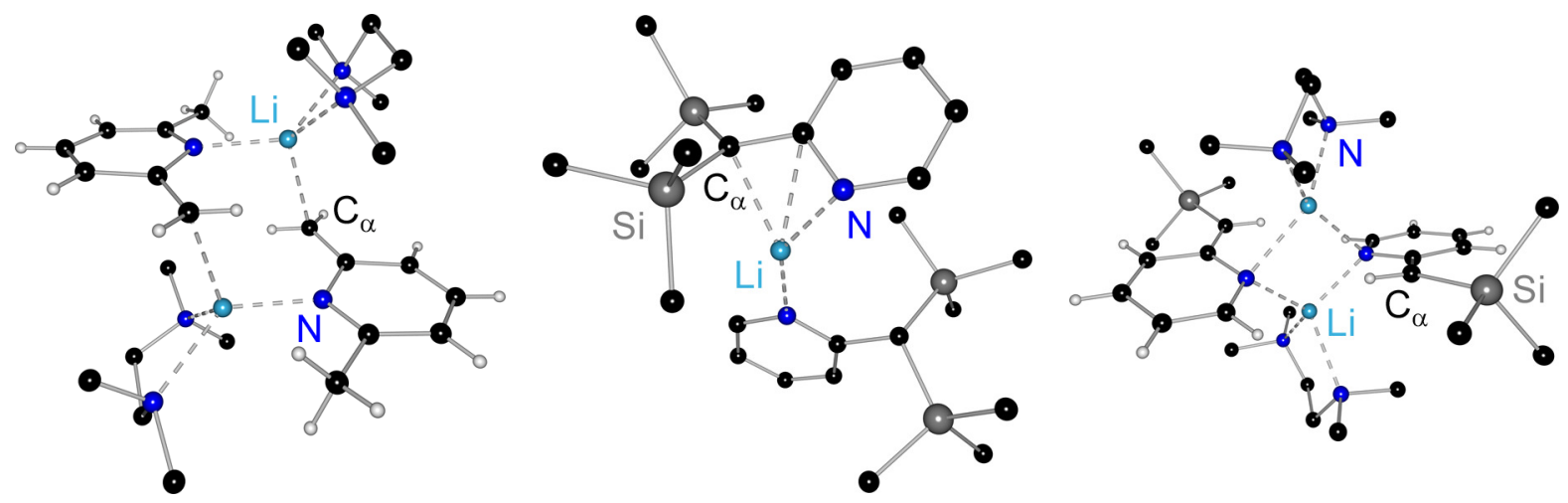

Figure 2-1. Bonding motifs of 2-picolyllithium derivatives: $\eta^{1}$-alky[ ${ }^{[133]}$ (left), $\eta^{3}$-azaallyl ${ }^{[134]}$ (centre) and $\eta^{1}$-enamide donor (right). ${ }^{[135]}$

Interestingly, the unsubstituted picolyl alkaline metal compounds were prepared in situ and instantly derivatised in almost all reported studies. The only exceptions (PicLi isolation) can be found in the pioneering NMR studies of $K$. Takahashi et al. on picolylic carbanions ${ }^{[136-141]}$ and the zirconium complex mentioned above. ${ }^{[129]}$ However, side-chain silylated methylpyridyllithium compounds for example were received as highly crystalline materials and structurally investigated in the presence of various donor atoms. ${ }^{[131]}$ Thus, the only experimental information in literature about picolyl alkaline metal aggregation is known from derivatives (Figure 2-1). 
These aggregates can be assigned to three different main structural bonding motifs. The following section will highlight key issues of the lithium species. Higher homologues are only mentioned if necessary. First, the picolyl anion can interact with the lithium atom as a $\eta^{1}$-alkyl donor and the nitrogen atom of a second anion serves as an intermolecular donor. The resulting dimer is in consequence consistent with the carbanionic resonance formula of the picolyl anion (A in Scheme 2-4).

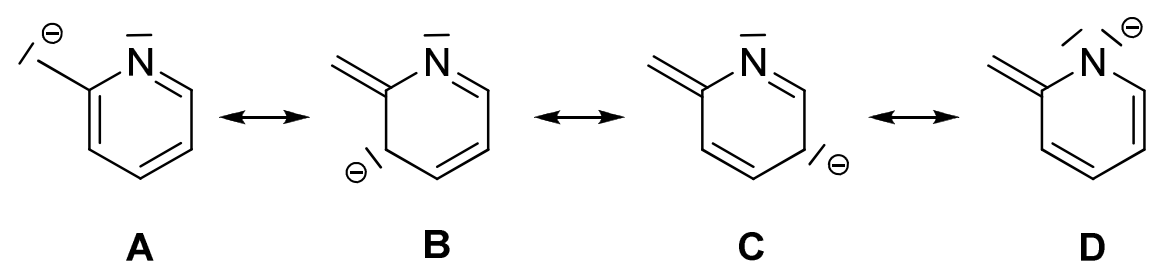

Scheme 2-4. Resonance formulae of the 2-picolyl anion

This aggregation mode was found in a 6-methyl-2-picolyllithium tmeda adduct $^{[133]}$ and in the bis(trimethylsilyl)(2-pyridyl)methyllithium ${ }^{[135,142]}$ dimer $\left(\left[2-\left(\mathrm{Me}_{3} \mathrm{Si}\right)_{2} \mathrm{CPyLi}\right]_{2}\right)$, in which the metal atom is only dicoordinated, possibly due to the steric demand of the silyl groups, which hampers the interaction of additional solvent molecules to the lithium cation. Second, a $\eta^{3}$-aza-allylic contact can be formed to the metal atom, either in monomeric or dimeric molecular structures. Monomers arise if the side-chain groups are rather bulky, as it is the case in [2-( $\left.\mathrm{Me}_{3} \mathrm{Si}\right)_{2} \mathrm{CPyLi}$ ttmeda] and [2-( $\left.\mathrm{Me}_{3} \mathrm{Si}\right)_{2} \mathrm{CPyLi} \cdot\left(\mathrm{Me}_{3} \mathrm{Si}\right)_{2} \mathrm{CHPy}$, or a sterically demanding donor is added such as (-)-sparteine (sp) in the monosilylated compound [2-(Me $\left.\left.{ }_{3} \mathrm{Si}\right) \mathrm{CHPyLi} \cdot \mathrm{sp}\right] .{ }^{[135]}$ This coordination type can be interpreted as an intermediate case between the carbanionic (A) and the enamidic (D) resonance formula (Scheme 2-4). Third, a dominant enamidic dimerisation is possible, in which no obvious interaction between the deprotonated carbon and the metal atom can be observed (D in Scheme 2-4). Examples are [2-(Me3Si)CHPyLi.tmeda $]_{2}^{[135]}$ and the phenyl(2-pyridy)। methyllithium tmeda dimer. ${ }^{[143]}$ This is also the favoured coordination mode for the trimethylsilyl(2-pyridyl)methylsodium and -potassium pmdeta aggregates. ${ }^{[144]}$

Structural investigations of 4-picolyllithium compounds are in contrast to those of the 2-isomer scarce. To the best of the author's knowledge, there are only two studies published, both by $E$. Anders et al. They could show that the metallation of ethyl- and iso-propylpyridine with di-iso-propylamide results in dimers (4-MeCHPyLi and 4-Me ${ }_{2} \mathrm{CPyLi}$ ) that are exclusively linked via the pyridine 
nitrogen atoms. ${ }^{[145,146]}$ This was postulated before by NMR studies on the metallation of 4-picoline with $n$-butyllithium and the competing addition of the metallation reagent. ${ }^{[141,147,148]}$ In the case of 3-picolyllithium and its derivatives, no molecular structure is known so far.

The focus of this work was set on unsubstituted picolyl metal compounds, since additional substituents on the side-chain increase the steric bulk dramatically. This is desirable in stabilising labile metal complexes (cf. ref. [131]) but a nucleophilic attack on a substrate will be consequently disfavoured. Both facts cannot be tolerated in ligand design, especially if a hemilabile character is desired. Unfortunately, the in situ preparation and derivatisation of picolyl metal compounds leads to product yields that range from very poor to excellent, which cannot be explained in a straightforward way. It can possibly originate from byproduct formation during the metallation, incomplete deprotonation or decomposition of the highly reactive deprotonated compounds. Moreover, PicM can be too reactive so that a degradation of the electrophile occurs or the reactivity is too low and only an incomplete conversion is observed. Thus, it is essential to isolate the picolyl metal compound, preferably as a crystalline solid of known composition, before further conversions will be undertaken. This also enables the storage of the valuable precursor. Additionally, the aggregation and influence of donor solvents can be understood from X-ray structure determination in the absence of side-chain substitution effects. Thus, the reactivity of a given solution can be estimated or even actively tuned.
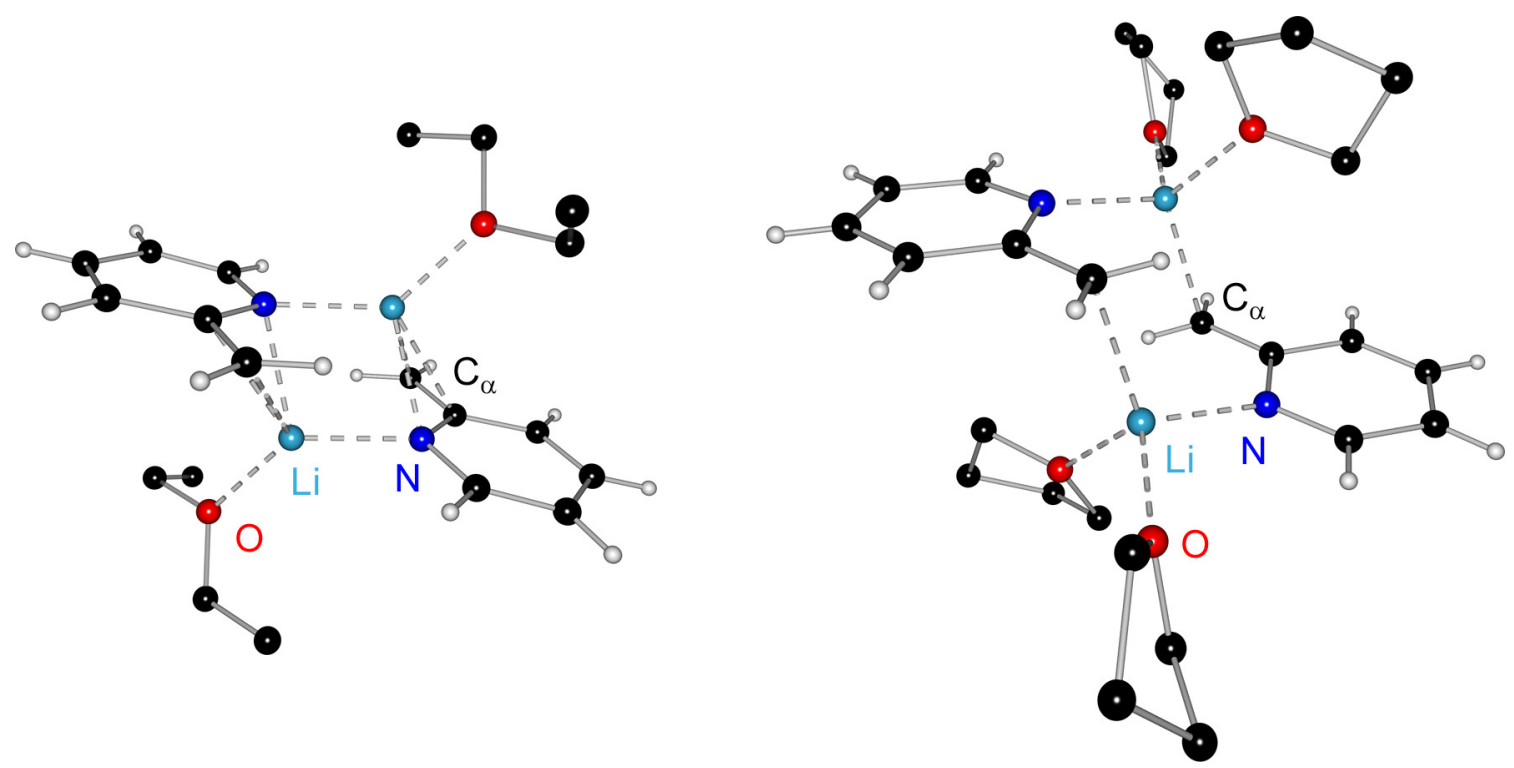

Figure 2-2. 2-Picolyllithium as adducts with diethyl ether (left) and thf (right). 
Initial success in that field were achieved by $U$. Pieper and the author who isolated and crystallised unsubstituted 2-picolyllithium with oxygen ${ }^{[107,149]}$ and nitrogen $^{[149]}$ donor molecules for the first time and gained insight into their aggregation situation (Figure 2-2). Also an unsubstituted 4-picolyllithium compound has been described. ${ }^{[149]}$ Following these results, the research was extended to elaborate adequate and simple syntheses for crystalline picolyl metal compounds in high yields and good purities. Standard structure determinations of these compounds should give direct insight into the coordination pattern of unsubstituted PicLi depending on the applied solvents. Furthermore, an experimental charge density study on an appropriate metallated compound was meant to elucidate the bonding situation in these aggregates more specifically. 


\subsection{Lithiation of 2-Picoline}

\subsection{1 $\quad \eta^{1}$-Carbanionic 2-Picolyllithium Aggregates}

2-Picoline was deprotonated with $n$-butyllithium in a dme/hexane solution at $-78^{\circ} \mathrm{C}$. The resulting brown crude product was recrystallised from diethyl ether at $-24{ }^{\circ} \mathrm{C}$ and bright red crystals were obtained. These were extremely sensitive to air and moisture but suitable for an X-ray structural analysis. They consisted of 2-picolyllithium as the dimethoxyethane adduct (1), which crystallises in the centrosymmetric space group $P 2_{1} / n$ with half of the dimer in the asymmetric unit. The full dimer is generated by an inversion operation at the origin (Figure 2-3).

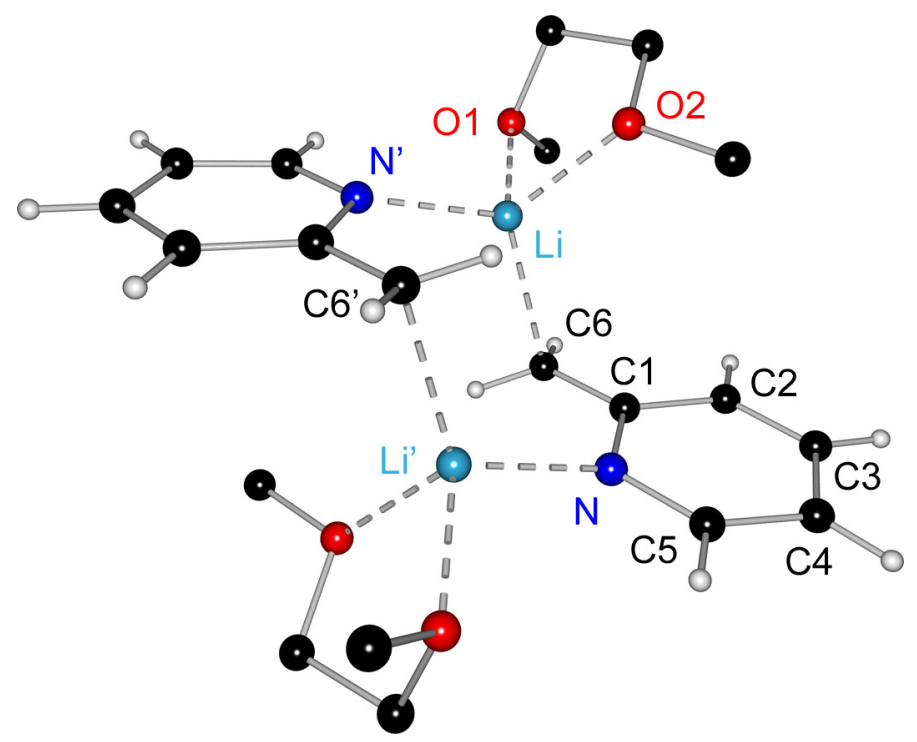

Figure 2-3. Molecular structure of $[2-\mathrm{PiCLi} \cdot \mathrm{dme}]_{2}(\mathbf{1})$. Hydrogen and disordered atoms of the donor molecules are omitted for clarity.

The dimerisation of $\mathbf{1}$ is achieved via two lithium contacts to the picolyl anions. Exemplarily, the lithium atom $\mathrm{Li}$ (inverted for $\mathrm{Li}^{\prime}$ ) bonds to the deprotonated carbon atom $\mathrm{C} 6$, the ring nitrogen atom of the second anion $\left(\mathrm{N}^{\prime}\right)$, and additionally to both oxygen atoms of one dme donor molecule $(01,02)$. Hence, the bidentate donor chelates the lithium cation and does not act as bridging reagent. An eight-membered $\left[\mathrm{LiC}\left(\mathrm{H}_{2}\right) \mathrm{CN}\right]_{2}$ ring is formed in a chair conformation.

The picolyl ring is still planar (mean deviation from ideal plane: $0.01 \AA$ ) after the deprotonation. Both hydrogen atoms at the deprotonated carbon atom $\mathrm{C} 6$ could be identified in a difference Fourier map and were refined with a riding model, but without geometrical constraints. The carbon-hydrogen distance 
refined to reasonable values (0.90(3) and 0.96(3) $\AA$ ) and the hybridisation state at $\mathrm{C} 6$ could be determined according to the hydrogen atom positions. Although an $\mathrm{sp}^{2}$ carbon atom is evident, a slight pyramidalisation towards the cation can be recognised. The hydrogen atoms at $\mathrm{C} 6$ do not ideally reside in the picolyl ring plane (deviation 0.23 and $0.16 \AA$ ) and the sum of the bond angles at $\mathrm{C} 6$ is $355.6^{\circ}$. Moreover, the hydrogen atoms are slightly rotated by $2.5^{\circ}$ relative to the ring plane. This small tilt maximises the interaction of the lithium cation with the lone pair density at $\mathrm{C} 6$ located in a p orbital almost orthogonal to the ring plane, which is in accordance with an $\mathrm{sp}^{2}$ hybridisation. Li forms an angle of $105.5(2)^{\circ}$ with the $\mathrm{C} 1-\mathrm{C} 6$ bond. Thus, it is not positioned on the plane normal through C6, which would result in the best overlap with the $\mathrm{p}$ orbital. In addition, the cation resides closer to the centre of the dimer, due to the interaction with the nitrogen atom of the second picolyl anion $\left(\mathrm{N}^{\prime}\right)$. The lone pair in the ring plane of the nitrogen atom also does not bond the cation in a straight line. Indeed, the lithium atom resides $0.38 \AA$ outside the pyridine ring plane and an angle of $11^{\circ}$ is enclosed with the $\mathrm{Li}-\mathrm{N}^{\prime}$ bonding vector. Nonetheless, the $\mathrm{Li}-\mathrm{N}^{\prime}$ bond length $(2.007(4) \AA)$ is shorter than the Li-C6 bond $(2.231(5) \AA)$. It is worthy of mention, that an additional aza-allylic interaction as alternative bonding mode

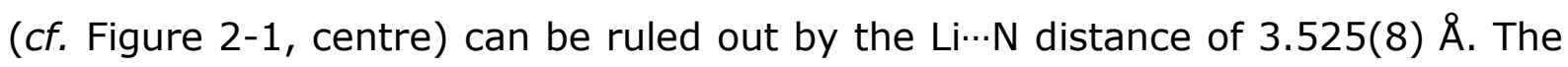
$\mathrm{Li}-\mathrm{O}$ bond lengths vary from $1.996(4)$ to $2.030(4) \AA$.

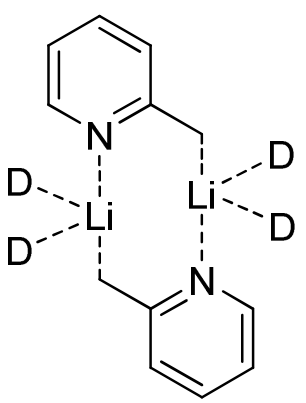

$[\text { PicLi-dme }]_{2}$ (1)

$\left.[\text { PicLi-(thf })_{2}\right]_{2}$

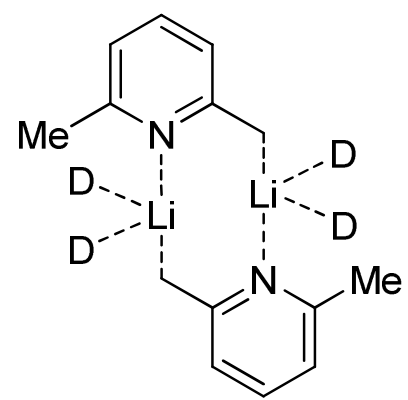

$[6-M e P i c L i \cdot t m e d a]_{2}$

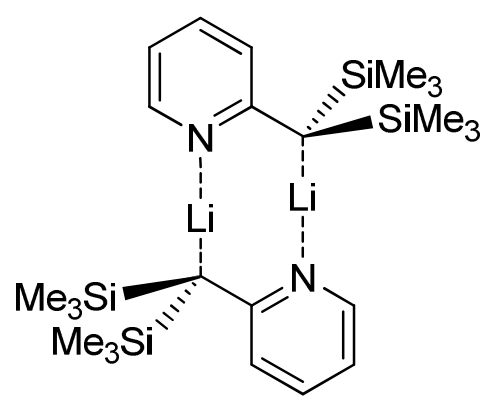

$\left[\left(\mathrm{Me}_{3} \mathrm{Si}_{2}\right)_{2} \mathrm{CPyLi}\right]_{2}$

Scheme 2-5. Lewis formulae of PicLi compounds with an $\eta^{1}$-carbanionic dimerisation pattern $(D=$ coordinating atom of the donor molecule(s); Pic $=2$-picolyl, Py $=2$-pyridyl).

The $\mathrm{Li}-\mathrm{C} / \mathrm{N}$ bond lengths are comparable to reported dimers showing the same bonding motif (Table 2-1). These are the thf adduct, ${ }^{[149]}$ the 6 -MePicLi-tmeda adduct, ${ }^{[133]}$ and $\left[2-\left(\mathrm{Me}_{3} \mathrm{Si}\right)_{2} \mathrm{CPyLi}\right]_{2}$ (Scheme $\left.2-5\right) \cdot{ }^{[135,142]}$ The $\mathrm{Li}-\mathrm{C}$ bond length in $\mathbf{1}(2.231(5) \AA)$ is the shortest in $\mathbf{1}$, the thf $(2.277(3) \AA)$ and the tmeda adduct 
(2.352(5) $\AA$ ). Compound $\mathbf{1}$ also shows the shortest Li-N contact (2.007(4) $\AA$ ) in this series (thf: $2.028(2) \AA$; tmeda: 2.075(4) $\AA$ ). The Li-O bonds in $\mathbf{1}$ are slightly longer than those in the thf adduct $(1.974(3), 1.982(2) \AA)$ but well in the range of other lithium organic compounds with dme donors (1.96-2.12 $\AA$; mean $2.01 \AA) \cdot{ }^{[48]}$

Table 2-1. Selected bond lengths $[\AA]$ and angles $\left[^{\circ}\right]$ in $[\mathrm{PicLi} \cdot \mathrm{dme}]_{2}(\mathbf{1})$ and related compounds $\left(\mathrm{Pic}=2\right.$-picolyl, $\mathrm{Py}=2$-pyridyl). ${ }^{[133,135,149,150]}$

\begin{tabular}{|c|c|c|c|c|c|}
\hline & $\begin{array}{c}{[\mathrm{PicLi} \cdot \mathrm{dme}]_{2}} \\
(\mathbf{1})\end{array}$ & {$\left[\mathrm{PicLi} \cdot(\text { thf })_{2}\right]_{2}$} & $\begin{array}{c}{[6-\mathrm{MePicLi} \cdot} \\
\text { tmeda }]_{2}\end{array}$ & $\begin{array}{c}{\left[\left[\left(\mathrm{Me}_{3} \mathrm{Si}\right)_{2} \mathrm{C}\right.\right.} \\
\mathrm{PyLi}]_{2}\end{array}$ & $\mathrm{PicH}$ \\
\hline $\mathrm{Li}-\mathrm{N}^{\prime}$ & $2.007(4)$ & $2.028(2)$ & $2.075(4)$ & $1.937(6)$ & - \\
\hline $\mathrm{Li}-\mathrm{C} 6$ & $2.231(5)$ & $2.277(3)$ & $2.352(5)$ & $2.213(7)$ & - \\
\hline $\mathrm{N}-\mathrm{C} 1$ & $1.387(3)$ & $1.387(2)$ & $1.381(3)$ & $1.354(3)$ & $1.343(3)$ \\
\hline $\mathrm{N}-\mathrm{C} 5$ & $1.342(3)$ & $1.343(2)$ & $1.365(4)$ & $1.337(4)$ & $1.342(3)$ \\
\hline $\mathrm{C} 1-\mathrm{C} 2$ & $1.440(3)$ & $1.444(2)$ & $1.446(5)$ & $1.414(4)$ & $1.394(3)$ \\
\hline $\mathrm{C} 2-\mathrm{C} 3$ & $1.355(4)$ & $1.351(2)$ & $1.354(3)$ & $1.361(3)$ & $1.384(3)$ \\
\hline C3-C4 & $1.409(4)$ & $1.402(2)$ & $1.388(2)$ & $1.369(3)$ & $1.375(4)$ \\
\hline C4-C5 & $1.364(4)$ & $1.375(2)$ & $1.370(5)$ & $1.373(2)$ & $1.379(3)$ \\
\hline $\mathrm{C} 1-\mathrm{C} 6$ & $1.378(3)$ & $1.381(2)$ & $1.380(4)$ & $1.475(4)$ & $1.502(3)$ \\
\hline $\mathrm{Li}-\mathrm{O} 1$ & $2.030(4)$ & $1.982(2)$ & - & - & - \\
\hline $\mathrm{Li}-\mathrm{O} 2$ & $1.996(4)$ & $1.974(3)$ & - & - & - \\
\hline $\mathrm{Li}-\mathrm{C} 6-\mathrm{C} 1$ & $105.5(2)$ & $107.2(1)$ & $105.3(3)$ & $122.2(3)$ & - \\
\hline$N^{\prime}-\mathrm{Li}-\mathrm{C} 6$ & $115.6(2)$ & $111.1(1)$ & $99.7(4)$ & $146.8(4)$ & - \\
\hline
\end{tabular}

Looking at Figure 2-4, it is obvious that there is hardly any difference between the dme and the thf dimer concerning the picolyllithium part. Nevertheless, the steric demand and influence of the donor molecules on the lithium bonds can be estimated. Both thf donor molecules attached to one lithium cation can bind closer to the metal atom than the non-flexible dimethoxyethane ligand, as they reduce their steric repulsion by a ring orientation in which the planar ring sides face each other. Thus, the donation to the cation is enhanced, it is better stabilised by the donor atoms, and the Li-N/C bond lengths become longer. This is even visible in Figure 2-4, in which the lithium atoms - represented by transparent spheres - of the thf adduct are positioned further outside the ring relative to the centre of inversion. The dme molecule is more rigid and the ethylene bridge prevents an ideal orientation of the oxygen atom lone pairs towards the cation 


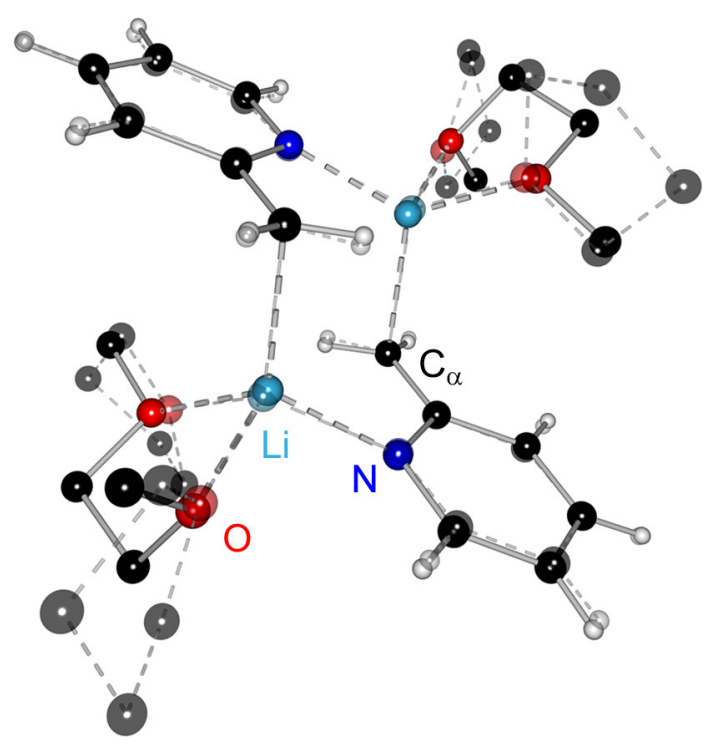

Figure 2-4. Molecular structure representation of [2-PicLi-dme $]_{2}(\mathbf{1}$, solid) and [2-PicLi. $\left.(\text { thf })_{2}\right]_{2}$ (dashed and transparent). Superposition reference atoms were those of the eight-membered rings. Hydrogen atom of the donor molecules are omitted for clarity.

and hence a closer contact to the lithium atom. In this case, the cation is consequently more closely bonded by the picolyl anions.

The disilylated picolyllithium (Figure 2-5), ${ }^{[142]}$ however, shows significant variations compared to $\mathbf{1}$. The bonds to the lithium atoms are much shorter ( $\mathrm{Li}-\mathrm{N}: 1.937(6) \AA ; \mathrm{Li}-\mathrm{C}: 2.213(7) \AA$ ). This can be attributed to the stabilising effect of both trimethylsilyl groups on the carbanion, ${ }^{[151]}$ which leads to an $\mathrm{sp}^{3}$ hybridised methylene carbon atom with a lone pair directed to the lithium atom.

Moreover, the silyl groups prevent the coordination of additional donor solvent molecules that would coordinatively saturate the cation. The bulky silyl groups

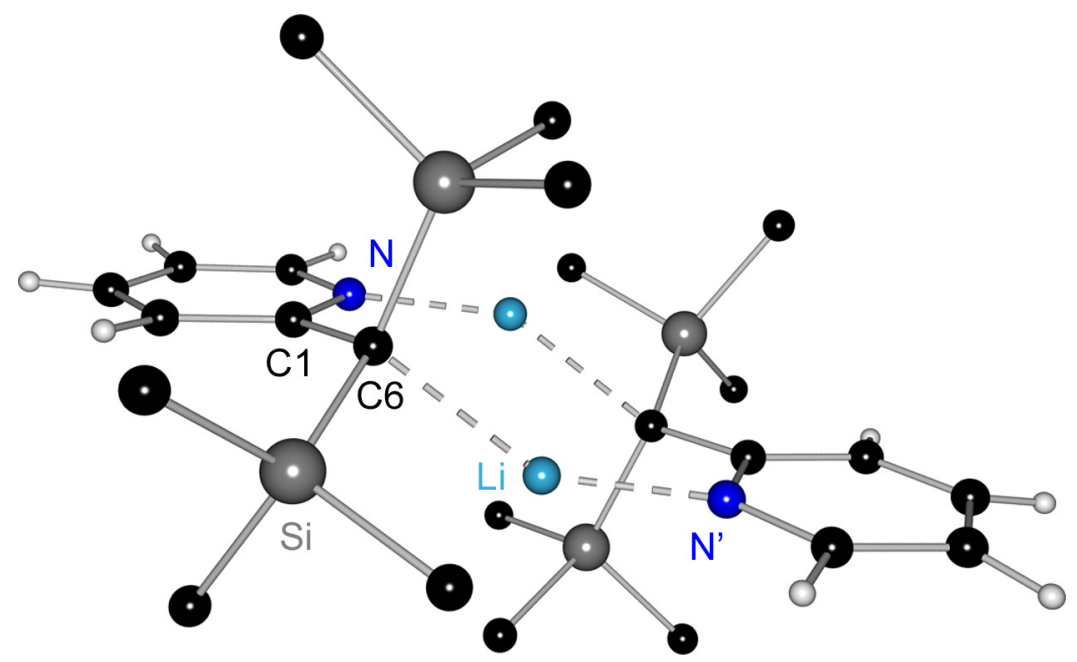

Figure 2-5. Molecular structure of the bis(trimethylsilyl)(2-pyridyl)methyllithium dimer $\left(\left[2-\left(\mathrm{Me}_{3} \mathrm{Si}\right)_{2} \mathrm{CPyLi}\right]_{2} ;\right.$ hydrogen atoms of methyl groups are omitted for clarity). 
effectively shield the dicoordinated lithium atom, and shorter bonds to the carbanion and the pyridine nitrogen atoms are formed to compensate for the extraordinary low number of donor atoms. This steric demand also results in larger bond angles at the lithium ( $\mathrm{N}^{\prime}-\mathrm{Li}-\mathrm{C} 6: 146.8(4)^{\circ}$ vs. $115.6(2)^{\circ}$ in $\left.\mathbf{1}\right)$ and the deprotonated carbon atom ( $\mathrm{Li}-\mathrm{C} 6-\mathrm{C} 1: 122.2(3)^{\circ}$ vs. $105.5(2)^{\circ}$ in 1). As a result, the parallel picolyl rings in the disilylated dimer are much closer to each other (plane distance: $1.42 \AA$ ) compared to 1 (plane distance: $2.55 \AA$ ).

By the first glance on the molecular structure, one might conclude that the dimethoxyethane adduct $\mathbf{1}$ is clearly a dimerised carbanion. The lone pair at the methylene carbon atom has a p orbital shape and bonds to the lithium cation, while the pyridine nitrogen atom acts as an amine donor. However, the bond lengths in the picolyl ring favour a different model view. A molecular structure determination of 2-picoline (2-PicH) at $120 \mathrm{~K}^{[150]}$ shall serve as reference system for the undisturbed starting material. The $\mathrm{N}-\mathrm{C}$ bonds in 2-PicH are equal in length $(1.343(3) \AA$ ), while the $C-C$ bond distances vary from $1.375(4)$ to $1.394(3) \AA$ without a detectable systematic pattern. An intact $N$-heteroaromatic system can be assumed. The bond distance to the methyl group is 1.502(3) $\AA$ which is almost the value for a standard $\mathrm{C}_{\mathrm{sp} 3}-\mathrm{C}_{\mathrm{sp} 2}$ single bond (1.510 $\AA$ ). ${ }^{[152]}$

The deprotonation of the methyl group has tremendous effects on the bonding situation in $\mathbf{1}$ (Table 2-1). Above all, the methylene bond length (C6-C1) is dramatically shortened $(1.378(3) \AA)$ and much closer to a standard double bond length $\left(C_{\mathrm{sp} 2}=\mathrm{C}_{\mathrm{sp} 2}: 1.335 \AA\right)$ than to a corresponding single bond $\left(\mathrm{C}_{\mathrm{sp2} 2}-\mathrm{C}_{\mathrm{sp2} 2}\right.$ : $1.466 \AA) .{ }^{[152]}$ Thus, the delocalised $\pi$-system is expanded to the deprotonated carbon atom C6. This also affects the aromatic system that is now seriously disturbed. The $\mathrm{N}-\mathrm{C} 1$ bond becomes longer (1.387(3) $\AA$ vs. N-C5: 1.342(3) $\AA$ ) and a strong trend towards the localisation of the double bonds is evident. The C2-C3 (1.355(4) $\AA$ ) as well as C4-C5 (1.364(4) $\AA$ ) bond lengths are shorter than in 2-picoline indicating additional double bond character. The $\mathrm{C} 1-\mathrm{C} 2$ and $\mathrm{C} 3-\mathrm{C} 4$ bond lengths on the other hand are elongated (1.440(3) and 1.409(4) $\AA$ respectively) and show a reduced bond order. Hence the preference of the enamidic resonance formula ( $\boldsymbol{D}$ in Scheme 2-4) can be deduced from the analysis of the picolyl bond lengths. A large delocalisation of the negative charge into the ring system leading to a charge concentration at the nitrogen atom seems to describe the electronic situation best. This comes along with a partial loss of aromaticity in the pyridine ring.

The disilylated 2-pyridylmethyllithium compound $\left[2-\left(\mathrm{Me}_{3} \mathrm{Si}\right)_{2} \mathrm{CPyLi}\right]_{2}$ (Figure 2-5), in contrast, shows only little delocalisation of charge into the aromatic ring 
as explained above. The methylene bond length is slightly reduced ( $\mathrm{C} 6-\mathrm{C} 1$ : $1.475(4) \AA$ ) compared to 2-picoline, while the ring bond lengths show little alternations. The lone pair of the carbanion is hence still localised in a $\mathrm{sp}^{3}$ hybrid orbital that interacts slightly with the aromatic system via negative hyperconjugation. ${ }^{[87,153-156]}$

Yet, an unambiguous statement concerning the electron density distribution in 1 cannot be made according to the analysis of the bond lengths. Unfortunately, the crystal quality was not sufficient for a charge density study, because of a slight disorder in the dme donor molecule. Thus, some doubts remain about the enamidic character of $\mathbf{1}$. Several studies of 2-picolyllithium compounds proved that a nucleophilic substitution solely occurs at the methylene carbon atom C6 (cf. chapter 2.1). Moreover, the claimed Li-N amide bond (2.007(4) $\AA$ ) is well in the range found in standard lithium amides $(1.97-2.10 \AA){ }^{[47]}$ which are however, just marginally shorter than a pure Li-N donor bond of non-deprotonated picoline rings in other picolyllithium compounds $\left(2.020(2) \AA^{[149]} ; 2.01(1) \AA^{[134]}\right)$. On the other hand, it can be argued whether the preferred Li-N interactions, which seem to be responsible for the observed dimerisation pattern, even increase the nucleophilicity at the methylene carbon atom. The deprotonated carbon atom is in consequence more loosely bound, which may facilitate an electrophilic attack. 


\subsection{2 $\eta^{1}$-Amidic 2-Picolyllithium Aggregates}

The amine chelating ligand tmeda was used in the deprotonation of 2-picoline with $n B u L i$ to activate the alkyl base by disaggregation. This was done to exclude a competing reaction path of the picoline hydrogen abstraction in which the lithium organic compound is added in 2-position to the nitrogen atom. ${ }^{[141,147]}$ Remarkably, the formation of that by-product (Scheme 2-6, bottom; $\mathrm{R}^{\prime}=$ 2/6-Me) seems to have almost no negative influence on several in situ conversions of 2-picolyllithium. ${ }^{\text {[99-102] }}$ The $\mathbf{R L i}$ addition is the initial step of the analogous Chichibabin reaction with alkyl nucleophiles, which was originally reported for sodium amide and pyridine (Scheme 2-6, top). ${ }^{[157]}$ But it can also be synthetically used in $\mathrm{C}-\mathrm{C}$ bond formations at heteroaromatic compounds, first shown by $K$. Ziegler and $H$. Zeiser with pyridine and $n B u L i^{[158]}$ (Scheme 2-6, bottom).

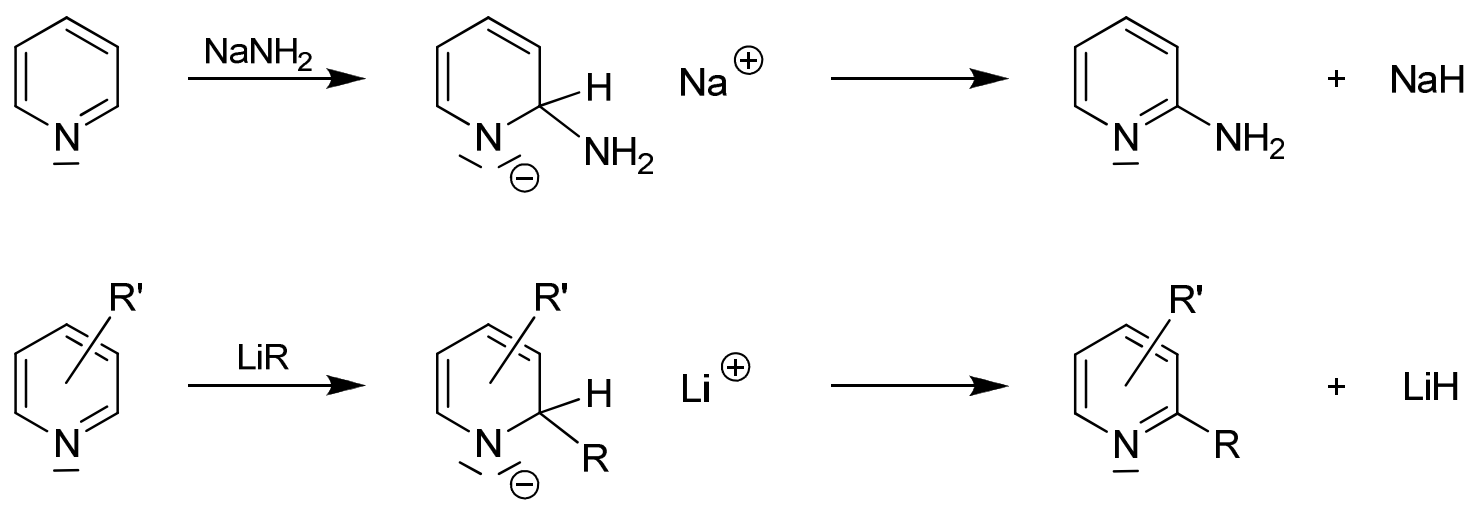

Scheme 2-6. Original Chichibabin reaction (top) and alkyl analogue (bottom).

Deprotonation of 2-picoline with $n B u L i$ and two equivalents of tmeda in hexane at $-78{ }^{\circ} \mathrm{C}$ resulted in an orange suspension. Recrystallisation of the isolated solid from hexane yielded red crystals. These consisted of a 2-picolyllithium.tmeda dimer that crystallises in the chiral monoclinic space group $P 2_{1}$ (Figure 2-6). The central structural motif is an almost planar four-membered [LiN] $]_{2}$ ring (mean deviation from plane: $0.015 \AA$ ). Each lithium atom $\mu$-bridges the picolyl anions and is coordinated by one tmeda donor. Four types of lithium-nitrogen bonds can be identified. The N1/2-Li1 bonds are 2.229(2) $\AA$ and 2.194(2) $\AA$ in length and accordingly much longer than those to Li2 (N1: 2.048(2) $\AA$, N2: 2.063(2) $\AA$ ). The same tendency can be found among the $\mathrm{Li}-\mathrm{N}$ distances to the tmeda donor (Table 2-2). Slightly longer bonds are formed to Li1 (N3: 2.211(2) $\AA$, N4: 2.201(2) $\AA$ ) than to Li2 (N5: 2.160(2) $\AA$, N6: 2.193(2) $\AA$ ). 


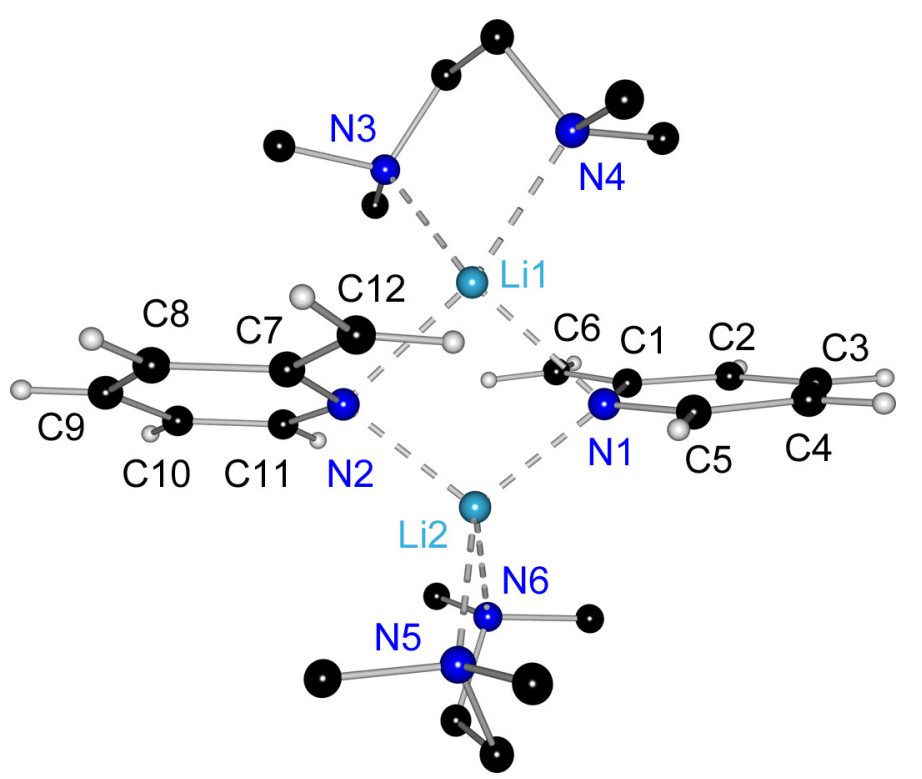

Figure 2-6. Molecular structure of [2-PicLi-tmeda $]_{2}$ (2). Hydrogen and disordered atoms of the donor molecules are omitted for clarity.

Unlike in other 2-picolyllithium dimers, the halves of the tmeda adduct are not related via a crystallographic symmetry operation and thus independent. The picolyl systems are almost planar (mean deviation from idealised plane: 0.024 and $0.012 \AA$ ) but not parallel as in the case of the $\eta^{1}$-carbanionic 2-PicLi compounds. They are skewed by $26^{\circ}$ relative to each other (rotation axis: $\sim N 1 \cdots N 2$ vector; Figure 2-7). Moreover, both methylene groups are orientated towards Li1 (Li1-C6/C12: $2.817 \AA / 2.725 \AA$; Li2-C6/C12: $3.144 \AA / 3.274 \AA$ ) but point to opposite directions relative to the $[\mathrm{LiN}]_{2}$ ring (trans configuration; Figure 2-6). Additionally, one ring is slightly tilted towards Li1 (angle between ring planes and the $\mathrm{N} 1 \cdots \mathrm{N} 2$ vector: $3^{\circ}$ (N1-ring) and $0^{\circ}$ (N2-ring)).

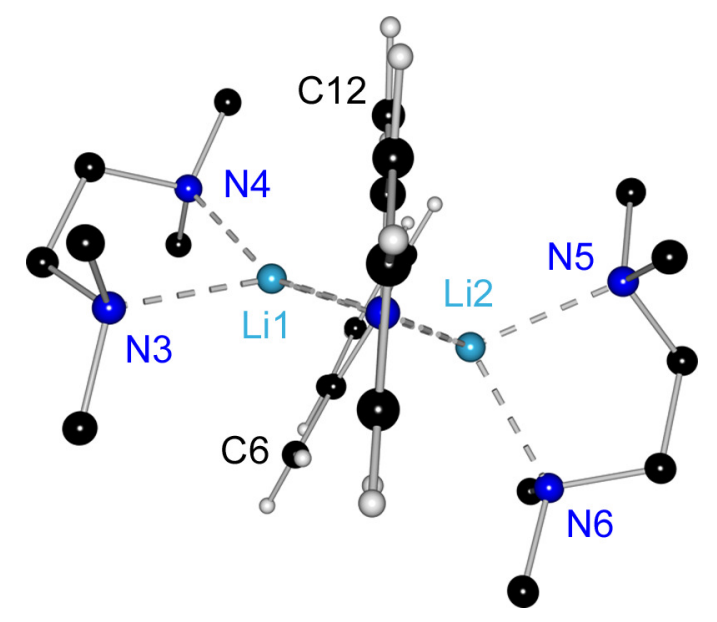

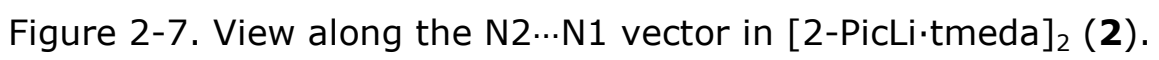


The lithium-carbon bond lengths are completely different to those in the dme adduct $1(2.231(5) \AA$ ) or aggregates with aza-allyl contacts (cf. chapter 2.3.6, e.g. $\mathrm{Et}_{2} \mathrm{O}: 2.297(2)$ and $\left.2.321(3) \AA\right)$. Therefore, no carbon-donor bonds are shown to Li1 in Figure 2-6. Yet, a relatively small coordinating effect of the azaallyl carbon atoms must be the reason for the observed asymmetric dimerisation mode in 2. The weak $\pi$-interactions of these carbon atoms with Li1 (Li1-C6/C12: $2.817 \AA / 2.725 \AA$ and Li1-C1/C7: $2.763 \AA / 2.703 \AA$ ) result in longer Li1-N bonds compared to Li2, which does not show equally "short" Li-C contacts (2.907 to $3.274 \AA$ ). Even though the Li1-C distances of the putative $\eta^{3}$-aza-allylic interaction appear extraordinarily long to be of attractive character, they can be discussed in the context of known $\eta^{3}$-allylic interactions. Polymeric benzyllithium as its triethylendiamine adduct shows a $\eta^{3}$-coordination to the lithium cation, in which an $\mathrm{Li}-\mathrm{C}$ bond length of $2.58(2) \AA$ was reported to the $\beta$-carbon atom. ${ }^{[159]}$ An even longer $\mathrm{Li}-\mathrm{C}$ contact could be found in bisquinuclidine fluorenyllithium with 2.794(13) $\AA_{.}{ }^{[160]}$ Moreover, an $\eta^{3}$-allylic stabilisation was claimed in the case of [(9-chloro-10-anthracenyl)lithium. $\left.\mathrm{OEt}_{2}\right]_{2}$ with interaction distances of 2.696(9) and $2.743(10) \AA$ (Figure 2-8) $\cdot{ }^{[161]}$ According to these facts, a stabilising effect on the lithium cation is reasonable, even if it is weak.

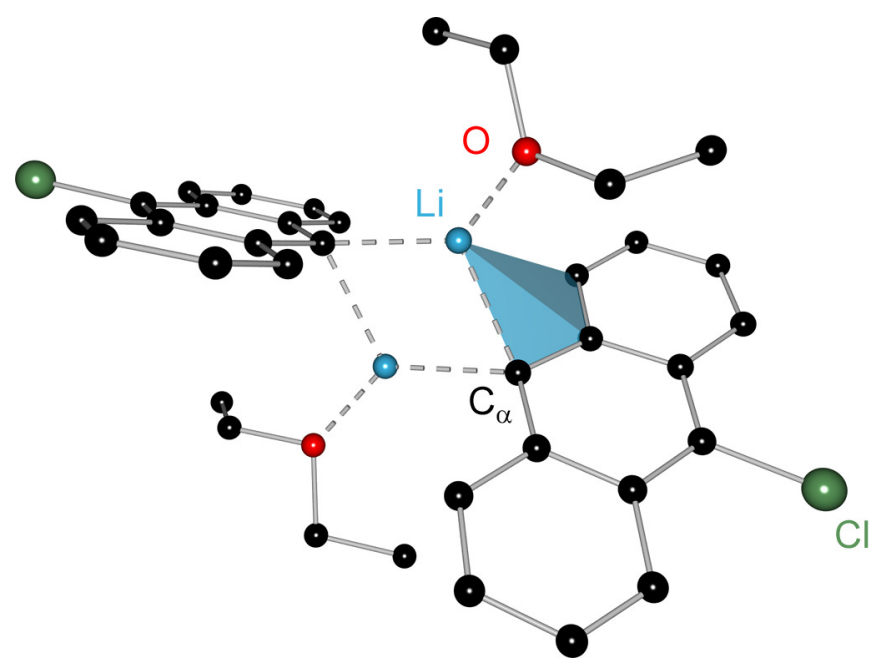

Figure 2-8. $\eta^{3}$-Allylic coordination of the anthracenyl anion to the lithium atom in [(9-chloro-10-anthracenyl)lithium. $\left.\mathrm{OEt}_{2}\right]_{2}$.

The main cation-anion interactions are the $\mathrm{Li}-\mathrm{N}$ bonds that definitely favour an enamidic description ( $\mathbf{D}$ in Scheme 2-4) of the picolyl anion. Another example of an anion, which exclusively coordinates the cation by a different atom than the deprotonated one, is diphenyl(2-pyridyl)methyllithium. ${ }^{[162]}$ An exclusive interaction to the pyridine nitrogen atom is found in this related monomer, too. The 
observed coordination mode in $\mathbf{2}$ does not involve the methylene groups in contrast to the dme adduct 1 . In comparison, the short Li-N bonds to Li2 (N1: 2.048(2) $\AA, N 2: 2.063(2) \AA$ ) in $\mathbf{2}$ are slightly longer than those in $\mathbf{1}$. However, they are well in the range of reported lithium amide distances (1.97-2.10 $\AA)$, ${ }^{[47]}$ whilst the Li1-N bonds are considerably longer (N1: 2.229(2) $\AA$, N2: 2.194(2) $\AA$ ) even compared to $\eta^{3}$-aza-allylic interactions of known picolyllithium compounds (e.g. $\mathrm{Et}_{2} \mathrm{O}$ adduct in chapter 2.3.6: 2.133(2) $\AA$ ). Accordingly, the inclusion of the methylene group into the lithium coordination sphere does not influence the $\mathrm{Li}-\mathrm{N}$ bond lengths considerably.

Table 2-2. Selected bond lengths $[\AA]$ and angles $\left[{ }^{\circ}\right]$ of $[\text { PicLi.tmeda }]_{2}$ (2) and related compounds $\left(\mathrm{Pic}=2\right.$-picolyl, $\mathrm{Py}=2$-pyridyl). ${ }^{[135,143,144]}$

\begin{tabular}{|c|c|c|c|c|}
\hline & $\begin{array}{l}{[\text { PicLi } \cdot \text { tmeda }]_{2}} \\
(\mathbf{2})^{[a]}\end{array}$ & $\begin{array}{c}{\left[\left(\mathrm{Me}_{3} \mathrm{Si}\right) \mathrm{CHPyLi} \cdot\right.} \\
\text { tmeda }]_{2}\end{array}$ & $\begin{array}{c}{\left[\left(t_{\mathrm{BuMe}} \mathrm{Si}\right) \mathrm{HC}\right.} \\
\text { PyLi·tmeda }]_{2}\end{array}$ & 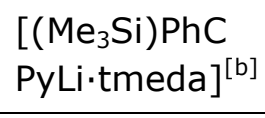 \\
\hline Li1-N1 & $2.229(2) / 2.194(2)$ & $2.25(1)$ & $2.181(4)$ & $1.978(9)$ \\
\hline Li2-N1 & $2.048(2) / 2.063(2)$ & $2.08(1)$ & $2.143(8)$ & - \\
\hline Li1-C1 & $2.763(2) / 2.703(2)$ & $2.82(2)$ & $2.98(1)$ & $3.01(1)$ \\
\hline Li1-C6 & $2.817(2) / 2.725(2)$ & $2.91(2)$ & $3.20(1)$ & $3.29(1)$ \\
\hline $\mathrm{N} 1-\mathrm{C} 1$ & $1.397(2) / 1.402(1)$ & $1.39(1)$ & $1.386(5)$ & $1.382(6)$ \\
\hline $\mathrm{N} 1-\mathrm{C} 5$ & $1.356(1) / 1.362(2)$ & $1.35(1)$ & $1.354(7)$ & $1.345(6)$ \\
\hline $\mathrm{C} 1-\mathrm{C} 2$ & $1.453(1) / 1.460(2)$ & $1.45(1)$ & $1.437(6)$ & $1.444(6)$ \\
\hline $\mathrm{C} 2-\mathrm{C} 3$ & $1.353(2) / 1.346(2)$ & $1.34(1)$ & $1.333(8)$ & $1.345(7)$ \\
\hline $\mathrm{C} 3-\mathrm{C} 4$ & $1.419(2) / 1.425(2)$ & $1.42(1)$ & $1.406(6)$ & $1.390(8)$ \\
\hline C4-C5 & $1.366(2) / 1.363(2)$ & $1.35(1)$ & $1.358(8)$ & $1.359(8)$ \\
\hline $\mathrm{C} 1-\mathrm{C} 6$ & $1.366(2) / 1.355(2)$ & $1.37(1)$ & $1.385(7)$ & $1.386(6)$ \\
\hline Li1-N3 & $2.211(2) / 2.201(2)$ & $2.18(2)$ & $2.217(5)$ & $2.13(1)$ \\
\hline Li2-N5 & $2.160(2) / 2.193(2)$ & $2.20(1)$ & $2.140(9)$ & - \\
\hline Li1-N1-Li2 & $81.1(1) / 81.6(1)$ & $81.7(7) / 81.7(7)$ & $83.5(5) / 83.5(5)$ & - \\
\hline N1-Li1-N2 & $103.5(1) / 93.7(1)$ & $103.6(7) / 93.1(1)$ & $95.4(2) / 97.6(5)$ & - \\
\hline N3-Li1-N4 & $83.1(1) / 84.6(1)$ & $83.7(7) / 83.2(6)$ & $84.7(3) / 85.2(5)$ & $86.5(3)$ \\
\hline
\end{tabular}

[a] Additional values refer to the corresponding bonds in the related independent second part of the molecule (e.g. Li1-N1 and Li1-N2). [b] This monomeric compound also shows a pure enamidic interaction to the cation. Values are averaged over two independent molecules in the asymmetric unit. 
It can be argued about the hybridisation of the ring nitrogen atoms, since the dimerisation shows characteristics of an amido ligand, in which two formal lone pairs are centred at the nitrogen atoms. These reside most probably in an $\mathrm{sp}^{2}$ orbital within the ring plane and a $\mathrm{p}$ orbital orthogonal to it. An alternative description with an $\mathrm{sp}^{3}$ hybridised nitrogen atom is unlikely, because it would seriously disturb the conjugated system. The donation to Li2 is slightly favoured according to the observed ring tilts, even if angles of $145.6^{\circ}$ (N1) and $144.2^{\circ}$ (N2) are enclosed with the ring planes. The corresponding angles to Li1 are $134.8^{\circ}$ (N1) and $138.1^{\circ}(\mathrm{N} 2)$. The dimerisation is consequently a compromise in sharing the anionic charge density at the nitrogen atoms. This is also reflected by the acute $\mathrm{Li}-\mathrm{N}-\mathrm{Li}$ angles of $81.1(1)^{\circ}(\mathrm{N} 1)$ and $81.6(1)^{\circ}(\mathrm{N} 2)$. Moreover, it was theoretically shown earlier that the $\mathrm{Li}-\mathrm{N}$ interactions are only slightly depending on the direction (cf. chapter 2.4).

Other reported picolyllithium derivatives (Scheme 2-7) also show a preferred enamidic structure in the crystal. This is also the case for the heavier alkaline metal derivatives. ${ }^{[144]}$ Trimethylsilyl(2-pyridyl)methyllithium as tmeda adduct forms a $C_{2}$ symmetrical dimer along the $\mathrm{Li}-\mathrm{Li}$ axis with a related structure. ${ }^{[135]}$ The short Li-N bond lengths are 2.08(1) $\AA$ while the longer ones are 2.25(1) $\AA$ (Table 2-2). Each of them is longer than the corresponding bond in $\mathbf{2}$, which can be explained by the steric demand of the silyl groups and their already discussed capability of stabilising carbanions in $\alpha$-position.

If the trimethylsilyl group is replaced by a phenyl group, the bond lengths are unaffected, in contrast to a $\mathrm{tBuMe}_{2} \mathrm{Si}$ substitution. ${ }^{[143]}$ The $\mathrm{Li}-\mathrm{N}$ distances are almost identical with the bulkier side chains (2.143(8) and 2.181(4) $\AA$, Table 2-2). Hence, a lithium-nitrogen bond length of around $2.16 \AA$ would be adopted in the imaginary case of a totally symmetric enamidic coordination to the cation without additional stabilisation by the $\pi$-system. This is again longer than the mean Li-N bond length of aza-allylic interactions (cf. chapter 2.3.6: 2.14 $\AA$ ).

A side-chain substitution with two bulky groups leads to monomer formation with a chelating donor base $\left(\left[2-\left(\mathrm{Me}_{3} \mathrm{Si}\right) \mathrm{PhCPyLi} \cdot\right.\right.$ tmeda $\left.]\right) .{ }^{[144]}$ The electron density at the pyridine nitrogen atom is concentrated towards only one lithium cation, so that a much shorter $\mathrm{Li}-\mathrm{N}$ bond is established (1.978(9) $\AA$ ). Monomeric picolyllithium compounds will be the topic of the following chapter 2.2.3, where they are discussed in detail. 
<smiles>[R]C=C1C=CC=CN1[Si]([2H])([2H])N1C=CC=CC1=C[R]</smiles>

$[\text { RHCPyLi-tmeda }]_{2}$<smiles></smiles>

[(Me $\left.{ }_{3} \mathrm{Si}\right) \mathrm{PhCPyLi-tmeda]}$

Scheme 2-7. Lewis formulae of PicLi compounds with an $\eta^{1}$-amidic dimerisation pattern $\left(\mathrm{R}=\mathrm{H},\left(\mathrm{SiMe}_{3}\right),\left(\mathrm{SiMe}_{2} \mathrm{tBu}\right) ; \mathrm{D}=\right.$ tmeda nitrogen atom; Py $=2$-pyridyl).

Interestingly, Li2 is distorted tetrahedrally coordinated in $\mathbf{2}$ while the coordination sphere at Li1 is better described as distorted square planar. A standard arrangement is adopted at Li2, because this side of the dimer is sterically wide open (Figure 2-9). The bond angles are quite different from the ideal tetrahedral angles, which is caused by the nitrogen anions N1/N2 and the more or less rigid coordination angle of the tmeda donor (reported $\mathrm{N}-\mathrm{Li}-\mathrm{N}$ angles: $83-86^{\circ}$; Table 2-2) to the lithium atom. However, the tmeda molecule is specifically orientated relative to the $[\mathrm{LiN}]_{2}$ ring (angle between N1-Li2-N2 and N5-Li2-N6 plane: $81^{\circ}$ ). In contrast, the coordination mode of Li1 shows more acute $\mathrm{N}-\mathrm{Li}-\mathrm{N}$ bond angles and the donor position is adjusted to the steric demand of the methylene groups facing the cation. An angle between the $\mathrm{N}-\mathrm{Li}-\mathrm{N}$ planes of $49^{\circ}$ illustrates this (Figure 2-9). Hence, the Li1-N3/4 bonds are longer due to the unusual square planar coordination of the cation and the additional interaction with the aza-allylic system.

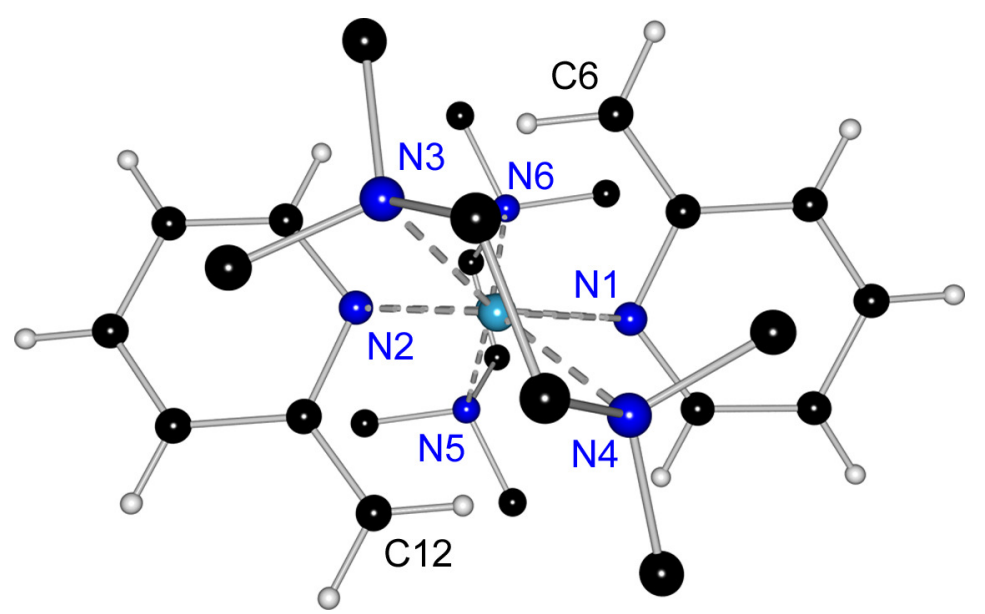

Figure 2-9. View along the Li1...Li2 vector in [2-PicLi·tmeda $]_{2}(\mathbf{2})$. 
By an examination of the bonding situation within the picolyl anions of $\mathbf{2}$, enamidic bond lengths should be evident. The picolyl rings are not crystallographically equivalent but show the same tendencies, hence, the discussion is limited to the ring containing $\mathrm{N} 1$ and values of the second ring are given in brackets. Again, the changes in bond lengths are compared with 2-picoline in order to study the effect of the deprotonation. ${ }^{[150]}$ The $\mathrm{N}-\mathrm{C}$ bond to the 2-carbon atom in 2 (C1: 1.397(2) $\AA, C 7: 1.402(1) \AA$ ) is much longer than in 2-picoline $(1.343(3) \AA)$. The second $\mathrm{N}-\mathrm{C}$ bond is also slightly longer this time (C5: $1.356(1) \AA, C 11: 1.362(2) \AA)$. The changes of the $C-C$ bonds are even more pronounced than in the dme adduct 1 . The shortest bond with 1.353(2) $\AA$ can be found between $\mathrm{C} 2$ and $\mathrm{C} 3$ (C8-C9: 1.346(2) $\AA$ ), while the C4-C5 (1.366(2) $\AA, C 10-C 11: 1.363(2) \AA)$ and C1-C6 bond lengths (1.366(2) $\AA$, C7C12: $1.355(2) \AA)$ do also confirm the strong tendency towards localisation of the double bonds in the conjugated system. The methylene bonds are just $0.03 \AA$ $(0.02 \AA)$ longer than a standard double bond of carbon atoms with a similar hybridisation state $\left(\mathrm{C}_{\mathrm{sp} 2}=\mathrm{C}_{\mathrm{sp} 2}: 1.335 \AA\right) .{ }^{[152]}$ Consequently, the remaining $\mathrm{C}-\mathrm{C}$ bonds are vastly elongated in comparison to the corresponding acid (2-PicH), as predicted by the enamidic Lewis formula of 2-PicLi (D in Scheme 2-4). The C1$\mathrm{C} 2$ atom distance of $1.453(1) \AA(1.460(2) \AA)$ does almost match the idealised value of a single bond $\left(\mathrm{C}_{\mathrm{sp} 2}-\mathrm{C}_{\mathrm{sp} 2}: 1.466 \AA\right) \cdot{ }^{[152]}$ Moreover, the $\mathrm{C} 3-\mathrm{C} 4$ bond (1.419(2) $\AA, C 9-C 10: 1.425(2) \AA)$ is longer than the same one in the dme adduct $1(1.409(4) \AA)$. Therefore, it can be concluded that all partially localised double bonds in $\mathbf{1}$ are even shorter in $\mathbf{2}$, while the situation is the other way round for the elongated bonds. With the tmeda adduct at hand, one might be willing to claim that the most pronounced delocalisation of negative charge into the pyridine ring is found with that donor base. Indeed, the bond lengths of other reported derivatives of 2-picolyllithium do not show a similar acute double bond localisation pattern, but the diethyl ether adduct of 2-picolyllithium (chapter 2.3.6) demonstrates to a certain extent similar features. Thus, care has to be taken with generalisation. 


\subsubsection{Monomeric 2-Picolyllithium}

As mentioned in the introduction, the complete disaggregation of lithium organic compounds results in the most potent reagents. Hence, this was attempted with 2-picolyllithium by the right choice of the donor base. Probably due to their extraordinary reactivity, only a few monomeric RLi are structurally characterised. ${ }^{[47,48]}$ These studies teach the use of polydentate ligands to reduce the aggregation. tert-Butyllithium, for example, was obtained as a monomer with the chiral diamine donor (-)-sparteine (sp), ${ }^{[163]}$ while monomeric phenyllithium $^{[164]}$ and trimethylsilylmethyllithium ${ }^{[165]}$ were stabilised with pmdeta (Figure 2-10). The much bulkier 2,4,6-tris(tert-butyl)phenyllithium monomer could be obtained with tmeda. ${ }^{[166]}$ Other reported monomers were already mentioned in the preceding chapters or are mainly based on cyclopentadienyl and extended conjugated systems. ${ }^{[47,48]}$
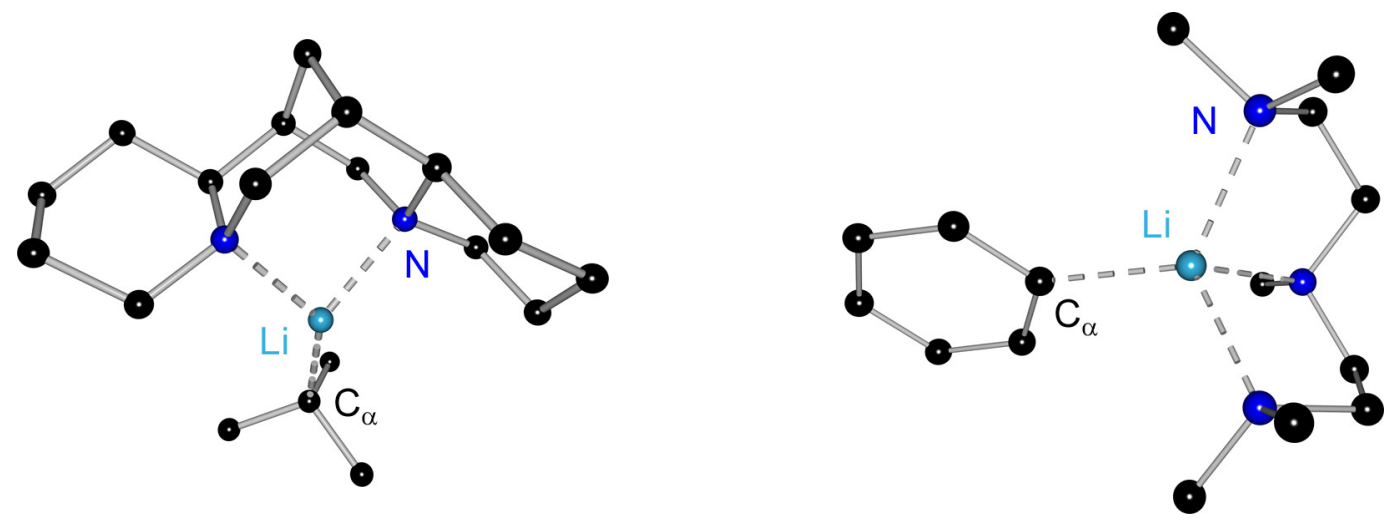

Figure 2-10. Examples of monomeric lithium organic compounds (left: [tBuLi·(sp)], right:

$[$ PhLi·pmdeta]).

Accordingly, the tridentate amine donor pmdeta was applied during the deprotonation of 2-picoline with $n$-butyllithium in hexane at $-78{ }^{\circ} \mathrm{C}$, since tmeda still gave a dimer. The resulting bright orange suspension was filtered and the storage of the saturated solution at $4{ }^{\circ} \mathrm{C}$ yielded orange-red crystals suitable for $X$-ray structural analysis. These were extraordinarily sensitive and decomposed instantly under air exposure. NMR spectra of the dissolved crystals and the filtered off solid proved the successful deprotonation and the coordination of the pmdeta donor. This was verified by the molecular structure determination of the 2-picolyllithium.pmdeta adduct ([2-PicLi·pmdeta], 3) that crystallises in the triclinic space group $P \overline{1}$. Two independent 2-PicLi·pmdeta monomers are present in the asymmetric unit, of which both show slight disorder in the tridentate 
ligand and one even in the picolyl part. Therefore, the following discussion of structural features will be limited to the non-disordered picolyllithium monomer. Furthermore, it has to be emphasised that the monomeric units in $\mathbf{3}$ show no interaction to their neighbours.

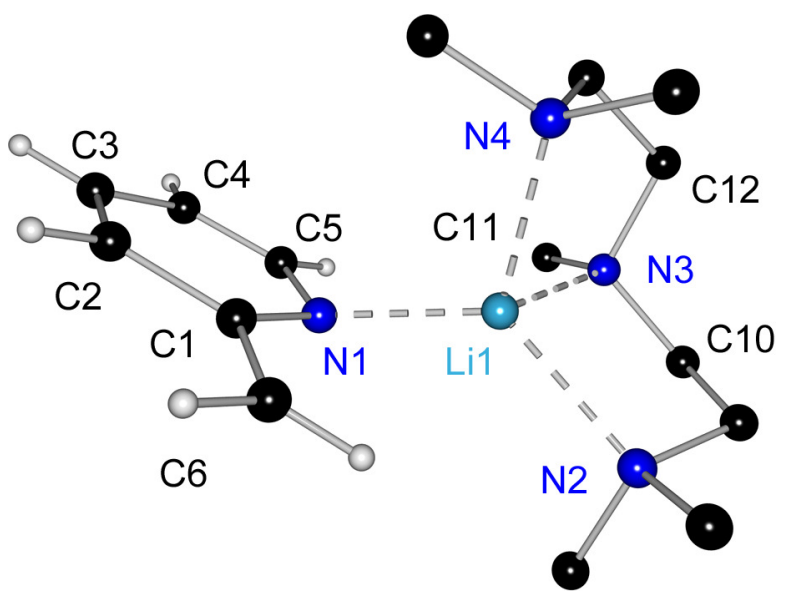

Figure 2-11. Molecular structure of one [2-PicLi-pmdeta] (3) moiety in the asymmetric unit. Hydrogen and disordered atoms of the donor molecule are omitted for clarity.

The picolyl anion mainly bonds to the lithium cation via the pyridyl nitrogen atom (Figure 2-11). The conclusion can be drawn that a dominant enamidic structure is present with the chelating ligand pmdeta. The lithium atom is tetracoordinated. The limited flexibility of the donor molecule results in acute $\mathrm{N}-\mathrm{Li}-\mathrm{N}$ bond angles to neighbouring donor nitrogen atoms of $85.2(1)^{\circ}$ (N2-Li1-N3) and $87.1(1)^{\circ}$ (N3-Li1-N4). The bond angles involving $\mathrm{N} 1$ are much wider with $130.0(1)^{\circ}(\mathrm{N} 2), 120.5(1)^{\circ}(\mathrm{N} 3)$ and $107.3(1)^{\circ}(\mathrm{N} 4)$. The metal atom accordingly possesses a quite open coordination face for a substrate approach. Moreover, the position of $\mathrm{N} 1$ relative to the donor molecule is rather unusual (Figure 2-12). It resides almost perfectly on the projection of the angle bisection of the $\mathrm{V}$-shaped coordinating pmdeta molecule (i.e. plane through the bisections of the N2-Li1$\mathrm{N} 4$ and $\mathrm{C} 10-\mathrm{N} 3-\mathrm{C} 12$ angle). Thus the N1-Li1-N3 and the Li1-N3-C11 planes are almost parallel (dihedral angle of $3.7(2)^{\circ}$ ). Interestingly, the methylene carbon atom is also positioned in the projection of the angle bisection and even almost in the projection of the N3-Li1 bond (N3-Li1 ‥C6 angle of $172.1^{\circ}$ ). Thus, the steric interference with the bulky donor is minimised with the pyridine ring pointing away from it and the methylene group extending into the open pocket of the pmdeta molecule. 


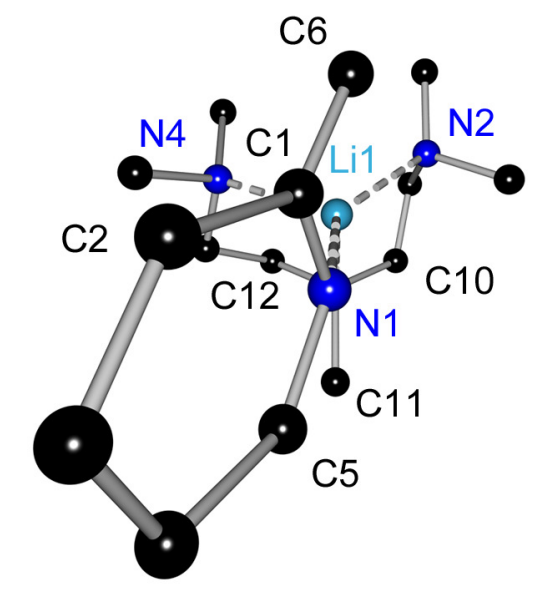

Figure 2-12. Picolyl group orientation relative to the pmdeta-lithium moiety in $\mathbf{3}$.

The shortest Li-N bond is formed to the pyridine nitrogen atom (2.010(2) $\AA$ ), while the donor bonds vary from $2.136(2) \AA$ (N4) to $2.153(2) \AA$ (N3). They are in the expected range of other known lithium organic compounds involving the pmdeta donor ${ }^{[48]}$ Remarkably, the Li1-N1 bond in $\mathbf{3}$ is not the shortest of all reported picolyllithium compounds (Table 2-3).<smiles>[2H][Al]([2H])([2H])N1C=CC=CC1=C</smiles>

[PicLi·pmdeta] (3)<smiles>[2H][13C]([2H])([2H])N1C(C)=CC=C/C1=C/[SiH3]</smiles><smiles></smiles>

[(Me $\left.\left.{ }_{3} \mathrm{Si}\right) \mathrm{PhCPyLi} \cdot \mathrm{tmeda}\right]$

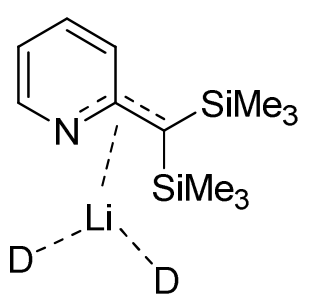

$\left[\left(\mathrm{Me}_{3} \mathrm{Si}\right)_{2} \mathrm{CPyLi} \cdot \mathrm{tmeda}\right]$

Scheme 2-8. Lewis formulae of monomeric PicLi compounds ( $\mathrm{Pic}=2$-picolyl, Py $=2$-pyridyl).

Similar short distances are found in the dme adduct $\mathbf{1}$ as well as the thf adduct and even smaller ones are reported for the $\left[2-\left(\mathrm{Me}_{3} \mathrm{Si}\right)_{2} \mathrm{CPyLi}\right]_{2} \operatorname{dimer}^{[142]}$ $(1.937(6) \AA)$ and the [2-(Me $3 \mathrm{Si})$ PhCPyLi.tmeda] monomer ${ }^{[143]}$ (1.978(9) $\AA$; Scheme 2-8). This can be understood if the number of bonded atoms to the cation is taken into account. The disilylated compound shows an uncommon dicoordinated lithium atom, while the coordination number is three in the silylphenyl substituted monomer. The lithium atom in the pmdeta adduct on the other hand is tetracoordinated and relatively well stabilised by the three donating nitrogen atoms of the pmdeta ligand. Another monomeric picolyllithium 
derivative that also shows a very short Li-N $\mathrm{N}_{\text {Pic }}$ bond $(1.96(2) \AA)$ is the bis(trimethylsilyl) substituted 2-picolyllithium tmeda adduct ([2-( $\left.\mathrm{Me}_{3} \mathrm{Si}\right)_{2} \mathrm{CPyLi}$. tmeda]). ${ }^{[135]}$ However, this molecule is a border line case between an enamidic and an aza-allylic bonding mode. The atom distances between the cation and the aza-allylic carbon atoms are 2.43(2) and 2.46(2) $\AA$, and much larger than in other picolyllithium compounds showing the $\eta^{3}$-coordination type (cf. chapter 2.3.6, e.g. $\mathrm{Et}_{2} \mathrm{O}$ dimer: 2.297(2) and 2.321(3) $\AA$ ). However, exactly this coordination moves the lithium atom towards $\mathrm{C} 6$ causing the shorter $\mathrm{Li}-\mathrm{N}$ bond, which is probably less stabilising than a contact in a plane perpendicular to the pyridine ring passing through $\mathrm{C} 3$ and $\mathrm{N} 1$. This is also manifested in the rather undisturbed aromaticity within the pyridine ring, which will be discussed later on.

Table 2-3. Selected bond lengths $[\AA]$ of [PicLi.pmdeta] (3) and related compounds (Pic $=2$-picolyl, Py $=2$-pyridyl).$^{[135,143,144]}$

\begin{tabular}{|c|c|c|c|c|}
\hline & $\begin{array}{c}{[\text { PicLi } \cdot \text { pmdeta }]} \\
(\mathbf{3})^{[a]}\end{array}$ & $\begin{array}{c}{\left[\left(\mathrm{Me}_{3} \mathrm{Si}\right) \mathrm{HC}-6-\mathrm{Me}-\right.} \\
\text { PyLi·pmdeta }]\end{array}$ & $\begin{array}{l}{\left[\left(\mathrm{Me}_{3} \mathrm{Si}\right) \mathrm{PhC}\right.} \\
\text { PyLi.tmeda }]^{[\mathrm{b}]}\end{array}$ & $\begin{array}{c}{\left[\left(\mathrm{Me}_{3} \mathrm{Si}\right)_{2} \mathrm{CPyLi} \cdot\right.} \\
\text { tmeda }^{\mathrm{cc}]}\end{array}$ \\
\hline Li1-N1 & $2.010(2)$ & $2.048(3)$ & $1.978(9)$ & $1.96(2)$ \\
\hline Li1-C1 & $2.799(2)$ & $2.649(3)$ & $3.009(9)$ & $2.46(2)$ \\
\hline Li1-C6 & $2.987(2)$ & $2.700(3)$ & $3.290(9)$ & $2.43(2)$ \\
\hline $\mathrm{N} 1-\mathrm{C} 1$ & $1.393(2)$ & $1.393(2)$ & $1.382(6)$ & $1.38(1)$ \\
\hline $\mathrm{N} 1-\mathrm{C} 5$ & $1.362(2)$ & $1.351(2)$ & $1.345(6)$ & $1.35(1)$ \\
\hline $\mathrm{C} 1-\mathrm{C} 2$ & $1.457(2)$ & $1.443(2)$ & $1.444(6)$ & $1.42(1)$ \\
\hline $\mathrm{C} 2-\mathrm{C} 3$ & $1.346(2)$ & $1.354(2)$ & $1.345(7)$ & $1.39(1)$ \\
\hline $\mathrm{C} 3-\mathrm{C} 4$ & $1.418(2)$ & $1.402(2)$ & $1.390(8)$ & $1.37(1)$ \\
\hline $\mathrm{C} 4-\mathrm{C} 5$ & $1.367(2)$ & $1.378(2)$ & $1.359(8)$ & $1.38(1)$ \\
\hline $\mathrm{C} 1-\mathrm{C} 6$ & $1.358(2)$ & $1.393(2)$ & $1.386(6)$ & $1.42(1)$ \\
\hline
\end{tabular}

[a] Values refer to the non-disordered formula unit in the asymmetric unit. [b] Values are averaged over two independent molecules in the asymmetric unit. [c] This molecule shows an aza-allylic interaction to the cation.

Yet again, the coordination of the picolyl ring to the cation in $\mathbf{3}$ is not achieved within the ring plane. The lithium atom resides $1.13 \AA$ outside the pyridine plane and an angle of $146.6^{\circ}$ is enclosed with the Li1-N1 bonding vector. The pyridine ring is almost planar (mean deviation from idealised plane $0.017 \AA$ ) and the methylene carbon atom resides $0.11 \AA$ outside of the ring plane (mean picolyl ring deviation $0.028 \AA$ ). This might be caused by the arrangement of the 
methylene group relative to the donor that was discussed above. The reason for the picolyl ring tilt could be an additional aza-allyl interaction, which seems to be quite small due to the observed atom distances (Li1-C1: 2.799 $\AA$, Li1-C6: $2.987 \AA$ ), or the interference of the pyridine ring with the methyl group at C11 (Figure 2-12). An even more pronounced deviation from an ideal $\sigma-\mathrm{N}$ - $\mathrm{Li}$ interaction was found in (6-methyl-2-pyridyl)trimethylsilylmethyllithium as pmdeta adduct ([( $\left.\left.\mathrm{Me}_{3} \mathrm{Si}\right) \mathrm{HC}-6-\mathrm{Me}-\mathrm{PyLi} \cdot \mathrm{pmdeta}\right]$; Scheme 2-8). ${ }^{[144]}$ The Li-N vector encloses an angle of $142.8^{\circ}$ with the ring plane as an effect of the steric demand of the substituted anion. Indeed, the non-deprotonated methyl group in 6-position is oriented towards the discussed pmdeta pocket (Figure 2-12), while the methylene group is pointing away from the donor. Nevertheless, the silyl group gets close to the methyl group at N3, anyway. The Li-N $\mathrm{N}_{\mathrm{Py}}$ bond is slightly longer $(2.048(3) \AA)$ than in $\mathbf{3}$, which is caused by the less pronounced delocalisation of the negative charge into the ring due to the silyl substitution.

A remarkable bond alternation is evident in the picolyl fragment of $\mathbf{3}$, which was already suggested by observed coordination mode. One of the shortest exocyclic double bonds can be found in $3(1.358(2) \AA)$. The other two localised double bond lengths (C2-C3: 1.346(2) $\AA$, C4-C5: 1.367(2) $\AA$ ) almost perfectly match those of the tmeda dimer $\mathbf{2}$. The same is true for the remaining bond lengths. Hence, the bonding situation in the picolyl anion is independent of the coordination to the cation(s) or the aggregation mode if the same mesomeric structure is adopted. This is exclusively determined by the applied donor (cf. chapter 2.4).

The other related picolyllithium compounds, except [2-( $\left.\mathrm{Me}_{3} \mathrm{Si}\right)_{2} \mathrm{CPyLi}$ tmeda] (Scheme 2-8), also show alternating $\mathrm{C}-\mathrm{C}$ bond lengths in the crystal, even when the methylene bond is longer because of the silyl substitution. The disilylated compound on the other hand shows this feature to a lesser extent. The bonds between $\mathrm{C} 2$ to $\mathrm{C} 5$ are even almost equal (1.38 $\AA$ ). Accordingly, the C1-C6 bond is the longest in the discussed series.

It should be further mentioned, that the $\mathrm{Li} \cdots \mathrm{H}$ distance to a methylene hydrogen atom in $\mathbf{3}$ gets quite short due to the bonding via the ring nitrogen atom $(2.610 \AA)$. This contact can be - and was in other cases - interpreted as lithium agostic interaction. But since the electron density study of $W$. Scherer et al. on bis(trimethylsilyl)(2-pyridyl)methyllithium, it is known that an agostic $\mathrm{Li}-\mathrm{H}$ bond can be excluded, even if shorter lithium hydrogen distances are observed in the reported case $(2.245(5) \AA){ }^{[87]}$ 


\subsection{Experimental Charge Density Study on Unsub- stituted 2-Picolyllithium}

Several structural features of 2-picolyllithium were highlighted in the previous chapters. These were based on standard low-temperature $\left(-173^{\circ} \mathrm{C}\right)$ or even $0{ }^{\circ} \mathrm{C}$ (cited molecules for comparison) X-ray diffraction experiments with single crystals. However, only atomic positions and thermal parameters are available with this method. The sticks between the atoms in the molecular structure figures throughout these examples are bond indicators that are solely derived from calculations of atomic difference vectors, bond radii considerations or the experience of the chemist. Hence, these are expected bonds or attractive interactions in an atomic arrangement without an explicit prove of existence.

Various ways to verify chemical bonds are known. Force constants of bonds can be calculated from infrared spectroscopy experiments and also the appearance of chemical coupling in a nuclear magnetic resonance spectrum indicates chemical bonding. Another method is the investigation of the electron density distribution (EDD) in a compound via topological analysis by use of R. F. W. Bader's Quantum Theory of Atoms in Molecules (QTAIM). ${ }^{[61]}$ The examined EDD may originate from diffraction experiments but also from quantum mechanical calculations. The following chapters shall give a brief introduction to standard structure determination procedures in order to understand the fundamental differences to the modelling of the electron density distribution in a crystal using the multipole model (MM) instead of a spherical atom approach. This highlights the progress from standard structure refinement techniques, with their inherent limitation of interpretation, to the multipole refinement procedure. In the further course some basics of QTAIM shall be explained as well as important properties that can be derived from the EDD. This is necessary to follow the discussion of topological features in the charge density related chapters of this thesis.

2-Picolyllithum shows an interesting variety of bonding modes in the solid state. The dimethoxyethane adduct exhibits a carbanionic dimerisation pattern (A in Scheme 2-4), while the interaction of the pyridine nitrogen atom with the lithium cation is dominant in the tmeda compound (D in Scheme 2-4). A situation in between is found in $\eta^{3}$-aza-allylic 2-picolyllithium compounds ( $c f$. Figure 2-1). The cation seems to interact with the formal carbanion as well as the nitrogen atom, which suggests that the negative charge is delocalised between those two atoms. A compound with this intermediate coordination mode 
is therefore an ideal candidate for a charge density study, since the bonding situation is most ambiguous and just the direct inspection of the electron density distribution itself can give a doubtless answer to the present coordination mode.

The standard structure of crystalline 2-picoline served as an external reference for the undisturbed starting material in the structural discussions above. Therefore, it was attempted to crystallise 2-picolyllithium with the free acid (2-PicH) as donor, which can serve as internal standard for the charge density analysis of the picolyl anion. This was accomplished by the synthesis and isolation of 2-picolyllithium as its 2-picoline adduct (4). ${ }^{[149]}$ Single crystals of excellent quality and appropriate size could be obtained suitable for an experimental charge density investigation (chapter 2.3.5 and 2.3.7).

\subsubsection{Principles of X-Ray Diffraction}

Ideal single crystals represent a regular three-dimensional periodic arrangement of atoms in the solid state (Figure 2-13). This arrangement can be described by the unit cell that reproduces the systematic packing in the crystal when it is merely repeated in all directions of space.

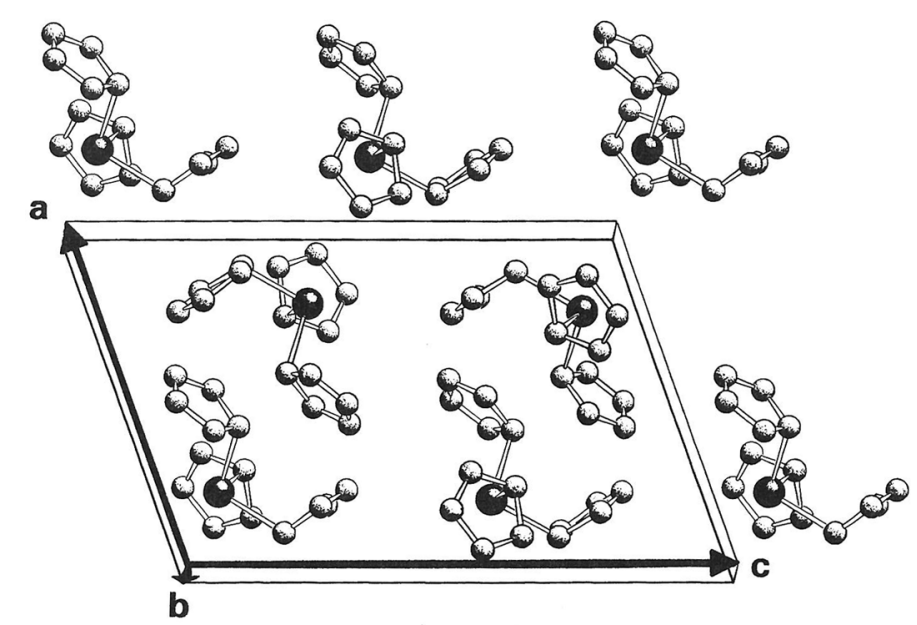

Figure 2-13. Unit cell representation of $\left[\mathrm{Cp}_{3} \mathrm{Sb}\right]$ including next molecules of neighbouring cells in $a$ - and $c$-direction. ${ }^{[167]}$

An even smaller increment, which is necessary to know in order to express the content of a crystal, is the asymmetric unit. By the application of the symmetry operations of the parent space group, the unit cell is obtained. Information about 
the space group, the asymmetric unit, and its content are available from the diffraction data.

Crystals show a regular arrangement of atoms and act as three-dimensional lattices, which have slit widths in the range of X-ray radiation wavelengths. An $X$-ray beam that passes through a crystal mainly interacts with the electron density of the atoms and is elastically scattered. The same phenomenon can be observed with neutron and electron beams, besides the fact that they interact with the atomic cores or the core and the electron density distribution, respectively. The scattering at the atoms results in plane waves that interfere destructively except for certain angles in which their path differences are a multiple of their wavelengths. This criterion is strictly obeyed in regular sized crystals $(0.1-0.5 \mathrm{~mm})$, because they contain a tremendous number of scattering centres $\left(\sim 10^{15}\right)$ so that even minimal wavelength differences lead to destructive interference of the diffracted waves. W. H. and W. L. Bragg have shown that the diffraction process can be interpreted as reflection of the primary beam at crystal lattice planes (Figure 2-14) denoted by their Miller indices $(\mathrm{hkl}) \cdot{ }^{[168]}$

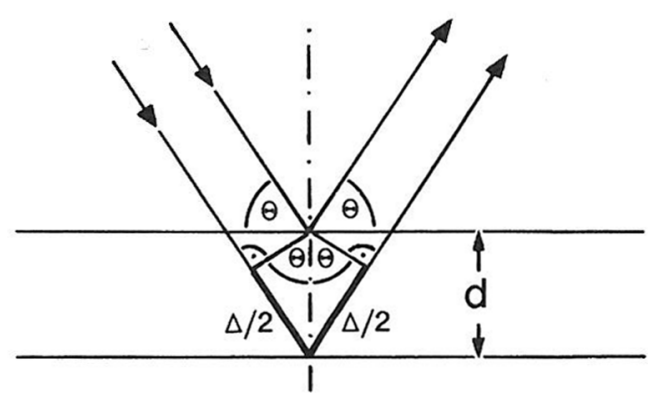

Figure 2-14. Reflection of X-ray beams at lattice planes. ${ }^{[167]}$

Therefore, the diffraction angles $\theta$ and, in consequence, the position of a reflection can be predicted if the radiation wavelength $(\lambda)$ and the distance between these planes $(d)$ are known (Eq. 2-1), which is normally not the case in novel compounds so that the diffraction angles have to be used to calculate $d$ as well as the dimensions and angles of the unit cell. This is achieved by the rotation of the crystal relative to the primary beam and the detection of positive scattering wave interferences. 
The intensity of the diffracted beam or the scattering amplitude is proportional to the "number of electrons" (the atomic number $Z$ of an element) it interacts with. However, a model of point scattering atoms is no longer valid in reality and the radial expansion of the atomic electron shell has to be taken into account. Indeed, the time-averaged three-dimensional electron density distribution (EDD) acts as source of the interaction and not solely the atom centre. The scattering amplitudes, derived from quantum-mechanical ab-initio calculations on isolated atoms in the gas phase (theoretical ground state atoms), are tabulated for almost all elements and many ions. The radial distribution of the electron density results in a phase shift of the diffraction wave, because of additional scattering centres deviating from the lattice planes in which the atomic cores are found. This shift is the more distinct the smaller the lattice plane distance $d$ gets. Hence, the atomic or scattering amplitudes (normalised to one electron known as atomic scattering factor $f$ ) decrease with increasing diffraction angle. As a result, the ability of an atom to diffract to higher Bragg angles depends on the number of electrons and their radial distribution (Figure 2-15).
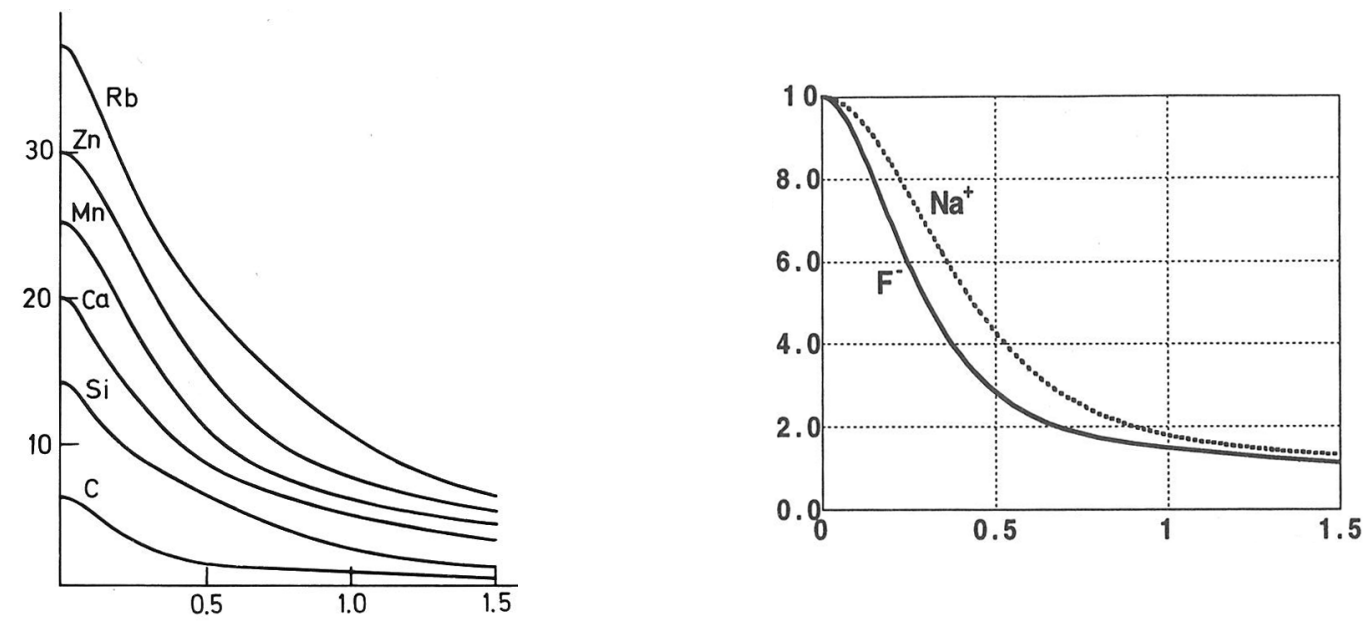

Figure 2-15. Diffraction angle dependence (x-axis in $\sin \theta \lambda^{-1}\left[\AA^{-1}\right]$ ) of some element $\left(\right.$ left ${ }^{[169]}$ or ion (right) ${ }^{[170]}$ scattering factors $[e]$.

Another effect resulting in a phase shift is the oscillation of the atoms in a crystal. This thermal motion leads to a weakening of the scattering power of an atom, which is taken into account by a normal probability distribution.

The weakening is proportional to the atomic displacement parameter $U$ and the reciprocal plane distance in an isotropical case (Eq. 2-2). A more complicated anisotropic description of the atomic thermal motion requires six $U_{x y}$ parameters 
which contribute individually depending on the Miller indices and the reciprocal cell dimensions.

$$
f^{\prime}=f \cdot \exp \left(-2 \pi^{2} U d^{* 2}\right)
$$

Furthermore, crystals normally contain several atoms in the asymmetric unit. Whenever one atom in the translation lattice is in diffracting position according to the Bragg equation (Eq. 2-1), the same is true for all other atoms in the cell. Each of them has an individual phase shift relative to an atom on the origin of the unit cell. The sum of all atomic contributions (scattering amplitudes) in the unit cell is determining the diffraction intensity and is called the structure factor $F_{h k l}$ (Eq. 2-3, the atomic position is given in fractional coordinates $(x, y, z)$ relative to the unit cell).

$$
F_{h k l}=\sum_{i} f_{i}^{\prime} \cdot \exp \left[2 \pi i\left(h x_{i}+k y_{i}+\mid z_{i}\right)\right]
$$

It is important to emphasise, that the whole electron density in the unit cell gives rise to scattering and not only isolated atoms without any interactions between each other. Indeed, the structure factors $F_{\text {hkl }}$ contain the complete information about the electron density distribution in a crystal. $F_{\text {hkl }}$ are the Fourier transformations of the static unit cell electron density $\rho_{x y z}$ according to Eq. 2-4.

$$
F_{h k l}=\int_{V} \rho_{x y z} \exp \left[2 \pi i\left(h x_{i}+k y_{i}+\mid z_{i}\right)\right] d V
$$

As a result, the electron density function can be obtained by the Fourier synthesis of $F_{\text {hkl }}$ (Eq. 2-5).

$$
\rho_{x y z}=V^{-1} \cdot \sum_{h k l} F_{h k l} \cdot \exp \left[-2 \pi i\left(h x_{i}+k y_{i}+z_{i}\right)\right] \quad \text { Eq. } 2-5
$$

The electron density distribution thus is an observable. However, the experimental determination of the structure factors is not practicable yet, since the phase information is lost during the detection process (phase problem). Only intensities $\left(I_{h k l}\right)$, which are proportional to the modulus of the squared structure factors, are measureable in practice. This limitation necessitates a modelling of the electron density. 


\subsubsection{Independent Atom Model}

The most common approach in modelling the total electron density is the reconstruction from atomic contributions (Eq. 2-6).

$$
\rho_{x y z}=\sum_{n} \rho_{n, x y z} \quad \text { Eq. } 2-6
$$

If only spherical atoms are considered to contribute to the total electron density and hence to the structure factor, a spherical or independent atom model (IAM) is applied. This fairly good assumption works well for heavy atoms and high Bragg order reflections. Their amplitudes originate almost exclusively from core electron densities of spherical shape. On the other hand, low order data are also influenced by valence densities which may be distributed between connected atoms, polarised to a certain extent, or accumulated in lone pair regions. This shortcoming is especially significant for hydrogen atoms, whose single electron is involved in the electron pair bonds to the bonding partners. Therefore, hydrogen bond lengths are normally much smaller from an X-ray IAM than those obtained from neutron diffraction experiments. ${ }^{[171]}$ In addition, all atoms are treated as neutral and no charge transfer is taken into account.

According to the assigned atom position in the IAM, theoretical structure factors of the modelled molecule can be calculated $\left(F_{\text {calc }}\right)$. These can be compared to the collected data $\left(F_{o b s}\right)$, in which the model phases were combined with the measured intensities. During the model refinement process, the differences between $F_{o b s}$ and $F_{c a l c}$ are minimised in a least squares procedure so that the model is constantly improved. Three coordinates and six anisotropic displacement parameters (one for isotropic atoms) can be adjusted for each atom with the aim of matching the observed intensities. For further information, especially on the generation of an initial model for the refinement, it has to be referred to known literature on single crystal diffraction in order to limit the extent of this introduction. ${ }^{[167,169,172,173]}$ 


\subsubsection{The Multipole Model}

The shortcomings of the IAM formalism mentioned above lead to a deficient model for the calculation of theoretical structure factors. This means that it is not possible to obtain a perfect set of calculated structure factors that match a hypothetical set of ideal observed intensities. This can be visualised to a certain extent by a difference Fourier map (residual density map from Eq. 2-5 with $\Delta F=F_{o b s}-F_{c a l c} ;$ phases for both cases are those obtained from the model) of a high resolution data set, in which the atom positions were assigned according to high order reflections.
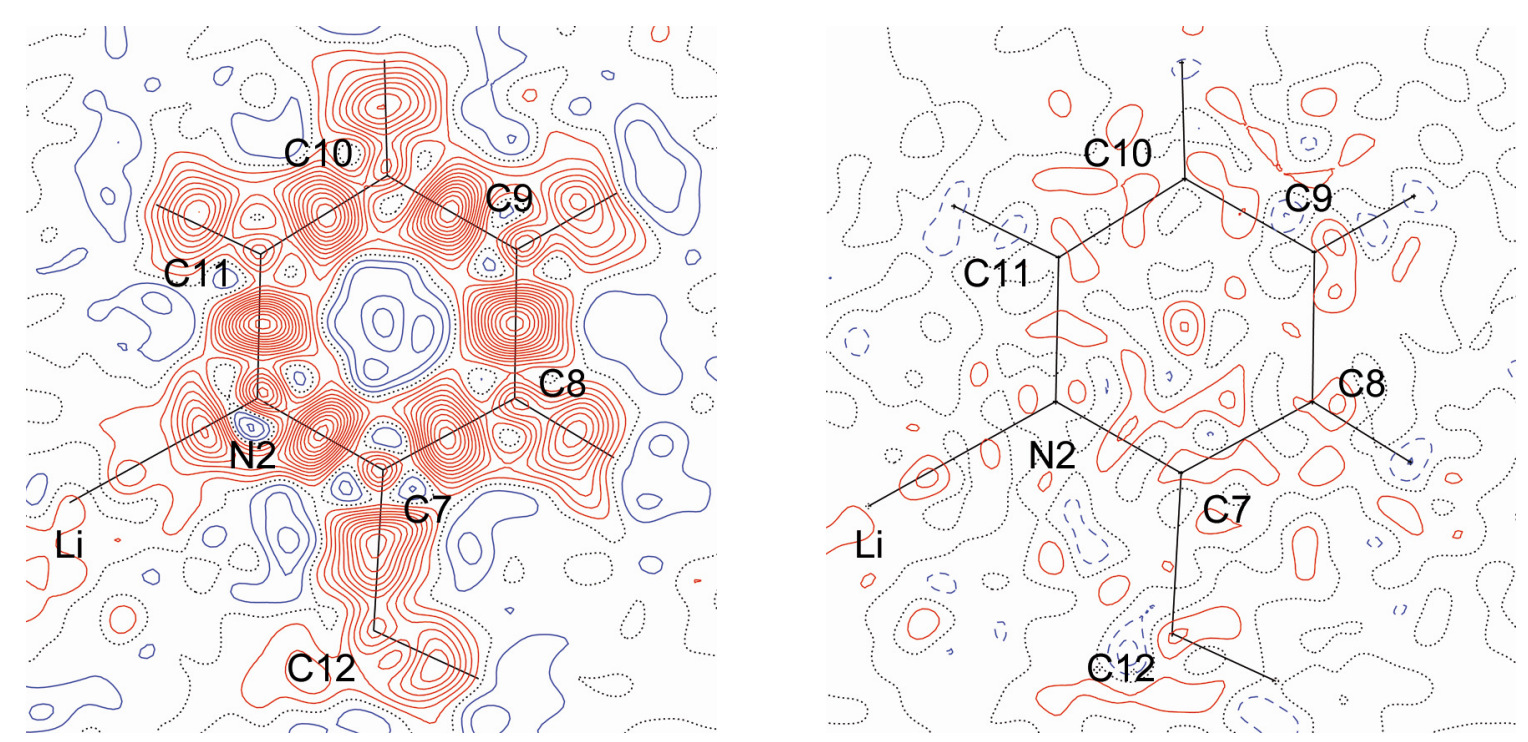

Figure 2-16. Residual density maps before (left) and after (right) the multipole refinement of the picoline ring in $[2-\mathrm{PicLi} \cdot \mathrm{PicH}]_{2}(\mathbf{4})$. Positive contour values are plotted solid red, zero dotted black, and negative dashed blue (step size 0.05 e $\AA^{-3}$ ).

A high order refinement (above $\sin \theta \lambda^{-1}=0.9 \AA^{-1}$ ) reobtains the "real" atomic positions in the asymmetric unit, because only spherical core electron densities diffract to high Bragg angles. This eliminates the influence of aspherical density contributions in the bonding regions. If all data were used to calculate the residual density map, the electron density difference reflects the discrepancies between the observed and the modelled structure factors. Significant features can be detected in a contour plot (Figure 2-16, left). These show that the bonding as well as the lone pair electron densities (N2) cannot be accounted for in the IAM.

The atom centred multipole model (still based on the reconstruction of $\rho$ from atomic contributions) as an improved scattering model accounts for these inadequacies, which is reflected by the smaller and randomly distributed 
residuals on the residual density map after the MM refinement (Figure 2-16, right). ${ }^{[174]}$ Each atom does now contribute to $\rho(\mathbf{r})$ (or $\rho_{n, x y z}$ ) with a fixed spherical core density term $\left(\rho_{c}\right)$, a spherical valence density term $\left(\rho_{v}\right)$, and an aspherical valence deformation density part (Eq. 2-7).

$$
\rho_{n}=\rho_{a t}(\boldsymbol{r})=\rho_{c}(r)+P_{v} \kappa^{3} \rho_{v}(\kappa r)+\sum_{l} \kappa^{\prime 3} R_{l}\left(\kappa^{\prime} r\right) \sum_{m=-l}^{l} P_{l m} Y_{l m}(\boldsymbol{r} / \mathrm{r})
$$

The populations $\left(P_{v}, P_{l m}\right)$ as well as the spatial expansions (radial scaling parameters $\kappa$ and $\kappa^{\prime}$ ) of the valence electron density can be adjusted. The radial functions $R_{l}$ are of Slater type orbital form (Eq. 2-8) with $\zeta_{l}$ exponents.

$$
R_{l}\left(\kappa^{\prime} r\right)=\frac{\zeta_{l}^{n_{1}+3}}{\left(n_{l}+2\right) !}\left(\kappa^{\prime} r\right)^{n_{l}} \exp \left(-\kappa^{\prime} \zeta, r\right)
$$

$Y_{I m}$ are density-normalized real spherical harmonics, usually referred to as multipoles, which account for the deformation of the electron density. Their shape resembles those of orbitals (Figure 2-17) but one must not be misled that the population of multipoles is equal to the population of orbitals.

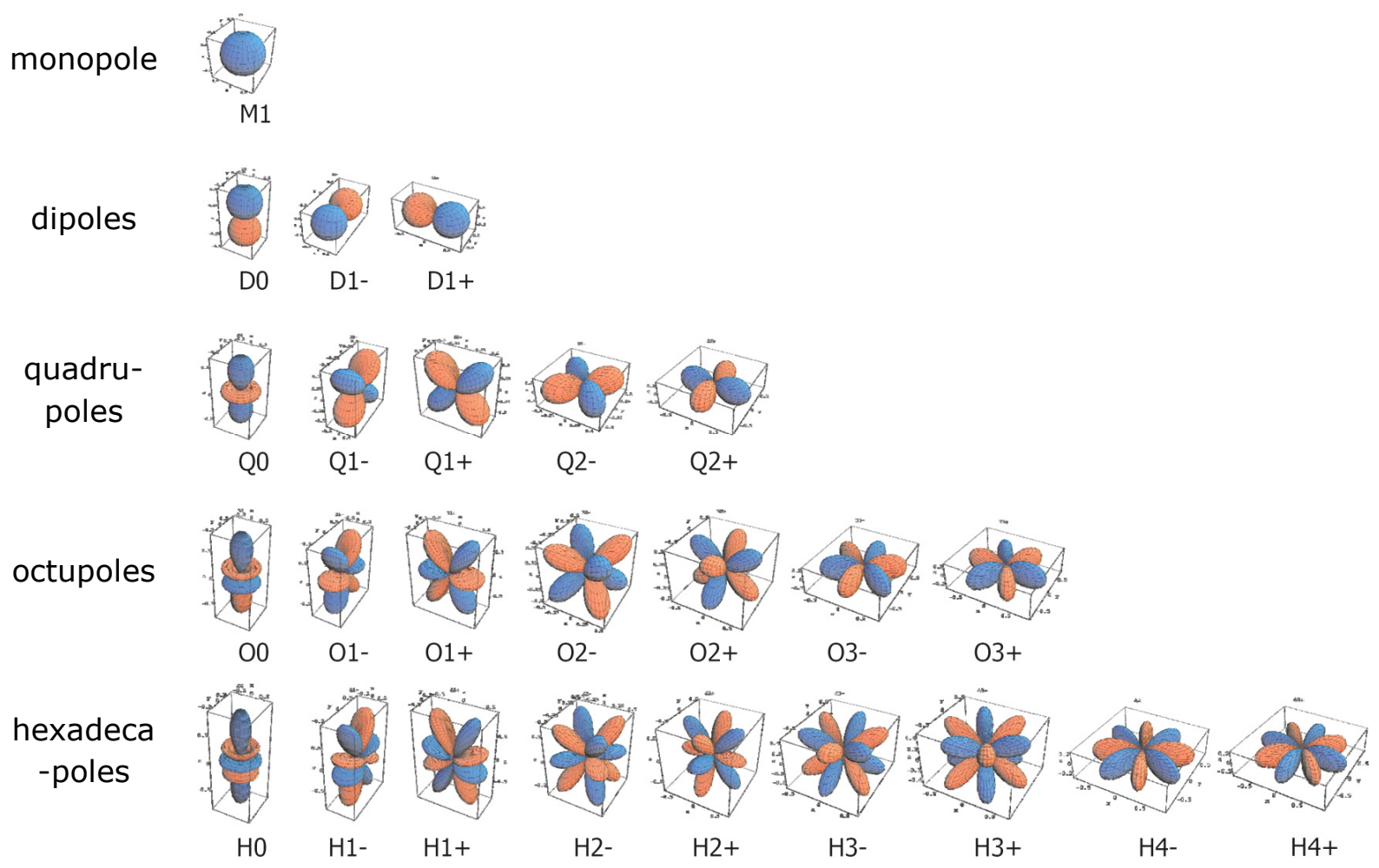

Figure 2-17. Shape, orientation and classification of multipoles. 
These functions are used to redistribute spherical electron density, e.g. into the bonding region. Their normalisation implies that one electron is transferred from the negative to the positive lobe if $P_{l m}$ equals 1 .

The atomic structure factors in the MM are the Fourier transformations of $\rho_{\text {at }}$ and as a result not as fixed as in the IAM (only influenced by $U$ ). They can be adjusted during the refinement process to mimic the real electronic distribution of an atom in the crystal which is nicely displayed in Figure 2-18.
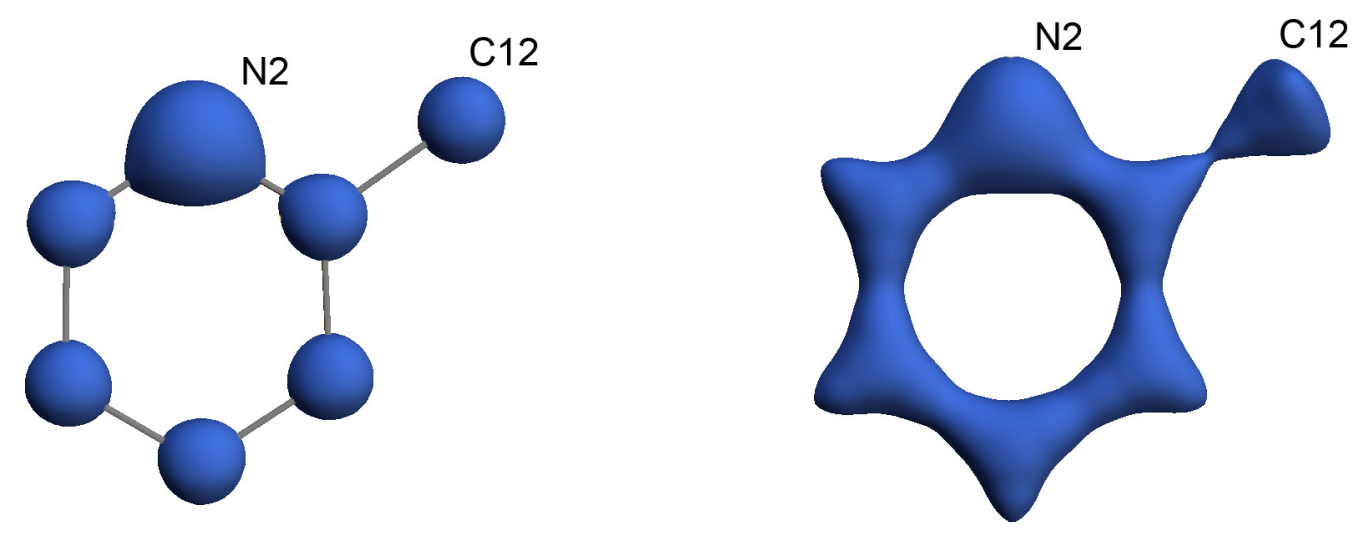

Figure 2-18. Modelled electron density of the 2-picoline ring in [2-PicLi $\cdot \mathrm{PicH}]_{2}(4)$ by a IAM (left) and MM (right). $\rho$ (r) shown on the same isosurface level.

Altogether up to 39 parameters for each atom can be refined and even more if anharmonic thermal motion of an atom is assumed. This high number compared to the 9 parameters (position and thermal motion) in an IAM refinement carries the inherent danger of strong parameter correlations and an incorrect electron density modelling. Therefore, the results of an MM refinement have to be checked for consistency.

One indicator for a successful refinement is a so called "flat and featureless" residual density map. The "measured" electron density in the crystal has to be completely modelled (besides experimental noise), so that neither large absolute values nor systematically distributed residual density peaks appear in the plot. K. Meindl and J. Henn developed a method to quantify the flatness and remaining features in residual density grids, because the judgement on these criteria was normally a subjective interpretation. ${ }^{[175]}$ Unfortunately, the residual density maps are until now biased by a correction of $F_{o b s}$ for the calculation. A systematic error is made during the treatment of anomalous dispersion effects and a detailed analysis of residual density maps is limited to structures with light atoms. It was shown for a manganese containing molecule ${ }^{[74]}$ in the research group of $D$. Stalke that the inclusion of anomalous dispersion during the MM refinement leads to a 
more featured residual density map with higher peaks than a refinement without the consideration of anomalous signal effects (theoretically inferior model). Thus, care has to be taken in the interpretation of residual density maps with heavy atoms. Nevertheless, these maps are an ideal tool to judge on the refinement of light atom structures such as [2-PicLi-PicH $]_{2}(\mathbf{4})$.

Further quality factors are the figures of merit that quantify the discrepancies between the observed and calculated structure factors. As mentioned above, these differences are minimised during the least-squares refinement procedure for the sum of all structure factors which were individually weighted and normally brought on the same scale by a single factor for all components. Commonly used criteria are $R 1$ (Eq. 2-9), wR2 (Eq. 2-10), and the GoF (goodness of fit, Eq. 2-11). The $R$-values should be minimal, while the GoF shall adopt values close to one.

$$
\begin{gathered}
R 1=\frac{\sum_{h k l}\left(\left|F_{\text {obs }}\right|-\left|F_{\text {calc }}\right|\right)}{\sum_{h k l}\left|F_{\text {obs }}\right|} \\
W R 2=\frac{\sum_{h k l} w_{h k l}\left(\left|F_{\text {obs }}\right|^{2}-\left|F_{\text {calc }}\right|^{2}\right)^{2}}{\sum_{h k l} w_{h k l}\left|F_{\text {obs }}\right|^{4}} \\
\text { GoF }=\sqrt{\frac{\sum_{h k l} w_{h k l}\left(\left|F_{\text {obs }}\right|-\left|F_{\text {calc }}\right|\right)^{2}}{N-n}}
\end{gathered}
$$

Especially the GoF is a critical value in multipole refinements, because an appropriate weighting scheme cannot be refined with XD. Statistical weights have to be used instead. Their values, determined during the integration procedure, are questionable, especially for high order data. As a consequence, the value of the GoF can differ significantly from 1 and normally ranges between 1.5 and 2.5 for data collected and processed on BRUKER equipment.

Additional criteria to check the reliability of a multipole refinement are rare. Only the investigation of properties derived from the modelled electron density distribution may provide indications of an erroneous refinement. 


\subsubsection{Topological Analysis According to QTAIM}

The obtained electron density distribution in a crystal from a multipole model can be analysed according to the quantum theory of atoms in molecules (QTAIM). ${ }^{[61]}$ The topological analysis of $\rho(\mathbf{r})$ provides much more information than the simple inspection of deformation density plots. These were readily used in the early days of charge density refinement to visualise the differences between a MM and an IAM. Although these plots normally show charge density differences in bonding or lone pair regions relative to the spherical model, there are also examples known in which no or even negative "bonding" electron density appeared. ${ }^{[170,176]}$ Therefore, descriptors based on the topology of $\rho(\mathbf{r})$ mainly replaced the deformation density in recent studies.

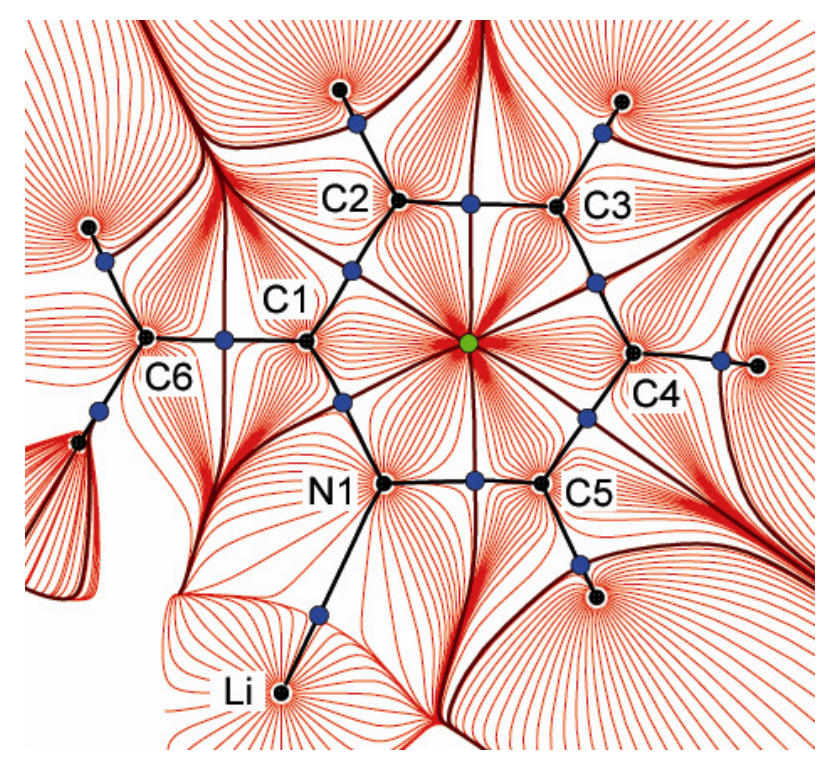

Figure 2-19. Trajectory plot of the picolyl ring in [2-PicLi.PicH $]_{2}(4)$. Red lines are gradient vectors, while blue/green dots indicate BCPs/RCPs. Black lines refer to bond paths between atomic positions (black dots) and zero flux surface border lines.

As the name QTAIM suggests, a criterion for the separation of atoms in a molecular assembly was found that is based on the electron density distribution. The first derivative of $\rho(\mathbf{r})$ defines a vector field in which the gradient vectors point in the direction of the greatest increase in $\rho(\mathbf{r})$. Thus, these trajectories are perpendicular to the contour lines of $\rho(\mathbf{r})$. They terminate at an attractor, which is the point of the atomic nucleus in $\nabla \rho(\mathbf{r})$. All gradient vectors that terminate at the same attractor belong to one basin. Every basin contains only one attractor and is confined by the gradient vectors that do not cross the boundary surface, which is accordingly referred to as zero flux surface (Figure 2-19). It is defined 
as $\nabla \rho(\mathbf{r}) \cdot \mathbf{n}(\mathbf{r})=0$, with $\mathbf{n}(\mathbf{r})$ being the normal vector on the surface. Nevertheless, gradient vectors will originate on the zero flux surface, if two atoms are bonded (this is explained later on).

The electron density enclosed by the atomic basins can be integrated. This results in physically meaningful atomic charges. These originate from charge transfer and polarisation effects in a molecule. They are more model independent compared to net charges, the difference between the number of valence electrons and the refined monopole population. However, it is known that integrated atomic charges tend to be larger than charges derived from other atom separation methods (e.g. Mulliken charges). ${ }^{[170]}$

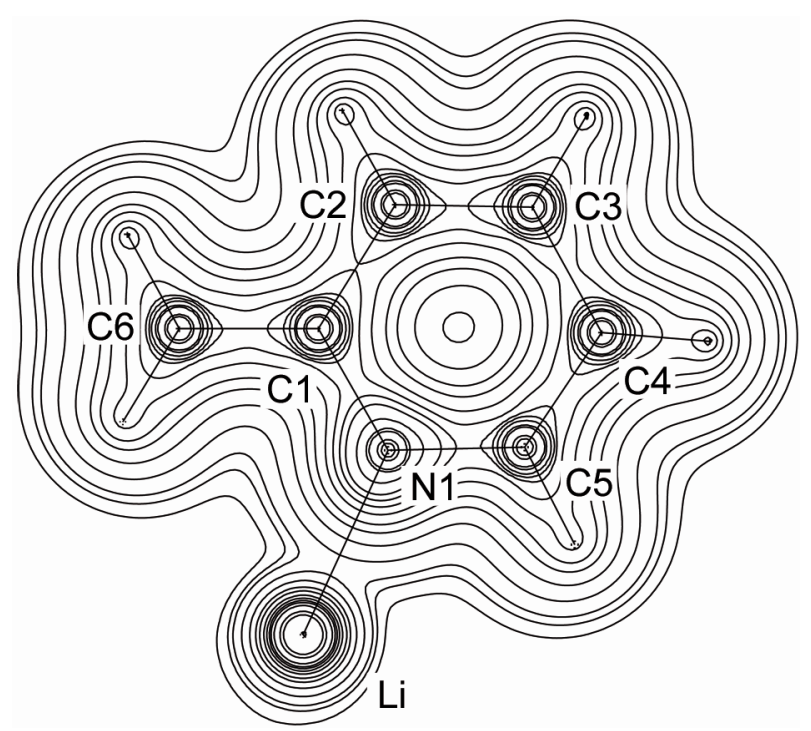

Figure 2-20. Contour plot of the electron density in the picolyl ring plane of [2-PicLi.PicH $]_{2}$ (4). Contours are drawn at $1 \times 10^{n}, 4 \times 10^{n}, 7 \times 10^{n}$ and $0.2,1.5,2.0,2.5,3.0 \mathrm{e}^{-3}$ with $\mathrm{n}=-2,-1,0,1$.

For the investigation of bonding features, the spatial distribution of the electron density in a molecule is not very helpful since hardly any features in the bonding region are visible (Figure 2-20). The atomic core densities dominate the $\rho(\mathbf{r})$ map and conceal the slender features of bond or lone pair electron density. The first and second derivatives of the electron density distribution are hence used to highlight characteristics of its topology.

Points at which $\nabla \rho(\mathbf{r})$ is equal to zero are extrema in $\rho(\mathbf{r})$. These are further classified by the signs of the diagonal elements (eigenvalues) of the Hessian matrix. The Hessian matrix $\mathbf{H}(\mathbf{r})$ is the $3 \times 3$ matrix of the second derivatives whose eigenvectors represent the curvatures at the extremum, if $\mathbf{H}(\mathbf{r})$ is diagonalised. Extrema are classified according to their rank (the number of 
nonzero eigenvalues) and the signature (sum of the signs of the eigenvalues). Local maxima in $\rho(\mathbf{r})$, where the electron density decreases in all directions of space, are $(3,-3)$ critical points and associated with nuclear/atomic positions (AP).

Saddle points in $\rho(\mathbf{r})$ are $(3,-1)$ critical points. This means that the electron density is maximal in two dimensions but minimal in the third. These points are so called bond critical points (BCPs) and always reside on the zero flux surface of two neighbouring atoms. Two gradient vector lines connect the BCP with the atomic positions and are called a bond path. The bond path thus represents the line along maximum density between two bonded atoms with respect to each neighbouring line and features a minimum at the BCP. $\rho(\mathbf{r})$ decreases orthogonal to the bond path. According to Bader, a BCP is a necessary and sufficient condition for the existence of a chemical bond. ${ }^{[61,177]}$ Only if a bond path between two atoms is quantified, a bond shall be drawn in molecular structures or Lewis diagrams. However, the existence of a bond in the chemical sense can sometimes still be discussed if a bond path is found (cf. chapter 2.3.7). ${ }^{[178,179]}$

The value of $\rho(\mathbf{r})$ at the BCP is an indicator for the interaction type. High electron density values point to covalent bonds (shared interaction), whereas low values can be found for ionic (closed shell) interactions. Moreover, it is connected with the bond order (BO), which can be calculated according to Eq. 2-12. ${ }^{[180]}$ The coefficients $A$ and $B$ are constants for given element bonds. In the case of carbon-carbon bonds, $\mathrm{B}$ is the $\rho\left(\mathbf{r}_{\mathrm{BCP}}\right)$ value of ethane and $\mathrm{A}$ is chosen to give a bond order of two for ethene and three for ethine.

$$
B O=\exp \left[A\left(\rho\left(r_{\mathrm{BCP}}\right)-B\right)\right]
$$

$(3,+1)$ critical points (ring critical points, RCP) can be found in closed rings, where the RCP is the point of minimal electron density in the ring plane but of maximal electron density orthogonal to the plane. Cage critical points (CCP, $(3,+3)$ critical points) are local minima in $\rho(\mathbf{r})$ and only appear in closed threedimensional molecules such as 1,4-diazabicyclo[2.2.2]octane.

The number of critical points in an isolated molecule can be used to validate the multipole refinement, since it has to obey the Poincaré-Hopf equation. This implies that the number of critical points $\left(N_{x}\right)$ is related by Eq. 2-13.

$$
N_{\mathrm{AP}}-N_{\mathrm{BCP}}+N_{\mathrm{RCP}}-N_{\mathrm{CCP}}=1
$$


Besides the judgement on extrema, the trace of the diagonalised Hessian matrix also known as the Laplacian $\left(\nabla^{2} \rho(\mathbf{r})\right)$ can be used to decide on bond characteristics. $^{[181]}$ It is related by the virial theorem to the local electronic energy density $E(\mathbf{r})$, as the sum of the potential $(V(\mathbf{r}))$ and kinetic energy density $(G(\mathbf{r}))$ according to Eq. $2-14 .^{[182]}$

$$
\left(\hbar^{2} / 4 m\right) \nabla^{2} \rho(\boldsymbol{r})=2 G(\boldsymbol{r})+V(\boldsymbol{r}) \quad \text { Eq. } 2-14
$$

As a consequence, the sign of $\nabla^{2} \rho(\mathbf{r})$ indicates if the negative potential energy or the positive kinetic energy dominates. Negative values show charge accumulations, which can be found for shared interactions, while positive values indicate charge depletions as in closed shell structures. However, it is well known, that this strict classification does not hold for very polar bonds or interactions with metal atoms. ${ }^{[72-74,183,184]}$ It has to be mentioned that the Laplacian as the second derivative of $\rho(\mathbf{r})$ is very sensitive to minimal changes in the electron density distribution and can also change rapidly in the bonding region. Therefore, the investigation of a single $\nabla^{2} \rho(\mathbf{r})$ value at the bond critical point to classify bonds gave way to the calculation and evaluation of $\nabla^{2} \rho(\mathbf{r})$ along the whole bond path in recent years. ${ }^{[185]}$

Moreover, local minima in $\nabla^{2} \rho(\mathbf{r})((3,+3)$ critical points $)$ specify points of local maximal charge accumulation. These can be found in the valence shell regions of atoms (valence shell charge concentrations, VSCCs), as bonding charge concentrations (BCCs) near the bond path of atoms that are bonded, and also as non-bonding charge concentrations (NBCCs) in the lone pair region.

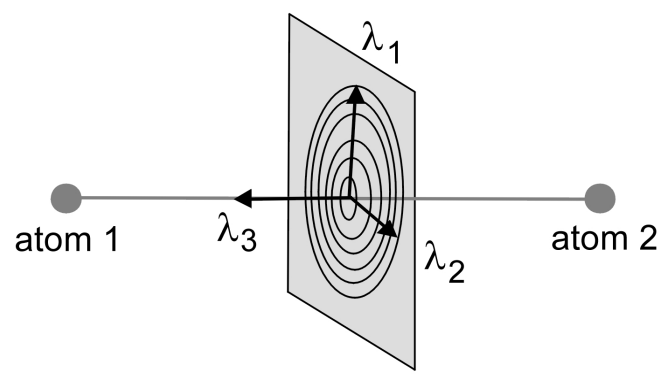

Figure 2-21. Orientation of the eigenvalues of the Hessian matrix.

The three eigenvalues of $\mathbf{H}(\mathbf{r})$ are called $\lambda_{1}$ to $\lambda_{3} . \lambda_{3}$ is associated with the direction of the bond path and accordingly possesses a positive sign (Figure 2-21). The remaining values have negative signs denoting charge concentrations. $\lambda_{1}$ is defined as largest negative value perpendicular to the bond, 
and its absolute value divided by $\lambda_{3}$ points to ionic bonding if the ratio is smaller than one. The ratio increases with the bond strength for covalent interactions.

The ratio of $\lambda_{1}$ and $\lambda_{2}$ minus one is called the ellipticity $\varepsilon$ of a bond. If both eigenvalues perpendicular to the bond path are of equal size, $\varepsilon$ becomes zero, which is the case for cylindrically symmetric bonds such as single or triple bonds between atoms of the same element. For $\pi$-interactions, which depend on their direction, higher $\varepsilon$ values are found such as 0.33 for ethene. ${ }^{[87]}$

\subsubsection{Experimental Details and Refinement Strategy}

The crystal application of the sensitive 2-picolyllithium.2-picoline material (Figure 2-22) was performed according to chapter 8.1. The high-resolution data were collected on a BRUKER TXS Mo rotating anode with INCOATEC HELIOS mirrors for focusing and monochromatisation and an APEX II CCD detector mounted on a D8 goniometer platform. This setup gives rise to the most powerful and brilliant molybdenum X-ray source available on the market for in-house facilities. The only alternatives for more intensive sources can be found at beam lines such as the EUROPEAN SYNCHROTRON RADIATION FACILITY in Grenoble (France) or the Swiss LIGHT SOURCE in Villingen (Switzerland).

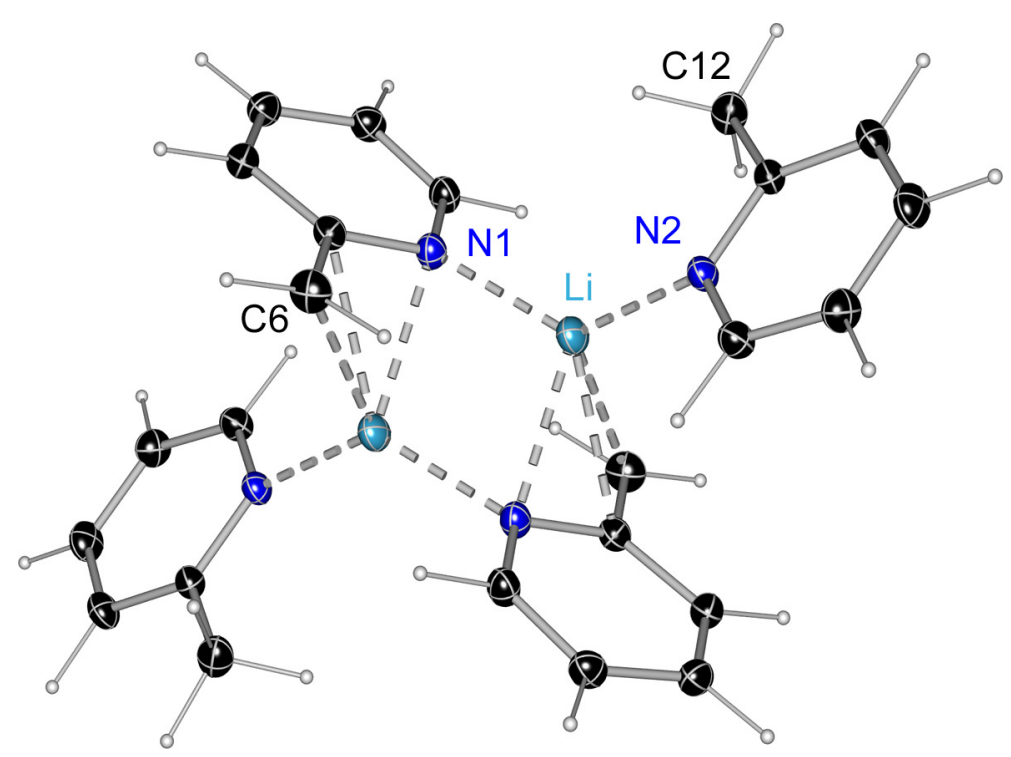

Figure 2-22. Molecular structure representation of $[2-\mathrm{PicLi} \cdot \mathrm{PicH}]_{2}(4)$ with anisotropic displacement parameters depicted at the $50 \%$ probability level.

The data set was collected at $100 \mathrm{~K}$ in an omega-scan mode $\left(\Delta \omega=0.3^{\circ}\right)$ at fixed $\varphi$-angles with a detector distance of $5 \mathrm{~cm}$ and an exposure time of $5 \mathrm{~s}$ (low- 
order data, 8 runs with 600 frames, $\left.d_{\min }=0.68 \AA\left(2 \theta_{\max }=63.0^{\circ}\right)\right)$ and at $4 \mathrm{~cm}$ distance with an exposure time of $90 \mathrm{~s}$ (high-order data, 6 runs with 600 frames, $\left.d_{\min }=0.45 \AA\left(2 \theta_{\max }=104.3^{\circ}\right)\right)$. Two separate detector positions had to be used due to the limited detector width of the APEX II CCD. After the determination of the orientation matrix and the unit cell with the APEX2 software package, ${ }^{[186]}$ the programme SAINT $v 7.23 \mathrm{~A}^{[187]}$ was used for data integration. The integration box size was fixed and the blend option used during the integration procedure. Two separate integrations were performed for the low and high order data. Afterwards, these were scaled, corrected for absorption, and merged with the new SADABS-2008/1 version, ${ }^{[188]}$ which was modified by G. M. Sheldrick for charge density data purposes. Three different output files were generated: an unmerged hkl file, a merged hkl file (both in the SHELXTL format) and a merged hkl file for XD. The unmerged file was only used to generate standard data quality statistics with XPREP. ${ }^{[189]}$

The structure was solved with SHELXS ${ }^{[190]}$ using the merged hkl file and an adapted IAM refinement with SHELXL ${ }^{[191]}$ supplied the starting model for the multipole refinement (XD hkl). The positional and anisotropic displacement parameters of the non-hydrogen atoms were refined with high-order data $\left(d_{\max }=\right.$ $\left.0.48 \AA\left(2 \theta_{\min }=95.5^{\circ}\right)\right)$. These parameters were kept fixed in the subsequent IAM refinement steps. The hydrogen atoms were identified by a difference Fourier analysis using the low-order data $\left(d_{\min }=1.00 \AA\left(2 \theta_{\max }=41.6^{\circ}\right)\right)$. Based on the same subset of data, the hydrogen atom positions were refined without the use of distance and bond angle restraints, but with an isotropic riding model (default values of SHELXL). Then, the hydrogen atoms were shifted along their bond vectors with the programme $\mathrm{XP}^{[192]}$ to neutron diffraction standard $\mathrm{C}-\mathrm{H}$ distances of $1.076 \AA$ except those of the $\mathrm{sp}^{3}$ hybridised carbon atom $\mathrm{C} 12$ $(1.085 \AA) \cdot{ }^{[171]}$

With the programme XDINI ${ }^{[193]}$ the SHELXTL instruction file was converted into XD format. The XD hkl file generated with SADABS was corrected for systematic absences and reflections with negative intensities or zero standard deviation were erased for the following multipole refinement. This was carried out on $F^{2}$ with the full-matrix-least-squares refinement programme XDLSM implemented in the XD2006 programme package. ${ }^{[193]}$ Statistical weights had to be used because the weighting scheme cannot be refined with XDLSM in contrast to SHELXL. The core and the spherical valence densities were composed of relativistic Dirac-Fock wave functions reported by Z. Su, P. Coppens, and P. Macchi (SCM bank file). ${ }^{[194,195]}$ Single-zeta functions with energy-optimized Slater exponents were 
used for the deformation density terms. ${ }^{[196,197]}$ The radial fit of these functions was optimised by refinement of the expansion-contraction parameter $\kappa^{\prime}$ and the monopole expansion by $\kappa$. The expansions over the spherical harmonics were truncated at the hexadecapolar level for all non-hydrogen atoms. All multipoles ( $n_{l}=1$ to 4$)$ of each atom shared the same $\kappa^{\prime}$-set (keep kappa constraint). The deformation densities of the hydrogen atoms were represented by bond-directed dipoles. To derive adequate parameters for the contraction of the hydrogen atoms, $\mathrm{K}$ and $\mathrm{K}^{\prime}$ values suggested by $A$. Volkov et al. were introduced and kept fixed during the refinement. ${ }^{[198]}$ Moreover, riding model constraints (cf. IAM refinement) were applied for the hydrogen atoms during the multipole refinement. The distance constraints from the starting model were maintained until the final refinement steps (reset bond). Then, the positions of the hydrogen atoms were refined against low order data $\left(d_{\min }=1.00 \AA\right)$ keeping all other parameters fixed. Since the $\mathrm{C}-\mathrm{H}$ distances stayed almost the same, the reset bond commands were updated to the new bond distances.

The final refinement model contained only a minimum number of chemical $(\mathrm{H} 3=\mathrm{H} 5 ; \mathrm{H6A}=\mathrm{H6B} ; \mathrm{H} 8=\mathrm{H} 9-\mathrm{H} 11 ; \mathrm{H} 12 \mathrm{~A}=\mathrm{H} 12 \mathrm{~B} / \mathrm{C})$ and no local-symmetry-constraints, since the modelling of the aza-allylic system should not be influenced by the applied constraints. These chemical constraints were maintained, because the monopole and multipole parameters did not significantly change when they were refined without constraints. The lithium atom was treated as a cation, as initial refinements (several $\mathrm{k}$-values tested) with the standard scattering factor of the alkaline metal lead to net charges that indicated a lack of valence density. Sometimes even negative monopole populations appeared for $\mathrm{k}$-values close to 1.0. A residual density analysis proved that the handling as a cation describes the lithium atom best. It has to be emphasised that the modelling of the lithium atom without valence density does not fix the properties of the atom to be completely ionic. Bonding electron density can also be provided by the neighbouring atoms.

Table 2-4. Selected crystallographic details after the multipole refinement of $[2-\mathrm{PiCLi} \cdot \mathrm{PiCH}]_{2}(4)$.

\begin{tabular}{ll|ll}
\hline independent reflections & $13125\left(R_{\text {int }}=0.020\right)$ & $R 1$ & 0.0204 \\
reflections above $(I>3 \sigma(I))$ & 10378 & WR2 & 0.0318 \\
parameters & 509 & GoF & 2.565 \\
largest diff. peak and hole & 0.150 and $-0.124 \mathrm{e}^{-3}$ & & \\
\hline
\end{tabular}


The whole set of density parameters were implemented in the refinement routines in a stepwise manner but in the final cycles all parameters except the $\kappa^{\prime}$ (due to the known effect of large correlations with other multipole parameters) were refined together using positive reflections with $I>3 \sigma(I)$ until convergence was reached. The multipole refinement led to a satisfactory fit with low $R$-values (Table 2-4) and a flat and featureless residual density map (Figure 2-23 and Appendix). The rigid bond test (DMSDA test) according to F. L. Hirshfeldt (Appendix) as well as the ADP representation (Figure 2-22) shows that the electron density distribution has been well separated from the thermal motion of the non-hydrogen atoms. ${ }^{[199]}$

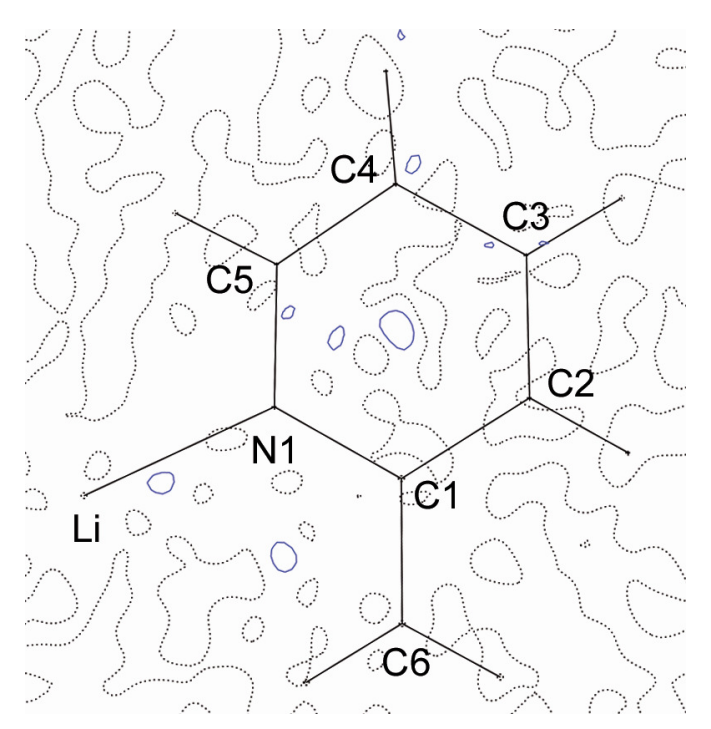

(a)

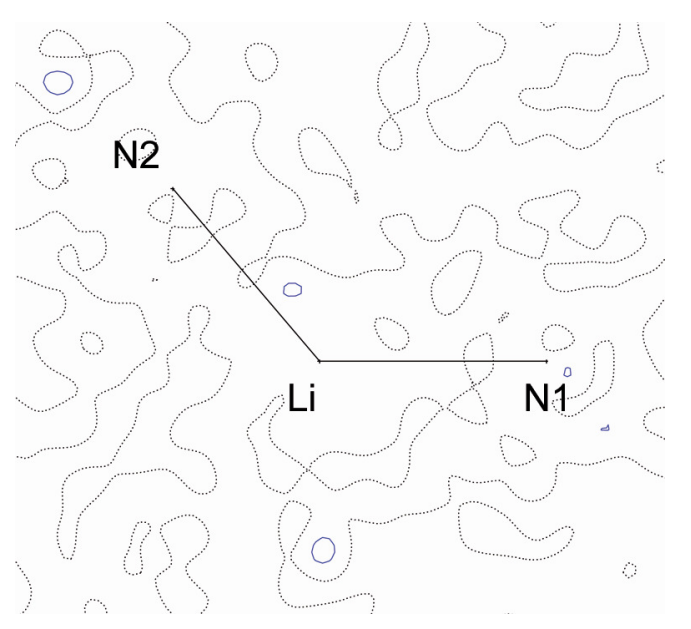

(c)

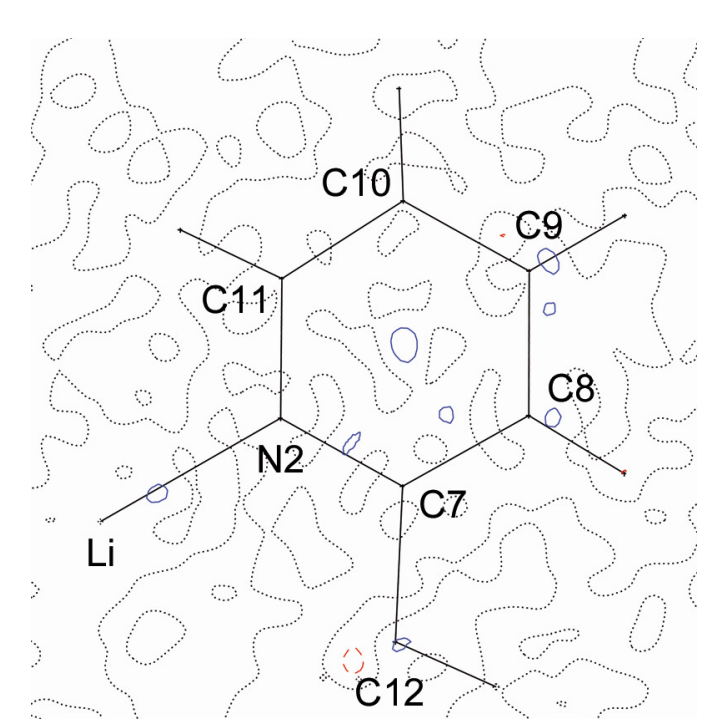

(b)

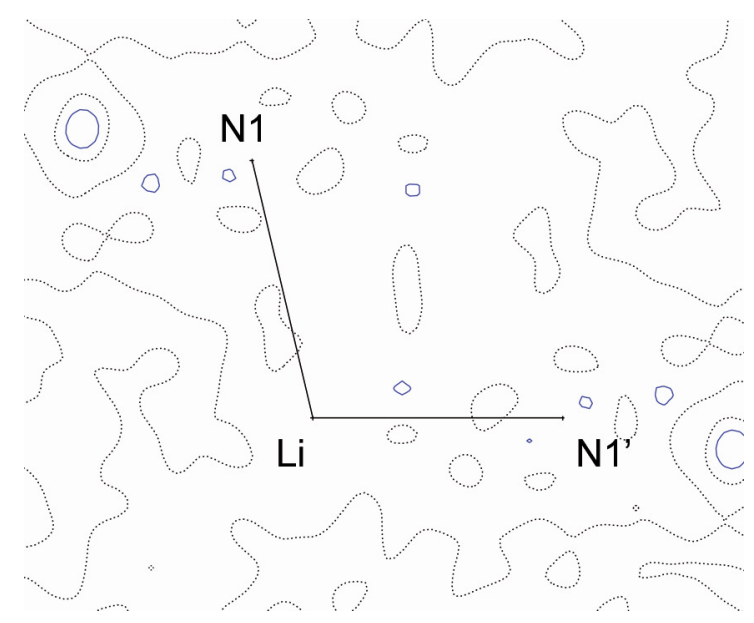

(d)

Figure 2-23. The step size of the contour lines is $0.1 \mathrm{e} \AA^{-3}$ (without a $\sin \theta \lambda^{-1}$ cut-off). Positive values are plotted as solid blue lines, negative values as dashed red ones and the zero value is drawn dotted black; (a) N1C2C4 plane, (b) N2C8C10 plane, (c) N1-Li$\mathrm{N} 2$ plane, and (d) N1-Li-N1' plane. 


\subsubsection{Molecular Structure of the 2-Picolyllithium. 2-Picoline Dimer}

The structural discussion of 2-PicLi.PicH (4) can be found in ref. [149]. Nevertheless, some major points shall be mentioned at the beginning of this chapter to facilitate the understanding of the charge density based results in relation to the geometrical appearance of $\mathbf{4}$. The presented bond lengths differ slightly from those reported before, because another data set of higher resolution has been measured. Moreover, the refinement model was changed from IAM to MM. The use of high resolution data and the improved structure model from the multipole refinement increase the accuracy of atomic descriptors significantly. Furthermore, the comparison with other picolyllithium compounds, sharing the motif of $\eta^{3}$-aza-allylic interaction with the cation, completes the discussion on structural features of unsubstituted 2-picolyllithium.

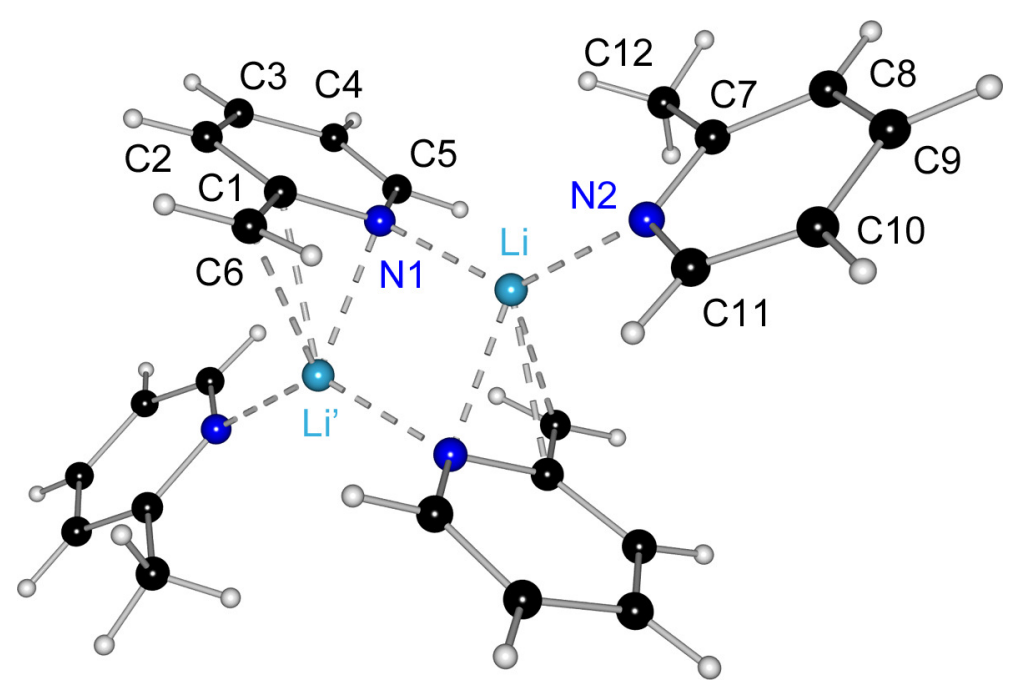

Figure 2-24. Molecular structure of $[2-\mathrm{PicLi} \cdot \mathrm{PiCH}]_{2}(4)$.

[2-PicLi-PicH] 2 (4) (Figure 2-24) crystallises in the monoclinic centrosymmetric space group $C 2 / C$ with one half of the dimer in the asymmetric unit. The full adduct is generated by an inversion operation at the centre of the fourmembered $[\mathrm{LiN}]_{2}$ ring. The dimer is linked via two different lithium-anion interactions: an $\mathrm{Li}-\mathrm{N}$ bond with the lithium atom located almost ideally in the pyridine ring plane (deviation: $0.20 \AA$; angle between $\mathrm{Li}-\mathrm{N}$ and the plane: $6^{\circ}$ ) and a $\eta^{3}$-aza-allylic contact in which the lithium cation is coordinated by the $\pi$-system of the methylene group $\mathrm{C} 6$, the 2-carbon atom $\mathrm{C} 1$, and the ring nitrogen atom N1. The coordination sphere of the lithium cation is completed by one 2-picoline donor molecule per metal atom. The in-plane $\mathrm{Li}-\mathrm{N}$ bond length 
(2.021(1) $\AA$ ) is about $0.12 \AA$ shorter than the contact to the respective aza-allylic nitrogen atom (2.136(1) $\AA$, Table 2-5). The lithium-carbon atomic distance is 2.284(1) $\AA$ to the 2-carbon atom and slightly larger to the methylene carbon atom C6 (2.328(1) $\AA)$.

Table 2-5. Selected bond lengths $[\AA]$ and angles $\left[^{\circ}\right]$ in $[\mathrm{PicLi} \cdot \mathrm{PiCH}]_{2}(4)$ and related compounds ( $\mathrm{Pic}=2$-picolyl, $\mathrm{Py}=2$-pyridyl) ${ }^{[134,135,200]}$

\begin{tabular}{l|ccccc}
\hline \multicolumn{7}{c}{} & $\begin{array}{c}{[\mathrm{PiCL} \cdot \mathrm{PiCH}]_{2}} \\
(4)\end{array}$ & $\begin{array}{c}{\left[\mathrm{PiCLi} \cdot \mathrm{OEt}_{2}\right]_{2}} \\
(5)\end{array}$ & $\begin{array}{c}{\left[\left(\mathrm{Me}_{3} \mathrm{Si}\right) \mathrm{CHPyLi} \cdot\right.} \\
\left.\left(\mathrm{Me}_{3} \mathrm{Si}\right)_{2} \mathrm{CHPy}\right]_{2}\end{array}$ & $\begin{array}{c}{\left[\left(\mathrm{Me}_{3} \mathrm{Si}\right) \mathrm{HC}\right.} \\
\left.\mathrm{PyLi} \mathrm{OEt}_{2}\right]_{2}\end{array}$ & $\begin{array}{c}{\left[\left(\mathrm{Me}_{3} \mathrm{Si}\right)_{2} \mathrm{CPyLi} \cdot\right.} \\
\left.\left(\mathrm{Me}_{3} \mathrm{Si}\right)_{2} \mathrm{CHPy}\right]\end{array}$ \\
\hline $\mathrm{Li}-\mathrm{N} 1$ & $2.021(1)$ & $2.031(2)$ & $2.06(1)$ & $2.04(1)$ & - \\
$\mathrm{Li}-\mathrm{N} 1$ & $2.136(1)$ & $2.133(2)$ & $2.14(1)$ & $2.19(1)$ & $2.00(1)$ \\
$\mathrm{Li}^{\prime}-\mathrm{C} 1$ & $2.284(1)$ & $2.297(2)$ & $2.39(1)$ & $2.34(1)$ & $2.32(1)$ \\
$\mathrm{Li}^{\prime}-\mathrm{C} 6$ & $2.328(1)$ & $2.321(3)$ & $2.39(1)$ & $2.36(1)$ & $2.22(1)$ \\
$\mathrm{Li}-\mathrm{D}$ [a] & $2.018(1)$ & $1.908(9)$ & $2.05(1)$ & $1.91(1)$ & $2.01(1)$ \\
$\mathrm{N} 1-\mathrm{C} 1$ & $1.394(1)$ & $1.401(2)$ & $1.38(1)$ & $1.39(1)$ & $1.38(1)$ \\
$\mathrm{N} 2-\mathrm{C} 7$ & $1.347(1)$ & & $1.36(1)$ & & $1.35(1)$ \\
$\mathrm{N} 1-\mathrm{C} 5$ & $1.358(1)$ & $1.358(2)$ & $1.36(1)$ & $1.36(1)$ & $1.35(1)$ \\
$\mathrm{N} 2-\mathrm{C} 11$ & $1.342(1)$ & & $1.36(1)$ & & $1.34(1)$ \\
$\mathrm{C} 1-\mathrm{C} 2$ & $1.448(1)$ & $1.453(2)$ & $1.44(1)$ & $1.43(1)$ & $1.43(1)$ \\
$\mathrm{C} 7-\mathrm{C} 8$ & $1.395(1)$ & & $1.38(1)$ & & $1.40(1)$ \\
$\mathrm{C} 2-\mathrm{C} 3$ & $1.361(1)$ & $1.349(2)$ & $1.35(1)$ & $1.32(1)$ & $1.35(1)$ \\
$\mathrm{C} 8-\mathrm{C} 9$ & $1.390(1)$ & & $1.37(1)$ & & $1.37(1)$ \\
$\mathrm{C} 3-\mathrm{C} 4$ & $1.423(1)$ & $1.417(2)$ & $1.42(1)$ & $1.40(1)$ & $1.37(1)$ \\
$\mathrm{C} 9-\mathrm{C} 10$ & $1.391(1)$ & & $1.39(1)$ & & $1.36(1)$ \\
$\mathrm{C} 4-\mathrm{C} 5$ & $1.372(1)$ & $1.364(3)$ & $1.36(1)$ & $1.36(1)$ & $1.36(1)$ \\
$\mathrm{C} 10-\mathrm{C} 11$ & $1.389(1)$ & & $1.34(1)$ & & $1.37(1)$ \\
$\mathrm{C} 1-\mathrm{C} 6$ & $1.382(1)$ & $1.371(2)$ & $1.38(1)$ & $1.40(1)$ & $1.43(1)$ \\
$\mathrm{C} 7-\mathrm{C} 12$ & $1.498(1)$ & & $1.52(1)$ & & $1.50(1)$ \\
\hline
\end{tabular}

[a] $\mathrm{D}=$ donor atom

Similarities of the bond lengths in the 2-picoline (4) and diethyl ether adduct (5) are evident (Table 2-5). The superposition plot of $\mathbf{4}$ and $\mathbf{5}$ demonstrates this (Figure 2-25). The atoms that are involved in the aza-allylic bonding of both adducts were used as reference atoms. They show only a small mean deviation $(0.016 \AA)$ for the aggregates. The only difference is the slightly changed picolyl ring position relative to the $[\mathrm{LiN}]_{2}$ ring, which is manifested by an angle of $3^{\circ}$ between both ring planes. Even the donor atom arrangement looks alike. The oxygen atom in $\mathbf{5}$ is located a little closer to the lithium atom than the 2-picoline 
nitrogen atom in $\mathbf{4}$, but the neighbouring carbon atom positions resemble each other. It is noteworthy, that the change from a nitrogen donor to an oxygen donor has only little influence on the aggregation pattern and also on the bond lengths of the anion, which will be discussed later.

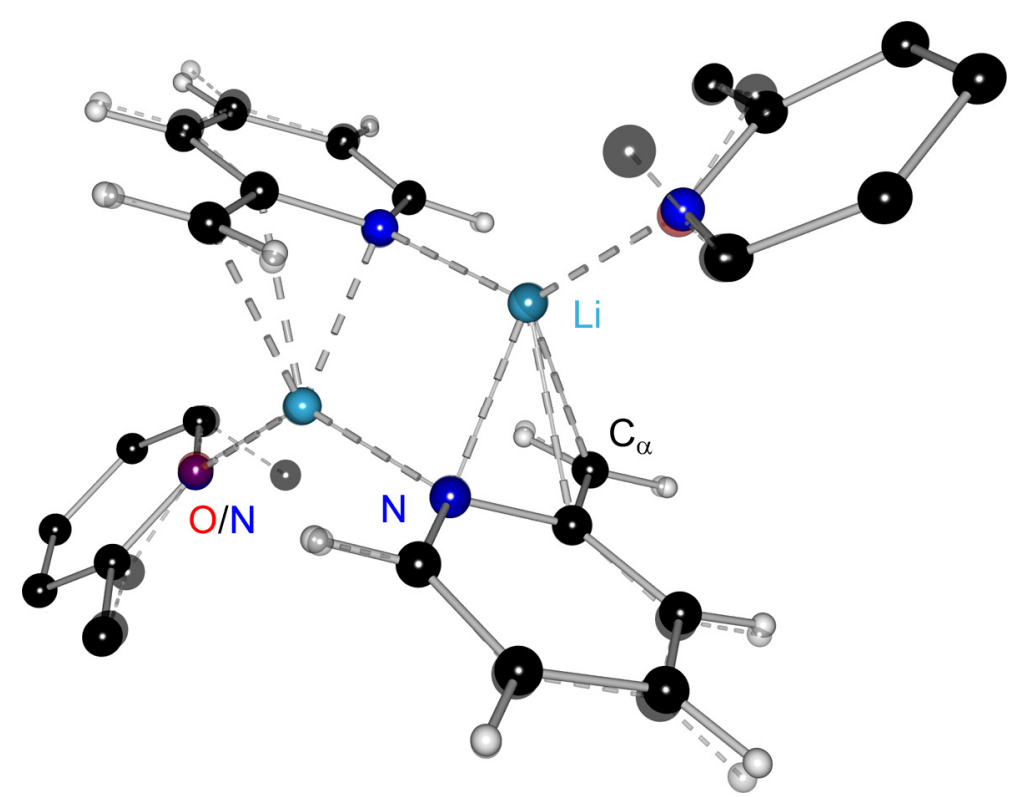

Figure 2-25. Molecular structure superposition plot of the 2-picolyllithium 2-picoline (4, solid) and diethyl ether adducts (5, dashed and transparent). Reference atoms were those involved in the aza-allyl bonding. Hydrogen atoms of the donor molecules are omitted for clarity.

Similar bonding situations are also reported in the (trimethylsilyl)(2-pyridyl)methyllithium diethyl ether ([2-( $\left.\left.\left.\mathrm{Me}_{3} \mathrm{Si}\right) \mathrm{CHPyLi}^{-\mathrm{OEt}_{2}}\right]_{2}\right)^{[134,135]}$ and the bis(trimethylsilyl)(2-pyridyl)methylpyridine adducts ([2-( $\left.\mathrm{Me}_{3} \mathrm{Si}\right) \mathrm{CHPyLi}$. $\left.2-\left(\mathrm{Me}_{3} \mathrm{Si}\right)_{2} \mathrm{CHPy}\right]_{2}^{[200]}$; Scheme 2-9). There, the aza-allyl nitrogen-lithium bonds (2.19(1) $\AA$; 2.14(1) $\AA$ ) are also longer than the in-plane lone pair based ones (2.04(1) $\AA$; 2.06(1) $\AA$ ), while the Li-C distances are slightly longer $(2.34-2.39 \AA)$ than in 4. Yet the tendency that the Li-C6 contact is the longest of the aza-allylic ones is maintained in the dimers ( $\mathrm{Li}-\mathrm{C} 1 / \mathrm{C} 6$ is equal in length in $\left[2-\left(\mathrm{Me}_{3} \mathrm{Si}\right) \mathrm{CH}\right.$ PyLi.2-( $\left.\left.\left(\mathrm{Me}_{3} \mathrm{Si}\right)_{2} \mathrm{CHPy}\right]_{2}\right)$. This situation is reversed in the bis(trimethylsilyl)(2-pyridyl)methyllithium monomers ${ }^{[134,135]}\left[2-\left(\mathrm{Me}_{3} \mathrm{Si}\right)_{2} \mathrm{CPyLi} \cdot 2-\left(\mathrm{Me}_{3} \mathrm{Si}\right)_{2} \mathrm{CHPy}\right]$ and [2-( $\left.\mathrm{Me}_{3} \mathrm{Si}\right)_{2} \mathrm{CPyLi}$.tmeda] (Table 2-3), which exclusively show aza-allylic lithium contacts. This can be explained by the rotation of the still $\mathrm{sp}^{2}$ hybridised bis(trimethylsilyl)methylene unit (sum of the bond angles: $358.5^{\circ}$ ) about the $\mathrm{C}_{6}$ C1 bonding vector relative to the pyridine plane $\left(41^{\circ}\right)$ in the picoline adduct. Thus the lone pair at C6 is oriented towards the cation, while the negative charge 
is delocalised to just a minor extent into the pyridine ring (long $\mathrm{C} 1-\mathrm{C} 6$ bond of $1.43(1) \AA)$. The tmeda donor of the second adduct on the other hand interferes too much with the silylated side chain, so that the lithium-carbon contacts of the aza-allylic system get significantly longer (2.46(2) and 2.43(2) $\AA$ ) and a strong $\mathrm{Li}-\mathrm{N}$ bond is formed (1.96(2) $\AA$ ), which is the shortest in the presented series.

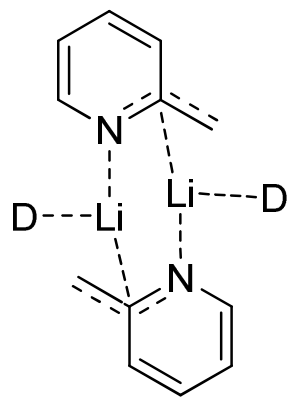

$[\mathrm{PiCLi} \cdot \mathrm{PiCH}]_{2}(4)$

[PicLi-OEt $\left.]_{2}\right]_{2}(5)$

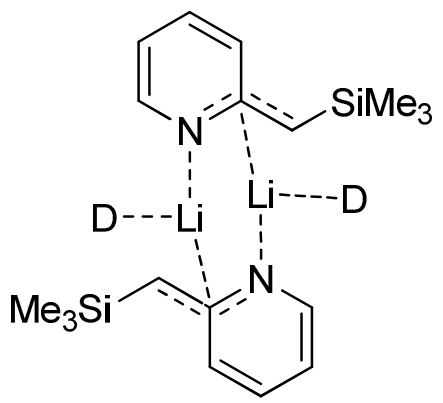

$\left[\left(\mathrm{Me}_{3} \mathrm{Si}\right) \mathrm{HCPyLi} \cdot\left(\mathrm{Me}_{3} \mathrm{Si}\right)_{2} \mathrm{HCPy}\right]_{2}$ $\left[\left(\mathrm{Me}_{3} \mathrm{Si}\right) \mathrm{HCPyLi} \cdot \mathrm{OEt}_{2}\right]_{2}$

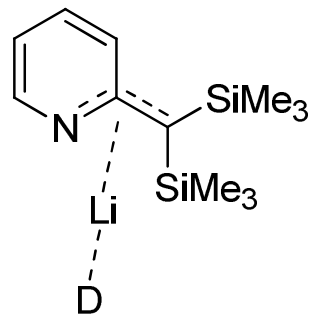

$\left[\left(\mathrm{Me}_{3} \mathrm{Si}\right)_{2} \mathrm{CPyLi} \cdot\right.$ $\left.\left(\mathrm{Me}_{3} \mathrm{Si}\right)_{2} \mathrm{HCPy}\right]$

Scheme 2-9. Lewis formulae of PicLi compounds with an $\eta^{3}$-aza-allylic coordination mode

$(\mathrm{D}=$ coordinating atom of the donor molecule; Pic $=2$-picolyl, $\mathrm{Py}=2$-pyridy $)$.

The lithium-donor bond in $\mathbf{4}$ is 2.018(1) $\AA$ long and comparable to those of the other picoline adducts (2.01(1) and 2.05 $\AA$, Table 2-5). The bis(trimethylsilyl) substitution has thereby no negative influence on the donor capacity of the pyridine ring.

2-Picoline already served as an external reference (Table 2-1) for the free acid but with 4 at hand the effect of the donation to the metal ion can be studied as well. When the 2-picoline molecule acts as a donor, rather similar but constantly larger bond lengths - compared to the free acid - are adopted (except for the C7-C12 bond). The N-C bond lengths are $1.345 \AA$ and that of the methyl group is $1.498(1) \AA$ (2-picoline: $1.502(3) \AA$ ), which is almost the value for a standard $\mathrm{C}_{\mathrm{sp} 3}-\mathrm{C}_{\mathrm{sp} 2}$ bond $(1.510 \AA) .{ }^{[152]}$ The aromatic $\mathrm{C}-\mathrm{C}$ bonds are herein almost identical (mean value 1.392(3) $\AA$ ) with the largest increase in the bond lengths involving atom $\mathrm{C} 4$. Thus, the donation to the metal atom elongates the bonds slightly, but does not affect the aromatic system. This is consistent with a donor concept stating that the lone pair of the nitrogen atom transfers some electron density to the metal atom.

By contrast, the deprotonation of the methyl group in 4 leads again to a tremendous change in the bonding situation of the aromatic ring. The nitrogen-

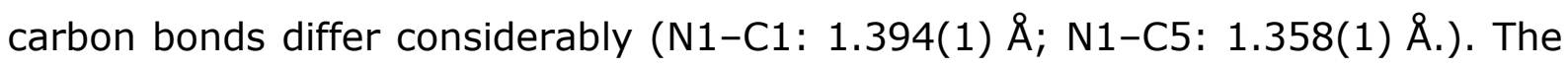


first is close to a standard $\mathrm{N}_{\mathrm{sp2} 2}-\mathrm{C}_{\mathrm{sp} 2}$ single bond $(1.40 \AA){ }^{[152]}$ while the second is slightly elongated compared to the respective bond in the donor. Moreover, there is a strong tendency towards the localisation of the double bonds. The methylene bond is shortened by $0.12 \AA$ compared to 2-picoline $(1.382(1) \AA)$ and is therefore much closer to a standard double bond length $\left(C_{\mathrm{sp} 2}=\mathrm{C}_{\mathrm{sp} 2}: 1.335 \AA\right)$ than to a corresponding single bond $\left(\mathrm{C}_{\mathrm{sp} 2}-\mathrm{C}_{\mathrm{sp} 2}: 1.466 \AA\right){ }^{[152]}$ This shortening is also observed for the $\mathrm{C} 2-\mathrm{C} 3$ and $\mathrm{C} 4-\mathrm{C} 5$ bonds (1.361(1) and 1.372(1) $\AA$ ). By contrast, $\mathrm{C} 1-\mathrm{C} 2$ and $\mathrm{C} 3-\mathrm{C} 4$ are closer to the values for a standard single bond (1.448(1) and 1.423(1) $\AA$ ). For that reason, the enamidic resonance formula seems once again to be most suitable to describe the electronic situation (cf. previous chapters).

The same is consistently true for $\mathbf{5}$. However, the influence of the diethyl ether donor is noticeable in the "localised" double bonds C2-C3, C4-C5, and C1-C6. All of them are shorter in the case of the superior oxygen donor molecule. Consequently, the negative charge can be distributed into the pyridine ring to a larger extent. Remarkably, almost the same bond lengths were reported for the monosilylated analogues of $\mathbf{4}$ and $\mathbf{5}$ as well. The trimethylsilyl group is apparently not stabilising the negative charge at the methylene group in those cases. The contrary is evident in the monomers. The disilyl substitution fixes the negative charge at the deprotonated carbon atom and a more or less undisturbed pyridine ring is observed (Table 2-5). This comes along with a rotation of the $\left(\mathrm{Me}_{3} \mathrm{Si}\right)_{2} \mathrm{C}$ group about the $\mathrm{C} 6-\mathrm{C} 1$ bonding vector relative to the ring $\left(41^{\circ}\right)$. Nonetheless, the carbanion is still almost planar (sum of the bond angles: $354.9^{\circ}$ ) and the lone pair interaction to the cation is preferred to the interaction of the $p$ orbital with the aromatic system.

Even though the bond lengths in $\mathbf{4}$ can serve as a first hint to the electronic properties, the investigation of the electron density distribution in the next chapter gives further evidence for the prevailed bonding situation. 


\subsubsection{Topological Analysis of the EDD in the 2-Picolyl- lithium-2-Picoline Dimer}

The topological analysis of the experimentally determined electron density distribution was performed with the programme XDPROP implemented in the XD2006 programme package. ${ }^{[193]}$

Supporting information to the experimental results was obtained from theoretical calculations. A gas phase geometry optimisation on a B3LYP $[201,202] /$ $6-311+G(d)^{[203,204]}$ level of theory, starting from the final atomic coordinates of the multipole model, was kindly performed by J. Henn. He also carried out the topological analysis of the theoretical EDD with the programme AIM2000. ${ }^{[205]}$ Nevertheless, the following discussion is mainly based on the result of the X-ray experiment. The theoretical results are used for comparison and the validation of the derived experimental density model. Values from theory are explicitly mentioned, if used, and written in italics throughout this chapter.

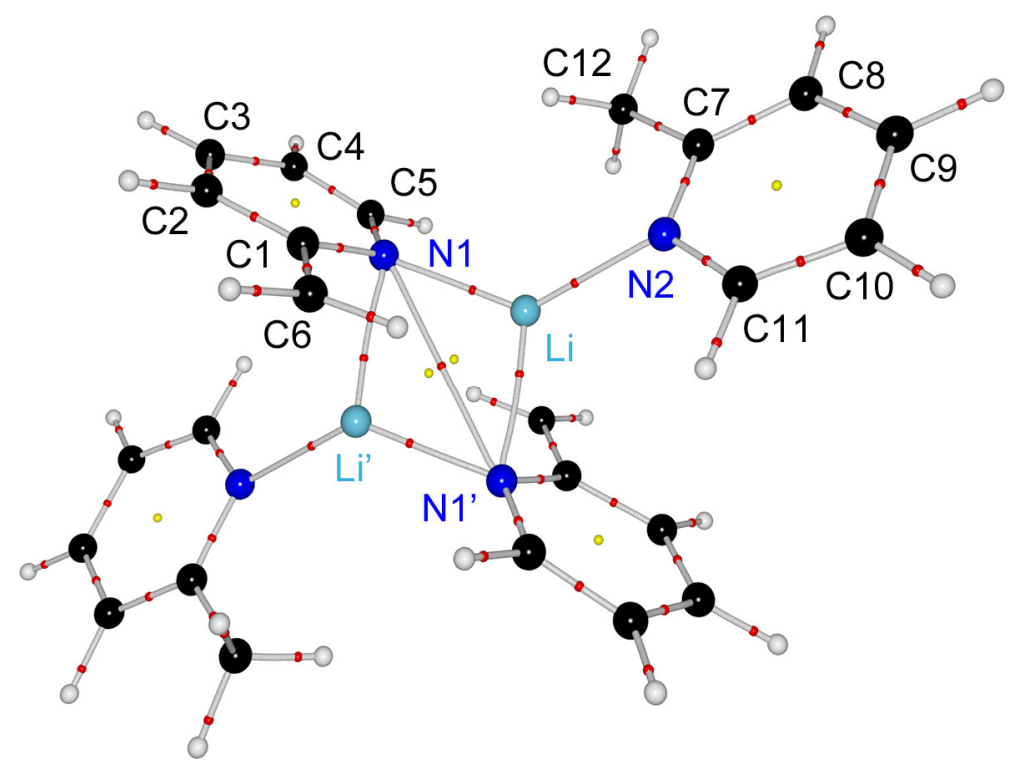

Figure 2-26. Molecular graph of [2-PiCLi·PicH $]_{2}$ (4) including BCPs (red dots) and RCPs (yellow dots). Hydrogen bond paths between the ring moieties are excluded for clarity.

First, all predicted critical points in the EDD were determined. The anticipated bond (BCPs) and ring critical points (RCPs) between the non-metal atoms were identified, as well as the bond paths between the bonded atoms. These are visualised in the molecular graph (Figure 2-26). In addition to the $\mathrm{N}-\mathrm{C}$ and $\mathrm{C}-\mathrm{C}$ bonds, only bond paths between the lithium and the nitrogen atoms could be found, but not to the aza-allylic carbon atoms (C6). This fuels the idea of dominant $\mathrm{Li}-\mathrm{N}$ bonds in the dimer and only auxiliary interactions to the formal 
anionic carbon atom C6. Moreover, a bond critical point between both picolyl anion nitrogen atoms ( $\left.\mathrm{N} 1, \mathrm{~N} 1^{\prime}\right)$ was found, but on a very low electron density level $\left(0.038 \mathrm{e}^{-3}\right.$, complete BCP list in ref. [206]). Similar weak attractive interactions between obviously non-bonded atoms in the view of a chemist are known and although no decisive point has been made so far in literature ${ }^{[88,179,207-209]}$ they should not be interpreted as a classical chemical bond. Nevertheless, their appearance strongly suggests an attractive character. ${ }^{[210,211]}$ For an exemplary case of an EDD analysis with a non-standard chemical bonding situation see J. Henn et al. ${ }^{[212]}$ In addition to the bond critical points, all corresponding RCPs were found, so that the Poincaré-Hopf equation (Eq. 2-13) is fulfilled.

Further, it has to be mentioned that additional hydrogen bonds between the picolyl and picoline rings in the dimer could be identified. These were not included in Figure 2-26 for clarity reasons. A complete molecular graph plot can be found in the appendix.

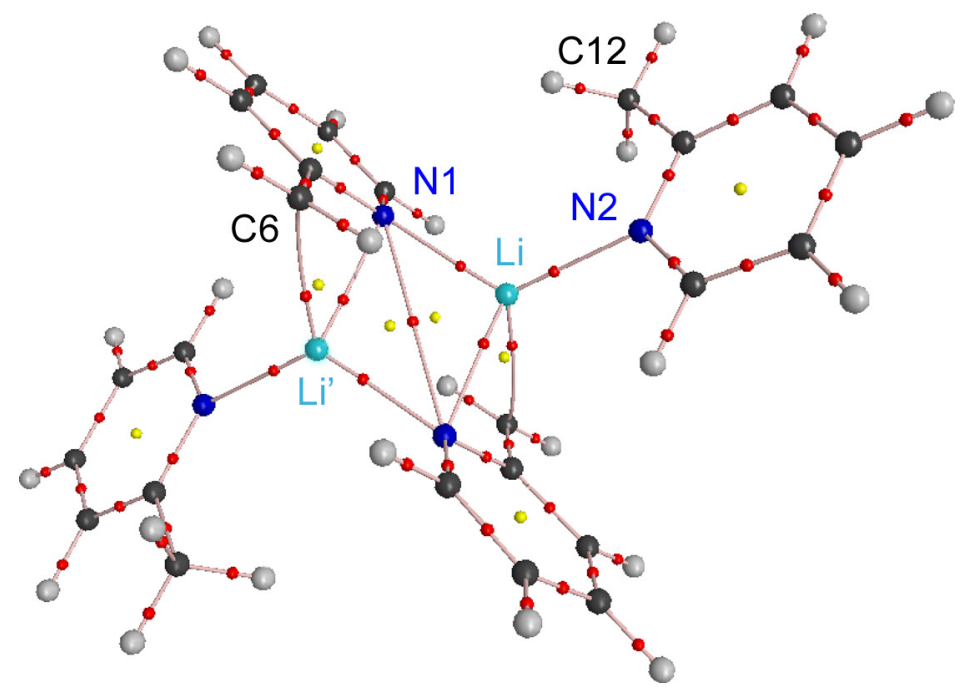

Figure 2-27. Molecular graph of the theoretical EDD in [2-PicLi-PicH $]_{2}$. The lines represent $\mathrm{BPs}$, red dots BCPs, and yellow dots RCPs.

All experimentally determined bond paths shown in Figure 2-26 were reproduced by theory (also the $\mathrm{N} 1-\mathrm{N1}^{\prime}$ path), but in addition a $\mathrm{BCP}$ between $\mathrm{Li}$ and $\mathrm{C} 6$ was detected there (Figure 2-27). This is most probably caused by geometrical changes of the ring positions relative to the $[\mathrm{LiN}]_{2}$ motif during the gas phase optimisation visualised in Figure 2-28 (almost no differences in the non-metal atom bond path lengths are observed; cf. Table 2-6). These also manifest in smaller Li-C6 distances in the isolated molecule $(2.302 \AA)$ than in the crystal $(2.328(1) \AA)$. This small difference is apparently sufficient for the formation of a bond path between the cation and the deprotonated carbon atom. 
The phenomenon clarifies that caution is advisable in the interpretation of missing or appearing bond paths in regions of a flat electron density distribution, as is the case for metal-metal, hydrogen-hydrogen, or long range bonding. This topic was perfectly tackled by $A$. M. Pendas et al., who stated in their article concerning bond paths, that "the existence or absence of a BP is related to the competition of different exchange channels, [...] when no competition is present, as in the He-He case, a BP will appear [...]. Moreover, there might be systems in which [...] a BP is not formed between two atoms because privileged channels of exchange interaction, other than the bond path, come into play, for example, those channels associated to lone pairs."[211] This seems plausible, if the lone pair at $\mathrm{N1}$, which is bonded to $\mathrm{Li}^{\prime}$ in accordance with the $\mathrm{BP}$, is considered (the existence or number of lone pairs at N1 and their influence on the molecular structure will be discussed later). The elucidation of the aza-allylic bonding mode, hence, cannot unambiguously be accomplished by an investigation of the bond paths to the lithium atom. Yet, the BCPs within the picolyl and picoline rings give further insight.

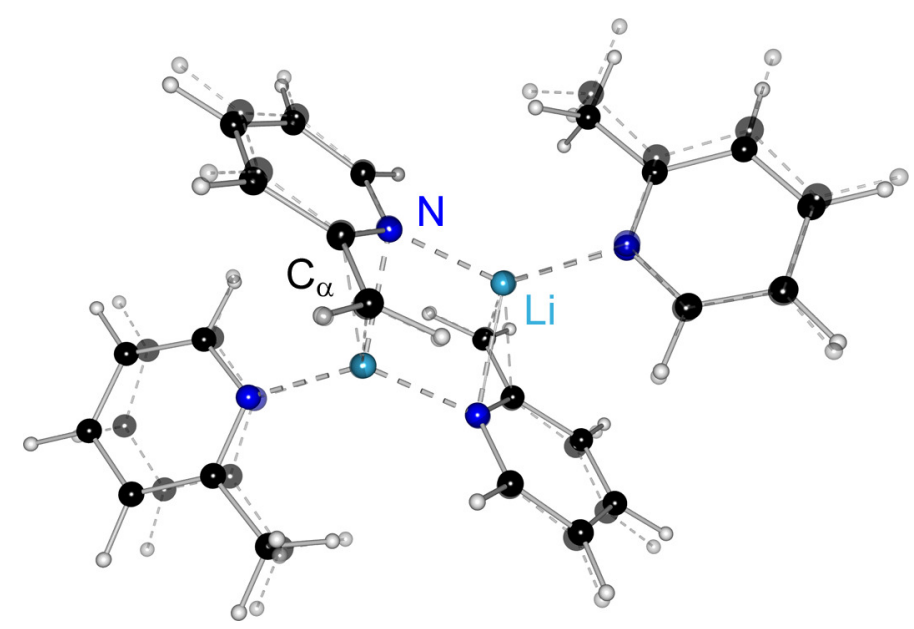

Figure 2-28. Superposition of the molecular structures of [2-PicLi.PicH $]_{2}(4)$ derived from $\mathrm{X}$-ray experiment (solid) and geometry optimisation (dashed and transparent).

The electron density values at the bond critical points of the donor molecule are an excellent internal reference for a non-deprotonated 2-picoline moiety (Table 2-6). The carbon-carbon bonds within the donor pyridine ring exhibit $\rho\left(\mathbf{r}_{\mathrm{BCP}}\right)$ values from 2.16 to $2.33 \mathrm{e}^{-3}$ that are in the range of phenyl ring systems cited in literature $\left(2.11\right.$ to $\left.2.26 \mathrm{e}^{-3}\right) \cdot{ }^{[213-217]}$ It has to be noted that even though the $\mathrm{C}-\mathrm{C}$ bond lengths, involving only $\mathrm{CH}$ moieties ( $\mathrm{C} 8-\mathrm{C} 11$ ), are the same $(1.391 \AA)$, significantly different electron density values at the bond critical points are observed $\left(2.21\right.$ to $\left.2.33 \mathrm{e}^{-3}\right)$. This is also true for the theoretical 
results. Therefore, $\rho\left(\mathbf{r}_{\mathrm{BCP}}\right)$ values cannot be directly inferred from atomic distances.

The $\mathrm{N}-\mathrm{C}$ bonds show elevated electron density values at the bond critical point ( 2.45 and $2.58 \mathrm{e}^{-3}$ ) due to the shift of the BCP towards the more electronegative nitrogen atoms. However, compared to previously investigated $\mathrm{N}-\mathrm{C}$ single bonds (1.54 to $\left.1.84 \mathrm{e}^{-3}\right){ }^{[185,213,217]}$ substantially higher $\rho\left(\mathbf{r}_{\mathrm{BCP}}\right)$ values are evident, obviously because of an increased bond order in the aromatic pyridine ring. Consistently, we find distinct $\varepsilon\left(\mathbf{r}_{\mathrm{BCP}}\right)$ values for the $\mathrm{C}-\mathrm{C}$ bonds $(0.13-0.18)$, which are typical for aromatic systems.

Table 2-6. Bond path lengths $[\AA]$, electron densities $\left[e \AA^{-3}\right]$, Laplacians $\left[e \AA^{-5}\right]$, and ellipticities at the BCPs of the $\mathrm{N}-\mathrm{C}$ and $\mathrm{C}-\mathrm{C}$ bonds in [2-PiCLi.PicH $]_{2}(4)$. Columns in italics refer to values from the B3LYP/6-311+G(d) optimisation with imposed $C_{i}$ symmetry.

\begin{tabular}{l|cccccccc}
\hline \multicolumn{3}{c}{ bond path } & \multicolumn{2}{c}{$\rho\left(\mathbf{r}_{\mathrm{BCP}}\right)$} & \multicolumn{2}{c}{$\nabla^{2} \rho\left(\mathbf{r}_{\mathrm{BCP}}\right)$} & \multicolumn{2}{c}{$\varepsilon\left(\mathbf{r}_{\mathrm{BCP}}\right)$} \\
\hline N1-C1 & 1.396 & 1.404 & $2.181(12)$ & 2.012 & $-17.7(1)$ & -20.5 & 0.07 & 0.08 \\
N2-C7 & 1.347 & 1.350 & $2.445(12)$ & 2.236 & $-23.6(1)$ & -23.0 & 0.09 & 0.10 \\
N1-C5 & 1.359 & 1.355 & $2.482(13)$ & 2.199 & $-27.7(1)$ & -22.9 & 0.10 & 0.10 \\
N2-C11 & 1.343 & 1.343 & $2.578(13)$ & 2.251 & $-27.3(1)$ & -22.5 & 0.05 & 0.09 \\
C1-C2 & 1.449 & 1.449 & $1.991(10)$ & 1.895 & $-16.0(1)$ & -17.5 & 0.09 & 0.14 \\
C7-C8 & 1.396 & 1.397 & $2.156(10)$ & 2.089 & $-18.9(1)$ & -20.7 & 0.14 & 0.22 \\
C2-C3 & 1.361 & 1.364 & $2.437(12)$ & 2.186 & $-24.7(1)$ & -22.2 & 0.16 & 0.27 \\
C8-C9 & 1.391 & 1.391 & $2.334(11)$ & 2.201 & $-23.1(1)$ & -20.9 & 0.13 & 0.19 \\
C3-C4 & 1.424 & 1.424 & $2.107(10)$ & 1.958 & $-18.8(1)$ & -18.5 & 0.08 & 0.16 \\
C9-C10 & 1.391 & 1.390 & $2.250(11)$ & 2.095 & $-19.9(1)$ & -21.0 & 0.14 & 0.19 \\
C4-C5 & 1.372 & 1.376 & $2.324(10)$ & 2.146 & $-21.6(1)$ & -21.6 & 0.18 & 0.27 \\
C10-C11 & 1.390 & 1.391 & $2.212(10)$ & 2.108 & $-20.1(1)$ & -21.2 & 0.18 & 0.21 \\
C1-C6 & 1.383 & 1.385 & $2.284(11)$ & 2.084 & $-21.3(1)$ & -20.0 & 0.26 & 0.34 \\
C7-C12 & 1.498 & 1.504 & $1.919(9)$ & 1.717 & $-18.1(1)$ & -14.7 & 0.02 & 0.05 \\
\hline
\end{tabular}

Two charge density studies of related molecules (Scheme 2-10) can be employed as a basis for comparisons. N. Kocher et al. used the lithiated iminophosphorane $\left[\left(\mathrm{Et}_{2} \mathrm{O}\right) \cdot \mathrm{Li}\left\{\mathrm{Ph}_{2}(\mathrm{CHPy}) \mathrm{P}\left(\mathrm{NSiMe}_{3}\right)\right\}\right]$ (6) to investigate the hypothetically hypervalent phosphorus atom and the coordination of the lithium atom. ${ }^{[71]}$ With respect to anionic picolyl compounds, 6 can be regarded as a phosphorus substituted picolyllithium compound without a direct contact of the deprotonated carbon atom to the metal. Second, the disilyl substituted picolyllithium dimer $\left[2-\left(\mathrm{Me}_{3} \mathrm{Si}\right)_{2} \mathrm{CPyLi}\right]_{2}$ (7), first synthesised by C. L. Raston and A. H. White et al., ${ }^{[134]}$ served W. Scherer et al. as a model compound to 
investigate putative $\mathrm{Li}-\mathrm{H}$ agostic interactions. ${ }^{[6,87]}$ Furthermore, the joint neutron and X-ray diffraction study mainly focussed on the quantification of negative hyperconjugation phenomena and not on the effect of the deprotonation on the pyridine ring.

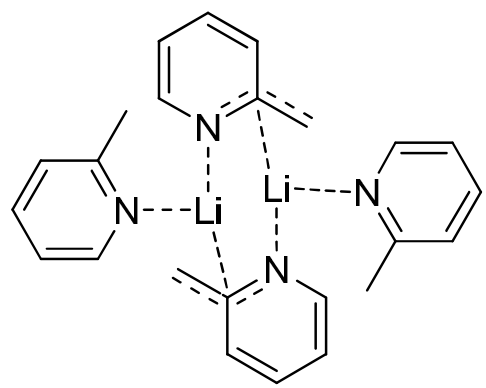

$[\mathrm{PiCLi} \cdot \mathrm{PiCH}]_{2}(\mathbf{4})$

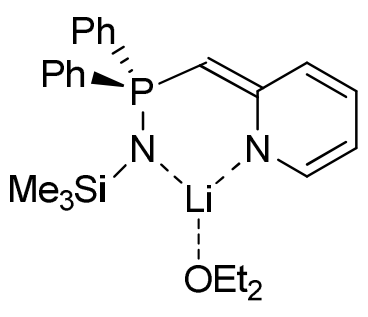

$\left[\left(\mathrm{Et}_{2} \mathrm{O}\right) \cdot \mathrm{Li}\left\{\mathrm{Ph}_{2}(\mathrm{HCPy}) \mathrm{P}\left(\mathrm{NSiMe}_{3}\right)\right\}\right](6)$

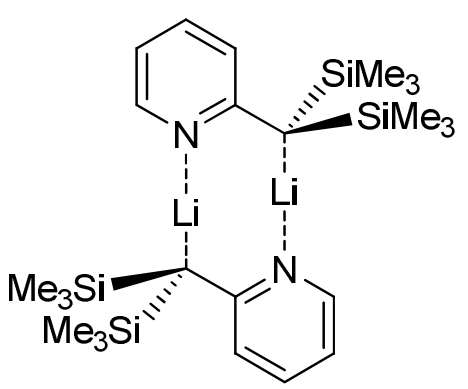

$\left[\left(\mathrm{Me}_{3} \mathrm{Si}\right)_{2} \mathrm{CPyLi}\right]_{2}(7)$

Scheme 2-10. Lewis formulae of PicLi compounds in charge density studies ( $D=$ donor atom; Pic $=2$-picolyl, Py $=2$-pyridyl).

[2-( $\left.\left.\mathrm{Me}_{3} \mathrm{Si}\right)_{2} \mathrm{CPyLi}\right]_{2}$ (7, Figure 2-5) was already mentioned at some stage in the structural discussion of $\eta^{1}$-carbanionic picolyllithium compounds in chapter 2.2.1. There, it was emphasised that $\left[2-\left(\mathrm{Me}_{3} \mathrm{Si}\right)_{2} \mathrm{CPyLi}\right]_{2}$ (7) shows much shorter $\mathrm{Li}-\mathrm{N}$ and $\mathrm{Li}-\mathrm{C}$ bonds than other dimers with the same coordination pattern, due to the stabilising effect of the silyl groups. This even leads to an $\mathrm{sp}^{3}$ hybridisation at the deprotonated carbon atom. Accordingly, the negative charge is hardly delocalised into the pyridine ring, which is evident from the $\rho\left(\mathbf{r}_{\mathrm{BCP}}\right)$ values of $\mathbf{7}$ given in Table 2-7. Just minor differences among the carbon-carbon bonds occur, so that the pyridine ring in $\mathbf{7}$ can be easily compared to the 2-picoline donor molecule in 4 . Significantly different $\rho\left(\mathbf{r}_{\mathrm{BCP}}\right)$ values were determined for $\left[\left(\mathrm{Et}_{2} \mathrm{O}\right) \cdot \mathrm{Li}\left\{\mathrm{Ph}_{2}(\mathrm{CHPy})\right.\right.$ $\left.\mathrm{P}\left(\mathrm{NSiMe}_{3}\right)\right\}$ ] (6), which indicate a disturbed aromatic system and will be discussed later on.

The methyl carbon bond C7-C12 in 4 reveals a reduced $\rho\left(\mathbf{r}_{\mathrm{BCP}}\right)\left(1.92 \mathrm{e} \AA^{-3}\right)$ and a negligible $\varepsilon\left(\mathbf{r}_{\mathrm{BCP}}\right)$ of 0.02 relative to the pyridine ring. These values are larger than those of a pure single bond ( $c$. ethane: $\rho\left(\mathbf{r}_{\mathrm{BCP}}\right)=1.63 \mathrm{e} \AA^{-3}$; $\left.\varepsilon\left(\mathbf{r}_{\mathrm{BCP}}\right)=0.00\right) .{ }^{[87]}$ But they are much closer to ethane than to ethene, the model compound of a pure carbon-carbon double bond $\left(\rho\left(\mathbf{r}_{\mathrm{BCP}}\right)=2.42\right.$ e $\AA^{-3}$; $\left.\varepsilon\left(\mathbf{r}_{\mathrm{BCP}}\right)=0.33\right) .{ }^{[87]}$ The theoretical value of the electron density at the C7-C12 bond critical point $\left(1.72 \mathrm{e} \AA^{-3}\right)$ as well as the ellipticity $\left(\varepsilon\left(\mathbf{r}_{\mathrm{BCP}}\right)=0.05\right)$ show the same trend towards a single bond. This is also true for the C1-C6 bond in the 
Table 2-7. Interatomic distances $d([\AA])$, electron densities $\left[\mathrm{e} \AA^{-3}\right]$, Laplacians $\left[\mathrm{e} \AA^{-5}\right]$, and ellipticities at the BCPs of the $\mathrm{N}-\mathrm{C}$ and $\mathrm{C}-\mathrm{C}$ bonds in $[2-\mathrm{PiCLi} \cdot \mathrm{PiCH}]_{2}(4),\left[\left(\mathrm{Et}_{2} \mathrm{O}\right)\right.$. $\left.\mathrm{Li}\left\{\mathrm{Ph}_{2} \mathrm{P}(\mathrm{CHPy})\left(\mathrm{NSiMe}_{3}\right)\right\}\right](6)$, and $\left[2-\left(\mathrm{Me}_{3} \mathrm{Si}\right)_{2} \mathrm{CPyLi}\right]_{2}(\mathbf{7}) .{ }^{[71,86,87]}$

\begin{tabular}{|c|c|c|c|c|c|c|c|c|}
\hline & & $\mathrm{N} 1-\mathrm{C} 1$ & $\mathrm{~N} 1-\mathrm{C} 5$ & $\mathrm{C} 1-\mathrm{C} 2$ & $\mathrm{C} 2-\mathrm{C} 3$ & $\mathrm{C} 3-\mathrm{C} 4$ & C4-C5 & $\mathrm{C} 1-\mathrm{C} 6$ \\
\hline \multirow{3}{*}{$d$} & 4 & 1.394 & 1.358 & 1.448 & 1.361 & 1.423 & 1.372 & 1.382 \\
\hline & 6 & 1.370 & 1.347 & 1.434 & 1.368 & 1.409 & 1.377 & 1.402 \\
\hline & 7 & 1.364 & 1.345 & 1.417 & 1.383 & 1.395 & 1.382 & 1.480 \\
\hline \multirow{3}{*}{$\rho\left(\mathbf{r}_{\mathrm{BCP}}\right)$} & 4 & $2.18(1)$ & $2.48(1)$ & $1.99(1)$ & $2.44(1)$ & $2.11(1)$ & $2.32(1)$ & $2.28(1)$ \\
\hline & 6 & $2.30(2)$ & $2.52(2)$ & $2.12(1)$ & $2.40(1)$ & $2.10(1)$ & $2.33(1)$ & $2.14(1)$ \\
\hline & 7 & $2.17(3)$ & $2.39(3)$ & $2.06(2)$ & $2.17(2)$ & $2.13(2)$ & $2.20(3)$ & $1.78(2)$ \\
\hline \multirow{3}{*}{$\nabla^{2} \rho\left(\mathbf{r}_{\mathrm{BCP}}\right)$} & 4 & $-17.7(1)$ & $-27.7(1)$ & $-16.0(1)$ & $-24.7(1)$ & $-18.8(1)$ & $-21.6(1)$ & $-21.3(1)$ \\
\hline & 6 & $-24.7(1)$ & $-30.5(1)$ & $-18.5(1)$ & $-22.9(1)$ & $-17.6(1)$ & $-23.2(1)$ & $-17.6(1)$ \\
\hline & 7 & $-17.3(1)$ & $-22.3(1)$ & $-16.5(1)$ & $-19.0(1)$ & $-18.1(1)$ & $-20.7(1)$ & $-11.2(1)$ \\
\hline \multirow{3}{*}{$\varepsilon\left(\mathbf{r}_{\mathrm{BCP}}\right)$} & 4 & 0.07 & 0.10 & 0.09 & 0.16 & 0.08 & 0.18 & 0.26 \\
\hline & 6 & 0.12 & 0.11 & 0.20 & 0.24 & 0.18 & 0.21 & 0.27 \\
\hline & 7 & 0.23 & 0.22 & 0.21 & 0.23 & 0.24 & 0.24 & 0.12 \\
\hline
\end{tabular}

anion $7\left(\rho\left(\mathbf{r}_{\mathrm{BCP}}\right)=1.78 \mathrm{e} \AA^{-3} ; \varepsilon\left(\mathbf{r}_{\mathrm{BCP}}\right)=0.12\right)$, supporting the point that the negative charge is not delocalised over the aromatic system.

Deprotonation at the methyl group changes the electronic situation in the whole ring drastically. The carbon bonds are no longer equal in length. As the bond length alteration already indicated, the charge density distribution supports the hypothesis that the double bonds are localised at the expense of the aromatic system. Consistent with the enamidic resonance formula, the highest $\rho\left(\mathbf{r}_{\mathrm{BCP}}\right)$ values are found between $\mathrm{C} 2-\mathrm{C} 3$ and $\mathrm{C} 4-\mathrm{C} 5\left(2.44\right.$ and $2.32 \mathrm{e}^{-3}$ ) that are accompanied by distinct $\varepsilon\left(\mathbf{r}_{\mathrm{BCP}}\right)$ values of 0.16 and 0.18 . Consequently, the electron density and the ellipticity at the BCP of C1-C2 (1.99 e $\left.\AA^{-3}, 0.09\right)$ and C3-C4 $\left(2.11 \mathrm{e}^{-3}, 0.08\right)$ are reduced compared to the donor molecule $(2.16$, $2.25 \mathrm{e}^{-3}$, and $0.14,0.14$ respectively). The $\mathrm{N}-\mathrm{C}$ bonds are also influenced. The perturbation of the aromaticity results in reduced $\rho\left(\mathbf{r}_{\mathrm{BCP}}\right)$ values of 2.18 and $2.48 \mathrm{e} \AA^{-3}$ for the nitrogen-carbon bonds ( 2.45 and $2.58 \mathrm{e} \AA^{-3}$ in the donor). The methylene $\mathrm{C} 1-\mathrm{C} 6$ bond is most affected by the deprotonation. Values typical for a bond order close to two (ethene as a prototype) are found in [2-PicLi.PicH] 2 (4) $\left(\rho\left(\mathbf{r}_{\mathrm{BCP}}\right)=2.28 \mathrm{e}^{-3} ; \varepsilon\left(\mathbf{r}_{\mathrm{BCP}}\right)=0.26\right)$.

The bond order can be approximated according to Eq. 2-12 in section 2.3.4. By using the determined $\rho\left(\mathbf{r}_{\mathrm{BCP}}\right)$ values of ethane and ethene, ${ }^{[87]}$ the coefficients 
A und B were calculated to be 0.88 and 1.63 , respectively. This gives a slightly raised bond order of 1.29 for the methyl bond in the donor. The methylene bond shows the $\pi$-conjugation with a $\mathrm{BO}$ of 1.79 . The most pronounced double bond character can be identified in the $\mathrm{C} 2-\mathrm{C} 3$ bond $(\mathrm{BO}=2.03)$. A mean bond order of 1.70 for the aromatic $\mathrm{C}-\mathrm{C}$ bonds in the donor molecule relativises the determined values to a certain extent. This deviation from the expected bond order of 1.5 for an aromatic system can be attributed to the known phenomenon of differing $\rho\left(\mathbf{r}_{\mathrm{BCP}}\right)$ values derived from experiment and theory of the same compound (reference for coefficients taken from theory). ${ }^{[72,183]}$ Systematic studies, dealing with the discrepancies between topological properties derived from X-ray experiments or computational chemistry, indicate that the limited flexibility of the multipole model as well as, to a minor extent, basis set shortcomings, electron correlation, and crystal field effects in theory are important sources of errors. ${ }^{[184,218,219]}$

A trend of elevated experimental $\rho\left(\mathbf{r}_{\mathrm{BCP}}\right)$ values is evident in [2-PicLi.PicH] $]_{2}$ (Table 2-6). A mean electron density at the bond critical points of $2.26 \mathrm{e}^{-3}$ is calculated for the experiment, while $2.08 e \AA^{-3}$ results from theory. Consequently, a mean $\mathrm{BO}$ of 1.54 is received using theoretical $\rho\left(\mathbf{r}_{\mathrm{BCP}}\right)$ values of [2-PicLi.PicH] $]_{2}$. This also leads to a better fit of the BO of the C7-C12 methyl bond (1.08). Hence, smaller values are also obtained for the "localised" double bonds (e.g. 1.63 for $\mathrm{C} 2-\mathrm{C} 3$ and 1.49 for $\mathrm{C} 1-\mathrm{C} 6)$. Yet, exactly the same conclusions can be drawn from the experiment and the theoretical calculations, even though their absolute values are on a slightly different scale. However, since experimental values for ideal single and double bonds are missing, it has to be reverted to theoretical ones. An electron density data bank with experimentally determined $\rho\left(\mathbf{r}_{\mathrm{BCP}}\right)$ values of a large number of compounds would be ideal for a statistical identification of such values.

The trend towards the localisation of double bonds can be further substantiated by the $\nabla^{2} \rho\left(\mathbf{r}_{\mathrm{BCP}}\right)$ values, which are more negative for the bonds with increased double bond character indicating more distinct charge accumulations in the bonding region. $\mathrm{C} 1-\mathrm{C} 6$ and $\mathrm{C} 4-\mathrm{C} 5$ show almost similar values $\left(-21.3\right.$ and $\left.-21.6 \mathrm{e} \AA^{-5}\right)$, while between $\mathrm{C} 2$ and $\mathrm{C} 3$ the most pronounced charge accumulation is detected with $-24.7 e \AA^{-5}$. This value is quite close to the one in ethene $\left(-27,2 \mathrm{e} \AA^{-5}\right)$, whereas ethane features a reduced negative value of $-13.4 \mathrm{e} \AA^{-5} \cdot{ }^{[87]}$ Related values are quantified between $\mathrm{C} 1-\mathrm{C} 2\left(-16.0 \mathrm{e} \AA^{-5}\right)$ and to a smaller extent between $\mathrm{C} 3-\mathrm{C} 4\left(-18.8 \mathrm{e}^{-5}\right)$. An identical pattern is observed in theory (Table 2-6). 
The same tendency of a localised $\mathrm{C} 1-\mathrm{C} 6$ double bond is observed in the lithiated iminophosphorane $\mathbf{6}$. The authors concluded that a delocalisation of the negative charge over the $\mathrm{C} 6-\mathrm{C} 1-\mathrm{N} 1$ residue occurs. This statement has to be slightly extended to the whole pyridine ring. Indeed, the N1-C1 bond shows even a reduced electron density at the BCP relative to 2-picoline $\left(2.30 \mathrm{e} \AA^{-3} \mathrm{vs}\right.$. $2.45 \mathrm{e}^{-3}$ ) and only those bonds with increased double bond character (C2-C3; C4-C5; C1-C6) exhibit elevated $\rho\left(\mathbf{r}_{\mathrm{BCP}}\right)$ values. Thus, $\left[\left(\mathrm{Et}_{2} \mathrm{O}\right) \cdot \mathrm{Li}\left\{\mathrm{Ph}_{2} \mathrm{P}(\mathrm{CHPy})\right.\right.$ $\left.\left.\left(\mathrm{NSiMe}_{3}\right)\right\}\right](6)$ is actually a monomeric picolyllithium compound with an intramolecular phosphorus imine donor site.

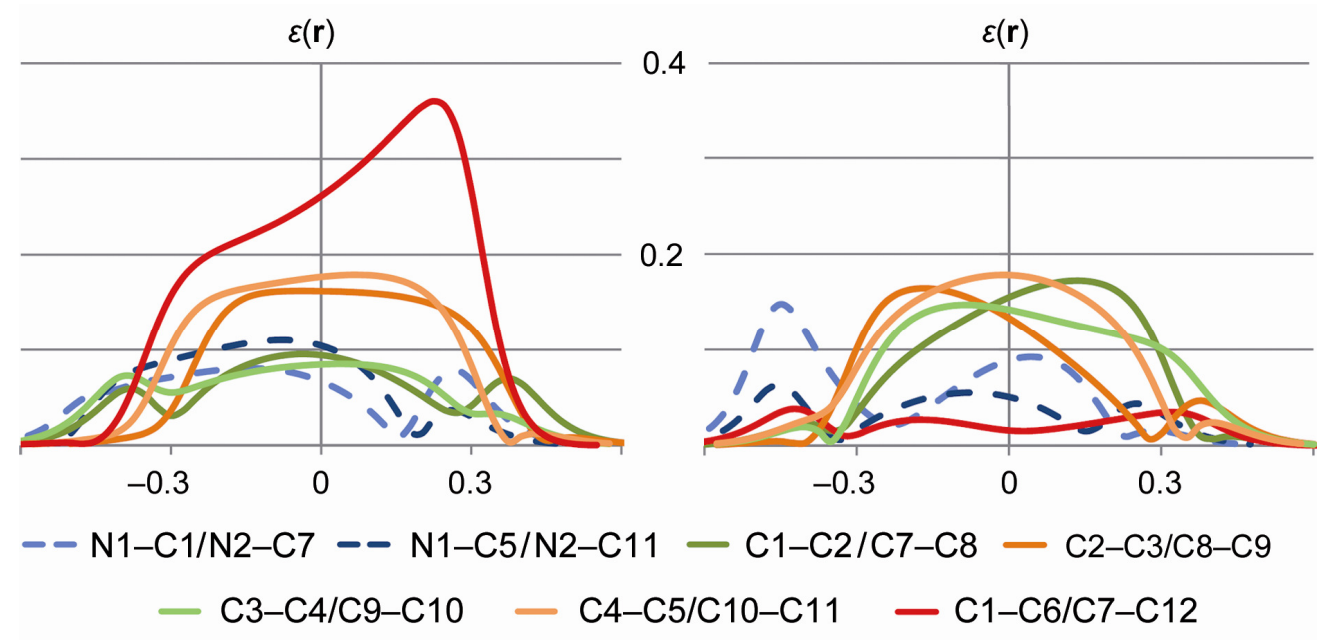

Figure 2-29. Ellipticities of the N/C-C bonds in $[2-\mathrm{PicLi} \cdot \mathrm{PicH}]_{2}(4)$ along the bond paths in the anion (left) and the donor (right) ( $x$-axis in $\AA, 0=B C P$ ).

An appropriate tool to distinguish a single from a double bond more specifically is the inspection of the ellipticities along the whole bond paths rather than the inspection of the $\varepsilon\left(\mathbf{r}_{\mathrm{BCP}}\right)$ values solely. This method was used by $W$. Scherer et al. to elucidate the manifestation of negative hyperconjugation in the electron density distribution of $\left[2-\left(\mathrm{Me}_{3} \mathrm{Si}\right)_{2} \mathrm{CPyLi}\right]_{2}(\mathbf{7}) .{ }^{[86,87]}$ The perturbation of the aromatic $\pi$-system in the anion and the different bonding types between the ipso carbon atoms C6 and C12 are evident (Figure 2-29). All C-C ring bonds in the donor show moderately elevated ellipticity values along the bond paths (mesomeric $\pi$-contribution) with gradual asymmetry relative to the BCP. The ellipticity of the exocyclic C7-C12 bond is close to zero, as expected for a single bond. In comparison, the $\mathrm{C} 1-\mathrm{C} 6$ bond in the anion reveals the highest $\varepsilon(\mathbf{r})$ value among all $\mathrm{C}-\mathrm{C}$ bonds under investigation. Therefore, the methylene carbon bond can be included into the series of localised formal double bonds in the conjugated $\pi$-system of the picolyl anion. $\varepsilon(\mathbf{r})$ gets more pronounced in the basin of $\mathrm{C} 6$, 
probably due to a charge accumulation at the methylene carbon atom. A related feature of increasing $\varepsilon(\mathbf{r})$ in the vicinity of a carbanion was reported by W. Scherer et al. ${ }^{[87]}$ However, the deformation can also be caused by an interaction to the lithium atom $\mathrm{Li}^{\prime}$.

The ellipticities along the carbon-carbon bonds in the anionic ring support the interpretation that $\mathrm{C} 2-\mathrm{C} 3$ and $\mathrm{C} 4-\mathrm{C} 5$ have appreciable double bond character, while $\mathrm{C} 1-\mathrm{C} 2$ as well as $\mathrm{C} 3-\mathrm{C} 4$ show about half the values and therefore less $\pi$-character. According to the $\varepsilon(\mathbf{r})$ distributions and the properties at the BCPs, the enamide form ( $\mathbf{D}$ in Scheme 2-4) of the picolyl anion seems to be most appropriate in describing the bonding properties in [2-PicLi.PicH $]_{2}(4)$.

A similar investigation of $\varepsilon(\mathbf{r})$ was performed for the $\mathrm{N} 1-\mathrm{C} 1$ and $\mathrm{C} 1-\mathrm{C} 6$ bonds in 6 (Figure 2-30). ${ }^{[220]}$ The methylene bond shows almost ideal $\varepsilon(\mathbf{r})$ values for a strong aromatic or even a double bond $\left(\rho\left(\mathbf{r}_{\mathrm{BCP}}\right)=0.27\right)$ with just a slight deformation towards the deprotonated carbon atom C6. The nitrogen bond on the other hand is hardly different to the one in the donating 2-picoline. This is another argument for the delocalisation of the negative charge over the whole ring system.

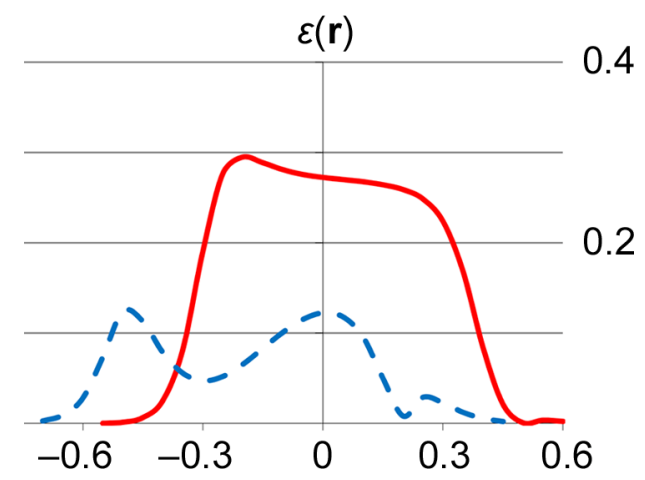

Figure 2-30. Ellipticities of the $\mathrm{N} 1-\mathrm{C} 1$ (blue) and the $\mathrm{C} 6-\mathrm{C} 1$ (red) bond along the bond paths in $\left[\left(\mathrm{Et}_{2} \mathrm{O}\right) \cdot \mathrm{Li}\left\{\mathrm{Ph}_{2} \mathrm{P}(\mathrm{CHPy})\left(\mathrm{NSiMe}_{3}\right)\right\}\right](6) .{ }^{[220]}$

A property that can also illustrate bond asymmetries is the deformation density. As mentioned in chapter 2.3.4, this is a relative electron density depiction, which displays the additionally modelled bonding electron density of the multipole model compared to the IAM. By a close inspection of the deformation density isosurface representation (isosurface value: $0.22 \mathrm{e} \AA^{-3}$ ), a uniform shape of the $\mathrm{C}-\mathrm{C}$ bonds can be observed in the donor (Figure 2-31). By contrast, dissimilarities can be seen in the anion. Most eye-catching are the differences between the $\mathrm{C} 4-\mathrm{C} 5$ and $\mathrm{C} 1-\mathrm{C} 2$ bond. A considerable extension of the deformation density perpendicular to the pyridine plane occurs in C4-C5. Less 


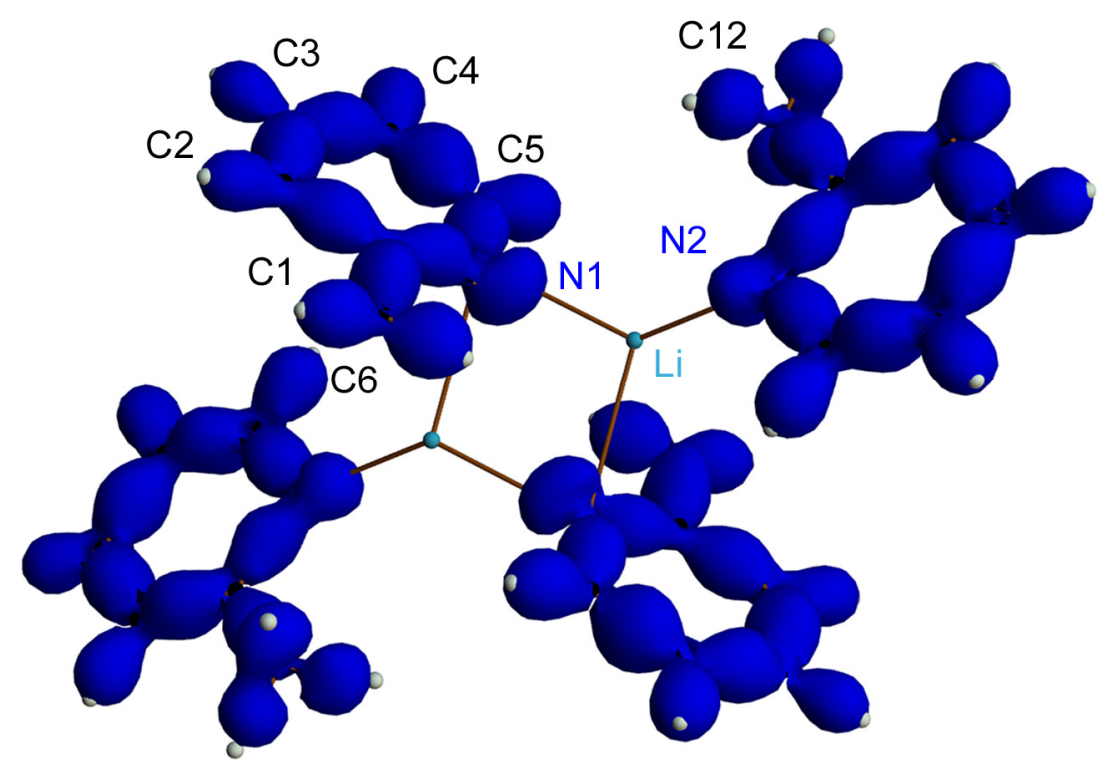

Figure 2-31. Deformation density representation of [2-PicLi.PicH $]_{2}(4)$ on a level of $0.22 \mathrm{e}^{-3}$.

obvious are the similar deformed lobes between the $\mathrm{C} 2-\mathrm{C} 3$ and $\mathrm{C} 1-\mathrm{C} 6$ bonds due to the orientation of the molecule in Figure 2-31.

In order to highlight the effect of the deprotonation at $\mathrm{C} 6$, a deformation density contour plot of the $\mathrm{C} 1-\mathrm{C} 6$ and $\mathrm{C} 7-\mathrm{C} 12$ bond can be drawn for comparison. The contour planes include the given atoms and were chosen to match the pyridine ring planes or to be exactly perpendicular to it (Figure 2-32). The methyl bond in the donor is almost equally distributed in both planes, while huge differences are present to the methylene bond of the anion. The deformation density is increased orthogonally to the ring plane indicating $\pi$-contributions (a).

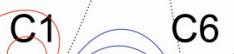

a)

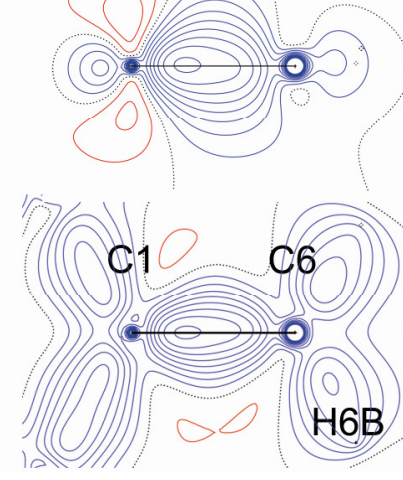

C7 $\quad \mathrm{C} 12$

b)

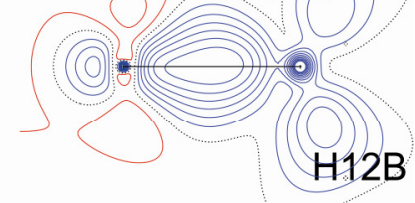

d)
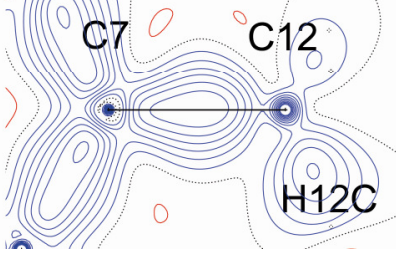

Figure 2-32. Deformation density contour plots orthogonal to the pyridine ring plane $(a+b)$ and in the plane $(c+d)$ of [2-PicLi.PicH $]_{2}(4)$. Positive contour lines are drawn blue and negative red (step size $0.1 \mathrm{e}^{-3}$ ). The dotted black line represents zero values. 
Regarding the resonance formulae of the picolyl anion (Scheme 2-4), the atomic charges should support the interpretation as an enamide according to the $\mathrm{C}-\mathrm{C}$ bond topology. The lithium atom exhibits a charge of $+0.93 \mathrm{e}$, and can accordingly be called a cation, which is counterbalanced by the anion (sum of atomic contributions: $-0.80 \mathrm{e})$ and the donor molecule ( -0.13 e group charge). Thus, the main portion of the negative charge is de facto located at the anion. Within the donor base, the negative charge is exclusively concentrated at the nitrogen ring atom $(-0.94 \mathrm{e})$. This results in a polarisation of the neighbouring carbon atoms (C7: $+0.37 \mathrm{e}, \mathrm{C} 11:+0.12 \mathrm{e})$, so that the negative charge is relativised.

Interestingly, despite the deprotonation, the charges in the anion show only a marginal increase of the negative charges at $\mathrm{N} 1(-1.04 \mathrm{e})$ and the methylene group ( $-0.19 \mathrm{e})$ compared to the donor ( $\mathrm{N} 2=-0.94 \mathrm{e}$ and methyl $=+0.02 \mathrm{e})$. Thus, the negative charge is distributed over the whole ring system with the largest increase at the methylene group $(\Delta=-0.21 \mathrm{e})$, while a more positive charge relative to 2 -picoline is observed only at C2 (0.17 e vs. 0.08 e). Hence, the carbanionic resonance formula $\mathbf{A}$ in Scheme 2-4 seems to be slightly favoured from the investigation of the group charges, even though the absolute values of the negative charges are relatively low. It is noteworthy that an atomic charge of about -1 e can be found at N1 of the carbanion, but also at N2 of the donor. Therefore, the nitrogen charge originates mainly from polarisation of the neighbouring atoms, instead of an elevated charge accumulation from the deprotonation at $\mathrm{C6}$.

Yet, the bonding properties derived from $\rho\left(\mathbf{r}_{\mathrm{BCP}}\right)$ and the ellipticity values at the BCPs as well as along the bond paths support the interpretation of PicLi as an enamide ( $\mathbf{D}$ in Scheme 2-4). The redistribution of electron density into the picolyl anion is hence not resulting in a predominant accumulation of negative charge at the nitrogen atom suggested by the resonance formula D. A more or less equal distribution of the charge over the conjugated system is observed instead.

The question remains, why the coordination of the anion to the lithium atom is accomplished first and foremost by the nitrogen atom N1, even though no prominent increase of charge compared to $\mathrm{N} 2$ is observed. The charge accumulation in the lone pair (LP) region seems to be the driving force for the arrangement of the four-membered $[\mathrm{LiN}]_{2}$ ring and the preferred coordination via the nitrogen atom. A search for minima in the Laplacian field $\nabla^{2} \rho(\mathbf{r})$ around both nitrogen atoms results in three valence shell charge concentrations (VSCCs) in each ring plane consistent with an $\mathrm{sp}^{2}$ hybridisation. An isosurface representation 
of $\nabla^{2} \rho(\mathbf{r})$ around N1 reveals the expected VSCC (maximum value -69.1 e $\AA^{-5}$ ) of a lithium-directed in-plane lone pair. Nonetheless, it is deformed towards the second "aza-allylic" lithium atom Li' (Figure 2-33), but no explicit fourth maximum in $-\nabla^{2} \rho(\mathbf{r})$ is present. By contrast, the donor nitrogen atom N2 exhibits a more symmetrical distribution of $\nabla^{2} \rho(\mathbf{r})$ on the same isosurface level and an even more pronounced maximum $\left(-73.5 \mathrm{e}^{-5}\right)$. It has to be emphasised that the term lone pair (or NBCC) is incorrect, since bond paths were determined to the lithium cation, which turns them into bonding charge concentrations. However, the notation is maintained, because of its common association in chemistry.
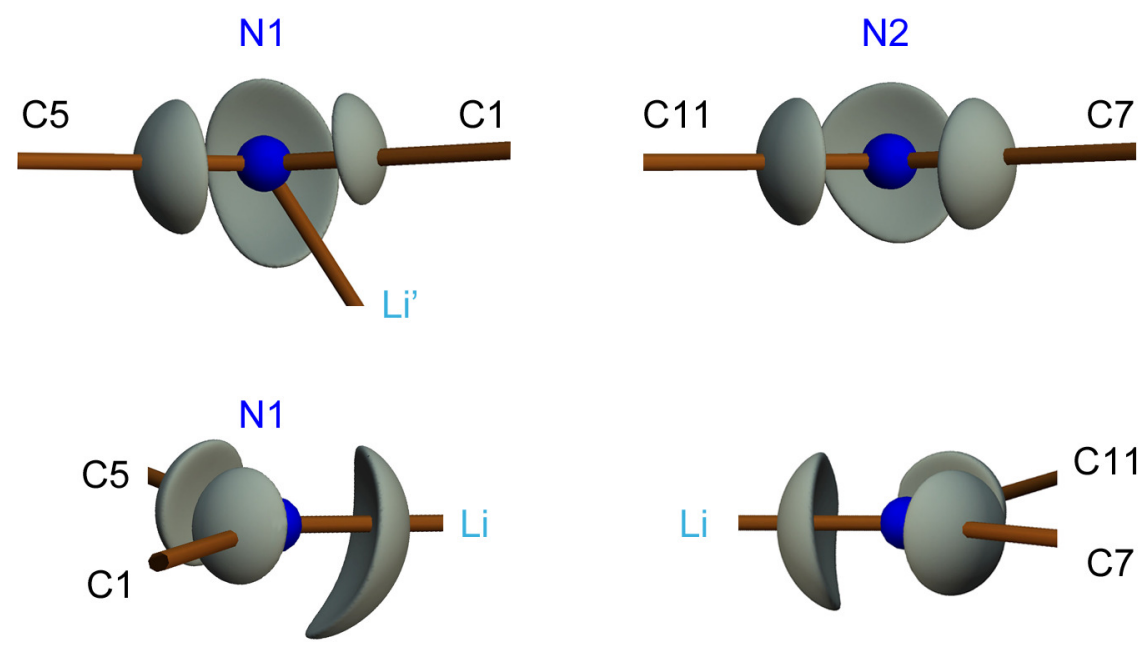

Figure 2-33. Isosurface representation of $\nabla^{2} \rho(\mathbf{r})$ around N1 (left) and N2 (right) on a -45 e $\AA^{-5}$ level.

No comparable accumulation was found around the methylene carbon atom C6. Three VSCCs towards the bonding partners could be identified, but no explicit concentration facing the "aza-allylically" coordinated lithium atom Li'. This means that the atomic orbitals at $\mathrm{C} 6$ are $\mathrm{sp}^{2}$ hybridised without the formation of a distinct lone pair density in an $\mathrm{sp}^{3}$ orbital lobe. Only a polarisation towards the cation on a much lower level is visible in the isosurface representation, but no well defined NBCC (Figure 2-34, $a+b$ ).

By contrast, this is observed in $\left[2-\left(\mathrm{Me}_{3} \mathrm{Si}\right)_{2} \mathrm{CPyLi}\right]_{2}$ (7). A fourth maximum in the negative Laplacian was quantified $\left(-18.0 \mathrm{e} \AA^{-5}\right)$ facing the lithium cation and proving an $\mathrm{sp}^{3}$ hybridisation at the deprotonated carbon atom (Figure 2-34, c) ${ }^{[221]}$. Therefore, a totally different electronic situation is adopted in $\mathbf{7}$ compared to the unsubstituted picolyllithium compound 4 . The deprotonation leaves a lone pair at $\mathrm{C} 6$ that is directed towards the lithium atom. This is stabilised according to the author by negative hyperconjugation ${ }^{[153-156]}$ by an $\mathrm{Si}-\mathrm{C}$ anti-bonding 
orbital with a minimal dihedral $\mathrm{C}-\mathrm{Si}-\mathrm{C}_{\alpha}-\mathrm{Li}$ angle, as well as by secondary electrostatic interactions with the trimethylsilyl groups. ${ }^{[87]}$ Hence, it is not surprising that this picolyl ring cannot be compared with those of $\mathbf{4}$ and $\mathbf{6}$. There, the pyridine rings act as the acceptor for the negative charge while in $\mathbf{7}$ the polarisable silicon atoms act stabilising.

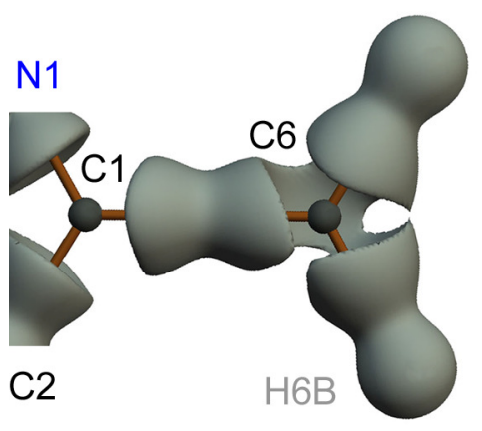

a)

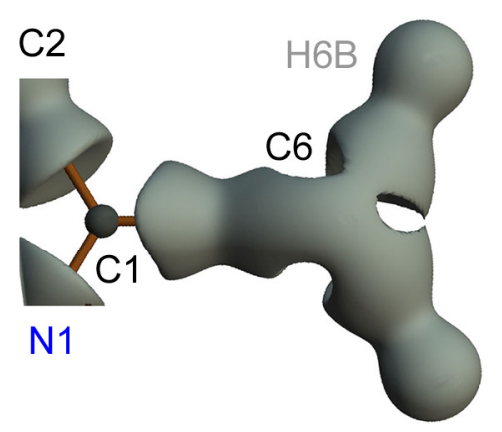

b)

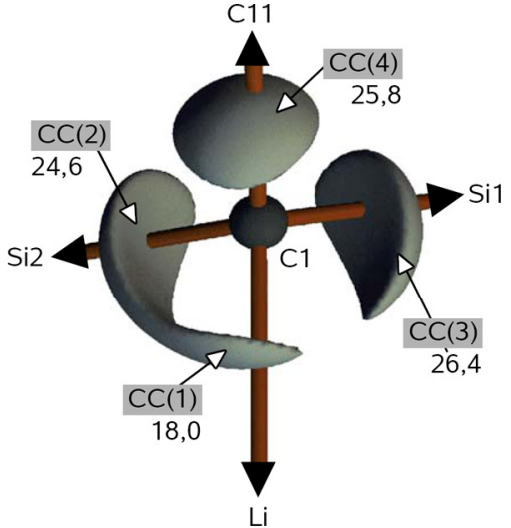

c)

Figure 2-34. Isosurface representation of $\nabla^{2} \rho(\mathbf{r})\left(-8.5 \mathrm{e}^{-5}\right)$ around $\mathrm{C} 6$ in [2-PicLi.PicH $]_{2}$ (4) with the backside (a) or the front side (b) facing $\mathrm{Li}^{\prime}$; c) $\nabla^{2} \rho(\mathbf{r})\left(-17.0 \mathrm{e} \AA^{-5}\right)$ around the related atom in $\left[2-\left(\mathrm{Me}_{3} \mathrm{Si}\right)_{2} \mathrm{CPyLi}\right]_{2}(\mathbf{7} ; \mathrm{C} 1=\mathrm{C} 6$ in 4$) .{ }^{[221]}$

Obviously, the Lewis basicity of C6 in 4 can neither be compared with the lone pair in $\mathbf{7}$ nor compete with the charge accumulation at N1. Nevertheless, the polarisation of the EDD around C6 towards $\mathrm{Li}^{\prime}$ suggests at least a co-coordination of the aza-allylic system, which is also in accordance with the observed bond path in the theoretical electron density distribution. These observations may also provide the key to understand the pattern in the aggregation modes of 2-picolyllithium compounds, which is the topic of the next chapter.

Moreover, the bonding charge concentration of C6 towards the 2-carbon atom $\mathrm{C} 1$ is another indicator for the electronic differences between 4 and 7 . The charge delocalisation in the 2-picoline adduct 4 results in a large BCC value of $-34.7 \mathrm{e} \AA^{-5}$ at $\mathrm{C} 6$, while only $-25.8 \mathrm{e} \AA^{-5}$ are observed in the disilylated compound 7. A contour plot of the Laplacian in the pyridine ring planes does likewise show these differences in the anion and the donor of [2-PicLi.PicH] 2 (Figure 2-35). Even the asymmetries in the charge accumulations of the anion relative to the reference are observed, as well as the more directed lone pair of N2. The lithium cation on the other hand shows persistent charge depletion in the whole bonding region. 

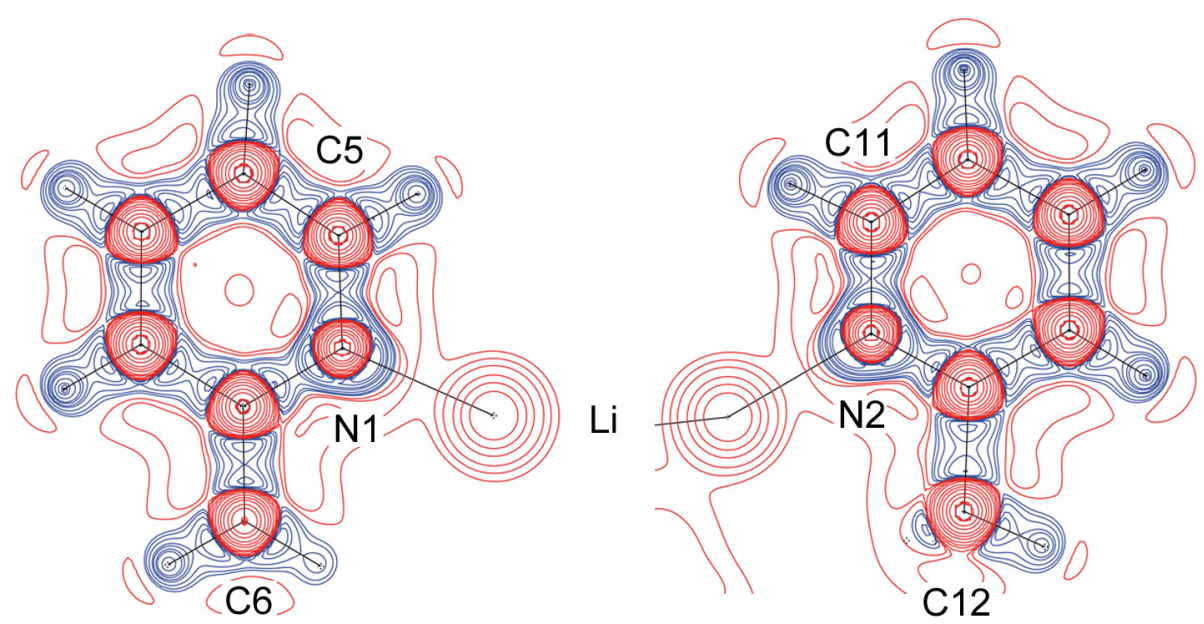

Figure 2-35. Laplacian distribution in the N1C2C4 plane (left) and the N2C8C10 plane (right) of [2-PicLi.PicH] $]_{2}(\mathbf{4})$. Negative values of $\nabla^{2} \rho(\mathbf{r})$ are depicted by blue and positive values by red lines. Contours are drawn at $\pm 2.0 \times 10^{n},-15,-30, \pm 4.0 \times 10^{n}, \pm 8.0 \times$ $10^{\mathrm{n}} \mathrm{e}^{-5}$ with $\mathrm{n}=0,1,2,3$.

The similarities of the nitrogen atoms in the donor and the anion concerning the charge and the VSCCs are also mirrored in the topological properties of the $\sigma$-donor Li-N1/2 bonds (Table 2-8). Low electron density values at the BCPs (N1: $\left.0.16, \mathrm{~N} 2: 0.15 \mathrm{e} \AA^{-3}\right)$, slightly positive $\nabla^{2} \rho\left(\mathbf{r}_{\mathrm{BCP}}\right)$ values $\left(4.5 \mathrm{e}^{-5}\right)$, and vanishing $\varepsilon\left(\mathbf{r}_{\mathrm{BCP}}\right)$ are found for both bonds. Even less electron density is accumulated in the $\mathrm{N} 1-\mathrm{Li}^{\prime}$ bond $\left(\rho\left(\mathbf{r}_{\mathrm{BCP}}\right)=0.11 \mathrm{e} \AA^{-3}\right.$ and $\left.\nabla^{2} \rho\left(\mathbf{r}_{\mathrm{BCP}}\right)=3.1 \mathrm{e}^{-5}\right)$, which proves the lone pair-Li $\sigma$-bond to be dominant. Thus, the $\rho\left(\mathbf{r}_{\mathrm{BCP}}\right)$ values derived from theory involving the lithium cation are consistently slightly higher than in the experiment $\left(\Delta \approx 0.022 \mathrm{e}^{-3}\right)$. The reason for that could be the treatment of the lithium atom as a cation during the multipole refinement. This also explains the small differences in the integrated charge of the lithium atom (exp.: $+0.93 \mathrm{e}$, theory: +0.84 e). Remarkably, the electron density value at the bond critical point between $\mathrm{Li}^{\prime}$ and $\mathrm{C} 6\left(0.116 \mathrm{e} \AA^{-3}\right)$ is just slightly smaller than that of the aza-allylic $\mathrm{Li}^{\prime}-\mathrm{N} 1$ bond $\left(0.129 \mathrm{e}^{-3}\right)$. The $\mathrm{Li}^{\prime}-\mathrm{C} 6$ bond path $(2.336 \AA)$ in theory is curved to some extent (Figure 2-27; $d=2.302 \AA$ ). However, the calculation of the experimental electron density along the straight line between $\mathrm{Li}^{\prime}$ and the deprotonated carbon atom gives a sufficient approximation for an imaginary $\rho\left(\mathbf{r}_{\mathrm{BCP}}\right)_{\exp }$. This is the minimal $\rho(\mathbf{r})$ value along the interatomic vector $\left(0.079 \mathrm{e}^{-3}\right)$, which is in a reasonably good agreement with the theoretically determined one, if $\Delta \rho(\mathbf{r})$ $\left(0.022 \mathrm{e}^{-3}\right)$ is taken into account. The only difference is that no bond path of maximum electron density can be found between $\mathrm{Li}^{\prime}$ and $\mathrm{C} 6$ in the experiment. 
Table 2-8. Bond path lengths $[\AA]$, electron densities $\left[\mathrm{e} \AA^{-3}\right]$, Laplacians $\left[\mathrm{e} \AA^{-5}\right]$, and ellipticities at the BCPs of the Li-N bonds in [2-PiCLi.PiCH $]_{2}(4),\left[\left(\mathrm{Et}_{2} \mathrm{O}\right) \cdot \mathrm{Li}_{2}\left\{\mathrm{Ph}_{2} \mathrm{P}(\mathrm{CHPy})\right.\right.$ $\left.\left.\left(\mathrm{NSiMe}_{3}\right)\right\}\right](6)$, and $\left[2-\left(\mathrm{Me}_{3} \mathrm{Si}\right)_{2} \mathrm{CPyLi}\right]_{2}(7) \cdot{ }^{[71,86,87]}$ Columns in italics refer to theoretical values.

\begin{tabular}{|c|c|c|c|c|c|c|c|c|c|}
\hline & & \multicolumn{2}{|c|}{ bond path } & \multicolumn{2}{|c|}{$\rho\left(\mathbf{r}_{\mathrm{BCP}}\right)$} & \multicolumn{2}{|c|}{$\nabla^{2} \rho\left(\mathbf{r}_{\mathrm{BCP}}\right)$} & \multicolumn{2}{|c|}{$\varepsilon\left(\mathbf{r}_{\mathrm{BCP}}\right)$} \\
\hline \multirow{4}{*}{4} & $\mathrm{Li}-\mathrm{N} 1$ & 2.022 & 2.051 & $0.155(1)$ & 0.189 & $4.6(1)$ & 4.0 & 0.02 & 0.08 \\
\hline & $\mathrm{Li}^{\prime}-\mathrm{N} 1$ & 2.141 & 2.153 & $0.107(1)$ & 0.129 & $3.1(1)$ & 2.9 & 0.39 & 0.28 \\
\hline & $\mathrm{Li}^{\prime}-\mathrm{C} 6$ & - & 2.336 & - & 0.116 & - & 2.1 & - & 0.78 \\
\hline & $\mathrm{Li}-\mathrm{N} 2$ & 2.019 & 2.110 & $0.152(1)$ & 0.173 & $4.5(1)$ & 3.7 & 0.02 & 0.05 \\
\hline 6 & $\mathrm{Li}-\mathrm{N} 1$ & 1.963 & - & $0.203(9)$ & - & $5.9(1)$ & - & 0.20 & - \\
\hline \multirow{2}{*}{7} & $\mathrm{Li}-\mathrm{N} 1$ & $1.951^{[a]}$ & $1.964^{[\mathrm{a}]}$ & $0.215(2)$ & 0.24 & $5.2(1)$ & 5.1 & 0.02 & 0.05 \\
\hline & $\mathrm{Li}^{\prime}-\mathrm{C} 6$ & $2.205^{[a]}$ & $2.176^{[\mathrm{a}]}$ & $0.150(2)$ & 0.19 & $2.5(1)$ & 2.8 & 0.12 & 0.10 \\
\hline
\end{tabular}

[a] The interatomic distance $d$ is given instead of the bond path length.

The electron density values of the $\mathrm{Li}-\mathrm{N}$ bonds suggest that the donor strength of $\mathrm{N} 1$ and $\mathrm{N} 2$ is equal. Nonetheless, one should keep in mind that the nitrogen atom in the anion is $\mu$-coordinating two lithium cations. This is an indicator for $\mathrm{N} 1$ to be a superior donor. The $\mathrm{Li}-\mathrm{N}$ bonds in $\mathbf{6}$ and $\mathbf{7}$ with only one metal contact can serve for comparison. The phosphorus substituted picolyllithium compound 6 was also identified as prevailing enamidic (see above), which is manifested in an even more negative charge at the ring nitrogen atom $(-1.17 \mathrm{e})$ compared to N1. Moreover, it is a chelating anionic ligand with a rigid coordination pocket. Both effects lead to a much closer $\mathrm{Li}-\mathrm{N}$ distance than in 4. This results in a larger $\rho\left(\mathbf{r}_{\mathrm{BCP}}\right)$ value of $0.20 \mathrm{e} \AA^{-3}$. A comparably short lithium nitrogen bond with similar $\rho\left(\mathbf{r}_{\mathrm{BCP}}\right)$ is observed for the disilylated picolyllithium compound 7. This might seem surprising, since it was concluded that the negative charge is stabilised at the carbon atom C6. However, it has to be noticed that each lithium atom is merely coordinated by the carbanion and the pyridine nitrogen atom (cf. chapter 2.2.1). A close coordination to both atoms is accordingly necessary to stabilise the cation.

The small electron density values at the bond critical points of the metal bonds also serve as evidence for their pronounced ionic nature. Yet $\rho(\mathbf{r})$ does not drop to zero between the atoms on the gradient vector of maximal electron density (bond path) as well as on the surrounding zero flux surface. This seems unlikely as the radial functions of the atomic form factor decreases with increasing $\mathbf{r}$, but does not become zero for distances that are in the range of interatomic 
interactions. Hence, small $\rho(\mathbf{r})$ values in the bonding regions are a strong hint for the ionic character of a bond but cannot be scaled to the extent of ionicity. Nevertheless, the distance from the nuclei to the bond critical point can be compared to reported ionic radii. R. D. Shannon determined a radius of $0.730 \AA$ for a lithium cation with a coordination number of four. ${ }^{[222]}$ This is just $0.02 \AA$ shorter than the distance from the metal ion to the BCP in the $\mathrm{Li}-\mathrm{N} 1 / 2$ bonds.

A property that is commonly used to judge on the bond character is $\nabla^{2} \rho(\mathbf{r})$ along the bond path (Figure 2-36). A charge separation $\left(\nabla^{2} \rho(\mathbf{r})>0\right.$ ) between the atoms can serve as another indication for a dominating closed shell interaction.

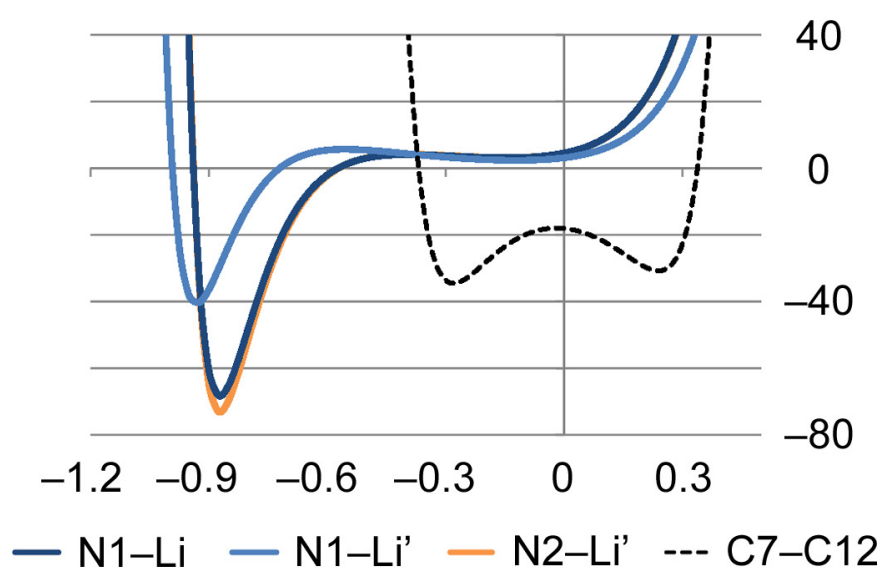

Figure 2-36. $\nabla^{2} \rho(\mathbf{r})\left[\mathrm{e} \AA^{-5}\right]$ of the $\mathrm{Li}-\mathrm{N}$ bonds in $[2-\mathrm{PiCLi} \cdot \mathrm{PiCH}]_{2}(\mathbf{4})$ along the bond path $[\AA]$ $(0=\mathrm{BCP})$. The $\mathrm{C} 7-\mathrm{C} 12$ bond is included for comparison reasons.

The methyl bond in the donor, as an example of a covalent bond, shows two distinct minima in the negative Laplacian field (BCCs) and also a minimum of charge accumulation near the bond critical point (maximum in $\nabla^{2} \rho(\mathbf{r})$ ). A negative Laplacian distribution is observed in the whole bonding region, which is typical for shared interactions. By contrast, the Li-N bonds show a totally different distribution. The Laplacian is positive in the whole lithium basins and also in the bonding areas. $\nabla^{2} \rho(\mathbf{r})$ does not change its sign until the lone pair regions of the nitrogen atoms are approached. Even slight maxima in the nitrogen basins (about $0.6 \AA$ from the BCP for $\mathrm{Li}^{\prime}-\mathrm{N} 1$ and $0.4 \AA$ for $\mathrm{Li}-\mathrm{N} 1 / 2$ ) can be detected. These features were also found for the Li-O diethyl ether donor bond in $\left[\left(\mathrm{Et}_{2} \mathrm{O}\right) \cdot \mathrm{Li}\left\{\mathrm{Ph}_{2} \mathrm{P}(\mathrm{CHPy})\left(\mathrm{NSiMe}_{3}\right)\right\}\right](6) \cdot{ }^{[71]}$ Hence, charge depletion is evident in the bonding to the lithium cation commonly associated with closed shell interactions. The examination of the Laplacian contour plot in Figure 2-35 supports this fact. The contour lines are of spherical shape, so that no asymmetries induced by covalent bonding can be detected. 
The same conclusion can be drawn according to the ratio of $\left|\lambda_{1}\right|$ and $\lambda_{3}$. These are close to zero for the $\mathrm{Li}-\mathrm{N}$ bonds in $4(\mathrm{Li}-\mathrm{N} 1: 0.16, \mathrm{Li}-\mathrm{N} 1: 0.14$, Li-N2: 0.16), which is typical for closed shell interactions. A similar small value is also present in $6(0.19)$ and was interpreted likewise. ${ }^{[220]}$

Moreover, the determined integrated atomic charges of the cation from the experiment and theory give rise to an ionic interpretation of the lithium atom interactions. However, some valence electron density is still accumulated at $\mathrm{Li}$ according to the separation of atoms in the QTAIM formalism. A slight charge transfer from the nitrogen donor atoms cannot be ruled out, which might be caused by small covalent contributions.

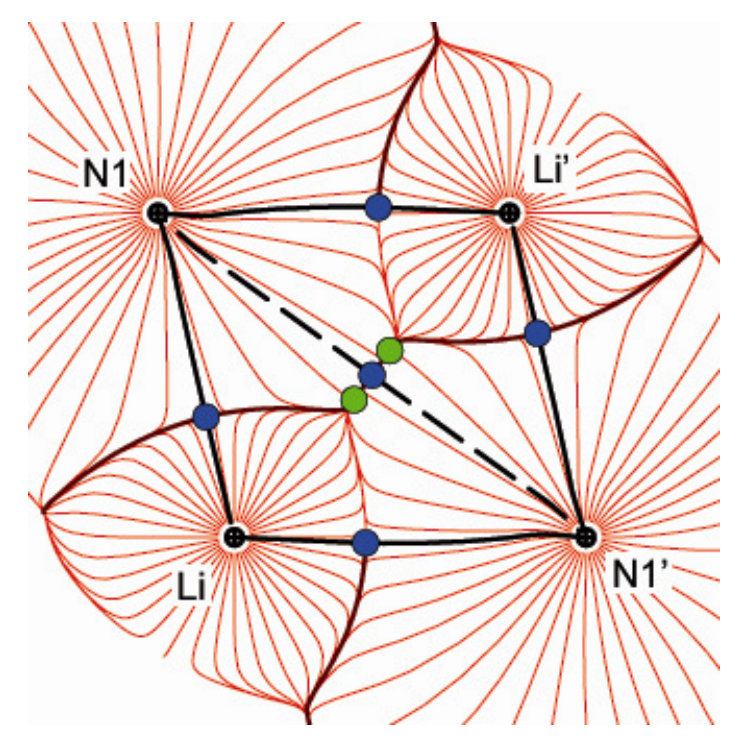

Figure 2-37. Trajectory plot of the $[\mathrm{LiN}]_{2}$ ring in $[2-\mathrm{PiCLi} \cdot \mathrm{PiCH}]_{2}(\mathbf{4})$. Red lines are gradient vectors, while blue/green dots indicate BCPs/RCPs. Black lines refer to bond paths between atomic positions (black dots) and zero flux surface border lines.

Shared interactions in a molecular orbital description of the $\mathrm{Li}-\mathrm{X}$ bonds are accompanied by hybrid orbitals at the lithium atoms that overlap with each other. This covalent Li-Li bonding was emphasised by F. M. Bickelhaupt et al. in methyllithium dimers and tetramers. ${ }^{[85]}$ This was also investigated in 4 . The interatomic lithium distances are $2.68 \AA$ in $[\mathrm{MeLi}]_{4}{ }^{[49]}$ and only $2.58 \AA$ in the 2 -picolyllithium dimer 4. Additionally, the trajectory plot in the $[\mathrm{LiN} 1]_{2}$ ring plane does not show an attractive interaction between the lithium atoms (Figure 2-37). No zero flux surface is shared between the metal atoms because of the extended nitrogen atom basins. An Li-Li bond can therefore be ruled out.

The question remains, why an electrophilic attack on 2-picolyllithium generally occurs at the methylene group, ${ }^{[99-102]}$ if the enamidic resonance form describes 
the bonding situation best ( $\boldsymbol{D}$ in Scheme 2-4), just low negative charge is accumulated at the methylene group, and the lone pair at N1 is determining the molecular structure. The three-dimensional distribution of the electrostatic potential (ESP) displayed in Figure 2-38 might provide an answer. The ESP at a given point in space is defined as the work required to bring a positive charge from infinite distance to the selected point and is therefore suitable for studying chemical reactivity. A nucleophilic reagent will be attracted by regions of positive ESP, while electrophiles are attracted by negative regions. This can be used to predict reaction sites of a molecule even in solution, if the molecular structure stays intact.

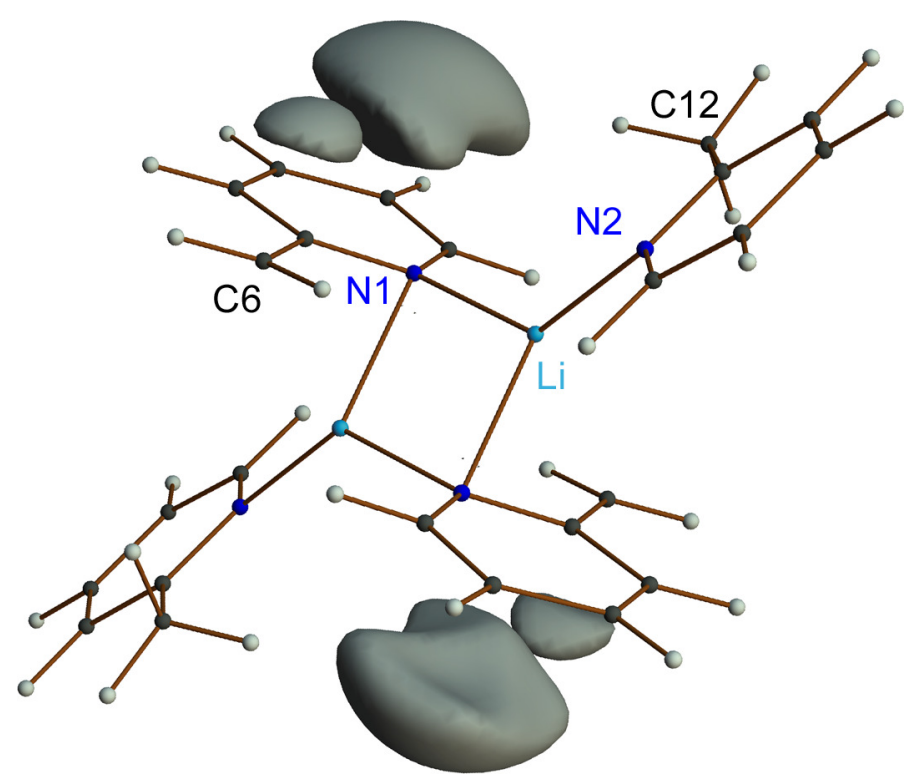

Figure 2-38. Isosurface representation of the electrostatic potential in $[2-\mathrm{PicLi} \cdot \mathrm{PicH}]_{2}(4)$ on a level of $-0.1 \mathrm{e}^{-1}$.

Opposite to the $\mathrm{Li}^{\prime}-\mathrm{N}$ bond in $\mathbf{4}$, we find a vast region of negative ESP above the picolyl anion plane (Figure 2-38). The spatial distribution suggests that electrophiles will be guided by the negative potential towards the nucleophilic carbon atom C6. Most probably it is the $\pi$-system of the anion as a whole that leads to the observed ESP, not a local charge concentration at the formal carbanion. If no solvent-separation of [2-PicLi.PicH $]_{2}(4)$ occurs in solution, it is reasonable to assume that similar attractive regions result in the $\mathrm{C}$ alkylation in solution. 


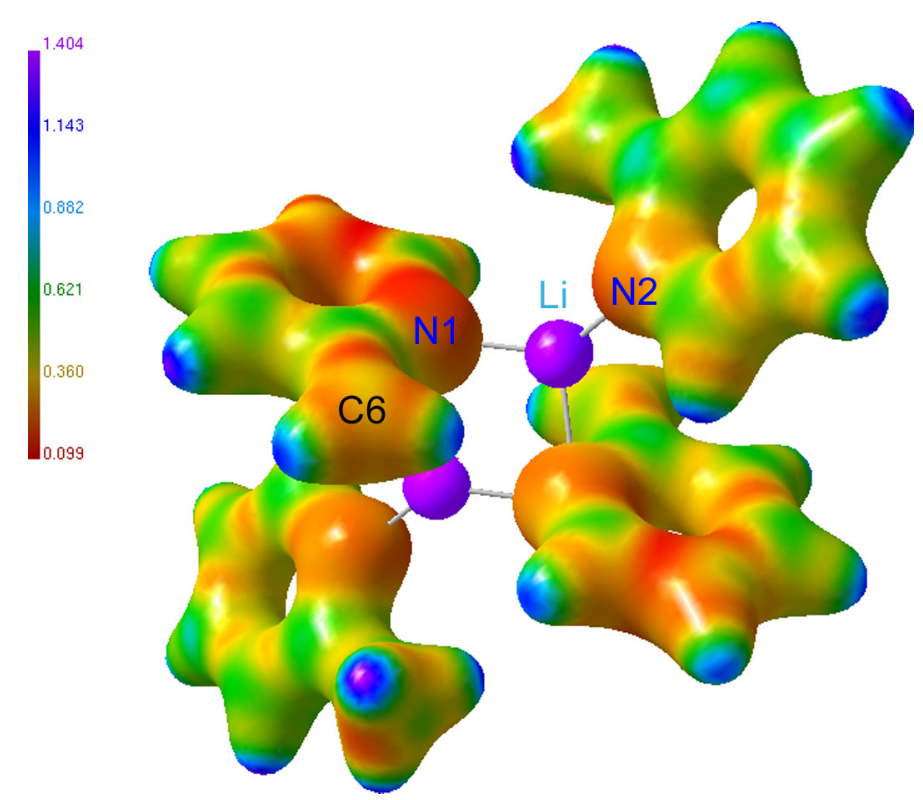

Figure 2-39. ESP $\left(\mathrm{e} \AA^{-1}\right)$ of $[2-\mathrm{PicLi} \cdot \mathrm{PicH}]_{2}$ (4) mapped on $\rho(\mathbf{r})\left(0.5 \mathrm{e}^{-3} ;\right.$ MoLISO $\left.^{[223]}\right)$

If the electrostatic potential is mapped on the electron density on a certain level, electronical differences of the anion and the donor are well displayed (Figure 2-39). The charge accumulation in the anion is represented by low values of the ESP, while the cations show a highly positive potential. The methylene side chain exhibits the most attractive region of all carbon atoms, also underlining the reactivity of the molecule.

Based on the results of the topological analysis of the aza-allylic 2-picolyllithium compound [2-PicLi.PicH $]_{2}$ (4) as well as on the observed molecular structures of 2-PicLi with various donor molecules, it could be argued about the taxonomy in the coordination pattern of these compounds. Several hypotheses are given in the next chapter. 


\subsection{On the Quest of the Coordination Pattern in 2-Picolyllithium Compounds}

Several systematic studies on 2-picolyllithium compounds including this work have shown their main aggregation motifs. In preceding investigations, one could speculate about the cause of the observed molecular structures in the crystal, because the influence of an additional side-chain or core substitution could not be distinguished from that of the different donor molecules applied. With the unsubstituted 2-picolyllithium aggregates at hand, the pristine effect of the coordinating solvent molecules can be determined and distinguished from the steric and electronic control of the added groups. But the discussion of the molecular appearance of the aggregates will be commenced with some considerations on theoretical results.

The first chemist to theoretically investigate the preferred lithium coordination by deprotonated 2-picoline was P. v. R. Schleyer et al. ${ }^{[133]}$ They compared the heats of formation from $\mathrm{MNDO}^{[224]}$ calculations of benzyllithium and 2-picolyllithium, which showed that the metallation of picoline is much more favourable, but only, if the lithium atom is coordinated closer to the ring plane (dihedral $\mathrm{N}$ $\mathrm{C}_{\beta}-\mathrm{C}_{\alpha}-\mathrm{Li}$ angle: $25.2^{\circ}$ ) than in the benzyl anion (dihedral $\mathrm{C}_{\gamma}-\mathrm{C}_{\beta}-\mathrm{C}_{\alpha}-\mathrm{Li}$ angle: $\left.45.5^{\circ}\right){ }^{[133]}$ Even a position of the lithium atom within the ring plane is only $0.4 \mathrm{kJmol}^{-1}$ less favoured than the aza-allylic arrangement on the flat potential energy surface of 2-PicLi. It was further stated that the $\mathrm{N}$ - $\mathrm{Li}$ chelation is presumably energetically more important than the benzyl type resonance stabilisation.

Eight years later, U. Pieper optimised the geometry of monomeric 2-picolyllithium without and with up to three ammonia donor molecules. ${ }^{[107]}$ As reported before, ${ }^{[133]}$ the aza-allylic coordination of the lithium atom is preferred. Afterwards, this was also reported for the monomeric trimethylsilyl(2-pyridyl)methyllithium and -sodium compounds in related calculations. ${ }^{[144]}$ However, in contrast to the dimeric 2-picolyllithium 2-picoline (4) and diethyl ether (5) adducts, the surroundings of the deprotonated carbon atom $\mathrm{C} 6$ in unsubstituted 2-PicLi is not planar. ${ }^{[107]} \mathrm{A}$ trend towards an $\mathrm{sp}^{3}$ hybridisation was detected (sum of the bond angles at $\mathrm{C} 6$ : $344.3^{\circ}$ ), which also leads to an increased $\mathrm{C} 1-\mathrm{C} 6$ bond length compared to the solid state $(\sim 0.06 \AA)$. The bonds to the lithium atoms are shorter than in the crystal with the Li-N distance being the shortest (1.94 $\AA$ ). In addition, the Li-C6 distance $(2.05 \AA)$ is reduced compared to the Li-C1 distance $(2.21 \AA)$ in the gas phase, which is consistent with an $\mathrm{sp}^{3}$ hybridised lone pair at 
C6. The same tendency, although not as distinct, is manifested in the theoretical calculation of $[2-\mathrm{PicLi} \cdot \mathrm{PiCH}]_{2} \quad(\mathrm{Li}-\mathrm{C} 1: 2.324 \AA$, Li-C6: $2.302 \AA)$, which is responsible for the observed bond path.

By successively adding ammonia molecules to the coordination sphere of the metal atom in 2-PicLi, the $\mathrm{C} 1-\mathrm{C} 6$ bond becomes shorter, the $\mathrm{Li}-\mathrm{N}_{\mathrm{Py}} / \mathrm{C}$ bonds longer, and the $\mathrm{sp}^{3}$ hybridisation at $\mathrm{C} 6$ less favourable. Even more interesting is the fact that the $\mathrm{Li}-\mathrm{C} 1 / \mathrm{C} 6$ bonds get incomparably longer than the $\mathrm{Li}-\mathrm{N}_{\mathrm{Py}}$ ones. One or two ammonia donor molecules leave the lithium nitrogen bond lengths almost unchanged $(1.95 \AA$ ) while the lithium carbon ones increase by $0.21 \AA$ (C1) and $0.32 \AA$ (C6). The differences between the $\mathrm{Li}-\mathrm{C}$ bonds are thus vanishing. Finally, Li-C6 (3.18 $\AA$ ) is even longer than Li-C1 (2.89 $\AA)$ in the case of three ammonia donor molecules. Thus, the coordination to the aza-allyl carbon atoms decreases when the lithium cation is sufficiently stabilised by ammonia. At that time, the picolyl anion solely bonds the metal via the nitrogen atom. An enamidic behaviour is adopted with reported picolyl bond lengths that are in good agreement with those in $[2-\mathrm{PicLi} \cdot \mathrm{OEt}]_{2}(\mathbf{5}) \cdot{ }^{[107]}$ However, the position of the lithium atom relative to the pyridine ring is far from being in plane (dihedral Li$\mathrm{N}-\mathrm{C}_{\beta}-\mathrm{C}_{\alpha}$ angle: $40^{\circ}$ ) in contrast to 4 and $\mathbf{5}$. The investigation of the energy differences with respect to the dihedral angle $\left(0-60^{\circ}\right)$ showed only small changes $\left(3 \mathrm{kJmol}^{-1}\right)$ for $\left[2\right.$-PicLi. $\left.\left(\mathrm{NH}_{3}\right)_{3}\right]$. Consequently, the position of the metal atom is in not rigid but easily changes without significant destabilisation of the aggregate as was already suggested by $P . V$. R. Schleyer et al. ${ }^{[133]}$

These results imply that the lithium atom preferably interacts with the best donor sites available. The four nitrogen atoms in [2-PicLi. $\left(\mathrm{NH}_{3}\right)_{3}$ ] sufficiently stabilise the cation and the weakly donating aza-allylic electron density (cf. chapter 2.3.7) is not required anymore. Moreover, the coordination to Li via the pyridyl nitrogen atom seems to depend barely on the direction. This is noteworthy, since the donation is expected to occur via the in-plane lone pair. The deviation from the ideal dihedral angle $\left(0^{\circ}\right)$ should reduce the orbital overlap with the cation. Hence, the interaction appears to be not orbital-controlled but of electrostatic nature.

With this knowledge at hand, the monomeric crystal structures of 2-picolyllithium are straightforwardly reasonable. The enamidic coordination mode is adopted with the tridentate pmdeta donor molecule. The four donor atoms readily stabilise the cation and the negative charge is delocalised into the pyridine ring, and a dramatic redistribution of the $\pi$-electron density occurs which results in the distinct bond length alternation observed. The deviation from the 
$\sigma$-donation of $\mathrm{N}_{\mathrm{py}}$ can now be rationalised, because it is barely decreasing the interaction to the cation. With bulky substituents at the methylene group, which do not sufficiently stabilise the negative charge at C6 (i.e. trimethylsilylphenyland diphenyl groups), even the coordination with two donor atoms (tmeda and diethyl ether) is sufficient to trigger the enamidic form ( $\mathbf{D}$ in Scheme 2-4). ${ }^{[162]}$

By contrast, a bis(trimethylsilyl) substitution and a bidentate donor (tmeda) result in an intermediate bonding mode between enamidic and aza-allylic. The polarisable silicon atoms stabilise ${ }^{[151]}$ and keep the negative charge at the deprotonated carbon atom, so that the aza-allyl unit is still attractive/sufficient for the lithium coordination. Moreover, the steric demand of two trimethylsilyl vs. two phenyl groups is different. The silyl substitution effectively shields the molecule from external attack, but the $\mathrm{C}-\mathrm{C}$ bond from the deprotonated carbon to the ipso-atom is shorter than the $\mathrm{Si}-\mathrm{C}$ bond. Consequently, there is less space for an aza-allyl interaction.

If the lithium atom is not sterically shielded by the anion and the donor atoms, the 2-picolyllithium compounds dimerise. This enables the interaction of the lithium atoms with two picolyl anions (steric demand) and a combination of stabilising coordination modes. The aza-allylic mode, in which $\mathrm{Li}-\mathrm{N}$ and $\mathrm{Li}-\left(\eta^{3}-\mathrm{N}-\right.$ $\mathrm{C}-\mathrm{C}$ ) contacts are present, is observed for less bulky anions and sterically demanding monodentate ligands. In this case, the lithium atom cannot be coordinated by a second ligand (Table 2-9). Therefore, the aza-allylic system has to stabilise the cation in accordance with the theoretical calculations of U. Pieper. ${ }^{[107]}$ Examples are [2-PicLi.PicH $]_{2}(4)$ and [2-PicLi.OEt $]_{2}$ (5).

Slightly more challenging is the discrimination of the enamidic and the carbanionic coordination pattern. Two oxygen donor atoms per lithium atom lead to a carbanionic dimerisation with a central eight-membered ring in which the $\mathrm{Li}-\mathrm{N}$ distances are smaller than the Li-C ones. These patterns appear with small, non-shielding donors such as dme and thf. Their donor strength is higher than that of the nitrogen donor molecules. With the less stabilising tmeda donor, the enamidic nitrogen atoms have to account for the discrepancy relative to the oxygen donor atoms. This means that with oxygen donors, the weaker carbon donation is sufficient for the stabilisation of the cation, while with tmeda more charge has to be accumulated at the nitrogen atoms at expense of the deprotonated carbon atom. That conclusion might also explain the only exception from the above mentioned rules for the coordination modes of 2-picolyllithium compounds. [2-(6-Me)PicLi-tmeda $]_{2}$ also exhibits the $\eta^{1}-\mathrm{C}$ pattern even if the $\eta^{1}-\mathrm{N}$ mode is predicted. However, the additional methyl ring substitution is 
increasing the electron density in the pyridine ring by its inductive effect, which might balance the deficient donation of the tmeda molecule to the lithium atom.

Table 2-9. Coordination modes of 2-picolyllithium compounds ( $\mathrm{Pic}=2$-picolyl, $\mathrm{Py}=$ 2-pyridyl TMS = trimethylsilyl).

\begin{tabular}{|c|c|c|c|c|}
\hline Aggregate $\mathrm{e}^{[\mathrm{a}]}$ & Anion & Donor & Coord. Mode $\mathrm{e}^{[\mathrm{b}]}$ & Reference \\
\hline M & PicLi & pmdeta & $\eta^{1}-\mathrm{N}$ & this work \\
\hline M & TMS-6-MePicLi & pmdeta & $\eta^{1}-\mathrm{N}$ & [144] \\
\hline M & TMSPhCPyLi & tmeda & $\eta^{1}-\mathrm{N}$ & [143] \\
\hline M & $\mathrm{Ph}_{2} \mathrm{CPyLi}$ & $\left(\mathrm{Et}_{2} \mathrm{O}\right)_{2}$ & $\eta^{1}-\mathrm{N}$ & [162] \\
\hline M & $\mathrm{TMS}_{2} \mathrm{CPyLi}$ & tmeda & $\eta^{1}-\mathrm{N} / \eta^{3}-\mathrm{NCC}$ & {$[134]$} \\
\hline M & $\mathrm{TMS}_{2} \mathrm{CPyLi}$ & $\mathrm{TMS}_{2} \mathrm{PicH}$ & $\eta^{3}$-NCC & {$[134]$} \\
\hline M & TMSCHPyLi & $(-)$-sparteine & $\eta^{3}-\mathrm{NCC}$ & {$[135]$} \\
\hline $\mathrm{D}$ & PicLi & tmeda & $\eta^{1}-\mathrm{N}$ & this work \\
\hline $\mathrm{D}$ & TMSCHPyLi & tmeda & $\eta^{1}-\mathrm{N}$ & [135] \\
\hline $\mathrm{D}$ & PhCHPyLi & tmeda & $\eta^{1}-\mathrm{N}$ & [143] \\
\hline $\mathrm{D}$ & $\left(t \mathrm{BuMe}_{2}\right) \mathrm{SiCHPyLi}$ & tmeda & $\eta^{1}-\mathrm{N}$ & [143] \\
\hline $\mathrm{D}$ & PicLi & dme & $\eta^{1}-\mathrm{C}$ & this work \\
\hline $\mathrm{D}$ & PicLi & $(\text { thf })_{2}$ & $\eta^{1}-\mathrm{C}$ & [149] \\
\hline $\mathrm{D}$ & 6-MePicLi & tmeda & $\eta^{1}-\mathrm{C}$ & [133] \\
\hline $\mathrm{D}$ & $\mathrm{TMS}_{2} \mathrm{CPyLi}$ & - & $\eta^{1}-\mathrm{C}$ & [142] \\
\hline $\mathrm{D}$ & PicLi & $\mathrm{PicH}$ & $\eta^{3}-\mathrm{NCC}$ & [149] \\
\hline $\mathrm{D}$ & PicLi & $\mathrm{Et}_{2} \mathrm{O}$ & $\eta^{3}-\mathrm{NCC}$ & {$[107,206]$} \\
\hline $\mathrm{D}$ & TMSCHPyLi & $\mathrm{Et}_{2} \mathrm{O}$ & $\eta^{3}-\mathrm{NCC}$ & {$[134]$} \\
\hline $\mathrm{D}$ & TMSCHPyLi & $\mathrm{TMS}_{2} \mathrm{PicH}$ & $\eta^{3}$-NCC & {$[200]$} \\
\hline
\end{tabular}

[a] $\mathrm{M}=$ monomer, $\mathrm{D}=$ dimer. [b] $\eta^{1}-\mathrm{N}=$ enamidic-,$\eta^{3}-\mathrm{NCC}=$ aza-allylic-,$\eta^{1}-\mathrm{C}=$ carbanionic coordination mode. 


\subsection{2-Picolyllithium Adducts in Solution}

The isolation of 2-picolyllithium adducts as crystalline materials enables the accurate determination of their solution NMR spectra after redissolving due to the defined crystal composition. Detailed NMR studies on the anions have already been reported by K. Konishi, K. Takahashi et al. in the 1970s and 80s. They published two series entitled "NMR Spectra of Carbanions" and "NMR Studies of Picolyl-type Carbanions" (altogether 18 papers) ${ }^{[225]}$ that dealt with various sidechain metallation products of pyridine derivatives. ${ }^{1} \mathrm{H}$ (Table $2-10$ ), ${ }^{7} \mathrm{Li},{ }^{13} \mathrm{C}$ (Table $2-11$ ), and ${ }^{15} \mathrm{~N}$ spectral data of 2-picolyllithium complexes with various donors solvents were reported. However, 2-PicLi was always prepared in hydrocarbons with the isolation of the crude product by removal of all volatiles in vacuo. The resulting solid was then redissolved in several polar non-deuterated solvents and the spectra were recorded on 60 to $200 \mathrm{MHz}$ NMR spectrometers. Large solvent peaks were present that sometimes overlapped with signals of the picolyl anion. Moreover, an excess of donating solvent influenced the behaviour of the anion in solution. This led to significantly different signals compared to those observed for the defined adducts in non-polar deuterated solvents (Table 2-10). Therefore, a systematic NMR study on dissolved crystalline material of known composition in the solid state may lead to further information on the aggregation of 2-picolyllithium in solution.

2-Picoline (2-PicH) has served as the reference for the assignment of the signals. Compared to the carbon analogue toluene $(\delta 7.13,7.02,2.11 \mathrm{ppm}$ in

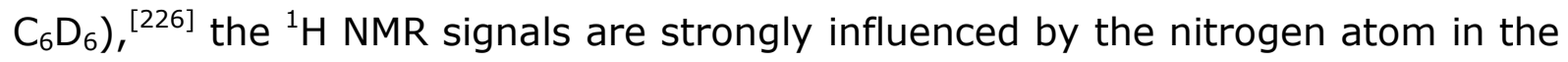
ring (Table 2-10). The neighbouring hydrogen atom (H5; Scheme 2-11) is further deshielded $(8.48 \mathrm{ppm})$, while the remaining ring protons are shifted to higher fields $(\delta 7.00,6.62,6.59 \mathrm{ppm})$.

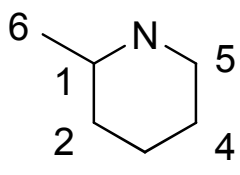

3

Scheme 2-11. Numbering scheme for the NMR assignment (lines denote connectivities only). 
Table 2-10. ${ }^{1} \mathrm{H}$ NMR signals $\delta$ [ppm] of 2-picolyllithium compounds at RT (Pic = 2-picolyl, Py $=2$-pyridyl, TMS = trimethylsilyl) with tetramethylsilane as external reference.

\begin{tabular}{|c|c|c|c|c|c|c|c|}
\hline Compound & Solvent & H5 & $\mathrm{H} 3$ & $\mathrm{H} 2$ & $\mathrm{H} 4$ & $\mathrm{H} 6$ & Reference \\
\hline$\left[\mathrm{PicLi} \cdot(\text { thf })_{2}\right]_{2}$ & $\mathrm{C}_{6} \mathrm{D}_{6}$ & 7.41 & 6.52 & 6.30 & 5.48 & 3.18 & [149] \\
\hline$[\mathrm{PicLi} \cdot \mathrm{dme}]_{2}$ & $\mathrm{C}_{6} \mathrm{D}_{6}$ & 7.30 & 6.54 & 6.26 & 5.46 & 3.06 & this work \\
\hline$[\mathrm{PicLi} \cdot \mathrm{dme}]_{2}$ & $\mathrm{C}_{6} \mathrm{D}_{5} \mathrm{CD}_{3}$ & 7.20 & 6.45 & 6.14 & 5.35 & 2.96 & this work \\
\hline$[\mathrm{PicLi} \cdot \text { tmeda }]_{2}$ & $C_{6} D_{6}$ & 7.11 & 6.41 & 6.10 & 5.32 & $3.15 / 3.06$ & this work \\
\hline$[\text { PicLi·tmeda }]_{2}$ & $\mathrm{C}_{6} \mathrm{D}_{5} \mathrm{CD}_{3}$ & 7.03 & 6.30 & 5.98 & 5.22 & $3.05 / 2.96$ & this work \\
\hline [PicLi·pmdeta] & $\mathrm{C}_{6} \mathrm{D}_{6}$ & 7.06 & 6.58 & 6.31 & 5.37 & $3.40 / 2.76$ & this work \\
\hline$\left[\mathrm{PiCLi} \cdot \mathrm{OEt}_{2}\right]_{2}$ & $\mathrm{C}_{6} \mathrm{D}_{6}$ & 6.76 & 5.92 & 5.55 & 4.67 & $2.55 / 2.41$ & [107] \\
\hline$[\mathrm{PiCLi} \cdot \mathrm{PiCH}]_{2}$ & $\mathrm{C}_{6} \mathrm{D}_{6}$ & 7.52 & 6.49 & 6.31 & 5.47 & 3.39 & [149] \\
\hline$\left[\mathrm{TMS}_{2} \mathrm{CPyLi}\right]_{2}$ & $\mathrm{C}_{6} \mathrm{D}_{6}$ & 7.78 & 6.92 & 6.92 & 6.27 & - & {$[135]$} \\
\hline $\begin{array}{l}{[\text { TMSCHPyLi }} \\
\text { tmeda }]_{2}\end{array}$ & $\mathrm{C}_{6} \mathrm{D}_{6}$ & 7.2 & 6.5 & 6.5 & 5.3 & 3.1 & [135] \\
\hline $\begin{array}{l}\text { [TMSCHPyLi } \\
\text { pmdeta] }^{[a]}\end{array}$ & $\mathrm{C}_{6} \mathrm{D}_{6}$ & 7.39 & 6.62 & 6.38 & 5.57 & 2.85 & [144] \\
\hline $\begin{array}{l}{[\mathrm{TMSCHPyLi} \cdot} \\
\left.\mathrm{OEt}_{2}\right]_{2}\end{array}$ & $\mathrm{C}_{6} \mathrm{D}_{6}$ & 7.8 & 6.7 & 6.7 & 5.9 & 2.9 & {$[135]$} \\
\hline [PicLi] & thf & 6.86 & 6.02 & 5.62 & 4.80 & 2.52 & {$[141]$} \\
\hline [PicLi] & dme & 6.82 & 6.01 & 5.58 & 4.77 & 2.52 & {$[137]$} \\
\hline [PicLi] & hmpa & 6.81 & 5.68 & 5.16 & 4.33 & {$[\mathrm{~b}]$} & [138] \\
\hline $\mathrm{PicH}$ & $\mathrm{C}_{6} \mathrm{D}_{6}$ & 8.48 & 7.00 & 6.62 & 6.59 & 2.40 & this work \\
\hline $\mathrm{PicH}$ donor & $C_{6} D_{6}$ & 8.58 & 6.83 & 6.54 & 6.42 & 2.43 & [149] \\
\hline
\end{tabular}

[a] Signals were reassigned to follow the common NMR assignment. [b] Signal covered by solvent peaks.

Deprotonation results in a general high-field shift of the ring hydrogen atoms consistent with an additional charge accumulation. The redistribution of the negative charge into the aromatic system is consequently mirrored by the proton shielding in solution. Each hydrogen atom is individually affected with shifts from -0.3 to $-2.3 \mathrm{ppm}$ for different donor solvents (Table $2-10$ ).

The methylene hydrogen atoms resonate further downfield compared to the methyl hydrogen atoms of picoline. This is remarkable since the deprotonation of an alkyl group normally results in a dramatic shift to higher magnetic field in accordance with the increased proton shielding by the negative charge. Examples are methyllithium $(\delta-1.97 \mathrm{ppm})$ and $n$-butyllithium $(\delta-0.95 \mathrm{ppm}) .^{[12]}$ However, 
the atomic orbitals of these carbanions are still $\mathrm{sp}^{3}$ hybridised while those of the picolyl carbanions are $\mathrm{sp}^{2}$ hybridised in the solid state. The delocalised system is accordingly also extended to $\mathrm{C} 6$ in solution and responsible for the deshielding. This was claimed by $K$. Takahashi et al. in an early publication, too. ${ }^{[136]}$ Thus, the rehybridisation of $\mathrm{C} 6$ opposes the effect of the charge accumulation so that the chemical shifts of the methylene hydrogen atoms cannot unambiguously serve as an indicator for the delocalisation of charge into the ring. However, a carbanion in a planar surroundings can be assumed ( $c$. preceding structural discussions) for the unsubstituted picolyl species, although this fact could not be unambiguously proven. Hence, the signals can be compared to one another.

Table 2 -11. ${ }^{13} \mathrm{C}\left\{{ }^{1} \mathrm{H}\right\}$ NMR signals $\delta$ [ppm] of 2-picolyllithium compounds (Pic = 2-picolyl, Py $=2$-pyridyl, TMS = trimethylsilyl) with tetramethylsilane as external reference.

\begin{tabular}{|c|c|c|c|c|c|c|c|c|}
\hline Compound & Solvent & $\mathrm{C} 1$ & $\mathrm{C} 5$ & $\mathrm{C} 3$ & $\mathrm{C} 2$ & C4 & C6 & Reference \\
\hline$\left[\mathrm{PicLi} \cdot(\text { thf })_{2}\right]_{2}$ & $\mathrm{C}_{6} \mathrm{D}_{6}$ & 164 & 148 & 133 & 117 & 100 & 56 & [149] \\
\hline$[\mathrm{PicLi} \cdot \mathrm{dme}]_{2}$ & $\mathrm{C}_{6} \mathrm{D}_{6}$ & 165 & 149 & 132 & 117 & 99 & 56 & this work \\
\hline$[\text { PicLi·tmeda }]_{2}$ & $\mathrm{C}_{6} \mathrm{D}_{5} \mathrm{CD}_{3}$ & 163 & 148 & 132 & 118 & 97 & 62 & this work \\
\hline [PicLi·pmdeta] & $\mathrm{C}_{6} \mathrm{D}_{6}$ & 163 & 148 & 131 & 116 & 95 & 61 & this work \\
\hline$\left[\mathrm{PicLi} \cdot \mathrm{OEt}_{2}\right]_{2}$ & $\mathrm{C}_{6} \mathrm{D}_{6}$ & 164 & 149 & 131 & 116 & 96 & 59 & {$[107]$} \\
\hline$[\mathrm{PiCLi} \cdot \mathrm{PiCH}]_{2}$ & $\mathrm{C}_{6} \mathrm{D}_{6}$ & 163 & 148 & 133 & 118 & 100 & 58 & [149] \\
\hline$\left[\mathrm{TMS}_{2} \mathrm{CPyLi}\right]_{2}$ & $\mathrm{C}_{6} \mathrm{D}_{6}$ & 176 & 147 & 136 & 129 & 113 & 105 & [135] \\
\hline $\begin{array}{l}{[\text { TMSCHPyLi. }} \\
\text { tmeda }]_{2}\end{array}$ & $\mathrm{C}_{6} \mathrm{D}_{6}$ & 169 & 148 & 134 & 118 & 101 & 66 & [135] \\
\hline $\begin{array}{l}\text { [TMSCHPyLi } \\
\text { pmdeta] }\end{array}$ & $\mathrm{C}_{6} \mathrm{D}_{6}$ & $163^{[a]}$ & 148 & 134 & 116 & 101 & 60 & [144] \\
\hline$\left[\mathrm{TMSCHPYLi} \mathrm{OEt}_{2}\right]_{2}$ & $\mathrm{C}_{6} \mathrm{D}_{6}$ & 167 & 148 & 134 & 118 & 103 & 66 & [135] \\
\hline [PicLi] & thf & 164 & 149 & 132 & 116 & 97 & 57 & [141] \\
\hline [PicLi] & dme & 164 & 149 & 131 & 116 & 96 & 58 & [139] \\
\hline [PicLi] & $\mathrm{Et}_{2} \mathrm{O}$ & 166 & 150 & 135 & 119 & 102 & 58 & [139] \\
\hline [PicLi] & hmpa & 162 & 149 & 130 & 114 & 93 & 59 & [138] \\
\hline [PicLi] & hmpa & 161 & 150 & 130 & 114 & 92 & 63 & [139] \\
\hline [PicLi] & $\operatorname{tg}^{[\mathrm{b}]}$ & 162 & 149 & 131 & 115 & 94 & 62 & [139] \\
\hline $\mathrm{PicH}$ & $\mathrm{C}_{6} \mathrm{D}_{6}$ & 159 & 149 & 136 & 123 & 121 & 24 & [227] \\
\hline $\mathrm{PicH}$ donor & $C_{6} D_{6}$ & 159 & 150 & 137 & 124 & 121 & 24 & [149] \\
\hline
\end{tabular}

[a] Changed from $153 \mathrm{ppm}$ because it is most probably a typing error. [b] tetraglyme 
The same trend for deprotonated carbon atoms in a planar arrangement is also evident in the ${ }^{13} \mathrm{C}$ spectra. A low-field shift of more than $30 \mathrm{ppm}$ can be observed for C6 in comparison to the methyl carbon atom in 2-picoline. The C4 (para atom in respect to the methylene group) shows a shift of $20 \mathrm{ppm}$ in the opposite direction. The deprotonation accordingly counteracts the charge accumulation at $\mathrm{C} 4$ by inductive or mesomeric effects of the side chain ( $\mathbf{C}$ in Scheme 2-4). Interestingly, among the deprotonated species (Table 2-11), the signals of each carbon atom vary much less than those of the protons (relative shift of up to $20 \%$ ).

After these introductory considerations, the 2-picolyllithium adducts are compared to each other ( $1^{\text {st }}$ part in Table 2-10 and Table 2-11), to related trimethylsilyl substituted compounds ( $2^{\text {nd }}$ part) and also to the polar solvent mixtures of K. Konishi, K. Takahashi et al. ( $3^{\text {rd }}$ part). The fourth part of the tables contains information on free or donating 2-picoline. For further information on the specific aggregation patterns of the solid state structures see to Table 2-9 in chapter 2.4.

The 2-PicLi adducts show ${ }^{1} \mathrm{H}$ signals roughly in the same region except for $\left[2-\mathrm{PicLi} \cdot \mathrm{OEt}_{2}\right]_{2}$. This compound was investigated by $U$. Pieper and the spectrum resembles those of the polar solvent mixtures ([PicLi], $3^{\text {rd }}$ segment). ${ }^{[107]}$ Therefore, it can be assumed that the diethyl ether dimer was not isolated as crystals prior to the measurement. The NMR sample was probably directly taken from the reaction mixture with an excess of donor solvent still present. The remaining compounds can be classified in two main categories. The thf, dme, and 2-picoline adducts give rise to comparable chemical shifts for the ring atoms (H5: $\delta$ 7.3-7.5, H3: 6.52, H2: 6.29, H4: 5.47 ppm), and only one signal for the methylene hydrogen atoms $(\mathrm{H} 6)$. The adducts with chelating nitrogen donor molecules - tmeda and pmdeta - are related. Both show two individual resonance peaks for each of the methylene hydrogen atoms.

It has to be emphasised that all spectra were recorded in non-polar and presumably also non-coordinating deuterated solvents (benzene, toluene). Consequently, an additional solvent stabilisation of the cations is unlikely. Therefore, only dimers, monomers, or solvent-separated ion pairs can be present in solution, if the solid state structure determines the highest achievable coordination numbers in solution (cf. chapter 1 ). However, a charge separation into ion pairs is unlikely in hydrocarbons, while the appearance of monomers and dimers will presumably be related to known solid state structures ( $\mathrm{N}$, aza-allyl, or $\mathrm{C}$ donation of the anion). 
The interpretation of the spectra starts with [2-PicLi.pmdeta], because its aggregation state is most likely monomeric in solution, too. The negative charge of the deprotonated species is largely delocalised into the aromatic ring, so that the hydrogen atom next to the nitrogen atom (H5) is shifted to higher fields ( $\delta 7.06 \mathrm{ppm}$ ) compared to the second category of substances (dme, thf, 2-PicH). An $\mathrm{N}$ coordination of the lithium cation can be assumed, so that the $\mathrm{CH}_{2}$ group barely interacts with the metal atom. The methylene group is consequently showing two peaks for the hydrogen atoms that integrate to one proton each. These are separated by notable $0.64 \mathrm{ppm}$ and show geminal proton coupling $\left({ }^{2} J\left(H 6_{\text {cis }}, H 6_{\text {trans }}\right)=1.5 \mathrm{~Hz}\right)$. The more shielded hydrogen atom additionally exhibits a coupling to one ring proton $(0.9 \mathrm{~Hz}$; Figure 2-40). This effect was already reported by $K$. Takahashi et al. for 2-PicLi in dme at $-18{ }^{\circ} \mathrm{C} .{ }^{[137]}$ They found a straight zigzag long-range coupling to $\mathrm{H} 3(0.7 \mathrm{~Hz})$, which could also be rationalised in [2-PicLi.pmdeta] by the complex coupling pattern of this proton. They assigned the less shielded proton to be $\mathrm{H}_{c i s}$ (cis to the nitrogen atom).
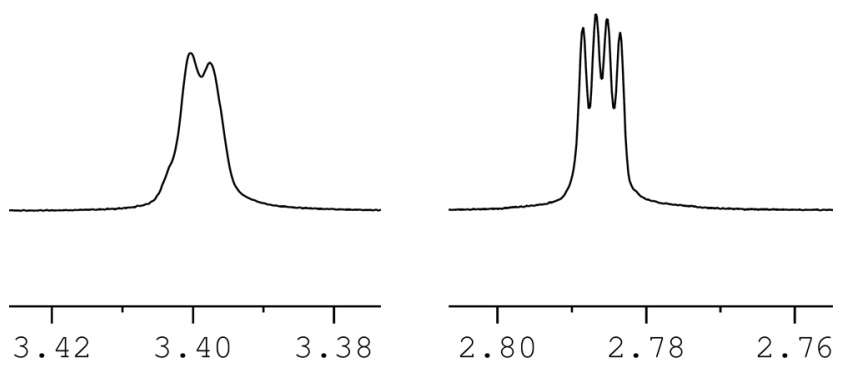

Figure 2-40. Methylene proton signals [ppm] of [2-PicLi·pmdeta] in $\mathrm{C}_{6} \mathrm{D}_{6}$ at RT (line broadening adjusted).

The magnetic non-equivalence of the methylene protons originates from an increased rotational barrier for the $\mathrm{C} 1-\mathrm{C} 6$ bond. The charge delocalisation is accompanied by an $\mathrm{sp}^{2}$ hybridisation of the atomic orbitals of $\mathrm{C} 6$ and a partial double bond to the pyridine ring. As suggested above, the lithium atom interacts mainly with the nitrogen atom, so that the postulated rotation mechanism of benzyl groups is hindered (on the NMR time scale). ${ }^{[228]}$ This involves an intermediate lithium induced $\mathrm{sp}^{3}$ hybridised carbanion that is free to rotate about the C1-C6 single bond. The chelating pmdeta donor apparently stabilises and sterically shields the cation in solution so that the negative charge can be distributed into the ring. A rotation is then already hindered at RT, while lower temperatures are needed for 2-PicLi in an excess of dme. Remarkably, all picolyllithium spectra with a surplus of donating solvent show smaller resonance values 
than the picolyllithium adducts. The better the donor, the more shielded the protons. As a result, hexamethylphosphoramide (hmpa) gives rise to NMR shifts indicating the most charge delocalisation (Table 2-10).

The ${ }^{13} \mathrm{C}$ signals for $\mathrm{C} 6$ and $\mathrm{C} 4$ also display values that differences the pmdeta and tmeda compounds from the remaining adducts (Table 2-11). C6 resonates further downfield ( $\delta 62 \mathrm{ppm}$ ) compared to the dme, thf, and 2-PicH adducts, while the C4 signals are shifted to higher magnetic fields ( $\delta 96 \mathrm{ppm})$. This can be interpreted as a sign for an increased charge accumulation at the nitrogen atom. However, the shifts for the C5 signals are almost invariant for all investigated compounds in contrast to the corresponding hydrogen signals, which are shifted to higher fields.

In accordance with the redistribution of the negative charge, no ${ }^{7} \mathrm{Li}-{ }^{13} \mathrm{C}$ coupling could be detected for the putatively enamidic compounds as well as for all other 2-PicLi compounds under investigation. This is not surprising, since it was mentioned before that all unsubstituted anions give signals of $\mathrm{sp}^{2}$ atomic orbital hybridisation of the carbon atoms. Yet, G. Fraenkel and K. V. Martin pointed out that a lithium-carbon coupling will only occur if the coplanarity at $\mathrm{C} 6$ is sufficiently disturbed (partial $\mathrm{sp}^{3}$ hybridisation). ${ }^{[229]}$

The similarities in the NMR signals of both nitrogen donor compounds suggest that the tmeda adduct is probably monomeric in solution as well. This is comprehensible because monomeric solid state structures are known with tmeda for substituted picolyllithium compounds (Table 2-9) and a dimerisation is hindered by the molecular movement of the solvent molecules. The only differences between the tri- and bidentate complexes are - as mentioned above - found at $\mathrm{H} 2$ and $\mathrm{H} 3$ as well as at the methylene hydrogen atoms. Their signals are only 0.09 ppm apart.

Since the tmeda adduct showed only minor decomposition after several hours in solution, it was chosen for low temperature ${ }^{1} \mathrm{H} /{ }^{7} \mathrm{Li}$ NMR and NOESY (Nuclear Overhauser Effect Spectroscopy) experiments as well as additional DOSY (diffusion-ordered spectroscopy), ${ }^{[230-232]}{ }^{1} \mathrm{H}-{ }^{15} \mathrm{~N}$ HMBC (Heteronuclear Multiple Bond Correlation) and ${ }^{1} \mathrm{H}-{ }^{7} \mathrm{Li}$ HOESY (Heteronuclear Overhauser Enhancement Spectroscopy) experiments.

First of all, repeated ${ }^{1} \mathrm{H}$ DOSY experiments showed only one species at RT (also true for the ${ }^{7} \mathrm{Li}$ DOSY), because all signals of the picolyl adduct shared the same diffusion coefficient. A mixture of different aggregates (monomer/dimer) could consequently be excluded. Furthermore, this proved that the tmeda donor is fixed to the cation in solution. $A{ }^{1} \mathrm{H}$ DOSY spectrum of a one day old sample 
already showed significant amounts of 2-picoline. This small molecule has a significantly different diffusion coefficient compared to the lithiated species due to the molecule (particle) size. Although picoline is not of spherical shape, the simple relation between the diffusion coefficient and the particle radius (StokesEinstein relation: $\left.D=k_{B} T\left(6 \pi \eta r_{S}\right)^{-1}\right)^{[233]}$ was used to estimate the size of the 2-picolyllithium aggregate relative to 2 -PicH. A radii ratio $\left(r_{\text {picLi }} / r_{\text {Pich }}\right)$ of more than 2 was quantified so that a larger aggregate can be assumed. However, no explicit assignment of the aggregation degree could be accomplished. The particle radius increases by the additional incorporation of a cation and the tmeda donor cannot be unambiguously distinguished from the involvement of a second anion in a hypothetical dimer.

The ${ }^{1} \mathrm{H}-{ }^{15} \mathrm{~N}$ HMBC spectrum on the other hand illustrates - as expected couplings for the tmeda nitrogen and hydrogen atoms. Furthermore, the pyridine nitrogen atom gives rise to cross peaks with the neighbouring ring proton $\mathrm{H} 5$ and with the low-field methylene hydrogen atom $\mathrm{H}_{\text {trans }}$ and to a much smaller extent with $\mathrm{HG}_{\text {cis }}$ owing to the torsion angle dependency of the coupling constant according to the Karplus equation (maximum at $180^{\circ}$ ). ${ }^{[234-236]}$

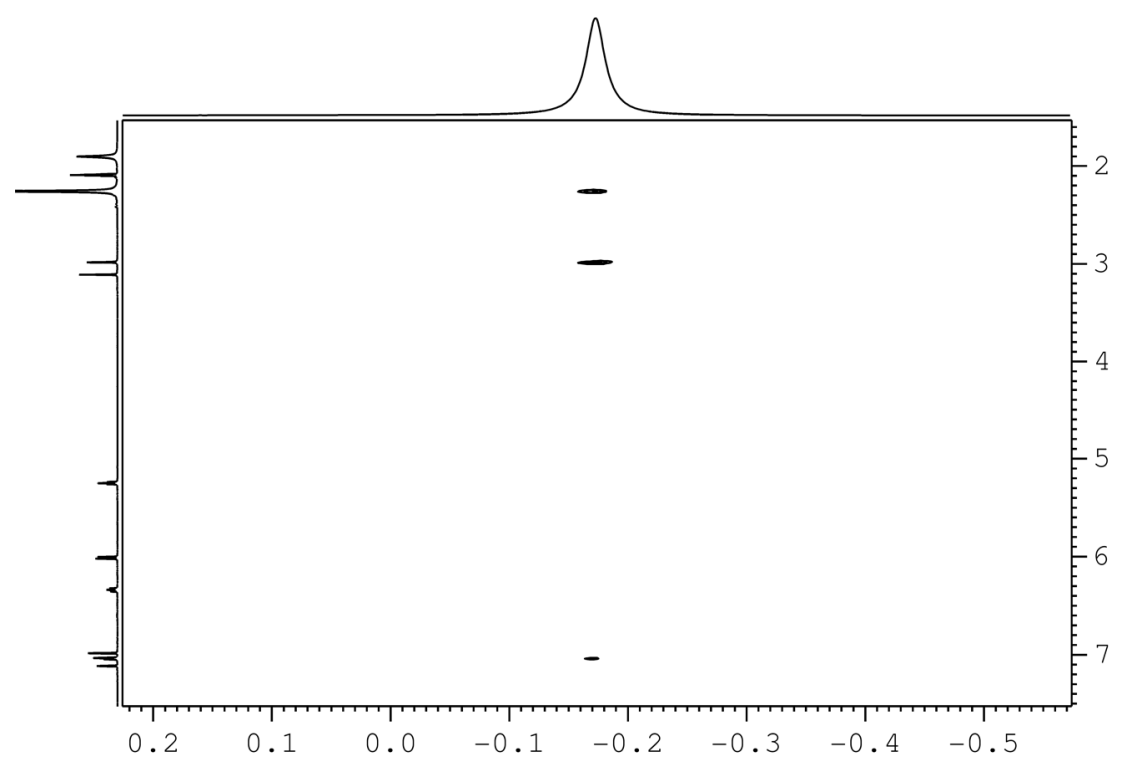

Figure 2-41. ${ }^{1} \mathrm{H}-{ }^{7} \mathrm{Li} \mathrm{HOESY}$ of [2-PicLi-tmeda $]_{2}$ (2).

This result verifies the assignment of $\mathrm{H}_{\text {cis }}$ and can be further substantiated by the ${ }^{1} \mathrm{H}-{ }^{7} \mathrm{Li}$ HOESY of [2-PicLi-tmeda $]_{2}(\mathbf{2})$. The cation is found in the proximity of $H 6_{\text {cis }}$ and the methyl groups of the tmeda donor. Additionally, a third signal is detected with $\mathrm{H} 5$ on a lower intensity (Figure 2-41). Hence, an enamidic bonding mode is reasonable as well as an aza-allylic one with the lithium atom positioned 
close to the pyridine ring, because no additional cross peaks could be detected to any other ring proton.

As mentioned above, only one species was found in solution and as a result only one signal is detected for the ${ }^{7} \mathrm{Li}$ atom at RT. The same is true at $-50{ }^{\circ} \mathrm{C}$, while a second sample showed three signals at $-85^{\circ} \mathrm{C}$ (red spectrum in Figure 2-42). Two peaks are symmetrical in respect to the signal at $-50{ }^{\circ} \mathrm{C}$ and one belongs most probably to a decomposition product of the aged sample (sharp resonance signal). Two distinguishable lithium species are formed at low temperature, which might be indicative for an asymmetrical dimer or the coexistence of dimer and monomer. However, further evidence has to be provided to verify this hypothesis. Yet, DOSY experiments cannot be used, because the sample cooling is not sufficiently constant in temperature for diffusion experiments of that kind.

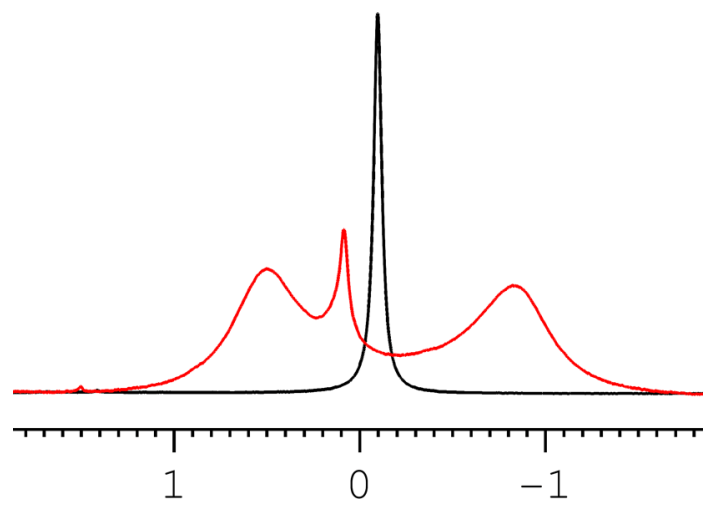

Figure 2-42. ${ }^{7} \mathrm{Li}$ spectra $[\mathrm{ppm}]$ of $[2-\mathrm{PiCLi} \cdot \mathrm{tmeda}]_{2}(2)$ at $-50^{\circ} \mathrm{C}$ (blue) and $-85^{\circ} \mathrm{C}$ (red).

More insight into the complex situation of [2-PicLi-tmeda] $]_{2}$ (2) in solution was gained by NOESY spectra at RT, $0^{\circ} \mathrm{C}$, and $-75^{\circ} \mathrm{C}$. Cross peaks for the $\mathrm{H} 6$ protons are only observed at RT and at very low temperatures. This is caused by two competing effects - the exchange rate and the cross-relaxation of the methylene protons. The methylene bond is rotating fast enough at RT that an off-diagonal signal can be observed in the height of the diagonal signals, while at $0{ }^{\circ} \mathrm{C}$ the exchange and the cross-relaxation effects exclude each other. At low temperature, the cross relaxation largely exceeds the exchange process (the cross relaxation contribution can also change the sign at low temperature so that both effects add) and a cross peak is once again observed. According to these results, the rotation rate of the methylene bond can be estimated or at least narrowed down. Because of the NOESY time scale and the two independent 
proton signals of the methylene group, the rotation rate must be between 2 and $40 \mathrm{~Hz}$.

The temperature dependence was also investigated by standard ${ }^{1} \mathrm{H}$ spectra. When the sample temperature is continuously lowered to $-90^{\circ} \mathrm{C}$, all signals are shifted to lower fields (Figure 2-43). Even more interestingly, the separation of the methylene hydrogen atoms ( $0.09 \mathrm{ppm}$ at RT, see above) is steadily increasing and finally becomes $0.27 \mathrm{ppm}$. Hence, almost half the value of the methylene hydrogen separation in the pmdeta compound at RT is reached at very low temperatures for the tmeda adduct. Moreover, the peak of the $H \sigma_{\text {cis }}$ proton is getting broader going down to a temperature of $-50^{\circ} \mathrm{C}$ and returns to the original shape when the cooling is continued. A change of the aggregation mode or degree can be assumed, which is in accordance with the ${ }^{7} \mathrm{Li}$ spectra (one signal at $-50^{\circ} \mathrm{C}$ and two at lower temperatures).

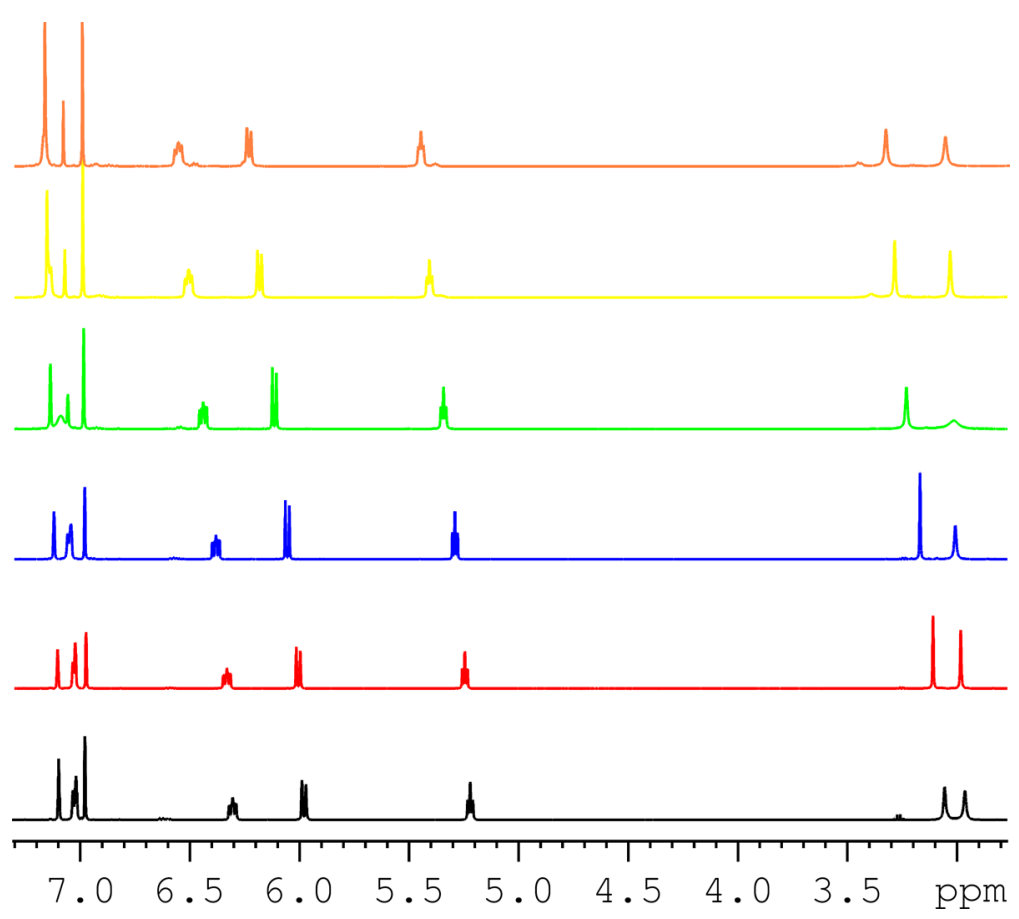

Figure $2-43 .{ }^{1} \mathrm{H}$ spectra of $[2-\mathrm{PicLi} \cdot \mathrm{tmeda}]_{2}(2)$ at various temperatures $(\mathrm{RT}, 0,-25,-50$, $-75,-90^{\circ} \mathrm{C}$, bottom to top).

A similar NMR investigation was also attempted for the dme adduct that was chosen as a key model for the second class of isolated 2-PicLi compounds. The ${ }^{1} \mathrm{H}$ DOSY spectrum gave only one diffusion coefficient for the picolyl anion, but there are some clues that the dme donor solvent is not firmly attached to the picolyl anion via the lithium cation, because a slightly different diffusion behaviour compared to the tmeda adduct was observed. This cannot be understood yet, 
since the donor strength of dme is supposed to be superior to the related nitrogen donor tmeda. However, the steric demand is different which might be the key to understand this phenomenon. Furthermore, the ${ }^{7} \mathrm{Li}$ DOSY is showing just a single lithium signal with a diffusion coefficient in the reported range of the tmeda adduct. The same is true for the proton spectrum, so that a similar size of the aggregate is assumed for both compounds.

By cooling the sample, the same effect as for the tmeda adduct is observed for $[2-\mathrm{PicLi} \cdot \mathrm{dme}]_{2}(\mathbf{1})$. All signals are shifted to lower field and the methylene proton signal splits up at $0^{\circ} \mathrm{C}$. This means that the rotation around the $\mathrm{C} 1-\mathrm{C} 6$ bond at this temperature is already slower than the NMR experiment. The proton separation is $0.05 \mathrm{ppm}$ at $-25^{\circ} \mathrm{C}$ and does not reach the value for the tmeda adduct at lower temperatures. Hence, the mentioned rotation is faster for the dme compound, but not dramatically, and the coordination mode should be related. This finding is also reflected by consistently downfield shifted proton signals indicating reduced electron density in the pyridine ring compared to [2-PicLi. tmeda $]_{2}$ (2).

The increased rotation rate is also reflected by the NOESY spectra. A large cross peak in the height of the diagonal signal is observed for the methylene protons at $0{ }^{\circ} \mathrm{C}$ as well as two signals for the $\mathrm{H} 2$ ring proton hardly differing in intensity. Accordingly, both $\mathrm{H} 6$ protons rotate faster than the time resolution of the NOESY experiment. At $-25^{\circ} \mathrm{C}$, the $\mathrm{H} 6_{\text {cis }} / \mathrm{H} 6_{\text {trans }}$ cross-peak intensity is reduced and the high-field methylene proton is showing a coupling with $\mathrm{H} 2$, while the second methylene hydrogen atom barely shows a comparable peak. This point to $\mathrm{HG}_{\text {trans }}$ being the high-field proton. This is different from the tmeda adduct, where this proton points to the nitrogen side and the lithium atom. The assignment was further proven by a HOESY spectrum.

The ${ }^{7} \mathrm{Li}$ HOESY at $-25^{\circ} \mathrm{C}$ shows the hydrogen atoms of the dme molecule in the proximity of the cation as well as $\mathrm{H} 5$ (hydrogen atom next to the nitrogen atom). Additionally, a cross peak is observed for the downfield shifted proton. Accordingly, the proton signal order is inversed for dme, so that $\mathrm{H}_{\text {cis }}$ is resonating at lower field than $\mathrm{H}_{\text {trans. }}$. This is in accordance with the results of $K$. Takahashi et al. for the same compound in an excess of dme at $-18{ }^{\circ} \mathrm{C} .{ }^{[137]}$

Next, some mono-silylated picolyllithium compounds shall be brought in context with the corresponding unsubstituted adducts. A comparison of the pmdeta compounds shows different tendencies for the ${ }^{1} \mathrm{H}$ signals. TMSCHPyLi is better assigned to the group of the dme, thf, and 2-PicH adducts due to the stabilising effect of the silicon atom (cf. Table 2-10). This leaves more charge on 
the carbanion and the lithium cation also interacts with C6. Even less charge delocalisation is observed with two diethyl ether donor molecules in [TMSCHPyLi. $\left.\mathrm{OEt}_{2}\right]_{2}$ and the disilylated dimer without donor solvent $\left[\mathrm{TMS}_{2} \mathrm{CPyLi}\right]_{2}$. Remarkably, the tmeda compounds resemble each other well. In contrast to the pmdeta adducts, the proton signals show little shifts besides for $\mathrm{H} 2$, which is shifted to lower fields ( $0.4 \mathrm{ppm})$ in the silylated compound. However, this can also be the outcome of the reported mean signals of $\mathrm{H} 2$ and $\mathrm{H} 3(6.5 \mathrm{ppm})$. It can be concluded that the tmeda adducts are widely alike, while a change in the aggregation pattern is reasonable for the pmdeta compounds.

In summary, further information on the structures of 2-PicLi in solution were detected by the use of defined aggregates in non-polar deuterated solvents, although the picolyllithium systems seemed to be well understood due to the work of K. Konishi and K. Takahashi. The separation into two general groups, which differ in the rotation rate around the methylene $\mathrm{C}-\mathrm{C}$ bond, could be accomplished and first hints toward the aggregation in solution were found. Nonetheless, many questions are not (finally) answered. Additional experiments are needed to clarify the exact involvement of the possible interaction sites ( $\mathrm{N}$ or $\mathrm{C}$ atom(s)) with the lithium ion and the aggregation state in solution. Although, the main interaction with the cation was presumably identified to be achieved via the pyridine nitrogen atom, the coordination pattern is still unknown ( $\eta^{1}$ enamide, $\eta^{3}$-aza-allyl, ( $\eta^{1}$-alkyl is unlikely)).

Further experiments are required to optimise the concentrations of the samples and their preparation. Even though isolated and thoroughly washed crystals were used, the presence of free picoline could not be ultimately excluded yet, which influences the results because it can act as a donor on its own. Furthermore, the deprotonation should also be performed with $n \mathrm{Bu}^{6} \mathrm{Li}$ in order to utilise the improved NMR characteristics of the spin one core. Even a ${ }^{13} \mathrm{C}$ enrichment at the methyl side chain is thinkable and may clarify a putatively partial $\mathrm{sp}^{3}$ hybridisation of the deprotonated carbon atom in the presence of oxygen donor molecules. Moreover, a comparison with NMR spectra in the solid state, in which the atomic arrangements are known from X-ray experiments, can give further references for the appearance of the 2-picolyllithium anions in solution depending on the applied donors. 


\subsection{2-Picolyl Complexes of Sodium}

In comparison to the vast application range of lithium organic compounds, their heavier alkaline metal congeners are scarcely used, due to their much higher reactivity compared to the lithium analogues. These compounds are more sensitive to air and moisture, can react with nitrogen protective gas, and thermally decompose more readily than their lithium counterparts. In addition, those metal organic compounds are poorly soluble even in highly polar solvents. The solvents are easily cleaved (ether) or metallated (donor bases such as tmeda or pmdeta). ${ }^{[237-239]}$ However, these metal organic compounds attracted some attention in synthetic chemistry ${ }^{[13,17,240,241]}$ since the pioneering work of $M$. Schlosser and L. Lochmann, who independently developed standard procedures for their preparation. ${ }^{[242-244]} \mathrm{A}$ metal-metal exchange reaction occurs in inert hydrocarbons with alkyl lithium compounds (LIC) and heavy alkaline metal alkoxides (e.g. potassium alkoxides, KOR), in which the resulting insoluble alkyl heavy metal compound can be filtered off, while the lithium alkoxides are still dissolved. The prominent metallation mixtures (LICKOR mixtures) were therefore called Schlosser- or Lochmann-bases or simply super bases. Other preparative routes are metathesis reactions with mercury organic compounds, the cleavage of ethers, and the deprotonation of $\mathrm{C}-\mathrm{H}$ acidic compounds with elemental metal. ${ }^{[8,245,246]}$

Super bases are used in reactions in which the reactivity of lithium compounds is not suitable. The enhanced metallation ability can be demonstrated with toluene as substrate. As already mentioned in chapter 1 , it cannot be deprotonated with pure $n B u L i$, but in the presence of tmeda. ${ }^{[55]}$ If a LICKOR mixture is employed, a complete conversion to benzylpotassium can be achieved without donor bases. ${ }^{[247]}$ In fact, the route via the heavy metal organic compound was used to generate tmeda-free benzyllithium representing an alternative to the dibenzylmercury route. ${ }^{[248]}$ This lithium organic compound can be more easily stored in contrast to the nitrogen donor adduct, which decomposes as isolated crystalline material within days.

Another prominent example of different degrees of metallation by variation of the reactivity of the deprotonating agent is ferrocene. It is mono-metallated with neat $n B u L i$, while the $1,1^{\prime}$-ferrocenyldilithium compound can be isolated in the presence of additional tmeda. ${ }^{[249-251]}$ The $1,1^{\prime}, 3,3^{\prime}$-ferrocenyltetraanion can only be obtained with the super basic mixture of $n$-butyl-sodium, dibutylmagnesium and di-iso-propylamine. ${ }^{[252]}$ Besides the enhanced basicity, heavy alkaline metal 
organic compounds can adopt different reaction routes. While RLi normally add to alkenes, LICKOR mixtures favour hydrogen abstraction. ${ }^{[241]}$ The deprotonation also proceeds highly regioselective (allylic methyl groups are preferred) and stereoselective in the case of cis/trans isomers (cis isomers react more rapidly). Due to their selectivity, they were applied in organic natural product syntheses. ${ }^{[253]}$ Therefore, it was attempted to isolate heavy alkaline metal analogues of 2-picolyllithium to tune the reactivity of the organic anion by the variation of the metal cation.

\subsubsection{2-Picolylsodium}

The standard preparation route for 2-picolylsodium in literature is the deprotonation of 2-picoline with sodium amide in liquid ammonia. ${ }^{[100,101]}$ The in situ conversions of 2-PicNa with organic electrophiles lead to fairly acceptable yields in the range of 50 to $60 \%$. However, H. C. Brown and W. A. Murphey revealed in a detailed study on picolyl sodium organic compounds that the metallation of picoline represents an equilibrium reaction. ${ }^{[96]}$ By slowly adding chloromethane over a period of $8 \mathrm{~h}$ to a 2-PicNa ammonia solution, the desired coupling product (EtPy) could be isolated in $60 \%$ yield. If the electrophile was added rapidly $(0.5 \mathrm{~h})$, the yield dropped to $7 \%$; the sodium organic compound (2-PicNa) could not be regenerated after consumption due to the reaction of the chloroalkane with sodium amide. Thus, this preparation procedure is not applicable for the isolation of the metallated species.

The deprotonation was therefore realised with the super base $n$-butylsodium. This precipitated from a hexane solution of sodium tert-butoxide and $n$-butyllithium as an orange solid, ${ }^{[254]}$ which was afterwards redissolved in a 1:4 mixture of tmeda and hexane. It has to be mentioned that extreme care had to be taken during the isolation of $n$ BuNa. Even small leakages in the Schlenk equipment instantly led to partial decomposition of the powdery material, which could have easily resulted in a violent ignition of the highly reactive compound. Subsequently, the $n B u N a$ tmeda solution was slowly added to an equimolar amount of 2-picoline in diethyl ether. Orange needles could be obtained from the reaction mixture at $-24^{\circ} \mathrm{C}$. These were of sufficient quality for an X-ray structural analysis.

2-Picolylsodium-tmeda (8) crystallises in the monoclinic centrosymmetric space group $\mathrm{C} 2 / \mathrm{m}$. The asymmetric unit contains one 2-PicNa moiety and half of 
a tmeda molecule. The picolyl ring resides on the crystallographic mirror plane and the complete dimer is generated by a mirror operation and a subsequent inversion operation at $(0,0.5,0)$. Alternatively, the second half of the dimer can be obtained by a $C_{2}$ rotation about the $\mathrm{Na} \cdots \mathrm{Na}^{\prime}$ axis. This leads to the highly symmetric [2-PicNa.tmeda] 2 adduct (8) in which all $\mathrm{Na}-\mathrm{N}_{\mathrm{Py}}, \mathrm{Na}-\mathrm{N}_{\text {donor, }}$ and $\mathrm{Na}-\mathrm{C}_{\alpha}$ bonds are equal in length among each other (Figure 2-44).

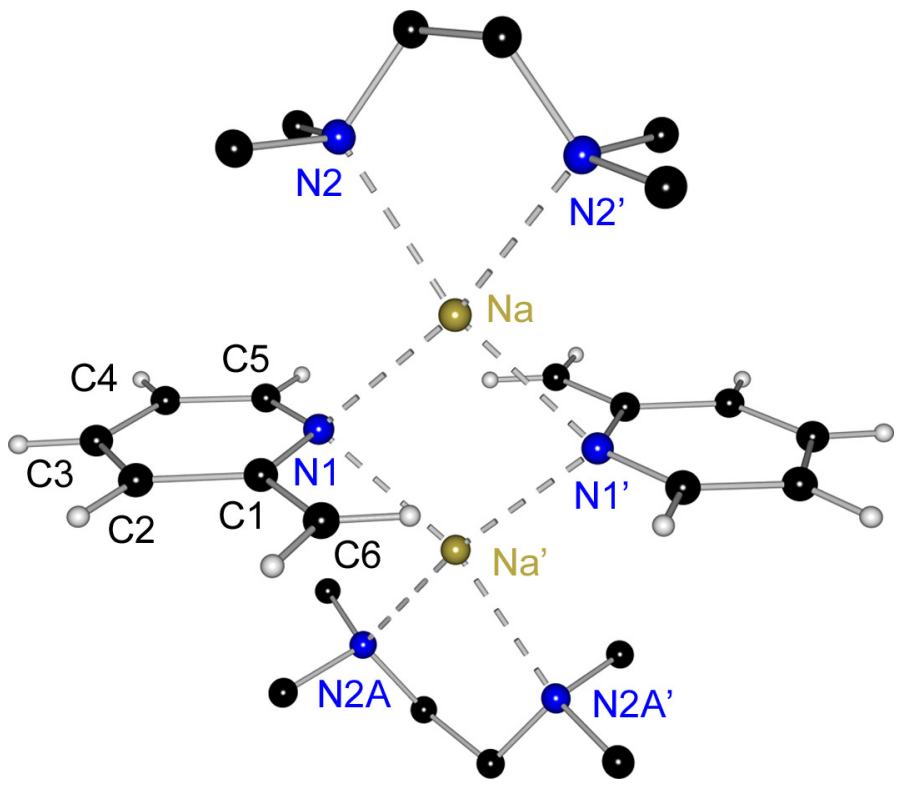

Figure 2-44. Molecular structure of [2-PicNa-tmeda $]_{2}(\mathbf{8})$. Hydrogen atoms of the donor molecule are omitted for clarity.

The dimer is linked via two sodium atom contacts to each pyridine nitrogen atom. A diamond shaped central $[\mathrm{NaN}]_{2}$ four-membered ring is formed with a trans configuration of the picolyl anions and an acute $\mathrm{Na}-\mathrm{N} 1-\mathrm{Na}^{\prime}$ angle of $84.9(1)^{\circ}$ (Table 2-12). Accordingly, the $\mathrm{N} 1-\mathrm{Na}-\mathrm{N} 1^{\prime}$ angle is $95.1(1)^{\circ}$ while the angle $\mathrm{N} 2-\mathrm{Na}-\mathrm{N} 2$ ' to the chelating tmeda nitrogen atoms is even more acute with $73.8(1)^{\circ}$. This is caused by the increased cation size in comparison to the smaller lithium ion ( $\mathrm{N}-\mathrm{Li}-\mathrm{N}$ tmeda angles: $\left.83-85^{\circ}\right){ }^{[255]}$ Therefore, the tmeda donor bonds to the metal atoms $(2.483(1) \AA)$ are much longer than in the lithium analogue $2(2.06 \AA)$. A similar effect is observed for the enamidic $\mathrm{Na}-\mathrm{N} 1$ bond lengths $(2.425(1) \AA)$ in 8 compared to the mean length of the corresponding lithium compounds ( 2.16 $\AA$, cf. chapter 2.2.2).

A pure enamidic interaction with the sodium cations can be assumed since the $\mathrm{Na}-\mathrm{C} 1 / \mathrm{C} 6$ distances are far too long for a noteworthy aza-allylic coordination (2.991(2) and 3.024(2) $\AA$ ). The lone pairs at the pyridine nitrogen atoms are not directed towards each other, as seen from the $\mathrm{N} 1^{\prime}-\mathrm{N} 1-\mathrm{C} 1$ angle of $103.0^{\circ}$ and 
the $\mathrm{N} 1^{\prime}-\mathrm{N} 1-\mathrm{C} 3$ angle of $163.8^{\circ}$ (Figure 2-45). The reason for that might be an electrostatic repulsion of the nitrogen lone pairs (N1-N1' distance $3.578 \AA$ ) or a small attractive interaction of the methylene hydrogen atom (cis in respect to N1) with the sodium cation ( $\left.\mathrm{Na}-\mathrm{H} 6_{c i s}: 2.619 \AA\right)$.

Table 2-12. Selected bond lengths $[\AA]$ and angles $\left[{ }^{\circ}\right]$ of $[\mathrm{PicNa} \cdot \mathrm{tmeda}]_{2}(\mathbf{8})$ and related compounds $\left(\mathrm{Pic}=2\right.$-picolyl, $\mathrm{Py}=2$-pyridyl) ${ }^{[144,162]}$

\begin{tabular}{|c|c|c|c|c|}
\hline & $\begin{array}{c}{[\mathrm{PicNa} \cdot} \\
\mathrm{tmeda}]_{2}(\mathbf{8})\end{array}$ & $\begin{array}{c}{\left[\left(\mathrm{Me}_{3} \mathrm{Si}\right) \mathrm{CHPyNa} \cdot\right.} \\
\text { tmeda }]_{2}^{[\mathrm{a}]}\end{array}$ & $\begin{array}{l}{\left[\left(\mathrm{Me}_{3} \mathrm{Si}\right) \mathrm{CHPyNa} .\right.} \\
\text { pmdeta }]_{2}^{[\mathrm{b}]}\end{array}$ & {$\left[\mathrm{Ph}_{2} \mathrm{CPyNa} \cdot(\text { thf })_{3}\right]^{[\mathrm{c}]}$} \\
\hline $\mathrm{Na}-\mathrm{N} 1$ & $2.425(1)$ & $2.39(1) / 2.51(1)$ & $2.475(2) / 2.586(3)$ & $2.414(7)$ \\
\hline $\mathrm{Na}-\mathrm{N} 2$ & $2.483(1)$ & $2.45(2) / 2.40(2)$ & $2.601(3) / 2.669(3)$ & $2.320(7) / 2.343(7)$ \\
\hline $\mathrm{N} 1-\mathrm{C} 1$ & $1.396(2)$ & $1.38(2)$ & $1.388(4)$ & $1.379(6)$ \\
\hline $\mathrm{N} 1-\mathrm{C} 5$ & $1.349(2)$ & $1.34(2)$ & $1.332(6)$ & $1.346(6)$ \\
\hline $\mathrm{C} 1-\mathrm{C} 2$ & $1.446(3)$ & $1.41(2)$ & $1.453(5)$ & $1.429(7)$ \\
\hline $\mathrm{C} 2-\mathrm{C} 3$ & $1.350(3)$ & $1.32(2)$ & $1.325(9)$ & $1.351(7)$ \\
\hline $\mathrm{C} 3-\mathrm{C} 4$ & $1.403(3)$ & $1.40(2)$ & $1.389(6)$ & $1.391(7)$ \\
\hline $\mathrm{C} 4-\mathrm{C} 5$ & $1.370(3)$ & $1.37(3)$ & $1.365(7)$ & $1.363(7)$ \\
\hline $\mathrm{C} 1-\mathrm{C} 6$ & $1.372(3)$ & $1.37(2)$ & $1.364(6)$ & $1.427(7)$ \\
\hline $\mathrm{Na}-\mathrm{N} 1-\mathrm{Na}^{\prime}$ & $84.9(1)$ & $81.8(3)$ & $88.8(1)$ & - \\
\hline $\mathrm{N} 1-\mathrm{Na}-\mathrm{N} 1^{\prime}$ & $95.1(1)$ & $101.3(4) / 95.2(4)$ & $91.2(1)$ & - \\
\hline $\begin{array}{l}\mathrm{N} 2-\mathrm{Na}-\mathrm{N} 2^{\prime} / \\
\mathrm{O}_{\text {thf }}-\mathrm{Na}-\mathrm{O}_{\text {thf }}\end{array}$ & $73.8(1)$ & $75.8(5) / 76.6(6)$ & $69.5(1) / 70.2(1)$ & $91.8(2) / 102.5(2)$ \\
\hline
\end{tabular}

[a] Additional values refer to the corresponding bonds in the independent second half of the dimer that is not related via the crystallographic $\mathrm{C2}$ axis along $\mathrm{Na} 1 \cdots \mathrm{Na} 2$. [b] Additional values refer to the corresponding bonds in the independent second half of the dimer that is not related via the inversion centre of the $[\mathrm{NaN}]_{2}$ ring. Only the shortest and longest $\mathrm{Na}-\mathrm{N}_{\text {donor }}$ bonds are listed. [c] Only the shortest and longest $\mathrm{Na}-\mathrm{O}_{\text {donor }}$ bonds are listed.

Similar short sodium hydrogen distances were detected in the trimethylsilyl substituted picolylsodium tmeda compound $(2.58 \AA) \cdot{ }^{[144]}$ The authors interpreted this contact as an indication for a "relatively strong compensatory agostic interaction". However, in view of the charge density results of W. Scherer et al., ${ }^{[86,87]}$ it is doubtful that effects other than electrostatic attractions play a role in the appearance of these short atom distances. Nonetheless, [2-PicNa.tmeda] 2 (8) would be an ideal candidate to verify this by a charge density study, because 
of the high crystal symmetry and the fixed atomic coordinates on crystallographic special positions.

The coordination sphere of the sodium metal is also special. A square-planar geometry (Figure 2-45) around the donating nitrogen atoms is adopted, which can be seen by the small angle between the $\mathrm{N} 1-\mathrm{Na}-\mathrm{N} 1^{\prime}$ and the N2-Na-N2' plane $\left(15^{\circ}\right)$. No reasonable explanation can be given for this observation, because no steric effects in the crystal packing were detected. A comparable feature was already found in [2-PicLittmeda $]_{2}$ (2, angle between related planes: $49^{\circ}$ ). The line between $\mathrm{N} 2 \cdots \mathrm{N} 2^{\prime}$ in the tmeda donor is almost parallel to the gap between the picolyl anions ( $\mathrm{C} 6-\mathrm{C} 1-\mathrm{C} 4$ line). Moreover, a steric cause of this arrangement was considered because of the short $\mathrm{Li}-\mathrm{N}$ bonds.

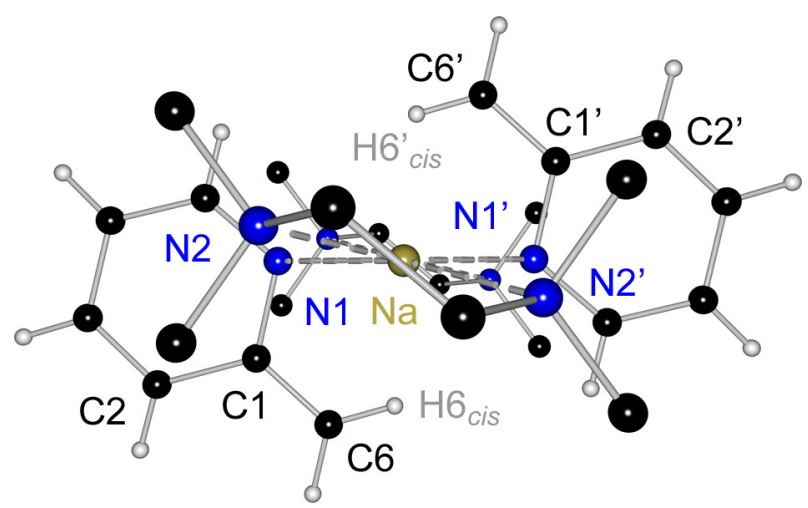

Figure 2-45. View along the crystallographic $C 2$ axis of [2-PicNa.tmeda $]_{2}(8)$.

The dimerisation pattern in [( $\left.\left.\mathrm{Me}_{3} \mathrm{Si}\right) \mathrm{CHPyNa} \cdot \mathrm{tmeda}\right]_{2}$ can be better compared to that of $\mathbf{2}$ rather than to the unsubstituted sodium analogue $\mathbf{8}^{[144]}$ The picolyl rings are also asymmetrically arranged with one side of the dimer slightly facing the sodium cation via the aza-allylic N1-C1-C6 unit. Accordingly, there is an open and a more shielded side of the picolyl anion, too, with shorter $\mathrm{Na}-\mathrm{N}_{\mathrm{Py}}$ bonds at the open end. The tmeda position relative to the $[\mathrm{NaN}]_{2}$ ring at the other side of the dimer is also more akin to the lithium adduct (angle between related planes: $44^{\circ}$ ). Thus, it can only be speculated about whether the short $\mathrm{Na}-\mathrm{H} 6_{\text {cis }}$ contact is the reason for the observed donor positions in $\mathbf{8}$. It can be seen in Figure 2-45 that the projection of the N2 $\cdots \mathrm{N} 2$ ' line on the crystallographic mirror plane containing the picolyl rings is almost at right angle to the similar projection of the $\mathrm{Na}-\mathrm{H} 6_{\text {cis }}$ contact (angle between $\mathrm{N} 2-\mathrm{Na}-\mathrm{N} 2^{\prime}$ and $\mathrm{NaH6}_{\text {cis }} \mathrm{Na}^{\prime}$ plane: $95^{\circ}$ ).

A comparison with other known 2-picolylsodium derivatives (Scheme 2-12) shows that the bonds of the picolyl anion to the sodium cation are on the short 
end of reported contacts (Table 2-12). The silylated tmeda adduct shows different $\mathrm{Na}-\mathrm{N}$ distances due to the asymmetrical coordination of the sodium cation. The same is true for the corresponding pmdeta adduct [ $\left(\mathrm{Me}_{3} \mathrm{Si}\right) \mathrm{CHPyNa}$. pmdeta $]_{2}{ }^{[144]}$ which shows differences between the dimer halves along the $\mathrm{Na} \cdot \mathrm{Na}^{\prime}$ axis and not along the $\mathrm{N} 1 \cdots \mathrm{N} 1^{\prime}$ vector, as in the tmeda aggregate. This is correlated with the steric demand of the tridentate nitrogen donor. In summary, all $\mathrm{Na}-\mathrm{N}$ bond lengths are increased compared to $\mathbf{8}$ or the tmeda adduct. Besides the steric aspect of the donor, the greater donating capacity of the pmdeta ligand also influences the strength of the $\mathrm{Na}-\mathrm{N}_{\mathrm{Py}}$ interactions. Interestingly, the pmdeta donor is not able to stabilise the monomeric species of the larger alkaline metal organic compound, which was the case for $\mathbf{3}$ and $\left[\left(\mathrm{Me}_{3} \mathrm{Si}\right) \mathrm{HC}-6-\mathrm{Me}-\mathrm{PyLi} \cdot \mathrm{pmdeta}\right]$.

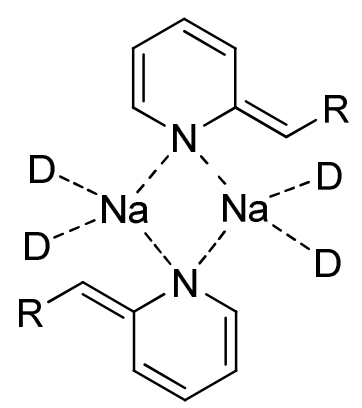

$[\mathrm{RHCPyNa} \cdot \mathrm{tmeda}]_{2}$

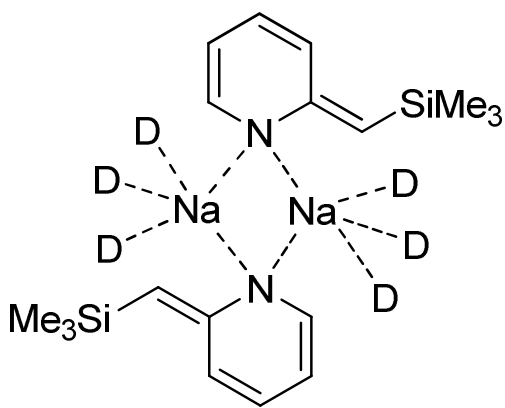

$\left[\left(\mathrm{Me}_{3} \mathrm{Si}\right) \mathrm{HCPyNa} \cdot \mathrm{pmdeta}\right]_{2}$<smiles>[2H]N([2H])[Al]([2H])N1C=CC=CC1=C(c1ccccc1)c1ccccc1</smiles>

$\left[\mathrm{Ph}_{2} \mathrm{CPyNa}(\text { thf })_{3}\right]$

Scheme 2-12. Lewis formulae of PicNa compounds with an $\eta^{1}$-amidic dimerisation pattern $\left(\mathrm{R}=\mathrm{H}, \mathrm{SiMe}_{3} ; \mathrm{D}=\right.$ tmeda/pmdeta/thf donor atom; Py $=2$-pyridyl).

The preferred coordination exclusively via the pyridine nitrogen atom is also reflected in the carbon-carbon bond lengths in the picolyl anion (Table 2-12). These are almost identical compared to the analogous lithium compound and show the systematics of the enamidic resonance formula of the picolyl anion (D in Scheme 2-4). The same tendency is observed for the silylated picolylsodium tmeda and pmdeta dimers as well. Only the values for diphenyl(2-pyridyl)methylsodium monomer are different. ${ }^{[162]}$ While the bond lengths of the pyridine ring match quite well, the $\mathrm{C} 1-\mathrm{C} 6$ bond is vastly longer $(1.427(7) \AA$ ) possibly due to a small stabilisation by the phenyl rings (not coplanar to the pyridine ring) and their steric demand. This is indeed much larger for carbon substituted picolyl derivatives in comparison to the silylated ones, because of the much longer $\mathrm{Si}-\mathrm{C}$ bonds (see also ref. [165]). 
In summary, it could be shown by the synthesis of [2-PicNa.tmeda $]_{2}$ (8) that the application of a super base also leads to the successful deprotonation of 2-picoline to form a defined, isolable product. A comparative study on the reactivity of crystalline picolyllithium and -sodium compounds should give further insights into the influence of defined nucleophiles on the yields of the reaction with organic as well as inorganic electrophiles.

The NMR spectrum of [2-PicNa.tmeda] 2 (8) in deuterated benzene shows all hydrogen atom signals slightly shifted to lower fields relative to the corresponding lithium compound $\mathbf{2}$ (Table 2-14). The protons are more deshielded in the sodium organic compound while $\mathrm{H}_{\text {cis }}$ is shifted to higher magnetic field. The splitting of the methylene hydrogen atoms is therefore just a little larger than in 2 and still far from the picolyllithium pmdeta adduct. Hence, no striking difference was detected that displays the varying ionicity by variation of the cation.

Table 2-13. ${ }^{1} \mathrm{H}$ NMR signals $[\mathrm{ppm}]$ of $\left[2-\mathrm{PicNa} \cdot \mathrm{tmeda}_{2}\right.$ (8) and related 2-picolyllithium compounds (Pic $=2$-picolyl).

\begin{tabular}{lccccccc}
\hline \multicolumn{1}{c}{ Compound } & Solvent & $\mathrm{H} 5$ & $\mathrm{H} 3$ & $\mathrm{H} 2$ & $\mathrm{H} 4$ & $\mathrm{H} 6$ & Reference \\
\hline$[\text { PicNa.tmeda }]_{2}$ & $\mathrm{C}_{6} \mathrm{D}_{6}$ & 7.25 & 6.51 & 6.29 & 5.37 & $3.15 / 2.97$ & this work \\
{$[\text { PicLi·tmeda }]_{2}$} & $\mathrm{C}_{6} \mathrm{D}_{6}$ & 7.11 & 6.41 & 6.10 & 5.32 & $3.15 / 3.06$ & this work \\
{$[\mathrm{PiCLi} \cdot \mathrm{pmdeta}]$} & $\mathrm{C}_{6} \mathrm{D}_{6}$ & 7.06 & 6.58 & 6.31 & 5.37 & $3.40 / 2.76$ & this work \\
{$\left[\mathrm{PiCLi} \cdot(\text { thf })_{2}\right]_{2}$} & $\mathrm{C}_{6} \mathrm{D}_{6}$ & 7.41 & 6.52 & 6.30 & 5.48 & 3.18 & {$[149]$} \\
{$[\mathrm{PiCLi} \cdot \mathrm{dme}]_{2}$} & $\mathrm{C}_{6} \mathrm{D}_{6}$ & 7.30 & 6.54 & 6.26 & 5.46 & 3.06 & this work \\
{$[\mathrm{PiCLi} \cdot \mathrm{PiCH}]_{2}$} & $\mathrm{C}_{6} \mathrm{D}_{6}$ & 7.52 & 6.49 & 6.31 & 5.47 & 3.39 & {$[149]$} \\
\hline
\end{tabular}




\subsubsection{Attempted One-pot Synthesis of 2-Picolylsodium/-potassium}

The simple one-pot synthesis of the heavy alkaline metal compound in the presence of a $\mathrm{C}-\mathrm{H}$ acidic substrate is a preparative variation to the isolation of $n$-butylsodium. The substrate is instantly metallated by the in situ generated sodium organic compound and butane emerges from the reaction mixture. The deprotonated organic compound normally precipitates from non-polar solvents and can be filtered off. This route avoids the dangerous handling of $n B u N a$ and also stints the additional isolation step in the synthesis. Therefore, an $n B u L i$ solution was slowly added to an equimolar mixture of 2-picoline and sodium tert-butoxide in hexane. A bright yellow solid started to precipitate immediately, which was filtered off at the end of the addition. The control NMR spectrum of the isolated compound still showed traces of tert-butyl protons that might originate from adherent lithium tert-butoxide or remainders of the starting material. Moreover, it has to be emphasised that the solid was hardly soluble in $\mathrm{d}_{8}$-thf and already showed decomposition after a short period of time.

The attempt to dissolve picolylsodium in thf in order to crystallise the sodium analogue of [2-PicLi.(thf $\left.)_{2}\right]_{2}$ was unsuccessful at low temperature, while during warm-up the reaction mixture started to foam, probably due to the decomposition of thf. Unfortunately, it was not possible to crystallise the resulting sodium enolate. Moreover, the utilisation of donor bases other than tmeda during the crystallisation of 2-picolylsodium complexed failed so far.

The same procedure was applied for the synthesis of 2-picolylpotassium. An orange solid could be isolated, whose NMR signals proved the successful metallation of 2-picoline. ${ }^{[129]}$ However, the potassium compound is barely soluble in inert solvents and readily decomposes polar donors. Hence, no suitable solvent mixture has been found by now from which crystals for an X-ray analysis could be grown.

In order to obtain crystalline material of 2-picolylpotassium, it could also be synthesised with potassium amide in liquid ammonia. M. J. Weiss and C. R. Hauser reported that the red solution turned into a dark red to black suspension on addition of diethyl ether and the formation of crystalline material. ${ }^{[256]}$ It can only be conjectured whether this material was picolylpotassium or a decomposition product (ether cleavage) according to the corresponding results of picolylsodium with thf described above. 
In addition, benzylsodium was synthesised via both synthetic routes (isolation and one-pot) for comparison reasons and for the use in addition reactions with inorganic electrophiles. If $n$-butylsodium was isolated, redissolved in tmeda, and subsequently mixed with an excess of toluene, a reddish-brown solution was obtained. Storage at $4^{\circ} \mathrm{C}$ resulted in bright red crystals suitable for an X-ray structural analysis. Even though the solid state structure of the benzylsodium tmeda adduct was already known, ${ }^{[257]}$ the crystal structure was reinvestigated. However, the unit cell determination showed cell parameters different from those of $[\mathrm{BzNa} \cdot \mathrm{tmeda}]_{4} \cdot{ }^{[257]}$

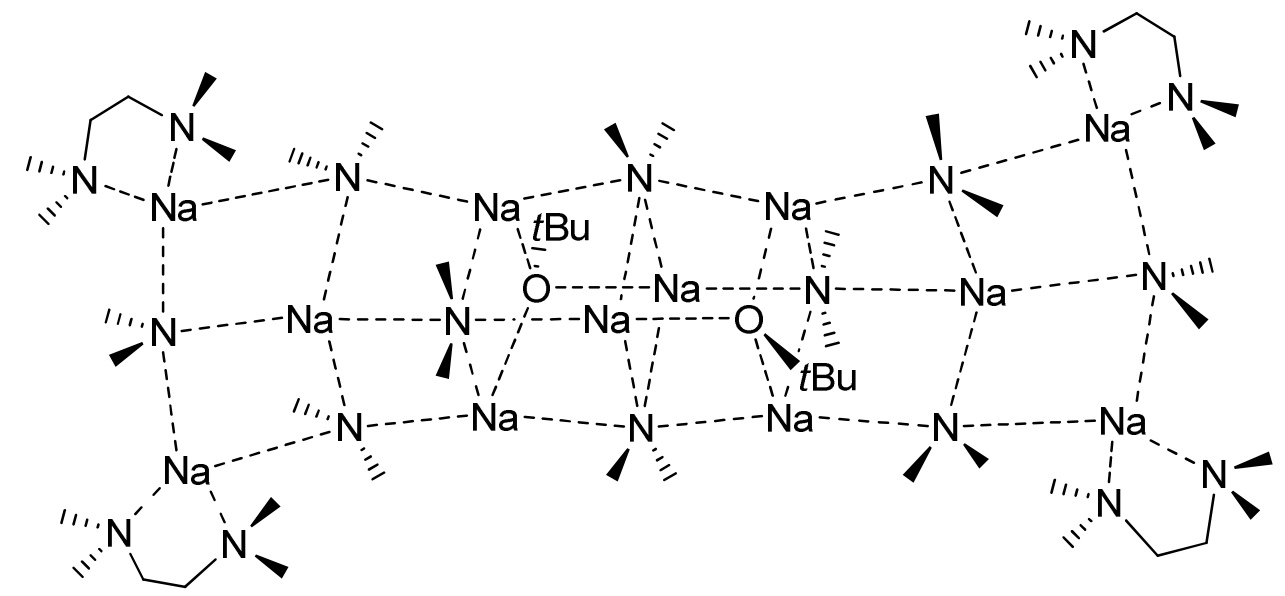

Scheme 2-13. Lewis diagram showing the constitution of $\left[\mathrm{Na}_{6}\left(\mathrm{NMe}_{2}\right)_{5}(\mathrm{OtBu}) \cdot(\mathrm{tmeda})_{2}\right]_{2}$.

Therefore, the data collection was continued and the structure solution revealed that the crystals contained no metallated toluene. In fact, a complicated structure of sodium dimethylamide was rationalised (Scheme 2-13 and Figure 2-46). The complete empirical formula of the crystal is $\left[\mathrm{Na}_{6}\left(\mathrm{NMe}_{2}\right)_{5}(\mathrm{OtBu})\right.$. $(\text { tmeda })_{2}($ tol $\left.)\right]_{2}$ (9) so that excess sodium tert-butoxide and lattice solvent were incorporated in the crystal. Sodium dimethylamide likely originated from the decomposition of tmeda (Scheme 2-14): Initially, $n$-butylsodium deprotonates a methylene carbon atom of the donor molecule. The following cleavage of the $\mathrm{N}-\mathrm{C}$ bond is analogous to the $\mathrm{O}-\mathrm{C}$ bond cleavage in the decomposition of ethers. ${ }^{[8,9]}$ The resulting products are sodium dimethylamide and dimethylaminoethene.

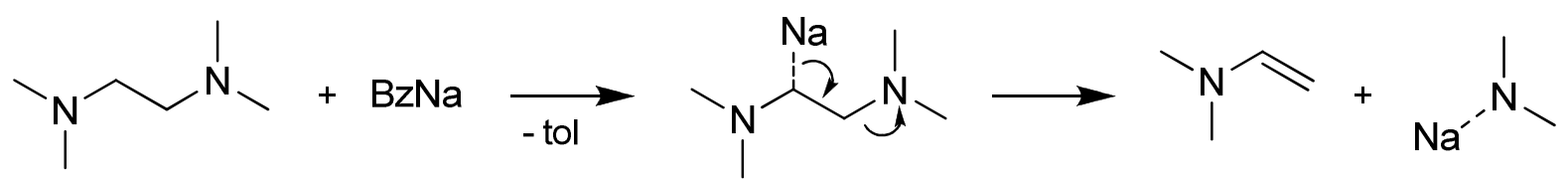

Scheme 2-14. Stepwise decomposition of tmeda by benzylsodium. 
Alternatively, this process can also take place with a concerted mechanism, already proposed for the decomposition of ethers with lithium organic compounds. ${ }^{[258]}$ However, the results of $F . H$. Köhler et al. showed that the preferred reaction of tert-butyllithium with tmeda is the deprotonation at a methyl rather than at a methylene carbon atom, while a mixture of $n B u L i$ and $t$ BuOK results in the cleavage of tmeda donor molecules similar to that observed in $\mathbf{9 .}{ }^{[237]}$

A search in literature revealed that an almost identical aggregate $\left(\left[\mathrm{Na}_{12}\left(\mathrm{NMe}_{2}\right)_{12}(\text { tmeda })_{4}\right]\right)$ was obtained during the metallation of 4-methylbiphenyl with $n$-butylsodium. ${ }^{[238]}$ The only differences to 9 are the absence of alkoxide anions and hexane instead of toluene as the lattice solvent. Both compounds incorporate twelve cations and show an interesting constitution, of which some features shall be discussed.

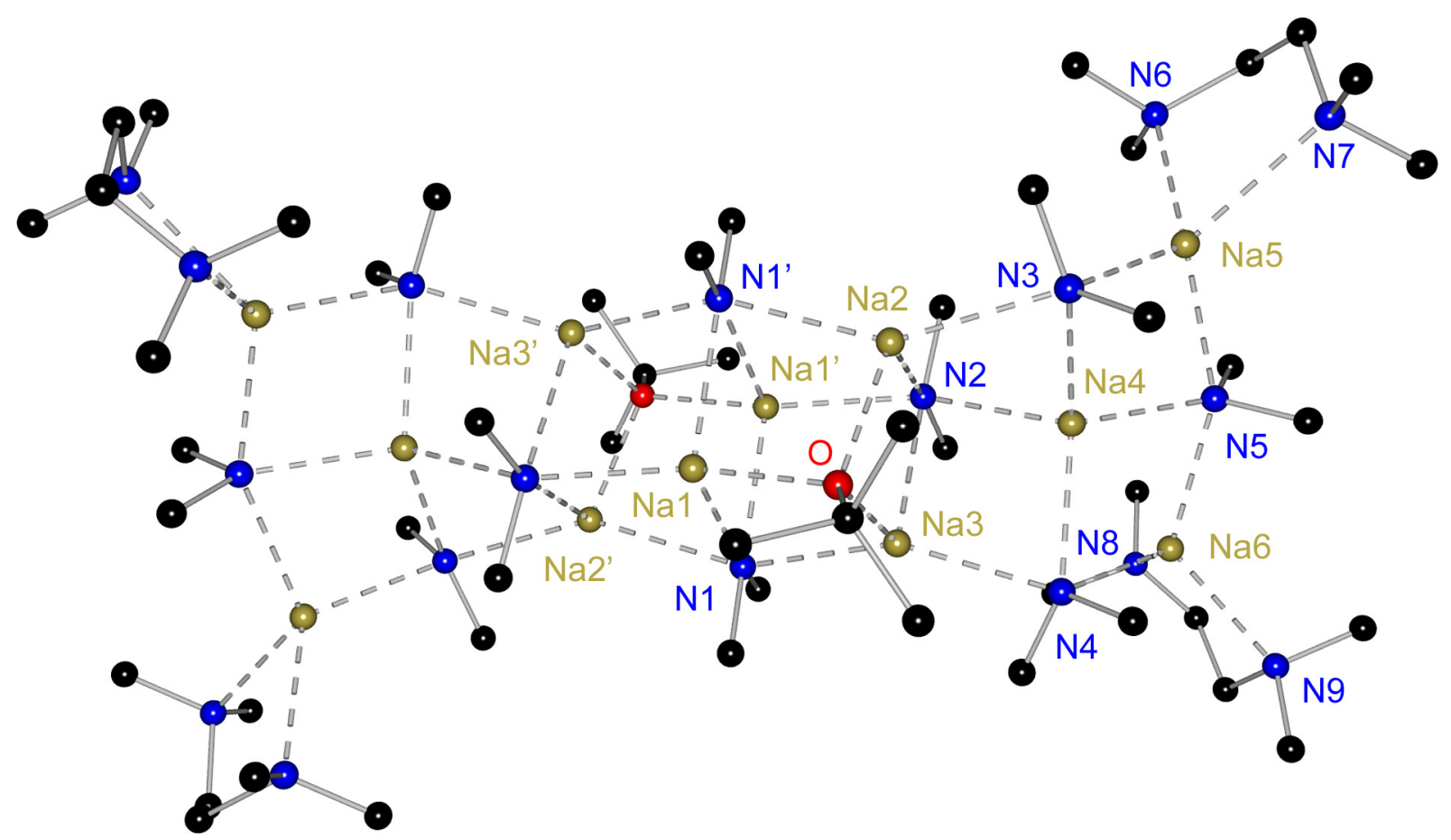

Figure 2-46. Molecular structure of $\left[\mathrm{Na}_{6}\left(\mathrm{NMe}_{2}\right)_{5}(\mathrm{OtBu}) \cdot(\mathrm{tmeda})_{2}(\mathrm{tol})\right]_{2}(\mathbf{9})$. Hydrogen atoms, disordered atoms of the donor molecules, and lattice solvent are omitted for clarity.

$\left[\mathrm{Na}_{6}\left(\mathrm{NMe}_{2}\right)_{5}(\mathrm{OtBu}) \cdot(\text { tmeda })_{2}(\mathrm{tol})\right]_{2}$ (9) crystallises in the triclinic centrosymmetric space group $P \overline{1}$ with one half of the aggregate in the asymmetric unit. The centre of inversion is located in the middle of the Na1-N1-Na1'-N1' ring. The complex molecular structure (Figure 2-46) consists of two known coordination motifs in amide chemistry: a central distorted double heterocubane formation, consisting of a triple-layered stack of $[\mathrm{NaN}]_{2}$ rings, and a ladder 
structure of NaN moieties on the outer sphere. These structural fragments have been mainly reported for metal amides containing both lithium and sodium ${ }^{\text {[259-261] }}$ or only lithium. ${ }^{[262,263]}$ In the present case no sign for the incorporation of the lighter alkaline metal has been observed.

Focussing on the central double heterocubane moiety, one planar $[\mathrm{NaN}]_{2}$ fourmembered ring ( $\mathrm{Na} 1$ and $\mathrm{N} 1$ ) is stacked with two slightly (about the $\mathrm{Na} 2 \cdots \mathrm{Na} 3$ vector) bent four-membered rings, in which one amide group is substituted by a tert-butoxide anion (Figure 2-47). All amide anions of the cubane fragment are bonded to four sodium atoms. Interestingly, the central nitrogen atoms N1 and $\mathrm{N} 1^{\prime}$ form three equally short $\mathrm{N}-\mathrm{Na}$ bonds $(2.512(2)$ to $2.520(2) \AA)$ and a longer one to $\mathrm{Na}^{\prime}(2.588(2) \AA)$. No explanation can be given for that fact, since both sides (above and below) of the amide appear similar.

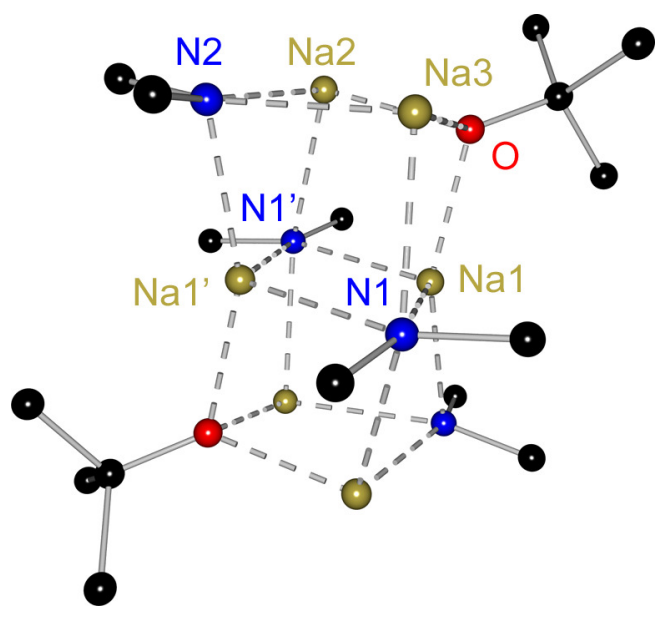

Figure 2-47. Double heterocubane fragment of $\left[\mathrm{Na}_{6}\left(\mathrm{NMe}_{2}\right)_{5}(\mathrm{OtBu}) \cdot(\text { tmeda })_{2}(\mathrm{tol})\right]_{2}(\mathbf{9})$.

The dimethylamide group at $\mathrm{N} 1$ is almost coplanar with the $[\mathrm{NaN}]_{2}$ fourmembered ring, so that an $\mathrm{sp}^{2}$ hybridisation at the nitrogen atom can be assumed. One lone pair resides accordingly in a $\mathrm{p}$ orbital pointing to $\mathrm{Na}^{\prime}$ and $\mathrm{Na3}$, while the second points towardds the inversion centre between $\mathrm{Na} 1$ and $\mathrm{Na1}{ }^{\prime}$. The same is also true for N2. Acute in-plane nitrogen bond angles (Na1N1-Na1': $\left.68.7(1)^{\circ}, \mathrm{Na2}-\mathrm{N} 2-\mathrm{Na3}: 71.4(1)^{\circ}\right)$ are the result of the diamondshaped four-membered rings, whereas those between the stacks are obtuse (Na3-N1-Na2': 154.3(1) $\left.{ }^{\circ}, \mathrm{Na}^{\prime}-\mathrm{N} 2-\mathrm{Na} 4: 149.7(1)^{\circ}\right)$. These rings are rotated by $90^{\circ}$ compared to the next stack. Differences between $\mathrm{N} 1$ and $\mathrm{N} 2$ can be quantified in the $\mathrm{Na}-\mathrm{N}$ bonds. The in-plane bonds $(2.570(2)$ and 2.603(1) $\AA$ ) of $\mathrm{N} 2$ are longer than those made up by the p orbital $(2.402(1)$ and $2.456(2) \AA)$. This is caused by the alkoxide anion, which is the better electron donor for $\mathrm{Na} 2$ 
and $\mathrm{Na3}$. Consequently, shorter N2-Na bonds to the remaining sodium atoms (Na1', Na4) are formed. Each alkoxide group coordinates three sodium cations and $\mathrm{Na}-\mathrm{O}$ bond lengths between 2.234(1) and 2.260(1) $\AA$ are evident. These are slightly smaller but still close to the range of reported $\mathrm{Na}-\mathrm{O}$ distances (2.27$2.42 \AA) .{ }^{[264,265]}$ The sodium-amide bonds are also of literature-known length. ${ }^{[238,261,266]}$

The transition from the cubane to the ladder structural motif is achieved by the missing fourth oxygen atom contact to a sodium cation (Figure 2-48). As a result, only three of four atoms in the ring have bonding partners in the next stack. This leads to a larger N3-Na4-N5 angle $\left(139.9(1)^{\circ}\right)$ in comparison to the related one two stacks further below (N1-Na1-N1': $\left.111.3(1)^{\circ}\right)$. The double ladder is composed of diamond shaped $[\mathrm{NaN}]_{2}$ rings too, so that the main building motif is continued.

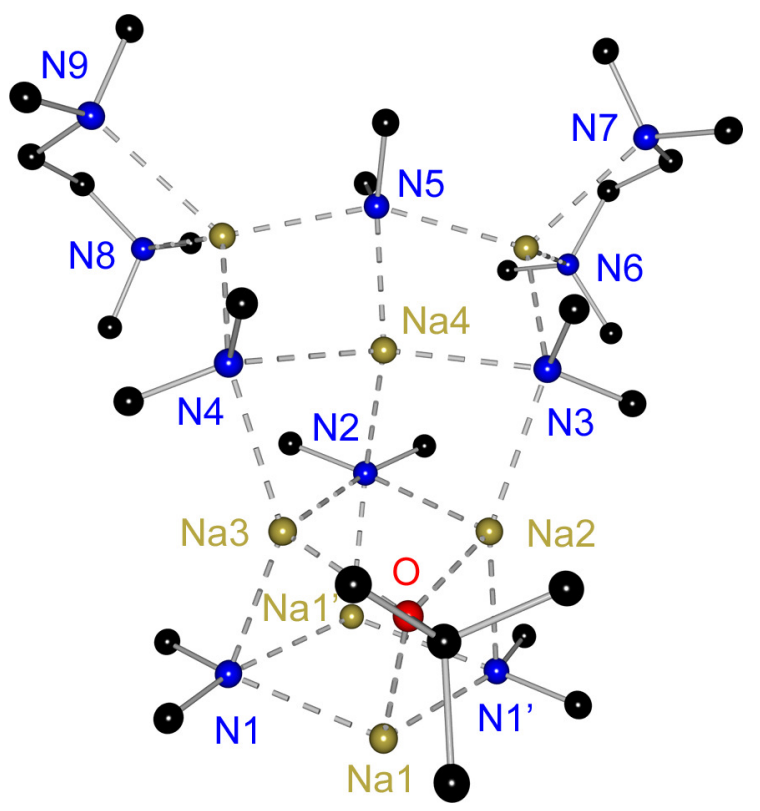

Figure 2-48. Ladder motif of $\left[\mathrm{Na}_{6}\left(\mathrm{NMe}_{2}\right)_{5}(\mathrm{OtBu}) \cdot(\text { tmeda })_{2}(\text { tol })\right]_{2}(\mathbf{9})$.

The amide groups are consequently only bonded to three cations, but still the contacts via the lone pair located in the p orbital seem to be favoured and only one interaction to a cation within the dimethylamide plane is present. Even the $\mathrm{sp}^{2}$ orbitals at N3 and N4 are not perfectly orientated towards the sodium ion. This is only the case for N5 that terminates the ladder and shows the shortest inplane $\mathrm{Na}-\mathrm{N}$ bond of $\mathbf{9}$ with $2.393(2) \AA$. The remaining bond lengths range between $2.422(2)$ and $2.469(2) \AA$. The ladder motif consequently leads to stronger $\mathrm{Na}-\mathrm{N}$ contacts than the cubane packing. The sodium atoms at the 
ladder termini ( $\mathrm{Na} 5, \mathrm{Na} 6)$ are each saturated by one tmeda donor molecule, with $\mathrm{Na}-\mathrm{N}$ bond lengths around $2.53 \AA$ and longer than those of the picolylsodium compounds reported in the previous chapter. This demonstrates the superior cation stabilising capabilities of the dimethylamide in comparison to the picolyl anion.

The isolation of the decomposition product of tmeda with benzylsodium 9 clearly indicates the reduced thermal stability of sodium organic intermediates and the challenging preparation requirements of heavy alkaline metal organic compounds. Nonetheless, picolyl as well as benzyl anions with sodium and potassium counterions are accessible by in situ deprotonation of the substrates with super bases. The products can then be filtered off from non-polar solvents and infinitely stored in the dry box if sealed in a Schlenk flask. 


\subsection{Unsubstituted 4-Picolyllithium}

The results in the field of unsubstituted 2-picolyl metal compounds encouraged the author to extend the work towards the deprotonation of 4-picoline. According to the reported proton acidities (cf. chapter 2.1), the metallation of the 4-isomer should be more facile. However, a strong tendency to form by-products was observed during the conversions with lithium bases. The first to report the exclusive addition of $n$-butyllithium to 4-picoline in a Chichibabin like reaction (cf. chapter 2.2.2) were H. Gilman and H. S. Broadbent in $1948 .{ }^{[267]} H$. Erlenmeyer et al. on the other hand observed a sequence of addition and deprotonation with phenyllithium on heating. ${ }^{[268]}$ He could identify (2-phenyl-4-pyridyl)methyllithium by a trapping reaction with an organic electrophile. These results were verified three years later in 1951 by . Osuch and R. Levine, who reported about $70 \%$ by-product formation during the metallation of 4-picoline with phenyllithium also including the addition of two equivalents of PhLi in 2-position. ${ }^{[269]}$ Recently, the intermediate of the $n B u L i$ addition to 4-picoline has been isolated (Figure 2-49: 2-n-Butyl-4-methyl-1,2-dihydropyridyllithium). ${ }^{[149]}$

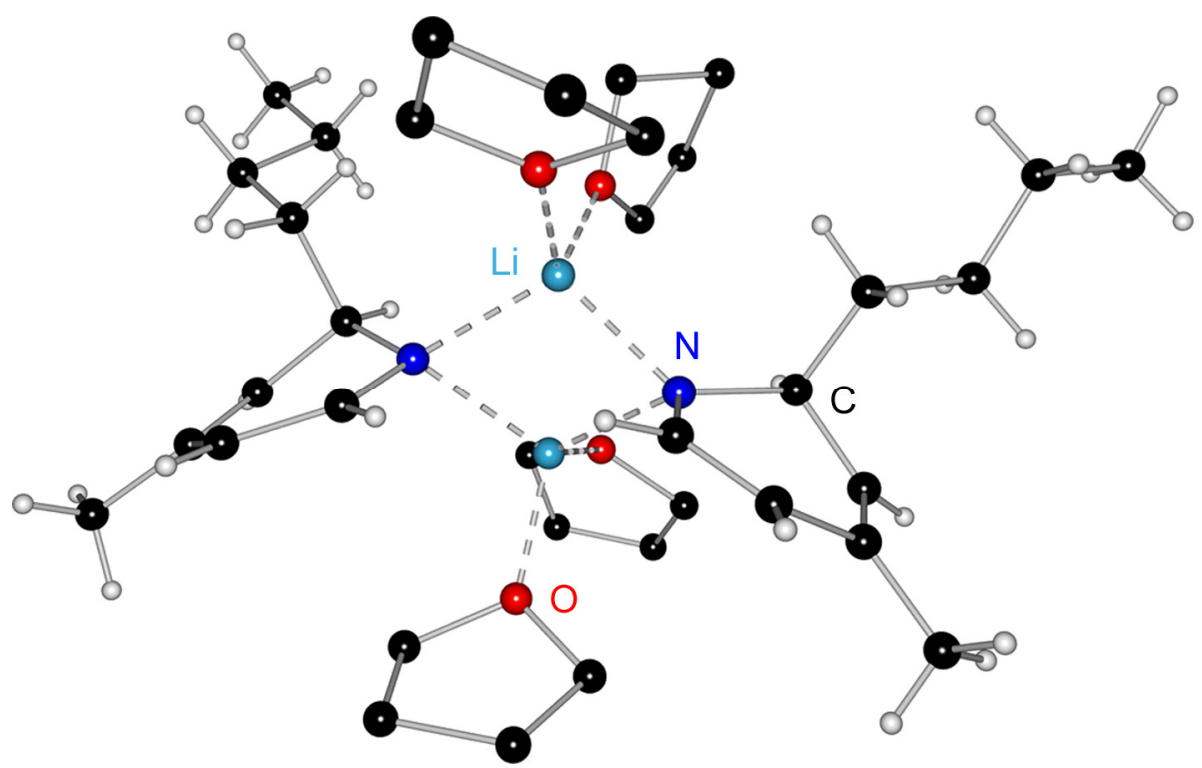

Figure 2-49. Addition product of the reaction of 4-picoline with $n B u L i$ at $-20^{\circ} \mathrm{C}$.

In contrast to $p K_{a}$ considerations, the competing nucleophilic addition of the metallation agent poses a serious challenge in the synthesis of 4-picolyllithium compared to the 2-isomer. ${ }^{[100]}$ Progress in the analysis of metallation mixtures was provided by a systematic NMR study carried out by E. M. Kaiser et al. ${ }^{[147]}$ Important information on the reaction conditions was gained by the quantifica- 
tion of both species (4-PicLi and dihydropyridyllithium) in solutions by varying the lithium organic compound or the solvent. As expect, an n-butyllithium 4-picoline mixture in thf yields about $70 \%$ addition product, whereas tert-butyllithium gives $80 \%$ lithiated picoline. In fact, the more basic and less nucleophilic $t B u L i$ was applied for the isolation of crystalline 4-picolyllithium (Figure 2-50). ${ }^{[149]}$ 4-Picoline needs to be present in excess to further activate the lithium base by disaggregation. Even though successful, the reaction conditions are drastic, so that the reaction solution cannot be cooled adequately even with a dry-ice bath.

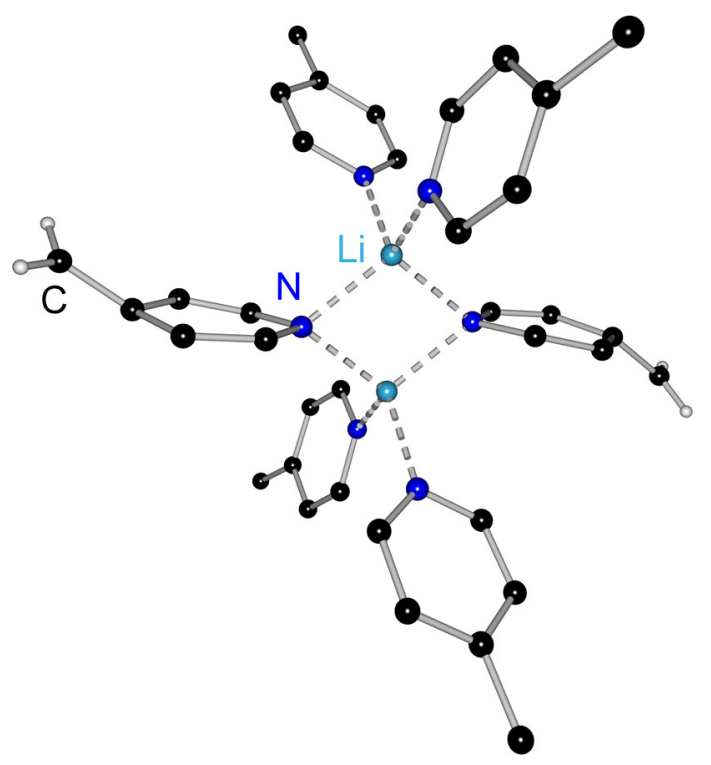

Figure 2-50. 4-Picolyllithium as 4-picoline adduct (constrained hydrogen atoms omitted for clarity).

Therefore, an alternative facile route to crystalline 4-picolyllithium had to be found, in order to extend the usability of this isomer. The results of E. M. Kaiser et al. suggested the deprotonation with methyllithium or lithium di-iso-propylamide, where almost complete conversions to 4-PicLi were observed. ${ }^{[147]}$ Hydrogen abstraction with $n B u L i$ on the other hand was only achieved with the carcinogenic donor base hexamethylphosphoramide that should be avoided in experimental laboratory work today. Not even an $n B u L i$ mixture with tmeda at RT was successful in deprotonating 4-picoline. According to these results, the reaction conditions were tuned to optimise the direct synthesis of 4-picolyllithium. 


\subsubsection{Dimeric Adducts}

The metallation of 4-picoline with methyllithium in thf successfully proceeded at $-78^{\circ} \mathrm{C}$. After covering the resulting red solution with a layer of pentane and storage at $4{ }^{\circ} \mathrm{C}$, reddish-brown crystals could be isolated. These consisted of the thf adduct of 4-picolyllithium (10), which crystallises in the centrosymmetric orthorhombic space group Pbca with one half of the dimer in the asymmetric unit. The dimerisation occurs via a central $[\mathrm{LiN}]_{2}$ ring in the shape of a parallelogram orthogonal to the picolyl ring planes (Figure 2-51). Two slightly different $\mathrm{Li}-\mathrm{N}$ bond lengths have been observed. Remarkably, the deprotonated carbon atom $\mathrm{C} 6$ has neither a direct contact to the intramolecular lithium atoms nor any possible attractive interaction to its next neighbours in the crystal. The nitrogen atoms are more attractive to the lithium cations, underlining the results of the charge density study on 2-picolyllithium concerning the donor strength and the reason for the dimerisation pattern (cf. chapter 2.3.7).

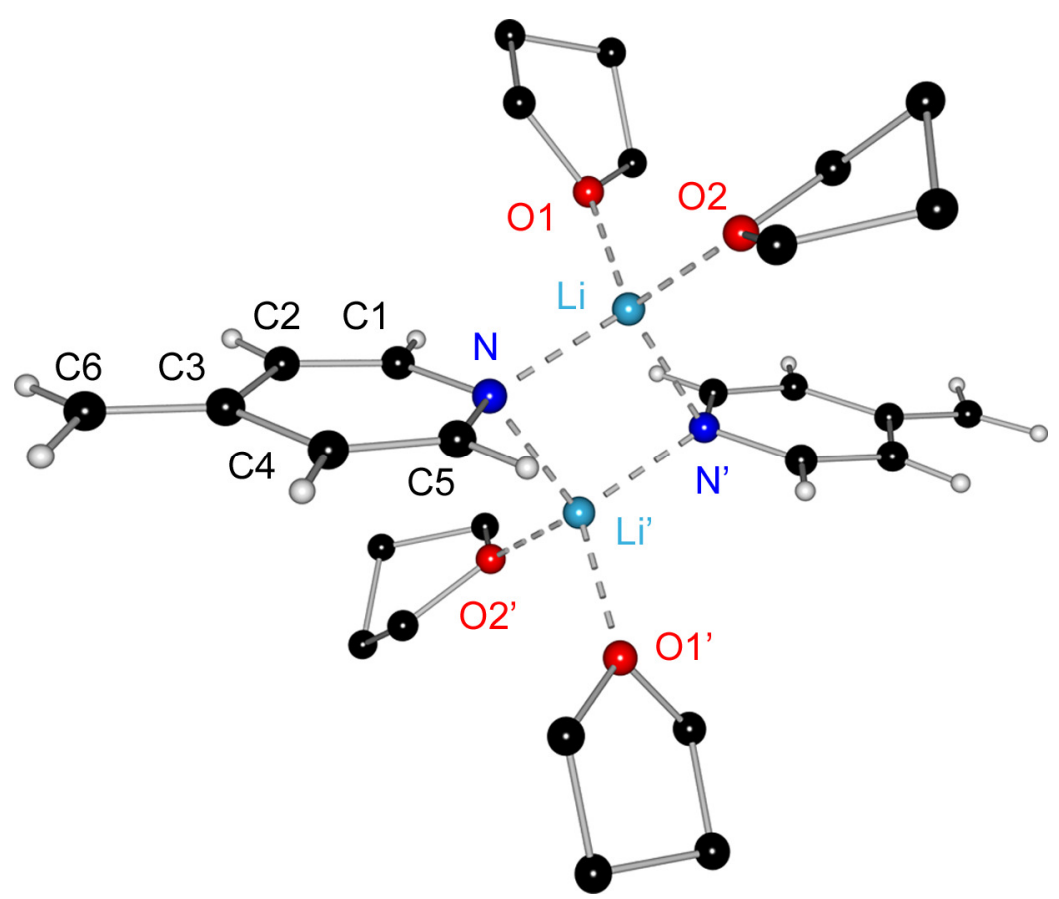

Figure 2-51. Molecular structure of $\left[4-\mathrm{PicLi} \cdot(\text { thf })_{2}\right]_{2}(\mathbf{1 0})$. Hydrogen atoms and disordered parts of the donor molecules are omitted for clarity.

The two planar picolyl rings (mean deviation from ideal plane $0.004 \AA$ ) are not coplanar but parallel with distance of the planes of $0.72 \AA$. The lithium atoms are positioned $0.90(\mathrm{Li})$ and $1.62 \AA\left(\mathrm{Li}^{\prime}\right)$ outside these planes resulting in $\mathrm{Li}-\mathrm{N}$ bond lengths of $2.047(2)$ and $2.095(2) \AA$. Those are comparable to lithium amide bonds showing this standard dimerisation pattern, even if they belong to the 
longer ones. ${ }^{[263]}$ However, the $\mathrm{Li}-\mathrm{N}-\mathrm{Li}^{\prime}$ bond angle at the pyridine nitrogen atom is markedly acute $\left(77.4(1)^{\circ}\right)$ in comparison to standard amides for which angles close to the tetrahedral one were reported. ${ }^{[263]}$ Thus, an $\mathrm{sp}^{3}$ hybridisation at N1 is disfavoured in $\mathbf{1 0}$ and the $\pi$-conjugation in the picolyl anion is maintained. Taking possible resonance formulae into account, the dimerisation pattern definitely points to the enamidic form with one lone pair within the ring plane in an $\mathrm{sp}^{2}$ orbital and the second one orthogonal to it (C in Scheme 2-15). This is also in compliance with the determined hydrogen atom positions at the deprotonated carbon atom $\mathrm{C} 6$. Both reside within the pyridine ring plane in a trigonal planar fashion $\left(\mathrm{H}-\mathrm{C} 6-\mathrm{H}\right.$ bond angle: $\left.121.7(1)^{\circ}\right)$. The interactions with the lithium cations are most likely charge-controlled as was theoretically shown for the 2-isomer (chapter 2.4).

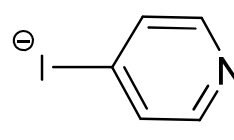

A

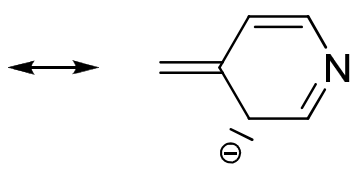

B

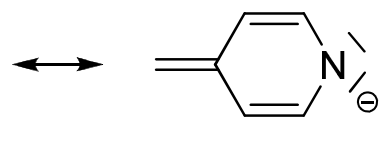

C

Scheme 2-15. Resonance formulae of the 4-picolyl anion.

The thf donor molecules bind more strongly to the lithium atoms than in the corresponding 2-picolyl analogue as seen from to the $\mathrm{Li}-\mathrm{O}$ bond lengths $(1.966(2) \text { and } 1.925(2) \AA \text { ). The differences to [2-PicLi-(thf) }]_{2}$ (av. Li-O distance: $1.978 \AA$ ) can be rationalised if aza-allylic contacts are considered. These stabilise the cation further so that the donor molecules are more loosely bound. Furthermore, the bond angles involving the lithium atoms in $\mathbf{1 0}$ show a slightly distorted tetrahedral coordination sphere commonly observed for these cations (Table 2-14).

The enamidic interactions via the pyridine nitrogen atoms also influence the bond lengths in the picolyl anion (Table 2-14). Crystalline 4-picoline can serve as external reference for bond length considerations. ${ }^{[270]}$ It shows an intramolecular crystallographic mirror plane through the nitrogen atom and the methyl carbon atom perpendicular to the pyridine ring so that only five independent nonhydrogen atoms are present in the asymmetric unit. The $\mathrm{N}-\mathrm{C}$ bonds $(1.344(3) \AA)$ are similar to those of 2-picoline, while the C-C bonds (1.390(4) and 1.392(2) $\AA$ ) show less alternation than in the related isomer. 
Table 2-14. Selected bond lengths $[\AA]$ and angles $\left[{ }^{\circ}\right]$ of $\left[\text { PicLi } \cdot(\text { thf })_{2}\right]_{2}(\mathbf{1 0}),\left[\right.$ PicLi $\cdot(\text { thf })_{2}$. 2-nBu-4-Me- $\left.\left(\mathrm{H}_{4} \mathrm{C}_{5} \mathrm{~N}\right) \mathrm{Li} \cdot(\text { thf })_{2}\right]$ (11) and related compounds (Pic =4-picolyl, $\mathrm{Py}=$ 4-pyridyl). ${ }^{[145,270]}$

\begin{tabular}{|c|c|c|c|c|}
\hline & $\begin{array}{c}{\left[\mathrm{PicLi} \cdot(\text { thf })_{2}\right]_{2}} \\
\quad(\mathbf{1 0})\end{array}$ & $\begin{array}{l}{[\text { MeCHPyLi } \cdot} \\
\text { tmeda }]_{2}\end{array}$ & $\begin{array}{l}{\left[\mathrm{PicLi} \cdot(\text { thf })_{2} \cdot 2-n \mathrm{Bu}-4-\mathrm{Me}-\right.} \\
\left.\left(\mathrm{H}_{4} \mathrm{C}_{5} \mathrm{~N}\right) \mathrm{Li} \cdot(\text { thf })_{2}\right](\mathbf{1 1})^{[\mathrm{a}]}\end{array}$ & $\mathrm{PicH}$ \\
\hline $\mathrm{Li}-\mathrm{N}$ & $2.047(2)$ & $2.096(9)$ & $2.080(4) / 2.025(4)$ & - \\
\hline$L i^{\prime}-N$ & $2.095(2)$ & $2.099(9)$ & $2.085(4) / 2.057(4)$ & - \\
\hline $\mathrm{Li}-\mathrm{D} 1^{[\mathrm{b}]}$ & $1.966(2)$ & $2.114(9)$ & $1.941(3) / 1.943(4)$ & - \\
\hline $\mathrm{Li}-\mathrm{D} 2^{[\mathrm{b}]}$ & $1.925(2)$ & $2.156(10)$ & $1.952(4) / 1.956(4)$ & - \\
\hline $\mathrm{N} 1-\mathrm{C} 1$ & $1.376(1)$ & $1.375(8)$ & $1.376(3) / 1.484(2)$ & $1.344(3)$ \\
\hline N1-C5 & $1.372(1)$ & $1.372(7)$ & $1.375(2) / 1.355(3)$ & $=\mathrm{N} 1-\mathrm{C} 1$ \\
\hline $\mathrm{C} 1-\mathrm{C} 2$ & $1.356(1)$ & $1.335(7)$ & $1.348(3) / 1.510(3)$ & $1.390(4)$ \\
\hline $\mathrm{C} 2-\mathrm{C} 3$ & $1.444(2)$ & $1.455(8)$ & $1.453(3) / 1.348(3)$ & $1.392(2)$ \\
\hline $\mathrm{C} 3-\mathrm{C} 4$ & $1.453(2)$ & $1.436(7)$ & $1.448(3) / 1.448(3)$ & $=\mathrm{C} 2-\mathrm{C} 3$ \\
\hline C4-C5 & $1.360(1)$ & $1.341(7)$ & $1.354(3) / 1.369(3)$ & $=\mathrm{C} 1-\mathrm{C} 2$ \\
\hline C3-C6 & $1.365(1)$ & $1.351(7)$ & $1.358(3) / 1.502(3)$ & $1.506(3)$ \\
\hline $\mathrm{Li}-\mathrm{N} 1-\mathrm{Li}^{\prime}$ & $77.4(1)$ & $76.4(3)$ & $74.3(1) / 76.1(1)$ & - \\
\hline N1-Li-N1' & $102.6(1)$ & $100.1(4)$ & $101.6(2) / 100.4(2)$ & - \\
\hline $\mathrm{D} 1-\mathrm{Li}-\mathrm{D} 2^{[\mathrm{b}]}$ & $102.8(1)$ & $87.9(3)$ & $104.0(2) / 102.9(2)$ & - \\
\hline $\mathrm{C} 1-\mathrm{N} 1-\mathrm{C} 5$ & $113.0(1)$ & $111.4(4)$ & $112.4(2) / 112.5(2)$ & $116.7(2)$ \\
\hline $\mathrm{C} 2-\mathrm{C} 3-\mathrm{C} 4$ & $112.1(1)$ & $111.0(4)$ & $111.7(2) / 118.3(2)$ & $117.0(2)$ \\
\hline
\end{tabular}

[a] Second values refer to corresponding bonds for the dihydropyridyl moiety. [b] $\mathrm{D}=$ donor atom.

The deprotonation of 4-picoline results in a symmetrical elongation of the $\mathrm{N}-\mathrm{C}$ bonds (1.376(1) and 1.372(1) $\AA$ ) and dramatic changes in the $\mathrm{C}-\mathrm{C}$ bonds. The presumed localised double bonds - according to resonance formula $\mathbf{C}$ in Scheme 2-15 - are shorter than those of the 2-isomers. Values between 1.356(1) and 1.365(1) $\AA$ for C1-C2, C4-C5 and the exocyclic methylene bond testify to the delocalisation of the negative charge generated at $\mathrm{C} 6$ to the pyridine nitrogen atom. It would be desirable to verify this by an experimental charge density study. However, no unsubstituted 4-picolyllithium compound known to date could be crystallised in sufficiently high quality.

While values close to standard double bond lengths are observed between the respective carbon atoms $\left(\mathrm{C}_{\mathrm{sp} 2}=\mathrm{C}_{\mathrm{sp} 2}: 1.335 \AA\right)$, the remaining carbon-carbon bonds $(1.444(2)$ and $1.453(2) \AA)$ show single bond character $\left(C_{\mathrm{sp} 2}-\mathrm{C}_{\mathrm{sp} 2}\right.$ : 
$1.466 \AA) \cdot{ }^{[152]}$ The deprotonation also leads to a ring deformation along the N $\cdots \mathrm{C} 3$ vector of the pyridine ring. Both bond angles - at the nitrogen atom and the carbon atom C3 - deviate significantly from the ideal $120^{\circ}$ and those observed for 4-picoline $\left(117^{\circ}\right)$. Angles between 112 and $113^{\circ}$ indicate an inclination of the opposing double bonds in the pyridine ring.

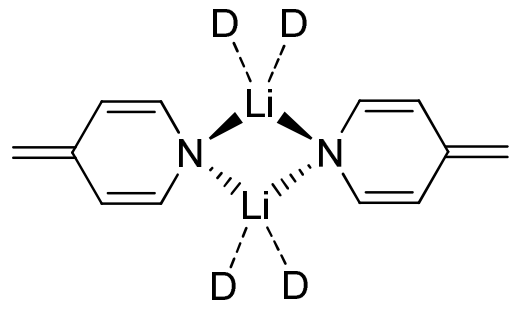

$[\text { PicLi-(thf) }]_{2}$ (10)

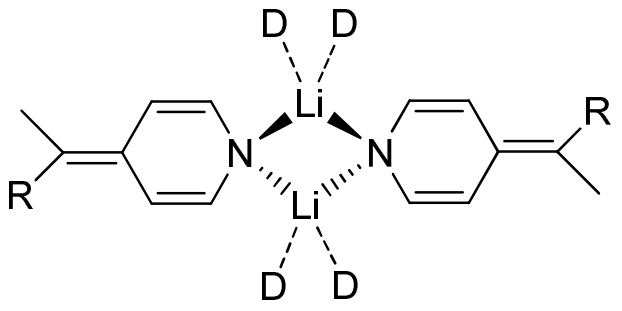

$\left.[\text { MeRCPyLi-tmeda/(thf })_{2}\right]_{2}$

Scheme 2-16. Lewis formulae of PicLi compounds $(R=H, M e ; D=$ tmeda or thf donor atom; Pic $=4$-picolyl, $\mathrm{Py}=4$-pyridyl).

The same phenomenon was reported for related aggregates of 4-picolyllithium derivatives (Scheme 2-16). ${ }^{[145,146]}$ They share an identical bond length scheme (Table 2-14) that was further studied by computational calculations. Interestingly, the results on isolated 4-picolyllithium moieties recognise the $\mathrm{Li}-\mathrm{N}$ interaction to be least stable in the series of possible coordination patterns. Almost equally in energy are the aggregates with a coordination via the allyl systems $\left(\eta^{3} ; \mathrm{C} 2-\mathrm{C} 3-\mathrm{C} 6\right.$ or $\left.\mathrm{C} 2-\mathrm{C} 3-\mathrm{C} 4\right)$, the pyridine ring $\left(\eta^{5} ; \mathrm{C} 2-\mathrm{C} 1-\mathrm{N}-\mathrm{C} 5-\mathrm{C} 4\right)$, and the methylene bond $\left(\eta^{2} ; \mathrm{C} 3-\mathrm{C} 6\right)$ to the lithium cation. Only a model for a dimer including lithium amide $\left(\mathrm{LiNH}_{2}\right)$ as second bonding partner reproduces the observed Li-N interactions ${ }^{[145]}$ and results in bond lengths exactly matching the standard carbon-carbon single and double bond lengths ${ }^{[152]}$ mentioned above. A perfect localisation of the conjugated system is hence observed in the gas phase.

It is evident that once again the nitrogen atom is the preferred coordination site for the lithium cations in the solid state and in solution, which was shown by E. Anders et al. ${ }^{[145]}$ This is also the reason for the addition of lithium nucleophiles in the vicinity of the nitrogen atom. 4-Picoline coordinates to the strong base (e.g. nBuLi) via the nitrogen atom and reduces the degree of aggregation of the lithium organic compound. Moreover, this directs the nucleophilic organic group of the base in the proximity of the 2-position of 4-picoline. The methyl hydrogen atoms on the other hand are located on the opposite side of the molecule. 
P. Thornton and K. J. Izod proclaimed this complex-induced proximity effect $\left(\mathrm{CIPE}^{[271]}\right.$ ) to explain the straight metallation of 2-pyridine. ${ }^{[272]}$

In contrast to the 2-isomer, it was experimentally proven that an alkylation at the nitrogen atom is achievable with 4-picolyllithium. ${ }^{[148,273]}$ Acid chlorides and even trimethylsilyl chloride yielded in an $\mathrm{N}-\mathrm{C}$ bond formation with lithium and sodium derivatives of the 4-picolyl anion. Nonetheless, the C-alkylation remains the standard reaction pathway of 4 -PicLi. ${ }^{[100,101]}$

In comparison to the 4-picolyllithium 4-picoline adduct (Figure 2-50) ${ }^{[149]}$ the methylene group is not bent away from the ring in $\mathbf{1 0}$. This points to a disorder effect in the previously reported structure. Unfortunately, no single crystals of improved quality could be obtained yet. However, some structural features shall be compared. The central four-membered ring is of a symmetrical diamond shape with an $\mathrm{Li}-\mathrm{N}$ bond length of $2.077(4) \AA$, which is close to the mean value $(2.071 \AA)$ in 10. The localised double bond lengths (1.346(3) $\AA$ ) are also well in the range of the thf adduct and so are the single bonds (1.446(4) and $1.462(4) \AA)$.

Alternatively to the synthesis with methyllithium, it was attempted to metallate 4-picoline with $n$-butyllithium at low temperatures $\left(-78{ }^{\circ} \mathrm{C}\right)$ to avoid the addition reaction, which is favoured at RT. However, NMR spectra of the crystalline material from the thf solution proved the coexistence of both species with a preference to the addition product ( $63 \%)$. Accordingly, a unit cell determination of the single crystals showed the characteristic values for the 2-n-butyl-4-methyl-1,2-dihydropyridyllithium thf dimer (Figure 2-49). ${ }^{[149]}$ Yet, a second batch of crystals slightly differing in colour and shape were detected under the microscope. These consisted of a fascinating hybrid (Scheme 2-17) between both products being the linker between the metallated compound $\mathbf{1 0}$ and the dihydropyridyllithium dimer.

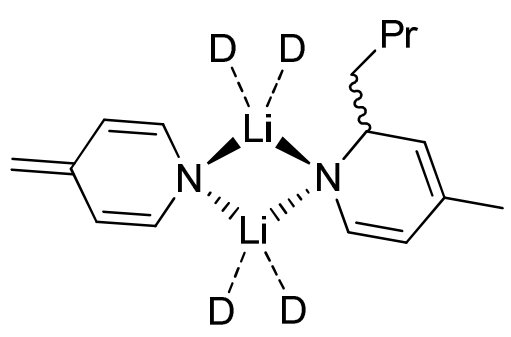

Scheme 2-17. Lewis formula of the mixed 4-picolyllithium 2-n-butyl-4-methyl1,2-dihydropyridyllithium dimer (11, D = thf). 
The asymmetric unit contains the full dimer [PicLi-(thf) $)_{2} \cdot 2-n \mathrm{Bu}-4-\mathrm{Me}-$ $\left.\left(\mathrm{H}_{4} \mathrm{C}_{5} \mathrm{~N}\right) \mathrm{Li} \cdot(\text { thf })_{2}\right]$ (11) and shows a central $[\mathrm{LiN}]_{2}$ four-membered ring (Figure

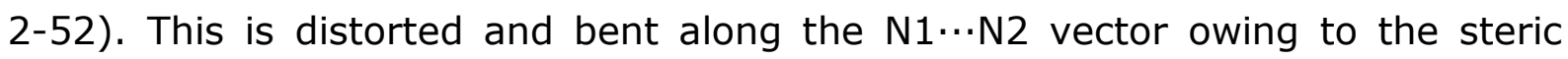
demand of the $n$-butyl side chain. The angle of inclination along the $\mathrm{N} \cdots \mathrm{N}$ vector is $154^{\circ}$. The two rings are tilted relative to this vector. The picolyl ring axis (N1 ‥C3) encloses an angle of $161^{\circ}$ with the vector, but the C1-N1-C5 plane points almost perfectly into the angle bisection of the $[\mathrm{LiN}]_{2}$ ring. The same is true for the dihydropyridyl ring (C7-N2-C11) but an even smaller angle to the $\mathrm{N} \cdots \mathrm{N}$ vector is observed $\left(144^{\circ}\right)$. Furthermore, the ring $\mathrm{N} \cdots \mathrm{C}_{\text {para }}$ vectors point to opposite sides of the central four-membered lithium nitrogen ring (trans arrangement, Figure 2-53).

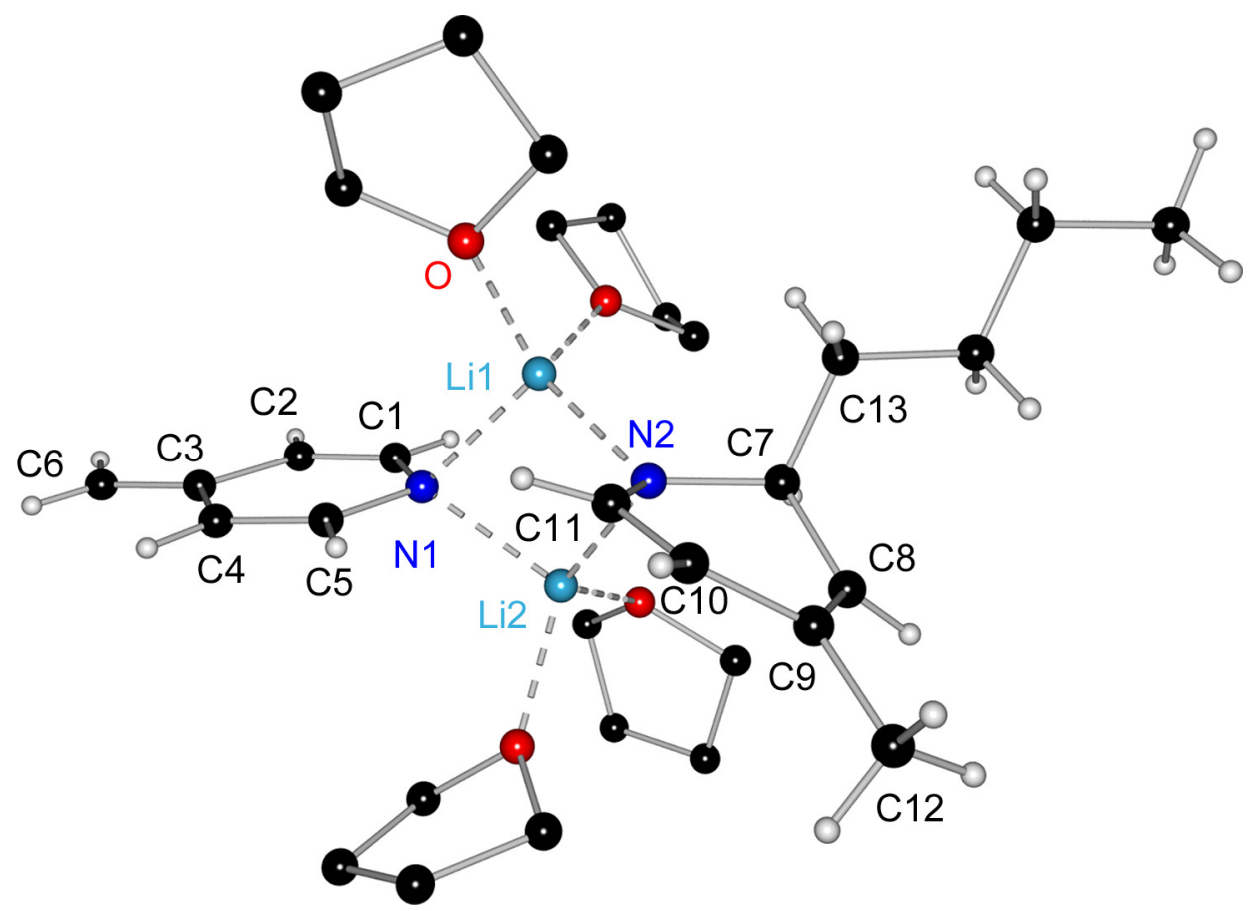

Figure 2-52. Molecular structure of [PicLi $\left.(\text { thf })_{2} \cdot 2-n \mathrm{Bu}-4-\mathrm{Me}-\left(\mathrm{H}_{4} \mathrm{C}_{5} \mathrm{~N}\right) \mathrm{Li} \cdot(\text { thf })_{2}\right](\mathbf{1 1})$. Hydrogen atoms and disordered parts of the donor molecules are omitted for clarity.

Each lithium atom is coordinated by both nitrogen atoms and two thf oxygen atoms in a slightly distorted tetrahedral assembly. Short $\mathrm{Li}-\mathrm{N}$ bonds are observed to the nitrogen atom N2 of the dihydropyridyl ring (2.025(4) and 2.057(4) $\AA$ ). The Li-N1 bond lengths are slightly larger with 2.080(4) and 2.085(4) $\AA$ due to the different electronic conditions in the rings. The anion is a perfectly resonance stabilised enamide originating from a carbanion, while N2 is unambiguously an amide that is far less stabilised because of the destruction of the delocalised system at the $\mathrm{sp}^{3}$ hybridised carbon atom $\mathrm{C7}$ (sum of the non- 
hydrogen bond angles: $\left.333.3^{\circ}\right)$. Yet again, the $\mathrm{Li}-\mathrm{N}-\mathrm{Li}$ bond angles of $74.3(1)^{\circ}$ or $76.1(1)^{\circ}$ (N2) are far from the ideal tetrahedron angle. This means that both nitrogen interactions to the cations hardly differ and an $\mathrm{sp}^{3}$ character of the two formal lone pairs can be most probably excluded.

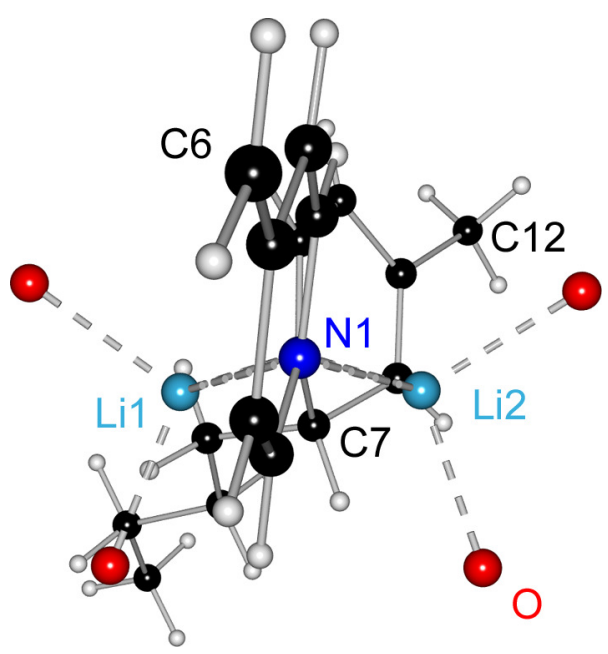

Figure 2-53. View along the N1 $\cdots \mathrm{N} 2$ vector of $\left[\mathrm{PicLi} \cdot(\text { thf })_{2} \cdot 2-n \mathrm{Bu}-4-\mathrm{Me}-\left(\mathrm{H}_{4} \mathrm{C}_{5} \mathrm{~N}\right) \mathrm{Li} \cdot(\text { thf })_{2}\right]$ (11; carbon atoms of thf molecules are omitted for clarity).

The picolyl anion is perfectly planar (mean deviation from ideal plane: $0.004 \AA$ ) and shows almost identical bond lengths compared to the pure 4-picolyllithium compound 10, while the second ring is profoundly deformed. The ring atoms of the $\mathrm{C}-\mathrm{N}-\mathrm{C}$ moiety reside $0.18 \AA$ (C7) to $0.56 \AA$ (N2) outside the planar $\mathrm{C} 8-\mathrm{C} 9(-\mathrm{C} 12)-\mathrm{C} 10$ fragment (mean deviation: $0.01 \AA$ ), while also the N2C11-C10-C9-C8 chain (mean deviation: $0.04 \AA$ ) is almost planar with the carbon atoms $\mathrm{C} 7(0.55 \AA)$ and $\mathrm{C} 12(0.46 \AA)$ positioned outside that plane (Figure 2-52). This is caused by the incorporation of the $\mathrm{sp}^{3}$ hybridised ipso carbon atom C7 into the former pyridine ring. This carbon atom represents a stereogenic centre, but the substance crystallises in the centrosymmetric space group Pbca as racemate. $U$. Pieper added in a related reaction $n$-butyllithium to unsubstituted pyridine and obtained enantiomerically pure crystals from the reaction mixture. ${ }^{[107]}$ This could neither be achieved with $\mathbf{1 1}$ nor with the associated pure dihydropyridine adduct (Figure 2-49).

Interestingly, the bonds between $\mathrm{N} 1, \mathrm{C} 11, \mathrm{C} 10$, and $\mathrm{C} 9$ deviate only little from those of the anion (Table 2-14). In that view, a conjugated $\pi$-system can be assumed (Scheme 2-17), showing alternating single and double bonds. This is even extended towards C8 (C8-C9: 1.348(3) $\AA$ ). 
However, it has to be concluded that a lowering of the reaction temperature did not avoid the side-product formation, even if a higher percentage of metallated product could be obtained compared to previously reported results. ${ }^{[147]}$.

\subsubsection{4-Picolyllithium Monomer and Polymer}

The metallation of 4-picoline with methyllithium in thf proceeds smoothly as was shown in the preceding chapter. The same happens in the presence of the tridentate donor base pmdeta. Already during the addition of the lithium base to the reaction mixture an orange solid starts to precipitate. After removal of all volatiles, the orange-red solid is redissolved in thf and layered with hexane. Purple crystals grew from that solution at $4^{\circ} \mathrm{C}$. The crystal mounting had to be carried out very quickly to prevent crystal decomposition. The molecular structure explains the high sensitivity to air and moisture of the crystals afterwards. Monomeric 4-picolyllithium as pmdeta adduct $\mathbf{1 2}$, which is more reactive than the dimers, has been isolated under these reaction conditions (Figure 2-54).

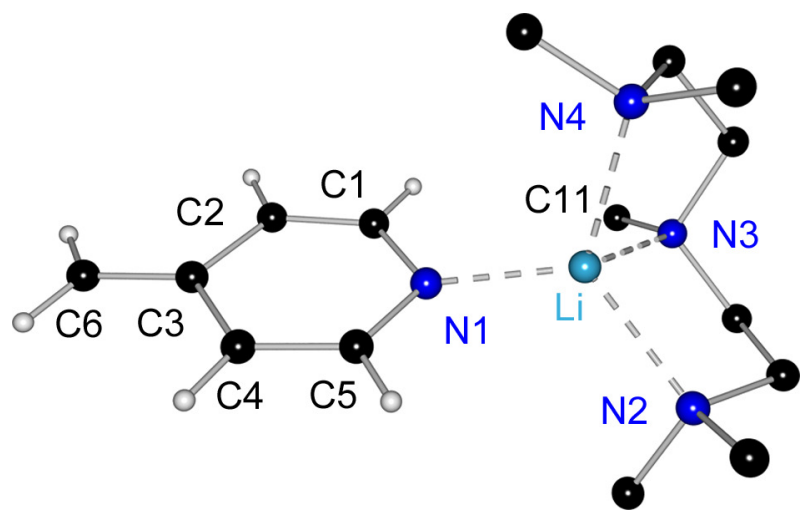

Figure 2-54. Molecular structure representation of [4-PicLi·pmdeta] (12). Hydrogen atoms of the donor molecule are omitted for clarity.

The lithium atom resides in the donor atom cavity of the pmdeta ligand and is tetracoordinated as in the corresponding 2-picolyllithium compound $\mathbf{3}$. Yet, the reduced steric demand of the 4-isomer allows a tighter coordination of the cation. This is evident by the short donor bonds that are almost equal in length (2.099(2) to 2.107(2) $\AA$ ) and a wider $\mathrm{N}-\mathrm{Li}-\mathrm{N}$ bond angles of neighbouring donor atoms compared to the 2-isomer. This is accompanied by the shortest $\mathrm{Li}-\mathrm{N}$ bond of all presented 4-picolyllithium compounds with 1.966(2) $\AA$ (Table 2-15). However, the coordination to the cation is again not achieved within the planar picolyl 
ring (mean deviation from ideal plane: $0.012 \AA$ ). The lithium atom resides $0.3 \AA$ outside the ring plane and an angle of $170.2^{\circ}$ is enclosed with the Li-N1 bond. The reason for that is unknown, but might be caused by steric interference with the methyl groups of the donor. A relatively small N1-Li-N3-C11 dihedral angle of $4.7(2)^{\circ}$ shows that the Li-N1 bond points to the angle bisection of the open pmdeta face (cf. chapter 2.2.3). The orientation of the picolyl ring to that open donor side is rotated about $37.5(2)^{\circ}$ (N3-Li-N1-C1 dihedral angle). As for the 2-picolyl isomer 3, a ring orientation with the least steric interplay with the donor is achieved in 12. Crystal packing effects do not seem to contribute to the ring arrangement but cannot ultimately be ruled out. Within the picolyl ring all important bond lengths and angles are in good agreement with the thf adduct 10. Only the $\mathrm{N}-\mathrm{C}$ bonds are shorter in the monomer (1.360 $\AA$ vs. $1.374 \AA$; Table 2-15), which is most probably an effect of the coordination to two lithium atoms in the dimer. A clear enamidic resonance structure is adopted ( $\mathbf{C}$ in Scheme 2-15).

Table 2-15. Selected bond lengths $[\AA]$ and angles $\left[{ }^{\circ}\right]$ of [PicLi.pmdeta] (12), $[\text { PicLi-tmeda }]_{\mathrm{n}}(\mathbf{1 3})$ and related adducts (Pic $=4$-picolyl, Py $=4$-pyridyl). ${ }^{[143]}$

\begin{tabular}{|c|c|c|c|c|}
\hline & $\begin{array}{c}\text { [PicLi·pmdeta] } \\
(\mathbf{1 2})\end{array}$ & $\begin{array}{c}{[\text { PicLi } \cdot \text { tmeda }]_{\mathrm{n}}} \\
(\mathbf{1 3})\end{array}$ & $\begin{array}{c}{\left[\mathrm{PicLi} \cdot(\text { thf })_{2}\right]_{2}} \\
(\mathbf{1 0})\end{array}$ & $\begin{array}{c}\text { [MeCHPyLi } \\
\text { tmeda }]_{2}\end{array}$ \\
\hline $\mathrm{Li}-\mathrm{N}$ & $1.966(2)$ & $2.012(11)$ & $2.047(2)$ & $2.096(9)$ \\
\hline Li'-N & - & - & $2.095(2)$ & $2.099(9)$ \\
\hline $\mathrm{Li}-\mathrm{D} 1^{[\mathrm{a}]}$ & $2.105(2)$ & $2.132(10)$ & $1.966(2)$ & $2.114(9)$ \\
\hline $\mathrm{Li}-\mathrm{D} 2^{[\mathrm{a}]}$ & $2.099(2)$ & $2.182(11)$ & $1.925(2)$ & $2.156(10)$ \\
\hline $\mathrm{Li}-\mathrm{D} 3^{[\mathrm{a}]}$ & $2.107(2)$ & - & - & - \\
\hline $\mathrm{N} 1-\mathrm{C} 1$ & $1.359(2)$ & $1.346(8)$ & $1.376(1)$ & $1.375(8)$ \\
\hline $\mathrm{N} 1-\mathrm{C} 5$ & $1.361(2)$ & $1.374(8)$ & $1.372(1)$ & $1.372(7)$ \\
\hline $\mathrm{C} 1-\mathrm{C} 2$ & $1.362(2)$ & $1.355(8)$ & $1.356(1)$ & $1.335(7)$ \\
\hline $\mathrm{C} 2-\mathrm{C} 3$ & $1.441(2)$ & $1.447(8)$ & $1.444(2)$ & $1.455(8)$ \\
\hline $\mathrm{C} 3-\mathrm{C} 4$ & $1.438(2)$ & $1.438(9)$ & $1.453(2)$ & $1.436(7)$ \\
\hline $\mathrm{C} 4-\mathrm{C} 5$ & $1.363(2)$ & $1.353(8)$ & $1.360(1)$ & $1.341(7)$ \\
\hline C3-C6 & $1.370(2)$ & $1.373(9)$ & $1.365(1)$ & $1.351(7)$ \\
\hline $\mathrm{D}-\mathrm{Li}-\mathrm{D}^{[\mathrm{b}]}$ & $86.5(1) / 88.5(1)$ & $85.3(4)$ & $102.8(1)$ & $87.9(3)$ \\
\hline $\mathrm{C} 1-\mathrm{N} 1-\mathrm{C} 5$ & $112.9(1)$ & $112.3(5)$ & $113.0(1)$ & $111.4(4)$ \\
\hline $\mathrm{C} 2-\mathrm{C} 3-\mathrm{C} 4$ & $112.0(1)$ & $111.8(5)$ & $112.1(1)$ & $111.0(4)$ \\
\hline
\end{tabular}

[a] $D=$ donor atom. [b] $D=$ neighbouring donor atoms. 
Due to the low solubility of the thf and pmdeta adduct in benzene their NMR spectra were recorded in $\mathrm{d}_{8}$-thf. Interestingly, both compounds show identical ${ }^{1} \mathrm{H}$ and ${ }^{13} \mathrm{C}$ spectra for the picolyl moiety. Hence, the surplus of oxygen donor molecules displaces the chelating pmdeta donor. This is also reflected by the proton signals of the tridentate ligand that indicate no coordination to the cation. The terminal methyl groups normally resonate further downfield in the case of a donation compared to the methylene bridge hydrogen atoms, which is not valid for 12. Even at low temperatures down to $-75^{\circ} \mathrm{C}$, the pmdeta molecule is not involved in the complexation of the cation. Furthermore, the ring proton signals are not changed by lowering the temperature. No species interchange is consequently detectable, and a 4-PicLi monomer with three thf molecules coordinating to the cation can be assumed that might be further stabilised by a second and third solvation shell.

The deprotonation of 4-picoline with neat $n$-butyllithium at reduced temperatures did not result in a pure product, as shown above. The use of tmeda during the metallation on the other hand is intended to increase the basicity and repel the addition in the 2-position. The lithium base is then pre-complexed by the chelating ligand and the direction of the butyl chain to the picolyl nitrogen atom might be prevented (cf. chapter 2.7.1). Accordingly, two equivalents of tmeda were added to favour the complexation of the lithium cations by the donor base and $n B u L i$ was added very slowly. The use of a large excess of tmeda was avoided because it could hamper the subsequent crystallisation of the product. Moreover, hexane was used as a non-polar solvent to support the precipitation of 4-picolyllithium as its tmeda adduct. This was indeed observed during the addition of $n B u L i$. A yellow solid has been obtained from the solution, which was filtered off and redissolved in thf. After a few days at $-24{ }^{\circ} \mathrm{C}$, red crystals of minor quality had formed. However, attempts to improve the crystallisation failed and only a low-quality X-ray diffraction data set could be measured so far. Criteria for the model accuracy did not fulfil standard requirements $(R 1=$ $0.1135 ; w R 2=0.2285$ ) due to the weak diffraction power of the crystals. Therefore, high and suspiciously oriented ADPs (cf. chapter 8.4.11) are observed in the model and the data quality was also not sufficient to resolve a possible disorder of the tmeda molecule. Nevertheless, the connectivity is resolved and at least trends for the bond lengths will be discussed in the following paragraph.

In contrast to all reported 4-picolyllithium compounds, the tmeda adduct did not form enclosed dimers but the chain structure shown in Figure 2-55. The 
chains are generated by the $c$ glide plane operation of the monoclinic space group $P Z_{1} / C$ passing almost through the $\mathrm{N} 1 \cdots \mathrm{C} 4$ vector. This results in picolyl ring planes in an almost parallel arrangement (ring plane angles $3^{\circ}$ ) with an average plane distance of $2.18 \AA$. The rings are rotated by $64^{\circ}$ relative to each other in respect of the plane normal vectors.

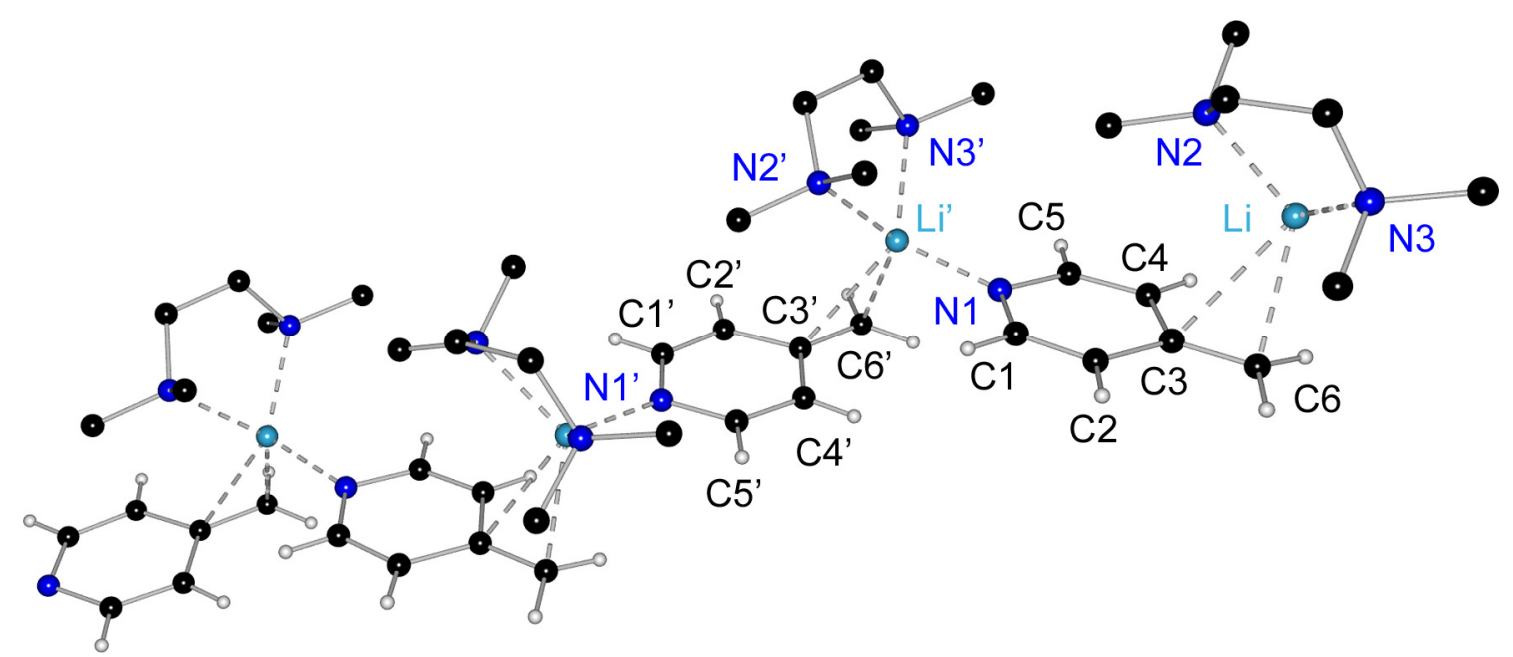

Figure 2-55. Polymeric chain structure of [4-PicLi.tmeda $]_{n}(\mathbf{1 3})$ showing four formula units. Hydrogen atoms of the donor molecules are omitted for clarity.

The polymeric chain is created via two different lithium interactions of the picolyl anion. The nitrogen atom of the planar ring (mean deviation from ideal plane: $0.006 \AA$ ) bonds to one cation in a $\sigma$-donor fashion already observed for the pmdeta monomer. Yet again, the coordination is not made up within the ring plane (deviation from plane: $0.23 \AA$ ), which encloses an angle of $171.2^{\circ}$ with the $\mathrm{Li}^{\prime}-\mathrm{N} 1$ bond vector. The $\mathrm{Li}^{\prime}-\mathrm{N}$ bond length (2.01(1) $\AA$ ) lies between those of the monomer and the dimers (Table 2-15).

The C3-C6 methylene bond interacts via the $\pi$-electron density of the localised double bond with the lithium atom (Scheme 2-18). It can be speculated about whether the contacts are of $\eta^{1}$ - or $\eta^{2}$-type. The shorter $\mathrm{Li}-\mathrm{C}$ contact is observed

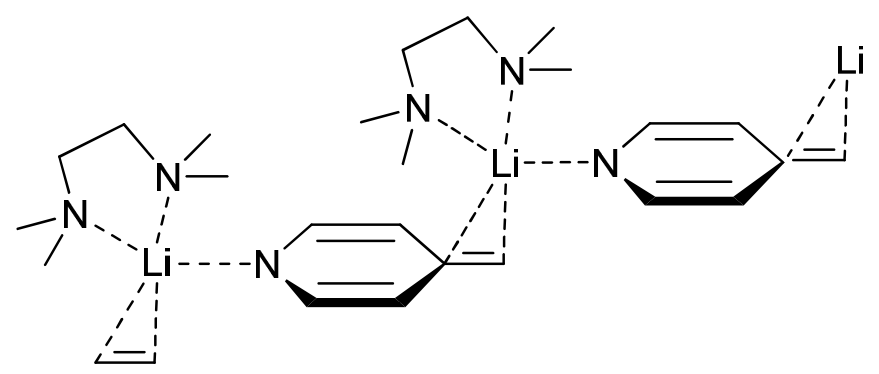

Scheme 2-18. Schematic representation of the polymeric chain in [4-PicLi-tmeda] $(13)$. 
to the deprotonated carbon atom C6 (2.40(1) $\AA$ ), while Li-C3 $(2.73(2) \AA)$ is in the range of the very weak interactions discussed in chapter 2.2.2. Also the position of the cation relative to the methylene bond favours a description of the bonding situation as $\eta^{1}$-carbanion. The distance of Li from the picolyl plane is $2.27 \AA$ so that the cation does not reside perfectly beneath C6 (Li-C6 distance is $2.40(1) \AA)$, which would result in the best overlap with its $\pi$-orbital. However, the systematic studies of picolyllithium compounds repeatedly showed that bonding to the alkaline metal atoms are only to a minor extent controlled by orbital interactions. A deviation from the ideal position normally leads only to a marginal increase in energy (cf. chapter 2.4).

The position of $\mathrm{Li}$ can be more precisely specified by the inspection of the asymmetric unit along the $\mathrm{C} 2 \cdots \mathrm{C} 4$ (or $\mathrm{C} 1 \cdots \mathrm{C} 5$ ) and the $\mathrm{C} 6-\mathrm{C} 3 \cdots \mathrm{N} 1$ vector (Figure 2-56), as seen from the side on the short and long diameter of the picolyl fragment. The view along $\mathrm{C} 2 \cdots \mathrm{C} 4$ shows that the lithium atom is positioned almost in a right angle to the ring (angle between the Li-C6 projection and the plane: $\sim 88^{\circ}$; Figure 2-56, left).
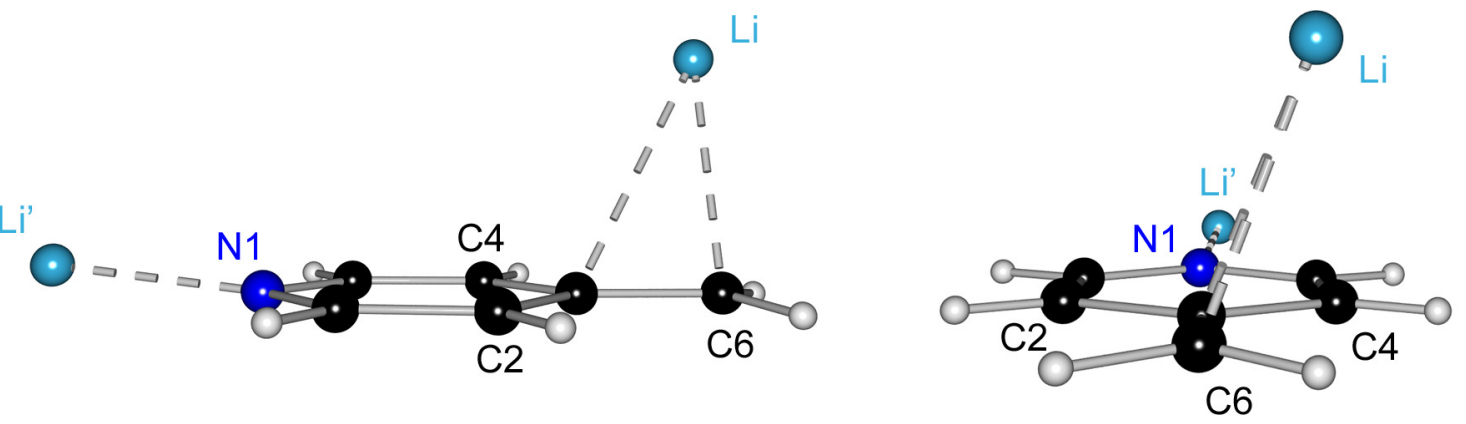

Figure 2-56. Side views on the picolyl anion in $\mathbf{1 3}$ to visualise the position of the lithium cation relative to the methylene bond.

A deviation from the ideal position is also observed in the second view. This time an $\mathrm{Li}-\mathrm{C} 6-\mathrm{C} 3$ angle of $\sim 70^{\circ}$ is enclosed (Figure 2-56, right). This is caused by the additional coordination of the tmeda donor to the lithium cation, involving an $\mathrm{N} 1^{\prime}-\mathrm{Li}-\mathrm{C} 6$ bond angle of $103.7(4)^{\circ}$. The coordination sphere of the metal atom can be characterised as distorted tetrahedrally due to the known acute bite angle of the tmeda donor $\left(85.3(4)^{\circ}\right)$.

The bond lengths within the picolyl anion show the known features of localised bonds and values in the expected range (Table 2-15). The effect of the $\eta^{1}$-carbanionic coordination to the cation cannot be unambiguously determined due to the low data quality. However, the C3-C6 bond length is equal in length compared to the monomer $\mathbf{1 2}$ within the standard deviations. 
Nonetheless, different crystallisation conditions have to be found in order to permit a detailed bond analysis of the 4-picolyllithium tmeda polymer. It might also be an interesting task to reproduce the observed coordination pattern by a donor exchange reaction starting from the crystalline thf adduct $\mathbf{1 0}$. Alternatively, this might result in the formation of dimers as reported for substituted picolyllithium compounds. ${ }^{[145]}$ Exactly this substitution might prevent the creation of a related polymeric chain due to the interference of the sterically demanding groups with the next picolyl ring (projection of the $\mathrm{C}^{\prime}-\mathrm{H} 6^{\prime}$ bond onto the next ring is almost parallel to the $\mathrm{C} 4-\mathrm{C} 5$ bond in 13).

Remarkably, the tmeda adduct exhibits significantly different chemical shifts compared to those measured in thf solutions. In contrast to other unsubstituted 4-PicLi compounds, $\mathbf{1 3}$ is only slightly soluble in deuterated benzene, but the tmeda signals proved the coordination of lithium. The methylene protons are still chemically equivalent but shifted further downfield by $1.2 \mathrm{ppm}$ in comparison to their analogues. Furthermore, also the meta hydrogen atoms are shifted in the same direction $(0.7 \mathrm{ppm})$, while the hydrogen atoms next to the heteroatom are barely influenced $(0.1 \mathrm{ppm})$. Charge density seems to be redistributed from the deprotonated carbon atom into the ring, which is also reflected by the downfield shift of the corresponding ${ }^{13} \mathrm{C}$ signal $(7 \mathrm{ppm})$. The chain structure is abandoned in solution and the coordination of the cations by $\mathrm{C} 6$ is no longer present.

The results in this and the preceding chapter show that side-product formation can be reduced by lowering the reaction temperature, by the addition of chelating donor molecules, and by the use of methyllithium instead of $n$-butyllithium. Even though the variations of the reaction conditions does not completely exclude the addition of the nucleophilic base to the pyridine ring, ensuing crystallisation of defined adducts assures the isolation of the pure 4-picolyllithium compounds, if the by-product yield is below approximately $30 \%$ (otherwise a mixed adducts like $\mathbf{1 1}$ might co-crystallise). 


\subsection{Conclusion}

The synthesis of three unsubstituted 2-picolyllithium adducts (dme, tmeda, pmdeta) completed the series of lead structural motifs in these metal organic compounds. The main coordination modes are $\eta^{1}$-alkyl, $\eta^{1}$-enamidic, and $\eta^{3}$-azaallylic. It was also possible to form monomers with the tridentate pmdeta donor to maximise the reactivity of the anion.

[2-PicLi.PicH $]_{2}$ (4) showing an aza-allylic coordination mode, was chosen for an experimental charge density study, which elucidated the preferred bonding interactions of the picolyl nitrogen atoms with the cations and the redistribution of the negative charge into the pyridine ring. The anion is accordingly better described as enamide rather than as carbanion. Furthermore, the spatial distribution of the electrostatic potential illustrated, why electrophiles are generally added to the side chain.

With the complete pool of 2-PicLi adducts at hand, their coordination modes could be understood in dependence of the applied donors or additional substituents, which was further supported by the charge density results of 4 and theoretical calculations on 2-PicLi reported before.

The 2-picolyllithium solid state structures are well established by now and their behaviour in solution was investigated by several NMR experiments, but the aggregation state and coordination mode to the cation could not be ultimately understood yet.

Furthermore, the first unsubstituted 2-picolyl anion with a heavier alkaline metal counterion was isolated as crystalline material and investigated by $\mathrm{X}$-ray diffraction. According to the known reactivity enhancement of the lithium homologues, it is possible to tune the reactivity of the organic picolyl group by the right choice of the cation and the donor base.

The work was also extended to 4-picolyllithium compounds that are far less applied in synthesis compared to the 2-isomer. Applicable synthetic routes to crystalline material were presented that also showed a preferred Li-N coordination in the solid state. A complete delocalisation of the negative charge into the ring has been observed with one exception: the polymeric 4-PicLi tmeda complex. 



\section{TRIMETHYLSILYLMETHYLLITHIUM}

\subsection{Introduction}

Trimethylsilylmethyllithium $\left(\mathrm{TMSCH}_{2} \mathrm{Li}\right)$ is the sila-analogue of neo-pentyllithium, in which the quaternary carbon atom is replaced by a silicon atom. It is synthesised by the metallation of trimethylsilylmethylchloride with elementary lithium $^{[274]}$ or by the deprotonation of tetramethylsilane with a $n$-butyllithium tmeda mixture. ${ }^{[275]}$ This compound is another excellent example of the enhanced reactivity of lithium organic compounds in low aggregation states already addressed in chapter 1 . Recently, $\mathrm{TMSCH}_{2} \mathrm{Li}$ was also included into the product portfolio of several chemical distributors. This supports the wide use of $\mathrm{TMSCH}_{2} \mathrm{Li}$, whose application potential is generally the same as those of common standard lithium organic compounds (cf. chapter 1 ). Therefore, only a few recent examples in synthesis or special applications shall be mentioned subsequently.

P. C. Gros et al. discovered in 2006 that a mixture of $\mathrm{TMSCH}_{2} \mathrm{Li}$ and $\mathrm{LiDMAE}$ (lithium dimethylaminoethoxide) reacted highly chemo- and regioselective in the ortho-lithiation of chloro- and fluoropyridine isomers (Scheme 3-1, A+B). ${ }^{[276]}$ The deprotonation always occurred in the vicinity of the ring nitrogen atom without the normally observed side-reactions. These are nucleophilic additions of the alkyl anion and metal-halide exchange reactions. Additionally, the reaction temperature could be raised from dry ice $\left(-78^{\circ} \mathrm{C}\right)$ to room temperature improving the preparation conditions. The same mixture gave also excellent results in the selective C3 functionalisation of 4-dimethylaminopyridine (DMAP), which is a

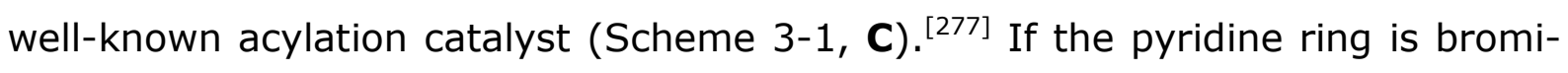
nated, metal-halide exchange is observed exclusively instead of the deprotonation. For the first time, 2,5-dibromopyridine could be regioselectively converted into the ortho-lithiated pyridyl derivative by the application of $\mathrm{TMSCH}_{2} \mathrm{Li}$ / LiDMAE (Scheme 3-1, D). ${ }^{[278]}$

But trimethylsilylmethyllithium does not only attract increasing attention as a component in mixtures of enhanced basicity. The metal-halide exchange reaction with $\mathrm{TMSCH}_{2} \mathrm{Li}$ neatly proceeds with bromopyridines (Scheme 3-1, E), ${ }^{[278]}$ while $(S)$-nicotine is selectively metallated in 4-position (Scheme $3-1, \mathbf{F}$ ). ${ }^{[279]}$ It also gained some recognition as an efficient anionic polymerisation initiator. ${ }^{[280]}$ 
A<smiles>[Al]c1cccc([Ti]Cl)n1</smiles>

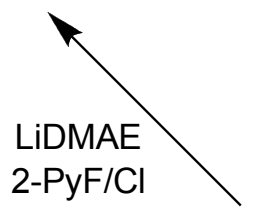

$D$<smiles>[AlH]c1ccc(Br)cn1</smiles><smiles>Fc1ccc(Cl)nc1</smiles>

B<smiles>CN(C)c1ccncc1Cl</smiles>

C

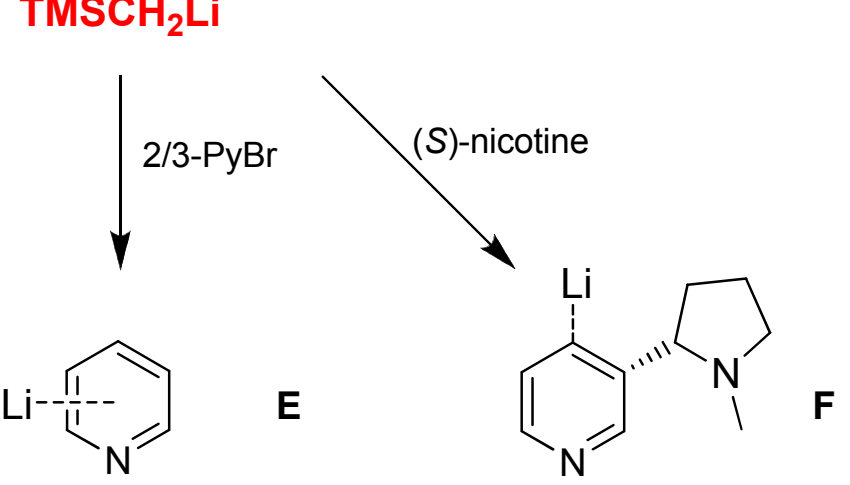

Scheme 3-1. Selected synthetic reactions using $\mathrm{TMSCH}_{2} \mathrm{Li}$.

The composition of trimethylsilylmethyllithium in solution and the solid state is known. In apolar solvents it exists as hexamers (cyclohexane) ${ }^{[40]}$ or tetramers (benzene $)^{[36]}$ and crystallises from pentane without lattice solvent as $\left[\mathrm{TMSCH}_{2} \mathrm{Li}\right]_{6}$, in which a $\mathrm{Li}_{6}$ octahedron as central unit (Figure 3-1). ${ }^{[281]}$ Therefore, it features one of the lead structural motifs in lithium organic chemistry. ${ }^{[48]}$

Even though the octahedral arrangement of the lithium atoms is normally emphasised in literature, the lines on the edges of the octahedron usually represent only topological indicators rather than attractive $\mathrm{Li}-\mathrm{Li}$ interactions. This is reasonable since the experimental charge density investigation of $S$. Deuerlein et al. on a lithium sulfur ylide in which a carbanion interacts with a lithium triangle via a lone pair pointing close to the centre of the lithium atoms (cf. Figure $1-7, a+b) .{ }^{[88]}$ The carbanion binds to all three cations and for that reason shows six bond paths to neighbouring atoms, while no metal-metal bonds could be detected between the cations ( $c f$. Figure 1-7, $c+d$ ). The same conclusions can be deduced from the study on [2-PicLi.PicH $]_{2}$ (4) (cf. chapter 2.3.7). Although the lithium atoms in $\mathbf{4}$ show a smaller atomic distance $(2.58 \AA)$ than the picolyl nitrogen atoms (3.26 $\AA$ ), an exchange channel between the non-metal atoms was found. These two features are strong signs for an anion mediated alignment of the lithium atoms - which interact with each other by electrostatic repulsion - 
that results in the repeatedly observed aggregation motifs in lithium organic chemistry. With the lithium ylide at hand, a tremendous step towards the elucidation of the $\mathrm{Li}_{3} \mathrm{C}$ interaction was made. However, it is a multi-functional compound containing a polarised S-C ylide bond and intramolecular nitrogen donor atoms, which might influence the coordination to the metal atoms. Therefore, simple model compounds possessing tetrameric or hexameric lithium motifs are necessary to study their undisturbed $\mathrm{Li}-\mathrm{C}$ bonding situation.

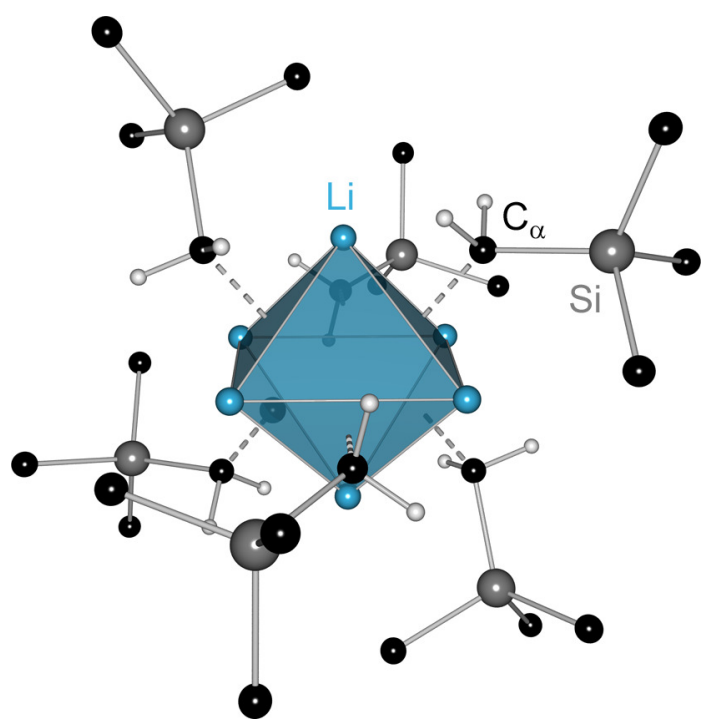

Figure 3-1. Molecular structure of $\left[\mathrm{TMSCH}_{2} \mathrm{Li}\right]_{6}$ with the lithium octahedron highlighted. Dashed bonds indicate the interaction of the carbanion with triangular lithium faces of the octahedron.

Trimethylsilylmethyllithium seems to be a perfect candidate for an experimental charge density study, because it can be crystallised in good quality and is not too sensitive to air and moisture in comparison to other substances featuring the same bonding mode (e.g. n-butyllithium). Moreover, it might be even possible to investigate a whole series of aggregates containing the same organic anion. T. Tatic et al. reported on the disaggregation of the hexameric $\left[\mathrm{TMSCH}_{2} \mathrm{Li}\right]_{6}$ with nitrogen donor bases to obtain dimeric or even monomeric species. ${ }^{[165]}$ Promising additional compounds for charge density studies were already provided, such as the trimethylsilylmethyllithium dimethoxymethane dimer ${ }^{[282]}$ or the chiral $(R, R)-N, N, N^{\prime}, N^{\prime}$-tetramethyl-1,2-diaminocyclohexane (tmcda) monomer, synthesised by the research group of $C$. Strohmann (Figure 3-2). ${ }^{[283]}$ Meanwhile, also a $\mathrm{TMSCH}_{2} \mathrm{Li}$ tetramer was crystallised in our group by T. Tatić. 

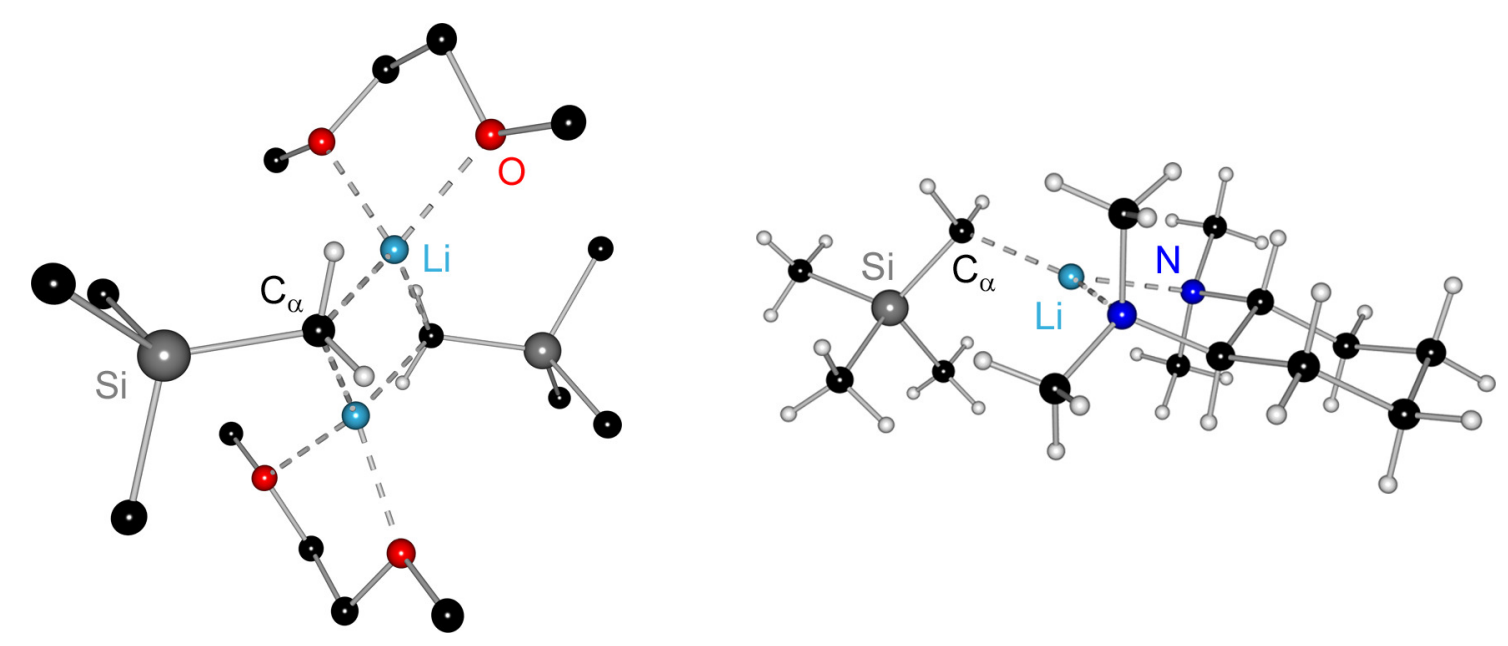

Figure 3-2. Promising candidates for an experimental charge density study: $\left[\mathrm{TMSCH}_{2} \mathrm{Li} \cdot \mathrm{dme}\right]_{2}$ (left) and $\left[\mathrm{TMSCH}_{2} \mathrm{Li}\right.$.tmcda] (right).

Hence, a series of different $\mathrm{TMSCH}_{2} \mathrm{Li}$ aggregates is available, which show all basic structural motifs of lithium organic compounds in possibly sufficient crystal quality to enable a high-resolution data set collection. Nevertheless, this work was primarily focused on the parent compound - the $\left[\mathrm{TMSCH}_{2} \mathrm{Li}\right]_{6}$ hexamer because it is completely free of donor solvent influences on the cations and can therefore serve as reference system for subsequent investigations. 


\subsection{Charge Density Study of a Hexameric Lithium Organic Compound}

\subsubsection{Preparative Work}

The starting material, of which even huge crystals could be obtained (Figure 3-3), was kindly provided by the CHEMETALL GMBH (Frankfurt) in hexane. This was of special importance since an application for beam time at the Heinz MAIERLEIBNITZ NeUtRon SouRCE (FRM II) in Garching was granted. As was already pointed out in chapter 2.3.1, neutron diffraction experiments enable the accurate determination of all atomic positions including hydrogen atoms. Additionally, their thermal motion can be described anisotropically using neutron data, so that the electron density modelling on X-ray diffraction data of the same compound (combined study) can be significantly improved for compounds with high hydrogen atom content.

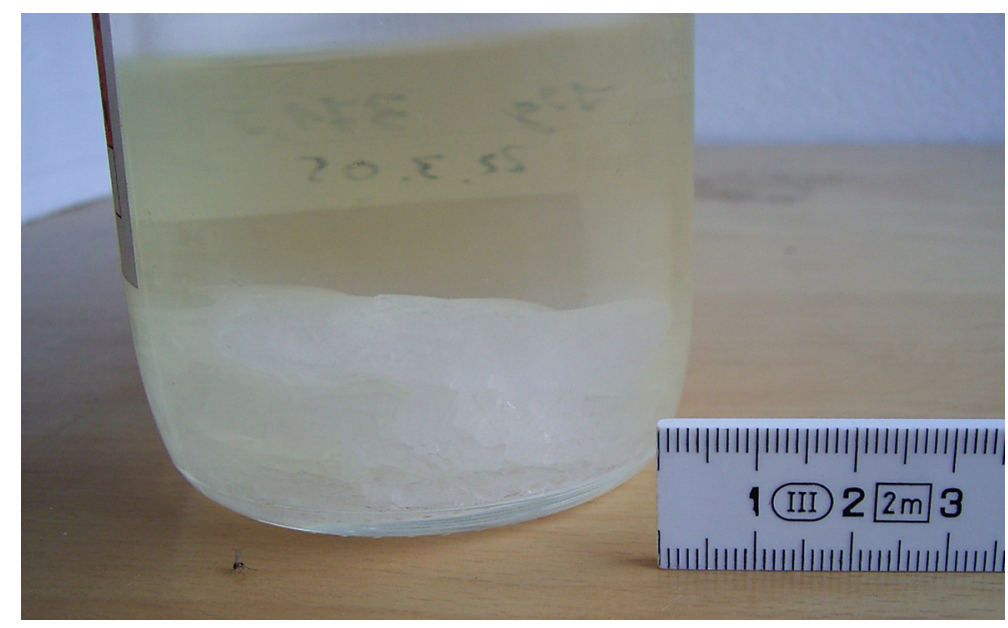

Figure 3-3. Septum bottle containing large $\left[\mathrm{TMSCH}_{2} \mathrm{Li}\right]_{6}$ crystals suitable for neutron diffraction experiments.

A preceding crystal structure determination on a "normal sized" $\left[\mathrm{TMSCH}_{2} \mathrm{Li}\right]_{6}$ single crystal showed that a different polymorph is obtained from hexane than that reported before. ${ }^{[281]}$ In contrast to the known modification that was crystallised from pentane in the monoclinic space group $P Z_{1} / C$ with one full hexamer in the asymmetric unit, the change in the solvent resulted in crystals of triclinic modification (15) with two independent hexamer halves in the asymmetric unit. The standard structure determination gave promising small anisotropic displacement parameters for all no-hydrogen atoms and residual density peaks exclusively in the bonding or lone pair region suggesting the suitability of these 
crystals for a charge density investigation (cf. chapter 8.4.13). A high-resolution data set was obtained on the BRUKER TXS Mo rotating anode with a very good data quality (Figure 3-4). Reflections up to a resolution of $0.45 \AA$ could be measured with an $R_{\text {int }}$ and $R_{\sigma}$ in the highest resolution shell $(0.55-045 \AA$ ) of 0.078 and 0.082 respectively. Exceptionally low $R$ values of $0.021 / 0.009$ were obtained over the whole data range suggesting the suitability of the data for a multipole refinement.

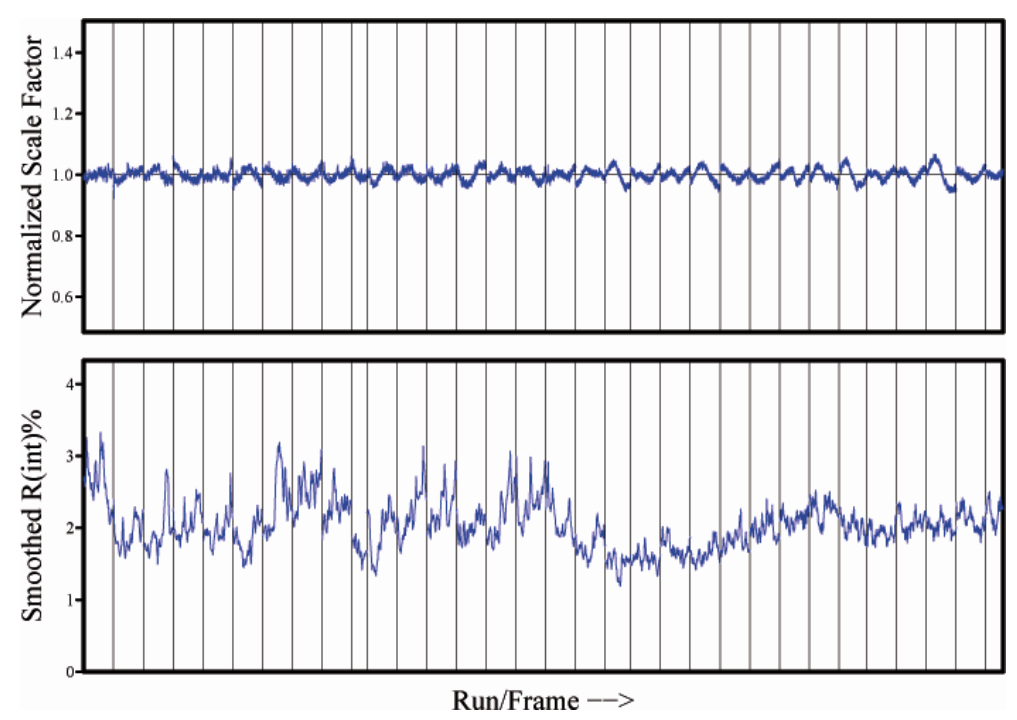

Figure 3-4. Scale and $R$ factors of the high-resolution data set of the triclinic $\left[\mathrm{TMSCH}_{2} \mathrm{Li}\right]_{6}$ modification derived from SADABS-2006/4 (each normal sized segment represents one run with 600 frames).

The starting model was generated according to chapter 2.3.5 and the subsequent multipole refinement was related to the strategy presented there. An $R 1$ value of below 0.03 was obtained, however, severe problems with the data manifested in two tremendously high residual density peaks (1.49 and $1.11 \mathrm{e}^{-3}$ ) during the multipole refinement. These are situated in the projection of two $\mathrm{Si}-\mathrm{C}_{\alpha}$ bonds - one in each moiety of the asymmetric unit - in the $\sigma^{*}$ molecular orbital region "behind" the silicon atoms. A closer inspection of an isotropic IAM also showed this feature and a modelling of a disorder involving the silicon atoms was attempted. This proceeded smoothly and successively and a whole second independent trimethylsilylmethyl fragment could be refined with a slightly higher distance to the $\mathrm{Li}_{3}$ face in each hexamer half. This was even possible without applying ADP stabilisation restraints such as DELU, SIMU, or ISOR and only distance and geometry restraints were used. Remarkably well shaped ADPs could be obtained for both moieties, which are displayed in the Crystallographic 
Section (chapter 8.4.14). It was presumed that decomposed $\mathrm{TMSCH}_{2} \mathrm{Li}$ as tetramethylsilane was incorporated to a minor extent into the crystal. It occupies to a certain extent (site occupation factor between 0.05 and 0.12 ) the position of one anion in the hexamer halves so that also a reduced lithium ratio must be present. This could not be verified during the refinement probably due to $a$ possible distribution of that defect over three lithium sites, which accordingly have a site occupation barely differing from one.

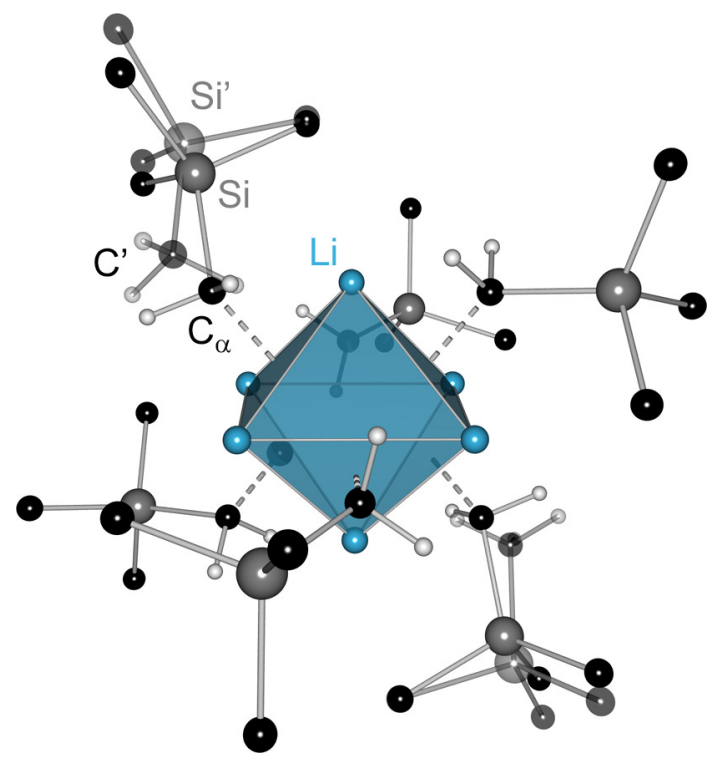

Figure 3-5. Disordered molecular structure of one hexamer in the triclinic [ $\left.\mathrm{TMSCH}_{2} \mathrm{Li}\right]_{6}$ modification (high-resolution data set). The less occupied tetramethylsilane is shown transparent $\left(\mathrm{Si}^{\prime}\right.$ and $\left.\mathrm{C}^{\prime}\right)$.

The hypothesis of incorporated tetramethylsilane was tested by NMR experiments of the isolated crystals. The NMR tubes were immediately sealed after the filling procedure under Schlenk conditions but a slight decomposition of the crystalline lithium organic compound during this operation cannot be completely precluded, since $T$. Tatić reported the appearance of tetramethylsilane peaks in the NMR spectra of disaggregated $\mathrm{TMSCH}_{2} \mathrm{Li}$ after a short period of time. ${ }^{[282]}$ The ${ }^{1} \mathrm{H}$ spectrum [ $\delta$ ] in deuterated toluene at $35^{\circ} \mathrm{C}$ showed signals for the trimethylsilylmethyl anion at $0.15 \mathrm{ppm}\left(\mathrm{CH}_{3}\right)$ and $-2.01 \mathrm{ppm}$ (broad, $\mathrm{CH}_{2}$ ) that integrated to a ratio of $9: 2$. The originally reported value is $\delta-2.16 \mathrm{ppm}$ for the methylene protons of $\mathrm{TMSCH}_{2} \mathrm{Li}$ in benzene. ${ }^{[274]}$ Additionally, a peak at $0.00 \mathrm{ppm}$ indicated the presence of tetramethylsilane with an integral of one proton. This amount is in the range of the determined decomposition product content in the crystals. Furthermore, signals at 3.36 and $0.09 \mathrm{ppm}$ could be assigned to the trimethylsilylmethoxide anion originating from the $\mathrm{TMSCH}_{2} \mathrm{Li}$ decomposition with 
oxygen. Interestingly, at $-50^{\circ} \mathrm{C}$ the methylene proton signal splits up into two independent signal groups at -1.70 and $-2.14 \mathrm{ppm}$ with an intensity ratio of 2:0.6. Two different $\mathrm{TMSCH}_{2} \mathrm{Li}$ species are present at low temperatures which are most probably the tetrameric and hexameric species. Since it was reported that the tetramer is present in benzene, ${ }^{[36]}$ this is almost certainly also the case for toluene at RT. By lowering the temperature, a change in the aggregation state is assumed, which is reflected by a low-field shift of the $\mathrm{CH}_{2}$ signal, accompanied by a splitting up of the trimethylsilylmethyl anion signals that are now resonating at 0.42 and $0.20 \mathrm{ppm}$.

The ${ }^{13} \mathrm{C}$ NMR spectrum [ $\delta$ ] at $35^{\circ} \mathrm{C}$ shows the methyl carbon signals at $3.70 \mathrm{ppm}$ and the deprotonated one as broad peak at $-4.58 \mathrm{ppm}$. This is caused by the coupling (not resolved) to both NMR active lithium isotopes ${ }^{6 / 7} \mathrm{Li}$. Similar ${ }^{13} \mathrm{C}$ shifts were reported for nitrogen donor adducts of $\mathrm{TMSCH}_{2} \mathrm{Li}^{[165]}$ Again, a peak at $0.01 \mathrm{ppm}$ points towards $\mathrm{SiMe}_{4}$ in solution. Additionally, a signal at $-2.97 \mathrm{ppm}$ occurs, which cannot unequivocally be assigned. Two different aggregates may even exist above RT, which was claimed in the master thesis of M. S. Medley, who reported the coexistence of a hexamer and an octamer in cyclopentane solutions. ${ }^{[284]}$ Even though the assignment as an octameric species is rather doubtful, two independent signal groups were identified. Further details on the aggregates in toluene could not be extracted because the lowtemperature spectra did show complex signals that defied a definite assignment. Also a low-temperature ${ }^{29} \mathrm{Si}$ NMR spectrum shed no further light on that problem, showing at least five signals of which some overlapped. The single broad ${ }^{7} \mathrm{Li}$ peak at $1.94 \mathrm{ppm}$ is in the expected range for $\mathrm{TMSCH}_{2} \mathrm{Li}$ but cannot serve as an indicator for the presence or absence of tetramethylsilane in the lithium aggregate at RT.

However, tetramethylsilane was detected in the NMR samples, although its origin from the crystals and not from their decomposition afterwards (or silicon grease) is still questionable. Nonetheless, a hydrolysation of almost $10 \%$ of a lithium organic compound during the NMR sample preparation is normally not observed, so that results of the crystal structure determination are supported by these experiments.

Thus, no successful experimental charge density determination can be undertaken with these crystals. Several independent batches showed the same disorder phenomenon. Interestingly, standard resolution $(0.84 \AA)$ data did not show any disorder signs at all. This feature could only be detected by an accurate high-resolution data acquisition. Thus, a judgement on crystal quality prior to the 
data collection cannot be convincingly given. Furthermore, it is quite remarkable that disorder effects cannot be modelled by the flexible multipole model. The population of multipoles is dominated by bonding effects so that the electron density description of disordered parts of a molecule is suppressed.

In summary, the triclinic modification of trimethylsilylmethyllithium was identified as inadequate for an experimental charge density study. Therefore, it was focussed on the known monoclinic polymorph in the further course.

\subsubsection{Experimental and Refinement Details}

Single crystals of the monoclinic $\left[\mathrm{TMSCH}_{2} \mathrm{Li}\right]_{6}$ (14) modification were received from pentane solutions at $4^{\circ} \mathrm{C}$. Several attempts to obtain a high-resolution X-ray data set were undertaken with varying success, so that numerous crystal batches had to be provided. These were crystallised in cooperation with G. Schwab or recently made available by $T$. Tatić. The crystals were of moderate sensitivity but tended to grow in layers, which either caused disorder effects or limited the diffracting power. Furthermore, they showed destructive phase transitions between 100 and $120 \mathrm{~K}$, a phenomenon they share with the triclinic polymorph. Therefore, the temperature of the crystal cooling device had be raised to prevent the cracking of the crystals. Approximately, ten complete or partial (reasons for that: abortion of the data collection due to insufficient data quality or instrumental problems) data sets were collected. Only two of them reached the quality criteria (i.e. $R$ values, ADP shape, residual density peaks) for a multipole refinement.

A complete data set (39420 unique reflections) with a mean redundancy of 6.2 - kindly measured by $D$. Kratzert - had reasonably low $R_{\text {int }}(0.022)$ and $R_{\sigma}$ (0.018) values up to a resolution of $0.48 \AA$. However, these values increased in the high resolution shell $(0.60-0.48 \AA$ ) up to 0.179 and 0.220 , respectively. Nevertheless, a multipole refinement was undertaken, which showed already in an early stage that severe problems with residual density peaks in the vicinity (0.03 to $0.08 \AA$ distance) of all independent six silicon atoms were present. Once again, it was not possible to describe them with the multipole parameters and the refinement was aborted.

The second data set was measured with a crystal of acceptable quality, but the crystal cooling device broke after one low-resolution and two high-resolution runs. For that reason, the completeness of the data was only $89 \%$ with a mean 
data redundancy of $1.5 . R_{\text {int }}(0.020)$ and $R_{\sigma}(0.029)$ values to a resolution of $0.46 \AA$ were promising and only less than 300 unique reflections were not measured (39139) compared to the high redundant first data set. Thus, a high data to parameter ratio was achieved during the multipole refinement, anyway (28.5). However, higher $R$ values can be expected after the refinement procedure owing to the higher standard deviations of each reflection, which could not all be averaged over many equivalent reflections. The data collection and refinement proceeded according to the described method outlined in details in chapter 2.3.5. Therefore, only differences will be mentioned below.

The exposure times were $8 \mathrm{~s}$ for the low-order and $120 \mathrm{~s}$ for the high-order data. The data processing was performed with the most recent SAINT $(v 7.46 A)^{[285]}$ and SADABS $(2008 / 2)^{[286]}$ versions. The multipole refinement started with a fully chemically constrained model, in which all silicon, lithium, methyl carbon, and methylene carbon atoms including the corresponding hydrogen atoms shared the same multipole parameters among each other. Moreover, only $C_{3}$ symmetric multipoles oriented along the Si-C bond were refined for the methyl carbon atoms and a bond directed dipole and quadrupole for the hydrogen atoms along the $\mathrm{C}-\mathrm{H}$ bonds. The chemical constraints were successively reduced during the refinement procedure. Finally, the silicon, lithium, and methylene carbon atoms were refined independently, while the methyl atoms were related via a non-crystallographic inversion centre in the middle of the lithium octahedron. Additionally, the two methylene hydrogen atoms at the deprotonated carbon atom were treated independently but remained constrained within the hexamer.

In contrast to the picolyllithium data set, the lithium atoms were handled as neutral lithium atoms in the scattering table and could be refined. Monopole populations between 0.04 and 0.24 resulted with a refined $\kappa$ value of 1.30 . The refinement proceeded stepwise. The atomic positions, thermal parameters, monopoles, and multipoles were independently refined from $\kappa$ and $\kappa^{\prime}$ parameters. Intermediately, the positions of the hydrogen atoms were refined against low order data $\left(d_{\min }=1.00 \AA\right)$ keeping all other parameters fixed. Subsequently, the standard reset bond commands were again applied.

The multipole refinement resulted in an acceptable model of the electron density distribution in $\left[\mathrm{TMSCH}_{2} \mathrm{Li}\right]_{6}(\mathbf{1 4})$, even though a similar model of highest quality standard as for [2-PicLi.PicH $]_{2}$ (4) could not be achieved. This is demonstrated by the following criteria (Table 3-1, Figure 3-6, and Appendix). 
Table 3-1. Selected crystallographic details after the multipole refinement of $\left[\mathrm{TMSCH}_{2} \mathrm{Li}\right]_{6}$ (14).

\begin{tabular}{ll|ll}
\hline independent reflections & $33338\left(R_{\text {int }}=0.020\right)$ & $R 1$ & 0.0307 \\
reflections above $(I>3 \sigma(I))$ & 20972 & wR2 & 0.0548 \\
parameters & 805 & GoF & 1.873 \\
largest diff. peak and hole & 0.365 and $-0.344 \mathrm{e}^{-3}$ & & \\
\hline
\end{tabular}

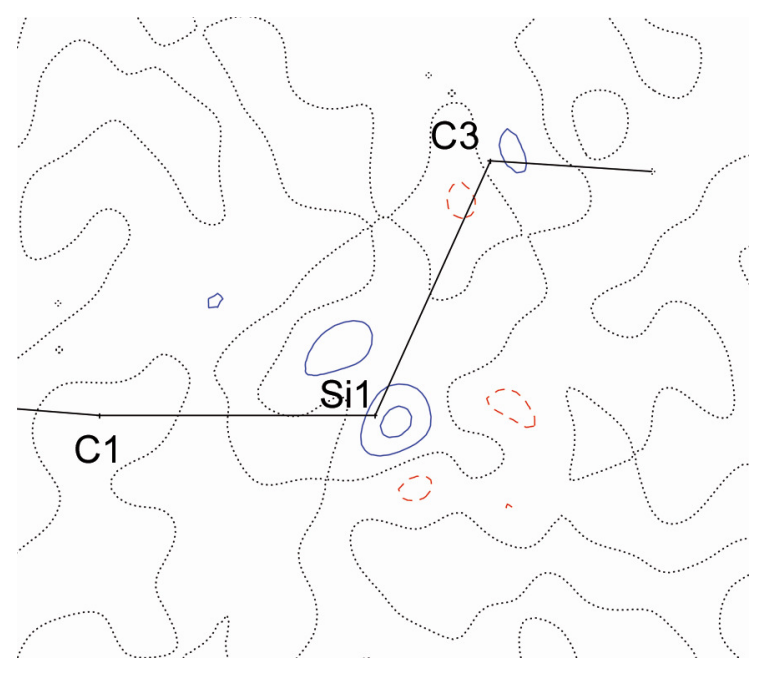

(a)

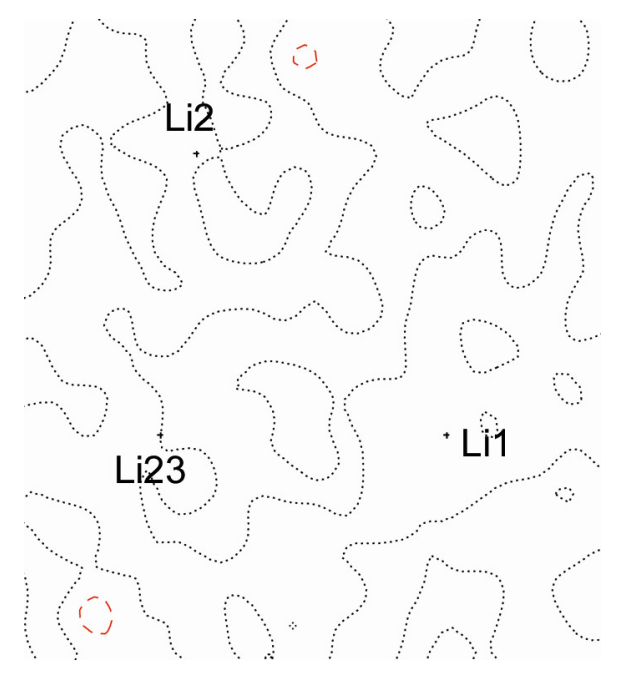

(c)

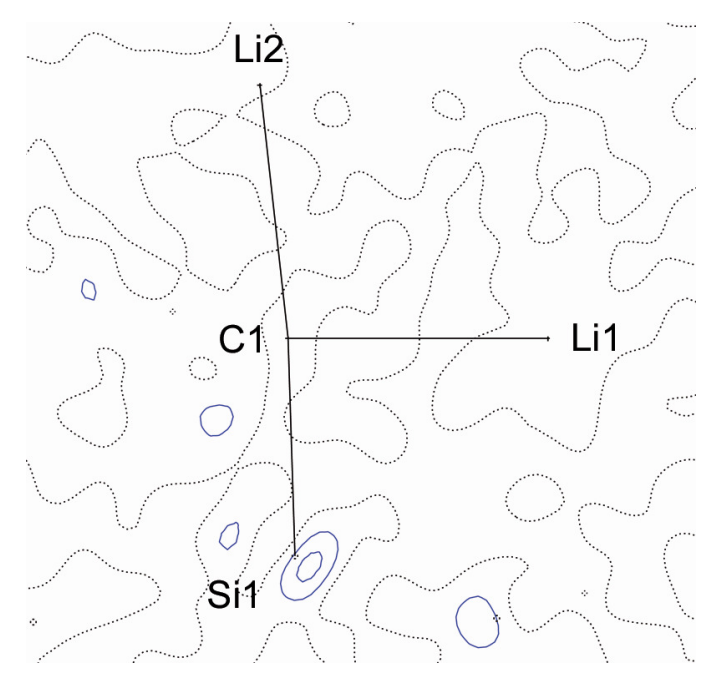

(b)

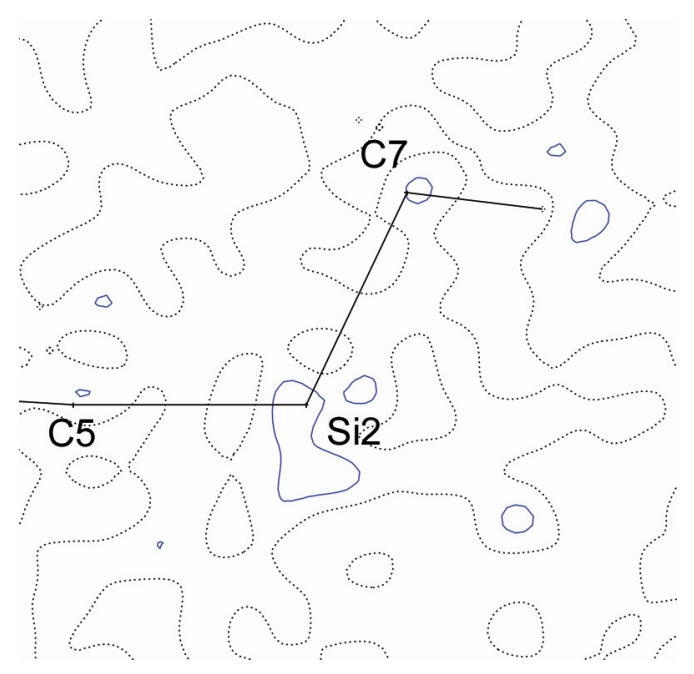

(d) 


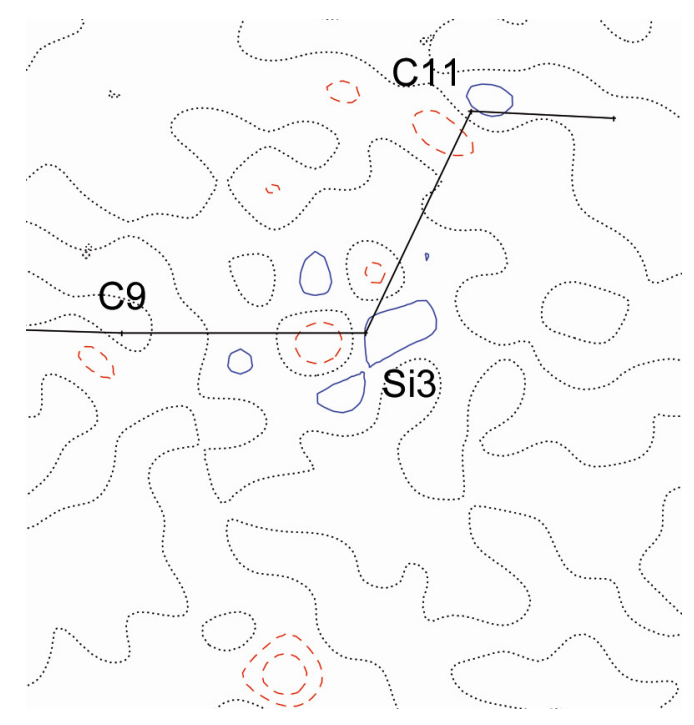

(e)

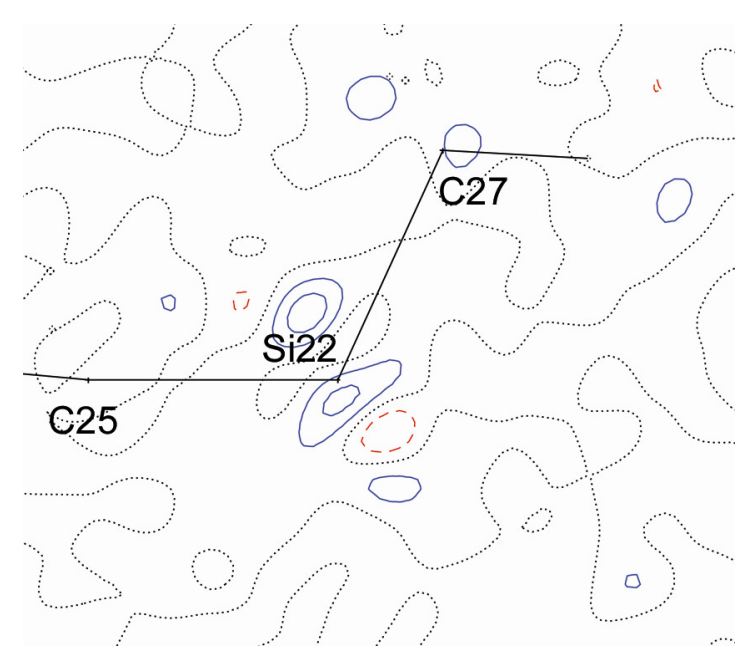

(g)

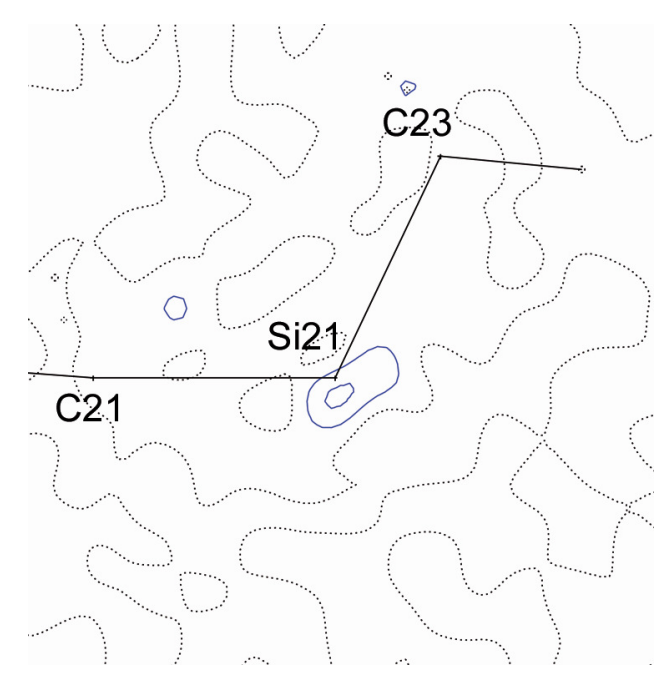

(f)

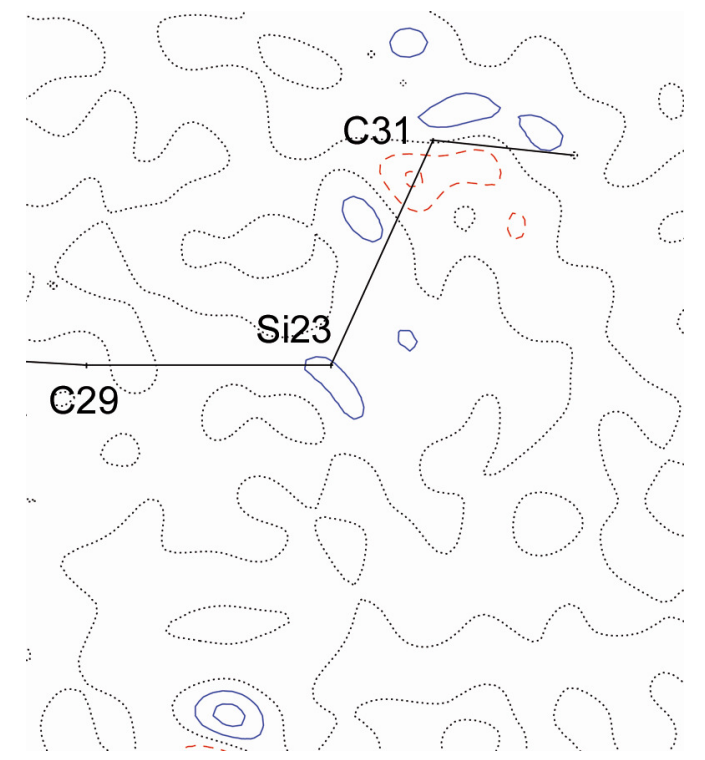

(h)

Figure 3-6. Residual density maps ( $\sin \theta \lambda^{-1}$ cut-off of $1.0 \AA^{-1}$ ) of $\left[\mathrm{TMSCH}_{2} \mathrm{Li}\right]_{6}$ (14). Step size of the contour lines is $0.1 \mathrm{e}^{-3}$. Positive values are plotted as solid blue lines, negative values as dashed red ones and the zero value is drawn dotted black; (a) C1Si1-C3 plane, (b) Li1-C1-Li2 plane, (c) Li1 $\cdots$ Li2 $\cdots$ Li23 plane, (d) C5-Si2-C7 plane, (e) C9-Si3-C11 plane, (f) C21-Si21-C23 plane, (d) C25-Si22-C27 plane, and (e) C29Si23-C31 plane. 


\subsubsection{Molecular Structure Considerations}

The following chapter deals with the discussion of structural features in the trimethylsilylmethyllithium hexamer (14) to gain insight into the coordination sphere of this octahedrally arranged lithium organic compound. Moreover, it is compared to the standard structure of the novel triclinic modification (15) and the most prominent representative of hexameric $\mathbf{R} \mathbf{L i}, n$-butyllithium. ${ }^{[41]}$

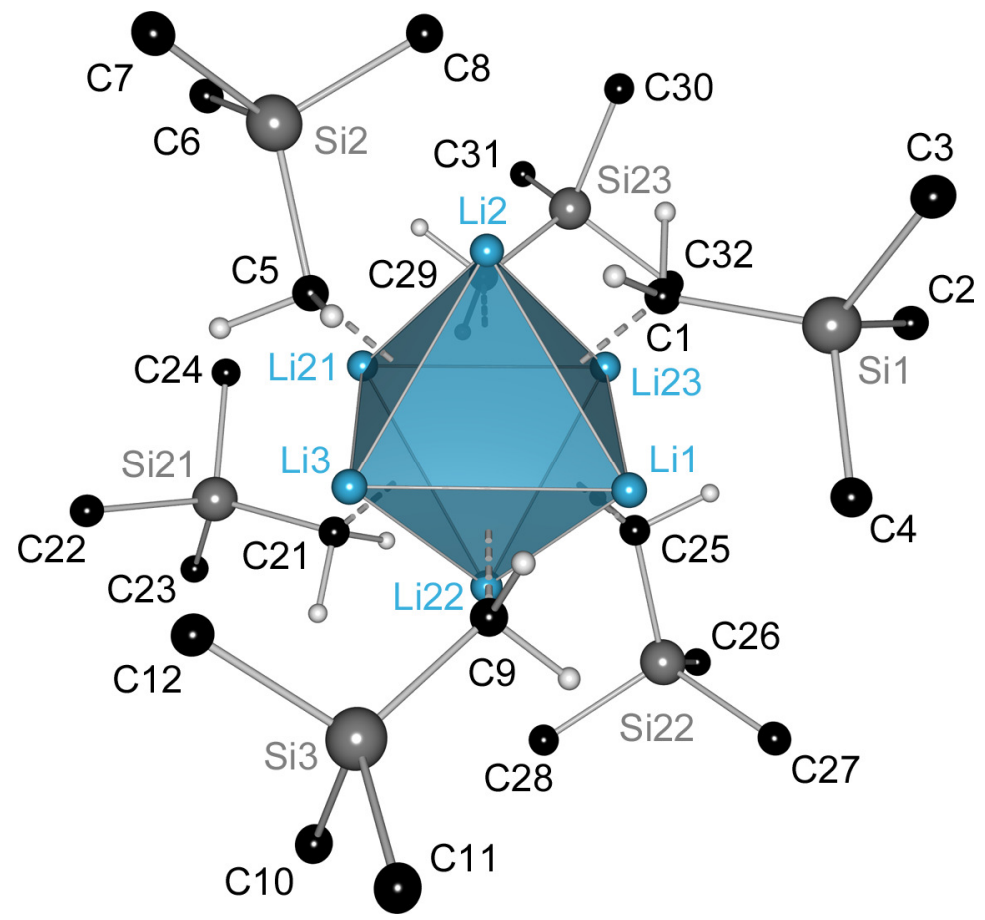

Figure 3-7. Molecular structure representation of $\left[\mathrm{TMSCH}_{2} \mathrm{Li}\right]_{6}(\mathbf{1 4})$ highlighting the motif of carbanion capped lithium atoms in an octahedral arrangement.

Focussing on the arrangement of the metal atoms in $\left[\mathrm{TMSCH}_{2} \mathrm{Li}\right]_{6}(\mathbf{1 4})$, the lithium octahedron is severely distorted. Its basis ( $\mathrm{Li} 1 \cdots \mathrm{Li} 3 \cdots \mathrm{Li} 21 \cdots \mathrm{Li} 23$ ) is almost

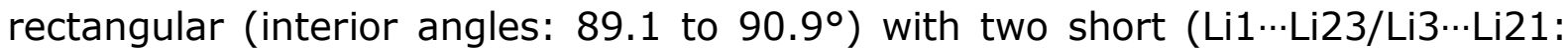

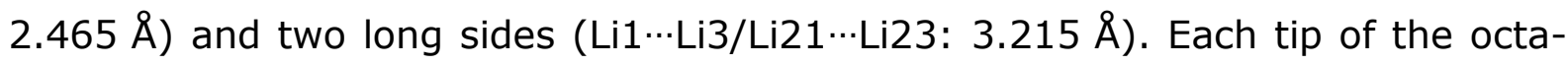
hedron ( $\mathrm{Li} 2 / \mathrm{Li} 22$ ) is shifted towards one side of the basis along the mid-line of the longer sides. This results in more or less isosceles lithium triangles that are capped by one anion. Six lithium faces are coordinated by carbon atoms, while two remain vacant (Li1Li2Li3/Li21Li22Li23), which are positioned opposite to each other. These lithium triangles show much longer $\mathrm{Li} \cdots \mathrm{Li}$ distances (3.123 to $3.238 \AA$ ) than their capped counterparts (electrostatic repulsion without the attraction of an anion). The triangle shown to the upper right of the octahedron in Figure 3-7 (Li1Li2Li23) for example has short Li23 ‥Li1/Li2 legs (2.467 and 
$2.445 \AA$ ) with a much longer basis. Exactly the same situation is evident in $[n B u L i]_{6}$ but with smaller Li...Li distances (a related triangle has sides of 2.413, 2.436, and $2.898 \AA) .{ }^{[41]}$

However, the positions of the deprotonated carbon atoms ( $\mathrm{C}_{\alpha}$ atoms) relative to the triangles are different in $\left[\mathrm{TMSCH}_{2} \mathrm{Li}\right]_{6}(\mathbf{1 4})$ compared to $[n \mathrm{BuLi}]_{6}$. In the projection of the lithium atom plane, $\mathrm{C} 1$ resides between Li1 $\cdots \mathrm{Li} 2$ in $\mathbf{1 4}$, while in $[n B u L i]_{6}$ the carbon atom is positioned more closely to the centre of the triangle (Figure 3-8). This has an effect on the Li-C bonds - discussed below - and the direction of the assumed lone pairs (further details for 14 in chapter 3.2.4). Moreover, the orientation of the organic group relative to the Li1 $\cdots$ Li2 vector differs. The Si-C bond in $\mathbf{1 4}$ is almost parallel to Li1 $\cdots$ Li2 resulting in an nearly straight Li2-C1-Si1 line (bond angle: $\left.175.2(1)^{\circ}\right)$. The corresponding $\mathrm{C}-\mathrm{C}$ bond in $[n B u L i]_{6}$ on the other hand is slightly rotated so that the $C_{\beta}$ atom resides above Li1. This particular position was interpreted by T. Kottke and D. Stalke as a secondary electrostatic interaction with the cation showing remarkably short $\mathrm{Li}-\mathrm{C}_{\beta}$ distances of only $2.280(3)$ to $2.295(3) \AA .{ }^{[41]}$
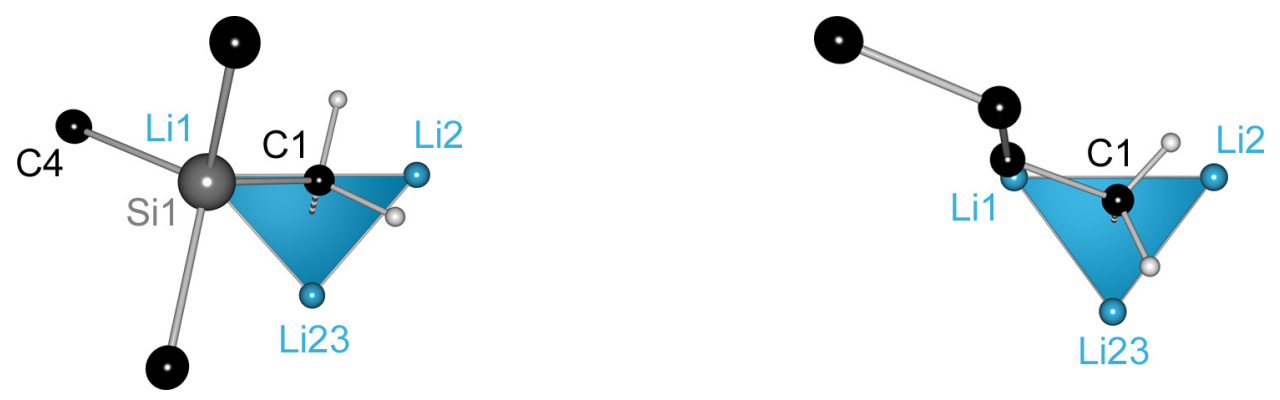

Figure 3-8. Exemplary position of the carbanion $\mathrm{C} 1$ relative to the lithium triangle in $\left[\mathrm{TMSCH}_{2} \mathrm{Li}\right]_{6}\left(\mathbf{1 4}\right.$, left) and $[\mathrm{nBuLi}]_{6}$ (right; view in both cases exactly parallel to the triangle plane normal).

Two types of $\mathrm{Li}-\mathrm{C}$ bonds can be distinguished according to the observed bond lengths in $\left[\mathrm{TMSCH}_{2} \mathrm{Li}\right]_{6}(\mathbf{1 4})$. Bonds to the atoms of the long basis of the isosceles triangles are shorter (e.g. Li1-C1: 2.199(1) $\AA$; Li2-C1: 2.158(1) $\AA$ ) than those to the top (e.g. Li23-C1: 2.260(1) $\AA$; av. $2.273 \AA$ ) throughout the lithium octahedron. On the other hand, no preference among the shorter $\mathrm{Li}-\mathrm{C}$ bonds could be detected. The "straight" $\mathrm{Li}-\mathrm{C}$ bonds are in three out of six cases shorter than the "rectangular" ones (bond lengths: Li2/22-C1/21 < Li1/21-C1/21, Li23C25 < Li22-C25; av. $2.186 \AA)$. The situation is inversed in the remaining cases (bond lengths: Li2-C5 < Li3-C5, Li3-C9 < Li1-C9, (Li21-C29 = Li23-C29); av. $2.193 \AA$ ). It is referred to Table 3-2 for more details. 
The same is valid for [nBuLi] 6 with average short $\mathrm{Li}-\mathrm{C}$ bond lengths of 2.156 and $2.161 \AA$. Interestingly, the longer $\mathrm{Li}-\mathrm{C}$ bonds (av. $2.273 \AA$ ) are identical in length compared to $\mathbf{1 4}$, while the remaining bonds are $0.3 \AA$ shorter than in the silyl compound, for yet unknown reasons. A stabilisation of the lone pairs in $\mathbf{1 4}$ by increased negative hyperconjugation effects seems plausible and will be further discussed in chapter 3.2.4. The similarity of the longer Li-C contacts on the other hand might be explained by electrostatic interactions of the lithium cation with the negatively charged carbanions without orbital control. It has to be noted, that the triclinic modification $\mathbf{1 5}$ is closely related to the monoclinic one (Table 3-2) and is consequently not discussed in further detail.
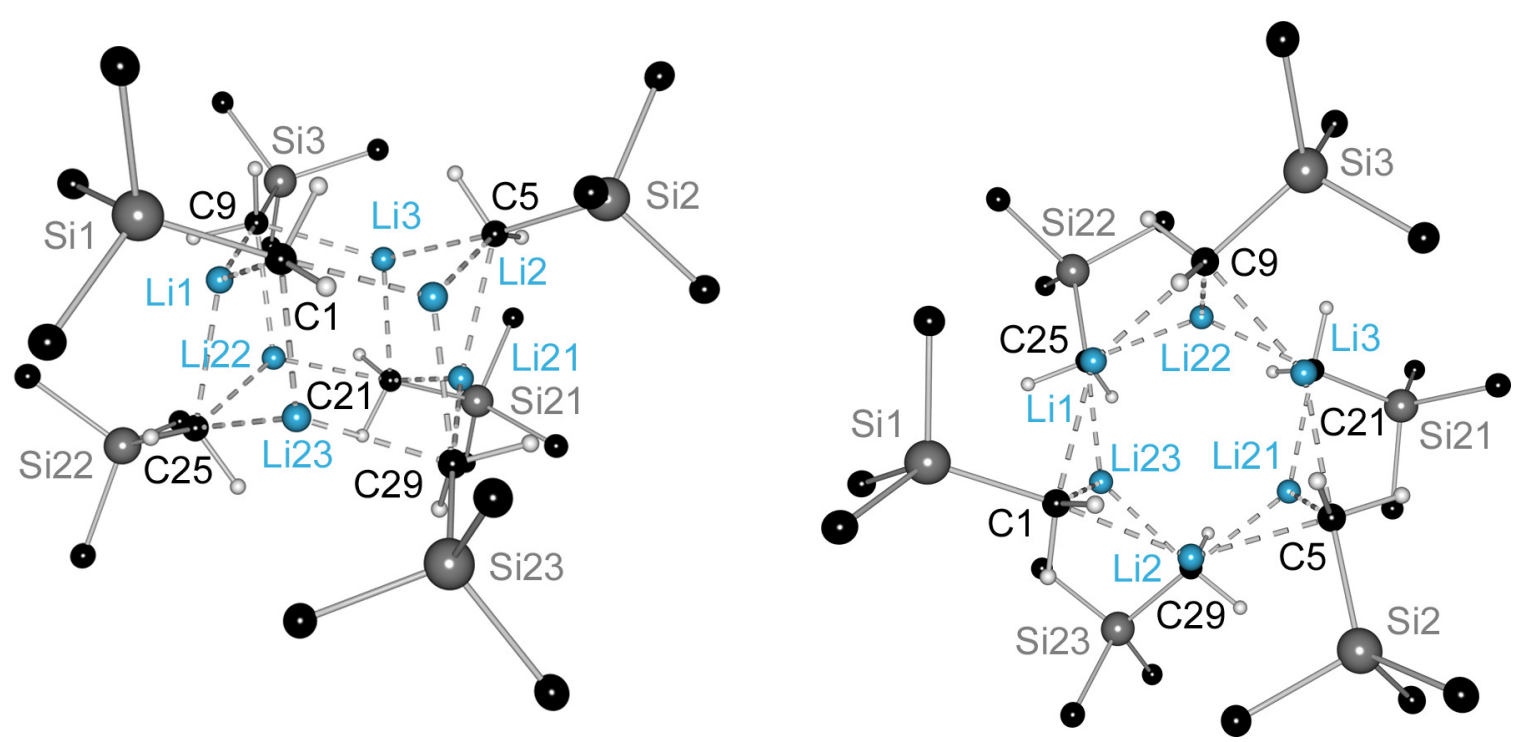

Figure 3-9. Alternative molecular structure representations of $\left[\mathrm{TMSCH}_{2} \mathrm{Li}\right]_{6}$ (14) clarifying the stacked tri-metalla-cyclohexane rings.

Although hexameric lithium organic compounds are normally displayed as lithium octahedrons with carbanions bonded to the lithium faces (cf. Figure 1-5 and Figure 3-7), in view of the potential Li-C bond connectivities they are better described as two stacked tri-metalla-cyclohexane rings linked by six bonds of $\mathrm{Li}-\mathrm{C}$ pairs "stacked on top of each other" (Figure 3-9). Consequently, each deprotonated carbon atom binds to three neighbouring lithium atoms in accordance with the charge density investigation on a lithium sulfur ylide by $S$. Deuerlein et al. (cf. Figure 1-7c). ${ }^{[88]}$ The $\mathrm{Li}-\mathrm{C}$ bonds representing the six-membered rings are always shorter than the interlinking ones between the stacks.

A view through the centres of both rings (Figure 3-9, right) shows that all trimethylsilyl groups are bent into the same direction of rotation. Within the silyl groups, short $\mathrm{Si}-\mathrm{C}_{\alpha}$ bonds are observed (1.845(1) to $1.857(1) \AA$ ) while the bonds 
to the methyl groups are longer $(1.867(1)$ to $1.890(1) \AA$ ). This could be due to an electrostatic reinforcement of the bonds to the deprotonated carbon atoms or by negative hyperconjugation, which will be further clarified in the next chapter. The orientation of the methyl compared to the $\mathrm{CH}_{2}$ groups seems to be ideal for the orbital controlled bond strengthening (Figure 3-10). The dihedral angles between the trans methyl carbon, the silicon, the methylene carbon atom, and the centre of the methylene hydrogen atoms are close to zero (C3/23-Si1/21$\mathrm{C} 1 / 21-\mathrm{H} 1 / 21: 11.2^{\circ} ; 13.7^{\circ}$ and $1.5^{\circ}$ respectively). It is noteworthy that the hydrogen atom positions at the deprotonated carbon atoms were identified by difference Fourier maps and not constrained to ideal positions.

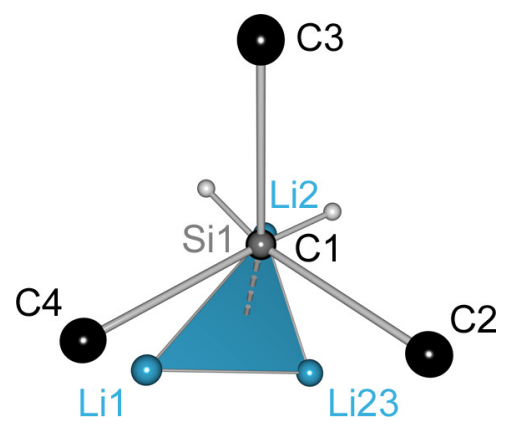

Figure 3-10. View along the $\mathrm{Si} 1-\mathrm{C} 1$ bond in $\left[\mathrm{TMSCH}_{2} \mathrm{Li}\right]_{6}(\mathbf{1 4})$.

Moreover, the parallel orientation of the trimethylsilyl groups relative to the long $\mathrm{Li} \cdots \mathrm{Li}$ bond vectors can be quantified by dihedral angles. The Li1-C1-Si1-C4 angle is only $20.0^{\circ}$ (averaged with the corresponding angle at C21) and those of the other two triangles are $17.9^{\circ}(\mathrm{C} 5 / \mathrm{C} 25)$ and $29.7^{\circ}$ ( $\left.\mathrm{C} 9 / \mathrm{C} 29\right)$. According to the dihedral angles, a special orientation of the anions at $\mathrm{C} 9$ and $\mathrm{C} 29$ relative to the lithium triangle could be identified.

The alignment of one methyl group of each trimethylsilyl moiety parallel to the $\mathrm{Li} \cdots \mathrm{Li}$ vectors causes small $\mathrm{Li}-\mathrm{H}$ distances of 2.135 ( $\mathrm{Li} 2)$ to $2.352 \AA$ (Li23). These are, however, on the large end of typically claimed $\mathrm{Li}-\mathrm{H}$ agostic interactions $(1.8-2.2 \AA) .{ }^{[287]}$ Some of them are shorter than those in bis(trimethylsilyl)(2-pyridyl)methyllithium (7; shortest one $2.25 \AA$ ), in which agostic interactions were excluded. ${ }^{[87]}$ According to the determined hydrogen atom positions of the methyl carbon atoms, no obvious rotation of the $\mathrm{CH}_{3}$ groups could be observed, which would result in a minimisation of the $\mathrm{Li}-\mathrm{H}$ distances. Each methyl group features one hydrogen atom exactly trans to the $\mathrm{Si}-\mathrm{C}_{\alpha}$ bond and the remaining atoms in an orientation associated with $\mathrm{sp}^{3}$ hybrid orbitals. Nevertheless, it was looked for indicators of agostic interactions during the inspection of the electron 
density distribution of $\left[\mathrm{TMSCH}_{2} \mathrm{Li}\right]_{6}(\mathbf{1 4})$, which is presented in the following chapter.

Table 3-2. Selected bond lengths $[\AA]$ of the monoclinic (14) and the triclinic (15) modification of $\left[\mathrm{TMSCH}_{2} \mathrm{Li}\right]_{6}$ as well as of $[n B u L i]_{6}{ }^{[41]}$

\begin{tabular}{l|clc}
\hline & {$\left[\mathrm{TMSCH}_{2} \mathrm{Li}_{6}(\mathbf{1 4})^{[\mathrm{a}]}\right.$} & {$\left[_{\mathrm{TMSCH}} \mathrm{Li}_{6}(\mathbf{1 5})^{[\mathrm{b}]}\right.$} & {$[n B u L i]_{6}$} \\
\hline Li1-C1 & $2.199(1) / 2.194(1)$ & $2.203(2) / 2.192(2)$ & $2.175(3)$ \\
Li1-C9 & $2.216(1) / 2.196(1)$ & $2.224(2) / 2.224(2)$ & $2.172(3)$ \\
Li1-C25 & $2.283(1) / 2.283(1)$ & $2.284(2) / 2.293(2)$ & $2.277(3)$ \\
Li2-C1 & $2.158(1) / 2.174(1)$ & $2.168(2) / 2.177(2)$ & $2.158(3)$ \\
Li2-C5 & $2.177(1) / 2.186(1)$ & $2.168(2) / 2.173(2)$ & $2.137(3)$ \\
Li2-C29 & $2.264(1) / 2.269(1)$ & $2.245(2) / 2.253(2)$ & $2.272(3)$ \\
Li3-C5 & $2.194(1) / 2.178(1)$ & $2.181(2) / 2.214(2)$ & $2.157(3)$ \\
Li3-C9 & $2.199(1) / 2.196(1)$ & $2.199(2) / 2.205(2)$ & $2.153(3)$ \\
Li3-C21 & $2.267(1) / 2.260(1)$ & $2.264(2) / 2.268(2)$ & $2.262(3)$ \\
Si1-C1 & $1.845(1) / 1.847(1)$ & $1.848(1) / 1.850(1)$ & - \\
Si2-C5 & $1.854(1) / 1.857(1)$ & $1.851(1) / 1.851(1)$ & - \\
Si3-C9 & $1.854(1) / 1.849(1)$ & $1.861(1) / 1.864(1)$ & - \\
Si1-C2 & $1.872(1) / 1.877(1)$ & $1.875(1) / 1.866(1)$ & - \\
Si2-C6 & $1.873(1) / 1.874(1)$ & $1.873(1) / 1.865(1)$ & - \\
Si3-C10 & $1.879(1) / 1.872(1)$ & $1.869(1) / 1.865(1)$ & - \\
Si1-C3 & $1.870(1) / 1.872(1)$ & $1.870(1) / 1.872(1)$ & - \\
Si2-C7 & $1.868(1) / 1.874(1)$ & $1.874(1) / 1.874(1)$ & - \\
Si3-C11 & $1.868(1) / 1.867(1)$ & $1.873(1) / 1.873(1)$ & - \\
Si1-C4 & $1.888(1) / 1.889(1)$ & $1.881(1) / 1.875(1)$ & - \\
Si2-C8 & $1.888(1) / 1.890(1)$ & $1.888(1) / 1.886(1)$ & - \\
Si3-C12 & $1.883(1) / 1.876(1)$ & $1.886(1) / 1.884(1)$ & - \\
\hline
\end{tabular}

[a] Each second value refers to the corresponding bond in the second half of the hexamer (a non-crystallographic inversion centre in the middle of the lithium octahedron is assumed; e.g. $\mathrm{Li} 1-\mathrm{C} 1=\mathrm{Li} 21-\mathrm{C} 21)$. [b] Each second value refers to the corresponding bond of the second independent hexamer half in the asymmetric unit. 


\subsubsection{Analysis of the Electron Density Distribution}

The determination of all bond critical points and the corresponding bond paths in the trimethylsilylmethyllithium hexamer $\mathbf{1 4}$ should clarify whether the interaction of a carbanion with a lithium triangle also leads to bonds connecting all lithium atoms with the deprotonated carbon atom (cf. [(thf) $\mathrm{Li}_{2}\left\{\mathrm{H}_{2} \mathrm{C}\right.$

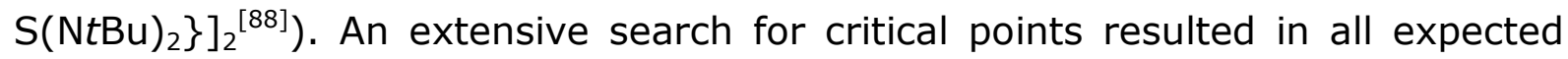
non-metal atom bonds as well as in three bond critical points of each carbanion to neighbouring lithium cations (Figure 3-11).
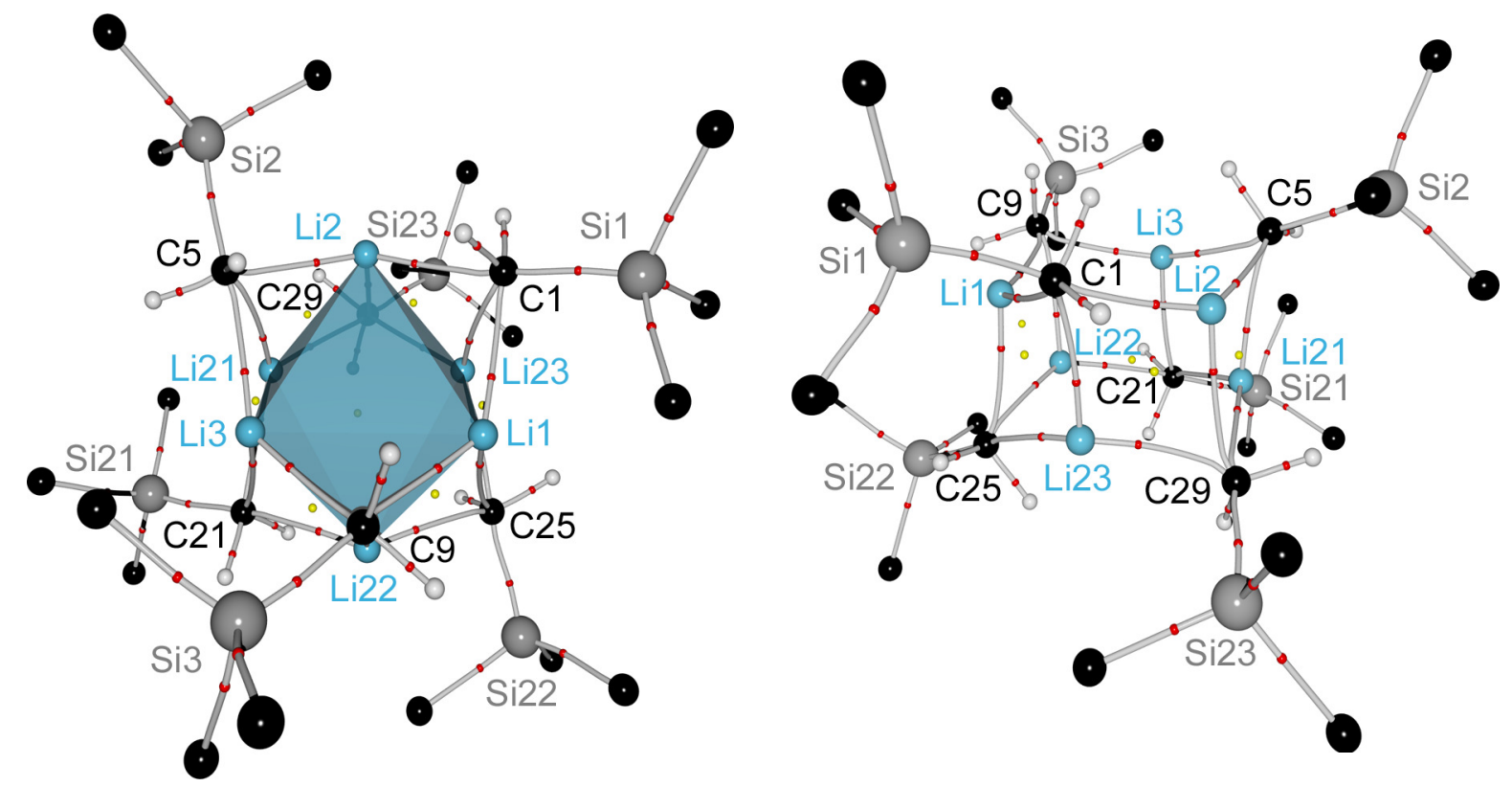

Figure 3-11. Molecular graph of $\left[\mathrm{TMSCH}_{2} \mathrm{Li}\right]_{6}$ (14) (left: lithium octahedron motif; right: tri-metalla-cyclohexane motif) including BCPs (red dots) and RCPs (yellow dots). Hydrogen bond paths of the methyl groups and between the trimethylsilylmethyl moieties are excluded for clarity reasons.

Each methylene carbon atom is hexacoordinated forming bonds to one silicon, two hydrogen, and three lithium atoms. Exactly the same situation was found for the ylidic carbon atom in $\left[(\text { thf }) \mathrm{Li}_{2}\left\{\mathrm{H}_{2} \mathrm{CS}(\mathrm{NtBu})_{2}\right\}\right]_{2}$ with the only difference that just two carbanions are present there (cf. Figure 1-7, c). ${ }^{[88]}$ In total 108 bond critical and 7 ring critical points could be quantified in $\mathbf{1 4}$ that are associated with the hexamer. Therefore, the Poincaré-Hopf equation (Eq. 2-13) is fulfilled taking the 102 atoms of the hexamer into account. Ring critical points were found slightly above each carbanion-capped lithium triangle as well as near the middle of the two metallacyclohexane rings. Furthermore, additional bond paths interlinking the trimethylsilylmethyl groups were determined. These show electron 
density values at the BCPs below $0.04 \mathrm{e}^{-3}$ and were not included in Figure 3-11 for clarity reasons (see also chapter 2.3.7). Additionally, a complete molecular graph plot can be found in the appendix.

Noteworthy, bond paths between lithium and hydrogen atoms in close proximity were neither detected in the presented (Figure 3-11) nor in the complete molecular graph. Consequently, no indications for possible lithium agostic interactions are detected in $\left[\mathrm{TMSCH}_{2} \mathrm{Li}\right]_{6}(\mathbf{1 4})$. These results are in good agreement with the studies of $W$. Scherer et al. ${ }^{[86,87]}$ Hence, even shorter $\mathrm{Li}-\mathrm{H}$ distances than those reported before do not lead to a formation of a bond path.

Table 3-3. Electron densities $\left[\mathrm{e}^{-3}\right]$ and Laplacians $\left[\mathrm{e} \AA^{-5}\right]$ at the $\mathrm{BCP}$, as well as bond path length $\mathrm{BP}[\AA]$ of the $\mathrm{Li}-\mathrm{C}$ bonds in $\left[\mathrm{TMSCH}_{2} \mathrm{Li}\right]_{6}(\mathbf{1 4})$.

\begin{tabular}{l|lll|l|llll}
\hline \multicolumn{3}{c}{$\rho\left(\mathbf{r}_{\mathrm{BCP}}\right)$} & $\nabla^{2} \rho\left(\mathbf{r}_{\mathrm{BCP}}\right)$ & \multicolumn{1}{c}{ BP } & \multicolumn{1}{c}{$\rho\left(\mathbf{r}_{\mathrm{BCP}}\right)$} & $\nabla^{2} \rho\left(\mathbf{r}_{\mathrm{BCP}}\right)$ & BP \\
\hline Li1-C1 & $0.137(7)$ & $2.9(1)$ & 2.212 & Li21-C21 & $0.148(7)$ & $2.8(1)$ & 2.196 \\
Li1-C9 & $0.124(8)$ & $2.5(1)$ & 2.234 & Li21-C29 & $0.125(7)$ & $2.6(1)$ & 2.202 \\
Li1-C25 & $0.143(7)$ & $2.4(1)$ & 2.291 & Li21-C5 & $0.132(7)$ & $2.3(1)$ & 2.294 \\
Li2-C1 & $0.158(8)$ & $2.9(1)$ & 2.163 & Li22-C21 & $0.129(8)$ & $2.7(1)$ & 2.177 \\
Li2-C5 & $0.146(7)$ & $3.0(1)$ & 2.183 & Li22-C25 & $0.142(7)$ & $2.7(1)$ & 2.197 \\
Li2-C29 & $0.139(6)$ & $2.3(1)$ & 2.277 & Li22-C9 & $0.139(6)$ & $2.4(1)$ & 2.284 \\
Li3-C5 & $0.126(8)$ & $2.6(1)$ & 2.201 & Li23-C25 & $0.140(8)$ & $2.7(1)$ & 2.183 \\
Li3-C9 & $0.144(8)$ & $2.8(1)$ & 2.208 & Li23-C29 & $0.150(7)$ & $2.7(1)$ & 2.214 \\
Li3-C21 & $0.136(6)$ & $2.4(1)$ & 2.275 & Li23-C1 & $0.145(7)$ & $2.3(1)$ & 2.273
\end{tabular}

Focussing on the lithium-carbon bonds, similar $\rho\left(\mathbf{r}_{\mathrm{BCP}}\right)$ values are evident for all interactions under investigation ranging from 0.12 to $0.16 \mathrm{e}^{-3}$. These do not correlate with the bond path lengths, so that the short $\mathrm{Li}-\mathrm{C}$ bonds to the atoms of the lithium triangle basis do not consistently have higher values than the remaining bonds. Interestingly, smaller $\rho\left(\mathbf{r}_{\mathrm{BCP}}\right)$ values $\left(0.08-0.11 \mathrm{e} \AA^{-3}\right)$ are found throughout the $\mathrm{Li}-\mathrm{C}$ bonds in $\left[(\text { thf }) \mathrm{Li}_{2}\left\{\mathrm{H}_{2} \mathrm{CS}(\mathrm{N} t \mathrm{Bu})_{2}\right\}\right]_{2}$ that might be caused by the additional nitrogen atoms, which are better donor atoms than the deprotonated carbon atoms. Compared to 14, $\left[2-\left(\mathrm{Me}_{3} \mathrm{Si}\right)_{2} \mathrm{CPyLi}\right]_{2}$ (7) shows a similar $\rho\left(\mathbf{r}_{\mathrm{BCP}}\right)$ of $0.15 \mathrm{e} \AA^{-3}$ in the $\mathrm{Li}-\mathrm{C}$ bonds, but unlike the hexamer, this compound only forms a single directed lithium bond per carbanion. Accordingly, the carbanion strength in $\mathbf{1 4}$ has to be classified as superior to the picolyl one, because of the three similarly strong interactions to lithium cations per carbanion. Their $\rho\left(\mathbf{r}_{\mathrm{BCP}}\right)$ values are even in range of $\mathrm{Li}-\mathrm{N}$ interactions $\left(0.15 \mathrm{e} \AA^{-3}\right)$ 
found in [2-PicLi.PicH $]_{2}(\mathbf{4})$, so that the $\mathrm{Li}-\mathrm{C}$ interactions in the hexamer are of remarkable strength, even though the simple numbers of $\rho\left(\mathbf{r}_{\mathrm{BCP}}\right)$ appear quite low.

J. P. Ritchie and S. M. Bachrach studied a huge number of lithium organic compounds theoretically in 1987 and applied topological analyses according to the QTAIM to investigate the nature of the lithium-carbon bonds. ${ }^{[288]}$ One example was the methyllithium tetramer, for which he could reproduce the geometry in the crystal and also found $\mathrm{Li}-\mathrm{C}$ bond paths to all lithium atoms of the capped triangle. The determined $\rho\left(\mathbf{r}_{\mathrm{BCP}}\right)$ values of $0.15 \AA^{-3}$ nicely agree with those of 14. Furthermore, he could quantify bond paths between the carbanions, comparable to the $\mathrm{N}-\mathrm{N}$ interaction in [2-PicLi.PicH] $]_{2}$ (4). This could be excluded for $\left[\mathrm{TMSCH}_{2} \mathrm{Li}\right]_{6}(\mathbf{1 4})$. As in $[\mathrm{MeLi}]_{4}$, no Li-Li bonds were detected. Trajectory plots of two capped and one non-capped lithium face (Figure 3-12) show that the metal atom basins do not share a common zero flux surface, which prohibits a bond path formation between the cations.
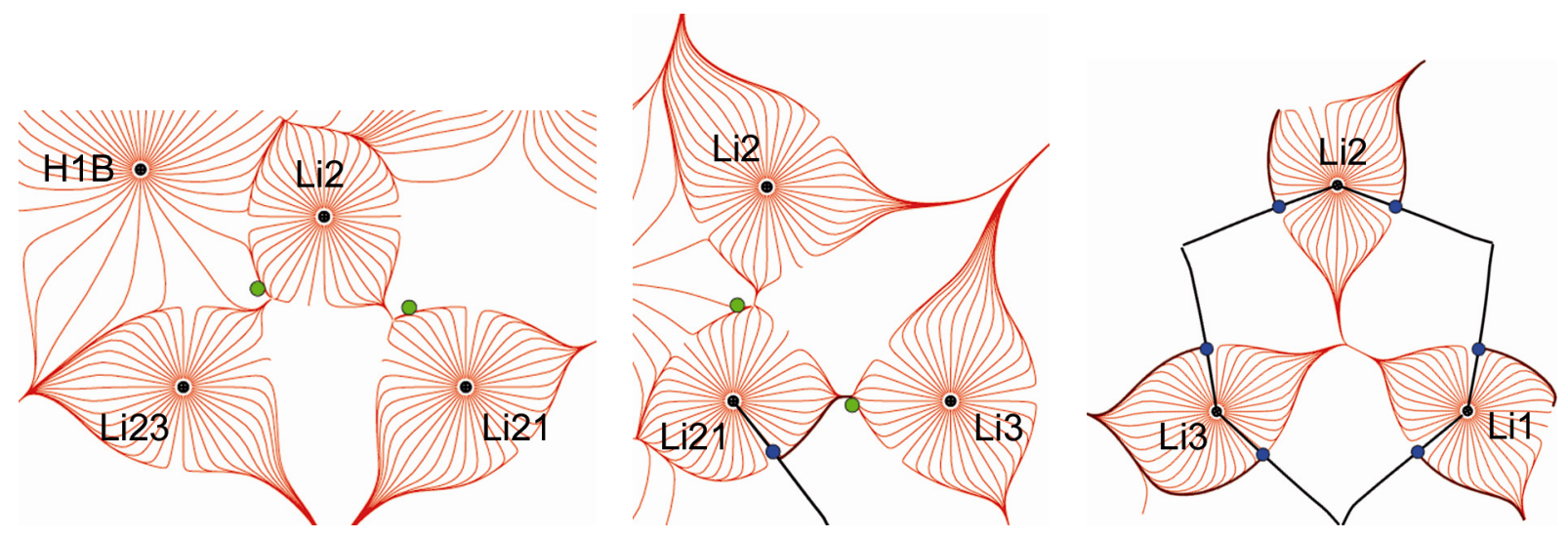

Figure 3-12. Trajectory plots of three $\mathrm{Li}_{3}$ faces in $\left[\mathrm{TMSCH}_{2} \mathrm{Li}\right]_{6}(\mathbf{1 4})$. Red lines are gradient vectors, while blue/green dots indicate BCPs/RCPs. Black lines refer to bond paths between atomic positions (black dots) or zero flux surface border lines.

However, C. Gatti et al. showed for planar lithium clusters $\left(\mathrm{Li}_{2}\right.$ to $\left.\mathrm{Li}_{6}\right)$ that this arrangement of non-neighbouring atomic basins can also be the result of nonnuclear attractors between the lithium atoms. ${ }^{[289]}$ Each basin in QTAIM includes one $(3,-3)$ critical point as attractor (cf. chapter 2.3.4), which is normally identical with the atomic position. Vice versa, local maxima in $\rho(\mathbf{r})$ were found in the middle of the triangular $\mathrm{Li}_{3}$ motifs in modelled $\mathrm{Li}_{4}, \mathrm{Li}_{5}$, and $\mathrm{Li}_{6}$ clusters. Accordingly, the basins associated with lithium atoms were separated by basins that do not include an atom. Bond paths are exclusively formed between the lithium atoms and the non-nuclear attractors and not directly between the lithium atoms 
(Figure 3-13, left). The origin of non-nuclear attractors is unknown, but was occasionally related to mobile metallic electrons. ${ }^{[289]}$

An inspection of $\rho(\mathbf{r})$ in the lithium triangles of $\left[\mathrm{TMSCH}_{2} \mathrm{Li}\right]_{6}$ (14) showed a local maximum in the electron density planes exemplarily displayed for Li1Li2Li23 in Figure 3-13 (right). Yet, the presence of a non-nuclear attractor could be excluded by plotting $\rho(\mathbf{r})$ between $\mathrm{C} 1$ and straight through the centre of the observed maximum. The electron density decreases monotonically along this line and towards the centre of the octahedron, so that the maximum in the contour plot is caused by the lone pair electron density directed to the space between the lithium cations. Hence, the electronic situation in the hexamer is not comparable to metal clusters of elemental lithium.
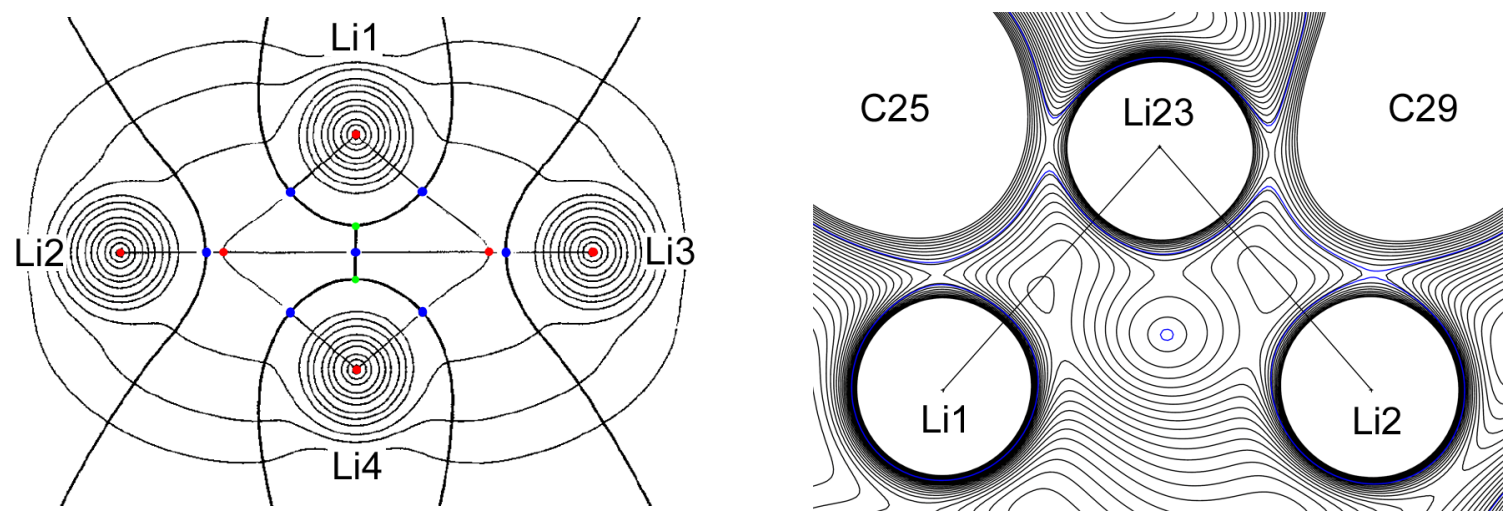

Figure 3-13. Left: $\rho(\mathbf{r})$ plot in a planar $\mathrm{Li}_{4}$ cluster including bond paths, $(3,-3)($ red), $(3,-1)$ (blue), and $(3,+1)$ (green) critical points. Right: $\rho(\mathbf{r})$ plot (step size $0.005 \mathrm{e}^{-3}$; blue line $=0.112 \mathrm{e \AA}^{-3}$ ) in the Li1Li2Li23 plane of $\left[\mathrm{TMSCH}_{2} \mathrm{Li}\right]_{6}(\mathbf{1 4})$.

In contrast to the more or less equal $\rho\left(\mathbf{r}_{\mathrm{BCP}}\right)$ values of the $\mathrm{Li}-\mathrm{C}$ bonds, a trend for the long $\mathrm{Li}-\mathrm{C}$ bonds is observed in $\nabla^{2} \rho\left(\mathbf{r}_{\mathrm{BCP}}\right)$. The longest bond shows the lowest positive value within each lithium face. All lithium-carbon bonds adopt values of prevailing ionic character, which can also be described by a Laplacian plot along the $\mathrm{Li}-\mathrm{C}$ bond paths (Figure 3-14). $\nabla^{2} \rho(\mathbf{r})$ is positive in the whole region of the bond critical point for all bonds and even shows a maximum for some of them, which is an indication for pronounced charge separation. Related $\nabla^{2} \rho(\mathbf{r})$ distributions were already found for the $\mathrm{Li}-\mathrm{N}$ bonds in [2-PicLi.PicH $]_{2}$ (4, Figure 2-36), the lithiated iminophosphorane $\left[\left(\mathrm{Et}_{2} \mathrm{O}\right) \cdot \mathrm{Li}\left\{\mathrm{Ph}_{2}(\mathrm{CHPy}) \mathrm{P}\left(\mathrm{NSiMe}_{3}\right)\right\}\right]$ $(6)^{[71]}$ and also the sulfur ylide $[$ (thf $\left.) \mathrm{Li}_{2}\left\{\mathrm{H}_{2} \mathrm{CS}(\mathrm{N} t \mathrm{Bu})_{2}\right\}\right]_{2}{ }^{[88]}$ including the $\mathrm{Li}-\mathrm{C}$ bonds. 

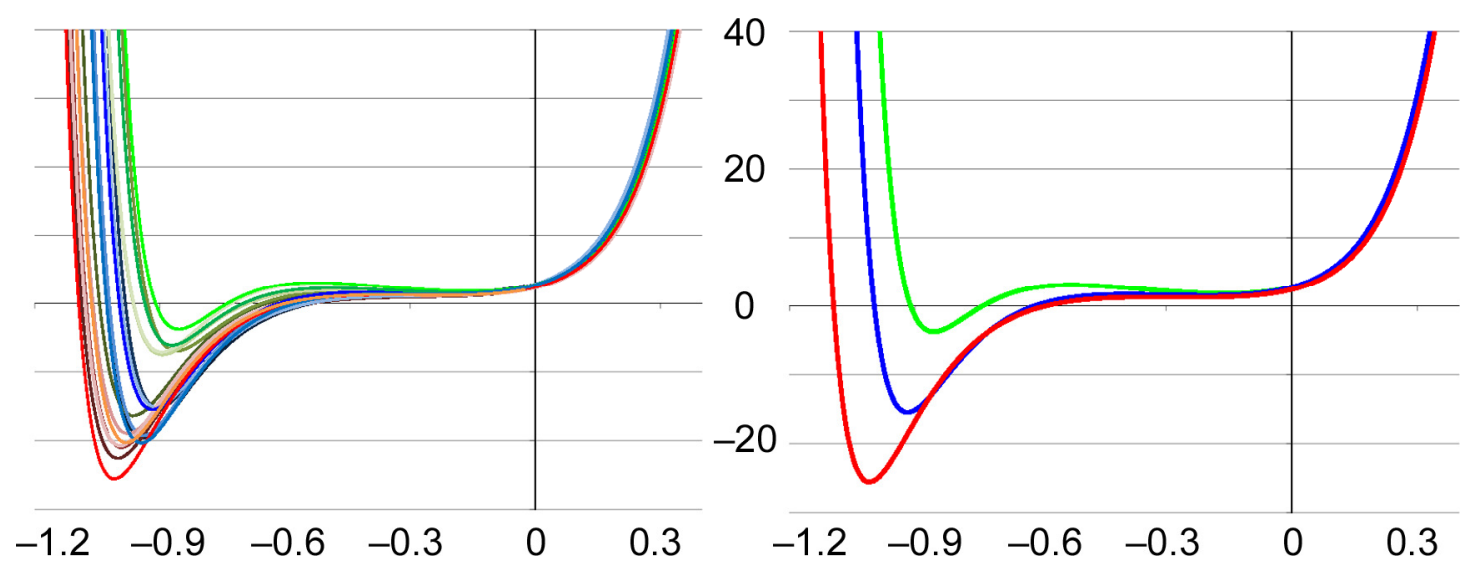

Figure 3-14. Laplacian $\left[\mathrm{e} \AA^{-5}\right]$ of the $\mathrm{Li}-\mathrm{C}$ bonds in $\left[\mathrm{TMSCH}_{2} \mathrm{Li}\right]_{6}(\mathbf{1 4})$ along the bond paths

$[\AA](0=\mathrm{BCP}$ with negative values towards the carbon atom; colour code: green shades $=\mathrm{Li} 1-\mathrm{C} 9 / \mathrm{Li} 2-\mathrm{C} 1 / \mathrm{Li} 3-\mathrm{C} 5$ etc. $;$ blue shades $=\mathrm{Li} 1-\mathrm{C} 1$ etc. $;$ red shades $=\mathrm{Li} 1-\mathrm{C} 25$ etc.; left: all bonds, right: Li22 exemplarily).

A systematic shape of the $\nabla^{2} \rho(\mathbf{r})$ graph can be distinguished for the three bond types (cf. chapter 3.2.3) by an investigation of all lithium bonds. These are "straight" $\mathrm{Li}-\mathrm{C}$ bonds (e.g. Li1-C9; $\mathrm{Li}-\mathrm{C}-\mathrm{Si}: ~ 180^{\circ}$ ), "rectangular" ones (e.g. $\mathrm{Li} 1-\mathrm{C} 1$; $\mathrm{Li}-\mathrm{C}-\mathrm{Si}: \sim 90^{\circ}$ ), and longer $\mathrm{Li}-\mathrm{C}$ interactions (e.g. Li1-C25). They are colour-coded in Figure 3-14 (left), and even though the figure might look confusing on the first sight, a trend can be recognised. This is further clarified by one example of three different interactions to one lithium atom (Figure 3-14, right). The $\mathrm{Li}-\mathrm{C}$ bonds, for which $\mathrm{Li}-\mathrm{C}-\mathrm{Si}$ bond angles close to $180^{\circ}$ are observed ("straight" $\mathrm{Li}-\mathrm{C}$ bonds, green shades), show the most distinct charge separation - mirrored by a maximum in $\nabla^{2} \rho(\mathbf{r})$ - with just a small charge accumulation in the vicinity of the carbon atom. The bonds to the lithium atoms, in which the Si$\mathrm{C}_{\alpha}$ bonds are orientated ("rectangular" $\mathrm{Li}-\mathrm{C}$ bonds, blue shades) and form bond angles close to $90^{\circ}$, feature higher charge accumulations at the deprotonated carbon atoms along the bond and less charge separation (almost horizontal course of $\nabla^{2} \rho(\mathbf{r})$ from BCP and midway to the CC). Within the longer Li-C interactions, similar $\nabla^{2} \rho(\mathbf{r})$ courses in respect to the "rectangular" $\mathrm{Li}-\mathrm{C}$ bonds are evident, while the largest negative values of the Laplacian in the carbon basins are observed. This might seem controversial to the largest $\mathrm{Li}-\mathrm{C}$ bond path lengths. However, this could be the reason for the similar electron density values at the Li-C BCPs. The charge accumulations at the $\mathrm{C}_{\alpha}$ atoms - the lone pairs are pointing towards the lithium atoms at the top of the isosceles lithium triangles.

As a consequence, the lone pairs (LPS) at the deprotonated carbon atoms should be orientated likewise. A search for VSCCs around the carbon atoms 
under investigation showed four charge concentrations for each of them: one in the direction of the silicon atom, two to the methylene hydrogen atoms, and one lone pair facing the lithium triangle. Once again, the term "Ione pair" - associated with non-bonding charge concentration - is wrong in this case due to the determined bond paths to the metal atoms. However, it is used for the sake of the established vocabulary of preparative chemists. The positions of the VSCCs are marked as green dots in Figure 3-15 and a deviation from the line to the centre of the lithium triangles can be observed for the lone pairs.
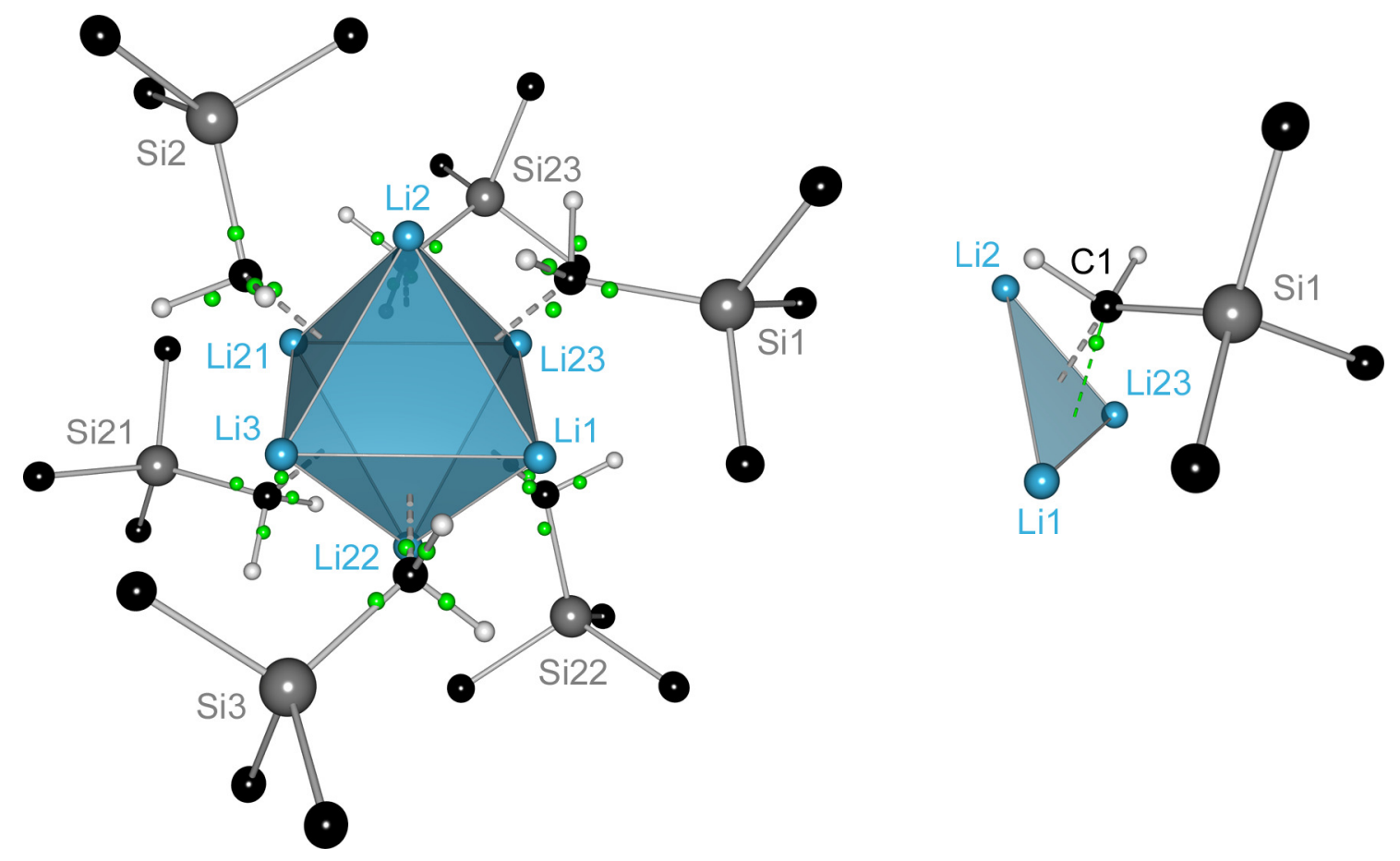

Figure 3-15. Left: Positions of the VSCCs (green dots) around the deprotonated carbon atoms in $\left[\mathrm{TMSCH}_{2} \mathrm{Li}\right]_{6}(\mathbf{1 4})$; Right: Lone pair at $\mathrm{C} 1$ and its projection to the lithium atom plane (thin dashed green line) relative to the centre of the triangle (dashed grey line).

Exemplarily, the line between C1 and the LP was extended to the Li1Li2Li23 plane in order to visualise its direction. Interestingly, it is deflected towards the

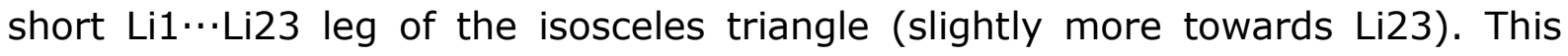
could be the reason why similar high $\rho\left(\mathbf{r}_{\mathrm{BCP}}\right)$ values are observed for all $\mathrm{Li}-\mathrm{C}$ bonds. The lithium atom with the longest $\mathrm{Li}-\mathrm{C}$ distance (Li23) does well interact with the lone pair, which is in accordance with the results from the inspection of the Laplacian distribution along the bond paths (Figure 3-14). The related lone pairs in the lithium sulfur ylide $\left[(\text { thf }) \mathrm{Li}_{2}\left\{\mathrm{H}_{2} \mathrm{CS}(\mathrm{N} t \mathrm{Bu})_{2}\right\}\right]_{2}$ also do not face the centre of the triangle, but point to only one lithium atom in contrast to $\mathbf{1 4}$ (Figure 1-7). ${ }^{[88]}$ 
The orientation of the VSCCs around each deprotonated carbon atom indicates a clear $\mathrm{sp}^{3}$ hybridisation. Even the concepts of the valence shell electron pair repulsion (VSEPR) theory ${ }^{[290-292]}$ seems to be obeyed. The angles between the LP and the remaining BCCs are generally higher than the ideal tetrahedral angle showing the increased spatial requirement of a lone pair (C1: $110.0-115.9^{\circ}$; C5: $110.7-117.9^{\circ}$; C9: $\left.106.8-121.0^{\circ}\right)$. As a result, a standard hybridisation state is adopted for the carbanions, even though they are involved in six bonds to neighbouring atoms. The carbon atoms are consequently not hypervalent as might be concluded by geometry considerations. The forth non-standard carbon interaction to three lithium cations is hence best described as a four centre two electron bond $(4 \mathrm{c}-2 \mathrm{e})$ following the nomenclature in borane chemistry.
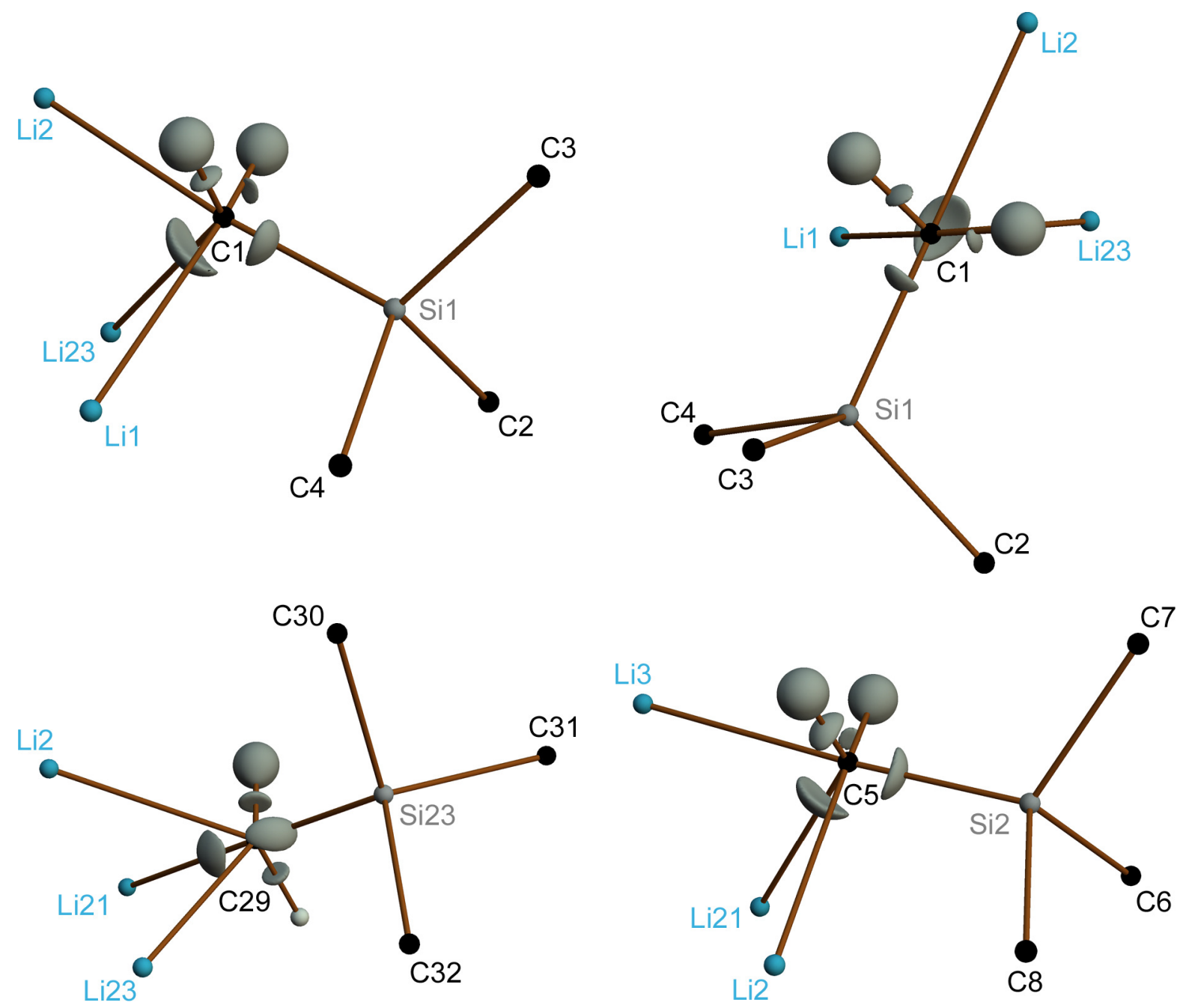

Figure 3-16. $\nabla^{2} \rho(\mathbf{r})$ isosurface representation of $\mathrm{C} 1$ (top, in two alternative orientations), C29 (bottom left), and C5 (bottom right) on a level of $-15 \mathrm{e} \AA^{-5}$. 
The orientation and spatial expansion of the VSCCs at the carbanions can be shown by an isosurface representation of $\nabla^{2} \rho(\mathbf{r})$ on a level of $-15 \mathrm{e} \AA^{-5}$ for the lithium triangles in the $\left[\mathrm{TMSCH}_{2} \mathrm{Li}\right]_{6}$ octahedron (half of the capped $\mathrm{Li}_{6}$ faces shown in Figure 3-16). The values of $\nabla^{2} \rho\left(\mathbf{r}_{\mathrm{LP}}\right)$ range from -21.5 to -28.3 e $\AA^{-5}$ for the deprotonated carbon atoms, which is more charge accumulation than in the $\mathrm{sp}^{3}$ hybridised carbanions in $\left[2-\left(\mathrm{Me}_{3} \mathrm{Si}\right)_{2} \mathrm{CPyLi}\right]_{2}$ (7) (-18 e $\AA^{-5}$; Figure 2-34, right). ${ }^{[221]}$ The related lone pairs in the sulfur ylide have $-23 \mathrm{e} \AA^{-5}$ and are well comparable to those of $14 .^{[88]}$ A higher $\nabla^{2} \rho\left(\mathbf{r}_{\mathrm{LP}}\right)$ value for an LP was only found for the carbanion in [tBuLi.(sp)] with $-34 \mathrm{e}^{-5}{ }^{[220,293]}$ This is not surprising, since the methyl substitution increases the electron density (inductive effect) at the deprotonated carbon atom that interacts only with a single lithium cation. It should be attempted in future work to scale the $\nabla^{2} \rho\left(\mathbf{r}_{\mathrm{LP}}\right)$ value to the reactivity of the parent compound. However, very little information is currently available on the electron density distributions of lithium organic compounds. This study may be the basis for a complete series of trimethylsilylmethyllithium molecules in different aggregation states, which can be investigated with experimental charge density methods. Suitable crystals for that task are already synthesised, but not even the experimental reactivity differences are known yet.

Focussing on the integration of all atomic basins in $\left[\mathrm{TMSCH}_{2} \mathrm{Li}\right]_{6}(\mathbf{1 4})$, the charges add up to zero and the electroneutrality of the compound is obeyed. The lithium atoms are in fact cations with a mean charge of +0.85 e (values range from +0.83 to $+0.86 \mathrm{e})$. Therefore, they are slightly less positive than in [2-PicLi.PicH $]_{2}(4,+0.93 \mathrm{e}),\left[(\text { thf }) \mathrm{Li}_{2}\left\{\mathrm{H}_{2} \mathrm{CS}(\mathrm{NtBu})_{2}\right\}\right]_{2}(+0.92 \mathrm{e})^{[88]}$ and close to the value of $\left[\left(\mathrm{Et}_{2} \mathrm{O}\right) \cdot \mathrm{Li}\left\{\mathrm{Ph}_{2}(\mathrm{CHPy}) \mathrm{P}\left(\mathrm{NSiMe}_{3}\right)\right\}\right](6,+0.87 \mathrm{e}) .{ }^{[71]} \mathrm{A}$ chargecontrolled nature of the $\mathrm{Li}-\mathrm{C}$ interaction can accordingly be assumed. However, a back donation of charge from the carbanions cannot be completely excluded. A survey of earlier theoretical QTAIM investigations on lithium organic compounds reveals values between +0.90 and +0.93 e for monomers and $+0.91 \mathrm{e}$ for $[\mathrm{MeLi}]_{4} \cdot{ }^{[288,294-297]}$ Furthermore, a charge of $+0.87 \mathrm{e}$ was determined for the cation in lithium fluoride as paradigm for a lithium containing inorganic salt. ${ }^{\text {[298] }}$ In summary, all examples presented here show no complete charge separation between the cations and the anions so that a proclamation of completely ionic $\mathrm{Li}-\mathrm{C}$ bonding situations would go too far. Nonetheless, a predominant ionic charge interaction with inferior covalent contributions seems to describe the lithium bonds best.

This is especially true since the deprotonated carbon atoms exhibit extraordinarily high negative charges. A mean value of $-1.57 \mathrm{e}$ is not just an outcome of 
the hydrogen abstraction but does also originate from the neighbouring polarisable silicon atoms (mean charge: $+2.15 \mathrm{e}$ ). The methylene hydrogen atoms contribute about +0.09 e each so that a group charge of $-1.39 \mathrm{e}$ is still concentrated on the $\mathrm{CH}_{2}$ fragment interacting with the lithium cations. A comparison with the corresponding anionic carbon atom of the lithium sulfur ylide supports the assumption of a large neighbouring atom effect in $\left[\mathrm{TMSCH}_{2} \mathrm{Li}\right]_{6}$ (14). The integrated charge is -0.78 e and accordingly half the value determined in the hexamer. [2-( $\left.\left.\mathrm{Me}_{3} \mathrm{Si}\right)_{2} \mathrm{CPyLi}\right]_{2}(\mathbf{7})$ is another related compound and fits the situation in $\mathbf{1 4}$ even better, because two trimethylsilyl groups are connected to the deprotonated carbon atom. However, integrated charges were neither reported for the carbanion nor for the silicon atoms. Nevertheless, the net charges signalise the same tendency, even though they are strongly model dependent and do not mirror polarisation effects that seem to be most important in the presented case. The magnitudes of the concentrated charges at the $\mathrm{C}_{\alpha}$ atoms are even more remarkable with regard to the observed heteroatomic charges in [2-PicLi.PicH $]_{2}$ (4). The more electronegative nitrogen atoms "only" feature charges around $-1 \mathrm{e}$. Another example of a carbanion in the environs of a silicon atom will thoroughly be presented in chapter 4 .

The effects of the polarisable semimetal atom Si on the neighbouring groups are also manifested at the methyl group carbon atoms. The charge of each trimethylsilyl carbon atom ranges from -0.61 to $-0.81 \mathrm{e}$, while the group charges including those of the hydrogen atoms vary from -1.50 to $-1.65 \mathrm{e}$. Thus, the electron deficiency of the silicon atom (mean charge: $+2.15 \mathrm{e}$ ) is largely caused or counterbalanced by the methyl groups. The charge is in fact evenly distributed over all four carbon atoms. Accordingly, the deprotonation at the $\mathrm{C}_{\alpha}$ atoms has no special impact on the silicon atom.

By taking a closer look at the methyl group charges, a systematic distribution is detected. The mean charge (averaged over all six anions) for each of the three individual methyl carbon atoms is between -0.65 and -0.68 e for those pointing into the direction of the lone pair, while the trans orientated methyl group carbon atoms exhibit a charge of $-0.81 \mathrm{e}$. This is most probably caused by an effect that is commonly referred to as negative hyperconjugation, even though the increased negative charge at $\mathrm{C}_{\text {trans }}$ is not comprehensible with the standard molecular orbital model, with which this interaction is normally clarified (Scheme 3-2). Additional evidence for the presence of this stabilising effect on the carbanion will be given further below. 


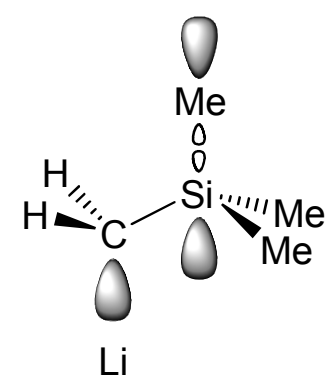

Scheme 3-2. Negative hyperconjugation in $\left[\mathrm{TMSCH}_{2} \mathrm{Li}\right]_{6}(\mathbf{1 4})$ : molecular orbital model of the carbanion stabilisation (Ione pair lobe to $\mathrm{Li}$ ) by the $\sigma^{*}$ orbital of the trans orientated Si-Me bond.

The observed charge depletion at all silicon atoms is also reflected in the Laplacian distribution around the heteroatoms. Large areas of positive $\nabla^{2} \rho(\mathbf{r})$ are evident in the silicon basins - and accordingly no VSCCs -exemplarily shown for $\mathrm{Si2}$ in Figure 3-17. Similar acute charge depletions were already reported for $\left[2-\left(\mathrm{Me}_{3} \mathrm{Si}\right)_{2} \mathrm{CPyLi}\right]_{2}(\mathbf{7}){ }^{\left[{ }^{[87]}\right.}$ cyclotetrasilazanes, ${ }^{[299]}$ and a hexacoordinated silicon complex, ${ }^{[183]}$ in which an even larger integrated charge of $+2.78 \mathrm{e}$ was determined for the silicon atom. However, all these atoms exhibited a spherical symmetrical Laplacian shape, while $\mathbf{1 4}$ features the most dented $\nabla^{2} \rho(\mathbf{r})$ distribution in this series.
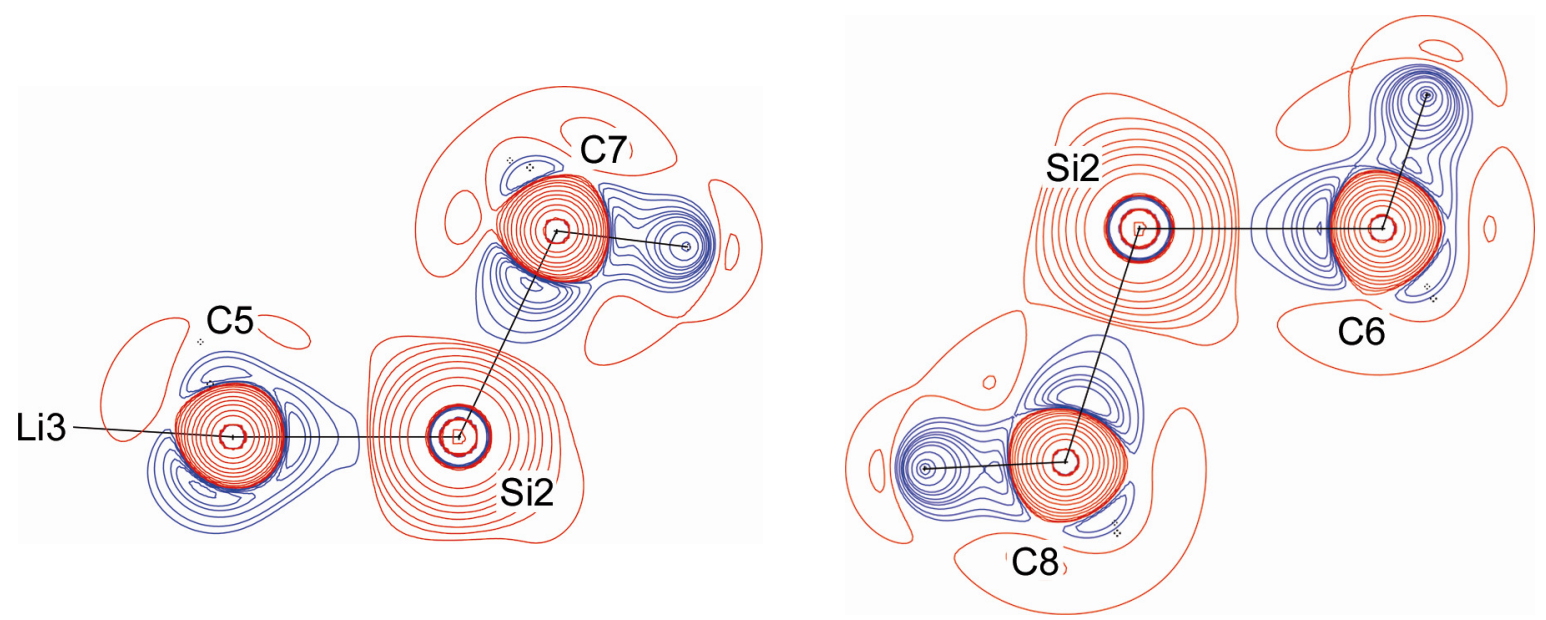

Figure 3-17. Laplacian distribution in the C5-Si2-C7 plane (left) and the C6-Si2-C8 plane (right) of $\left[\mathrm{TMSCH}_{2} \mathrm{Li}\right]_{6}(\mathbf{1 4})$. Negative values of $\nabla^{2} \rho(\mathbf{r})$ are depicted by blue and positive by red lines. Contours are drawn at $\pm 2.0 \times 10^{n},-12,-15,-30, \pm 4.0 \times 10^{n}$, $\pm 8.0 \times 10^{\mathrm{n}} \mathrm{e}^{-5}$ with $\mathrm{n}=0,1,2,3$.

The silicon atoms in $\mathbf{1 4}$ accordingly have less ionic character than in the other three mentioned compounds. The connected carbon atoms in $\mathbf{1 4}$ on the other hand reflect an even more polarised character of the $\mathrm{Si}-\mathrm{C}$ bonds compared to $\left[2-\left(\mathrm{Me}_{3} \mathrm{Si}\right)_{2} \mathrm{CPyLi}\right]_{2}(\mathbf{7})$ and octamethylcyclotetrasilazane. ${ }^{[299]}$ The BCC shapes 
towards the silicon atoms resemble lone pair charge accumulations rather than standard shared interactions (cf. Figure 2-35).

The electron density values at the $\mathrm{Si}-\mathrm{C}$ bond critical points are also influenced by the electron deficiency of the heteroatoms. Almost halved $\rho\left(\mathbf{r}_{\mathrm{BCP}}\right)$ values are observed in comparison to standard carbon-carbon single bonds (cf. chapter 2.3.7). These range from $0.75(3)$ to $1.00(3) \mathrm{e}^{-3}$ for the $\mathrm{Si}-\mathrm{C}$ bonds in $\left[\mathrm{TMSCH}_{2} \mathrm{Li}\right]_{6}(\mathbf{1 4})$ with a mean value of $0.87 \mathrm{e}^{-3}$. No consistent distribution for specific bonds could be detected, although the $\mathrm{Si}-\mathrm{C}_{\alpha}$ bonds show the highest $\rho\left(\mathbf{r}_{\mathrm{BCP}}\right)$ values with $0.91 \mathrm{e} \AA^{-3}$ on average. The putatively weakened bonds trans to the lone pairs at $C_{\alpha}$ (negative hyperconjugation) on the other hand demonstrate no peculiarities with a mean $\rho\left(\mathbf{r}_{\mathrm{BCP}}\right)$ value of $0.87 \mathrm{e}^{-3}$.

Additionally, even higher variations are evident for the corresponding $\nabla^{2} \rho\left(\mathbf{r}_{\mathrm{BCP}}\right)$ values $\left(-5.4(1)\right.$ to $\left.2.7(1) e \AA^{-5}\right)$. The bond critical points of the silicon-carbon bonds are lying in the intermediate bond region, where the outer sphere of the bonding charge concentrations of the carbon atoms interchange with charge depleted areas of the silicon atoms (Figure 3-17). This can be further clarified by the graphical representation of the Laplacian distributions along the bonds paths (Figure 3-18).
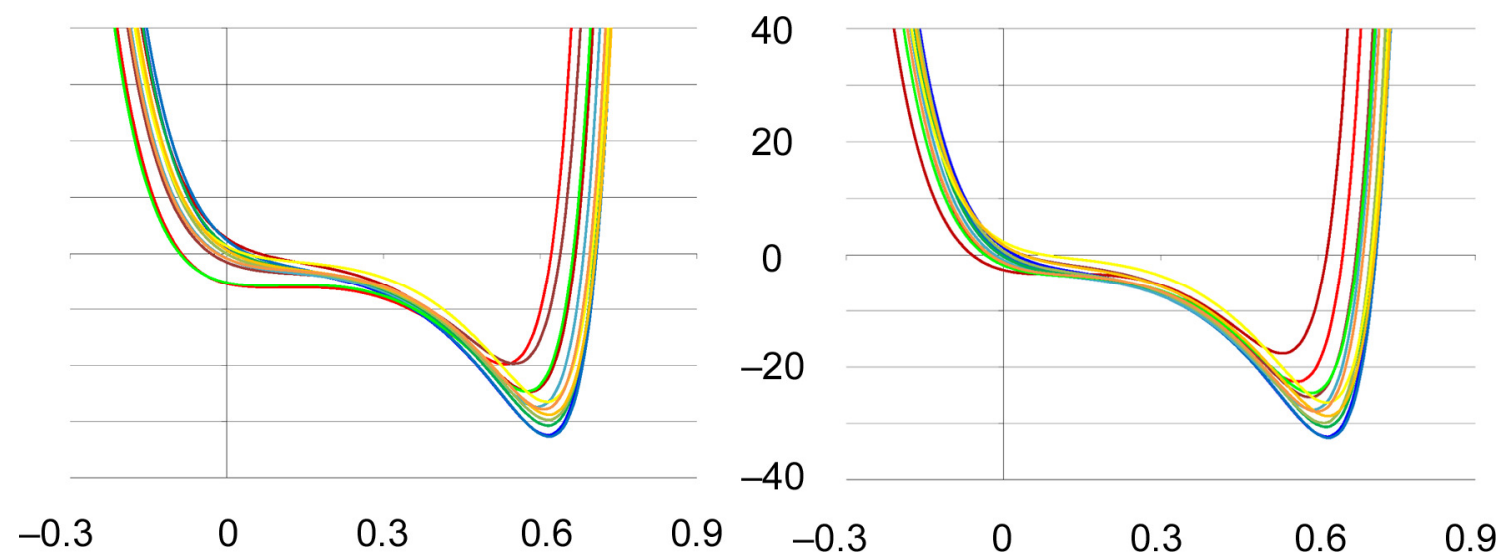

Figure 3-18. $\nabla^{2} \rho(\mathbf{r})\left[\mathrm{e} \AA^{-5}\right]$ along the $\mathrm{Si}-\mathrm{C}$ bond paths $[\AA]$ in $\left[\mathrm{TMSCH}_{2} \mathrm{Li}\right]_{6}$ (14) (left: upper half of the octahedron; right: lower half; $0=\mathrm{BCP}$; Si basin to negative $\mathrm{x}$ values). Colour code: red shades $=\mathrm{C} 1$ (or related bonds); blue $=\mathrm{C} 3\left(\mathrm{C}_{\text {trans }}\right)$; green $=\mathrm{C} 2$; yellow $=\mathrm{C} 4$.

As expected, $\nabla^{2} \rho(\mathbf{r})$ rapidly changes around the bond critical points for all silicon bonds. In summary, the same trend of a strongly polarised covalent bond can be recognised for all Si-C bonds. The sign of $\nabla^{2} \rho(\mathbf{r})$ changes near the BCP, while $\nabla^{2} \rho(\mathbf{r})$ is negative in the whole carbon basin except in the vicinity of the bond critical point. The only differences that can be rationalised are the dissimilar bond lengths and the absolute values of the BCCs at the carbon atoms. These 
show that the methylene carbon atoms exhibit the lowest $\nabla^{2} \rho\left(\mathbf{r}_{\mathrm{vscc}}\right)$ values to the silicon atoms of all bonds under investigation.

Consequently, the examination of $\nabla^{2} \rho(\mathbf{r})$ is inadequate to describe the effect of negative hyperconjugation in $\left[\mathrm{TMSCH}_{2} \mathrm{Li}\right]_{6}(\mathbf{1 4})$. The bond lengths of the possibly involved $\mathrm{Si}-\mathrm{C}$ bonds as well as their orientation relative to each other already favour this bond reinforcement explanation. However, the shortening of the methylene carbon bond can also be attributed to electrostatic effects. Therefore, $W$. Scherer et al. used a combination of properties derived from the electron density distribution to investigate the occurrence of negative hyperconjugation. ${ }^{[87]}$

First, they took the reduced VSCC value of the carbon lone pair $\left(-18.0 \mathrm{e} \AA^{-5}\right)$ compared to the BCC to the silicon atom $\left(-26.4 \mathrm{e} \AA^{-5}\right)$ as an indicator for a charge delocalisation by conjugation effects. Yet, the quantified values for the lone pair VSCCs in $\mathbf{1 4}$ are larger than those of the BCCs in four out of six cases. Moreover, the mean difference between the CCs is only about $3 \mathrm{e} \AA^{-5}$.

Second, they found that the LP is diffuse towards the BCC to the silicon atom in two and three dimensional representations of $\nabla^{2} \rho(\mathbf{r})$ around the carbanion. Once again, a similar situation is not present in the hexamer, because the VSCCS exhibit a $\mathrm{sp}^{3}$ hybridised arrangement and are well separated (Figure 3-16). Nevertheless, deformation density plots around the fragments under investigation $\left(\mathrm{C}_{\alpha}-\mathrm{Si}-\mathrm{C}_{\text {trans }}\right)$ - exemplarily shown for $\mathrm{C} 25$ in Figure 3-19 - display connected electron density between the lone pair, the $\mathrm{C}_{\alpha}-\mathrm{Si}$, and the $\mathrm{Si}-\mathrm{C}_{\text {trans }}$ bond.

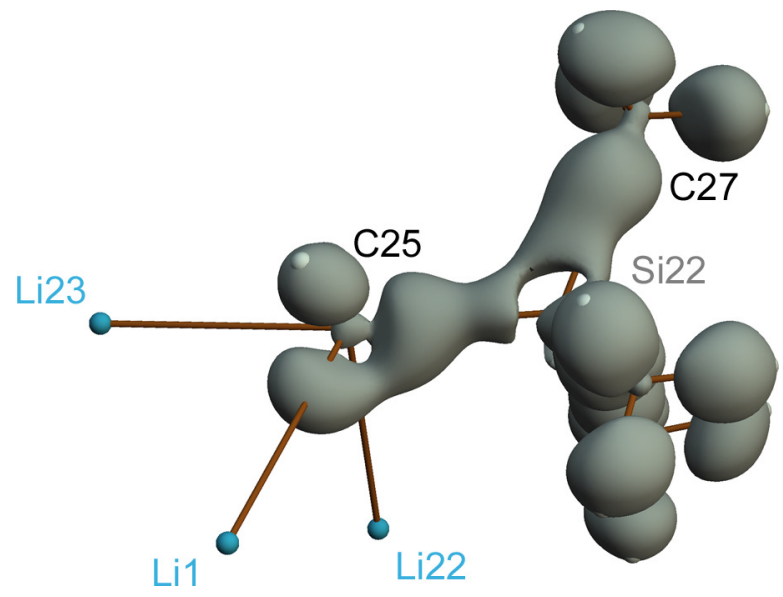

Figure 3-19. Deformation density representation of the trimethylsilyl group around Si22 in $\left[\mathrm{TMSCH}_{2} \mathrm{Li}\right]_{6}(\mathbf{1 4})$ on a level of $0.20 \mathrm{e}^{-3}$. 
Finally, W. Scherer et al. investigated the course of the bond ellipticities along the $\mathrm{Si}-\mathrm{C}_{\alpha}$ paths and compared them to theoretically calculated reference systems (Figure 3-20). These were methylsilane $\left(\mathrm{H}_{3} \mathrm{C}-\mathrm{SiH}_{3}\right)$, the corresponding anion $\left(\mathrm{H}_{2} \mathrm{C}-\mathrm{SiH}_{3}{ }^{-}\right)$, and methylenesilane $\left(\mathrm{H}_{2} \mathrm{C}=\mathrm{SiH}_{2}\right)$. Hence, model compounds for a standard silicon-carbon single and double bond were investigated, as well as a related carbanion without counterbalancing cation. The single bond is perfectly symmetrical along the bond path and $\varepsilon(\mathbf{r})$ values of zero are observed as expected. The heteroatomic double bond, in contrast, shows pronounced $\varepsilon(\mathbf{r})$ values in the bonding region with a distinct maximum near the bond critical point. The course of a standard carbon-carbon double bond is in comparison completely symmetric in respect to the BCP with generally lower ellipticity values (cf. chapter 2.3.7).

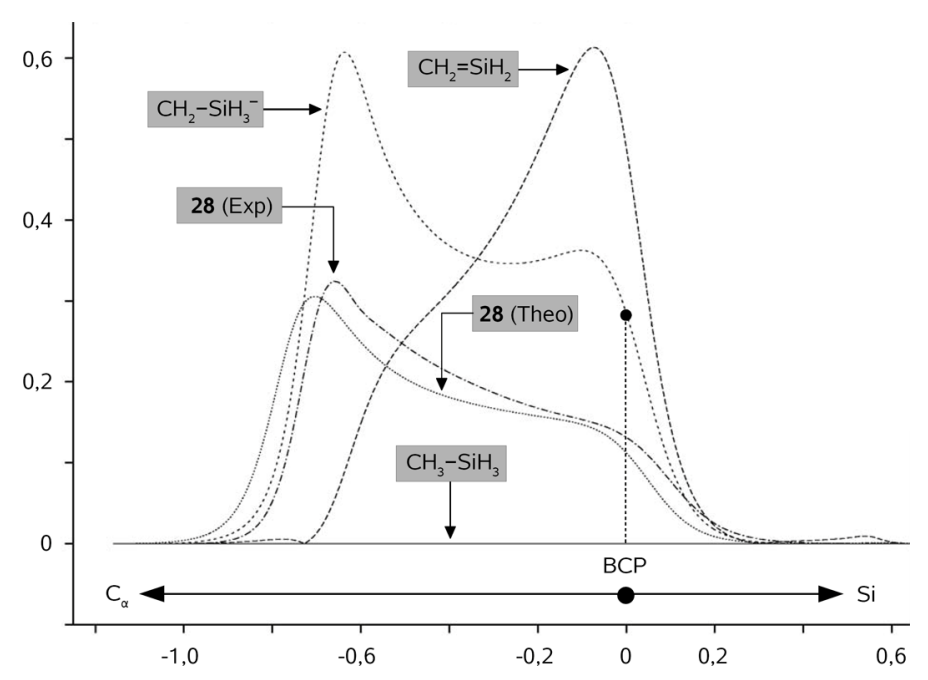

Figure 3-20. Bond ellipticities along the bond paths $[\AA]$ in $\left[2-\left(\mathrm{Me}_{3} \mathrm{Si}\right)_{2} \mathrm{CPyLi}\right]_{2}(\mathbf{7}$; labelled as $\mathbf{2 8}$ in the original publication) and related model compounds. ${ }^{[221]}$

The deprotonated methylsilane on the other hand exhibits a high $\varepsilon(\mathbf{r})$ value in the carbon basin that is commonly observed for isolated carbanionic $\mathrm{C}-\mathrm{C}$ bonds. ${ }^{[87]}$ The height of this maximum in the symmetrical $\mathrm{C}-\mathrm{C}$ case is reduced if an appropriate counterion is present. However, a second maximum on a lower level near the BCP appears for the $\mathrm{Si}-\mathrm{C}_{\alpha}$ bond in the deprotonated silane. This was interpreted as a sign for a partial double bond character originating from negative hyperconjugation in the anion. The experimental molecule under investigation, $\left[2-\left(\mathrm{Me}_{3} \mathrm{Si}\right)_{2} \mathrm{CPyLi}\right]_{2}(\mathbf{7})$, adopted a similar - although less pronounced distribution of $\varepsilon(\mathbf{r})$, so that a bond reinforcement by negative hyperconjugation was assumed. 
A related examination of the ellipticities along all $\mathrm{Si}-\mathrm{C}$ bond paths was performed for $\left[\mathrm{TMSCH}_{2} \mathrm{Li}\right]_{6}(\mathbf{1 4})$ in order to compare them to the results of W. Scherer et al. ${ }^{[87]}$ The $\mathrm{Si}-\mathrm{C}_{\alpha}$ bonds, printed in bold red shades in Figure 3-21, display remarkable similarities to $\left[\mathrm{H}_{2} \mathrm{C}-\mathrm{SiH}_{3}\right]^{-}$(Figure 3-20). All bonds feature a distinct maximum in the carbon atom basin and four out of six also a smaller second one in the $\mathrm{BCP}$ region. The remaining two $\mathrm{Si}-\mathrm{C}_{\alpha}$ graphs look more like the one for $\left[2-\left(\mathrm{Me}_{3} \mathrm{Si}\right)_{2} \mathrm{CPyLi}\right]_{2}$ (7) with only a slight $\varepsilon(\mathbf{r})$ variation in the vicinity of the bond critical point. Consequently, the same conclusions can be drawn for $\left[\mathrm{TMSCH}_{2} \mathrm{Li}\right]_{6}$ (14) as for the model compound 7. Negative hyperconjugation is in fact playing an important role in the stabilisation of the negative charge at the deprotonated carbon atoms in the hexamer. This leads to a shortening of the silicon bonds to the methylene carbon atoms and an elongation of the trans orientated $\mathrm{Si}-\mathrm{C}$ bond.

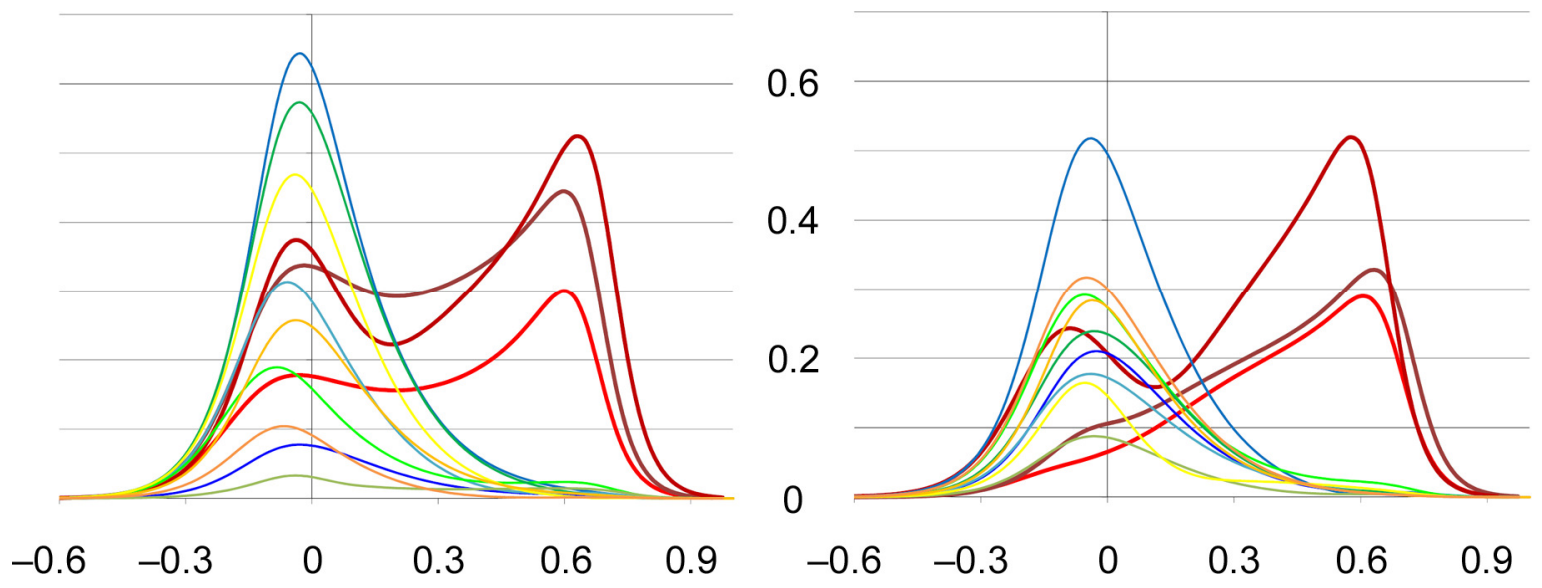

Figure 3-21. Bond ellipticities along the Si-C bond paths $[\AA]$ in $\left[\mathrm{TMSCH}_{2} \mathrm{Li}\right]_{6}$ (14) (left: upper half of the octahedron; right: lower half; $0=B C P$; Si basin to negative $x$ values; same colour code used as in Figure 3-18).

Interestingly, the $\mathrm{Si}-\mathrm{C}_{\mathrm{Me}}$ bonds barely resemble the ideal $\varepsilon(\mathbf{r})$ distribution of methylsilane $\left(\mathrm{H}_{3} \mathrm{C}-\mathrm{SiH}_{3}\right) \cdot \varepsilon(\mathbf{r})$ is rather symmetrically distributed in respect to the BCPs, but some bonds show quite high ellipticity values near the critical points. No systematic pattern could be detected for the three distinguishable methyl groups so that the reason for this phenomenon is unknown. Discrepancies of the model due to chemical or local symmetry constraints of the multipole parameters might cause a slight deformation of the Laplacian along the bond paths resulting in pronounced ellipticity values. Nevertheless, these constraints were maintained to limit the number of refined parameters and stabilise the multipole refinement. 
The carbanions and their neighbouring non-hydrogen atoms on the other hand were described without any constraints (cf. chapter 3.2.2).

Even though the methyl carbon bond characteristics deviate from the expected values, the common course of the $\mathrm{Si}-\mathrm{C}_{\alpha}$ bonds is evident. $\mathrm{A} \varepsilon(\mathbf{r})$ maximum in the carbon atom basin exactly in the same distance from the BCP as in the reference graph for carbanions (Figure 3-20) is observed in $\left[\mathrm{TMSCH}_{2} \mathrm{Li}\right]_{6}$ (14). Moreover, a second maximum close to the bond critical point resembles the situation in silicon-carbon double bonds and indicates a partial multiple bond character of those bonds. This can be explained by the interaction of the lone pair ( $\mathrm{sp}^{3}$ orbital facing an $\mathrm{Li}_{3}$ triangle) with the $\sigma^{*}$ molecular orbital of the Si-C $\mathrm{C}_{\text {trans }}$ bond (Scheme 3-2).

\subsection{Conclusion}

Trimethylsilylmethyllithium was crystallised in two modifications of which one was suitable for an experimental charge density study. Therefore, it was possible to gain detailed information on the bonding situation in a hexameric lithium organic compound showing the standard $\mathrm{Li}_{3}-\mathrm{C}$ motif. The main interactions of the carbanions with the lithium cations were identified to be made up by a lone pair facing the lithium triangle. This leads to a bond path of the $\mathrm{C}_{\alpha}$ atoms to each of the three lithium atoms of each capped $\mathrm{Li}_{3}$ triangle. However, it was shown that the bonding is not exclusively controlled by orbital interactions but strongly influenced by the charge localised on the deprotonated carbon atom. The Li-C bonds were thus identified as predominantly ionic with only subsidiary covalent contributions. Furthermore, no indications for potential agostic $\mathrm{Li}-\mathrm{H}$ contacts were detected. The small $\mathrm{Li}-\mathrm{H}$ distances are first and foremost the result of the orientation of the trimethylsilylmethyl groups with respect to the lithium octahedron and the staggered arrangement of all substituents throughout the anions. This conformation is controlled by the lone pairs at the carbanions, which interact with the $\sigma^{*}$ molecular orbital of the silicon-carbon bond in trans position. The anion is hence further stabilised by negative hyperconjugation. 


\section{A Chiral Benzyllithium Derivative}

\subsection{Asymmetric Lithium Organic Compounds}

The synthesis of enantiomerically or diastereomerically enriched lithium organic compounds, in which the metallated carbon atom is a stereogenic centre, has been intensively studied for more than 20 years. ${ }^{[26,27,300-305]}$ In general, they have been synthesised by deprotonation reactions employing strong lithium bases like butyllithium. Although the configuration of the metallated chiral carbon atom is only stable at low temperatures $\left(-78^{\circ} \mathrm{C}\right)$ for most of these compounds, D. Hoppe and C. Strohmann were able to characterise key compounds that maintain their configuration at higher temperatures at least for minutes. ${ }^{\text {[306-309] }}$

A frequently proposed mechanism for the racemisation implies an intermediate $\mathrm{sp}^{2}$ hybridised carbanion in a planar environment $(\mathbf{C})$, which proceeds via separated ion pairs (B and ent-B) shown in Scheme 4-1.

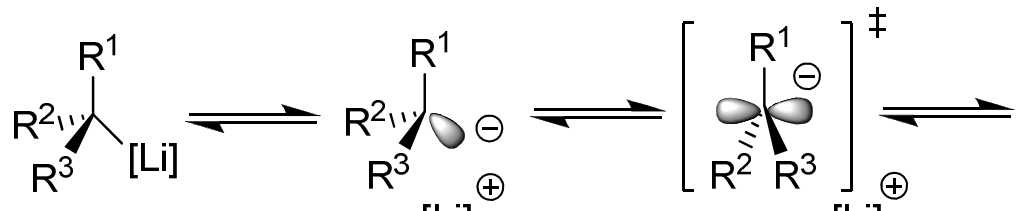

[Li]
[Li]<smiles>[R]C([R])([R])[C-]C</smiles>

[Li]

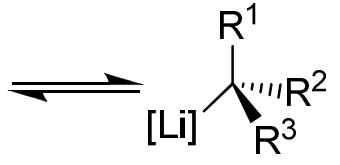
C
ent-B
ent-A
A
B
C

fithium organic compounds bearing a stereogenic centre.

Although this racemisation process occurs fast even at low temperatures in polar solvents, strategies have been developed to increase the configurational stability of the lithiated species. An electronegative substituent in $\alpha$-position to the deprotonated carbon atom can raise the inversion barrier due to the increased p-character of the corresponding bond in accordance with Bent's rule. ${ }^{[310,311]}$ Organosulfur substituents on the other hand stabilise the configuration via negative hyperconjugation. ${ }^{[87,153-156]}$ An inversion at the carbanion would also require a rotation about the $\mathrm{C}-\mathrm{S}$ bond, which is indeed the rate determining step with an activation energy of about $50 \mathrm{kJmol}^{-1}$. $^{[312]}$ Finally, a strong fixation of the metal to the carbon atom by side-chain complexation leads to an increased inversion barrier of up to $100-120 \mathrm{kJmol}^{-1}$. $^{[303,309,313,314]}$

For several years, enantiomerically enriched lithium organic compounds have been subject of discussion concerning the complete stereochemical course of 
their formation by deprotonation and the trapping reactions with different electrophiles like carbonyl reagents or group 14 halides (e.g. alkyl, silyl, and stannyl halides). The determination of the absolute configuration at the metallated stereogenic centre and the clarification of the stereochemical course of further transformations are of major interest. In order to elucidate the absolute configurations of both the anion and the product, single crystal X-ray diffraction provides the most unambiguous assignments, even though many of the lithium containing compounds are difficult to handle because of their thermal instability and sensitivity towards air and moisture (cf. chapter 1).

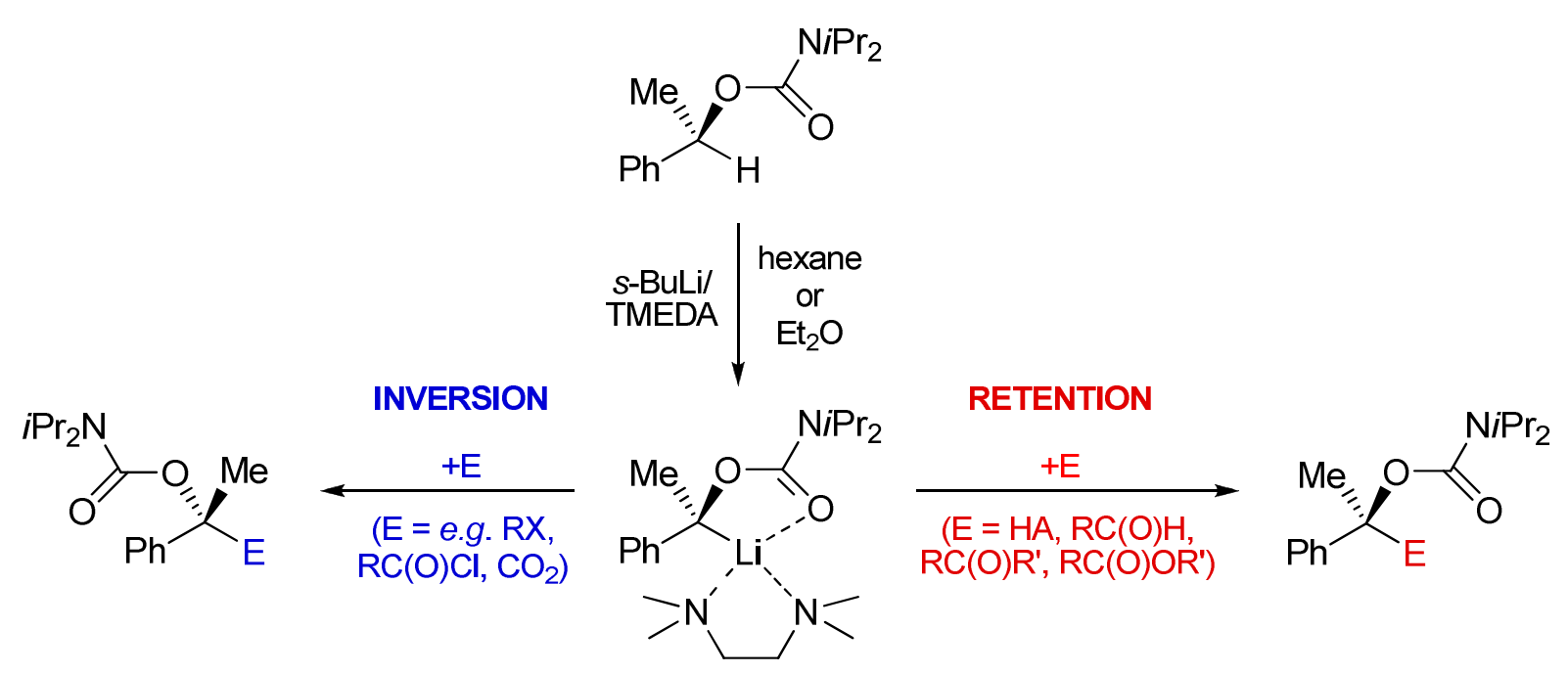

Scheme 4-2. Stereochemical course of the reaction of an enantiomerically enriched benzyllithium compound with different electrophiles.

In 1994, A. Carstens and D. Hoppe reported several reactions of a highly enantiomerically enriched benzyllithium compound with different electrophiles (Scheme 4-2). ${ }^{[315]}$ In contrast to non-mesomerically stabilised lithium organic compounds containing carbanions with $\mathrm{sp}^{3}$ hybrid orbitals that react with almost all electrophiles under strict stereo-retention, the reaction of this system can either result in retention or inversion of the absolute configuration at the parent carbon atom depending on the attack of the electrophile from the front side or the backside of the metallated atom. Most of the trapping reactions occur under inversion (alkyl, silyl and stannyl halides, acid chlorides, carbon dioxide, carbon disulphide, and alkyl-iso-cyanates) and only the reactions with protic acids, aliphatic aldehydes, ketones, or esters occur under retention. ${ }^{[27]}$

The preference of the stereo-inversion and the observed retention in reactions with carbonyl compounds can be rationalised if pre-complexation of the electrophiles is taken into account. Electrophiles that are capable of a strong coordina- 
tion to the lithium atom, like carbonyl compounds, result in products with stereoretention. Almost all others yield in inversion products by attacking the vacant backside of the nucleophile. Unfavourable steric interactions interfere only in few cases with the inversion process and lead to the retention product. ${ }^{[301]}$ In addition to the influence of the electrophile employed, it is obvious that the substitution and geometry of the carbanionic atom has a crucial control on the reaction pathway. If the carbanion is surrounded planar, the reaction with the electrophile can occur from the less hindered, uncoordinated backside of the molecule to cause inversion. If the carbanionic centre is pyramidalised, retention should be preferred as a consequence of the easier access to the front side of the molecule (Figure 4-1).
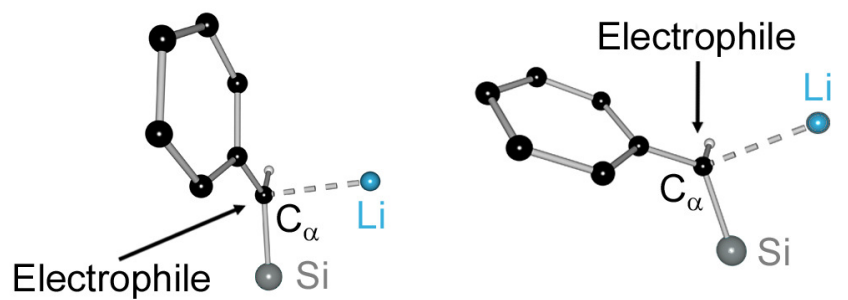

Figure 4-1. Preferred reaction of an electrophile with a carbanion in planar (left) or tetrahedral (right) surroundings.

Along with this geometrically imposed behaviour of the carbanion, there is an electronical reason for the preferred stereo-retention of $\mathrm{sp}^{3}$ hybridised compounds and the stereo-inversion of planar ones, first pointed out by $A$. Carstens and $D$. Hoppe. ${ }^{[315]}$ The $\mathrm{sp}^{2}$ character increases the electron density at the backside of the $\mathrm{Li}-\mathrm{C}$ bond and therefore the attractiveness for an approaching electrophile to interact with that side of the molecule. Especially electrophiles with a low-energy LUMO rapidly react with these carbanions through an inversion pathway (orbital controlled reaction). ${ }^{[301]}$

Although all these effects are very important to rationalise the suggested reaction patterns of benzyllithium compounds, the coordination sphere of the lithium atom has not attracted much interest yet. ${ }^{[229,316,317]}$ In 2005, C. Strohmann et al. reported on a highly diastereomerically enriched silyl substituted lithium organic compound in various substitution reactions. ${ }^{[314]}$ Depending on the applied solvents, different products could be isolated (Scheme 4-3). If the reaction was carried out in non-coordinating toluene/cyclohexane solutions, one coordination side of the lithium atom remained vacant and the trialkyl stannyl chloride was able to pre-coordinate. The reaction follows a retention path- 

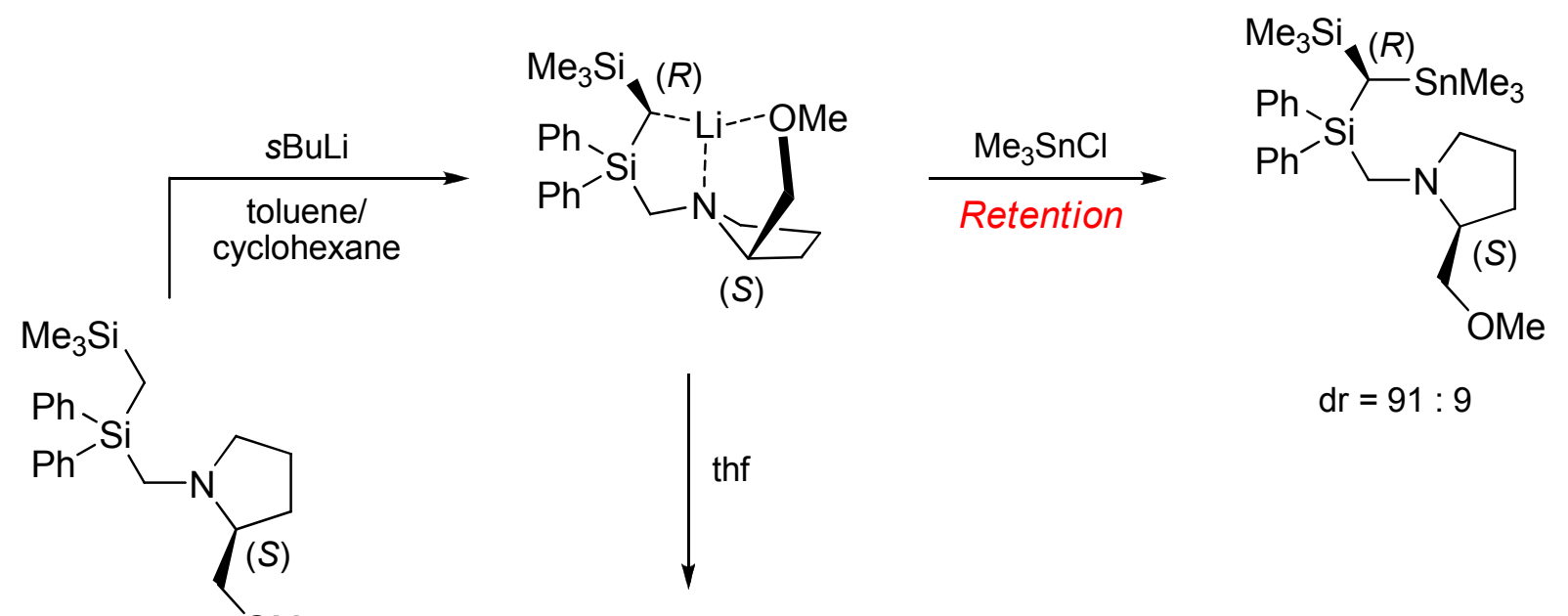

$\mathrm{dr}=91: 9$
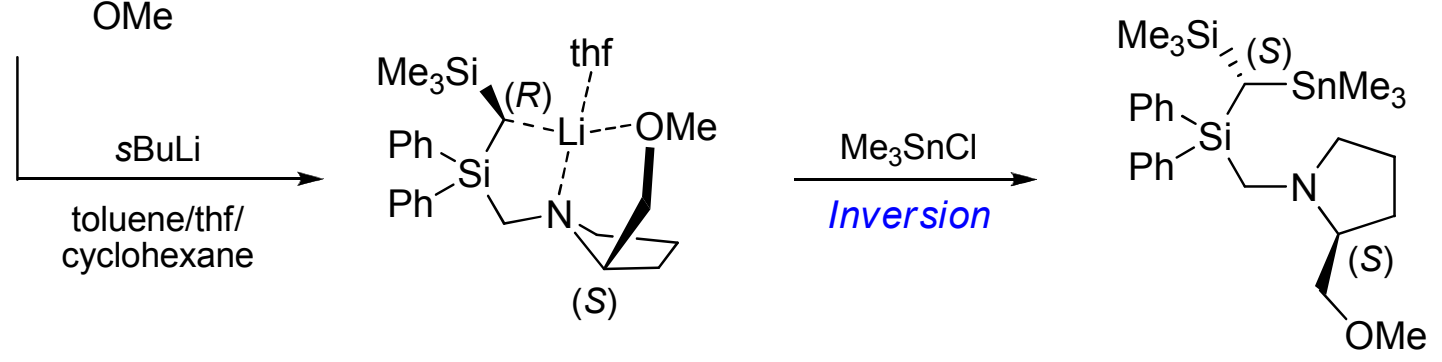

$\mathrm{dr}=92: 8$

Scheme 4-3. Solvent dependant reaction pathway of a highly diastereomerically enriched lithium organic compound.

pathway ( $\mathrm{dr}=91: 9)$. If thf was donating to the lithium atom, pre-coordination of the electrophile was impossible and the attack occurred from the backside of the molecule causing inversion ( $\mathrm{dr}=92: 8)$.

A related system was chosen for a combined study on diastereomerically enriched lithium organic compounds. Experimental NMR and derivatisation results were to be joined with an experimental charge density study to understand key features in $(R)$ - $[\{(S)$-2-(methoxymethyl)pyrrolidinomethyl $\}$ dimethylsilyl]benzyllithium (16). These are the stabilisation of the negative charge by the substituents, the influence of the surrounded planar carbanion on the electron density distribution, and the characteristics of the lithium bonds amongst others. 


\subsection{Preliminary Work}

The diastereomerically enriched lithium organic compound under investigation ([PhCH( $\left.\mathrm{Li}) \mathrm{Si}(\mathrm{Me})_{2} \mathrm{CH}_{2}\left\{\mathrm{NC}_{4} \mathrm{H}_{7}\left(\mathrm{CH}_{2} \mathrm{OMe}\right)\right\}\right]$ (16), was prepared by C. Däschlein in the research group of $C$. Strohmann. It could be crystallised in high quality with the donor bases quinuclidine and dabco. ${ }^{[308]}$ The quinuclidine adduct 17 was finally chosen for the experimental charge density investigation due to the well diffracting crystals of appropriate size. Moreover, this substituted benzyllithium compound is an ideal model compound because several experimental and theoretical results were already available including the previous knowledge of the stereochemical pathway on the deprotonation and the subsequent substitution. These results are summarised below.
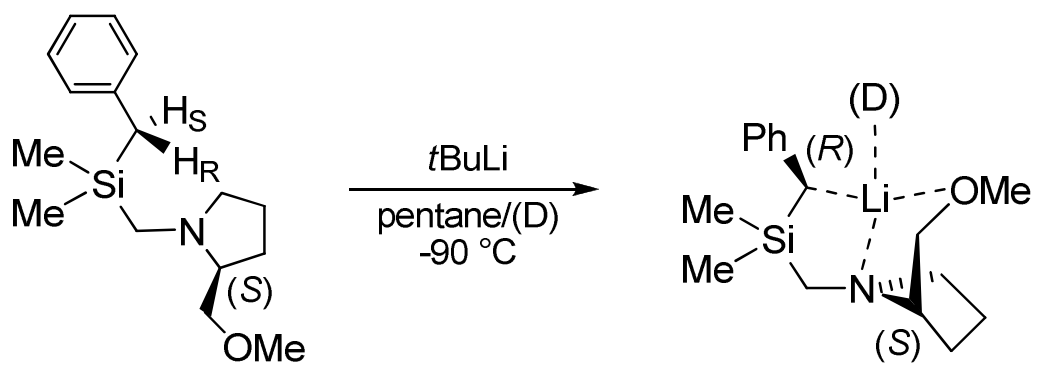

(16)

Scheme 4-4. Synthesis of the highly diastereomerically enriched benzyllithium compound 16 ( $D$ = optional donor molecule).

The lithiated compound is prepared by the deprotonation of the corresponding benzylsilane with tert-butyllithium in toluene at $-90{ }^{\circ} \mathrm{C}$ (Scheme 4-4). ${ }^{[309,318]}$ The resulting lithium organic compound is of remarkable diastereomeric purity. Only the $(R)$ enantiomer at the deprotonated carbon atom could be observed in solution by NMR (so that less than $4 \%$ of the second diastereomer could have been present at most) ${ }^{[319]}$ and in the crystal. ${ }^{[318]}$ In fact, the determination of the absolute configuration was accomplished by X-ray diffraction experiments. Moreover, G. Fraenkel et al. could show that the configuration of the carbanion is even maintained at elevated temperatures up to $27^{\circ} \mathrm{C} .^{[319]}$ Accordingly, it is not necessary to crystallise the lithiated species in order to obtain the highly diastereomerically enriched starting material. The side-chain fixation of the nitrogen and oxygen donor atoms is consequently directing the abstraction of the $(R)$ proton, so that the diastereomeric ratios of the substitution products are independent of the preceding isolation of crystalline material of 16. ${ }^{[318]}$ 
${ }^{13} \mathrm{C}$ NMR-spectra were also recorded for the quinuclidine adduct (17) under investigation to get similar information about the configurational stability of the lithiated carbon atom. Based on the observed ${ }^{13} \mathrm{C}-{ }^{7} \mathrm{Li}$ coupling at RT in deuterated toluene, $\mathbf{1 7}$ has is at least configurationally stable on the NMR time scale and therefore on the reaction time scale at $-78^{\circ} \mathrm{C}$ as well. Moreover, the ${ }^{13} \mathrm{C}$ NMR spectrum [ $\delta$ ] showed only one set of signals indicating the presence of only one diastereomer. The resonance signal for the metallated carbon atom is observed at $36.5 \mathrm{ppm}$. The signal consists of a quartet (nuclear spin of ${ }^{7} \mathrm{Li}$ : $\mathrm{I}=3 / 2 ;{ }^{13} \mathrm{C}-{ }^{7} \mathrm{Li}$-coupling: $9 \mathrm{~Hz}$ ), indicating only one fixed $\mathrm{C}-\mathrm{Li}$ contact in solution, thus representing a monomer. A quartet with four equal peaks is expected for a ${ }^{13} \mathrm{C}-{ }^{7} \mathrm{Li}$ coupling. However, the observed signal shape was not symmetric, which could be caused by an overlap with the corresponding signal of the second possible diastereomer with $(S, S)$ configuration or by the ${ }^{13} \mathrm{C}-{ }^{6} \mathrm{Li}$ coupling (natural abundance ${ }^{7} \mathrm{Li}:{ }^{6} \mathrm{Li} \approx 0.93: 0.07$ ). The ${ }^{13} \mathrm{C}$-Li-coupling was simulated in order to rationalise the peak shape (Figure 4-2).
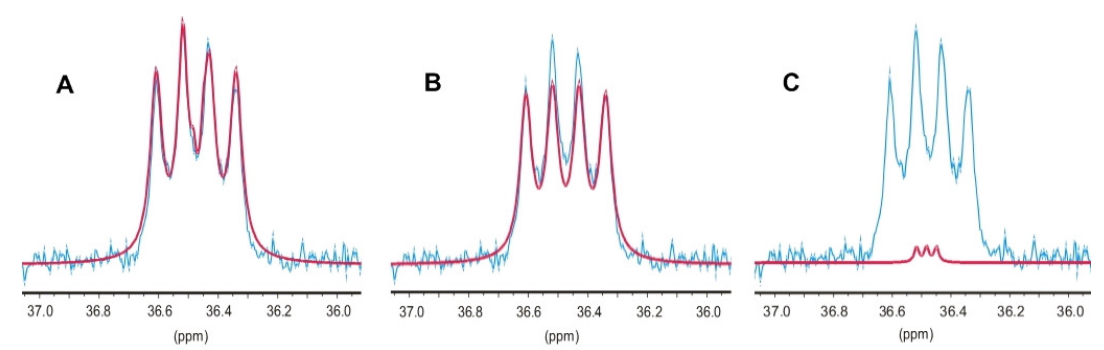

Figure 4-2. Section of the ${ }^{13} \mathrm{C}-\mathrm{NMR}$-spectrum of 17: blue line shapes: experimental

${ }^{13} \mathrm{C}-\mathrm{Li}$ coupling; red line shapes: simulated ${ }^{13} \mathrm{C}-\mathrm{Li}$ coupling; A) ${ }^{13} \mathrm{C}-{ }^{7} \mathrm{Li}$ and ${ }^{13} \mathrm{C}-{ }^{6} \mathrm{Li}$ coupling add-up; B) ${ }^{13} \mathrm{C}-{ }^{7} \mathrm{Li}$ coupling; C) ${ }^{13} \mathrm{C}-{ }^{6} \mathrm{Li}$ coupling.

A in Figure 4-2 shows the superimposed simulated spectra of the ${ }^{13} \mathrm{C}-{ }^{7} \mathrm{Li}-$ and ${ }^{13} \mathrm{C}-{ }^{6} \mathrm{Li}$-coupling, $\mathbf{B}$ shows the same for the ${ }^{13} \mathrm{C}-{ }^{7} \mathrm{Li}$ coupling and $\mathbf{C}$ shows the ${ }^{13} \mathrm{C}-{ }^{6} \mathrm{Li}$ coupling. In all three cases the red lines refer to the simulated spectra and the blue line to the experimental one. Obviously, the experimental line shape of the non-symmetric quartet is a result of the coupling between ${ }^{13} \mathrm{C}$ and ${ }^{6} \mathrm{Li}(\mathbf{C})$. The addition of the symmetric line shape of the simulated ${ }^{13} \mathrm{C}-{ }^{7} \mathrm{Li}$-coupling (B) results in the observed experimental shape $(\mathbf{A}=\mathbf{B}+\mathbf{C})$.

The reaction of $\mathbf{1 6}$ with electrophiles generally proceeds under inversion of the configuration at the nucleophilic carbon atom using a variety of substrates (e.g. methyl iodide, mercury chloride). ${ }^{[309,318]}$ The choice of the solvent is quite uncritical for these reactions. It is even possible to use diethyl ether or a mixture of toluene and one equivalent of thf to get a reasonable good diastereomeric 
ratio in the reaction sequence - only an excess of thf lowered the dr. Hence, as long as the contact ion pair stays intact, the donor solvent does not matter. Thf with a dipole moment of $1.75 \mathrm{D}$ has a stronger donor-capacity compared to $\mathrm{Et}_{2} \mathrm{O}$ $(\mu=1.15 \mathrm{D})^{[320]}$ so that the intramolecular donor sites are more easily displaced (solvent-separated ion pairs), which releases the cation from its fixed position and the racemisation process can take place.

The stereoselective substitution with a variety of substrates is synthetically important, because the side chain that transfers the stereo-information can be cleaved at the $\mathrm{Si}-\mathrm{C}$ bond. This enables the synthesis of enantiomerically pure benzyl alcohols. ${ }^{[303]}$

The standard structure determination of $\mathbf{1 6}$ without additional donor solvents showed that the deprotonated carbon atom is $\mathrm{sp}^{2}$ hybridised and in consequence planar. ${ }^{[318]}$ In principle, it could be attacked by electrophiles from two sides: from the front side (where the lithium atom is located) and from the open backside. The general reaction pathway of $\mathbf{1 6}$ was also verified for the isolated quinuclidine adduct 17. It was reacted with 1.1 equivalents of trimethylchlorostannane at $-90{ }^{\circ} \mathrm{C}$. Warming to RT caused a complete decolourisation of the previously yellow solution. ${ }^{13} \mathrm{C}$ NMR studies showed a dr of the product of more than 98:2. A subsequent treatment of the compound with one equivalent of methyl iodide in acetone resulted in the $\mathrm{N}$-methylated product (Scheme 4-5), which was necessary to obtain crystals for the determination of the absolute configuration (Figure 4-3).

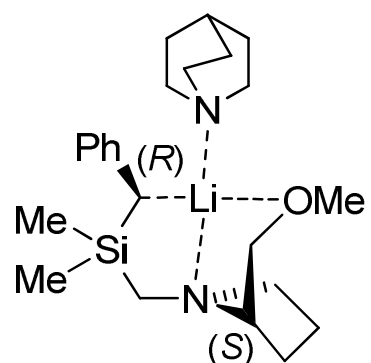

(S)
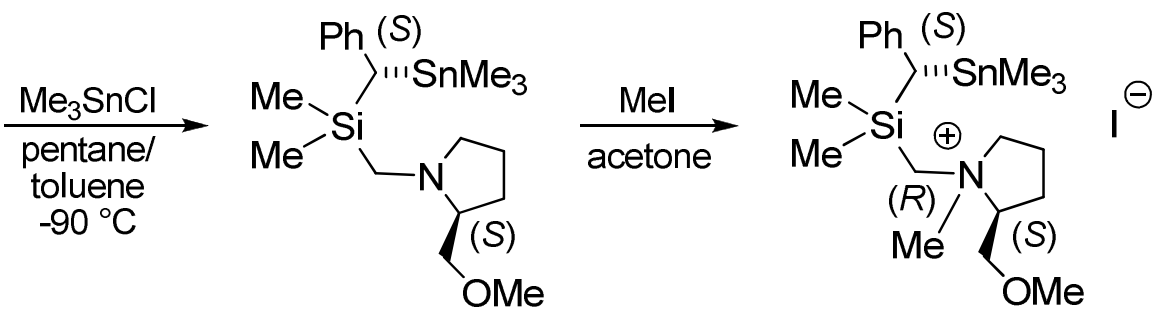

17

Scheme 4-5. Reaction of $\mathbf{1 7}$ with an electrophile and subsequent derivatisation to determine the stereo-chemical pathway.

The methyl iodide salt has in consequence three stereogenic centres: two at carbon atoms and one at a nitrogen atom. Both stereogenic carbon atoms were determined to have $(S)$-, the nitrogen atom to have $(R)$ configuration. This was unambiguously determined by the Flack $x$ parameter ${ }^{[321,322]}(0.00(3)$; see also 
remarks in chapter 7.3.2) of the structure refinement and on the basis of the known configuration at the pyrrolidine carbon atom. Hence, the trapping reaction of $\mathbf{1 7}$ with trimethylchlorostannane also proceeded under inversion of the absolute configuration, as was expected with having the results for $\mathbf{1 6}$ in mind.

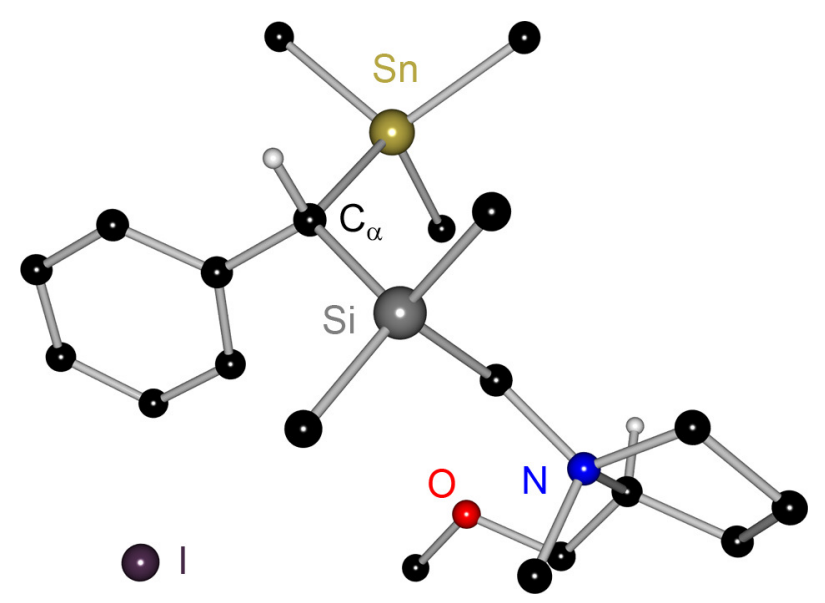

Figure 4-3. Molecular structure of the conversion product of $\mathbf{1 7}$ with $\mathrm{Me}_{3} \mathrm{SnCl}$ as $\mathrm{MeI}$ salt (hydrogen atoms are omitted except those at stereogenic centres).

Initial information on the reason for the observed stereochemical course of $\mathbf{1 6}$ was gained from theoretical calculations. ${ }^{[309,318]}$ The highest occupied molecular orbital is mainly concentrated at the deprotonated carbon atom (Figure 4-4). However, a preferred backside attack of an electrophile cannot be deduced from its symmetrical shape. Therefore, geometrical reasons and the easy accessibility from the side opposite to the $\mathrm{Li}-\mathrm{C}$ interaction were accounted for the selective inversion at the carbanion.

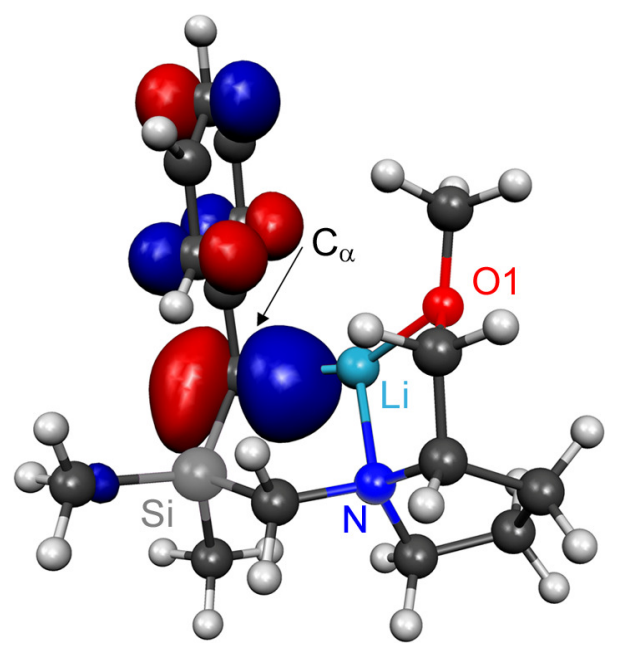

Figure 4-4. Visualisation of the HOMO in $\mathbf{1 6 .}$ 
Additional indicators are consequently needed to explain the excellent selectivity of the configurationally stable benzyllithium compounds $(\mathbf{1 6}, \mathbf{1 7})$. The inspection of the electron density distribution in this class of compounds seems to be appropriate to provide further information. An experimental charge density study with supporting theoretical calculations was therefore undertaken on the quinuclidine adduct $\mathbf{1 7}$ to unravel the reason for the experimental results.

\subsection{Experiment and Refinement}

The principle routines of the X-ray experiment, data processing, and the refinement were already outlined in details (cf. chapter 2.3.5). However, some differences for 17 (Figure 4-5) are worth mentioning, which are summarised below.

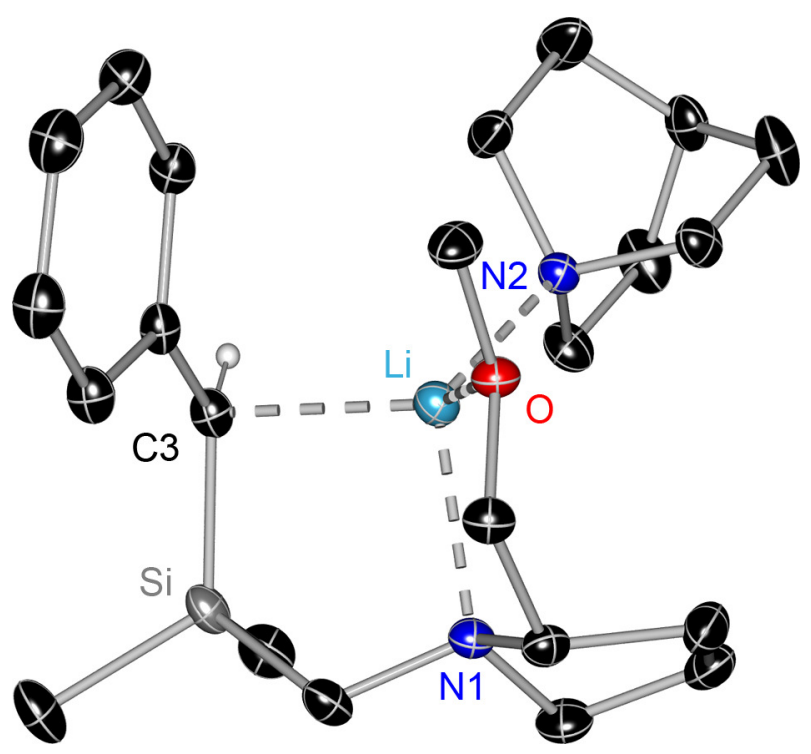

Figure 4-5. Molecular structure representation of the chiral benzyllithium compound 17 with anisotropic displacement parameters depicted at the $50 \%$ probability level (only the benzylic hydrogen atom shown for clarity reasons).

The charge density data set was measured with detector distances of $6 \mathrm{~cm}$ (low-order data) and $5 \mathrm{~cm}$ (medium- and high-order data), respectively. Two low-angle batches at exposure times of $10 \mathrm{~s}$ ( 8 runs with 600 frames each at positive and negative $2 \theta$ detector positions, $d_{\min }=0.8 \AA\left(2 \theta_{\max }=52.7^{\circ}\right)$ ), one medium-angle batch ( 7 runs with 600 frames each, $30 \mathrm{~s}, d_{\min }=0.55 \AA$ $\left(2 \theta_{\max }=80.5^{\circ}\right)$ ) and one high-angle batch (6 runs with 600 frames, $90 \mathrm{~s}$, $\left.d_{\min }=0.464 \AA\left(2 \theta_{\max }=100.0^{\circ}\right)\right)$ were collected. The integration of the data was 
performed with SAINT V7.23A. ${ }^{[187]}$ These were corrected for absorption, scaled and merged with SADABS-2006/3 $3^{[323]}$, in which Friedel pairs were not included into the merging process to take the anomalous dispersion in the chiral space group $P 2_{1} 2_{1} 2_{1}$ into account.

During the multipole refinement, relativistic Dirac-Fock wave functions (SCM bank file) were used for the core and spherical valence densities. The deformation density terms of the silicon atom were modelled with optimised $n_{\text {I }}$ values $(2,4,6,8 \text { for } I=1,2,3,4)^{[183]}$ and a $\zeta$ of 2.9598 au. The expansions over the spherical harmonics were truncated at the hexadecapolar level for all heteroatoms, the deprotonated carbon atom $\mathrm{C} 3$ and the carbon atoms with a reduced number of parameters (local non-crystallographic symmetry constraints of the multipoles; see below). All remaining carbon atoms were modelled to the octapolar level. Moreover, the known riding model and distance constraints ${ }^{[171]}$ were applied for the hydrogen atoms during the multipole refinement, even though the refinement without distance constraints led to reasonable carbonhydrogen bond lengths in the range of $1.01-1.09 \AA$. Nevertheless, this did not improve the model significantly and the constraints were maintained to lower the number of refined parameters and avoid correlations. It is noteworthy that the hydrogen atom constraints were switched off during an intermediate refinement step and were again fixed to neutron distances afterwards. This was of special importance for the determination of the benzylic hydrogen atom position.

Several models were refined and compared, differing in the degree of applied chemical constraints and local non-crystallographic symmetry. A maximum amount of chemical constraints and symmetry restrictions for the multipole functions stabilised the refinement procedures in the non-centrosymmetric space group and reduced correlations. On the other hand, the model had to be flexible enough to account for small differences of supposed chemically equivalent atoms, e.g. carbon atoms in the phenyl ring were refined without constraints. Special care was taken to model the electronic state of the lithium atom. In an initial refinement, the standard scattering factor of the alkaline metal was used and its monopole population was fixed until the final refinement steps. The subsequent refinement of the lithium monopole parameter using different $\mathrm{k}$ values $(1.4,1.6,1.8$, and 2.0$)$ resulted in a net charge close to $+1 \mathrm{e}$. Therefore, the lithium atom was treated as $\mathrm{Li}^{+}$with the monopole population set to zero. For electroneutrality reasons, the resulting charge was counterbalanced by increasing the initial monopole populations about 0.25 e for the donor atoms in 
the starting model. The influence of the starting model on the final multipole parameters was checked and found negligible.

The final refinement cycle included all parameters except $\kappa^{\prime}$ (due to known correlation effects with other multipole parameters) using positive reflections with $I>3 \sigma(I)$. Chemically equivalent or similar atoms (methyl carbon atoms at the silicon atom, carbon atoms at the 3- and 4-position of the pyrrolidine ring) and atoms, which were related by local molecular non-crystallographic symmetry (three-fold axis in the quinuclidine moiety) were constrained to share the same expansion/contraction parameters, monopole, and multipole populations. Hydrogen atoms with similar chemical environment were modelled with one set of parameters each: methyl hydrogen atoms at the silicon atom; phenyl ring hydrogen atoms; pyrrolidine hydrogen atoms at the 3- and 4-position; all quinuclidine hydrogen atoms; hydrogen atoms on $\mathrm{CH}_{2}$; and remaining $\mathrm{CH}_{3}$ groups. Several additional local non-crystallographic symmetry restrictions were applied for the angular functions (three-fold symmetry at the methyl carbon atoms and the quinuclidine nitrogen atom, a mirror plane at the silicon atom relating the methyl groups and at the $\mathrm{CH}_{2}$ bridges $\mathrm{C} 10$ and $\mathrm{C} 15$ ). The multipole refinement led to a very satisfactory fit. The electron density is not biased by and well separated from the thermal motion of the non-hydrogen atoms. This was justified by the DMSDA test (Appendix). ${ }^{[199]}$ The final model, which included only those constraints that were chemically sensible, led to the lowest $R$-values (Table 4-1) and a flat and featureless residual density (Figure 4-6 and Appendix). Therefore, this model was selected for the subsequent discussion.

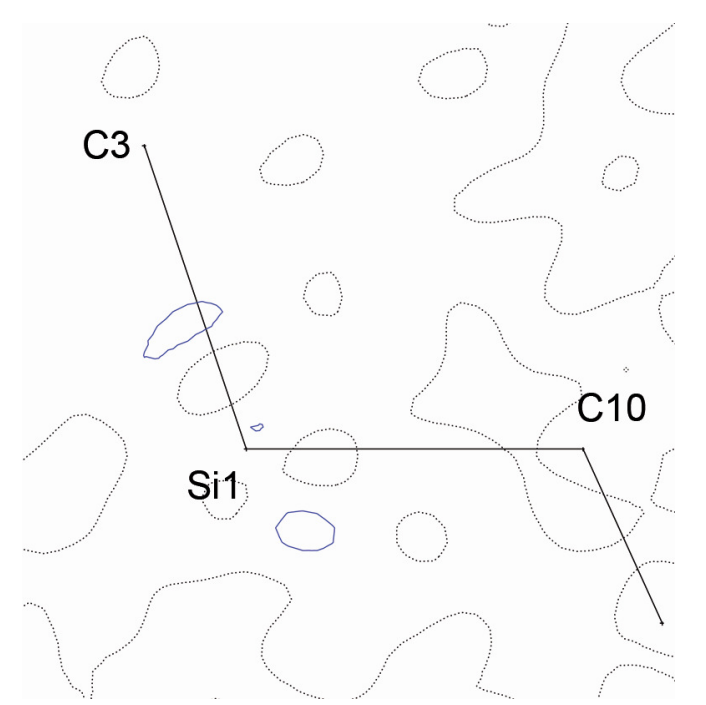

(a)

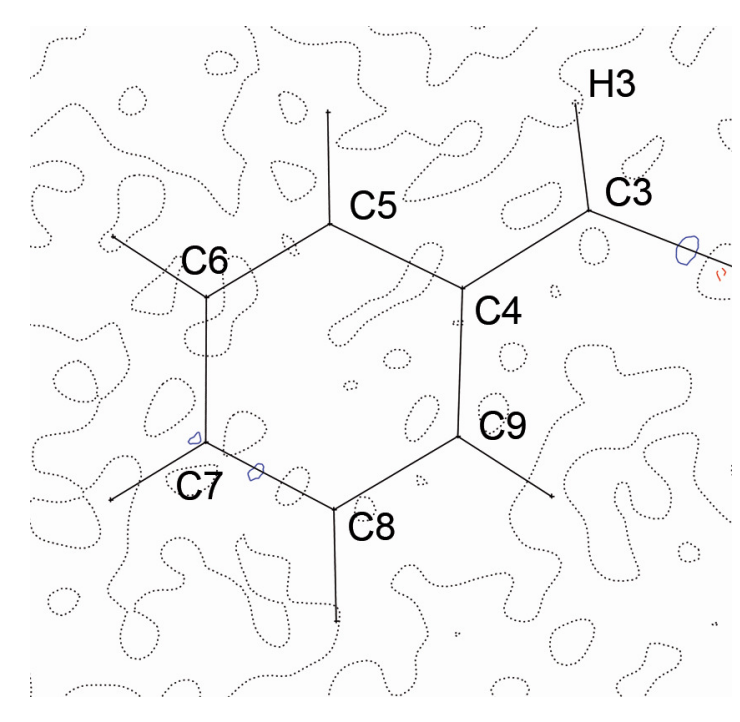

(b) 


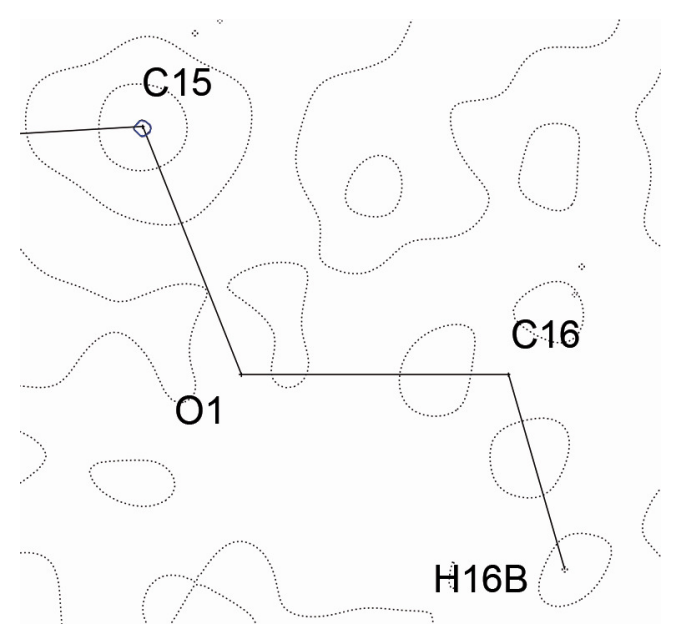

(c)

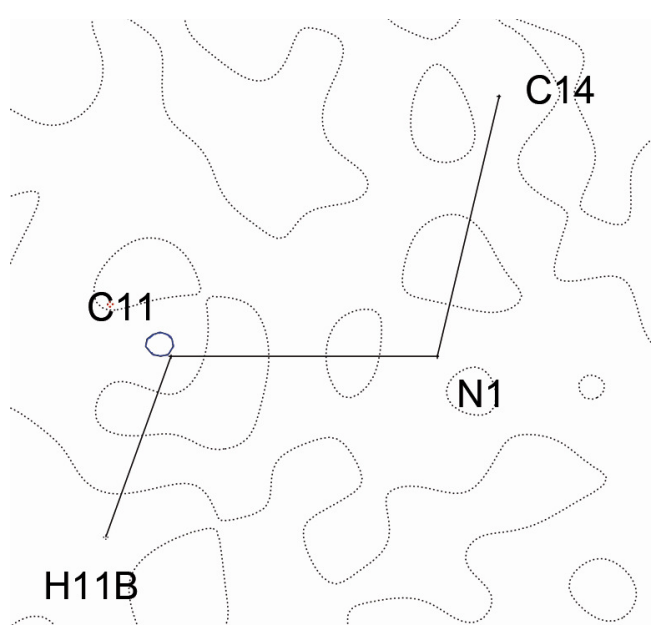

(d)

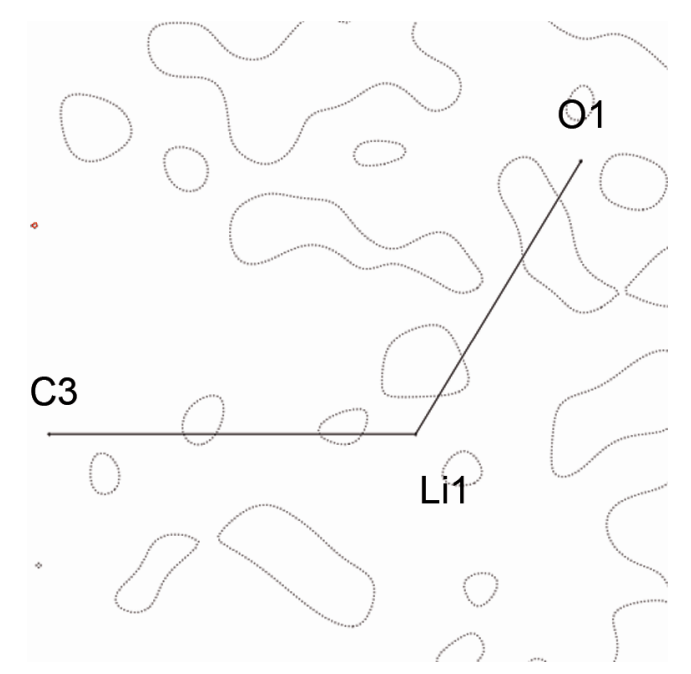

(e)

Figure 4-6. Residual density maps $\left(\sin \theta \lambda^{-1}=1.0 \AA^{-1}\right.$ ) of 17. Step size of the contour lines is $0.1 \mathrm{e}^{-3}$. Positive values are plotted as solid blue lines, negative values as dashed red ones and the zero value is drawn dotted black; (a) C3-Si1-C10 plane, (b) C4C6C8 plane, (c) C15-O1-C16 plane, (d) C11-N1-C14 plane and (e) C3-Li1-O1 plane.

Table 4-1. Selected crystallographic details after the multipole refinement of $\mathbf{1 7}$.

\begin{tabular}{ll|ll}
\hline independent reflections & $24554\left(R_{\text {int }}=0.023\right)$ & $R 1$ & 0.0203 \\
reflections above $(I>3 \sigma(I))$ & 21027 & wR2 & 0.0312 \\
parameters & 616 & GoF & 2.595 \\
largest diff. peak and hole & 0.211 and $-0.134 \mathrm{e}^{-3}$ & & \\
\hline
\end{tabular}




\subsection{Multipole Model and the Electron Density Distribution in 17}

The silyl substituted benzyl lithium compound $\mathbf{1 7}$ crystallises in the chiral orthorhombic space group $P 2_{1} 2_{1} 2_{1}$ (Figure 4-7). Its absolute structure could be determined unambiguously based on the known configuration at C14 as well as on the refined Flack $x$ parameter $(0.01(3)){ }^{[321,322]}$

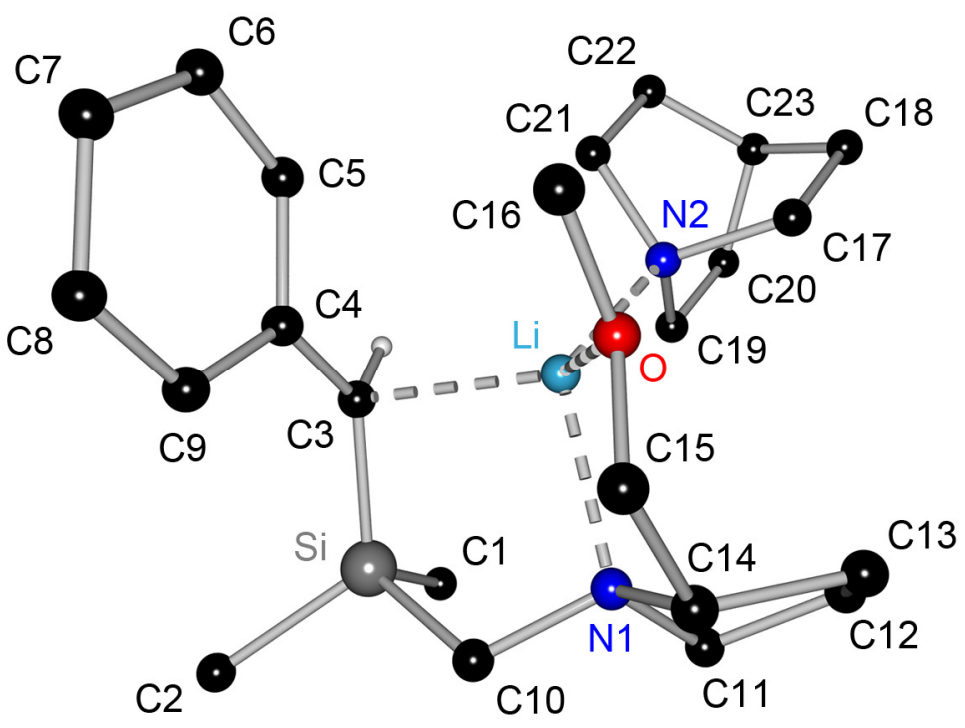

Figure 4-7. Molecular structure of $\mathbf{1 7}$ (hydrogen atoms except the benzylic one are omitted for clarity).

The slightly distorted tetrahedral coordination sphere of the lithium atom arises from contacts to the benzylic carbon atom, to the (methoxymethyl)pyrrolidine side-chain oxygen (C3-Li-O: 121.2(1) ${ }^{\circ}$ ) and nitrogen atoms (C3-Li1$\left.\mathrm{N} 1: 99.4(1)^{\circ} ; \mathrm{O}-\mathrm{Li}-\mathrm{N} 1: 83.3(1)^{\circ}\right)$, and to the nitrogen atom of the quinuclidine molecule (C3-Li-N2: $\left.125.6(1)^{\circ}\right)$. As anticipated, this arrangement keeps the lithium atom in a fixed position.

The $(R)$ configured stereogenic carbon atom C3 features an almost planar surrounding with respect to the three neighbouring non-metal atoms (sum of bond angles: $358.5^{\circ}$ ) while the lithium-carbon distance of $2.273(1) \AA$ is in the expected range. ${ }^{[4]}$ The Si-C3 bond length of $1.813(1) \AA$ is clearly smaller compared to that of a standard Si-C single bond $(1.87 \AA)^{[152]}$ whereas the SiC10 bond $(1.907(1) \AA)$ is slightly elongated. This is normally attributed to the $\alpha$-stabilising effect of the silicon atom. ${ }^{[133,154,200,324,325]}$ 


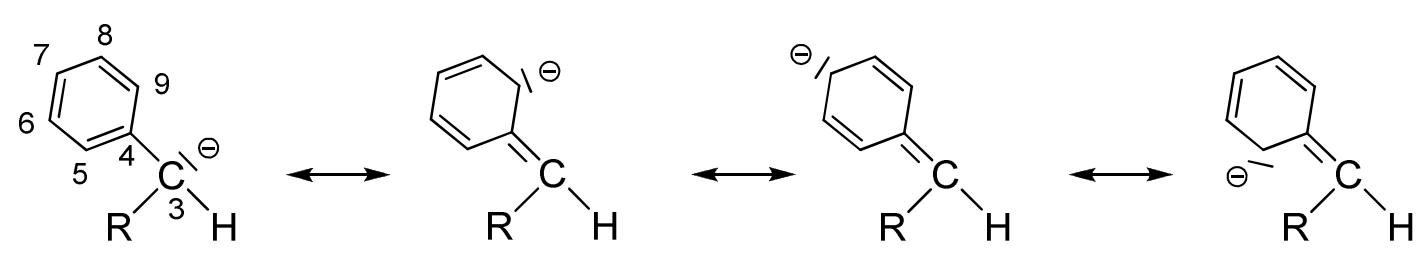

Scheme 4-6. Mesomeric formulae of the metallated carbon atom and the phenyl substituent in $\mathbf{1 7}$.

Furthermore, the phenyl substituent is believed to exert a stabilising effect on the metallated carbon atom. Four different mesomeric formulae are conceivable for the delocalisation of the negative charge (Scheme 4-6). Indications for that can be found by analysing the bond lengths of the phenyl ring. The bond between C3 and C4 is significantly shortened (1.434(1) $\AA$ ) in comparison to the corresponding $\mathrm{CH}_{2}$ group (e.g. 1.508(6) $\AA$ in the tin compound shown in Figure 4-3). Nevertheless, the bond length is more in the range of a single than of $a$ double bond $\left(\mathrm{C}_{\mathrm{sp} 2}-\mathrm{C}_{\mathrm{sp} 2}: 1.466 \AA ; \mathrm{C}_{\mathrm{sp} 2}=\mathrm{C}_{\mathrm{sp2} 2}: 1.335 \AA\right){ }^{[152]}$ Moreover, the $\mathrm{C}-\mathrm{C}$ bonds between the 1 - and the 2-carbon atoms are slightly longer (C4-C5/ C4-C9: $1.427(1) \AA)$ compared to C8-C9 (1.390(1) $\AA)$ and C5-C6 (1.386(1) $\AA)$. The bonds to the 4-carbon atom are of intermediate length (C6-C7: 1.395(1) $\AA$; C7-C8: $1.399(1) \AA)$.

The two nitrogen-lithium bond lengths of 2.162(1) (Li-N1) and 2.118(1) $\AA$ (Li-N2) differ significantly. The shortened bond between the lithium and the nitrogen atom of the quinuclidine might suggest that quinuclidine is a better donor than the substituent or that the N1 coordination to the cation is limited by the side-arm flexibility. While the donor can interact with the lithium atom from a rather open side, the second nitrogen atom has to adapt to the coordination geometry of the tridentate benzyl anion.

The topological analysis of the experimentally derived electron density distribution commenced with the determination of critical points. All expected bond critical points as well as the corresponding ring and cage critical points could be quantified (Figure 4-8). 72 BCPs above a $\rho\left(\mathbf{r}_{\mathrm{BCP}}\right)$ threshold of $0.045 \mathrm{e}^{-3}$ were found. 7 RCPs and one CCP are related to these bond critical points. Accordingly, the Poincaré-Hopf equation (cf. Eq. 2-13) is fulfilled taking the 67 nuclear attractors into account. Additional bond paths were detected on lower electron density values involving secondary hydrogen bonds, which are shown in the appendix together with their corresponding RCPs and CCPs. 


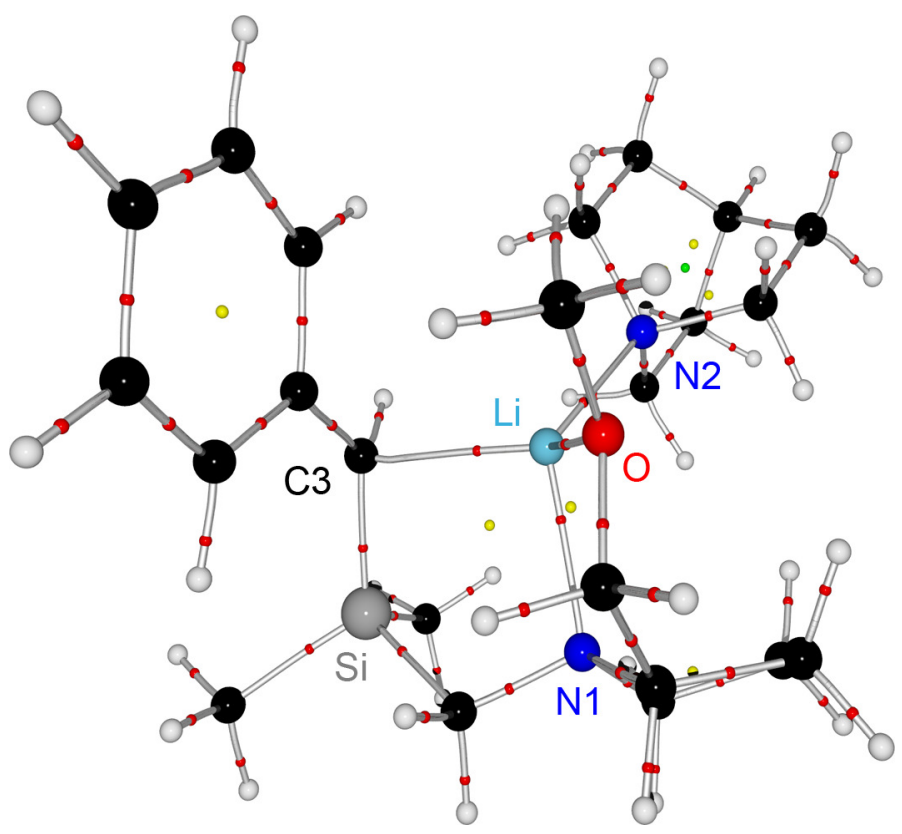

Figure 4-8. Molecular graph of $\mathbf{1 7}$ showing the determined bond paths and relevant BCPS (red), RCPs (yellow), and CCPs (green).

The electron densities at the BCPs of the carbon-carbon bonds within the $\mathrm{C}_{7}$-benzyl moiety ( $\mathrm{C} 3$ to $\mathrm{C} 9$; Table 4 -2) feature the highest values of the whole molecule. Their mean value $\left(2.15 \mathrm{e}^{-3}\right)$ indicates the increased bond order owing to the aromaticity in the phenyl ring. However, the phenyl ring features no increased $\rho\left(\mathbf{r}_{\mathrm{BCP}}\right)$ compared to known systems that are not believed to have external $\pi$-interactions (experimental values range from 2.11 to $2.26 \mathrm{e}^{-3}$ ). ${ }^{[214-217,326]}$ The same is true for the Laplacian (Figure 4-10), which ranges from -16.8 to $-20.4 e \AA^{-5}$ (literature: -16.0 to $-20.7 e \AA^{-5}$ ) and the rather similar $\varepsilon\left(\mathbf{r}_{\mathrm{BCP}}\right)$ values in the phenyl ring. Therefore, the influence of the deprotonated atom $\left(C_{\alpha}\right)$ on the ring system is rather small according to these results.

Table 4-2. Bond path lengths $[\AA]$, electron densities $\left[\mathrm{e} \AA^{-3}\right]$, Laplacians $\left[\mathrm{e} \AA^{-5}\right]$, and ellipticities at the BCPs, as well as integrated atomic charges of the benzyl fragment in $\mathbf{1 7 .}$

\begin{tabular}{l|ccccccc}
\hline \multicolumn{1}{c}{ A-B } & C3-C4 & C4-C5 & C5-C6 & C6-C7 & C7-C8 & C8-C9 & C9-C4 \\
\hline bond path & 1.437 & 1.428 & 1.387 & 1.398 & 1.401 & 1.392 & 1.427 \\
$\rho\left(\mathbf{r}_{\mathrm{BCP}}\right)$ & $2.02(1)$ & $2.12(1)$ & $2.25(1)$ & $2.20(2)$ & $2.18(2)$ & $2.22(1)$ & $2.05(1)$ \\
$\nabla^{2} \rho\left(\mathbf{r}_{\mathrm{BCP}}\right)$ & $-16.8(1)$ & $-17.9(1)$ & $-20.4(1)$ & $-19.1(1)$ & $-18.2(1)$ & $-19.9(1)$ & $-17.5(1)$ \\
$\varepsilon\left(\mathbf{r}_{\mathrm{BCP}}\right)$ & 0.19 & 0.18 & 0.22 & 0.24 & 0.24 & 0.25 & 0.22 \\
charge A & -0.97 & +0.07 & -0.19 & -0.22 & -0.33 & 0.00 & -0.29 \\
\hline
\end{tabular}


The negative charge, formally generated at $\mathrm{C} 3$ by the hydrogen abstraction with $t B u L i$, is therefore not delocalised into the aromatic ring as normally assumed by organic chemists ( $-M$ effect). According to Scheme 4-6, the mesomeric structures show two extreme cases for the $\mathrm{C} 3-\mathrm{C} 4$ bond: a $\mathrm{C}-\mathrm{C}$ single bond if the negative charge is located at $\mathrm{C} 3$ or a partial double bond if charge delocalisation into the phenyl ring system is assumed. A standard $\mathrm{C} 3-\mathrm{C} 4$ single bond can be precluded by the topological properties of $\rho\left(\mathbf{r}_{\mathrm{BCP}}\right)\left(2.02 \mathrm{e} \AA^{-3}\right)$, the Laplacian $\left(-16.8 \mathrm{e} \AA^{-5}\right)$, and the ellipticity of 0.19 (cf. ethane: $\rho\left(\mathbf{r}_{\mathrm{BCP}}\right)=1.63 \mathrm{e} \AA^{-3}$, $\varepsilon\left(\mathbf{r}_{\mathrm{BCP}}\right)=0.00$; ethene: $\left.\rho\left(\mathbf{r}_{\mathrm{BCP}}\right)=2.42 \mathrm{e}^{-3}, \varepsilon\left(\mathbf{r}_{\mathrm{BCP}}\right)=0.33\right){ }^{[87]}$ The observed values reflect an intermediate situation: increased compared to a single bond but diminished with respect to the aromatic ring.

Integrated charges were determined to estimate a possible charge transfer to the benzylic unit that could not be verified with properties at the bond critical points. Interestingly, a charge of almost minus one $(-0.97 \mathrm{e})$ is accumulated at the deprotonated carbon atom. In contrast, the atomic charges in the phenyl ring including the hydrogen atoms add to a charge of $-0.1 \mathrm{e}$ in total. As a consequence, it is not surprising that the aromatic ring does not show any features of increased electron density, since the charge seems to remain on $\mathrm{C} 3$.

Moreover, an asymmetrically electron density distribution is found in the aromatic ring. The $\mathrm{C} 4-\mathrm{C} 5 / \mathrm{C} 4-\mathrm{C} 9$ bonds are significantly elongated compared to the remaining ring bonds, which is accompanied by the lowest $\rho\left(\mathbf{r}_{\mathrm{BCP}}\right)$ values in the phenyl ring ( 2.12 and $2.05 \mathrm{e}^{-3}$ ). This seems to be the electronic consequence of the inductive effect of the ring substitution and, to a much smaller extent, the delocalisation of the negative charge depicted in Scheme 4-6. The 2- and 4 -carbon atoms are also affected. They reveal negative charges $(-0.19,-0.29$, and $-0.33 \mathrm{e}$ ), while the 1 - and one 3 -carbon atoms possess a slightly positive $(+0.09 \mathrm{e})$ or no charge. It is noteworthy that the different atomic charges originate solely from the carbon atoms, because all hydrogen atoms have almost identical integrated charges $(+0.17 \mathrm{e})$. However, the meta-carbon atom $\mathrm{C} 6$ is also negatively charged $(-0.22 \mathrm{e})$ contradicting a straight explanation with inductive or mesomeric effects. Not mentioned so far, this could be caused by the trans orientation of the silicon group relative to that carbon atom ( $\mathrm{C} 8$ is positioned on the same side as $\mathrm{Si}$ ). Although the asymmetry in the integrated charges seems to be not worth mentioning - and might be even ascribed to model discrepancies - this feature can be found again in the crystal packing of the donor free crystal structure of 16. Intermolecular $\eta^{2}$-coordination of $\mathrm{C} 6$ and C7 to a neighbouring lithium atom can be observed (Figure 4-9), so that chains 


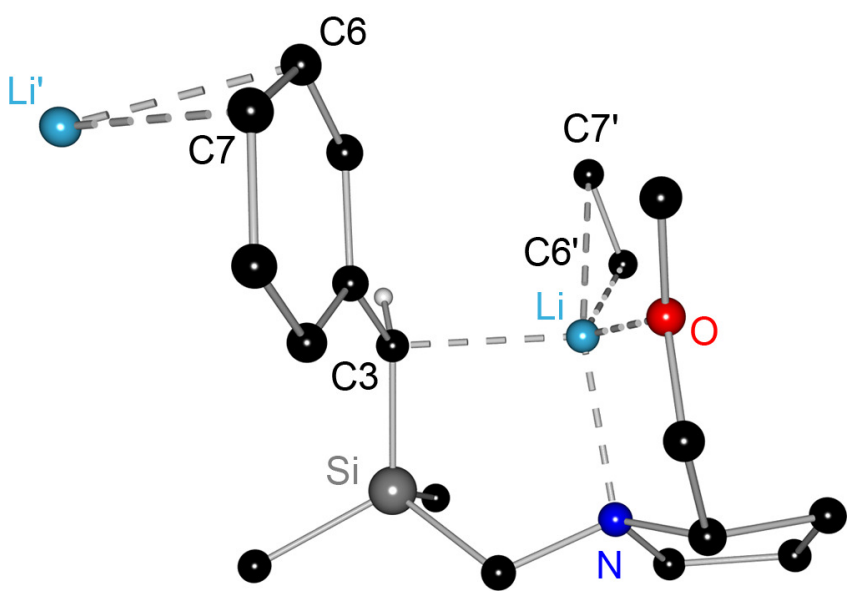

Figure 4-9. Formation of a chain structure in $\mathbf{1 6}$ via $\eta^{2}$-interactions of C6 and C7 with neighbouring lithium atoms.

are built up. ${ }^{[309,318]}$ Accordingly, these charge accumulations seem to be an intrinsic property of $\mathbf{1 6}$, also present in the adduct $\mathbf{1 7 .}$

The charge distribution within the phenyl ring has also an effect on the bonding charge concentrations of the carbon-carbon bonds. Those at the four negatively charged ring atoms range from -33.4 to $-43.1 \mathrm{e} \AA^{-5}$ compared to -28.6 to -34.8 e $\AA^{-5}$ for C4 and C9 (Figure 4-10), which reflect the systematics of the integrated charges. The $\mathrm{C} 3-\mathrm{C} 4\left(-32.8\right.$ and $\left.-32.1 \mathrm{e}^{-5}\right)$ bond on the other hand does not indicate any peculiarities of $\nabla^{2} \rho\left(\mathbf{r}_{\mathrm{BCC}}\right)$.

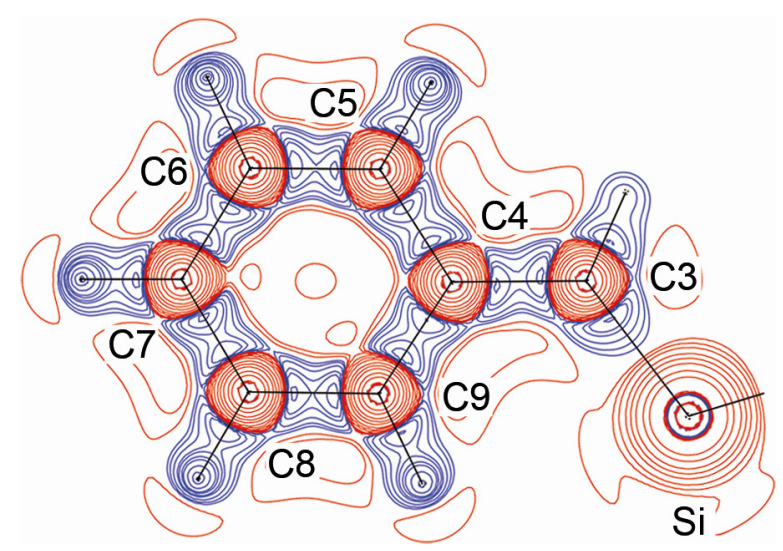

Figure 4-10. Laplacian distribution in the C3C6C8 plane of 17. Positive values of $\nabla^{2} \rho\left(\mathbf{r}_{\mathrm{BCP}}\right)$ are depicted by red and negative by blue lines. Contours are drawn at $\pm 2.0 \cdot 10^{n},-15$, $-30, \pm 4.0 \cdot 10^{n}, \pm 8.0 \cdot 10^{n} e^{-5}$ with $n=0,1,2,3$.

In order to further investigate the $\mathrm{C} 3-\mathrm{C} 4$ bond in comparison to other carboncarbon bonds in 17, the bond ellipticities along the bond paths were determined (Figure 4-11), a procedure that was already used for the discrimination of single and double bonds in $[2-\mathrm{PiCLi} \cdot \mathrm{PiCH}]_{2}$ (4). Two different shapes can be distin- 
guished within the phenyl ring. The ellipticities along $\mathrm{C} 4-\mathrm{C} 9, \mathrm{C} 8-\mathrm{C} 9$, and $\mathrm{C} 6-\mathrm{C} 7$ display pronounced maxima in the carbon atom basins of C7 and C9 that revealed the highest negative charges $(-0.33$ and -0.29 e). This was found even more pronounced by $W$. Scherer et al. in theoretical calculations of charged C-C interactions. ${ }^{[87]}$ The remaining phenyl bonds give ellipticities that are more commonly associated with aromatic carbon-carbon bonds (cf. Figure 2-29 and ref. [87]), although the C7-C8 bond also reflect a slight polarisation towards C7. For comparison purposes, the single bond between $\mathrm{C} 12-\mathrm{C} 13$ is depicted in Figure 4-11, also. Obviously, C3-C4 can be assigned to neither of the mentioned bonding types. It adopts an intermediate shape between the aromatic and the single bonds. The absolute values of $\varepsilon(\mathbf{r})$ are closer to an aromatic bond, while the shape is similar to a single bond with a maximum in each atomic basin, even though this is not well expressed at C4. Again, the C3-C4 bond defies a straightforward classification in the framework of textbook examples. Having the $\varepsilon(\mathbf{r})$ shape of the $\mathrm{C} 1-\mathrm{C} 6$ bond in $[2-\mathrm{PicLi} \cdot \mathrm{PicH}]_{2}$ (4) and those reported for charged $\mathrm{C}-\mathrm{C}$ interactions ${ }^{[87]}$ in mind, the appearance of $\mathrm{C} 3-\mathrm{C} 4$ is even more remarkable. $\lambda_{1}$ is constantly larger in the bonding region than $\lambda_{2}$ so that $\varepsilon(\mathbf{r})$ does not exhibit a distinct maximum approaching the $\mathrm{C} 3$ atom. The reason for that is discussed further below.

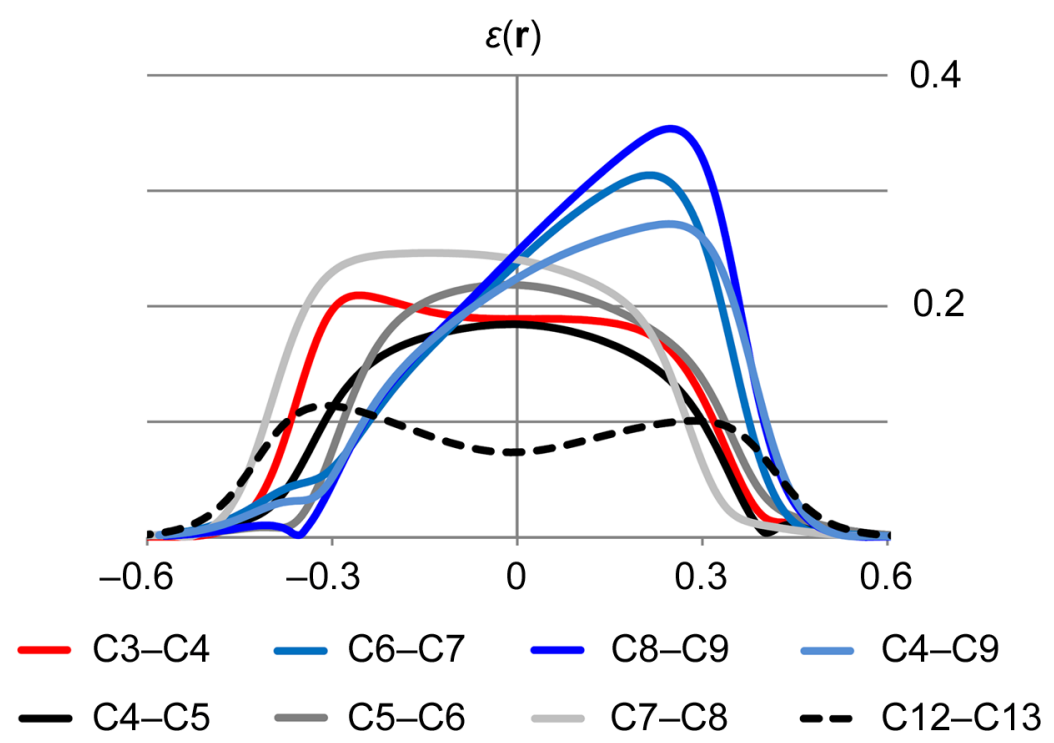

Figure 4-11. Ellipticities along selected carbon-carbon bond paths in $\mathbf{1 7}$.

According to the integrated charges, the deprotonation of C3 leaves the negative charge at the carbon atom, which is bonded to the lithium cation (+0.94 e). However, other pronounced charges are determined in $\mathbf{1 7}$ and a more complicated situation than just a cation-anion pair is found. Group charges could 
be assigned by partitioning the whole molecule into subunits. The quinuclidine donor group charge is slightly positive with +0.16 e, so that the donor molecule is only little influenced by the interaction with the cation (cf. [2-PicLi-PicH] $]_{2}, 4$ ). The dimethylsilyl moiety on the other hand contributes substantially with $+1.22 \mathrm{e}$. This is mainly balanced by the benzyl $(-0.87 \mathrm{e})$ and the pyrrolidine group ( -1.08 e of which -1.22 e are located at N1) and to a small extent by the methoxymethyl side chain $(-0.39$ e). This gives rise to a more complex situation of the charge distribution in 17. The soft Lewis acid and easily polarisable silicon atom forms a second cationic centre. Therefore, two positively charged atoms ( $\mathrm{Li}$ and $\mathrm{Si}$ ) surround the deprotonated carbon atom and stabilise its negative charge.

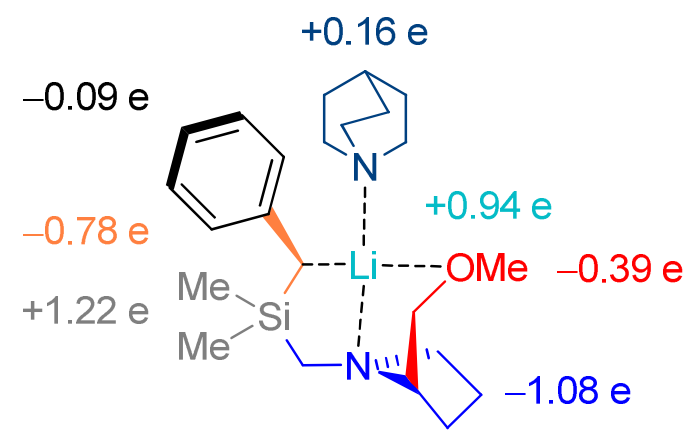

Scheme 4-7. Group charges in 17.

This situation resembles that in $\left[\mathrm{TMSCH}_{2} \mathrm{Li}\right]_{6}(\mathbf{1 4})$, although these carbanions are even more negatively charged when interacting with three cations and having only one non-hydrogen substituent (cf. chapter 3.2.4). An even more impressive example of the dominant stabilising effect of the silicon atom over an aromatic ring can be found in $\left[2-\left(\mathrm{Me}_{3} \mathrm{Si}\right)_{2} \mathrm{CPyLi}\right]_{2}(\mathbf{7}) .{ }^{[87]}$ The disilylated side chain keeps the negative charge at the deprotonated carbon atom in contrast to almost all other reported 2-PicLi compounds, in which the charge is delocalised into the pyridine ring ( $c f$. chapter 2 ). The carbanion is $\mathrm{sp}^{3}$ hybridised and barely interacts with the more electron withdrawing heteroaromatic ring compared to the phenyl group. In summary, the $\alpha$-effect of the silicon atom is also displayed in $\mathbf{7}$ yet largely disregarded by the authors.

The distinct charges at Si and C3 also affect their bond. As mentioned above, it is significantly shortened compared to a standard Si-C single bond. The bond strengthening is also reflected by an increased $\rho\left(\mathbf{r}_{\mathrm{BCP}}\right)$ value of $0.90 \mathrm{e}^{-3}$ in comparison to the other $\mathrm{Si}-\mathrm{C}$ bonds $\left(0.81 \mathrm{e} \AA^{-3}\right.$; Table $\left.4-3\right)$. This is accompanied by the highest positive Laplacian $\left(4.2 \mathrm{e}^{-5}\right)$ of a carbon bond in $\mathbf{1 7}$ and the smallest $\mathrm{Si}-\mathrm{BCP}$ distance. This expansion of the $\mathrm{C} 3$ basin reflects the charge accumulation 
at the deprotonated carbon atom. Compared to the carbon-carbon bonds in 17, the bond characteristics of all $\mathrm{Si}-\mathrm{C}$ bonds are low $\rho\left(\mathbf{r}_{\mathrm{BCP}}\right)$ values, that are halved with respect to a $\mathrm{C}-\mathrm{C}$ single bond and well in the range of the $\mathrm{Si}-\mathrm{C}$ bonds in $\left[\mathrm{TMSCH}_{2} \mathrm{Li}\right]_{6}(\mathbf{1 4})$. The $\nabla^{2} \rho\left(\mathbf{r}_{\mathrm{BCP}}\right)$ values are all positive resembling silicon-carbon contacts with ionic contributions. This is supported by the spherical shape of the positive Laplacian around the silicon atom (Figure 4-12, left), which was found to a lesser extent in $\left[\mathrm{TMSCH}_{2} \mathrm{Li}\right]_{6}(\mathbf{1 4} ; \mathrm{cf}$. chapter 3.2.4), and its positive atomic charge $(+2.15 \mathrm{e})$.

Table 4-3. Bond path lengths $[\AA]$, electron densities $\left[e \AA^{-3}\right]$, Laplacians $\left[e \AA^{-5}\right]$, and ellipticities at the BCPs of the Si-C bonds in $\mathbf{1 7}$.

\begin{tabular}{l|cccc}
\hline \multicolumn{1}{c}{ A-B } & $\mathrm{Si}-\mathrm{C} 3$ & $\mathrm{Si}-\mathrm{C} 1$ & $\mathrm{Si}-\mathrm{C} 2$ & $\mathrm{Si}-\mathrm{C} 10$ \\
\hline bond path & 1.814 & 1.882 & 1.887 & 1.907 \\
$\rho\left(\mathbf{r}_{\mathrm{BCP}}\right)$ & $0.90(1)$ & $0.82(1)$ & $0.81(1)$ & $0.81(1)$ \\
$\nabla^{2} \rho\left(\mathbf{r}_{\mathrm{BCP}}\right)$ & $4.2(1)$ & $3.3(1)$ & $3.2(1)$ & $2.5(1)$ \\
$\varepsilon\left(\mathbf{r}_{\mathrm{BCP}}\right)$ & 0.08 & 0.09 & 0.09 & 0.05
\end{tabular}

Accordingly, the silicon atom is profoundly depleted of charge, which was also reported in other charge density studies on silicon containing compounds. ${ }^{[183,299,324,327]}$ The Laplacian distribution of the bonded carbon atoms is pointing to polarised interactions (Figure 4-12, left) resembling those in $\left[\mathrm{TMSCH}_{2} \mathrm{Li}\right]_{6}$ (14; Figure 3-17).
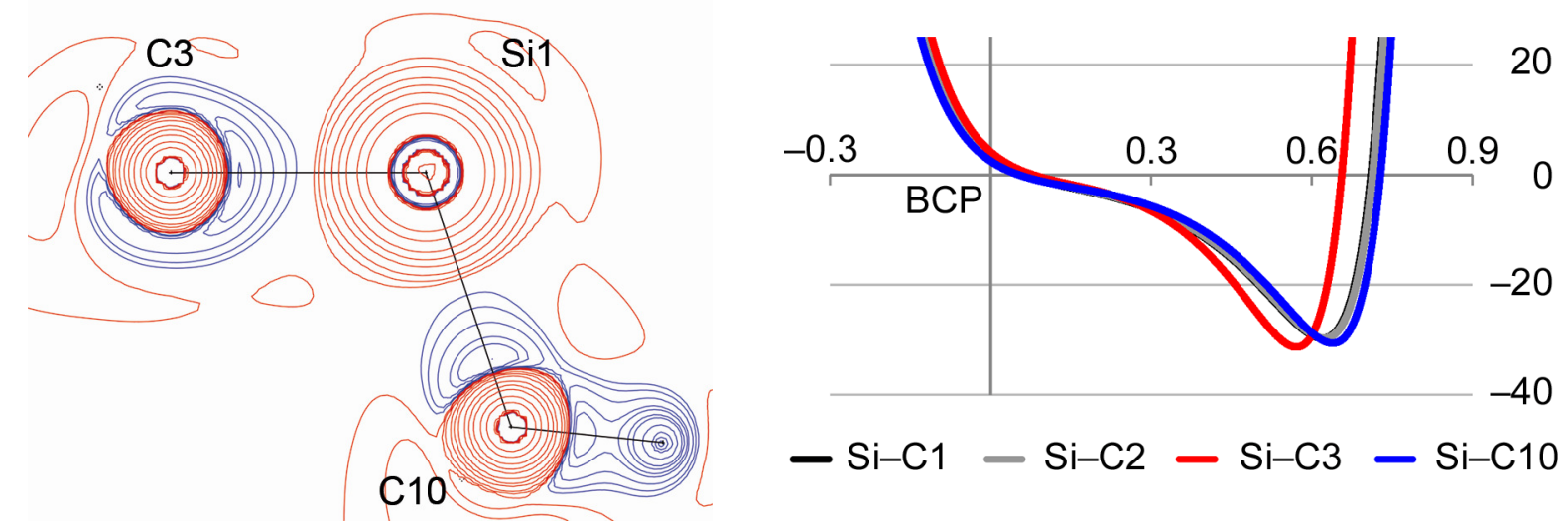

Figure 4-12. Contour plot of $\nabla^{2} \rho(\mathbf{r})$ in the $\mathrm{C} 3-\mathrm{Si}-\mathrm{C} 10$ plane (left; contour values identical to Figure 4-10) and $\nabla^{2} \rho(\mathbf{r})$ along the Si-C bond paths in $\mathbf{1 7}([\AA] ; 0=\mathrm{BCP}$ with negative values to the Si basin). 
Furthermore, the charge accumulations in the bonding regions are limited to the carbon basins. This is supported by a $\nabla^{2} \rho(\mathbf{r})$ plot along the $\mathrm{Si}-\mathrm{C}$ bonds (Figure 4-12, right). The same principal distribution is revealed for all bonds, while the minimum of the $\mathrm{Si}-\mathrm{C} 3$ bond is shifted towards the BCP due to the bond shortening. The shapes of the graphs are typical for distinctly polarised interactions showing charge depletions over the whole basin of the electropositive silicon atom and a charge concentration close to the carbon atoms (BCC).

A look at the bond ellipticities is shedding further light on the bond characteristics, as was shown for $[2-\mathrm{PicLi} \cdot \mathrm{PicH}]_{2}(\mathbf{4})$ and $\left[\mathrm{TMSCH}_{2} \mathrm{Li}\right]_{6}$ (14) before. Again, negative hyperconjugation might play an important role in the $\mathrm{Si}-\mathrm{C}_{\alpha}$ bond reinforcement, which is indicated by an $\mathrm{Si}-\mathrm{C} 3$ bond shortening and an $\mathrm{Si}-\mathrm{C} 10$ bond elongation. However, the $\mathrm{Li}-\mathrm{C} 3-\mathrm{Si}-\mathrm{C} 10$ dihedral angle $\left(32.8^{\circ}\right)$ is not ideal for this kind of interaction. It has to be noted that the stabilising orbital overlap in $\mathbf{1 7}$ would be between the $\sigma^{*}$ anti-bonding $\mathrm{Si}-\mathrm{C} 10$ orbital and the supposed $\mathrm{sp}^{2}$ hybridised lone pair lobe at $\mathrm{C} 3$ opposite to the $\mathrm{Li}-\mathrm{C} 3$ bond. The same kind of orbital arrangement was found in $\left[2-\left(\mathrm{Me}_{3} \mathrm{Si}\right)_{2} \mathrm{CPyLi}\right]_{2}(\mathbf{7})$ with a related torsion angle of $27^{\circ},{ }^{[87]}$ while a different situation was found in $\mathbf{1 4}$, in which an lone pair in an $\mathrm{sp}^{3}$ hybrid orbital lobe is involved with an $\mathrm{Li}-\mathrm{C}-\mathrm{Si}-\mathrm{C}$ dihedral angle of $180^{\circ}$.

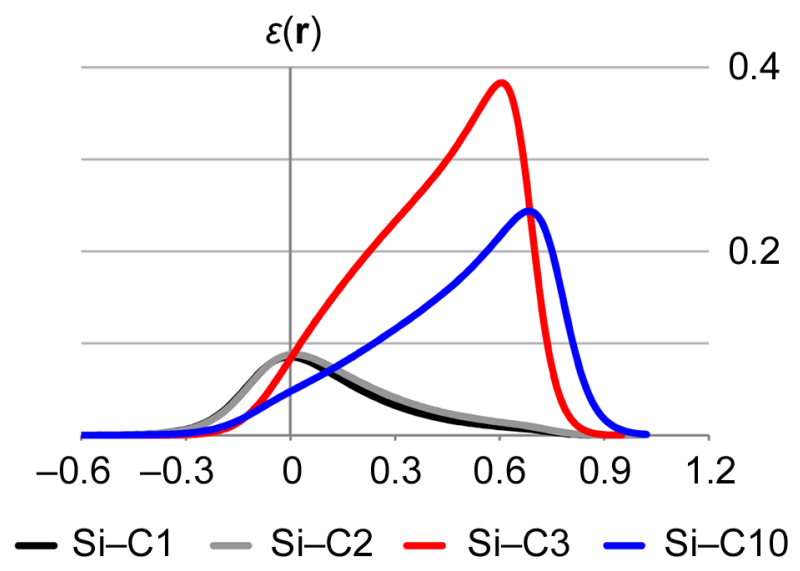

Figure 4-13. Bond ellipticities along the $\mathrm{Si}-\mathrm{C}$ bond paths $[\AA]$ in $\mathbf{1 7}(0=\mathrm{BCP}$; Si basin to negative $x$ values).

Two types of $\varepsilon(\mathbf{r})$ distributions along the $\mathrm{Si}-\mathrm{C}$ bond paths are distinguishable (Figure 4-13). The methyl carbon atoms $C 1$ and $C 2$ reveal maxima at the $B C P$, although on a very low level $\left(\varepsilon\left(\mathbf{r}_{\mathrm{BCP}}\right)=0.09\right)$ in the range of a $\mathrm{C}-\mathrm{C}$ single bond $\left(\mathrm{C} 12-\mathrm{C} 13: \varepsilon\left(\mathbf{r}_{\mathrm{BCP}}\right)=0.07\right)$. The two remaining $\mathrm{Si}-\mathrm{C}$ bonds have comparably low values at the BCPs $(0.08$ and 0.05$)$ but their profiles are completely different. Distinct maxima are observed at $\mathrm{C} 3$ and $\mathrm{C} 10$, while the ellipticity decreases in 
the proximity of the silicon atom. The deprotonated carbon atom gives rise to the most pronounced $\varepsilon(\mathbf{r})$ maximum in agreement with the results of $W$. Scherer et al. on charged C-C interactions. ${ }^{[87]}$ The main course of $\varepsilon(\mathbf{r})$ is, however, not comparable to those of $\mathbf{7}$ and $\mathbf{1 4}$, since no maximum of the ellipticity near the bond critical point could be detected. Hence, the involvement of negative hyperconjugation in the stabilisation of the negative charge at C3 can neither be affirmed nor definitely ruled out. Moreover, it is remarkable that the Si-C10 bond also exhibits an asymmetric $\varepsilon(\mathbf{r})$ distribution analogous to the $\mathrm{Si}-\mathrm{C} 3$ bond. The reason for that might be a distinct polarisation of the methylene bridge atom $\mathrm{C} 10$ by the more electronegative nitrogen atom $\mathrm{N} 1$, which is transferred to the polarisable silicon atom.

According to the results above, the negative charge originating from the hydrogen abstraction is still concentrated on C3 owing to the stabilising effect of the neighbouring silicon atom, which is highly charged and strengthens the $\mathrm{Si}-\mathrm{C} 3$ bond by electrostatic forces. Yet, the localisation of the negative charge at the deprotonated carbon atom has not been elucidated so far. A BCP search around C3 gave bond critical points and bond paths to all neighbouring atoms including the cation. A bond to the carbon lone pair (discrepancies in the use of the term LP were already discussed in chapter 2.3.7 and 3.2.4) has to be assumed, which is associated with a p orbital orthogonal to the plane of $\mathrm{C} 3$ and its surrounding bonding partners.

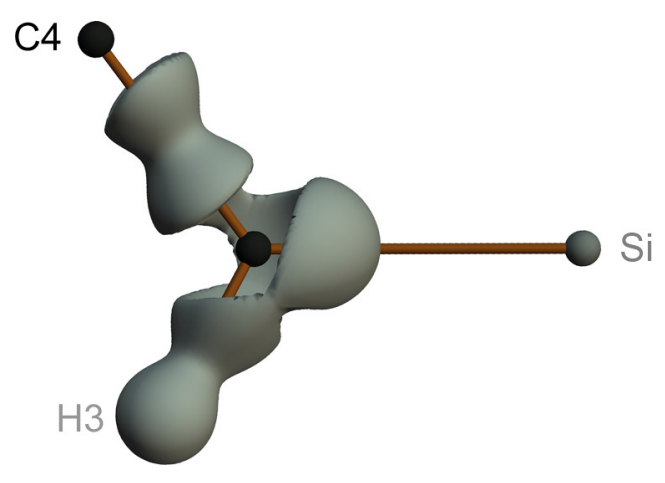

a)

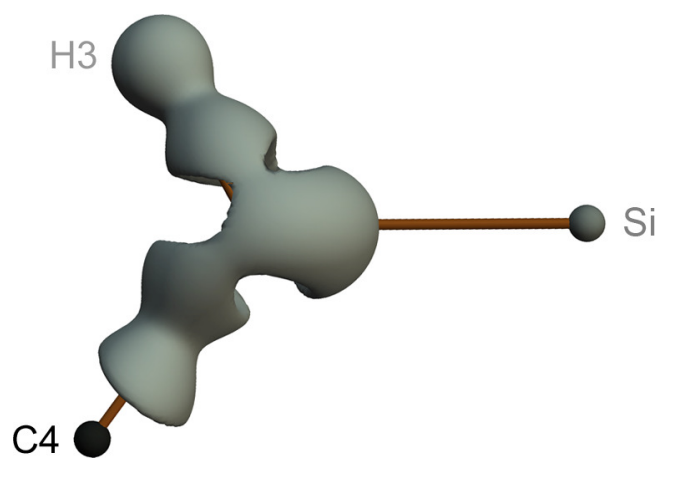

b)

Figure 4-14. Isosurface representation of $\nabla^{2} \rho(\mathbf{r})\left(-10.0 \mathrm{e}^{-5}\right)$ around $\mathrm{C} 3$ in $\mathbf{1 7}$ with the backside (a) or the front side (b) facing $\mathrm{Li}$.

However, an intense search for VSCCs around C3 resulted in only three BCCs to the non-metal atoms. No additional CC could be detected neither facing the lithium atom nor located on the opposite side. This result resembles the situation at the methylene carbon atom in $[2-\mathrm{PicLi} \cdot \mathrm{PiCH}]_{2}(\mathbf{4})$. Therefore, the spatial 
distribution of $\nabla^{2} \rho(\mathbf{r})$ is depicted to find indicators for a charge polarisation to the cation (Figure 4-14). Indeed, pronounced differences between both sides of the $\mathrm{C} 4-\mathrm{C} 3(-\mathrm{H} 3)-\mathrm{Si}$ plane are visible. Again, the cation induces a slightly asymmetric charge accumulation around the anion but no VSCC.

The question arises as to why the carbanion is surrounded planar, if a $\mathrm{sp}^{3}$ rehybridisation maximises the interaction of the negative charge with the cation. Previous results (chapter 2 and 3 ) clarified that the bonding to lithium cations is barely controlled by orbital interactions but mainly based on charges. Furthermore, the lone pair in the p orbital is interacting with the $\pi$-electron density of the aromatic ring, which is reflected by $\rho\left(\mathbf{r}_{\mathrm{BCP}}\right)$ and the ellipticity along the C3-C4 bond path (conjugation), although it is not delocalised. Another argument is the just slightly asymmetric negative charge distribution around the anion that maximises the electrostatic interaction with the silicon cation.

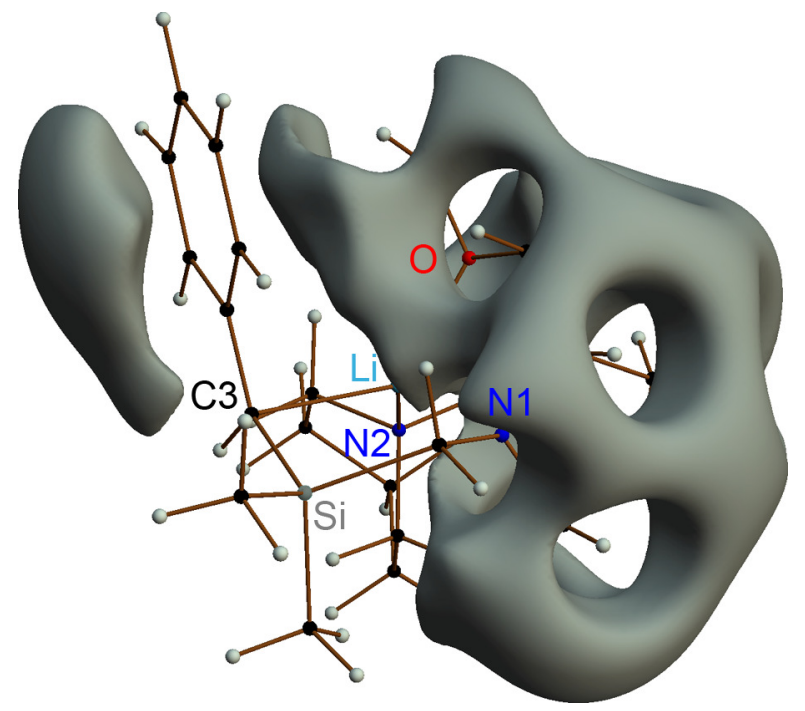

Figure 4-15. Isosurface representation of the ESP on a level of $-0.1 \mathrm{e} \AA^{-1}$ of $\mathbf{1 7}$.

The slight charge polarisation at C3 is definitely not sufficient to direct an electrophile into the sterically blocked pocket near the cation, which is maintained in solution (cf. NMR results). However, there was also no indication found for the preferred inversion reaction with electrophiles at the stereogenic centre so far (no charge accumulation opposite to the Li-C bond). Again ( $c f$. Figure 2-38), the electrostatic potential can explain the stereogenic pathway of substitution reactions. Two areas of possible electrophilic attacks can be identified (Figure 4-15). The first covers the pyrrolidine ring including the methoxymethyl side chain. This is irrelevant for substitution reactions because appropriate reactive centres are missing. The second area is located at the benzylic unit 
opposite to the lithium atom. This surface is extended towards $\mathrm{C} 3$ and resembles the ESP distribution in [2-PicLi-PicH $]_{2}$ (4). Electrophiles are literally guided to the deprotonated carbon atom on the opposite side of the $\mathrm{Li}-\mathrm{C}$ interaction. Consequently, the backside attack of $\mathrm{C} 3$ under inversion of the configuration in high diastereomeric ratios is straightforwardly explained by the ESP.

Table 4-4. Bond path lengths $[\AA]$, electron densities $\left[\mathrm{e}^{-3}\right]$, Laplacians $\left[\mathrm{e} \AA^{-5}\right]$, and ellipticities at the BCPs of the Li-X bonds $(X=C, O, N)$ in 17.

\begin{tabular}{l|cccc}
\hline \multicolumn{1}{c}{$\mathrm{A}-\mathrm{B}$} & $\mathrm{Li}-\mathrm{C} 3$ & $\mathrm{Li}-\mathrm{O}$ & $\mathrm{Li}-\mathrm{N} 1$ & $\mathrm{Si}-\mathrm{N} 2$ \\
\hline bond path & 2.279 & 1.983 & 2.164 & 2.118 \\
$\rho\left(\mathbf{r}_{\mathrm{BCP}}\right)$ & $0.09(1)$ & $0.15(1)$ & $0.11(1)$ & $0.13(1)$ \\
$\nabla^{2} \rho\left(\mathbf{r}_{\mathrm{BCP}}\right)$ & $2.2(1)$ & $4.8(1)$ & $3.2(1)$ & $3.7(1)$ \\
$\nabla^{2} \rho\left(\mathbf{r}_{\mathrm{LP}(\mathrm{B})}\right)$ & - & $-133.1(1)$ & $-73.0(1)$ & $-74.7(1)$
\end{tabular}

Focussing on the lithium bonds, three different donor atom types can be compared to each other. Since, the nature of the Li-O bond is commonly accepted to be predominantly ionic - other than the controversially discussed lithium-carbon bonds ( $c f$. chapter 1) - it can serve as internal reference. The bond characteristics in respect to ionic or covalent contributions are reflected by the course of the Laplacian along the whole bond paths. Even the positive values of $\nabla^{2} \rho\left(\mathbf{r}_{\mathrm{BCP}}\right)$ (Table $\left.4-4\right)$ already indicate closed shell interactions for all contacts to the lithium cation.

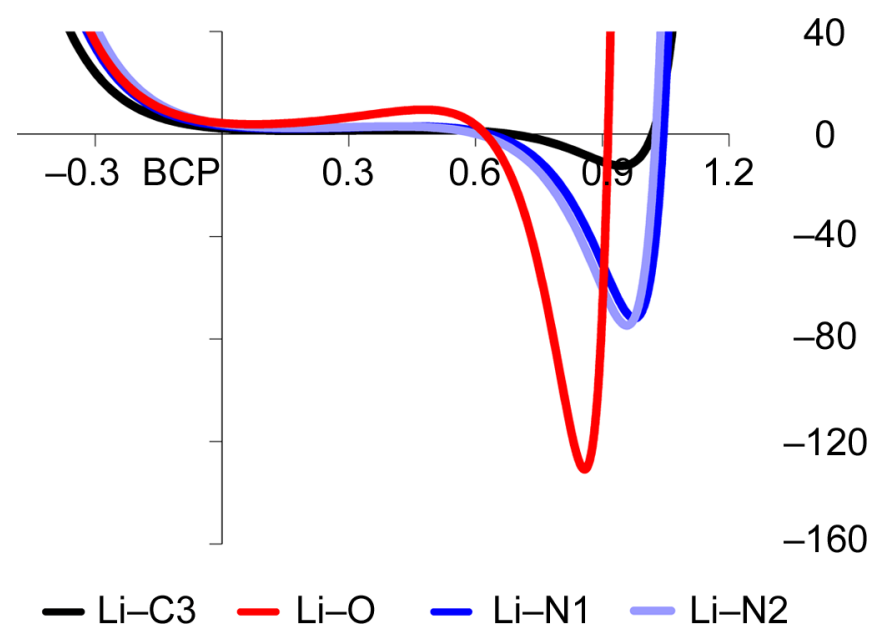

Figure 4-16. $\nabla^{2} \rho(\mathbf{r})$ along the Li-X bond paths $(\mathrm{X}=\mathrm{C}, \mathrm{O}, \mathrm{N})$ in $\mathbf{1 7}([\AA] ; 0=\mathrm{BCP}$ with negative values towards $\mathrm{Li}$ ). 
A charge depletion in the whole bond region is evident for all $\mathrm{Li}-\mathrm{X}$ bonds $(\mathrm{X}=$ $\mathrm{C}, \mathrm{O}, \mathrm{N})$ in $\mathbf{1 7}$ with a qualitatively similar shape of $\nabla^{2} \rho(\mathbf{r})$ along the Li-N/C bonds (Figure 4-16). $\nabla^{2} \rho(\mathbf{r})$ is positive in the lithium basin and all the way to the carbon atom up to the VSCCS $(\mathrm{N})$ or the charge accumulation of the deprotonated carbon atom ( $C$; cf. Figure 4-13) are reached. Only the absolute values of the negative Laplacians differ in the known way. The nitrogen donors with their lone pairs facing the cation exhibit more pronounced minima in comparison to the merely polarised carbanion.

An even more distinct charge separation is evident in the $\mathrm{Li}-\mathrm{O}$ bond. $\nabla^{2} \rho(\mathbf{r})$ adopts a maximum when approaching the valence shell of the oxygen atom, where it forms an extremely pronounced minimum, typical for a lone pair. With these results at hand and those of $[2-\mathrm{PiCLi} \cdot \mathrm{PiCH}]_{2}(\mathbf{4})$ and $\left[\mathrm{TMSCH}_{2} \mathrm{Li}\right]_{6}(\mathbf{1 4})$, it can currently be concluded that the lithium-carbon bond belongs to the group of ionic Li-X interactions showing comparable characteristics with lower absolute values in the carbon basin compared to the nitrogen donor atoms and even similarities to the Li-O contact.

As mentioned above, all bond paths to the four donor sites were found (Table 4-4). The electron density values can be related to the donor strength. The lithium-oxygen bond is the shortest, leading to the highest electron density value of $0.15 \mathrm{e}^{-3}$. This is supported by the highest atomic charge $(-1.32 \mathrm{e})$ and the most distinct VSCC $\left(-133.1 \mathrm{e}^{-5}\right)$. Interestingly, only three VSCCs were found at the oxygen atom. Two BCCs are directed towards the neighbouring carbon atoms and one $\mathrm{CC}$ is oriented to $\mathrm{Li}$ forming an acute $\mathrm{Li}-\mathrm{O}-\mathrm{LP}$ angle of $51.3^{\circ}$. The Laplacian distribution on a level of -100 e $\AA^{-5}$ displays that the charge accumulation of the two lone pairs cannot be resolved and a banana shaped belt facing the metal ion is form (Figure 4-17, left). This effect is well known and currently interpreted as a reorientation of the LPs towards the acceptor site strengthening the donor capability of the atom. ${ }^{[71,72,299]}$
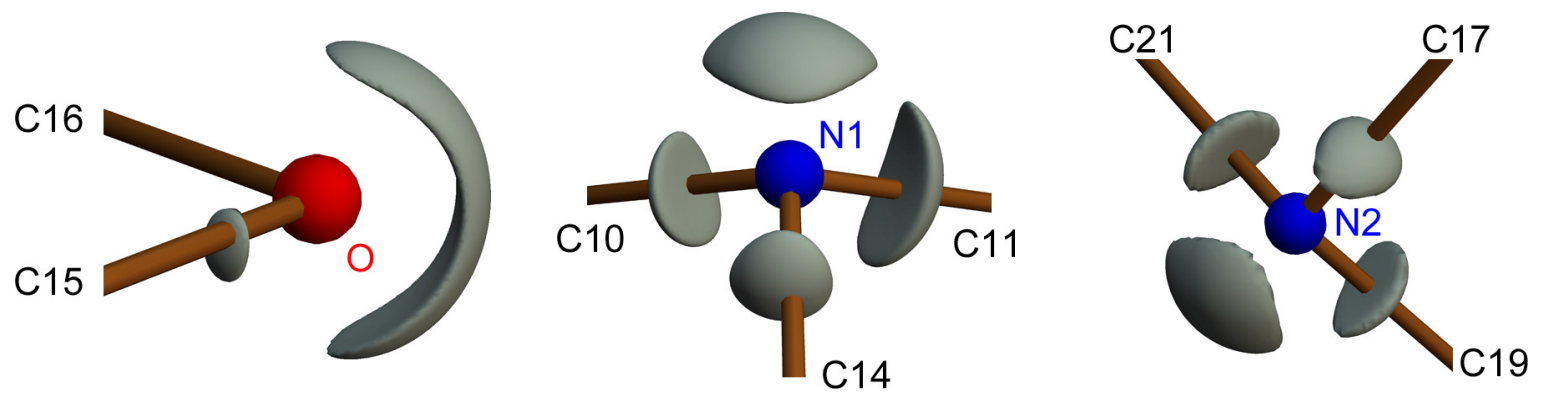

Figure 4-17. Laplacian distribution around the oxygen atom (left; isosurface value: $-110 \mathrm{e} \AA^{-5}$ ) and the nitrogen atoms (centre and right; isosurface value: $-50 \mathrm{e} \AA^{-5}$ ) in $\mathbf{1 7}$. 
The nitrogen atoms share almost the same charges $(-1.13$ and $-1.22 \mathrm{e})$ and $\nabla^{2} \rho\left(\mathbf{r}_{\mathrm{LP}}\right)\left(-73.0\right.$ and $\left.-74.7 \mathrm{e} \AA^{-5}\right)$ (Figure $4-17$, centre and right). But the Li-N bond lengths differ by $0.044 \AA$ causing a lower $\rho\left(\mathbf{r}_{\mathrm{BCP}}\right)$ for the longer bond $\left(0.11 \mathrm{e} \AA^{-3}\right)$ compared to the $\mathrm{Li}-\mathrm{N} 2$ bond $\left(0.13 \mathrm{e} \AA^{-3}\right)$. These values are close to related nitrogen donor bonds (cf. Table 2-8) in [2-PicLi-PicH $]_{2}(4)$, and slightly smaller than those in $\left[\left(\mathrm{Et}_{2} \mathrm{O}\right) \cdot \mathrm{Li}\left\{\mathrm{Ph}_{2}(\mathrm{CHPy}) \mathrm{P}\left(\mathrm{NSiMe}_{3}\right)\right\}\right]$ (6) and $\left[2-\left(\mathrm{Me}_{3} \mathrm{Si}\right)_{2} \mathrm{CPyLi}\right]_{2}(\mathbf{7})$.

The weakest interaction was identified for $\mathrm{Li}-\mathrm{C} 3$. This is expressed by an electron density of $0.09 \mathrm{e}^{-3}$ at the $B C P$, the largest bond length $(2.273(1) \AA)$, and the absence of a lone pair at the deprotonated carbon atom. Moreover, this trend of decreasing donor strength is also reflected by an elongated bond path. While the two stronger interactions ( 01 and $\mathrm{N} 2$ ) have no deviation of atom distance from path length, the weaker bonds are more curved (difference $0.002 \AA$ for N1 and $0.007 \AA$ for $(3)$.

Remarkably, the surrounding atoms of the positively charged lithium atom $(+0.94$ e) display distinct negative atomic charges $(-0.96$ to -1.32 e) in $\mathbf{1 7}$ (Figure 4-18). Each donor site alone equalises the total charge at the cation accompanied by a strong polarisation at all neighbouring atoms. This charge accumulation is one argument for the stability of this complex, in which the lithium atom is fixed in a pocket of pronounced electron density. The absolute structure can consequently be maintained even in solution up to RT.

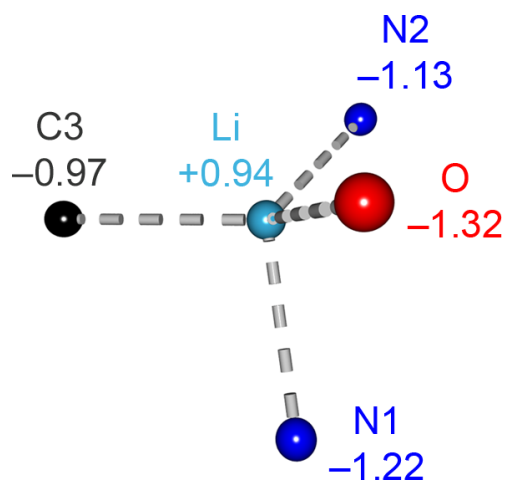

Figure 4-18. Integrated charges [e] of Li and its coordination sphere in $\mathbf{1 7}$.

$\rho\left(\mathbf{r}_{\mathrm{BCP}}\right)$ of the Li-C bond can be compared to the theoretical value in [2-PicLi.PicH $]_{2}$, to those bonds in $\left[\mathrm{TMSCH}_{2} \mathrm{Li}\right]_{6}(\mathbf{1 4})$, and to the only two published experimental charge density investigations - by $W$. Scherer et al. and S. Deuerlein et al. - containing $\mathrm{Li}-\mathrm{C}$ bonds. ${ }^{[87,88]} \mathrm{A}$ similar bonding situation involving a carbon atom in a planar surroundings is found in $[2-\mathrm{PiCLi} \cdot \mathrm{PiCH}]_{2}$ 
whose electron density value of the $\mathrm{Li}-\mathrm{C}$ contact $\left(0.11 \mathrm{e} \AA^{-3}\right)$ is close to the one in 17. Similar values are also reported for the sulfur ylide (mean value: $0.09 \mathrm{e} \AA^{-3}$ ), while significant differences are observed for those bonds involving an $\mathrm{sp}^{3}$ hybridised carbanion. Both, the $\mathrm{TMSCH}_{2} \mathrm{Li}$ hexamer and the substituted picolyllithium dimer $\mathbf{7}$, have a $\rho\left(\mathbf{r}_{\mathrm{BCP}(\mathrm{Li}-\mathrm{C})}\right)$ of around $0.14 \mathrm{e} \AA^{-3}$ originating from the participation of discrete lone pairs in the bonding. In summary, $(R)-[\{(S)$ 2-(methoxymethyl)pyrrolidinomethyl\}dimethylsilyl]benzyllithium as its quinuclidine adduct (17) adds four other $\mathrm{Li}-\mathrm{X}$ bonds to the small group of experimentally investigated (ED) lithium-donor bonds, thus providing further information of the bonding situation in lithium organic compounds.

\subsection{Theoretical EDD of the Benzyllithium Derivative}

It was refrained from comparing the theoretical (18) with the experimental (17) charge density results of the substituted benzyllithium compound in a parallel way similar to $[2-\mathrm{PicLi} \cdot \mathrm{PicH}]_{2}(4)$. The reason for that are the different geometrical parameters in the gas phase (Figure 4-19). Bond lengths and angles could not be adequately reproduced by the calculations on a B3LYP $[201,202]$ / $6-31+G(d)^{[203,204]}$ level.

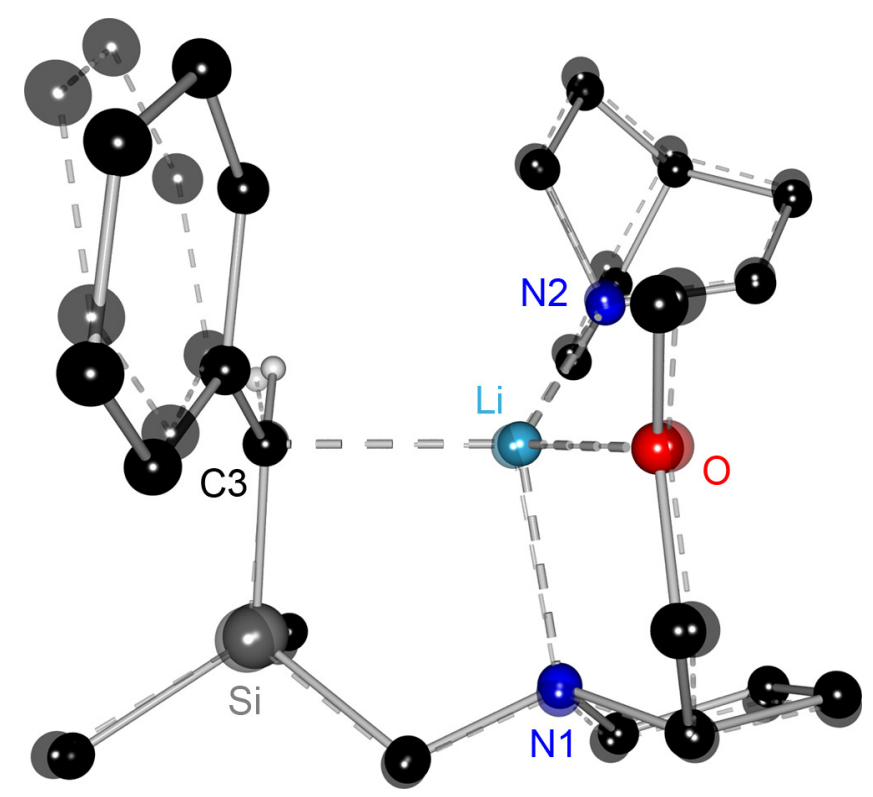

Figure 4-19. Superposition of the molecular structures of $\mathbf{1 7}$ and $\mathbf{1 8}$ (X-ray: solid; theory: dashed and transparent). Reference atoms are all atoms involved in $\mathrm{Li}-\mathrm{X}$ bonds $(X=C, O, N)$. 
This is crucial at the deprotonated carbon atom, which is pyramidalised in theory (values from theory are written in italics). This results in a totally different orientation of the phenyl ring in comparison to the solid state as shown in Figure 4-19.

The sum of the bond angles of the formally planar $\mathrm{C} 4-\mathrm{C} 3(-\mathrm{H} 3)-\mathrm{Si}$ fragment is now $351.3^{\circ}$ ( $358.5^{\circ}$ in $\mathbf{1 7}$ ). The partial $\mathrm{sp}^{3}$ hybridisation of the atomic orbitals at $\mathrm{C} 3$ in 18 has also an influence on its bond lengths. The Si-C3 (1.832 vs. $1.813 \AA$ ) and C3-C4 (1.451 vs. $1.434 \AA$ ) bonds are longer, while the lithium contact is shorter (2.213 vs. $2.273 \AA$ ) compared to 17 . This is also mirrored in the $\rho\left(\mathbf{r}_{\mathrm{BCP}}\right)$ values of the determined bond paths. All bonds as well as the corresponding RCPs and CCPs could be also found in theory (Figure 4-20). However, theory and experiment are once again on a slightly different scale (cf. chapter 2.3.7) and a one-to-one comparison of electron density values is not possible. Nevertheless, a mean difference can be estimated from an examination of the carbon-carbon single bonds in the 3/4-position of the pyrrolidine ring, which should not be influenced too much by the geometry changes. Indeed, the $\rho\left(\mathbf{r}_{\mathrm{BCP}}\right)$ values vary by only 0.03 and $0.07 \mathrm{e}^{-3}$.

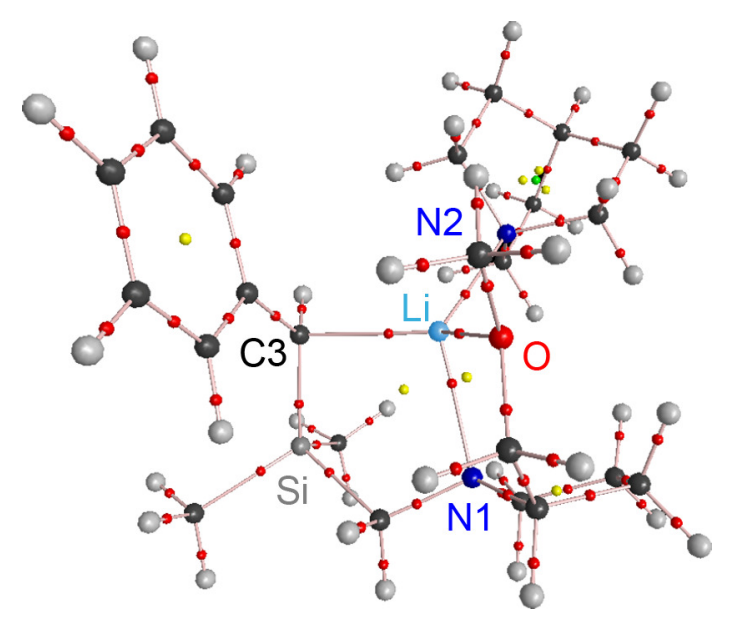

Figure 4-20. Molecular graph of $\mathbf{1 8}$ (theoretical EDD). The lines represent BPs, red dots BCPs, yellow dots RCPs, and green dots CCPs.

By contrast, an almost doubled electron density at the BCP is quantified for the $\mathrm{Li}-\mathrm{C} 3$ bond $\left(0.17\right.$ vs. $0.09 \mathrm{e}^{-3}$ ) indicating a stronger lithium-carbon interaction. In contrast, the remaining $\mathrm{Li}-\mathrm{X}$ bonds are all longer than in $\mathbf{1 7} \mathrm{( \textrm {Li } -}$

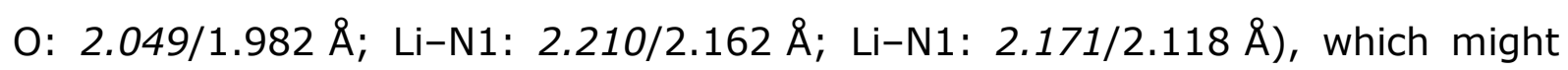
be caused by the improved donating capacity of C3. But only the Li-O bond features a marginal reduced $\rho\left(\mathbf{r}_{\mathrm{BCP}}\right)\left(0.14 e \AA^{-3}\right)$ compared to $\mathbf{1 7}$, while the $\rho\left(\mathbf{r}_{\mathrm{BCP}}\right)$ of the Li-N bonds are slightly increased (Li-N1: $0.13 e \AA^{-3}$; Li-N2: $0.14 e \AA^{-3}$ ). As 
a result, no significant differences have been detected for the $\mathrm{Li}-\mathrm{O} / \mathrm{N}$ bonds in respect to the experimental results.

Moreover, the rehybridisation at $\mathrm{C} 3$ also affects $\rho\left(\mathbf{r}_{\mathrm{BCP}}\right)$ of the C3-C4 bond. A dramatically lowered density value $\left(1.85 e \AA^{-3}\right)$ in comparison to $17\left(2.02 \mathrm{e} \AA^{-3}\right)$ mirrors the reduced $\pi$ interaction to the aromatic system owing to the partial $\mathrm{sp}^{3}$ hybridisation of the atomic orbitals at the deprotonated carbon atom. Furthermore, the pyramidalisation of C3 leads to a fourth VSCC - a lone pair facing the cation (Figure 4-21, left; Figure 4-22). The value of $\nabla^{2} \rho\left(\mathbf{r}_{\mathrm{LP}}\right)$ is $-14.9 e \AA^{-5}$, which is the smallest value of all reported lone pairs at a carbanion in this work (cf. chapter 3.2.4), but it is already close to those found for $\left[2-\left(\mathrm{Me}_{3} \mathrm{Si}\right)_{2} \mathrm{CPyLi}\right]_{2}$ (7; $\left.-18.0 \mathrm{e} \AA^{-5}\right) .{ }^{[87]}$ The spatial distribution of $\nabla^{2} \rho(\mathbf{r})$ was inspected on the same isosurface level as in Figure 4-14 in order to visualise differences to the experimental results (Figure 4-21; left). A broad $\nabla^{2} \rho(\mathbf{r})$ belt from one BCC $\left(-18.2 e \AA^{-5}\right.$ to $\mathrm{Si}$ ) to the $\mathrm{BCC}$ towards the cation is observed. This splits up into two well separated VSCCs on a more negative $\nabla^{2} \rho(\mathbf{r})$ isosurface value.
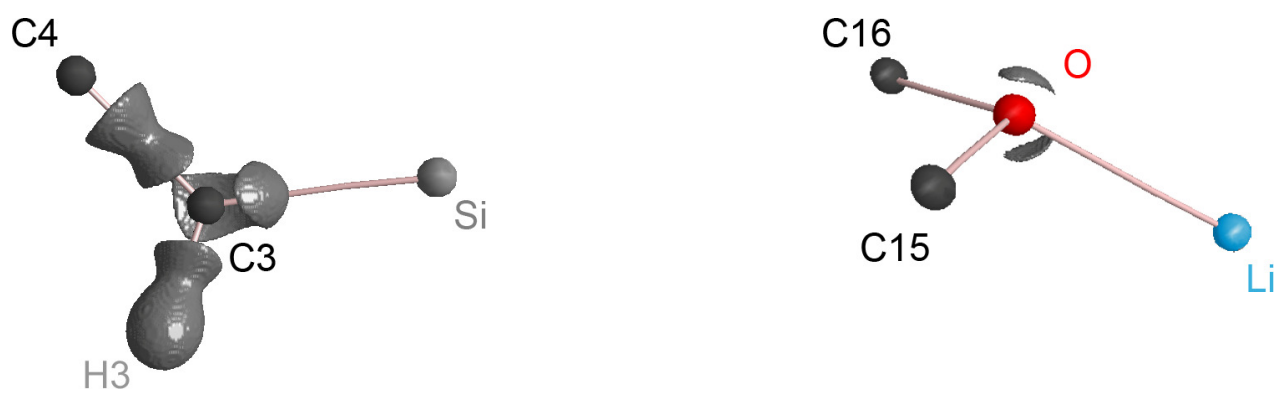

Figure 4-21. Isosurface representation of $\nabla^{2} \rho(\mathbf{r})$ around C3 (left; view along the C3-Li bond; isosurface value: $-10.0 \mathrm{e} \AA^{-5}$ ) and $\mathrm{O}$ (right; $-130.0 \mathrm{e} \AA^{-5}$ ) based on 18.

The exact position of these VSCCs can be seen from Figure 4-22. It can be concluded that the $\mathrm{Li}-\mathrm{C}$ interaction is maximised in the gas phase by a change of the hybridisation state at the deprotonated carbon atom C3 and the formation of a lone pair directed to the cation.

A search for VSCCs in $\mathbf{1 8}$ revealed all expected charge concentrations (Figure 4-22). Two BCCs are found for each $\mathrm{C}-\mathrm{C} / \mathrm{N} / \mathrm{O}$ bond and one for the $\mathrm{C}-\mathrm{H}$ bonds. Similar to 17, no VSCCs of the silicon atom are detected owing to its distinct charge depletion. However, two peculiarities are observed in the theoretical VSCC plot in contrast to 17. Four VSCCs are present at the oxygen atom. Three BCCs to the bonding partners including the lithium cation are observed and one "real" lone pair ( $c f$. definition: $L P=N B C C$ ). An isosurface representation of $\nabla^{2} \rho(\mathbf{r})$ around the oxygen atom illustrates the positions of the two LPs (Figure 
4-21; right). Both exhibit very high negative values (-154.7 and -160.2 e $\AA^{-5}$ ) and are widely separated, while a banana shape distribution was found in $\mathbf{1 7}$ (cf. Figure 4-17, left).

Moreover, a fifth VSCC can be found at N1 opposite to the Li-N bond in its projection. This phenomenon cannot be explained so far, but it has to be mentioned that a similar VSCC distribution was also observed for the nitrogen atoms in the theoretical EDD of tert-butyllithium as (-)-sparteine adduct and is currently under further investigation. ${ }^{[328]}$

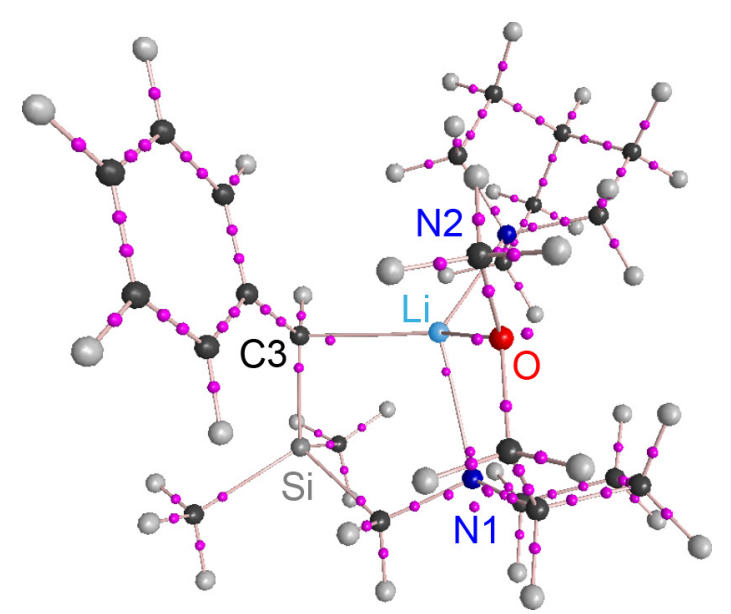

Figure 4-22. Positions of the VSCCs (magenta dots) projected on a molecular graph showing BPs of $\mathbf{1 8 .}$

Focussing on some main results of the experimental electron density distribution in the crystal of 17, many consistencies are detected. A Laplacian plot in the $\mathrm{C} 4-\mathrm{C} 3-\mathrm{Si}$ as well as $\mathrm{C} 3-\mathrm{Si}-\mathrm{C} 10$ plane is also showing a tremendous charge depletion of the silicon atom (Figure 4-23; cf. Figure 4-10 and Figure $4-12$, left). This affects the determined silicon charge as well. An even more positive atomic charge is found in the gas phase $(+2.84 \mathrm{e})$ compared to the crystal $(+2.15 \mathrm{e})$. The deprotonated carbon atom on the other hand is more negatively charged $(-1.12 \mathrm{vs}$. $-0.97 \mathrm{e})$. The charge accumulation at the deprotonated carbon atom is hence also present in theory. The remaining donor atoms exhibit charges comparable to $\mathbf{1 7}$. They bear a charge of more than minus one electron $(01:+1.12 \mathrm{e} ; \mathrm{N} 1:+1.04 \mathrm{e} ; \mathrm{N} 2:+1.02 \mathrm{e})$ and a similar electrostatic pocket is evident as in $\mathbf{1 7}$ that stabilises the cation $(+0.88 \mathrm{e})$.

In summary, the theoretical results provide some additional information, although the geometrical changes at the deprotonated carbon atom prevent a comprehensive comparison of the electron density distributions. 

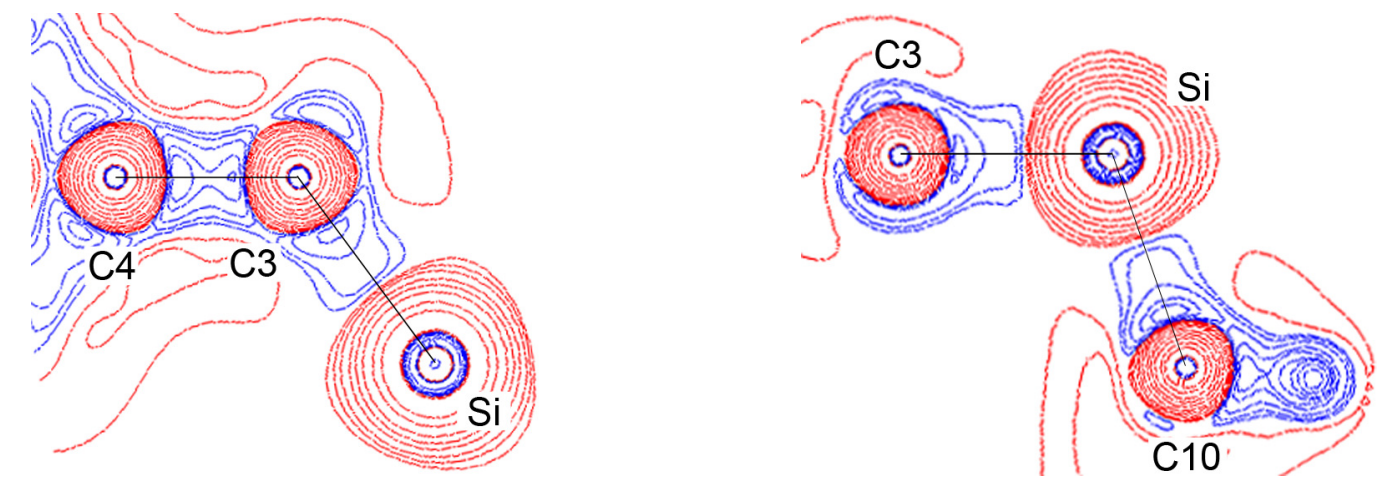

Figure 4-23. Contour plot of $\nabla^{2} \rho(\mathbf{r})$ in the C4-C3-Si (left) and $\mathrm{C} 3-\mathrm{Si}-\mathrm{C} 10$ (right) planes of 18 (contour values identical to Figure 4-10).

\subsection{Conclusion}

The highly enantiomerically enriched benzylsilane, $(R)$-[ $[\{(S)-2-($ methoxymethyl)pyrrolidinomethyl\}dimethylsilyl]benzyllithium·quinuclidine (17), was selected as a model compound to elucidate, among others, the reasons for the observed stereochemical pathway by an experimental charge density study. The absolute configuration at the metallated carbon atom C3 of this compound is fixed even at RT in solution so that high diastereomeric ratios of above 98:2 are obtained after the reaction with electrophiles under inversion of the configuration.

This is caused by a strong fixation of the lithium cation by four highly negatively charged donor atoms. Furthermore, the influence of the phenyl ring with respect to the stabilisation of the negative charge at C3 was surprisingly less significant than expected. Yet, the neighbouring silicon atom proved to counterbalance the charge accumulation at the deprotonated atom by a distinct positive charge. The $\alpha$-effect of the silicon atom is therefore not just caused by a polarisation of the electron density but also by electrostatic bond reinforcement. The stereo-chemical course of electrophilic substitution reactions under stereoinversion could be well understood by the charge density distribution and the electrostatic potential of the lithium organic compound. Furthermore, the lithium-carbon bond could be consistently integrated into the set of other polar donor bonds, of which three others were added to the small group of lithiumdonor bonds that have been investigated by experimental charge density studies. 



\section{Conclusions and Future Objectives}

Two major goals have been pursued and finally attained in the course of this thesis. The first was to synthesise and crystallise novel unsubstituted picolyl metal adducts for structural analyses and the second was to investigate these aggregates and other lithium organic compounds by experimental charge density methods in order to obtain detailed information on their bonding situations.

The preparative work in the field of unsubstituted 2-picolyllithium compounds extended the series of lead structural motifs in these benzyl type anions. The main coordination modes are $\eta^{1}$-alkyl, $\eta^{1}$-enamidic, and $\eta^{3}$-aza-allylic. It was also possible to isolate a 2-PicLi monomer with the tridentate pmdeta donor to maximise its reactivity. The compounds were obtained in acceptable yields as crystalline materials, storable in argon dry boxes for months. This makes them, together with their easy accessibility from cheap starting materials in laboratory quantities, valuable synthetic precursors. The tmeda and pmdeta adducts proved crystallise easily, so that it is advised to focus on these compounds if donor bases do not prevent subsequent synthetic steps. The isolation of crystalline material is of importance, because various in situ conversions with picolyllithium compounds only led to low yields and by-products. In close cooperation with the author of this thesis, I. Objartel was able to increase the reported yield of trimethylsilyl(2-pyridyl)methane $(42 \%)$ to above $80 \%$ by using crystalline [2-PicLi-tmeda $]_{2}$ (2) rather than a 2-PicLi reaction mixture in the conversion with $\mathrm{TMSCl}^{[111]}$ 2-TMSCH${ }_{2} \mathrm{Py}$ is an important precursor to phenyldipicolylphosphane $\left(\mathrm{PhPPic}_{2}\right)$, which is a versatile ligand in complex chemistry and catalysis.

One of the anionic picolyl metal organic compounds crystallised in sufficient quality to perform an experimental charge density study. [2-PicLi.PicH] 2 (4) has an aza-allylic coordination mode of the metal ion and also includes 2-picoline as internal reference. Preferred bonding interactions of the picolyl nitrogen atoms to the cations can be detected, while the formal anionic carbon atom plays only a minor role in the coordination of the lithium cation. The negative charge is readily delocalised over the aromatic ring, causing a severe disturbance of the $\pi$-system, even though the highest relative increase of the negative charge was observed at the deprotonated carbon atom. Partially localised double bonds are present, so that an enamidic description of the anion is favoured. In addition, the spatial distribution of the electrostatic potential indicates, why the enamide is 
generally attacked by electrophiles at the side chain. These are literally guided to the exocyclic carbon atom by a large area of negative ESP at the picolyl anions.

With all the isolated adducts at hand, it has been possible to investigate the effect of the donor molecules on the coordination mode of 2-picolyllithium compounds, free from interfering side chain or core substituents. This enables the understanding - and looking ahead, maybe also the prediction - of the coordination modes in the crystal according to the applied donor and its donor strength as well as steric bulk. This study was further supported by the charge density results of $\mathbf{4}$ and by theoretical calculations on 2-PicLi reported before. ${ }^{[107,133]}$

However, additional 2-picolyllithium coordination patterns are conceivable. These are solvent-separated ion pairs, which might arise from the use of 12-crown-4-ether, or polymeric chain structures with polydentate ligands such as di- or tetraglyme. However, several attempts to crystallise 2-PicLi with these donors failed until now. The same applies to donor-free 2-picolyllithium prepared in hexane, which turned out to be insoluble in various non-coordinating hydrocarbons. The molecular structure of this compound would be a valuable benchmark, because an aza-allylic coordination mode can be expected. If it turns out that no single crystals can be obtained, powder diffraction might provide the solid state structure by applying an aza-allylic structure model as starting point for the refinement.

The 2-picolyllithium solid state structures are well established by now, but their composition and structure of the aggregates in solution were unknown and had to be studied by several NMR experiments. Unprecedented effects and evidence for clarifying the aggregation state and the coordination mode to the cation in the different adducts were discovered, but have not yet been ultimately understood.

Furthermore, the research was extended to heavier alkaline metal compounds. The first step was accomplished with the 2-picolylsodium tmeda adduct, which was isolated as crystalline material and investigated by X-ray diffraction. According to the known reactivity enhancement of the lithium congeners, it is now possible to tune the reactivity of the picolyl group by the appropriate choice of the cation and the donor base. More efforts have to be undertaken to crystallise the potassium, rubidium, and caesium analogues. The crystallisation might be simplified in comparison to the benzyl analogues by the improved solubility characteristics due to the intramolecular donor site of the picolyl ring. Moreover, the synthesised picolylsodium compound showed the potential for an experimental charge density study and would be an ideal model compound to 
elucidate the differences in the bonding modes caused by the exchange of the alkaline metal and to compare the enamidic to the aza-allylic coordination pattern.

The experimental work was also extended to 4-picolyllithium, which is far less applied in synthesis compared to the 2-isomer, due to by-product formations during preparation. In order to overcome the concomitant ring addition of the strong base during the deprotonation of 4-picoline, various metal organic compounds were screened. Methyllithium proved to be the adequate choice for the hydrogen abstraction and the pmdeta monomer was identified as adduct that can readily be crystallised. All isolated aggregates showed a preferred coordination to the cations via the ring nitrogen atoms, as was expected by the results from the research on 2-picolyllithium. However, an additional coordination mode was detected for this species. The deprotonation of 4-picoline with $n B U L i$ in the presence of tmeda resulted in a chain structure of the anions, in which the picolyl nitrogen and the deprotonated carbon atoms interact with the lithium cations. In all the other adducts a complete delocalisation of the negative charge into the ring could be observed without any interaction of the methylene carbon atom with the cations. Furthermore, the first sodium and potassium analogues were synthesised with the super base approach and were isolated as powdery materials. However, the crystallisation with an appropriate donor solvent was not successful up to now, so that these results were not included in this work. Nonetheless, this research is promising and to be carried on.

It would also be desirable to find a 4-picolyllithium adduct that could be crystallised in excellent quality. This was not the case so far and no experimentally derived electron density distribution could be analysed and compared to that of the 2-isomer. The differences in the delocalisation degree and the accumulated charge on the ring nitrogen atom would give deeper insight into the precise bonding situation in the deprotonated 4-picoline compounds.

With all these results, the next step to be made is the extension of the work to the much less acidic 3-picoline compounds that were barely employed in synthesis yet, even though a shift in the nitrogen ring position would enable to regulate the coordination angles of a ligand. First efforts were made in the deprotonation with super bases, but the isolated material could not be crystallised so far. However, the NMR spectra point in the right direction. Hence, there is still scope for subsequent research, which should also include reactivity studies on picolyl metal compounds concerning the yields of substitution or 
addition products depending on the use of different alkaline metal cations, donor molecules and crystalline material.

In order to gain deeper insight into the bonding situation in lithium organic compounds, hexameric trimethylsilylmethyllithium was selected owing to its standard aggregation pattern with six lithium atoms in an octahedral arrangement in the solid state. It could be crystallised in two modifications, one of which was recognised to be not applicable for an experimental charge density study. The second polymorph showed variations in the crystal quality. Nevertheless, it was managed to collect a data set of sufficient quality for a multipole refinement.

A suitable model for the electron density distribution of $\left[\mathrm{TMSCH}_{2} \mathrm{Li}\right]_{6}$ was determined and topologically analysed in the QTAIM framework. A survey of the bond paths and the Laplacian field explains the interactions of the carbanions with the capped lithium triangles. These were identified to be made up by a lone pair facing the $\mathrm{Li}_{3}$ motif. Chemical bonds to each of the three metal ions were quantified so that six attractive interactions to neighbouring atoms are formed for each deprotonated carbon atom showing $\mathrm{sp}^{3}$ hybrid orbitals. It was shown that the $\mathrm{Li}-\mathrm{C}$ bonding is not exclusively orbital controlled but strongly influenced by negative charge accumulations at the methylene carbon atoms. Furthermore, no indications for possible $\mathrm{Li}-\mathrm{H}$ agostic contacts were detected. The small atomic distances between those atoms are caused by the staggered arrangement of all substituents throughout the anions and the orientation of the trimethylsilylmethyl groups with respect to the lithium octahedron. This conformation was proven to be mediated by the lone pairs. The electron pair is localised in an $\mathrm{sp}^{3}$ hybrid orbital and interacts with the $\sigma^{*}$ molecular orbital of the silicon-carbon bond in trans position by negative hyperconjugation.

A major step towards the understanding of the bonding situation in lithium organic aggregates was made and further studies on trimethylsilylmethyllithium donor aggregates will elucidate the influence of the aggregation state on the carbanion and the $\mathrm{Li}-\mathrm{C}$ bonds. It might even be possible to scale the reactivity on properties derived from the electron density distribution such as the $\nabla^{2} \rho(\mathbf{r})$ value of the lone pairs.

The explanation of the stereochemical pathway of a highly enantiomerically enriched nucleophile in substitution reactions was accomplished by an experimental charge density study on $(R)-[\{(S)-2-($ methoxymethyl)pyrrolidinomethyl $\}$ - 
dimethylsilyl]benzyllithium-quinuclidine (17). This benzylsilane contains a metallated carbon atom with three non-metal bonds in a plane and a $\mathrm{Li}-\mathrm{C}$ bond made up by a lone pair in a p orbital orthogonal to it and the lithium cation. Remarkably, the absolute configuration at this atom is even fixed at RT in solution so that high diastereomeric product ratios of above 98:2 are observed in conversions with electrophiles under inversion of the configuration. This is caused by a strong fixation of the lithium cation by four highly negatively charged donor atoms ( -1 e). Surprisingly, the charge at the deprotonated carbon atom is not delocalised into the neighbouring phenyl ring, as commonly expected by organic chemists, but is stabilised by the polarisable silicon atom. The $\alpha$-effect of the silicon atom is therefore not only caused by polarisation effects but also by electrostatic bond interactions of the positively charged heteroatom (above $+2 \mathrm{e}$ ) with the carbanion. The stabilisation capabilities of silyl groups are thus superior to those of aromatic phenyl substituents. Moreover, the stereo-chemical course of conversions with electrophiles under stereo-inversion was understood by the charge density distribution and the electrostatic potential of the benzyllithium derivative. Furthermore, the lithium-carbon bond could be consistently integrated into the set of other polar donor bonds.

It can thus be concluded that the experimentally challenging and time consuming method of experimental charge density determination proved to be an excellent tool to get detailed insights into bonding situations and molecular properties of lithium organic compounds, which are not available by any other experiment. Several lithium-donor bonds were investigated by this method in the course of the dissertation. They represent benchmark systems for the characterisation of these steadily discussed interactions. According to QTAIM indicators, the $\mathrm{Li}-\mathrm{X}(\mathrm{X}=\mathrm{C}, \mathrm{N}, \mathrm{O})$ bonds are of prevailing ionic character and only feature minor covalent contributions. This proposition should be object of future research using charge density studies on the fascinating class of lithium organic compounds. 


\section{Zusammenfassung Und Ausblick}

Im Rahmen dieser Doktorarbeit wurden hauptsächlich zwei Ziele verfolgt: Erstens sollten neue unsubstituierte Alkalimetallpicolylverbindungen zur Strukturaufklärung synthetisiert und kristallisiert werden. Zweitens sollten diese Aggregate und weitere lithiumorganische Verbindungen mittels experimenteller Elektronendichtebestimmungsmethoden untersucht werden, um detaillierte Informationen über ihre Bindungssituationen zu erlangen.

Die Synthesebemühungen auf dem Gebiet der unsubstituierten 2-Picolyllithiumverbindungen erweiterten die Liste der strukturellen Leitmotive dieser benzylischen Anionen. Die grundsätzlichen Koordinationsmotive sind von $\eta^{1}$-alkylischer, $\eta^{1}$-enamidischer und $\eta^{3}$-aza-allylischer Natur. Durch Einsatz des dreizähnigen Donormoleküls Pmdeta war es darüber hinaus möglich, eine monomeres 2-PicLi Donoraddukt zu erhalten. Die Verbindungen wurden in akzeptablen Ausbeuten als kristalline Materialien isoliert, die über Monate hinweg in Argontrockenboxen gelagert werden können. Dies, wie auch der leichte Zugang aus handelsüblichen Ausgangsverbindungen, machen sie zu wertvollen Synthesebausteinen. Die Tmeda- und Pmdeta-Addukte erwiesen sich als leicht zu kristallisieren, so dass die Verwendung dieser Verbindungen sinnvoll ist für weitere Umsetzungen, falls die Donorbasen im weiteren Syntheseverlauf nicht stören. Die Isolierung von reinen kristallinen Edukten ist wichtig, da unzählige Umsetzung aus Reaktionsmischungen zu niedrigen Ausbeuten und Nebenprodukten führten. Beispielweise war es $I$. Objartel in enger Zusammenarbeit mit dem Autor möglich, die Ausbeute an Trimethylsilyl(2-pyridyl)methan auf über $80 \%$, verglichen zu den vormals berichteten $42 \%$, zu steigern. ${ }^{[111]}$ Dies gelang durch den Umsatz von vorher isoliertem [2-PicLi.tmeda] 2 (2) mit TMSCl, während zuvor eine in situ dargestelltes 2-PicLi eingesetzt wurde. Diese Ausbeutenoptimierung ist bedeutsam, da es sich bei 2- $\mathrm{TMSCH}_{2} \mathrm{Py}$ um eine wichtige Vorstufe zum vielseitig einsetzbaren Phenyldipicolylphosphanliganden (PhPPic 2 ) handelt.

Eine der Picolyllithiumverbindungen kristallisierte in der nötigen Qualität um eine experimentelle Elektronendichteuntersuchung durchzuführen. [2-PicLi·PicH $]_{2}$ (4) weist eine $\eta^{3}$-aza-allylische Bindung zum Metall auf und beinhaltet 2-Picolin als interne Referenz, um die Auswirkungen der Deprotonierung vergleichen zu können. Es konnten bevorzugte Wechselwirkungen zwischen dem Kation und den Picolylstickstoffatomen beobachtet werden, wohingegen das formale Carbanion 
nur eine untergeordnete Rolle bei der Koordination des Metalls spielt. Die negative Ladung am Kohlenstoffatom ist in den aromatischen Ring delokalisiert und verursacht dort eine Störung des $\pi$-Systems. Es kommt zu teilweise lokalisierten Doppelbindungen, die eine enamidische Beschreibung des Anions unterstützen. Allerdings zeigt sich der höchste relative Ladungsanstieg im Vergleich zu 2-Picolin am deprotonierten Kohlenstoffatom. Zusätzlich macht die räumliche Ausdehnung des elektrostatischen Potenzials deutlich, warum das Enamid von Elektrophilen grundsätzlich an der Seitenkette angegriffen wird. Diese werden durch ein ausgedehntes negatives ESP an den Picoylanionen zum exocyclischen Kohlenstoffatom dirigiert.

Mit diesen Addukten war es möglich die Auswirkungen der Donormoleküle auf die Koordinationsmuster von 2-PicLi zu untersuchen, ohne Einflüsse von weiteren Seitenketten- oder Ringsubstituenten berücksichtigen zu müssen. Die unterschiedlichen Koordinationstypen im Kristall können so in Abhängigkeit der eingesetzten Donoren und ihrer Donorstärke bzw. ihres sterischen Anspruchs verstanden oder zukünftig vorausgesagt werden. Diese Erkenntnisse wurden durch die Ergebnisse der Elektronendichteuntersuchung an $\mathbf{4}$ und durch bereits bekannte theoretische Berechnungen gestützt. ${ }^{[107,133]}$

Jedoch sind weitere Koordinationsmodi denkbar. Solvensgetrennte Ionenpaare könnten mit 12-Krone-4 und Kettenstrukturen mit mehrzähnigen Liganden wie etwa Diglyme oder Tetraglyme entstehen. Zahlreiche Bemühungen, 2-PicLi mit diesen Donorreagenzien zu kristallisieren, scheiterten bislang. Das Gleiche trifft auch auf donorfreies 2-Picolyllithium zu, das zwar in Hexan darstellbar, aber in nicht-koordinierenden Lösungsmitteln weitestgehend unlöslich ist. Gerade dessen Molekülstruktur wäre aber aufgrund fehlender Donoren ein wichtiges Bezugssystem, für das eine aza-allylische Struktur vorhergesagt wird. Sollten keine Einkristalle von dieser Verbindung zu erhalten sein, so könnten Pulverdiffraktogramme die Festkörperstruktur unter Zuhilfenahme bekannter Strukturmotive aufklären.

Während die Molekülstrukturen von 2-Picolyllithium inzwischen durch die gezeigten Ergebnisse bekannt sind, ist deren Zusammensetzung und Struktur in Lösung weitestgehend unbekannt. Deshalb wurden zahlreiche NMR-Experimente durchgeführt, welche interessante Ergebnisse zur Aufklärung des Aggregatzustands und der Koordination zum Kation aufwiesen. Jedoch konnten noch keine endgültigen Aussagen getroffen werden, weitere Untersuchungen sind dazu notwendig. 
Darüber hinaus wurde die Forschung auf schwere Alkalimetallverbindungen ausgedehnt. Der erste erfolgreiche Schritt in diese Richtung war das 2-Picolylnatrium-Tmeda-Addukt, welches kristallin erhalten und strukturanalytisch untersucht wurde. Durch die bekannte Reaktivitätssteigerung beim Übergang zu den schweren Homologen des Lithiums ist es somit möglich, die Reaktivität des Picolylanions durch Wahl des Kations und der Donorbase einzustellen. Weitere Anstrengungen müssen unternommen werden um auch die Kalium-, Rubidiumund Cäsiumanaloga zu kristallisieren. Dies sollte im Vergleich zu den Benzylverbindungen durch die bessere Löslichkeit aufgrund der intramolekularen Donorstelle im Picolylring leichter vonstatten gehen. Weiterhin zeigte die Picolylnatriumverbindung das Potenzial für eine experimentelle Elektronendichteuntersuchung. Sie wäre eine ideale Modellverbindung, um die Unterschiede in den Bindungen der Alkalimetalle und der enamidischen und azaallylischen Koordination zu studieren.

Die experimentellen Arbeiten wurden auf 4-Picolyllithium ausgedehnt, das weitaus seltener zur Synthese eingesetzt wird als das 2-Isomer. Dies liegt im Auftreten von Nebenprodukten bei der Darstellung begründet. Eine Änderung des Deprotonierungsreagenzes sollte dies ausschließen, wobei Methyllithium die besten Ergebnisse lieferte. Im Zusammenspiel mit Pmdeta ist 4-Picolyllithium einfach in kristalliner Form zu erhalten und weiter umzusetzen. Alle isolierten Aggregate zeigten eine bevorzugte Koordination der Kationen über die Ringstickstoffatome, was bereits anhand der Ergebnisse mit 2-Picolyllithiums zu erwarten war. Jedoch wurde ein weiterer Koordinationsmodus bei der Deprotonierung von

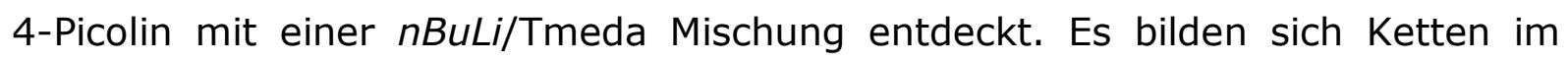
Festkörper, bei denen das Picolylstickstoff- und das deprotonierte Kohlenstoffatom mit den Lithiumatomen wechselwirken. Darüber hinaus konnten die Natrium- und Kaliumanaloga mittels Superbasen als Feststoffe isoliert werden. Jedoch waren Kristallisationsversuche mit geeigneten Donorbasen bislang nicht erfolgreich, weshalb die Ergebnisse in dieser Arbeit nicht berichtet werden. Die erzielten Erfolge stimmen aber zuversichtlich im Bezug auf weitere Forschungsergebnisse.

Es wäre wünschenswert ein probates 4-Picolyllithium-Addukt darzustellen, das sich in ausgezeichneter Qualität kristallisieren lässt. Anhand einer experimentellen Elektronendichtestudie im Vergleich zum 2-Isomer könnten Unterschiede im Delokalisierungsgrad und der Ladungsakkumulation an den Ringstickstoffatomen aufgeklärt werden. Einblicke in die Bindungsverhältnisse von deprotoniertem 4-Picolin wären dadurch möglich. 
Aufbauend auf den bisherigen Ergebnissen ist der nächste logische Schritt eine Ausweitung der Forschung auf das noch weniger saure 3-Picolin, das bisher keine Beachtung in der Synthesechemie fand. Eine Veränderung der Stickstoffposition innerhalb des Picolinrings bietet die Möglichkeit, den Bisswinkel eines Liganden zu regulieren. Erste Versuche zur Deprotonierung mit Superbasen wurden unternommen, jedoch konnten die isolierten Substanzen bis jetzt noch nicht zur Kristallisation gebracht werden. Die zugehörigen NMR-Spektren deuten aber auf die erfolgte Abstraktion des benzylischen Protons hin. Weiterführende Untersuchungen sollten Reaktivitätsstudien in Abhängigkeit von den eingesetzten Metallkationen, Donoren und der Art der Isolierung (Verbindung ausgefällt, kristallisiert oder in situ umgesetzt) beinhalten.

Um weiterführende Einblicke in die Bindungsverhältnisse von lithiumorganischen Verbindungen zu erlangen, wurde das hexamere Trimethylsilylmethyllithium ausgewählt. Es weist ein Leitmotiv der Organolithiumchemie auf, in dem sechs Lithiumatomen in oktaedrischer Anordnung von sechs Carbanionen überkappt werden. [ $\left.\mathrm{TMSCH}_{2} \mathrm{Li}\right]_{6}$ konnte in zwei Modifikationen kristallisiert werden, wobei eine den hohen Anforderungen einer experimentellen Elektronendichteuntersuchung nicht genügte. Das zweite Polymorph wies zwar Schwankungen in der Kristallqualität auf, ein Datensatz konnte jedoch erfolgreich aufgenommen werden.

Es war damit möglich, ein Modell der Elektronendichteverteilung in $\left[\mathrm{TMSCH}_{2} \mathrm{Li}\right]_{6} \mathrm{zu}$ verfeinern und nach QTAIM topologisch zu analysieren. Eine Untersuchung der Bindungspfade und des Laplace-Felds verdeutlichte dabei die Wechselwirkungen der Carbanionen mit den überkappten Dreiecken aus Lithiumatomen. Jeweils ein freies Elektronpaar an den Carbanionen in einer $\mathrm{sp}^{3}$-Hybridorbitalanordung zeigt auf eine $\mathrm{Li}_{3}$-Fläche, wodurch drei chemische Bindungen zu den Metallkationen eingegangen werden. Insgesamt konnten sechs attraktive Wechselwirkungen zu benachbarten Atomen beziffert werden. Es wurde darüber hinaus gezeigt, dass die $\mathrm{Li}-\mathrm{C}$-Bindungen nicht ausschließlich orbitalkontrolliert sind, sondern stark durch die negativen Ladungen an den Methylenkohlenstoffatomen beeinflusst werden. Anzeichen für agostische Li-H-Wechselwirkungen wurden nicht gefunden. Die kurzen Li-H-Atomabstände kommen vielmehr durch die ausnahmslos gestaffelte Anordnung der Substituenten an den Anionen zustande. Diese Konformation wird allerdings durch die freien Elektronenpaare bestimmt, welche mit den $\sigma^{*}$-Molekülorbitalen der trans-ständigen Si-CBindungen mittels negativer Hyperkonjugation wechselwirken. 
Somit wurde ein Schritt zum Verständnis der Bindungssituation in lithiumorganischen Verbindungen unternommen. Darauf aufbauende Studien an Addukten des Trimethylsilylmethyllithiums können den Einfluss des Aggregatzustands auf die Carbanionen und die $\mathrm{Li}-\mathrm{C}$-Bindungen verdeutlichen. So sollte es prinzipiell möglich sein, die Reaktivität dieser Verbindungen in Beziehung zu Werten aus der Elektronendichteverteilung zu setzen, wie beispielsweise $\nabla^{2} \rho\left(\mathbf{r}_{L P}\right)$ der freien Elektronenpaare.

Eine experimentelle Elektronendichtebestimmung an $(R)$ - $[\{(S)$-2-(Methoxymethyl)pyrrolidinomethyl\}dimethylsilyl]benzyllithium.Chinuclidin (17) konnte weitere Erkenntnisse über lithiumorganische Verbindungen liefern. Dieses weist ein metalliertes Kohlenstoffatom in planarer Umgebung mit einem freien Elektronenpaar in einem p-Orbital senkrecht dazu auf, welches die Li-C-Bindung zum Kation bildet. Die absolute Konfiguration an diesem Kohlenstoffatom bleibt erstaunlicherweise auch bei Raumtemperatur in Lösung erhalten, wodurch Diasteromerenverhältnisse der Substitutionsprodukte unter Stereoinversion von über 98:2 möglich sind. Die starke Fixierung des Kations wird durch vier negativ geladene Donoratome $(-1 \mathrm{e})$ erreicht. Interessanterweise ist die Ladung des deprotonierten Kohlenstoffatoms nicht wie allgemein erwartet im benachbarten Phenylring delokalisiert, sondern wird durch das polarisierbare Siliziumatom stabilisiert. Der $\alpha$-Effekt des Siliziums ist demnach nicht nur der Polarisierbarkeit des Atoms zuzuschreiben. Elektrostatische Wechselwirkungen des positiv geladenen Heteroatoms (über $+2 \mathrm{e}$ ) mit dem Carbanion sind ebenfalls beteiligt, wodurch der Einfluss der Silylgruppe auf die negative Ladung im Vergleich zum Einfluss des Phenylsubstituenten überwiegt. Darüber hinaus wurde der stereochemischen Verlauf der Umsetzung von 17 mit Elektrophilen anhand der Elektronendichteverteilung und des elektrostatischen Potenzials verständlich, während die Lithium-Kohlenstoffbindung in die Gruppe der polaren Donorbindungen eingereiht werden konnte.

Abschließend darf angemerkt werden, dass die experimentell herausfordernde und zeitintensive Methode der experimentellen Elektronendichtebestimmung sich als hervorragendes Instrument zur Untersuchung der Bindungssituation und der molekularen Eigenschaften lithiumorganischer Verbindungen erwiesen hat. Ähnlich detaillierte Einblicke sind durch kein anderes Experiment möglich und folglich wurden verschiedene Lithium-Donorbindungen im Verlauf dieser Arbeit mit dieser Methode untersucht. Es konnten wichtige Referenzdaten für die 
Charakterisierung dieser Wechselwirkungen gesammelt werden, wonach Li-XBindungen $(X=C, N, O)$ ein überwiegend ionischer Charakter mit nur geringen kovalenten Beiträgen zugesprochen wird. Diese Aussage sollte in zukünftigen Elektronendichteuntersuchungen an der Klasse der lithiumorganischen Verbindungen untermauert werden. 



\section{EXPERIMENTAL SECTION}

\subsection{General Procedures}

All experiments were carried out in a purified argon atmosphere using modified Schlenk techniques. An argon glove box was used for manipulations of solid air sensitive compounds. Glass vessels were dried for several hours at $120^{\circ} \mathrm{C}$ prior to use and immediately assembled while still hot. Traces of air were removed under vacuum. All solvents were freshly distilled from sodiumpotassium alloy (diethyl ether, pentane) or potassium (dme, thf, hexane) and degassed by passing argon through the solvent for about 30 minutes. The starting materials were commercially available or synthesised according to published procedures. The picoline isomers were dried over calcium hydride, distilled, degassed, and stored in Schlenk flasks prior to the metallation.

\subsection{Analytical Methods}

\subsubsection{NMR Spectroscopy}

Crystalline materials were transferred into NMR tubes in an argon glove box and dissolved in an appropriate deuterated solvent (benzene; thf; toluene) in order to obtain solutions of about $10 \%$ concentration. Liquid samples were filled in NMR tubes using a syringe and an NMR filling station with a specially designed seal so that the tubes can be evacuated and handled under protective gas. The NMR spectra were recorded on BRUKER AVANCE 200, 300, and 500 spectrometers. The exact frequency, temperature and solvent for the measurements are given in the analytical part of each compound. The chemical shifts $\delta$ are quoted in ppm with positive values for low-field shifts against tetramethylsilane $\left({ }^{1} \mathrm{H},{ }^{13} \mathrm{C}\right)$, lithium chloride in $\mathrm{D}_{2} \mathrm{O}\left({ }^{7} \mathrm{Li}\right)$ or nitromethane $\left({ }^{15} \mathrm{~N}\right)$ as external standards. Signals from the NMR solvents were used as internal reference in ${ }^{1} \mathrm{H}$ and ${ }^{13} \mathrm{C}$ spectra. Coupling constants $\mathrm{J}$ are given in $\mathrm{Hz}$. 


\subsubsection{Mass Spectrometry}

Mass spectra were recorded with the electron ionization method (EI MS: $70 \mathrm{eV}$ ) on a FinNIGAN MAT 95 spectrometer. The mass-to-charge ratios $(\mathrm{m} / \mathrm{z})$ of the fragment ions are based on the molecular masses of the isotopes with the highest natural abundance. The presented metal organic aggregates all show molecular peaks without metal ions and donor solvents.

\subsubsection{Melting and Decomposition Points}

Melting and decomposition points were determined on a BüchI MELTING POINT B-540 in sealed glass capillaries $\left(\varnothing=1.4 \mathrm{~mm}\right.$ ) with a heating rate of $5^{\circ} \mathrm{C} / \mathrm{min}$.

\subsubsection{Elemental Analyses}

Elemental analyses were carried out by the Mikroanalytische Labor des Instituts für Anorganische Chemie der Georg-August-Universität Göttingen with an ELEMENTAR VARIO EL3. Even though several attempts were made to obtain correct elemental analyses of the metallated products, this was only successful in two cases. The determined values for all other compounds deviated more than $1.0 \%$ from the theoretical ones, which can be explained by the loss of solvent molecules of the aggregates due to the sample preparation procedure (vacuum drying) or by the sensitivity against oxygen and moisture. Even traces of argon from the preparation and the handling of the substances in the glove box affected the results and led to known systematic errors. 


\subsection{Syntheses and Characterisations}

\subsubsection{2-Picolyllithium·Dme (1, H81/H116)}

A $2.26 \mathrm{M}$ solution of $n$-butyllithium in hexane $(8.9 \mathrm{~mL}, 20.2 \mathrm{mmol}, 1$ eq.) was slowly added to a 2-picoline $(2.0 \mathrm{~mL}, 20.2 \mathrm{mmol}, 1 \mathrm{eq.})$ solution in dimethoxyethane $(20 \mathrm{~mL})$ and hexane $(30 \mathrm{~mL})$ at $-78^{\circ} \mathrm{C}$. After $2.5 \mathrm{~h}$ of stirring, the solution was reduced in volume and the resulting yellow solid filtered off. $2.13 \mathrm{~g}$ of crude product has been isolated $(5.7 \mathrm{mmol}, 56 \%)$. Recrystallisation from diethyl ether $(25 \mathrm{~mL})$ at $-24^{\circ} \mathrm{C}$ yielded $\mathbf{1}$ as red crystals.

Empirical formula: $\mathrm{C}_{20} \mathrm{H}_{32} \mathrm{Li}_{2} \mathrm{~N}_{2} \mathrm{O}_{4}$. Molecular Mass: $387.36 \mathrm{~g} \mathrm{~mol}^{-1}$.

${ }^{1} \mathbf{H}$ NMR (500.13 MHz, $\left.\mathbf{C}_{6} \mathbf{D}_{6}, \quad 25^{\circ} \mathbf{C}\right): \delta 7.30$ (ddd, ${ }^{3} J(H 5, H 4)=5.6$, $\left.{ }^{4} J(\mathrm{H} 5, \mathrm{H} 3)=1.0,{ }^{5} J(\mathrm{H} 5, \mathrm{H} 2)=1.0,1 \mathrm{H}, \mathrm{H} 5\right), 6.54 \quad\left(\mathrm{ddd},{ }^{3} J(\mathrm{H} 3, \mathrm{H} 2)=9.0\right.$, $\left.{ }^{3} \mathrm{~J}(\mathrm{H} 3, \mathrm{H} 4)=6.2,{ }^{4} \mathrm{~J}(\mathrm{H3}, \mathrm{H} 5)=1.9,1 \mathrm{H}, \mathrm{H3}\right), 6.26 \quad\left(\mathrm{ddd},{ }^{3} \mathrm{~J}(\mathrm{H} 2, \mathrm{H} 3)=9.0\right.$, $\left.{ }^{4} \mathrm{~J}(\mathrm{H} 2, \mathrm{H} 4)=1.1,{ }^{5} \mathrm{~J}(\mathrm{H} 2, \mathrm{H} 5)=1.0,1 \mathrm{H}, \mathrm{H} 2\right), 5.46 \quad\left(\mathrm{ddd},{ }^{3} \mathrm{~J}(\mathrm{H} 4, \mathrm{H} 3)=6.2\right.$, $\left.{ }^{3} \mathrm{~J}(\mathrm{H} 4, \mathrm{H} 5)=5.6,{ }^{4} \mathrm{~J}(\mathrm{H} 4, \mathrm{H} 2)=1.1,1 \mathrm{H}, \mathrm{H} 4\right), 3.20\left(\mathrm{~s}, 6 \mathrm{H}, \mathrm{OCH}_{3}\right), 3.06(\mathrm{~s}, 2 \mathrm{H}$, H6), $2.95\left(\mathrm{~s}, 4 \mathrm{H}, \mathrm{OCH}_{2}\right) \mathrm{ppm}$.

${ }^{7}$ Li NMR (194.37 $\left.\mathbf{M H z}, \mathbf{C}_{6} \mathbf{D}_{6}, \mathbf{2 5}^{\circ} \mathbf{C}\right): \delta 0.24 \mathrm{ppm}$.

${ }^{13} \mathrm{C}$ NMR (125.76 MHz, $\left.\mathbf{C}_{6} \mathbf{D}_{6}, 25{ }^{\circ} \mathbf{C}\right): \delta 164.7$ (C1), 148.5 (C5), 132.3 (C3), $116.5(\mathrm{C} 2), 98.6(\mathrm{C} 4), 70.4\left(\mathrm{OCH}_{2}\right), 59.3\left(\mathrm{OCH}_{3}\right), 56.3(\mathrm{C} 6) \mathrm{ppm}$.

Elemental analysis calculated for $\mathrm{C}_{20} \mathrm{H}_{32} \mathrm{Li}_{2} \mathrm{~N}_{2}$ : C 63.49, H 8.52, N 7.40; found C 62.6, H 8.0, N $7.4 \%$.

\subsubsection{2-Picolyllithium·Tmeda (2, H113)}

A $1.86 \mathrm{~m}$ solution of $n$-butyllithium in hexane $(44 \mathrm{~mL}, 81.8 \mathrm{mmol}, 1.1$ eq.) was slowly added to a 2-picoline $(7.3 \mathrm{~mL}, 74.0 \mathrm{mmol}, 1.0$ eq.) tmeda (20 mL, $133.2 \mathrm{mmol}, 1.8 \mathrm{eq}$.) mixture in hexane $(30 \mathrm{~mL})$ at $-78^{\circ} \mathrm{C}$. After $2.0 \mathrm{~h}$ of stirring the solution was stored at $4{ }^{\circ} \mathrm{C}$ for one day and the resulting orange solid was filtered off. $9.70 \mathrm{~g}$ of product could be isolated $(45.1 \mathrm{mmol}, 61 \%)$. Red crystals suitable for an X-ray structure determination were grown from the saturated filtrate at $4{ }^{\circ} \mathrm{C}$.

Empirical formula: $\mathrm{C}_{24} \mathrm{H}_{44} \mathrm{Li}_{2} \mathrm{~N}_{6}$. Molecular Mass: $430.53 \mathrm{~g} \mathrm{~mol}^{-1}$.

M.p.: $114^{\circ} \mathrm{C}$ (decomp.). 
${ }^{1} \mathbf{H}$ NMR (500.13 $\left.\mathbf{~ M H z}, \mathbf{C}_{6} \mathbf{D}_{5} \mathbf{C D}_{3}, \mathbf{2 5}^{\circ} \mathbf{C}\right): \delta 7.03\left(\mathrm{ddd},{ }^{3} J(\mathrm{H} 5, \mathrm{H} 4)=5.7\right.$, $\left.{ }^{4} \mathrm{~J}(\mathrm{H} 5, \mathrm{H} 3)=1.9, \quad{ }^{5} \mathrm{~J}(\mathrm{H} 5, \mathrm{H} 2)=0.9, \quad 1 \mathrm{H}, \quad H 5\right), 6.30 \quad\left(\mathrm{ddd},{ }^{3} J(\mathrm{H} 3, \mathrm{H} 2)=9.0\right.$, $\left.{ }^{3} \mathrm{~J}(\mathrm{H} 3, \mathrm{H} 4)=6.2,{ }^{4} \mathrm{~J}(\mathrm{H} 3, \mathrm{H} 5)=1.9, \quad 1 \mathrm{H}, \quad \mathrm{H} 3\right), \quad 5.98 \quad\left(\mathrm{ddd},{ }^{3} \mathrm{~J}(\mathrm{H} 2, \mathrm{H} 3)=9.0\right.$, $\left.{ }^{4} \mathrm{~J}(\mathrm{H} 2, \mathrm{H} 4)=1.9,{ }^{5} \mathrm{~J}(\mathrm{H} 2, \mathrm{H} 5)=0.9, \quad 1 \mathrm{H}, \quad \mathrm{H} 2\right), \quad 5.22 \quad\left(\mathrm{ddd},{ }^{3} \mathrm{~J}(\mathrm{H} 4, \mathrm{H} 3)=6.2\right.$, $\left.{ }^{3} \mathrm{~J}(\mathrm{H} 4, \mathrm{H} 5)=5.7,{ }^{4} \mathrm{~J}(\mathrm{H} 4, \mathrm{H} 2)=1.9,1 \mathrm{H}, H 4\right), 3.05(\mathrm{~s}, 1 \mathrm{H}, H 6), 2.96(\mathrm{~s}, 1 \mathrm{H}, H 6)$, $2.28\left(\mathrm{~s}, 12 \mathrm{H}, \mathrm{NCH}_{3}\right), 1.96\left(\mathrm{~s}, 4 \mathrm{H}, \mathrm{NCH}_{2}\right) \mathrm{ppm}$.

${ }^{1} \mathbf{H} \quad$ NMR $\quad\left(200.13 \mathbf{~ M H z}, \quad \mathbf{C}_{6} \mathbf{D}_{6}, \quad 25^{\circ} \mathbf{C}\right): \delta 7.11$ (ddd, ${ }^{3} J(\mathrm{H} 5, \mathrm{H} 4)=5.8$, $\left.{ }^{4} \mathrm{~J}(\mathrm{H} 5, \mathrm{H} 3)=2.0,{ }^{5} \mathrm{~J}(\mathrm{H} 5, \mathrm{H} 2)=0.8, \quad 1 \mathrm{H}, \quad H 5\right), \quad 6.41 \quad\left(\mathrm{ddd},{ }^{3} \mathrm{~J}(\mathrm{H} 3, \mathrm{H} 2)=9.0\right.$, $\left.{ }^{3} \mathrm{~J}(\mathrm{H} 3, \mathrm{H} 4)=6.1,{ }^{4} \mathrm{~J}(\mathrm{H} 3, \mathrm{H} 5)=2.0, \quad 1 \mathrm{H}, \quad H 3\right), \quad 6.10 \quad\left(\mathrm{ddd},{ }^{3} \mathrm{~J}(\mathrm{H} 2, \mathrm{H} 3)=9.0\right.$, $\left.{ }^{4} J(\mathrm{H} 2, \mathrm{H} 4)=1.7,{ }^{5} J(\mathrm{H} 2, \mathrm{H} 5)=0.8, \quad 1 \mathrm{H}, \quad H 2\right), \quad 5.32 \quad\left(\mathrm{ddd},{ }^{3} \mathrm{~J}(\mathrm{H} 4, \mathrm{H} 3)=6.1\right.$, $\left.{ }^{3} \mathrm{~J}(\mathrm{H} 4, \mathrm{H} 5)=5.8,{ }^{4} \mathrm{~J}(\mathrm{H} 4, \mathrm{H} 2)=1.7,1 \mathrm{H}, H 4\right), 3.15(\mathrm{~s}, H 6), 3.06(\mathrm{~s}, H 6), 2.26(\mathrm{~s}$, $\left.12 \mathrm{H}, \mathrm{NCH}_{3}\right), 1.94\left(\mathrm{~s}, 4 \mathrm{H}, \mathrm{NCH}_{2}\right) \mathrm{ppm}$.

${ }^{7}$ Li NMR (194.37 MHz, $\left.\mathbf{C}_{6} \mathbf{D}_{5} \mathrm{CD}_{3}, \mathbf{2 5}^{\circ} \mathbf{C}\right)$ : $\delta-0.10 \mathrm{ppm}$.

${ }^{13} \mathbf{C}$ NMR (125.76 MHz, $\left.\mathbf{C}_{6} \mathbf{D}_{5} \mathbf{C D}_{\mathbf{3}}, 25{ }^{\circ} \mathbf{C}\right): \delta 162.9(C 1), 148.1$ (C5), 131.7 (C3), $117.5(C 2), 97.1(C 4), 61.5(C 6), 57.5\left(\mathrm{NCH}_{2}\right), 46.0\left(\mathrm{NCH}_{3}\right)$ ppm.

${ }^{15}$ N NMR (50.69 MHz, $\left.\mathbf{C}_{6} \mathbf{D}_{5} \mathbf{C D}_{3}, 25{ }^{\circ} \mathbf{C}\right): \delta-184\left(N_{P y}\right),-355\left(N_{\text {tmeda }}\right)$ ppm.

EI-MS: $\mathrm{m} / \mathrm{z}(\%): 183(3)\left[\left(\mathrm{PyCH}_{2}\right)_{2}-\mathrm{H}^{+}, 116(23)[\mathrm{tmeda}]^{+}, 93\right.$ (15) $\left[\mathrm{PyCH}_{3}\right]^{+}$, $78(8)[\mathrm{Py}]^{+}, 58(100)\left[\mathrm{Me}_{2} \mathrm{NCH}_{2}\right]^{+}$.

Elemental analysis calculated for $\mathrm{C}_{24} \mathrm{H}_{44} \mathrm{Li}_{2} \mathrm{~N}_{6}$ : C 66.95, $\mathrm{H}$ 10.30, N 19.52; found C 66.33, H 10.58, N 20.03.

\subsubsection{2-Picolyllithium-Pmdeta (3, H114/H172)}

A solution of $n$-butyllithium in hexane $(30 \mathrm{~mL}, 60 \mathrm{mmol}, 1.1 \mathrm{eq}$.) slowly added to 2-picoline ( $5.1 \mathrm{~g}, 55 \mathrm{mmol}, 1.0$ eq.) and pmdeta (11.3 g, $65 \mathrm{mmol}, 1.2$ eq.) in thf $(5 \mathrm{~mL})$ and hexane $(20 \mathrm{~mL})$ at $-78^{\circ} \mathrm{C}$. An orange solid precipitated from the red solution at $4{ }^{\circ} \mathrm{C}$ and was filtered off $(6.7 \mathrm{~g}, 45 \%)$. The filtrate was further concentrated and red crystals $(0.8 \mathrm{~g}, 5 \%)$ were obtained at $-24^{\circ} \mathrm{C}$.

Empirical formula: $\mathrm{C}_{15} \mathrm{H}_{29} \mathrm{LiN}_{4}$.

Molecular Mass: $272.36 \mathrm{~g} \mathrm{~mol}^{-1}$.

M.p.: $83^{\circ} \mathrm{C}$ (decomp.).

${ }^{1} \mathbf{H} \quad$ NMR $\quad\left(\mathbf{5 0 0 . 1 3} \mathbf{~ M H z}, \quad \mathbf{C}_{6} \mathbf{D}_{6}, \quad 25{ }^{\circ} \mathbf{C}\right): \delta 7.06$ (ddd, ${ }^{3} J(\mathrm{H} 5, \mathrm{H} 4)=5.4$, $\left.{ }^{4} J(\mathrm{H} 5, \mathrm{H} 3)=1.9,{ }^{5} J(\mathrm{H} 5, \mathrm{H} 2)=0.9,1 \mathrm{H}, \quad H 5\right), 6.58 \quad\left(\mathrm{dddd},{ }^{3} \mathrm{~J}(\mathrm{H} 3, \mathrm{H} 2)=8.8\right.$, $\left.{ }^{3} J(\mathrm{H} 3, \mathrm{H} 4)=6.3,{ }^{4} J(\mathrm{H} 3, \mathrm{H} 5)=1.9,{ }^{5} J(\mathrm{H} 3, \mathrm{H} 6)=0.9,1 \mathrm{H}, \mathrm{H3}\right), 6.31$ (ddd, $\left.{ }^{3} J(\mathrm{H} 2, \mathrm{H} 3)=8.8,{ }^{4} J(\mathrm{H} 2, \mathrm{H} 4)=1.0,{ }^{5} J(\mathrm{H} 2, \mathrm{H} 5)=0.9,1 \mathrm{H}, \mathrm{H} 2\right), 5.37 \quad(\mathrm{ddd}$, 
$\left.{ }^{3} J(\mathrm{H} 4, \mathrm{H} 3)=6.3,{ }^{3} \mathrm{~J}(\mathrm{H} 4, \mathrm{H} 5)=5.4,{ }^{4} \mathrm{~J}(\mathrm{H} 4, \mathrm{H} 2)=1.0, \quad 1 \mathrm{H}, \quad H 4\right), \quad 3.40 \quad(\mathrm{~d}$, $\left.{ }^{2} J(\mathrm{H} 6, \mathrm{H} 6)=1.5,1 \mathrm{H}, \mathrm{H}_{\text {trans }}\right), 2.79\left(\mathrm{dd},{ }^{2} \mathrm{~J}(\mathrm{H} 6, \mathrm{H} 6)=1.5,{ }^{5} \mathrm{~J}(\mathrm{H} 6, \mathrm{H} 3)=0.9,1 \mathrm{H}\right.$, $\left.H 6_{\text {cis }}\right), 2.06\left(\mathrm{~s}, 12 \mathrm{H}, \mathrm{N}\left(\mathrm{CH}_{3}\right)_{2}\right), 1.88\left(\mathrm{~s}, 3 \mathrm{H}, \mathrm{NCH}_{3}\right) 1.73\left(\mathrm{~s}, 8 \mathrm{H}, \mathrm{NCH}_{2}\right) \mathrm{ppm}$.

${ }^{7}$ Li NMR (116.64 MHz, $\left.\mathbf{C}_{6} \mathbf{D}_{6}, 2^{\circ}{ }^{\circ} \mathbf{C}\right): \delta 0.79$ ppm.

${ }^{13} \mathrm{C}$ NMR (125.76 MHz, $\left.\mathbf{C}_{6} \mathrm{D}_{6}, 25^{\circ} \mathrm{C}\right): \delta 162.4(C 1), 148.4$ (C5), 131.3 (C3), $115.6(\mathrm{C} 2), 95.0(\mathrm{C} 4), 60.8(\mathrm{C} 6), 56.6\left(\mathrm{NCH}_{2}\right), 53.2\left(\mathrm{NCH}_{2}\right), 45.0\left(\mathrm{~N}_{\left.\left(\mathrm{CH}_{3}\right)_{2}\right)}\right)$, $44.3\left(\mathrm{NCH}_{3}\right)$ ppm.

EI-MS: $\mathrm{m} / \mathrm{z}(\%): 201(26)\left[\left(\mathrm{Me}_{2} \mathrm{~N}\left(\mathrm{CH}_{2}\right)_{2} \mathrm{NMe}\right)_{2}-\mathrm{H}\right]^{+}, 184(80)\left[\left(\mathrm{PyCH}_{2}\right)_{2}\right]^{+}, 183$ (58) $\left[\left(\mathrm{PyCH}_{2}\right)_{2}-\mathrm{H}\right]^{+}, 135$ (50) $\left[\mathrm{PyCH}_{2} \mathrm{NMe}_{2}-\mathrm{H}\right]^{+}, 106$ (100) $[\mathrm{PyEt}]^{+}, 85$ (80) $\left[\mathrm{Et}_{2} \mathrm{NMe}\right]^{+}, 78(15)[\mathrm{Py}]^{+}$.

\subsubsection{2-Picolylsodium-Tmeda (8, H67)}

An $n$-butylsodium ${ }^{[254]}(1.04 \mathrm{~g}, 13 \mathrm{mmol}, 1$ eq.) solution in tmeda (11 mL) and hexane $(50 \mathrm{~mL})$ was slowly added to an equimolar amount of 2-picoline $(1.3 \mathrm{~mL}$, $13 \mathrm{mmol}, 1$ eq. $)$ in diethyl ether $(20 \mathrm{~mL})$ at $-78 \mathrm{C}$. An orange solid could be filtered off after storage of the reaction mixture at $-24^{\circ} \mathrm{C}(1.4 \mathrm{~g}, 47 \%)$. Orange needles suitable for $\mathrm{X}$-ray structural analysis have been obtained from the filtrate in the freezer $(0.4 \mathrm{~g}, 13 \%)$.

M.p.: $\sim 245^{\circ} \mathrm{C}$ (decomp.).

Empirical formula: $\mathrm{C}_{24} \mathrm{H}_{44} \mathrm{~N}_{6} \mathrm{Na}_{2}$.

Molecular Mass: $462.63 \mathrm{~g} \mathrm{~mol}^{-1}$.

${ }^{1} \mathbf{H}$ NMR (200.13 MHz, $\left.\mathbf{C}_{6} \mathbf{D}_{6}, \quad 25^{\circ} \mathbf{C}\right): \delta 7.25$ (ddd, ${ }^{3} J(H 5, H 4)=5.6$, $\left.{ }^{4} J(\mathrm{H} 5, \mathrm{H} 3)=1.9,{ }^{5} J(\mathrm{H} 5, \mathrm{H} 2)=0.9,1 \mathrm{H}, \mathrm{H} 5\right), 6.51 \quad\left(\right.$ ddd,${ }^{3} J(\mathrm{H} 3, \mathrm{H} 2)=8.9$, $\left.{ }^{3} J(\mathrm{H} 3, \mathrm{H} 4)=6.2,{ }^{4} J(\mathrm{H} 3, \mathrm{H} 5)=1.9,1 \mathrm{H}, \mathrm{H3}\right), 6.20 \quad\left(\mathrm{ddd},{ }^{3} J(\mathrm{H} 2, \mathrm{H} 3)=8.9\right.$, $\left.{ }^{4} J(\mathrm{H} 2, \mathrm{H} 4)=0.9,{ }^{5} J(\mathrm{H} 2, \mathrm{H} 5)=0.9, \quad 1 \mathrm{H}, \mathrm{H} 2\right), 5.37 \quad\left(\mathrm{ddd},{ }^{3} J(\mathrm{H} 4, \mathrm{H} 3)=6.2\right.$, $\left.{ }^{3} \mathrm{~J}(\mathrm{H} 4, \mathrm{H} 5)=5.6,{ }^{4} \mathrm{~J}(\mathrm{H} 4, \mathrm{H} 2)=0.9,1 \mathrm{H}, \mathrm{H} 4\right), 3.15(1 \mathrm{H}, \mathrm{H} 6), 2.97(1 \mathrm{H}, \mathrm{H} 6), 2.10$ $\left(\mathrm{s}, 12 \mathrm{H}, \mathrm{NCH}_{3}\right), 2.08\left(\mathrm{~s}, 4 \mathrm{H}, \mathrm{NCH}_{2}\right) \mathrm{ppm}$.

EI-MS: $m / z(\%): 184(83)\left[\left(\mathrm{PyCH}_{2}\right)_{2}\right]^{+}, 183(50)\left[\left(\mathrm{PyCH}_{2}\right)_{2}-\mathrm{H}\right]^{+}, 106$ (100) $[\text { PyEt }]^{+}, 93(20)[\mathrm{PiCH}]^{+}, 78(18)[\mathrm{Py}]^{+}$.

2-Picolylsodium.Tmeda (8) is poorly soluble in benzene, toluene, and thf when it is isolated. Two phases separate so that NMR spectra of the pure compound could not be measured. The NMR spectrum of a sample taken from the reaction mixture on the other hand could be analysed. 


\subsection{5 $\left[\mathrm{Na}_{6}\left(\mathrm{NMe}_{2}\right)_{5}(\mathrm{OtBu}) \cdot(\text { tmeda })_{2}(\mathrm{tol})\right]_{2}(9, \mathrm{H} 47)$}

Sodium tert-butoxide $(3.0 \mathrm{~g}, 31 \mathrm{mmol}, 1 \mathrm{eq}$.$) was suspended in toluene$ ( $5 \mathrm{~mL}, 47 \mathrm{mmol}, 1.5$ eq.) and hexane (30 mL) and slowly added with a $2.26 \mathrm{M}$ solution of $n$-butyllithium in hexane $\left(13.7 \mathrm{~mL}, 31 \mathrm{mmol}, 1\right.$ eq.) at $0{ }^{\circ} \mathrm{C}$. Afterwards, tmeda $(15 \mathrm{~mL})$ was added and an orange solid precipitated from the red solution. The filtrate was stored at $-24^{\circ} \mathrm{C}$ and orange crystals have been obtained.

Empirical formula: $\mathrm{C}_{66} \mathrm{H}_{158} \mathrm{~N}_{18} \mathrm{Na}_{12} \mathrm{O}_{2}$. Molecular Mass: $1511.98 \mathrm{~g} \mathrm{~mol}^{-1}$.

\subsubsection{4-Picolyllithium-Thf (10, H150)}

4-Picoline ( $2.7 \mathrm{~mL}, 27.5 \mathrm{mmol}, 1.0$ eq.) in thf ( $50 \mathrm{~mL}$ ) was cooled to $-78^{\circ} \mathrm{C}$ and a $1.6 \mathrm{M}$ solution of methyllithium in thf $(21 \mathrm{~mL}, 33.6 \mathrm{mmol}, 1.2 \mathrm{eq}$.) was slowly added over a period of $1.5 \mathrm{~h}$. The orange solution was stirred overnight and the solvent removed. The red viscous residue was redissolved in thf $(15 \mathrm{~mL})$, filtered and pentane was added. Red crystals $(2.4 \mathrm{~g}, 10 \mathrm{mmol}, 36 \%)$ have been obtained from the solution at $4{ }^{\circ} \mathrm{C}$.

Empirical formula: $\mathrm{C}_{28} \mathrm{H}_{44} \mathrm{Li}_{2} \mathrm{~N}_{2} \mathrm{O}_{4}$. Molecular Mass: $486.53 \mathrm{~g} \mathrm{~mol}^{-1}$.

M.p.: $99^{\circ} \mathrm{C}$ (decomp.).

${ }^{1} \mathbf{H}$ NMR (500.13 MHz, d8-thf, $\left.25^{\circ} \mathbf{C}\right): \delta 6.31\left(\mathrm{~d},{ }^{3} J(H 1, H 2)=6.8,2 \mathrm{H}, H 1\right)$, $5.22\left(\mathrm{~d},{ }^{3} \mathrm{~J}(\mathrm{H} 2, \mathrm{H} 1)=6.8,2 \mathrm{H}, \mathrm{H} 2\right), 3.62\left(\mathrm{~m}, 8 \mathrm{H}, \mathrm{OCH}_{2}\right), 2.66(\mathrm{~s}, 2 \mathrm{H}, H 6), 1.78$ $\left(\mathrm{m}, 8 \mathrm{H}, \mathrm{CH}_{2}\right) \mathrm{ppm}$.

${ }^{7}$ Li NMR (116.64 $\mathbf{M H z}, \mathbf{d}_{\mathbf{8}}$-thf, $\left.25^{\circ} \mathbf{C}\right): \delta 0.18 \mathrm{ppm}$.

${ }^{13}$ C NMR (125.76 MHz, d8-thf, $\left.25{ }^{\circ} \mathbf{C}\right): \delta 147.8$ (C3), 143.6 (C1), 109.6 (C2), $68.2\left(\mathrm{OCH}_{2}\right), 67.1(\mathrm{C} 6), 26.3\left(\mathrm{CH}_{2}\right) \mathrm{ppm}$.

EI-MS: m/z (\%): $184(10)\left[\left(\mathrm{PyCH}_{2}\right)_{2}\right]^{+}, 93(100)\left[\mathrm{PyCH}_{3}\right]^{+}, 92(28)\left[\mathrm{PyCH}_{2}\right]^{+}, 78$ (8) $[\mathrm{Py}]^{+}, 72(17)[\mathrm{thf}]^{+}, 66(26)\left[\mathrm{C}_{4} \mathrm{H}_{4} \mathrm{~N}\right]^{+}, 42(32)\left[\text { thf }-\mathrm{OCH}_{2}\right]^{+}$. 


\subsubsection{4-Picolyllithium·2-n-Butyl-4-methyl- 1,2-dihydropyridyllithium Dimer (11, H56)}

To a solution of 4 -Picoline ( $6 \mathrm{~mL}, 61 \mathrm{mmol}, 1$ eq.) in thf (40 mL) cooled to $-78^{\circ} \mathrm{C}$, a $2.26 \mathrm{M}$ solution of $n$-butyllithium in hexane ( $27 \mathrm{~mL}, 61 \mathrm{mmol}, 1$ eq.) was slowly added. The clear red solution was stirred for $16 \mathrm{~h}$ at RT and stored at $-24{ }^{\circ} \mathrm{C}$. Red crystals have been obtained $(8.3 \mathrm{~g}, 27 \mathrm{mmol}, 44 \%)$ after three days.

Empirical formula: $\mathrm{C}_{36} \mathrm{H}_{62} \mathrm{Li}_{2} \mathrm{~N}_{2} \mathrm{O}_{5}$.

Molecular Mass: $616.76 \mathrm{~g} \mathrm{~mol}^{-1}$.

M.p.: $135^{\circ} \mathrm{C}$ (decomp.).

${ }^{1} \mathbf{H}$ NMR (200.13 MHz, d8 -thf, $\left.25^{\circ} \mathbf{C}\right): \delta 6.49\left(\mathrm{~d},{ }^{3} J(\mathrm{H} 11, \mathrm{H} 10)=5.6,1 \mathrm{H}\right.$, $H 11), 6.25\left(\mathrm{~d},{ }^{3} \mathrm{~J}(\mathrm{H} 1, \mathrm{H} 2)=6.7,1.2 \mathrm{H}, H 1\right), 5.19\left(\mathrm{~d},{ }^{3} \mathrm{~J}(\mathrm{H} 2, \mathrm{H} 1)=6.7,1.2 \mathrm{H}\right.$, $H 2), 4.16\left(\mathrm{dd},{ }^{3} \mathrm{~J}(\mathrm{H} 10, \mathrm{H} 11)=5.6,{ }^{4} \mathrm{~J}(\mathrm{H} 10, \mathrm{H} 8)=1.9,1 \mathrm{H}, \mathrm{H} 10\right), 3.93$ (dd, $\left.{ }^{3} \mathrm{~J}(\mathrm{H} 8, \mathrm{H} 7)=4.3,{ }^{4} \mathrm{~J}(\mathrm{H} 8, \mathrm{H} 10)=1.9,1 \mathrm{H}, \mathrm{H8}\right), 3.60\left(\mathrm{~m}, 8 \mathrm{H}, \mathrm{OCH}_{2}\right), 3.49(\mathrm{td}$, $\left.{ }^{3} \mathrm{~J}(\mathrm{H7}, \mathrm{H} 13)=4.8,{ }^{3} \mathrm{~J}(\mathrm{H7}, \mathrm{H} 8)=4.3,1 \mathrm{H}, \mathrm{H7}\right), 2.66(\mathrm{~s}, 1.2 \mathrm{H}, \mathrm{H6}), 1.72(\mathrm{~m}, 8 \mathrm{H}$, $\left.\mathrm{CH}_{2}\right), 1.52(\mathrm{~s}, 3 \mathrm{H}, \mathrm{H12}), 1.35-1.05(\mathrm{~m}, 6 \mathrm{H}, \mathrm{H} 13-\mathrm{H} 15), 0.81$ (t, $\left.{ }^{3} \mathrm{~J}(\mathrm{H} 16, \mathrm{H} 15)=7.0,3 \mathrm{H}, \mathrm{H16}\right) \mathrm{ppm}$.

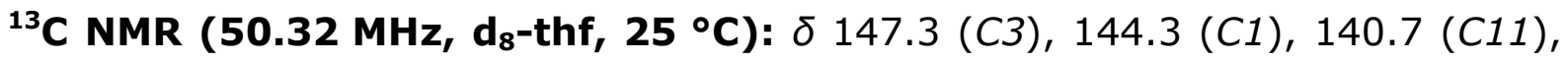
130.7 (C9), 106.9 (C2), 93.1 (C8), 89.1 (C10), 65.6 (C6), 55.4 (C7), 32.9 (C13), 25.7 (C14), 23.7 (C12), 18.7 (C15), 12.1 (C16) ppm.

\subsubsection{4-Picolyllithium.Pmdeta (12, H154/H161)}

A solution of 4-picoline (3.0 mL, $30.6 \mathrm{mmol}, 1.0$ eq.) in thf (50 mL) and pmdeta $\left(6.4 \mathrm{~mL}, 30.7 \mathrm{mmol}, 1.0\right.$ eq.) was cooled to $-78{ }^{\circ} \mathrm{C}$ and a $1.6 \mathrm{M}$ solution of methyllithium in thf ( $20 \mathrm{~mL}, 32 \mathrm{mmol}, 1.1$ eq.) was slowly added over a period of $1.5 \mathrm{~h}$. The solution was concentrated and orange-red crystals started to grow. After complete removal of all volatiles the residue was redissolved in thf and hexane was added. Red crystals $(4.9 \mathrm{~g}, 18.1 \mathrm{mmol}, 59 \%)$ have been obtained from the solution at $-24^{\circ} \mathrm{C}$.

Empirical formula: $\mathrm{C}_{15} \mathrm{H}_{29} \mathrm{LiN}_{4}$.

Molecular Mass: $272.36 \mathrm{~g} \mathrm{~mol}^{-1}$.

M.p.: 145 - $150^{\circ} \mathrm{C}$ (decomp.).

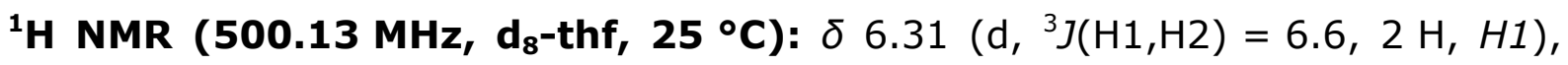
$5.22\left(\mathrm{~d},{ }^{3} \mathrm{~J}(\mathrm{H} 2, \mathrm{H} 1)=6.6,2 \mathrm{H}, \mathrm{H} 2\right), 2.68(\mathrm{~s}, 2 \mathrm{H}, H 6), 2.45\left(\mathrm{t},{ }^{3} J(H, H)=6.5\right.$, 
$\left.6 \mathrm{H}, \mathrm{NCH}_{2}\right), 2.34\left(\mathrm{t},{ }^{3} \mathrm{~J}(\mathrm{H}, \mathrm{H})=6.5,6 \mathrm{H}, \mathrm{NCH}_{2}\right), 2.25\left(\mathrm{~s}, 3 \mathrm{H}, \mathrm{NCH}_{3}\right) 2.18(\mathrm{~s}$, $\left.12 \mathrm{H}, \mathrm{N}\left(\mathrm{CH}_{3}\right)_{2}\right) \mathrm{ppm}$.

${ }^{13}$ C NMR (125.76 MHz, d8-thf, $\left.25^{\circ} \mathbf{C}\right): \delta 147.7$ (C3), 143.5 (C1), 109.7 (C2), 67.3 (C6 overlaps with solvent), $58.6\left(\mathrm{NCH}_{2}\right), 56.6\left(\mathrm{NCH}_{2}\right), 46.1\left(\mathrm{~N}\left(\mathrm{CH}_{3}\right)_{2}\right), 43.7$ $\left(\mathrm{NCH}_{3}\right) \mathrm{ppm}$.

EI-MS: m/z (\%): $184(100)\left[\left(\mathrm{PyCH}_{2}\right)_{2}\right]^{+}, 115(10)\left[\mathrm{Me}_{2} \mathrm{~N}\left(\mathrm{CH}_{2}\right)_{2} \mathrm{~N}(\mathrm{Me}) \mathrm{CH}_{2}\right]^{+}, 92$ (80) $\left[\mathrm{PyCH}_{2}\right]^{+}, 58(24)\left[\mathrm{Me}_{2} \mathrm{NCH}_{2}\right]^{+}$.

\subsection{9 [4-PicLi-tmeda] $(13, \mathrm{H} 69 / \mathrm{H102})$}

4-Picoline ( $4 \mathrm{~mL}, 41 \mathrm{mmol}, 1$ eq.), tmeda (12 mL, $81 \mathrm{mmol}, 2$ eq.), and hexane $(65 \mathrm{~mL})$ were cooled to $-78^{\circ} \mathrm{C}$, and a $2.26 \mathrm{M}$ solution of $n$-butyllithium in hexane (18 mL, $41 \mathrm{mmol}, 1$ eq.) was slowly added over a period of $2 \mathrm{~h}$. The mixture was stirred for $16 \mathrm{~h}$ at RT and the resulting yellow solid was filtered off. It was redissolved in thf and stored at $-24{ }^{\circ} \mathrm{C}$ to yield red crystals $(3.7 \mathrm{~g}$, $17 \mathrm{mmol}, 42 \%$ )

M.p.: $>300{ }^{\circ} \mathrm{C}$.

Empirical formula: $\mathrm{C}_{12} \mathrm{H}_{22} \mathrm{LiN}_{3}$. Molecular Mass: $215.27 \mathrm{~g} \mathrm{~mol}^{-1}$.

${ }^{1} \mathbf{H}$ NMR (500.13 MHz, $\left.\mathbf{C}_{6} \mathbf{D}_{6}, 25^{\circ} \mathbf{C}\right): \delta 6.39\left(\mathrm{~d},{ }^{3} J(H 1, H 2)=6.6,2 \mathrm{H}, H 1\right)$, $5.96\left(\mathrm{~d},{ }^{3} \mathrm{~J}(\mathrm{H} 2, \mathrm{H} 1)=6.6,2 \mathrm{H}, \mathrm{H} 2\right), 3.90(\mathrm{~s}, 2 \mathrm{H}, \mathrm{H6}), 1.89\left(\mathrm{~s}, 12 \mathrm{H}, \mathrm{NCH}_{3}\right), 1.62$ $\left(\mathrm{s}, 4 \mathrm{H}, \mathrm{NCH}_{2}\right)$ ppm.

${ }^{7}$ Li NMR (116.64 $\left.\mathbf{M H z}, \mathbf{C}_{6} \mathbf{D}_{6}, 2^{\circ}{ }^{\circ} \mathbf{C}\right): \delta 0.16 \mathrm{ppm}$.

${ }^{13} \mathrm{C}$ NMR (125.76 MHz, $\left.\mathbf{C}_{6} \mathrm{D}_{6}, 25^{\circ} \mathbf{C}\right): \delta 146.0(C 3), 143.2(C 1), 111.2(C 2)$, $73.8(\mathrm{C6}), 56.8\left(\mathrm{NCH}_{2}\right), 46.3\left(\mathrm{NCH}_{3}\right) \mathrm{ppm}$.

EI-MS: $m / z(\%): 184(100)\left[\left(\mathrm{PyCH}_{2}\right)_{2}\right]^{+}, 92(60)[\mathrm{PiCH}]^{+}, 58(80)\left[\mathrm{Me}_{2} \mathrm{NCH}_{2}\right]^{+}$. 


\section{Crystallographic Section}

\subsection{Crystal Application}

The crystal selection was carried out at a specially designed moveable table that was invented by $U$. Flierler. It consists of a fully functional Schlenk line including a high vacuum rotary vane pump, argon gas supply with adjacent gas drying unit and oxygen absorber as well as a polarisation microscope equipped with a video camera for demonstration purposes and an XTEMP2 ${ }^{[44]}$ crystal cooling device (Figure 8-1).

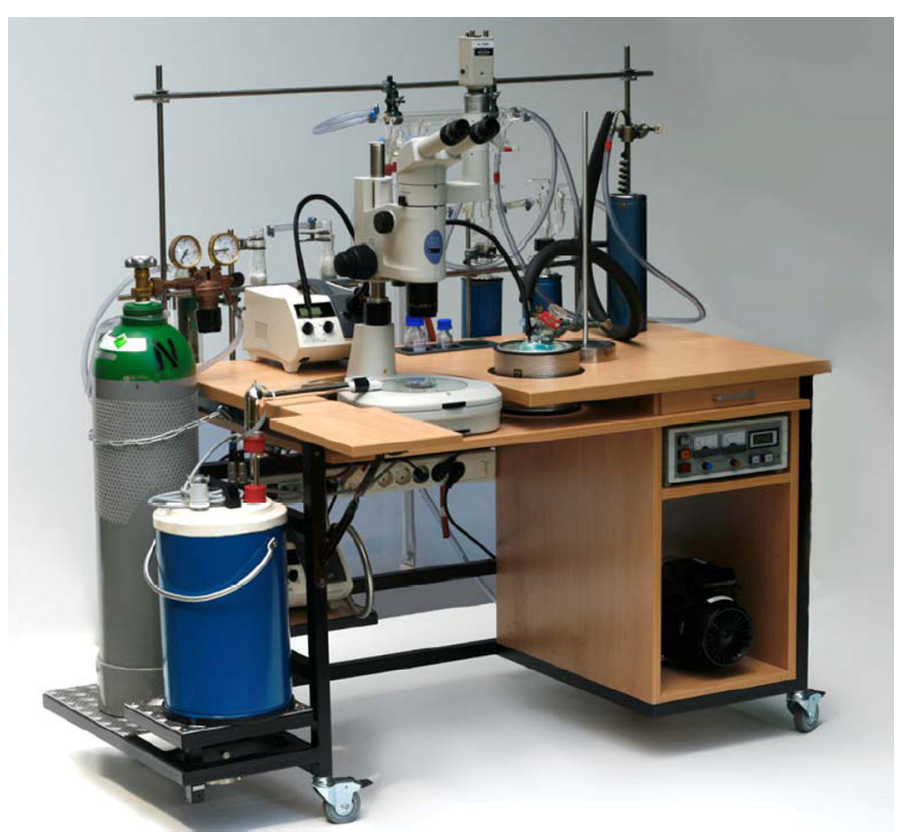

Figure 8-1. Crystal mounting table

First, the crystals were removed from their mother liquor in the Schlenk flask, which was attached to the Schlenk line under a slight argon stream with a small spoon or needle. The manipulation instrument was covered with a drop of perfluorinated polyether oil to protect the crystal from decomposition on the short way to the microscope slide, which carried a small amount of perfluorinated oil in which the crystals were placed and was cooled by the X TEMP2 device in the case of highly sensitive crystals. Thus, the crystal could be handled without degradation and specifically selected according to their size (crystal size should fit the X-ray beam size) and quality. This was checked by a standardised $1 \mathrm{~cm}$ scale in the microscope optics and a polarisation filter. The appropriate crystal was subsequently mounted on the tip of a glass fibre or into a MITEGEN cryo loop 
both attached to a magnetic pin. Subsequently, the crystal was rapidly transferred to the goniometer head equipped with a magnetic base for the pins and the compounds was immediately shock-cooled in the cryogenic stream of the KRYOFLEX open flow nitrogen cooling device of the diffractometer. Thus, the crystal was well fixed in the amorphously solidified oil and protected from air and moisture. The cooling temperature was normally $100 \mathrm{~K}$, but varied sometimes because of destructive phase transitions of some compounds on cooling. Details can be taken from the crystallographic tables of each compound.

\subsection{Data Collection, Integration, and Reduction}

Several improvements of the diffractometer pool were made during these studies so that different set-ups were used for the determination of molecular structures. Various X-ray sources, monochromators, and computer programmes for the operation of the diffractometers and the data processing were used, depending on the diffractometer type and manufacturer. However, all compounds were measured with monochromatic $\mathrm{MoK}_{\alpha}$ radiation (wave length: $0.71073 \AA$ ) and more specific details are summarised below:

\section{BRUKER TXs rotating anode}

The diffractometer is powered by a most recent generation table top rotating anode (TXS). The INCOATEC HELIOS mirror optics serves as monochromator and collimator. A beam size diameter of $0.35 \mathrm{~mm}$ is achieved by an off-focus operation of the X-ray source. The goniometer is of D8 three-cycle geometry and the detector is an APEX II CCD with a detector chip operation temperature of $-60{ }^{\circ} \mathrm{C}$.

The APEX2 software package ${ }^{[186]}$ was used for the data collections and the determination of unit cells and orientation matrices on BRUKER diffractometers. The collected data were integrated with SAINT ${ }^{[187]}$ and subsequently corrected for absorption and scales with SADABS. ${ }^{[188,329]}$ The determination of space groups and the data merging was performed with XPREP. ${ }^{[330]}$

\section{BRUKER SMART APEX II with D8 goniometer}

The three cycle diffractometer is equipped with an APEX II CCD camera and a SIEMENS sealed tube (st) with a graphite monochromator and a MONOCAP 
collimator (beam size: $0.5 \mathrm{~mm}$ ). A second X-ray source was implemented in 2007. The novel INCOATEC MICROSOURCE (I $\boldsymbol{\mu S}$ ) with X-ray mirror optics (Figure 8-2) provides a much smaller $(<0.16 \mathrm{~mm}$ ) and brighter beam that is even suitable for very small crystals $(<0.05 \mathrm{~mm})$.

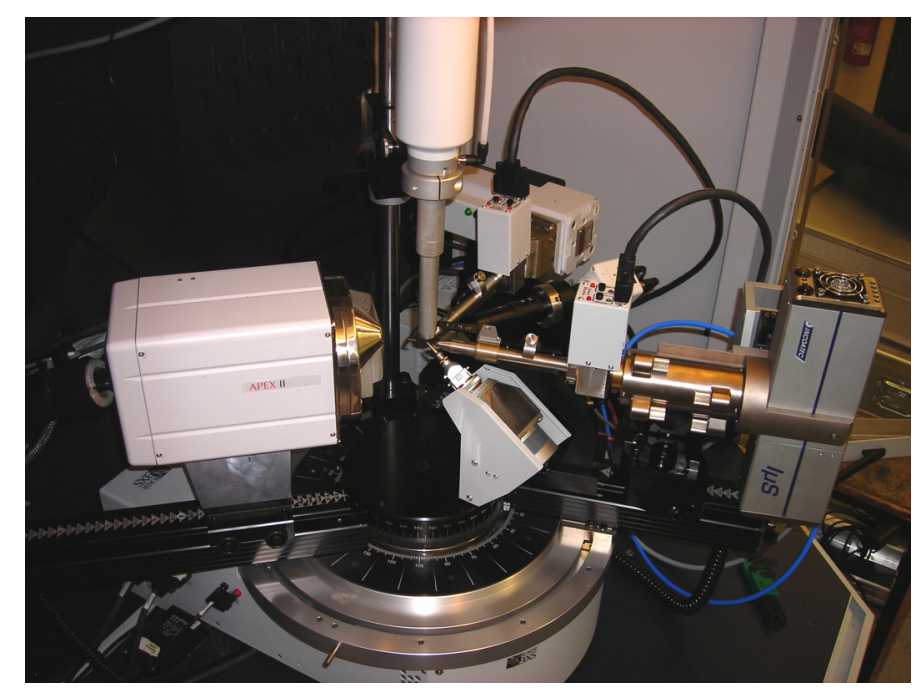

Figure 8-2. InCOATEC Mo-Microsource on a BRUKER SMART APEX II with D8 goniometer

\section{StOE IPDS II}

A sealed tube with graphite monochromator is used as X-ray source. The detector is an image plate (ip), which is $34 \mathrm{~cm}$ in diameter. This detector system has the advantage of a much lower background and a larger detection area compared to CCD cameras at the expense of much higher read out and exposure times. The X-AREA software package ${ }^{[331]}$ was used for the data collections, cell determinations, and data integrations. 


\subsection{Structure Solution and Refinement}

All structures were solved with direct or Patterson methods using SHELXS, ${ }^{[190]}$ while the subsequent IAM refinement was performed with SHELXL. ${ }^{[191]}$ If not stated otherwise, all hydrogen atoms were calculated to ideal positions and refined using a riding model (hydrogen motion fixed to 1.2 times that of the connected atom; 1.5 times for methyl carbon atoms). Further details concerning quality criteria were already outlined in chapter 2.3.3.

Structures containing disordered groups were refined using constraints and restraints. While constraints fix structural parameters to certain values, restraints introduce additional chemical or crystallographical information in the model and have to be observed within their standard deviations.

The geometries of chemically equivalent but crystallographically independent fragments can be fitted to each other by distance restraints. Especially the 1,2 distances (DFIX) and 1,3 distances (DANG) can be set to be equal within their standard deviations. This may help to refine disordered positions by introduction of chemical information that stabilises the refinement.

Restraints affecting the anisotropic displacement parameters are often necessary for the anisotropic refinement of disordered atomic positions. The rigid bond restraints (DELU) fit the components of the anisotropic displacement parameters along the bonds. Similarity restraints (SIMU) adjust the ADPs of neighbouring atoms within a certain radius to be equal. The ISOR command forces the ADPs to be of spherical shape, which is sometimes necessary to refine positions with low occupation factors. All restraints of this type are applied as weak restraints with relatively large standard deviation, as the affected parameters are not perfectly equal. Noteworthy, the first non-disordered atoms next to atoms with split occupancies were also refined with the observed site occupation factor of the disorder, but fixed to have the same atomic position (EXYZ) and ADP (EADP). This procedure enabled to correct description of the hydrogen positions at these atoms. In the subsequent chapters, disordered atoms of the minor domain in the asymmetric units were labelled with apostrophes and their bonds are drawn dotted.

Anisotropic displacement parameters are shown at a $50 \%$ probability level and were plotted with the programme XSHELL V4.01. ${ }^{[332]}$ 


\subsection{Crystallographic Details for 1-5, 8-15, and 17}

\subsubsection{2-Picolyllithium-Dme (1)}

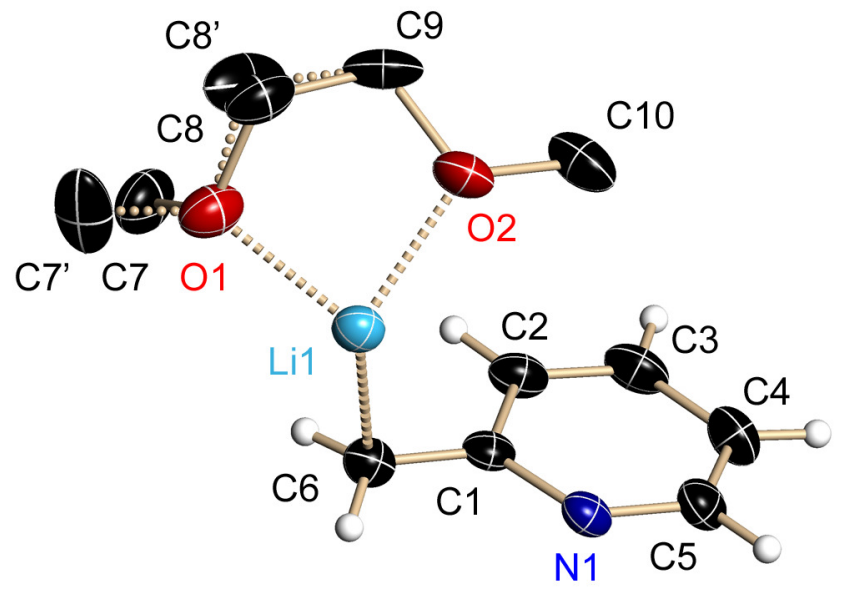

Figure 8-3. Asymmetric unit of [2-PicLi-dme $]_{2}(\mathbf{1})$. Hydrogen atoms of the dme donor are omitted for clarity.

Table 8-1. Crystallographic data for 2-PicLi·dme (1).

\begin{tabular}{lc|lc}
\hline identification code & HOTT0010/st & $F(000)$ & 408 \\
empirical formula & $\mathrm{C}_{20} \mathrm{H}_{32} \mathrm{Li}_{2} \mathrm{~N}_{2} \mathrm{O}_{4}$ & min./max. transmission & $0.77 / 0.99$ \\
molar mass $\left[\mathrm{g} \mathrm{mol}{ }^{-1}\right]$ & 378.36 & $\theta$ range $\left[^{\circ}\right]$ & $2.58-25.09$ \\
crystal size $[\mathrm{mm}]$ & $0.20 \times 0.15 \times 0.10$ & completeness to $\theta_{\max }$ & 0.99 \\
temperature $[\mathrm{K}]$ & $100(2)$ & redundancy to $\theta_{\max }$ & 6.15 \\
crystal system & monoclinic & reflections collected & 12847 \\
space group & $P 2_{1} / \mathrm{C}$ & independent reflections & 1933 \\
$a[\AA]$ & $10.8372(19)$ & $R_{\text {int }} / R_{\sigma}$ & $0.0401 / 0.0268$ \\
$b[\AA]$ & $9.3561(16)$ & restraints/parameters & $45 / 155$ \\
$c[\AA]$ & $10.8739(19)$ & GoF & 1.075 \\
$\beta\left[{ }^{\circ}\right]$ & $93.216(2)$ & $R 1($ all data $)$ & 0.0739 \\
$V\left[\AA^{3}\right]$ & $1100.8(3)$ & $R 1(I>2 \sigma(I))$ & 0.0586 \\
$\mathrm{Z}$ & 2 & $w R 2($ all data $)$ & 0.1481 \\
$\rho_{\text {calc }}\left[\mathrm{g} \mathrm{cm}^{-3}\right]$ & 1.141 & $w R 2(I>2 \sigma(I))$ & 0.1410 \\
$\mu\left[\mathrm{mm}^{-1}\right]$ & 0.077 & diff. peak/hole $\left[\mathrm{e} \AA^{-3}\right]$ & $0.293 /-0.243$ \\
\hline
\end{tabular}


2-Picolyllithium as dme adduct crystallises in the monoclinic centrosymmetric space group $P Z_{1} / n$ (Figure 8-3). The dimer is obtained by an inversion at the origin and a translation operation about $(1,0,2)$. The methylene hydrogen atoms were refined without geometrical constraints but with a riding model. A disorder in the dme donor molecule could be refined using DFIX, DELU, and SIMU restraints. The sof of the minor domain refined to 0.14 .

\subsubsection{2-Picolyllithium-Tmeda (2)}

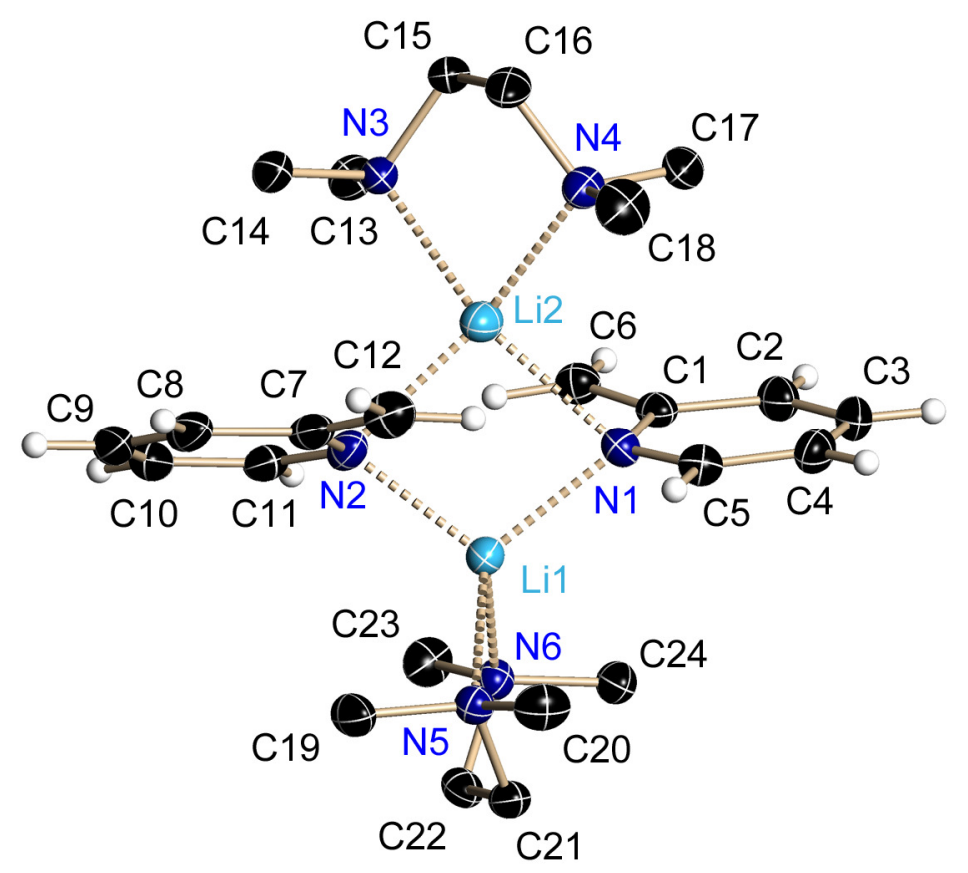

Figure 8-4. Asymmetric unit of [2-PicLi-tmeda $]_{2}$ (2). Hydrogen atoms of the donor are omitted for clarity.

The tmeda adduct of 2-picolyllithium crystallises in the monoclinic chiral space group $P 2_{1}$. The asymmetric unit contains a complete dimer (Figure 8-4). The absolute structure could not be determined due to the lack of a heavy atom. This would act as an anomalous scatterer near its absorption edge. An additional direction dependent complex component would then be included in the atomic structure factor and Friedel's law not strictly valid anymore. This enables the determination of the absolute structure by reliability indicators such as the Flack $x$ parameter ${ }^{[321,322]}$ or a novel $y$ value from Bayesian statistics of Bijvoet pairs. ${ }^{[333]}$ 
Table 8-2. Crystallographic data for 2-PicLi-tmeda (2).

\begin{tabular}{|c|c|c|c|}
\hline identification code & HOTТ0012/I $\mathbf{\mu S}$ & $F(000)$ & 472 \\
\hline empirical formula & $\mathrm{C}_{24} \mathrm{H}_{44} \mathrm{Li}_{2} \mathrm{~N}_{6}$ & min./max. transmission & $0.94 / 0.98$ \\
\hline molar mass $\left[\mathrm{g} \mathrm{mol}^{-1}\right]$ & 430.53 & $\theta$ range $\left[^{\circ}\right]$ & $2.40-26.73$ \\
\hline crystal size [mm] & $0.22 \times 0.15 \times 0.10$ & completeness to $\theta_{\max }$ & 1.00 \\
\hline temperature $[\mathrm{K}]$ & $100(2)$ & redundancy to $\theta_{\max }$ & 5.28 \\
\hline crystal system & monoclinic & reflections collected & 31316 \\
\hline space group & $P 2_{1}$ & independent reflections & 5717 \\
\hline Flack $\mathrm{x}$ & $-0.2(10)$ & $R_{\text {int }} / R_{\sigma}$ & $0.0304 / 0.0165$ \\
\hline$a[\AA]$ & $8.4964(9)$ & restraints/parameters & $1 / 309$ \\
\hline$b[\AA]$ & $14.0289(15)$ & GoF & 1.047 \\
\hline$c[\AA]$ & $11.3499(12)$ & $R 1$ (all data) & 0.0296 \\
\hline$\beta\left[^{\circ}\right]$ & $93.437(2)$ & $R 1(I>2 \sigma(I))$ & 0.0294 \\
\hline$V\left[\AA^{3}\right]$ & $1350.4(2)$ & $w R 2$ (all data) & 0.0801 \\
\hline Z & 2 & $w R 2(I>2 \sigma(I))$ & 0.0799 \\
\hline$\rho_{\text {calc }}\left[\mathrm{g} \mathrm{cm}^{-3}\right]$ & 1.059 & diff. peak/hole $\left[\mathrm{e} \AA^{-3}\right]$ & $0.152 /-0.175$ \\
\hline$\mu\left[\mathrm{mm}^{-1}\right]$ & 0.063 & & \\
\hline
\end{tabular}

\subsubsection{2-Picolyllithium-Pmdeta (3)}

The 2-picolyllithium monomer crystallises in the centrosymmetric triclinic space group $P \overline{1}$ with two independent molecules in the asymmetric unit (Figure 8-5 and Figure 8-6). Both show extensive disorder in the pmdeta donor and monomer number one even in the picolyl anion part. Even in the second monomer ring system residual density peaks could be detected, which point to a disorder below $3 \%$ and could not be refined. The sof for the picolyl disorder is 0.14 while the one in the amine donors is consistently 0.08 . The refinement of the disordered parts was stabilised with SADI, SIMU, and DELU restraints. Moreover, both picolyl anion fragments in the first monomer were geometrically restraint with a SAME command. Altogether 596 restraints had to be used to describe the molecular structure of $\mathbf{3}$ properly. Interestingly, after the recrystallisation or a second synthesis of $\mathbf{3}$, exactly the same disorder pattern could be found. 


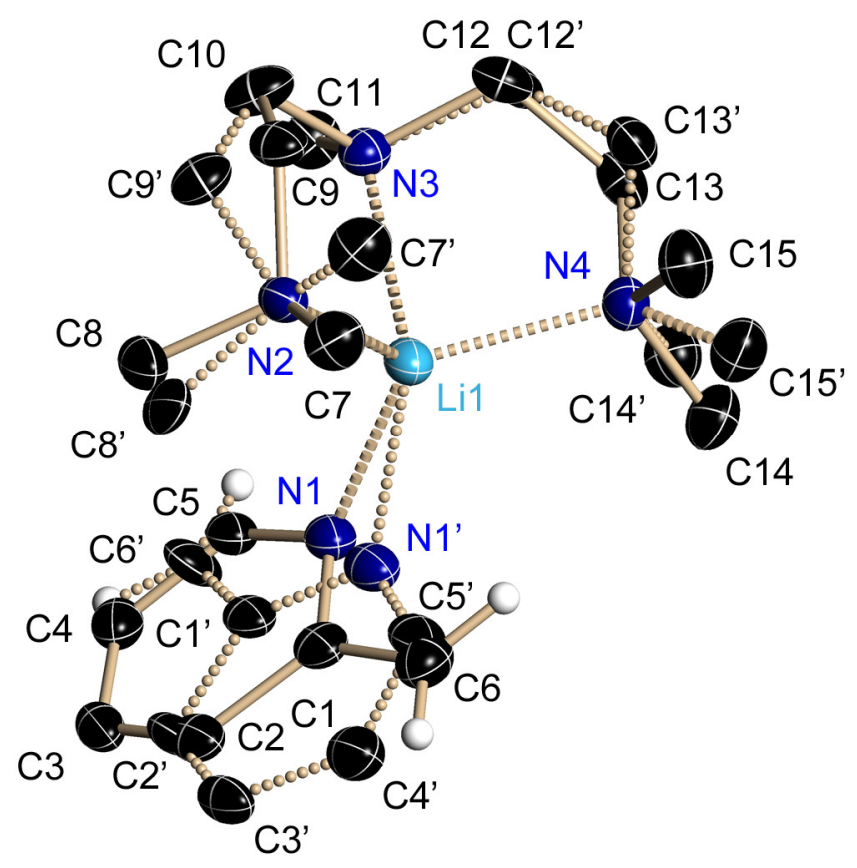

Figure 8-5. First monomer in the asymmetric unit of 2-PicLi.pmdeta (3). All constrained hydrogen atoms are omitted for clarity.

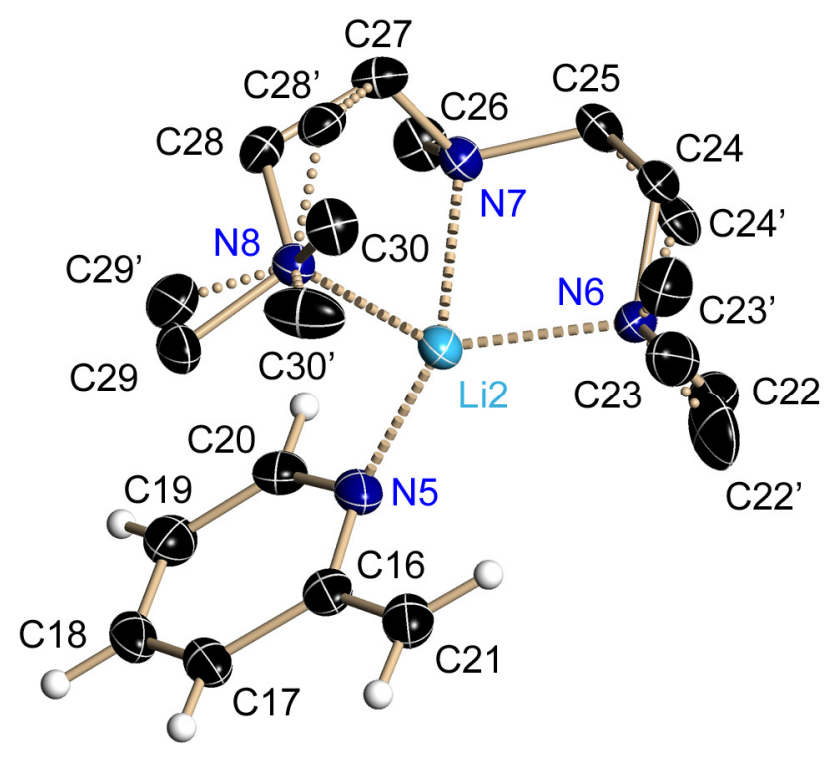

Figure 8-6. Second monomer in the asymmetric unit of 2-PicLi·pmdeta (3). Hydrogen atoms of the donor are omitted for clarity. 
Table 8-3. Crystallographic data for 2-PicLi·pmdeta (3).

\begin{tabular}{lc|lc}
\hline identification code & HOTT0017/I $\mathbf{S S}$ & $\mu\left[\mathrm{mm}^{-1}\right]$ & 0.064 \\
empirical formula & $\mathrm{C}_{15} \mathrm{H}_{29} \mathrm{LiN}_{4}$ & $F(000)$ & 600 \\
molar mass $\left[\mathrm{g} \mathrm{mol}^{-1}\right]$ & 272.36 & min./max. transmission & $0.88 / 0.98$ \\
crystal size $[\mathrm{mm}]$ & $0.24 \times 0.16 \times 0.04$ & $\theta$ range $\left.{ }^{\circ}\right]$ & $2.22-25.02$ \\
temperature $[\mathrm{K}]$ & $100(2)$ & completeness to $\theta_{\max }$ & 1.00 \\
crystal system & triclinic & redundancy to $\theta_{\max }$ & 3.77 \\
space group & $P \overline{1}$ & reflections collected & 28377 \\
$a[\AA]$ & $9.2380(13)$ & independent reflections & 5985 \\
$b\left[^{\AA}\right]$ & $13.0766(18)$ & $R_{\text {int }} / R_{\sigma}$ & $0.0273 / 0.0214$ \\
$c\left[^{\AA}\right]$ & $14.7308(19)$ & restraints/parameters & $596 / 557$ \\
$\alpha\left[^{\circ}\right]$ & $105.399(5)$ & GoF & 1.046 \\
$\beta\left[^{\circ}\right]$ & $91.043(4)$ & $R 1($ all data $)$ & 0.0430 \\
$\gamma\left[^{\circ}\right]$ & $96.893(3)$ & $R 1(I>2 \sigma(I))$ & 0.0378 \\
$V\left[^{3}\right]$ & $1700.9(4)$ & $w R 2($ all data $)$ & 0.0982 \\
$Z$ & 4 & $w R 2(I>2 \sigma(I))$ & 0.0951 \\
$\rho_{\text {calc }}\left[\mathrm{g} \mathrm{cm}^{-3}\right]$ & 1.064 & diff. peak/hole $\left[\mathrm{e} \AA^{-3}\right]$ & $0.338 /-0.200$ \\
\hline
\end{tabular}




\subsubsection{2-Picolyllithium·2-Picoline (4)}

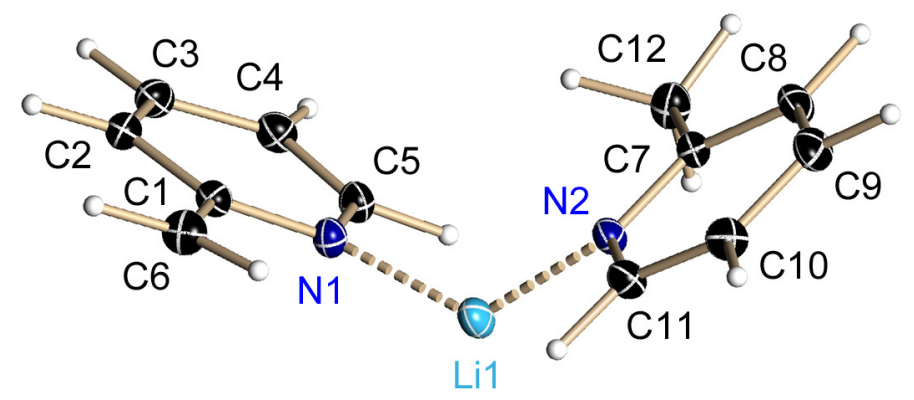

Figure 8-7. Asymmetric unit of $[2-\mathrm{PicLi} \cdot 2-\mathrm{PiCH}]_{2}(4)$.

2-Picolyllithium·2-picoline crystallises in the monoclinic centrosymmetric space group $C 2 / C$ (Figure 8-7). The dimer is generated by the inversion at the origin and a translation about $(2,0,2)$. All hydrogen atoms were refined only with a riding model. For further details see chapter 2.3.5.

Table 8-4. Crystallographic data for $[2-\mathrm{PicLi} \cdot 2-\mathrm{PiCH}]_{2}(\mathbf{4})$.

\begin{tabular}{lc|lc}
\hline identification code & PicLiPic_ED01/Txs & $\mu\left[\mathrm{mm}^{-1}\right]$ & 0.069 \\
empirical formula & $\mathrm{C}_{24} \mathrm{H}_{26} \mathrm{Li}_{2} \mathrm{~N}_{4}$ & $F(000)$ & 816 \\
molar mass $\left[\mathrm{g} \mathrm{mol}{ }^{-1}\right]$ & 384.37 & min./max. transmission & $0.90 / 0.98$ \\
crystal size $[\mathrm{mm}]$ & $0.55 \times 0.35 \times 0.30$ & $\theta$ range $\left.{ }^{\circ}\right]$ & $2.42-53.03$ \\
temperature $[\mathrm{K}]$ & $100(2)$ & completeness to $\theta_{\max }$ & 0.99 \\
crystal system & monoclinic & redundancy to $\theta_{\text {max }}$ & 7.28 \\
space group & $C 2 / c$ & reflections collected & 96235 \\
$a[\AA]$ & $11.0512(10)$ & independent reflections & 13125 \\
$b[\AA]$ & $14.2838(13)$ & $R_{\text {int }} / R_{\sigma}$ & $0.020 / 0.009$ \\
$c[\AA]$ & $14.5536(16)$ & data/restr./param. & $10378 / 0 / 509$ \\
$\beta\left[{ }^{\circ}\right]$ & $109.014(1)$ & GoF & 2.565 \\
$V\left[\AA^{3}\right]$ & $2172.0(4)$ & $R 1(I>3 \sigma(I))$ & 0.0204 \\
$Z$ & 4 & $w R 2(I>3 \sigma(I))$ & 0.0318 \\
$\rho_{\text {calc }}\left[\mathrm{g} \mathrm{cm}^{-3}\right]$ & 1.175 & diff. peak/hole $\left[\mathrm{e} \AA^{-3}\right]$ & $0.150 /-0.124$ \\
\hline
\end{tabular}




\subsubsection{2-Picolyllithium·Diethyl Ether (5)}

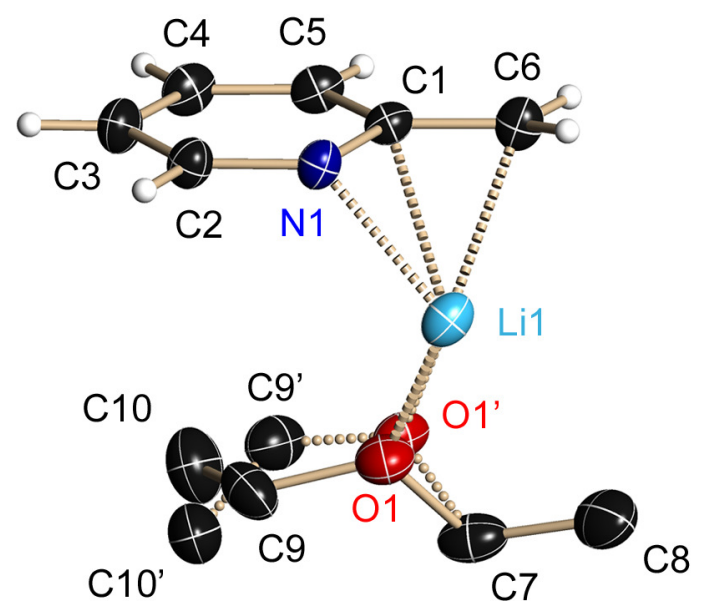

Figure 8-8. Asymmetric unit of $\left[2-\mathrm{PicLi} \cdot \mathrm{OEt}_{2}\right]_{2}(\mathbf{5})$. Hydrogen atoms of the donor molecule are omitted for clarity.

The diethyl ether adduct of 2-picolyllithium crystallises in the triclinic centrosymmetric space group $P \overline{1}$ (Figure $8-8$ ). The dimer is generated by an inversion and a translation about $(2,1,1)$. The site occupation factor of the disordered donor solvent molecule was refined to a value of 0.57 .

Table 8-5. Crystallographic data for $\left[2-\mathrm{PicLi} \cdot \mathrm{OEt}_{2}\right]_{2}(\mathbf{5})$.

\begin{tabular}{|c|c|c|c|}
\hline identification code & НОТТ0018/I $\mathbf{s}$ & $\mu\left[\mathrm{mm}^{-1}\right]$ & 0.068 \\
\hline empirical formula & $\mathrm{C}_{20} \mathrm{H}_{32} \mathrm{Li}_{2} \mathrm{~N}_{2} \mathrm{O}_{2}$ & $F(000)$ & 188 \\
\hline molar mass $\left[\mathrm{g} \mathrm{mol}^{-1}\right]$ & 346.36 & min./max. transmission & $0.85 / 0.98$ \\
\hline crystal size $[\mathrm{mm}]$ & $0.22 \times 0.15 \times 0.01$ & $\theta$ range $\left[{ }^{\circ}\right]$ & $2.37-25.09$ \\
\hline temperature $[\mathrm{K}]$ & $100(2)$ & completeness to $\theta_{\max }$ & 0.99 \\
\hline crystal system & triclinic & redundancy to $\theta_{\max }$ & 2.53 \\
\hline space group & $P \overline{1}$ & reflections collected & 4775 \\
\hline$a[\AA]$ & $8.102(2)$ & independent reflections & 1868 \\
\hline$b[\AA]$ & $8.115(2)$ & $R_{\text {int }} / R_{\sigma}$ & $0.0250 / 0.0314$ \\
\hline$c[\AA]$ & $8.613(3)$ & restraints/parameters & $1 / 154$ \\
\hline$\alpha\left[^{\circ}\right]$ & $88.512(5)$ & GoF & 1.035 \\
\hline$\beta\left[^{\circ}\right]$ & $87.700(5)$ & $R 1$ (all data) & 0.0588 \\
\hline$\gamma\left[^{\circ}\right]$ & $69.465(5)$ & $R 1(I>2 \sigma(I))$ & 0.0407 \\
\hline$V\left[\AA^{3}\right]$ & $529.8(3)$ & $w R 2$ (all data) & 0.1054 \\
\hline Z & 1 & $w R 2(I>2 \sigma(I))$ & 0.0991 \\
\hline$\rho_{\text {calc }}\left[\mathrm{g} \mathrm{cm}^{-3}\right]$ & 1.086 & diff. peak/hole $\left[\mathrm{e}^{-3}\right]$ & $0.165 /-0.192$ \\
\hline
\end{tabular}




\subsubsection{2-Picolylsodium-Tmeda (8)}

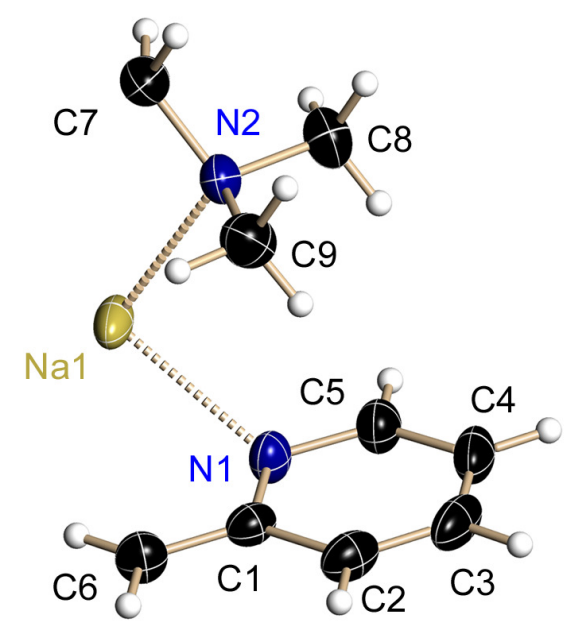

Figure 8-9. Asymmetric unit of $[2-\mathrm{PicNa} \cdot \mathrm{tmeda}]_{2}(\mathbf{8})$.

The tmeda adduct of 2-picolylsodium crystallises in the monoclinic centrosymmetric space group $C 2 / m$ (Figure 8-9). The dimer is generated by the symmetry operation of a two-fold axis along $b$ and a mirror plane perpendicular to $b$ containing the picolyl ring. The methylene hydrogen atoms were refined freely.

Table 8-6. Crystallographic data for [2-PicNa-tmeda $]_{2}(\mathbf{8})$.

\begin{tabular}{lc|lc}
\hline identification code & HOTT0007/ip & $F(000)$ & 504 \\
empirical formula & $\mathrm{C}_{24} \mathrm{H}_{44} \mathrm{~N}_{4} \mathrm{Na}_{2}$ & min./max. transmission & - \\
molar mass $\left[\mathrm{g} \mathrm{mol}^{-1}\right]$ & 462.63 & $\theta$ range $\left.{ }^{\circ}\right]$ & $2.32-24.82$ \\
crystal size $[\mathrm{mm}]$ & $0.4 \times 0.3 \times 0.2$ & completeness to $\theta_{\text {max }}$ & 1.00 \\
temperature $[\mathrm{K}]$ & $133(2)$ & redundancy to $\theta_{\max }$ & 8.00 \\
crystal system & monoclinic & reflections collected & 10005 \\
space group & $C 2 / m$ & independent reflections & 1245 \\
$a[\AA]$ & $11.945(2)$ & $R_{\text {int }} / R_{\sigma}$ & $0.0614 / 0.0201$ \\
$b[\AA]$ & $14.031(3)$ & restraints/parameters & $0 / 92$ \\
$c[\AA]$ & $8.7079(17)$ & GoF & 1.040 \\
$\beta\left[{ }^{\circ}\right]$ & $109.17(3)$ & $R 1($ all data $)$ & 0.0487 \\
$V\left[\AA^{3}\right]$ & $1378.6(5)$ & $R 1(I>2 \sigma(I))$ & 0.0348 \\
$\mathrm{Z}$ & 2 & $w R 2($ all data $)$ & 0.0922 \\
$\rho_{\text {calc }}\left[\mathrm{g} \mathrm{cm}^{-3}\right]$ & 1.115 & $w R 2(I>2 \sigma(I))$ & 0.0877 \\
$\mu\left[\mathrm{mm}^{-1}\right]$ & 0.095 & diff. peak/hole $\left[\mathrm{e} \AA^{-3}\right]$ & $0.249 /-0.171$ \\
\hline
\end{tabular}




\subsection{7 $\left[\mathrm{Na}_{6}\left(\mathrm{NMe}_{2}\right)_{5}(\mathrm{OtBu}) \cdot(\text { tmeda })_{2}(\text { tol })\right]_{2}(9)$}

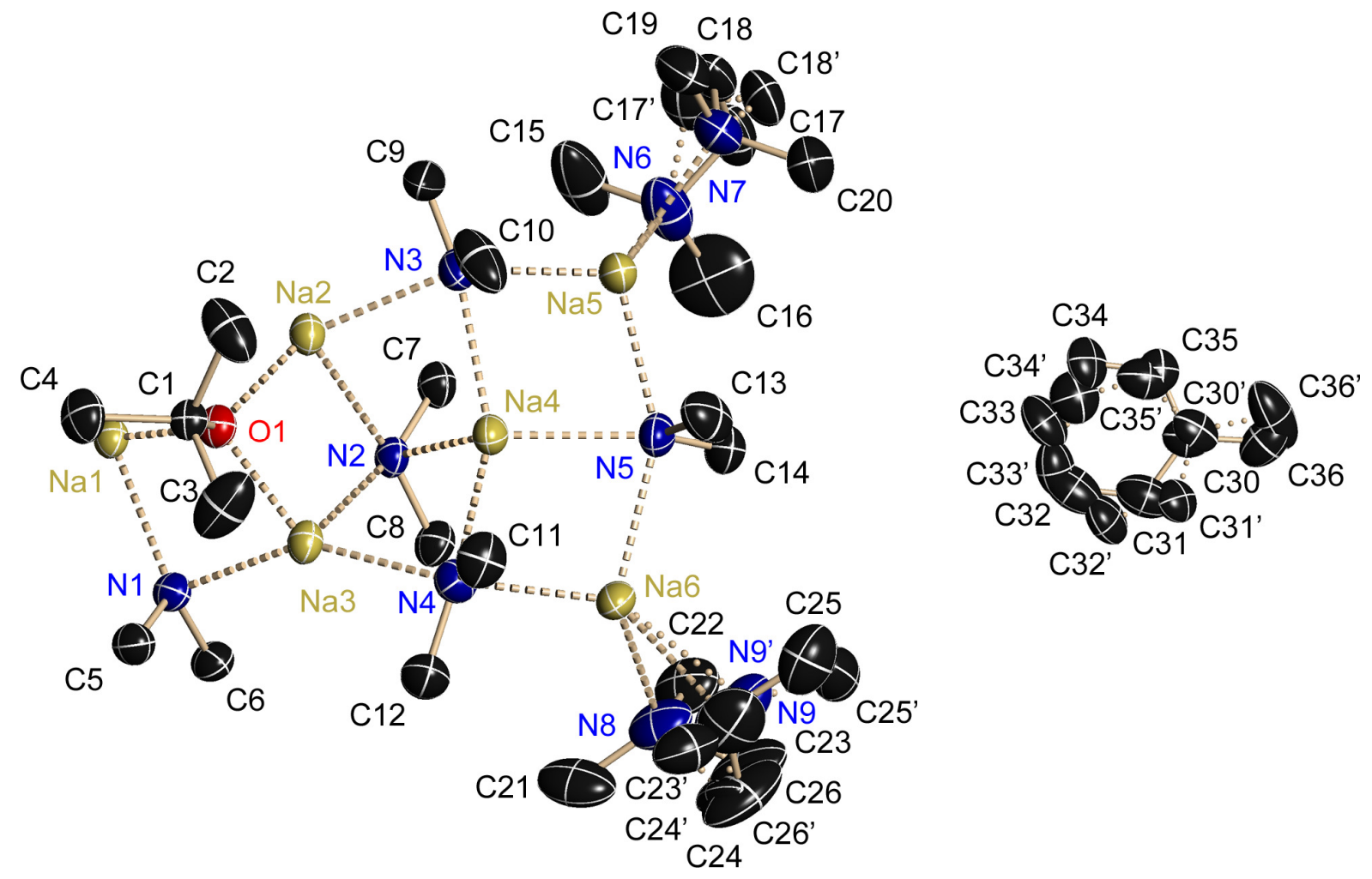

Figure 8-10. Content of the asymmetric unit of $\left[\mathrm{Na}_{6}\left(\mathrm{NMe}_{2}\right)_{5}(\mathrm{OtBu}) \cdot(\text { tmeda })_{2}(\text { tol })\right]_{2}(\mathbf{9})$ (left: sodium amide part; right: lattice solvent). Hydrogen atoms are omitted for clarity.

The decomposition by-product 9 of a benzylsodium tmeda/hexane mixture crystallises in the triclinic centrosymmetric space group $P \overline{1}$. Half of the complex aggregate is present in the asymmetric unit as well as one disordered toluene solvent molecule (Figure 8-10). The second half of the salt is generated by an inversion operation at the origin and a translation about $(2,1,1)$. Each of the tmeda donor showed an individual occupation of the main domain ( 0.79 for N6/7; 0.70 for N8/9). The disorder had to be stabilised by SIMU, DELU, ISOR, SADI, and SAME commands. However, some ADPs are still quite large, but no additional disorder could be consistently refined. The two toluene domains, on the other hand, are almost equally distributed (sof 0.56) and well refined with only SIMU and DELU commands. 
Table 8-7. Crystallographic data for $\left[\mathrm{Na}_{6}\left(\mathrm{NMe}_{2}\right)_{5}(\mathrm{OtBu}) \cdot(\text { tmeda })_{2}(\text { tol })\right]_{2}(\mathbf{9})$.

\begin{tabular}{|c|c|c|c|}
\hline identification code & HOTT0011/I $\mathbf{S}$ & $\mu\left[\mathrm{mm}^{-1}\right]$ & 0.113 \\
\hline empirical formula & $\mathrm{C}_{66} \mathrm{H}_{158} \mathrm{~N}_{18} \mathrm{Na}_{10} \mathrm{O}_{2}$ & $F(000)$ & 828 \\
\hline molar mass $\left[\mathrm{g} \mathrm{mol}^{-1}\right]$ & 1511.98 & min./max. transmission & $0.94 / 0.98$ \\
\hline crystal size $[\mathrm{mm}]$ & $0.25 \times 0.18 \times 0.10$ & $\theta$ range $\left[^{\circ}\right]$ & $2.63-27.11$ \\
\hline temperature $[\mathrm{K}]$ & $100(2)$ & completeness to $\theta_{\max }$ & 0.99 \\
\hline crystal system & triclinic & redundancy to $\theta_{\max }$ & 4.36 \\
\hline space group & $P \overline{1}$ & reflections collected & 46555 \\
\hline$a[\AA]$ & $13.555(2)$ & independent reflections & 10355 \\
\hline$b[\AA]$ & $13.918(2)$ & $R_{\text {int }} / R_{\sigma}$ & $0.0208 / 0.0150$ \\
\hline$c[\AA]$ & $15.797(2)$ & restraints/parameters & $530 / 568$ \\
\hline$\alpha\left[^{\circ}\right]$ & $65.038(1)$ & GoF & 1.060 \\
\hline$\beta\left[^{\circ}\right]$ & $86.112(2)$ & $R 1$ (all data) & 0.0561 \\
\hline$\gamma\left[{ }^{\circ}\right]$ & $62.442(1)$ & $R 1(I>2 \sigma(I))$ & 0.0492 \\
\hline$V\left[\AA^{3}\right]$ & $2364.9(5)$ & $w R 2$ (all data) & 0.1453 \\
\hline Z & 1 & $w R 2(I>2 \sigma(I))$ & 0.1401 \\
\hline$\rho_{\text {calc }}\left[\mathrm{g} \mathrm{cm}^{-3}\right]$ & 1.062 & diff. peak/hole $\left[\mathrm{e}^{-3}\right]$ & $0.667 /-0.366$ \\
\hline
\end{tabular}




\subsubsection{4-Picolyllithium-Thf (10)}

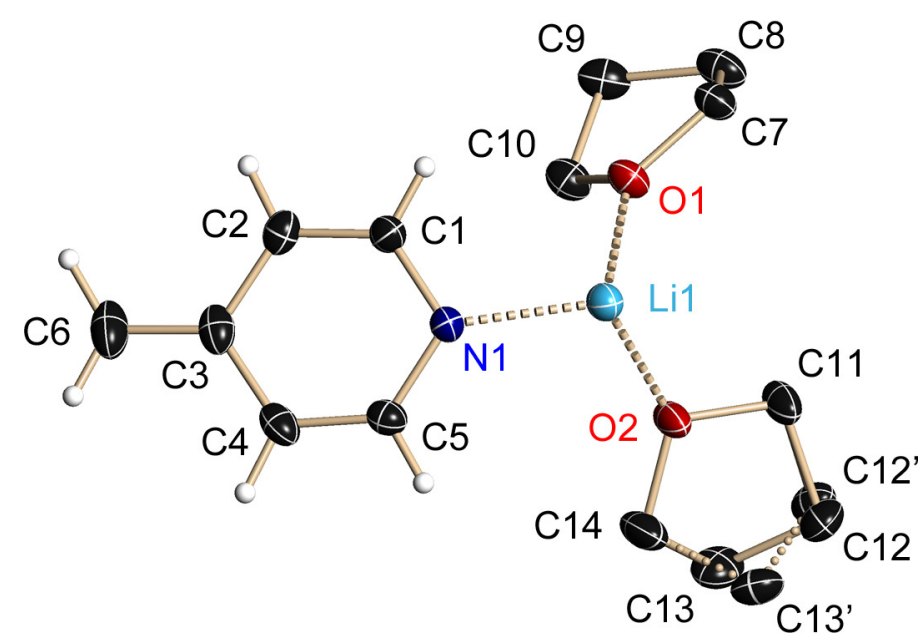

Figure 8-11. Asymmetric unit of $\left[4-\mathrm{PicLi} \cdot(\text { thf })_{2}\right]_{2}(\mathbf{1 0})$. Hydrogen atoms of the donor molecule are omitted for clarity.

Table 8-8. Crystallographic data for $\left[4-\mathrm{PicLi} \cdot(\text { thf })_{2}\right]_{2}(\mathbf{1 0})$.

\begin{tabular}{lc|lc}
\hline identification code & HOTT0015/Txs & min./max. transmission & $0.87 / 0.98$ \\
empirical formula & $\mathrm{C}_{28} \mathrm{H}_{44} \mathrm{Li}_{2} \mathrm{~N}_{2} \mathrm{O}_{4}$ & $\theta$ range $\left.{ }^{\circ}\right]$ & $2.36-29.12$ \\
molar mass $\left[\mathrm{g} \mathrm{mol}{ }^{-1}\right]$ & 486.53 & completeness to $\theta_{\max }$ & 1.00 \\
crystal size $[\mathrm{mm}]$ & $0.18 \times 0.16 \times 0.10$ & redundancy to $\theta_{\max }$ & 5.72 \\
temperature $[\mathrm{K}]$ & $100(2)$ & reflections collected & 25512 \\
crystal system & orthorhombic & independent reflections & 3802 \\
space group & $P b c a$ & $R_{\text {int }} / R_{\sigma}$ & $0.0227 / 0.0154$ \\
$a[\AA]$ & $13.934(2)$ & restraints/parameters & $10 / 188$ \\
$b[\AA]$ & $11.831(1)$ & GoF & 1.055 \\
$c[\AA]$ & $17.229(2)$ & $R 1$ (all data) & 0.0468 \\
$V\left[\AA^{3}\right]$ & $2840.2(5)$ & $R 1(I>2 \sigma(I))$ & 0.0406 \\
$Z$ & 4 & $w R 2($ all data) & 0.1182 \\
$\rho_{\text {calc }}\left[\mathrm{g} \mathrm{cm}^{-3}\right]$ & 1.138 & $w R 2(I>2 \sigma(I))$ & 0.1143 \\
$\mu\left[\mathrm{mm}^{-1}\right]$ & 0.074 & diff. peak/hole $\left[\mathrm{e} \AA^{-3}\right]$ & $0.361 /-0.201$ \\
$F(000)$ & 1056 & & \\
\hline
\end{tabular}


The 4-picolyllithium thf adduct crystallises in the orthorhombic centrosymmetric space group Pbca with half of the dimer in the asymmetric unit (Figure 8-11). The dimer is generated by an inversion operation and a translation about $(1,1,2)$. One thf molecule shows a known crossed disorder pattern of the 3/4-carbon atoms with an occupation factor of 0.52 .

\subsubsection{4-Picolyllithium 2-n-Butyl-4-methyl- 1,2-dihydropyridyllithium thf dimer (11)}

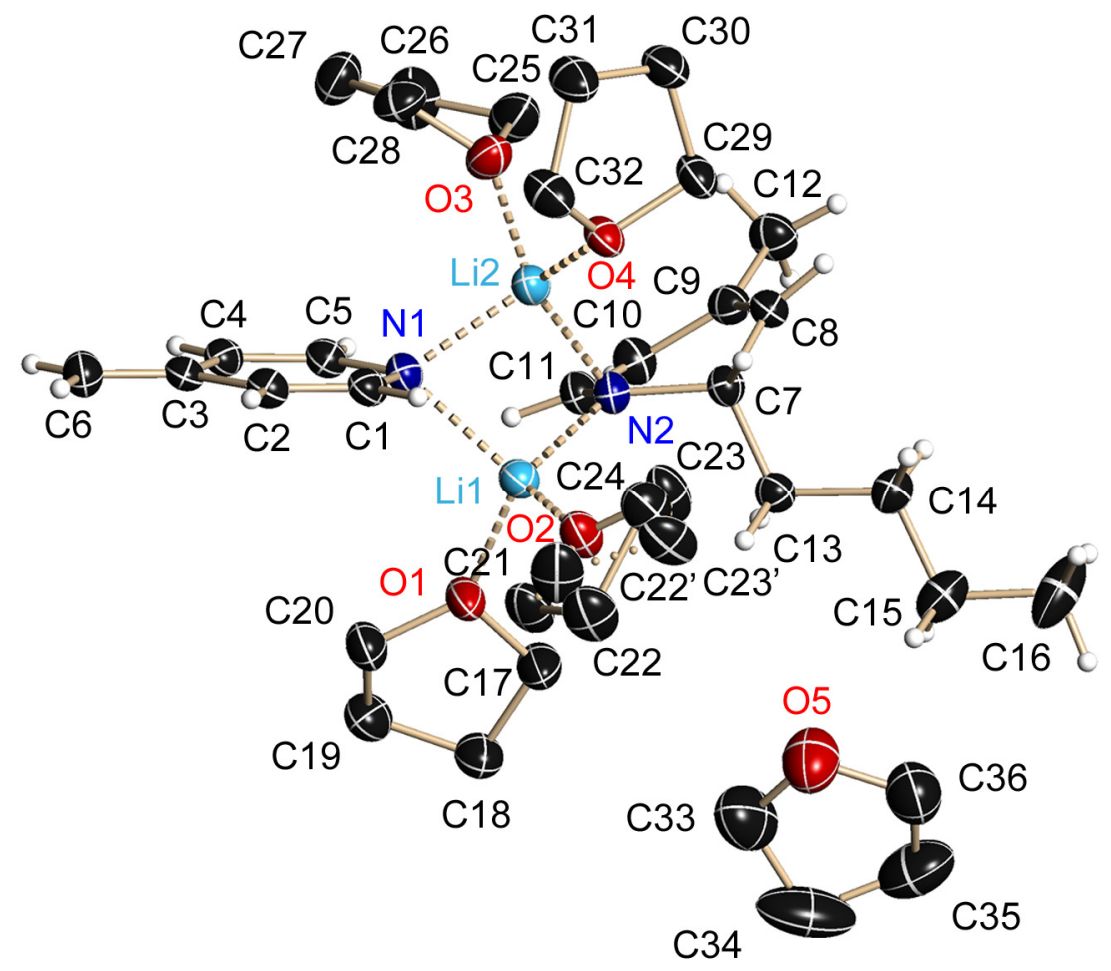

Figure 8-12. Asymmetric unit of [[PicLi·(thf $\left.)_{2} \cdot 2-n \mathrm{Bu}-4-\mathrm{Me}-\left(\mathrm{H}_{4} \mathrm{C}_{5} \mathrm{~N}\right) \mathrm{Li} \cdot(\text { thf })_{2}\right](\mathbf{1 1})$. Hydrogen atoms of the donor molecules are omitted for clarity.

The mixed 4-picolyllithium 2-n-butyl-4-methyl-1,2-dihydropyridyllithium adduct crystallises in the orthorhombic centrosymmetric space group Pbca with the full unsymmetrical dimer in the asymmetric unit (Figure 8-12). Additionally, one thf molecule is incorporated as lattice solvent without direct contact to the cations. Besides, one donating thf molecule (O2) displayed a disorder of the 3/4-carbon atoms that could be refined without any constraints or restraints. The occupation ratio was $62: 38$. 
Table 8-9. Crystallographic data for [PicLi·(thf $\left.)_{2} \cdot 2-n \mathrm{Bu}-4-\mathrm{Me}-\left(\mathrm{H}_{4} \mathrm{C}_{5} \mathrm{~N}\right) \mathrm{Li} \cdot(\text { thf })_{2}\right](\mathbf{1 1})$.

\begin{tabular}{lc|lc}
\hline identification code & HOTT0014/I $\mathbf{S S}$ & min./max. transmission & $0.88 / 0.98$ \\
empirical formula & $\mathrm{C}_{36} \mathrm{H}_{62} \mathrm{Li}_{2} \mathrm{~N}_{2} \mathrm{O}_{5}$ & $\theta$ range $\left.{ }^{\circ}\right]$ & $2.34-25.12$ \\
molar mass $\left[\mathrm{g} \mathrm{mol}^{-1}\right]$ & 616.76 & completeness to $\theta_{\text {max }}$ & 1.00 \\
crystal size $[\mathrm{mm}]$ & $0.30 \times 0.28 \times 0.25$ & redundancy to $\theta_{\max }$ & 25.13 \\
temperature $[\mathrm{K}]$ & $100(2)$ & reflections collected & 186166 \\
crystal system & orthorhombic & independent reflections & 6626 \\
space group & $P b c a$ & $R_{\text {int }} / R_{\sigma}$ & $0.0387 / 0.0115$ \\
$a[\AA]$ & $10.6991(11)$ & restraints/parameters & $0 / 433$ \\
$b[\AA]$ & $15.9433(15)$ & GoF & 1.112 \\
$c[\AA]$ & $43.702(4)$ & $R 1$ (all data) & 0.0626 \\
$V\left[\AA^{3}\right]$ & $7454.7(13)$ & $R 1(I>2 \sigma(I))$ & 0.0593 \\
$Z$ & 8 & $w R 2($ all data) & 0.1399 \\
$\rho_{\text {calc }}\left[\mathrm{g} \mathrm{cm}^{-3}\right]$ & 1.099 & $w R 2(I>2 \sigma(I))$ & 0.1377 \\
$\mu\left[\mathrm{mm}^{-1}\right]$ & 0.071 & diff. peak/hole $\left[\mathrm{e} \AA^{-3}\right]$ & $0.287 /-0.246$ \\
$F(000)$ & 2704 & & \\
\hline
\end{tabular}




\subsubsection{4-Picolyllithium·Pmdeta (12)}

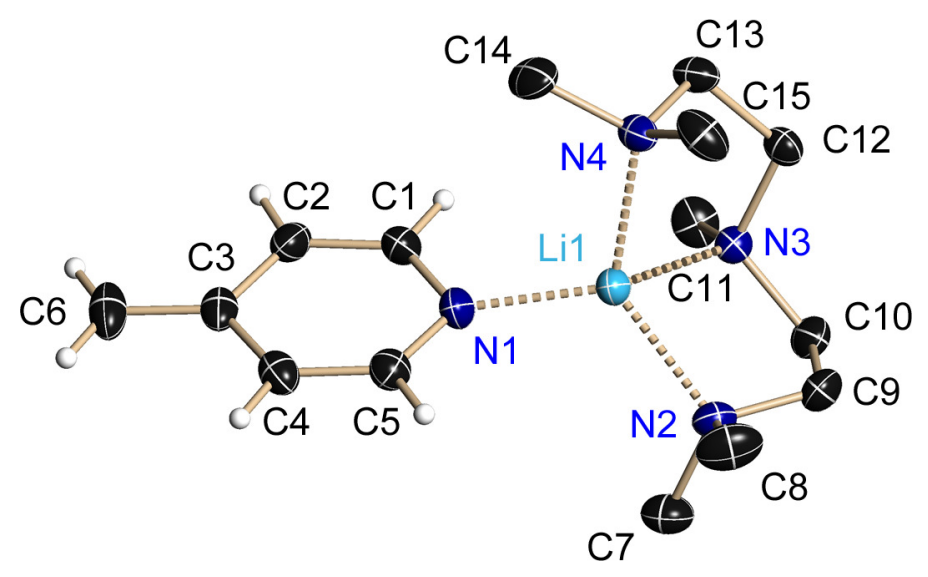

Figure 8-13. Asymmetric unit of [4-PicLi·pmdeta] (12). Hydrogen atoms of the donor molecule are omitted for clarity.

4-Picolyllithium as pmdeta adduct crystallises in the monoclinic space group $P 2_{1} / C$ with one monomer in the asymmetric unit (Figure 8-13).

Table 8-10. Crystallographic data for [4-PicLi·pmdeta] (12).

\begin{tabular}{lc|lc}
\hline identification code & HOTT0016/TxS & $F(000)$ & 600 \\
empirical formula & $\mathrm{C}_{15} \mathrm{H}_{29} \mathrm{LiN}_{4}$ & min./max. transmission & $0.90 / 0.99$ \\
molar mass $\left[\mathrm{g} \mathrm{mol}^{-1}\right]$ & 272.36 & $\theta$ range $\left.{ }^{\circ}\right]$ & $1.59-26.75$ \\
crystal size $[\mathrm{mm}]$ & $0.40 \times 0.23 \times 0.10$ & completeness to $\theta_{\text {max }}$ & 1.00 \\
temperature $[\mathrm{K}]$ & $100(2)$ & redundancy to $\theta_{\max }$ & 6.76 \\
crystal system & monoclinic & reflections collected & 26718 \\
space group & $P 2_{1} / \mathrm{C}$ & independent reflections & 3689 \\
$a[\AA]$ & $12.864(3)$ & $R_{\text {int }} / R_{\sigma}$ & $0.0259 / 0.0148$ \\
$b[\AA]$ & $8.6637(17)$ & restraints/parameters & $0 / 192$ \\
$c[\AA]$ & $15.692(3)$ & GoF & 1.071 \\
$\beta\left[{ }^{\circ}\right]$ & $96.029(5)$ & $R 1($ all data $)$ & 0.0478 \\
$V\left[\AA^{3}\right]$ & $1739.2(6)$ & $R 1(I>2 \sigma(I))$ & 0.0428 \\
$\mathrm{Z}$ & 4 & $w R 2($ all data) & 0.1223 \\
$\rho_{\text {calc }}\left[\mathrm{g} \mathrm{cm}^{-3}\right]$ & 1.040 & $w R 2(I>2 \sigma(I))$ & 0.1189 \\
$\mu\left[\mathrm{mm}^{-1}\right]$ & 0.062 & diff. peak/hole $\left[\mathrm{e} \AA^{-3}\right]$ & $0.411 /-0.201$ \\
\hline
\end{tabular}




\subsubsection{4-Picolyllithium·Tmeda Polymer (13)}

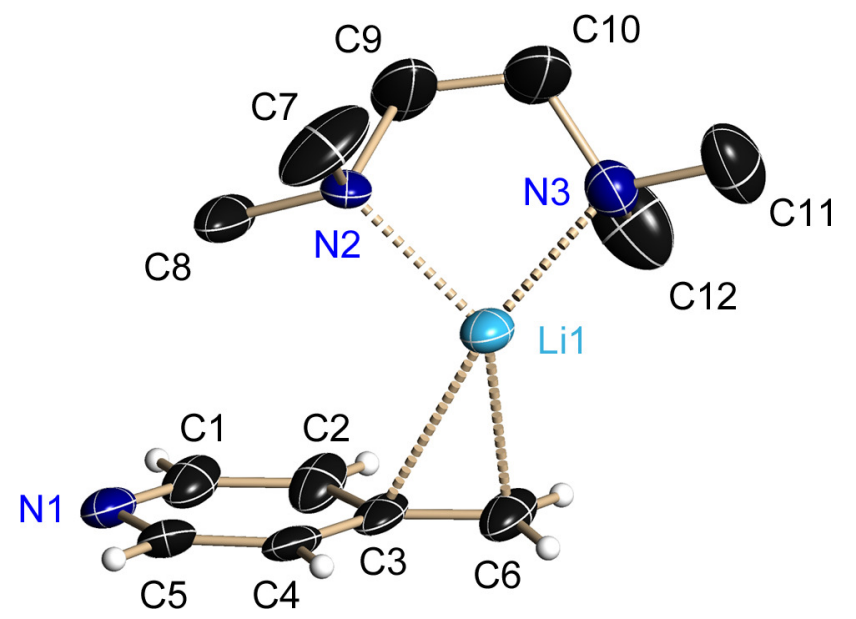

Figure 8-14. Asymmetric unit of [4-PicLi-tmeda $]_{n}(13, \mathrm{H}$-atoms of tmeda excluded).

4-Picolyllithium as tmeda adduct crystallises in the monoclinic space group $P Z_{1} / C$ with one unit of the chain structure in the asymmetric unit (Figure 8-14). The chains are generated by the $c$ glide plane and a translation about $(0,2,0)$ for Li1' and $(0,2,1)$ for $\mathrm{N} 1^{\prime}$.The ADPs of 13 were stabilised with SIMU and DELU instructions, while an ISOR command was additionally applied for C7.

Table 8-11. Crystallographic data for [4-PicLi-tmeda $]_{\mathrm{n}}(\mathbf{1 3})$.

\begin{tabular}{lc|lc}
\hline identification code & HOTT0019/st & $F(000)$ & 472 \\
empirical formula & $\mathrm{C}_{12} \mathrm{H}_{22} \mathrm{LiN}_{3}$ & min./max. transmission & $0.66 / 0.75$ \\
molar mass $\left[\mathrm{g} \mathrm{mol}{ }^{-1}\right]$ & 215.27 & $\theta$ range $\left[^{\circ}\right]$ & $2.37-23.89$ \\
crystal size $[\mathrm{mm}]$ & $0.20 \times 0.10 \times 0.05$ & completeness to $\theta_{\max }$ & 0.88 \\
temperature $[\mathrm{K}]$ & $100(2)$ & redundancy to $\theta_{\max }$ & 5.24 \\
crystal system & monoclinic & reflections collected & 11532 \\
space group & $P 2_{1} / \mathrm{C}$ & independent reflections & 1832 \\
$a[\AA]$ & $8.3668(14)$ & $R_{\text {int }} / R_{\sigma}$ & $0.0547 / 0.0426$ \\
$b[\AA]$ & $15.662(3)$ & restraints/parameters & $140 / 157$ \\
$c[\AA]$ & $11.895(2)$ & GoF & 1.254 \\
$\beta\left[{ }^{\circ}\right]$ & $120.122(2)$ & $R 1($ all data $)$ & 0.1316 \\
$V\left[\AA^{3}\right]$ & $1348.3(4)$ & $R 1(I>2 \sigma(I))$ & 0.1178 \\
$\mathrm{Z}$ & 4 & $w R 2($ all data $)$ & 0.2346 \\
$\rho_{\text {calc }}\left[\mathrm{g} \mathrm{cm}^{-3}\right]$ & 1.060 & $w R 2(I>2 \sigma(I))$ & 0.2282 \\
$\mu\left[\mathrm{mm}^{-1}\right]$ & 0.063 & diff. peak/hole $\left[\mathrm{e} \AA^{-3}\right]$ & $0.447 /-0.285$ \\
\hline
\end{tabular}




\subsubsection{Monoclinic Trimethylsilylmethyllithium Modifi- cation (14)}

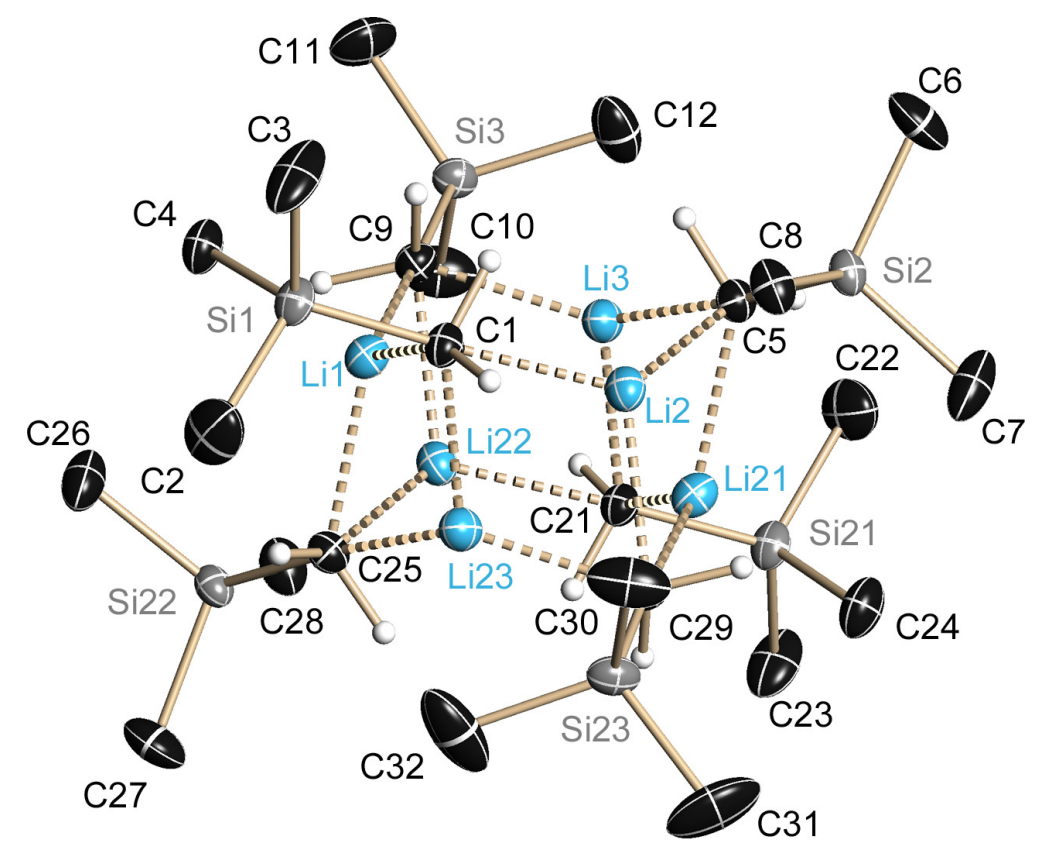

Figure 8-15. Asymmetric unit of $\left[\mathrm{TMSCH}_{2} \mathrm{Li}\right]_{6}$ (14; non-methylene hydrogen atoms excluded)

[TMSCH $\mathrm{TLi}_{6}$ (14) crystallises in the monoclinic space group $P 2_{1} / n$ with the full hexamer in the asymmetric unit (Figure 8-15).

Table 8-12. Crystallographic data for $\left[\mathrm{TMSCH}_{2} \mathrm{Li}\right]_{6}$ (14).

\begin{tabular}{lc|lc}
\hline identification code & Tmsmeli_ED01/TXs & $F(000)$ & 1248 \\
empirical formula & $\mathrm{C}_{24} \mathrm{H}_{66} \mathrm{Li}_{6} \mathrm{Si}_{6}$ & $\mu\left[\mathrm{mm}^{-1}\right]$ & 0.213 \\
molar mass $\left[\mathrm{g} \mathrm{mol}^{-1}\right]$ & 564.95 & min./max. transmission & $0.89 / 0.96$ \\
crystal size $[\mathrm{mm}]$ & $0.33 \times 0.20 \times 0.20$ & $\theta$ range $\left[^{\circ}\right]$ & $1.48-50.05$ \\
temperature $[\mathrm{K}]$ & $100(2)$ & completeness to $\theta_{\max }$ & 0.89 \\
crystal system & monoclinic & redundancy to $\theta_{\text {max }}$ & 1.51 \\
space group & $P 2_{1} / n$ & reflections collected & 66407 \\
$a[\AA]$ & $10.7863(6)$ & independent reflections & 33338 \\
$b[\AA]$ & $18.1049(9)$ & $R_{\text {int }} / R_{\sigma}$ & $0.0198 / 0.0292$ \\
$c[\AA]$ & $21.2034(11)$ & restraints/parameters & $0 / 737$ \\
$\beta\left[{ }^{\circ}\right]$ & $96.9560(10)$ & GoF & 1.873 \\
$V\left[\AA^{3}\right]$ & $4110.2(4)$ & $R 1(I>2 \sigma(I))$ & 0.0307 \\
$\mathrm{Z}$ & 4 & $w R 2(I>2 \sigma(I))$ & 0.0548 \\
$\rho_{\text {calc }}\left[\mathrm{g} \mathrm{cm}^{-3}\right]$ & 0.913 & diff. peak/hole $\left[\mathrm{e} \AA^{-3}\right]$ & $0.365 /-0.344$ \\
\hline
\end{tabular}




\subsubsection{Standard Structure of the Triclinic Trimethyl- silylmethyllithium Modification (15)}

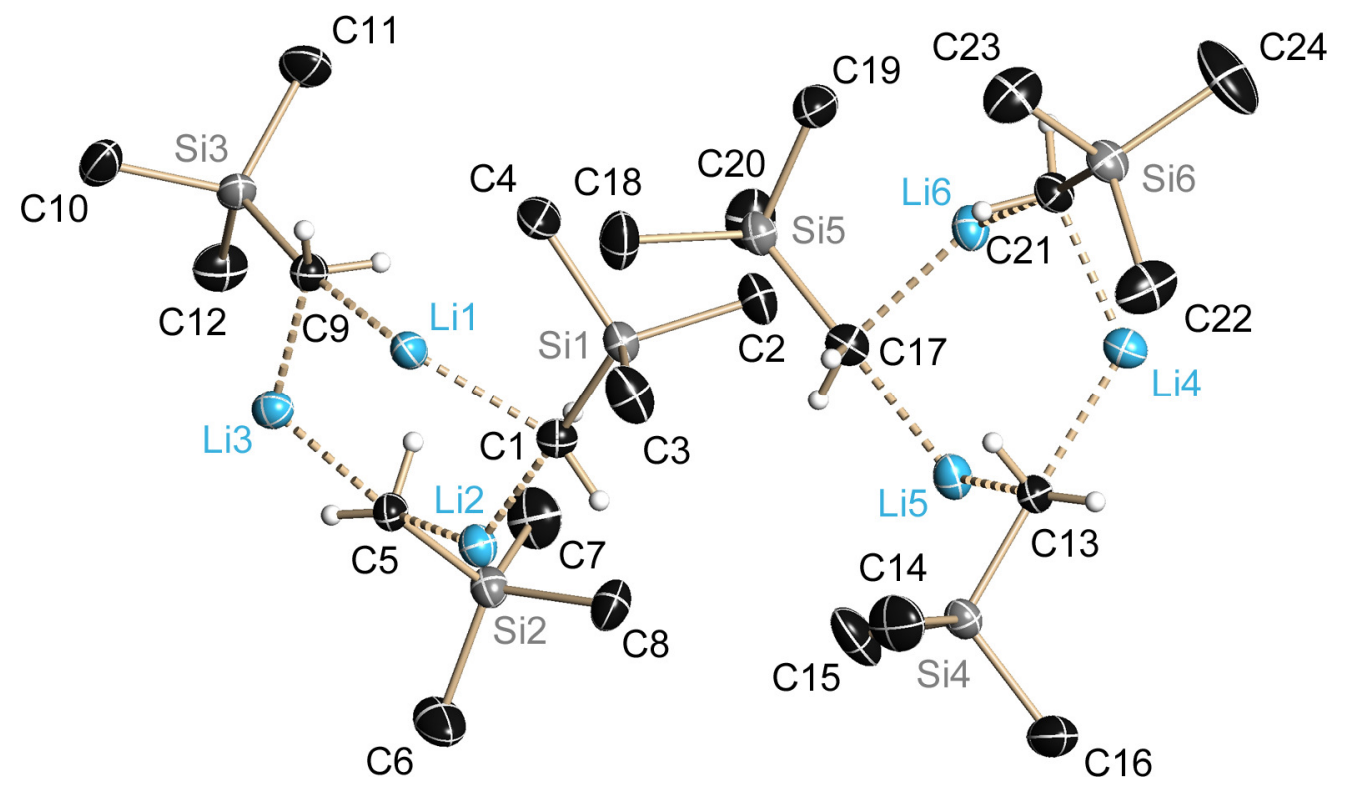

Figure 8-16. Asymmetric unit of $\left[\mathrm{TMSCH}_{2} \mathrm{Li}\right]_{6}(\mathbf{1 5}$; non-methylene hydrogen atoms omitted for clarity)

Table 8-13. Crystallographic data for $\left[\mathrm{TMSCH}_{2} \mathrm{Li}\right]_{6}(\mathbf{1 5})$.

\begin{tabular}{lc|lc}
\hline identification code & HOTTS005/st & $F(000)$ & 624 \\
empirical formula & $\mathrm{C}_{24} \mathrm{H}_{66} \mathrm{Li}_{6} \mathrm{Si}_{6}$ & $\mu\left[\mathrm{mm}^{-1}\right]$ & 0.29 \\
molar mass $\left[\mathrm{g} \mathrm{mol}^{-1}\right]$ & 564.95 & min./max. transmission & $0.88 / 0.92$ \\
crystal size $[\mathrm{mm}]$ & $0.60 \times 0.50 \times 0.40$ & $\theta$ range $\left.{ }^{\circ}\right]$ & $1.96-26.42$ \\
temperature $[\mathrm{K}]$ & $143(2)$ & completeness to $\theta_{\max }$ & 1.00 \\
crystal system & triclinic & redundancy to $\theta_{\text {max }}$ & 5.11 \\
space group & $P \overline{1}$ & reflections collected & 43008 \\
$a[\AA]$ & $12.2704(8)$ & independent reflections & 8395 \\
$b\left[^{\AA}\right]$ & $12.5022(8)$ & $R_{\text {int }} / R_{\sigma}$ & $0.0217 / 0.0139$ \\
$c\left[^{\AA}\right]$ & $14.3860(10)$ & restraints/parameters & $0 / 457$ \\
$\alpha\left[^{\circ}\right]$ & $74.2330(10)$ & GoF & 1.058 \\
$\beta\left[^{\circ}\right]$ & $74.6750(10)$ & $R 1($ all data $)$ & 0.0323 \\
$\gamma\left[^{\circ}\right]$ & $85.9160(10)$ & $R 1(I>2 \sigma(I))$ & 0.0292 \\
$V\left[^{3}\right]$ & $2048.4(2)$ & $w R 2($ all data) & 0.0832 \\
$\mathrm{Z}$ & 2 & $w R 2(I>2 \sigma(I))$ & 0.0808 \\
$\rho_{\text {calc }}\left[\mathrm{g} \mathrm{cm}^{-3}\right]$ & 0.913 & diff. peak/hole $\left[\mathrm{e} \AA^{-3}\right]$ & $0.425 /-0.200$ \\
\hline
\end{tabular}


$\left[\mathrm{TMSCH}_{2} \mathrm{Li}\right]_{6}(\mathbf{1 5})$ crystallises in the triclinic space group $P \overline{1}$. Two independent halves of the hexamer are present in the asymmetric unit (Figure 8-16). The full dimers are generated by an inversion at the origin and a translation operation about $(2,2,0)$ for the first and $(1,1,1)$ for the second moiety.

\subsubsection{High Resolution Data Set of the Triclinic TrimethylsilyImethyllithium Modification (15)}

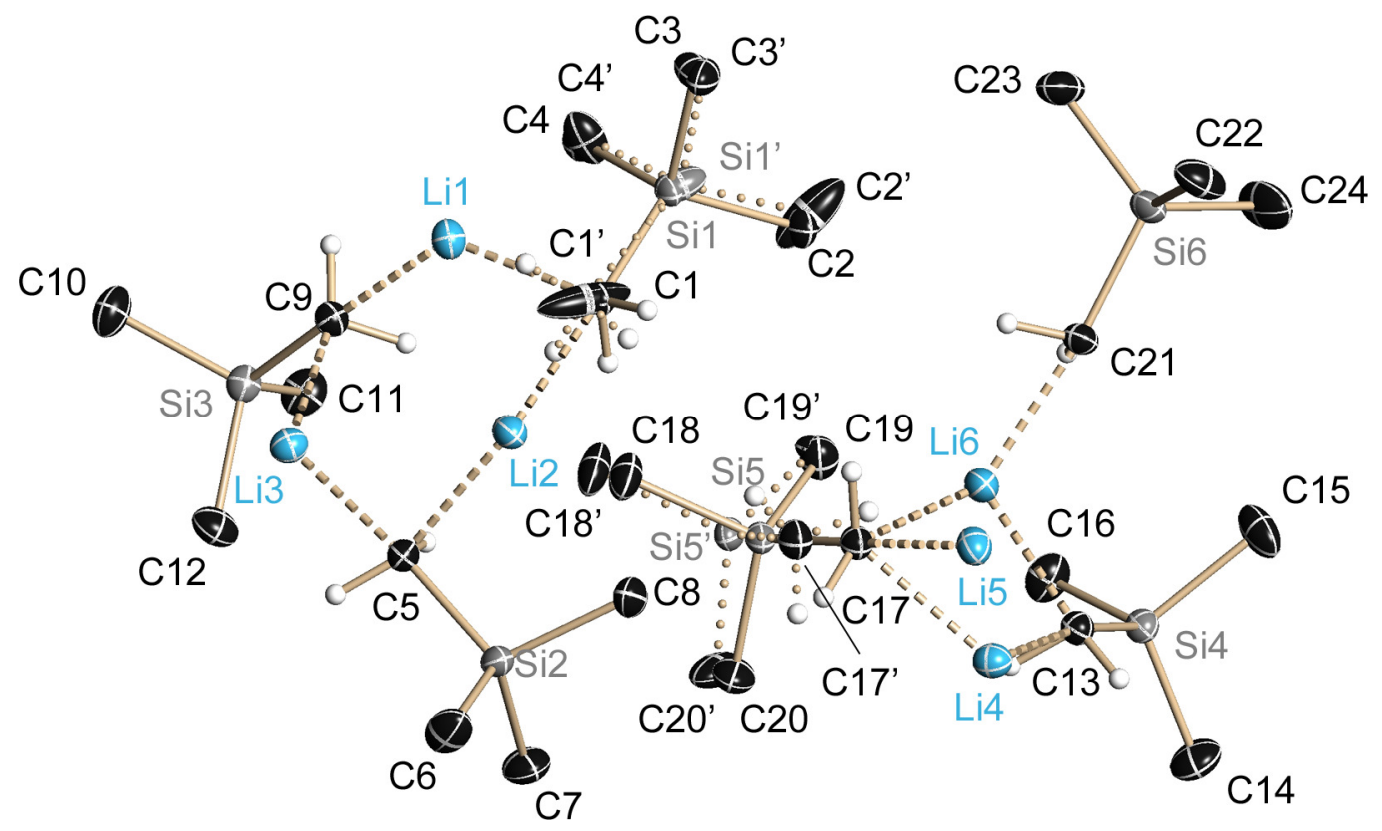

Figure 8-17. Asymmetric unit of the high resolution data set of $\left[\mathrm{TMSCH}_{2} \mathrm{Li}\right]_{6}(\mathbf{1 5}$; nonmethylene hydrogen atoms omitted for clarity).

Table 8-14. Crystallographic data for the high resolution data set of $\left[\mathrm{TMSCH}_{2} \mathrm{Li}\right]_{6}(\mathbf{1 5})$.

\begin{tabular}{lc|lc}
\hline identification code & Chemet_ED03/TXs & independent reflections & 45695 \\
crystal size $[\mathrm{mm}]$ & $0.40 \times 0.40 \times 0.30$ & restraints/parameters & $28 / 443$ \\
temperature $[\mathrm{K}]$ & $100(2)$ & GoF & 1.019 \\
$\theta$ range $\left[^{\circ}\right]$ & $1.52-52.06$ & $R 1$ (all data) & 0.0435 \\
completeness to $\theta_{\max }$ & 0.98 & $R 1(I>2 \sigma(I))$ & 0.0319 \\
redundancy to $\theta_{\max }$ & 6.93 & $w R 2($ all data) & 0.1061 \\
reflections collected & 323003 & $w R 2(I>2 \sigma(I))$ & 0.0951 \\
$R_{\text {int }} / R_{\sigma}$ & $0.0205 / 0.0093$ & diff. peak/hole $\left[\mathrm{e} \AA^{-3}\right]$ & $1.722 /-0.291$ \\
\hline
\end{tabular}


The site occupation factors of the disordered parts of the molecule (Figure 8-17) were refined to 0.12 (residue of Li1) and 0.05 (residue of Li4). The refinement was stabilised by applying only DFIX restraints for the $\mathrm{C}-\mathrm{H}$ bonds as well as $\mathrm{H} \cdots \mathrm{H}$ at the methyl group pointing towards the lithium triangles. However, there were still two high residual density peaks near the disordered methylene/methyl carbon atoms present. These could not be accurately included into the refinement model.

\subsubsection{5 (R)-[ $\{(S)-2-($ methoxymethyl)pyrrolidino- methyl\}dimethylsilyl]benzyllithium-Quinuclidine (17)}

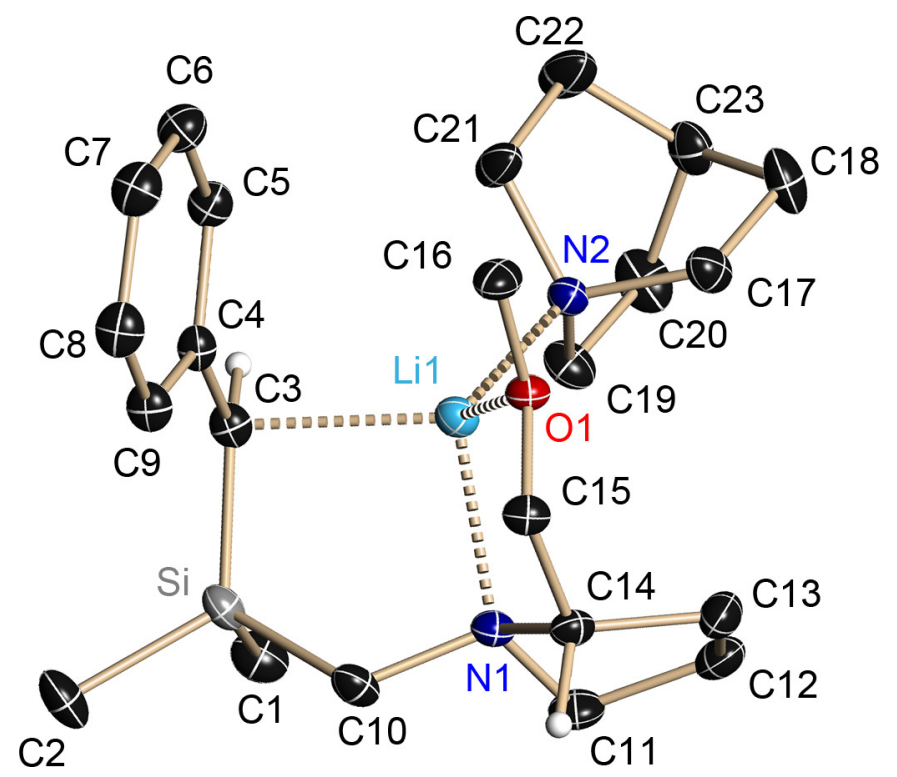

Figure 8-18. Asymmetric unit of (17; only hydrogen atoms at stereogenic centres included)

$(R)-[\{(S)-2-($ methoxymethyl)pyrrolidinomethyl\}dimethylsilyl]benzyllithium quinuclidine crystallises in the orthorhombic chiral space group $P 2_{1} 2_{1} 2_{1}$ with the whole molecule in the asymmetric unit (Figure 8-18). The absolute structure has been unambiguously determined which is indicated by the Flack $\mathrm{x}$ parameter of $0.01(3) \cdot{ }^{[321,322]}$ All hydrogen atoms were refined applying a riding model and distance restraints. For further details see chapter 3.2.2. 
Table 8-15. Crystallographic data for 17.

\begin{tabular}{lc|lc}
\hline identification code & Stroh_ED01b/TXs & $\mu\left[\mathrm{mm}^{-1}\right]$ & 0.116 \\
empirical formula & $\mathrm{C}_{23} \mathrm{H}_{39} \mathrm{LiN}_{2} \mathrm{OSi}$ & $F(000)$ & 864 \\
molar mass $\left[\mathrm{g} \mathrm{mol}^{-1}\right]$ & 394.59 & min./max. transmission & $0.96 / 0.98$ \\
crystal size $[\mathrm{mm}]$ & $0.30 \times 0.30 \times 0.25$ & $\theta$ range $\left.{ }^{\circ}\right]$ & $1.87-50.09$ \\
temperature $[\mathrm{K}]$ & $100(2)$ & completeness to $\theta_{\max }$ & 1.00 \\
crystal system & orthorhombic & redundancy to $\theta_{\max }$ & 10.8 \\
space group & $P 2_{1} 2_{1} 2_{1}$ & reflections collected & 264882 \\
Flack x & $-0.2(10)$ & independent reflections & 24554 \\
$a[\AA]$ & $9.5424(7)$ & $R_{\text {int }} / R_{\sigma}$ & $0.0228 / 0.0103$ \\
$b[\AA]$ & $11.2137(8)$ & restraints/parameters & $0 / 616$ \\
$c[\AA]$ & $21.7553(16)$ & GoF & 2.595 \\
$V\left[\AA^{3}\right]$ & $2327.94(29)$ & $R 1(I>3 \sigma(I))$ & 0.0203 \\
$Z$ & 4 & $w R 2(I>3 \sigma(I))$ & 0.0312 \\
$\rho_{\text {calc }}\left[\mathrm{g} \mathrm{cm}^{-3}\right]$ & 1.126 & diff. peak/hole $\left[\mathrm{e} \AA^{-3}\right]$ & $0.211 /-0.134$ \\
\hline
\end{tabular}




\subsection{Cooperation Projects}

Several service structures were determined in cooperation with other research groups. These were the groups of Prof. Dr. H. W. Roesky and Prof. Dr. U. Klingebiel. The synthetic chemists were Dr. S. Nembenna, Dr. A. Fischer, Dr. M. Görth, and Dr. C. Matthes.

\subsection{1 $N, N^{\prime}$-Bis(1,3-disila-2-lithiumamido)ethylene- silylamine-2 Thf}

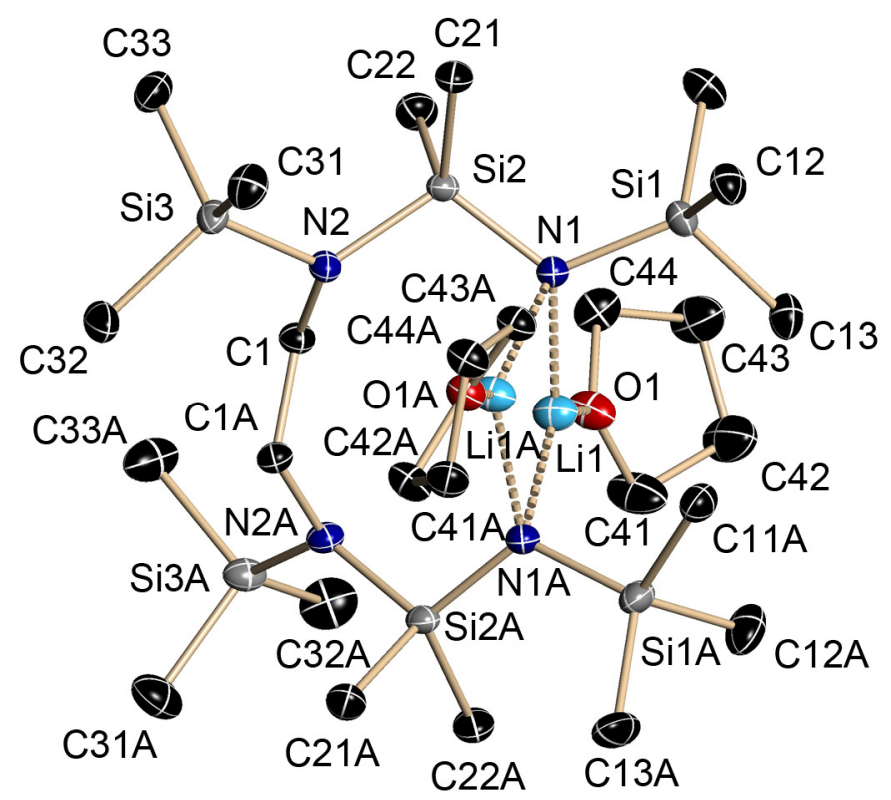

Figure 8-19. Asymmetric unit of $\left[\mathrm{H}_{2} \mathrm{CN}\left(\mathrm{SiMe}_{3}\right) \mathrm{Si}\left(\mathrm{Me}_{2}\right) \mathrm{N}\left(\mathrm{SiMe}_{3}\right) \mathrm{Li} \cdot \text { thf }\right]_{2}$. Hydrogen atoms are omitted for clarity.

$\left[\mathrm{H}_{2} \mathrm{CN}\left(\mathrm{SiMe}_{3}\right) \mathrm{Si}\left(\mathrm{Me}_{2}\right) \mathrm{N}\left(\mathrm{SiMe}_{3}\right) \mathrm{Li} \cdot \mathrm{thf}\right]_{2}$ crystallises in the triclinic centrosymmetric space group $P \overline{1}$ with the whole aggregate in the asymmetric unit. 
Table 8-16. Crystallographic data for $\left[\mathrm{H}_{2} \mathrm{CN}\left(\mathrm{SiMe}_{3}\right) \mathrm{Si}\left(\mathrm{Me}_{2}\right) \mathrm{N}\left(\mathrm{SiMe}_{3}\right) \mathrm{Li} \cdot \text { thf }\right]_{2}$.

\begin{tabular}{lc|lc}
\hline identification code & HOTTS006/st & $\mu\left[\mathrm{mm}^{-1}\right]$ & 0.237 \\
empirical formula & $\mathrm{C}_{26} \mathrm{H}_{68} \mathrm{Li}_{2} \mathrm{~N}_{4} \mathrm{O}_{2} \mathrm{Si}_{6}$ & $F(000)$ & 716 \\
molar mass $\left[\mathrm{g} \mathrm{mol}^{-1}\right]$ & 651.26 & min./max. transmission & $0.91 / 0.99$ \\
crystal size $[\mathrm{mm}]$ & $0.20 \times 0.15 \times 0.10$ & $\theta$ range $\left.{ }^{\circ}\right]$ & $2.37-26.37$ \\
temperature $[\mathrm{K}]$ & $100(2)$ & completeness to $\theta_{\text {max }}$ & 0.99 \\
crystal system & triclinic & redundancy to $\theta_{\text {max }}$ & 4.16 \\
space group & $P \overline{1}$ & reflections collected & 95381 \\
$a[\AA]$ & $11.1379(8)$ & independent reflections & 8046 \\
$b\left[^{\AA}\right]$ & $12.1262(8)$ & $R$ int $/ R_{\sigma}$ & $0.0225 / 0.0169$ \\
$c\left[^{\AA}\right]$ & $16.7053(14)$ & restraints / parameters & $0 / 377$ \\
$\alpha\left[^{\circ}\right]$ & $95.5870(10)$ & GoF & 1.040 \\
$\beta\left[^{\circ}\right]$ & $107.4550(10)$ & $R 1$ (all data) & 0.0322 \\
$\gamma\left[^{\circ}\right]$ & $109.1610(10)$ & $R 1(I>2 \sigma(I))$ & 0.0293 \\
$V\left[^{3}\right]$ & $1984.5(3)$ & $w R 2$ (all data) & 0.0817 \\
$\mathrm{Z}$ & 2 & $w R 2(I>2 \sigma(I))$ & 0.0787 \\
$\rho_{\text {calc }}\left[\mathrm{g} \mathrm{cm}{ }^{-3}\right]$ & 1.090 & diff. peak/hole $\left[\mathrm{e} \AA^{-3}\right]$ & $0.462 /-0.237$ \\
\hline
\end{tabular}

\subsubsection{N,N'-Bis(1,3-disila-2-lithiumamido)ethylene- silylamine. 2 tmeda}

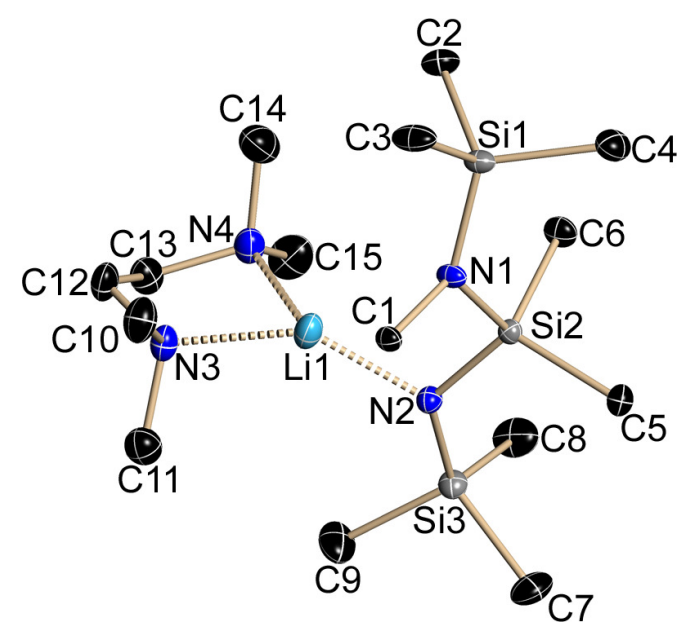

Figure 8-20. Asymmetric unit of $\left[\mathrm{Me}_{3} \mathrm{SiN}(\mathrm{Li} \cdot \mathrm{tmeda}) \mathrm{SiMe}_{2} \mathrm{~N}\left(\mathrm{SiMe}_{3}\right) \mathrm{CH}_{2}\right]_{2}$. Hydrogen atoms are omitted for clarity. 
The dilithiated amide $\left[\mathrm{Me}_{3} \mathrm{SiN}\left(\mathrm{Li} \text {-tmeda) } \mathrm{SiMe}_{2} \mathrm{~N}\left(\mathrm{SiMe}_{3}\right) \mathrm{CH}_{2}\right]_{2}\right.$ crystallises with one tmeda donor molecule per lithium atom and shows half of the aggregate in the asymmetric unit. The dimer in generated by an inversion at the origin and a translation about $(0,0,1)$.

Table 8-17. Crystallographic data for $\left[\mathrm{Me}_{3} \mathrm{SiN}(\mathrm{Li} \cdot \mathrm{tmeda}) \mathrm{SiMe}_{2} \mathrm{~N}\left(\mathrm{SiMe}_{3}\right) \mathrm{CH}_{2}\right]_{2}$.

\begin{tabular}{lc|lc}
\hline identification code & HOTTS009/st & $F(000)$ & 820 \\
empirical formula & $\mathrm{C}_{30} \mathrm{H}_{84} \mathrm{Li}_{2} \mathrm{~N}_{8} \mathrm{Si}_{6}$ & min./max. transmission & $0.93 / 0.99$ \\
molar mass $\left[\mathrm{g} \mathrm{mol}{ }^{-1}\right]$ & 739.47 & $\theta$ range $\left.{ }^{\circ}\right]$ & $2.21-28.28$ \\
crystal size $[\mathrm{mm}]$ & $0.40 \times 0.30 \times 0.20$ & completeness to $\theta_{\max }$ & 0.99 \\
temperature $[\mathrm{K}]$ & $100(2)$ & redundancy to $\theta_{\max }$ & 9.69 \\
crystal system & monoclinic & reflections collected & 62481 \\
space group & $P 2_{1} / n$ & independent reflections & 5789 \\
$a[\AA]$ & $10.2284(2)$ & $R_{\text {int }} / R_{\sigma}$ & $0.0336 / 0.0159$ \\
$b[\AA]$ & $22.8681(4)$ & restraints/parameters & $0 / 220$ \\
$c[\AA]$ & $10.5095(2)$ & GoF & 1.066 \\
$\beta\left[{ }^{\circ}\right]$ & $106.6680(10)$ & $R 1($ all data $)$ & 0.0318 \\
$V\left[\AA^{3}\right]$ & $2354.93(8)$ & $R 1(I>2 \sigma(I))$ & 0.0280 \\
$\mathrm{Z}$ & 2 & $w R 2($ all data $)$ & 0.0774 \\
$\rho_{\text {calc }}\left[\mathrm{g} \mathrm{cm}^{-3}\right]$ & 1.043 & $w R 2(I>2 \sigma(I))$ & 0.0750 \\
$\mu\left[\mathrm{mm}^{-1}\right]$ & 0.205 & diff. peak/hole $\left[\mathrm{e} \AA^{-3}\right]$ & $0.427 /-0.192$ \\
\hline
\end{tabular}

\subsubsection{Di(iso-propyl)amino-( $N$-2,6-tri(iso-propyl)- anilino)-fluoroborane}

The asymmetric unit of the diaminofluoroborane $\left(i \mathrm{Pr}_{2} \mathrm{Ph}\right) \mathrm{N}(i \mathrm{Pr}) \mathrm{B}(\mathrm{F}) \mathrm{N}(i \mathrm{Pr})_{2}$ contains four independent formula units and crystallises in the centrosymmetric monoclinic space group $C 2 / c$. Higher crystal symmetry could not be observed, so that 32 molecules are present in the unit cell. 


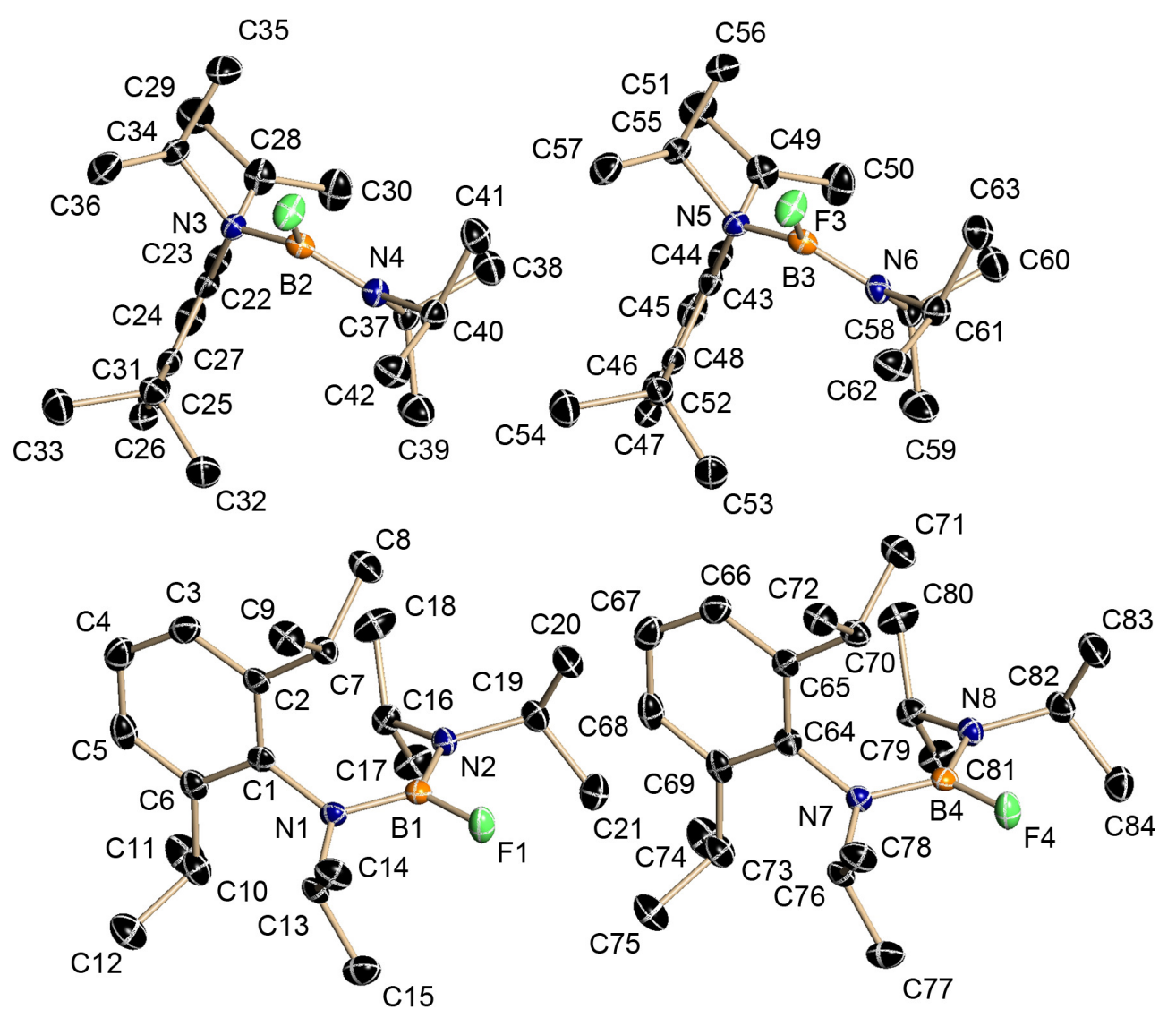

Figure 8-21. Asymmetric unit of $\left(i \mathrm{Pr}_{2} \mathrm{Ph}\right) \mathrm{N}(i \mathrm{Pr}) \mathrm{B}(\mathrm{F}) \mathrm{N}(i \mathrm{Pr})_{2}$. Hydrogen atoms are omitted for clarity.

Table 8-18. Crystallographic data for $\left(i \mathrm{Pr}_{2} \mathrm{Ph}\right) \mathrm{N}(i \mathrm{Pr}) \mathrm{B}(\mathrm{F}) \mathrm{N}(i \mathrm{Pr})_{2}$.

\begin{tabular}{lc|lc}
\hline identification code & HOTTS007/st & $F(000)$ & 6144 \\
empirical formula & $\mathrm{C}_{21} \mathrm{H}_{38} \mathrm{BFN}_{2}$ & min./max. transmission & $0.86 / 0.99$ \\
molar mass $\left[\mathrm{g} \mathrm{mol}^{-1}\right]$ & 348.34 & $\theta$ range $\left.{ }^{\circ}\right]$ & $2.21-25.36$ \\
crystal size $[\mathrm{mm}]$ & $0.20 \times 0.15 \times 0.10$ & completeness to $\theta_{\max }$ & 1.00 \\
temperature $[\mathrm{K}]$ & $100(2)$ & redundancy to $\theta_{\max }$ & 3.89 \\
crystal system & monoclinic & reflections collected & 64214 \\
space group & $C 2 / c$ & independent reflections & 15952 \\
$a[\AA]$ & $32.324(1)$ & $R_{\text {int }} / R_{\sigma}$ & $0.0574 / 0.0526$ \\
$b[\AA]$ & $18.306(1)$ & restraints/parameters & $0 / 941$ \\
$c[\AA]$ & $30.161(2)$ & GoF & 1.010 \\
$\beta\left[{ }^{\circ}\right]$ & $102.514(1)$ & $R 1($ all data $)$ & 0.1060 \\
$V\left[\AA^{3}\right]$ & $17423.1(12)$ & $R 1(I>2 \sigma(I))$ & 0.0517 \\
$\mathrm{Z}$ & 32 & $w R 2($ all data $)$ & 0.1494 \\
$\rho_{\text {calc }}\left[\mathrm{g} \mathrm{cm}^{-3}\right]$ & 1.062 & $w R 2(I>2 \sigma(I))$ & 0.1226 \\
$\mu\left[\mathrm{mm}^{-1}\right]$ & 0.067 & diff. peak/hole $\left[\mathrm{e} \AA^{-3}\right]$ & $0.469 /-0.189$ \\
\hline
\end{tabular}




\subsubsection{Di(iso-propyl)amino-2,6-di(iso-propyl)anilino- fluoroborane}

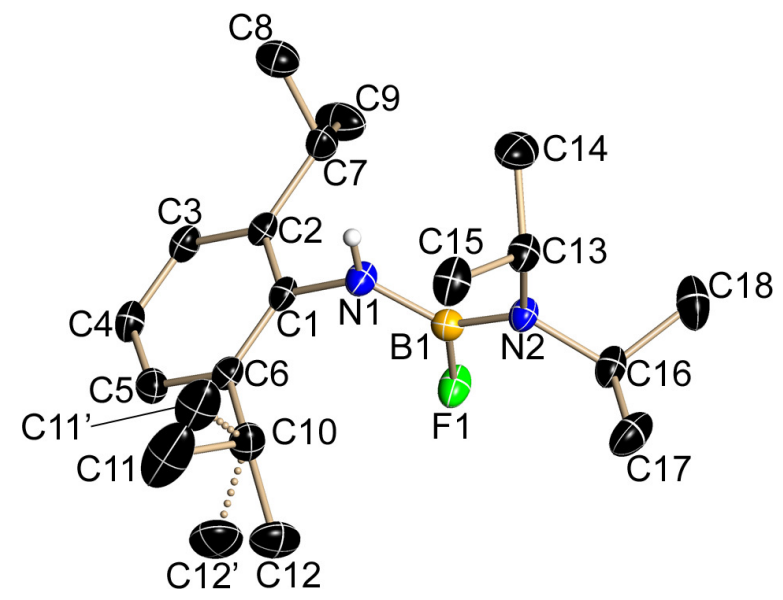

Figure 8-22. Asymmetric unit of $\left(i \mathrm{Pr}_{2} \mathrm{Ph}\right) \mathrm{N}(\mathrm{H}) \mathrm{B}(\mathrm{F}) \mathrm{N}(i \mathrm{Pr})_{2}$. Constrained hydrogen atoms are excluded for clarity reasons.

A disorder of one di-iso-propyl group (sof 0.33) was detected in the diaminofluoroborane $\left(i \mathrm{Pr}_{2} \mathrm{Ph}\right) \mathrm{N}(\mathrm{H}) \mathrm{B}(\mathrm{F}) \mathrm{N}(\mathrm{Pr})_{2}$. This could be well described without applying constraints or restraints. The asymmetric unit contains one formula unit.

Table 8-19. Crystallographic data for $\left(i \mathrm{Pr}_{2} \mathrm{Ph}\right) \mathrm{N}(\mathrm{H}) \mathrm{B}(\mathrm{F}) \mathrm{N}(i \mathrm{Pr})_{2}$.

\begin{tabular}{lc|lc}
\hline identification code & HOTTS008/st & $F(000)$ & 1344 \\
empirical formula & $\mathrm{C}_{18} \mathrm{H}_{32} \mathrm{BFN}_{2}$ & min./max. transmission & $0.77 / 0.99$ \\
molar mass $\left[\mathrm{g} \mathrm{mol}{ }^{-1}\right]$ & 306.27 & $\theta$ range $\left[^{\circ}\right]$ & $2.25-25.35$ \\
crystal size $[\mathrm{mm}]$ & $0.20 \times 0.10 \times 0.02$ & completeness to $\theta_{\max }$ & 1.00 \\
temperature $[\mathrm{K}]$ & $100(2)$ & redundancy to $\theta_{\max }$ & 3.68 \\
crystal system & monoclinic & reflections collected & 13521 \\
space group & $C 2 / \mathrm{C}$ & independent reflections & 3470 \\
$a[\AA]$ & $17.147(1)$ & $R_{\text {int }} / R_{\sigma}$ & $0.0373 / 0.0341$ \\
$b[\AA]$ & $11.003(1)$ & restraints/parameters & $0 / 232$ \\
$c[\AA]$ & $21.701(2)$ & GoF & 1.009 \\
$\beta\left[{ }^{\circ}\right]$ & $112.593(1)$ & $R 1($ all data $)$ & 0.0639 \\
$V\left[\AA^{3}\right]$ & $3780.2(6)$ & $R 1(I>2 \sigma(I))$ & 0.0401 \\
$\mathrm{Z}$ & 8 & $w R 2($ all data $)$ & 0.0982 \\
$\rho_{\text {calc }}\left[\mathrm{g} \mathrm{cm}^{-3}\right]$ & 1.076 & $w R 2(I>2 \sigma(I))$ & 0.0877 \\
$\mu\left[\mathrm{mm}^{-1}\right]$ & 0.069 & diff. peak/hole $\left[\mathrm{e} \AA^{-3}\right]$ & $0.169 /-0.187$ \\
\hline
\end{tabular}




\subsection{5 $\left(i \mathrm{Pr}_{2} \mathrm{Ph}\right) \mathrm{N}\left(\mathrm{SiMe}_{3}\right) \mathrm{B}(\mathrm{F}) \mathrm{N}(\mathrm{iPr})_{2}$}
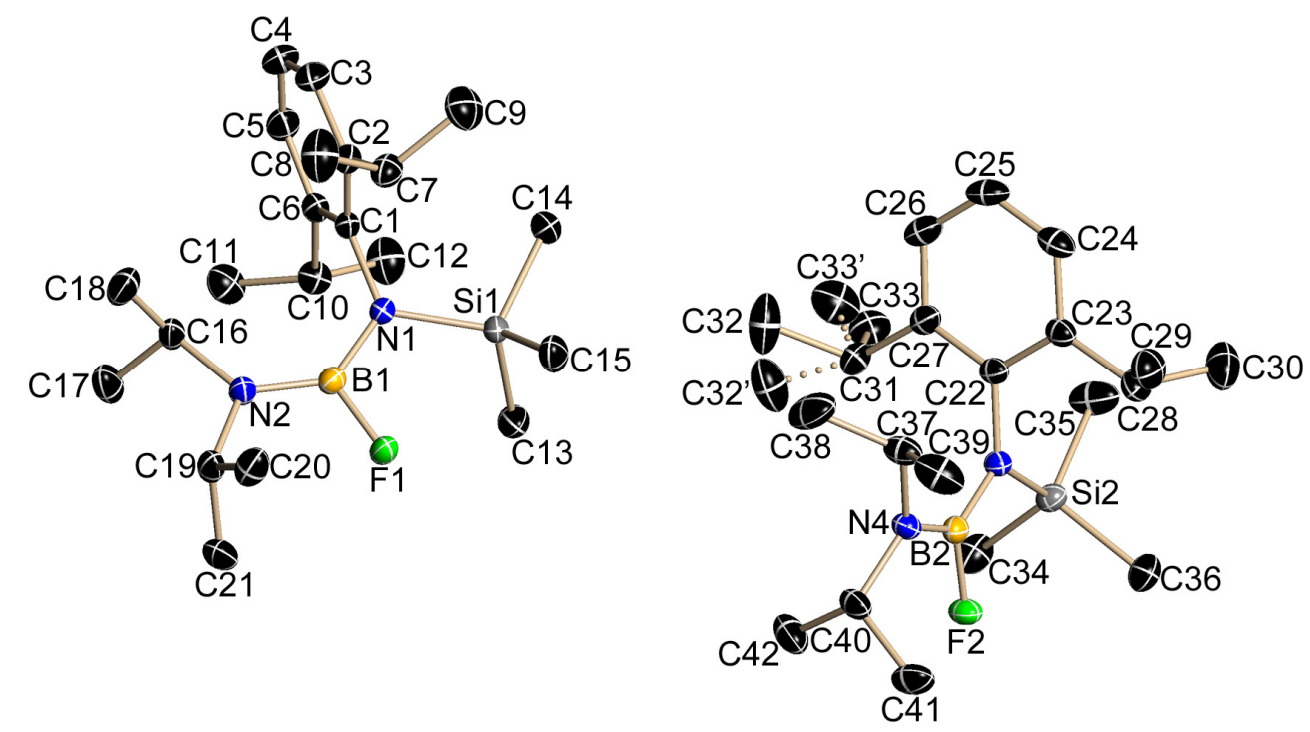

Figure 8-23. Asymmetric unit of $\left(i \mathrm{Pr}_{2} \mathrm{Ph}\right) \mathrm{N}\left(\mathrm{SiMe}_{3}\right) \mathrm{B}(\mathrm{F}) \mathrm{N}(\mathrm{Pr})_{2}$ (hydrogen atoms omitted).

Two independent molecules are present in the symmetric unit of $(i \mathrm{Pr} 2 \mathrm{Ph}) \mathrm{N}$ $\left(\mathrm{SiMe}_{3}\right) \mathrm{B}(\mathrm{F}) \mathrm{N}(\mathrm{iPr})_{2}$. One iso-propyl group is equally disordered over two positions (sof 0.5 ), which are interrelated by a rotation about the $\mathrm{HC}-\mathrm{C}_{\mathrm{Ph}}$ axis.

Table 8-20. Crystallographic data for $\left(i \mathrm{Pr}_{2} \mathrm{Ph}\right) \mathrm{N}\left(\mathrm{SiMe}_{3}\right) \mathrm{B}(\mathrm{F}) \mathrm{N}(i \mathrm{Pr})_{2}$.

\begin{tabular}{lc|lc}
\hline identification code & HOTTS012/st & $F(000)$ & 1664 \\
empirical formula & $\mathrm{C}_{21} \mathrm{H}_{40} \mathrm{BFN} \mathrm{N}_{2} \mathrm{Si}$ & min./max. transmission & $0.91 / 0.99$ \\
molar mass $\left[\mathrm{g} \mathrm{mol}^{-1}\right]$ & 378.45 & $\theta$ range $\left.{ }^{\circ}\right]$ & $1.87-26.04$ \\
crystal size $[\mathrm{mm}]$ & $0.20 \times 0.15 \times 0.05$ & completeness to $\theta_{\text {max }}$ & 1.00 \\
temperature $[\mathrm{K}]$ & $100(2)$ & redundancy to $\theta_{\text {max }}$ & 5.20 \\
crystal system & monoclinic & reflections collected & 66018 \\
space group & $P 2_{1} / n$ & independent reflections & 9648 \\
$a[\AA]$ & $16.9894(9)$ & $R_{\text {int }} / R_{\sigma}$ & $0.0394 / 0.0254$ \\
$b[\AA]$ & $14.1946(8)$ & restraints/parameters & $4 / 509$ \\
$c[\AA]$ & $19.4395(11)$ & GoF & 1.071 \\
$\beta\left[{ }^{\circ}\right]$ & $90.3826(10)$ & $R 1$ (all data) & 0.0514 \\
$V\left[\AA^{3}\right]$ & $4687.9(4)$ & $R 1(I>2 \sigma(I))$ & 0.0392 \\
$\mathrm{Z}$ & 8 & $w R 2($ all data) & 0.1022 \\
$\rho_{\text {calc }}\left[\mathrm{g} \mathrm{cm}^{-3}\right]$ & 1.072 & $w R 2(I>2 \sigma(I))$ & 0.0971 \\
$\mu\left[\mathrm{mm}^{-1}\right]$ & 0.115 & diff. peak/hole $\left[\mathrm{e} \AA^{-3}\right]$ & $0.277 /-0.272$ \\
\hline
\end{tabular}




\subsection{6 $\left(i \mathrm{Pr}_{2} \mathrm{PhNH}\right)_{2} \mathrm{BNiPr}_{2}$}

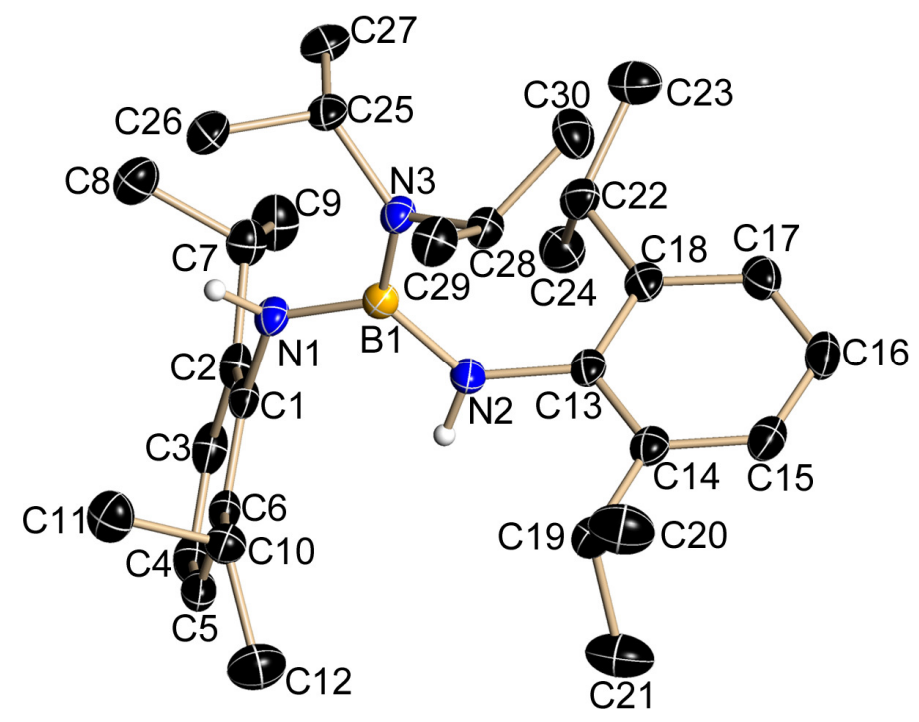

Figure 8-24. Asymmetric unit of $\left(i \mathrm{Pr}_{2} \mathrm{PhNH}\right)_{2} \mathrm{BNiPr}$. Constrained hydrogen atoms are excluded.

The triaminoborane crystallises in the orthorhombic centrosymmetric space group Pbca with one molecule in the asymmetric unit. The amine hydrogen atoms were detected in a residual map and refined with a riding model.

Table 8-21. Crystallographic data for $\left(\mathrm{Pr}_{2} \mathrm{PhNH}\right)_{2} \mathrm{BNiPr}_{2}$.

\begin{tabular}{lc|lc}
\hline identification code & HOTTS013/st & min./max. transmission & $0.82 / 0.99$ \\
empirical formula & $\mathrm{C}_{30} \mathrm{H}_{50} \mathrm{BN}_{3}$ & $\theta$ range $\left.{ }^{\circ}\right]$ & $1.98-25.35$ \\
molar mass $\left[\mathrm{g} \mathrm{mol}{ }^{-1}\right]$ & 463.54 & completeness to $\theta_{\max }$ & 1.00 \\
crystal size $[\mathrm{mm}]$ & $0.28 \times 0.18 \times 0.14$ & redundancy to $\theta_{\max }$ & 5.66 \\
temperature $[\mathrm{K}]$ & $100(2)$ & reflections collected & 45363 \\
crystal system & orthorhombic & independent reflections & 5944 \\
space group & $P b c a$ & $R_{\text {int }} / R_{\sigma}$ & $0.0438 / 0.0255$ \\
$a[\AA]$ & $18.7014(9)$ & restraints/parameters & $0 / 325$ \\
$b[\AA]$ & $15.6041(8)$ & GoF & 1.074 \\
$c[\AA]$ & $20.0935(10)$ & $R 1($ all data $)$ & 0.0531 \\
$V\left[\AA^{3}\right]$ & $5863.7(5)$ & $R 1(I>2 \sigma(I))$ & 0.0392 \\
$Z$ & 8 & $w R 2($ all data $)$ & 0.0985 \\
$\rho_{\text {calc }}\left[\mathrm{g} \mathrm{cm}^{-3}\right]$ & 1.050 & $w R 2(I>2 \sigma(I))$ & 0.0926 \\
$\mu\left[\mathrm{mm}^{-1}\right]$ & 0.060 & diff. peak/hole $\left[\mathrm{e} \AA^{-3}\right]$ & $0.161 /-0.168$ \\
$F(000)$ & 2048 & & \\
\hline
\end{tabular}




\subsection{7 $\left(\mathrm{Me}_{3} \mathrm{Si}\right)_{2} \mathrm{~B}(\mathrm{~F}) \mathrm{N}(\mathrm{tBu}) \mathrm{SiF}(\mathrm{tBu})_{2}$}

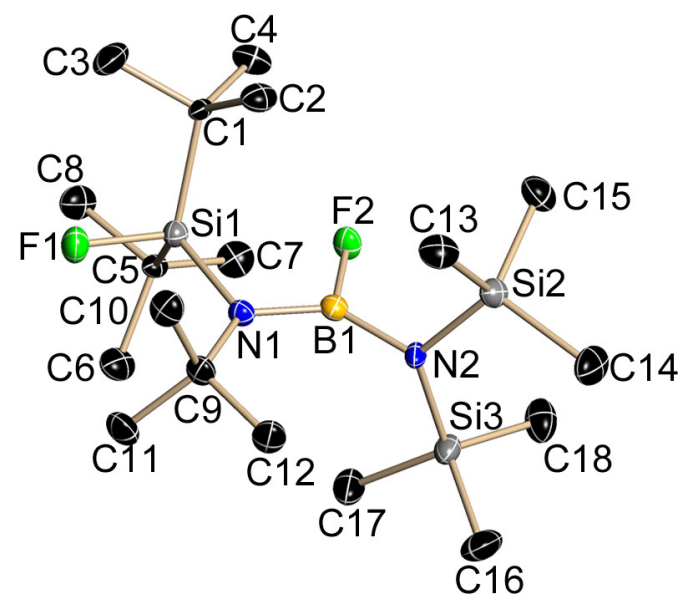

Figure 8-25. Asymmetric unit of $\left(\mathrm{Me}_{3} \mathrm{Si}\right)_{2} \mathrm{~B}(\mathrm{~F}) \mathrm{N}(t \mathrm{Bu}) \mathrm{SiF}(t \mathrm{Bu})_{2}$. Hydrogen atoms are omitted for clarity.

$\left(\mathrm{Me}_{3} \mathrm{Si}\right)_{2} \mathrm{~B}(\mathrm{~F}) \mathrm{N}(t \mathrm{Bu}) \operatorname{SiF}(t \mathrm{Bu})_{2}$ crystallises in the triclinic space group $P \overline{1}$ and the asymmetric unit contains one formula unit.

Table 8-22. Crystallographic data for $\left(\mathrm{Me}_{3} \mathrm{Si}\right)_{2} \mathrm{~B}(\mathrm{~F}) \mathrm{N}(t \mathrm{Bu}) \operatorname{SiF}(t \mathrm{Bu})_{2}$.

\begin{tabular}{|c|c|c|c|}
\hline identification code & HOTTS014/st & $\mu\left[\mathrm{mm}^{-1}\right]$ & 0.207 \\
\hline empirical formula & $\mathrm{C}_{18} \mathrm{H}_{45} \mathrm{BF}_{2} \mathrm{~N}_{2} \mathrm{Si}_{3}$ & $F(000)$ & 464 \\
\hline molar mass $\left[\mathrm{g} \mathrm{mol}^{-1}\right]$ & 422.64 & min./max. transmission & $0.88 / 0.99$ \\
\hline crystal size $[\mathrm{mm}]$ & $0.50 \times 0.40 \times 0.20$ & $\theta$ range $\left[{ }^{\circ}\right]$ & $2.18-26.73$ \\
\hline temperature [K] & $100(2)$ & completeness to $\theta_{\max }$ & 0.96 \\
\hline crystal system & triclinic & redundancy to $\theta_{\max }$ & 1.87 \\
\hline space group & $P \overline{1}$ & reflections collected & 13654 \\
\hline$a[\AA]$ & $8.8838(4)$ & independent reflections & 5342 \\
\hline$b[\AA]$ & $10.4050(4)$ & $R_{i n t} / R_{\sigma}$ & $0.0259 / 0.0245$ \\
\hline$c[\AA]$ & $14.6805(6)$ & restraints/parameters & $0 / 250$ \\
\hline$\alpha\left[^{\circ}\right]$ & $73.8300(10)$ & GoF & 1.036 \\
\hline$\beta\left[^{\circ}\right]$ & $89.8900(10)$ & $R 1$ (all data) & 0.0412 \\
\hline$\gamma\left[^{\circ}\right]$ & $77.5100(10)$ & $R 1(I>2 \sigma(I))$ & 0.0390 \\
\hline$V\left[\AA^{3}\right]$ & $1270.04(9)$ & $w R 2$ (all data) & 0.1135 \\
\hline Z & 2 & $w R 2(I>2 \sigma(I))$ & 0.1118 \\
\hline$\rho_{\text {calc }}\left[\mathrm{g} \mathrm{cm}^{-3}\right]$ & 1.105 & diff. peak/hole $\left[\mathrm{e}^{-3}\right]$ & $0.624 /-0.283$ \\
\hline
\end{tabular}




\subsection{8 $\left[\left(\mathrm{Me}_{3} \mathrm{Si}\right)_{2} \mathrm{NB}(\mathrm{F}) \mathrm{N}\left(\mathrm{SiMe}_{3}\right) \mathrm{Si}(\mathrm{Me})_{2} \mathrm{~N}\left(\mathrm{SiMe}_{3}\right) \mathrm{CH}_{2}\right]_{2}$}

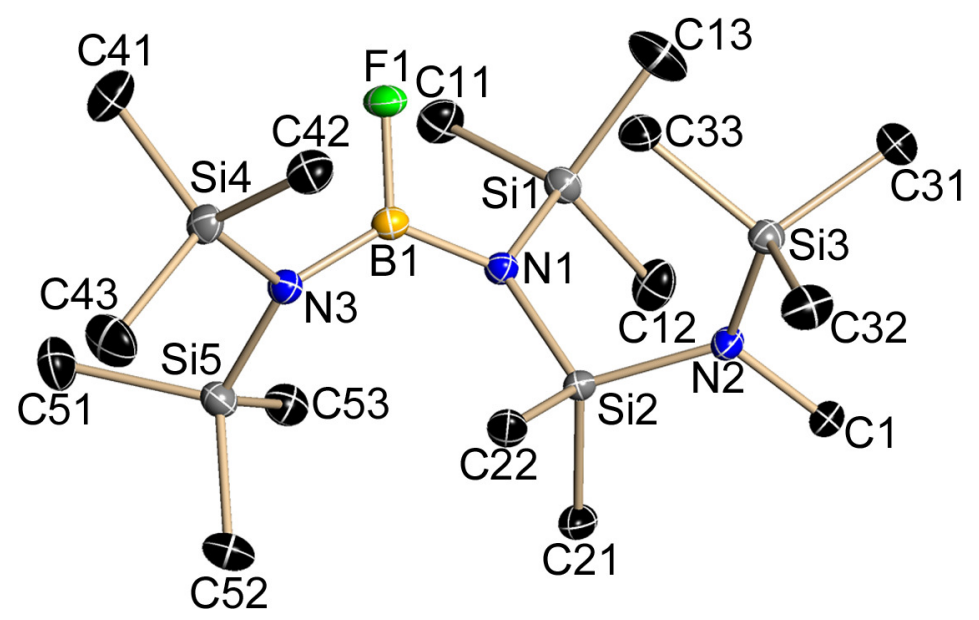

Figure 8-26. Asymmetric unit of $\left[\left(\mathrm{Me}_{3} \mathrm{Si}\right)_{2} \mathrm{NB}(\mathrm{F}) \mathrm{N}\left(\mathrm{SiMe}_{3}\right) \mathrm{Si}(\mathrm{Me})_{2} \mathrm{~N}\left(\mathrm{SiMe}_{3}\right) \mathrm{CH}_{2}\right]_{2}$.

The fluoroborane $\left[\left(\mathrm{Me}_{3} \mathrm{Si}\right)_{2} \mathrm{NB}(\mathrm{F}) \mathrm{N}\left(\mathrm{SiMe}_{3}\right) \mathrm{Si}(\mathrm{Me})_{2} \mathrm{~N}\left(\mathrm{SiMe}_{3}\right) \mathrm{CH}_{2}\right]_{2}$ shows one well separated molecule in the asymmetric unit.

Table 8-23. Crystallographic data for $\left[\left(\mathrm{Me}_{3} \mathrm{Si}\right)_{2} \mathrm{NB}(\mathrm{F}) \mathrm{N}\left(\mathrm{SiMe}_{3}\right) \mathrm{Si}(\mathrm{Me})_{2} \mathrm{~N}\left(\mathrm{SiMe}_{3}\right) \mathrm{CH}_{2}\right]_{2}$.

\begin{tabular}{lc|lc}
\hline identification code & HOTTS015/st & $F(000)$ & 956 \\
empirical formula & $\mathrm{C}_{15} \mathrm{H}_{4} \mathrm{BFN}_{3} \mathrm{Si}_{5}$ & min./max. transmission & $0.85 / 0.93$ \\
molar mass $\left[\mathrm{g} \mathrm{mol}{ }^{-1}\right]$ & 873.58 & $\theta$ range $\left[^{\circ}\right]$ & $2.18-28.27$ \\
crystal size $[\mathrm{mm}]$ & $0.35 \times 0.32 \times 0.25$ & completeness to $\theta_{\max }$ & 0.95 \\
temperature $[\mathrm{K}]$ & $100(2)$ & redundancy to $\theta_{\max }$ & 5.36 \\
crystal system & monoclinic & reflections collected & 62560 \\
space group & $P 2_{1} / \mathrm{C}$ & independent reflections & 6749 \\
$a[\AA]$ & $18.8101(5)$ & $R_{\text {int }} / R_{\sigma}$ & $0.0220 / 0.0114$ \\
$b[\AA]$ & $9.5179(3)$ & restraints/parameters & $0 / 240$ \\
$c[\AA]$ & $14.6172(4)$ & GoF & 1.043 \\
$\beta\left[{ }^{\circ}\right]$ & $95.6020(10)$ & $R 1($ all data $)$ & 0.0292 \\
$V\left[\AA^{3}\right]$ & $2604.46(13)$ & $R 1(I>2 \sigma(I))$ & 0.0267 \\
$Z$ & 4 & $w R 2($ all data $)$ & 0.0774 \\
$\rho_{\text {calc }}\left[\mathrm{g} \mathrm{cm}^{-3}\right]$ & 1.114 & $W R 2(I>2 \sigma(I))$ & 0.0757 \\
$\mu\left[\mathrm{mm}^{-1}\right]$ & 0.287 & diff. peak/hole $\left[\mathrm{e} \AA^{-3}\right]$ & $0.426 /-0.348$ \\
\hline
\end{tabular}




\subsection{9 $\left(i \mathrm{Pr}_{2} \mathrm{Ph}\right) \mathrm{N}(\mathrm{H}) \mathrm{B}(\mathrm{F}) \mathrm{N}(\mathrm{Me}) \mathrm{SiMe}_{3}$}

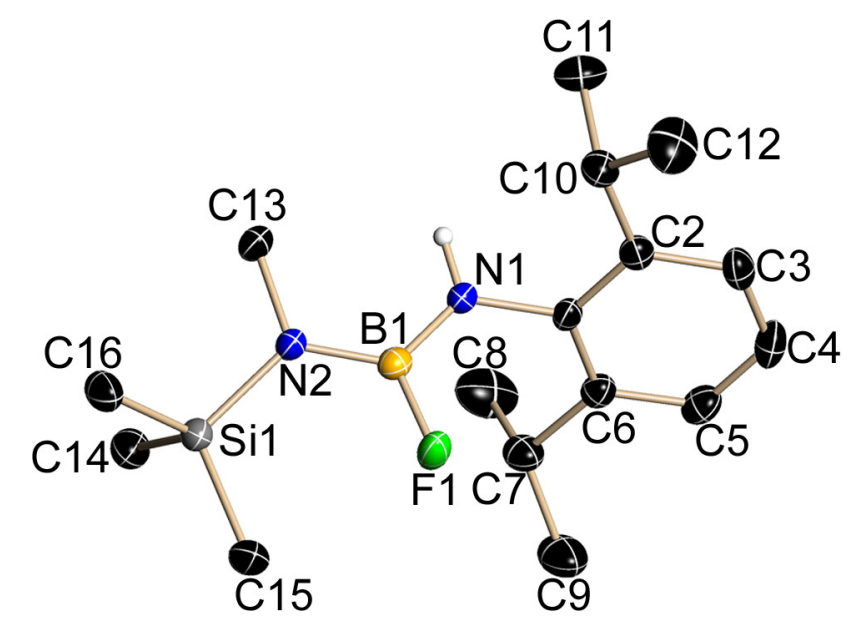

Figure 8-27. Asymmetric unit of $\left(\mathrm{Pr}_{2} \mathrm{Ph}\right) \mathrm{N}(\mathrm{H}) \mathrm{B}(\mathrm{F}) \mathrm{N}(\mathrm{Me}) \mathrm{SiMe}_{3}$. Constrained hydrogen atoms are excluded.

The diaminofluoroborane $\left(\mathrm{iPr}_{2} \mathrm{Ph}\right) \mathrm{N}(\mathrm{H}) \mathrm{B}(\mathrm{F}) \mathrm{N}(\mathrm{Me}) \mathrm{SiMe}_{3}$ crystallises as colourless blocks in the monoclinic space group $P 2_{1} / c$.

Table 8-24. Crystallographic data for $\left(\mathrm{iPr}_{2} \mathrm{Ph}\right) \mathrm{N}(\mathrm{H}) \mathrm{B}(\mathrm{F}) \mathrm{N}(\mathrm{Me}) \mathrm{SiMe}_{3}$.

\begin{tabular}{lc|lc}
\hline identification code & HOTTS017/st & $F(000)$ & 672 \\
empirical formula & $\mathrm{C}_{16} \mathrm{H}_{30} \mathrm{BFN}{ }_{2} \mathrm{Si}$ & min./max. transmission & $0.84 / 0.99$ \\
molar mass $\left[\mathrm{g} \mathrm{mol}^{-1}\right]$ & 308.32 & $\theta$ range $\left.{ }^{\circ}\right]$ & $2.50-25.33$ \\
crystal size $[\mathrm{mm}]$ & $0.18 \times 0.15 \times 0.10$ & completeness to $\theta_{\text {max }}$ & 0.99 \\
temperature $[\mathrm{K}]$ & $100(2)$ & redundancy to $\theta_{\text {max }}$ & 3.54 \\
crystal system & monoclinic & reflections collected & 16421 \\
space group & $P 2_{1} / \mathrm{c}$ & independent reflections & 3610 \\
$a[\AA]$ & $6.6315(9)$ & $R_{\text {int }} / R_{\sigma}$ & $0.0568 / 0.0488$ \\
$b[\AA]$ & $9.9553(13)$ & restraints/parameters & $1 / 201$ \\
$c[\AA]$ & $28.329(4)$ & GoF & 1.066 \\
$\beta\left[{ }^{\circ}\right]$ & $90.720(2)$ & $R 1$ (all data) & 0.0738 \\
$V\left[\AA^{3}\right]$ & $1870.1(4)$ & $R 1(I>2 \sigma(I))$ & 0.0431 \\
$\mathrm{Z}$ & 4 & $w R 2($ all data) & 0.1055 \\
$\rho_{\text {calc }}\left[\mathrm{g} \mathrm{cm}^{-3}\right]$ & 1.095 & $w R 2(I>2 \sigma(I))$ & 0.0958 \\
$\mu\left[\mathrm{mm}^{-1}\right]$ & 0.130 & diff. peak/hole $\left[\mathrm{e} \AA^{-3}\right]$ & $0.237 /-0.243$ \\
\hline
\end{tabular}




\subsubsection{0 $\mathrm{Ph}_{3} \operatorname{SiN}(\mathrm{H}) \mathrm{B}(\mathrm{F}) \mathrm{N}(\mathrm{iPr})_{2}$}

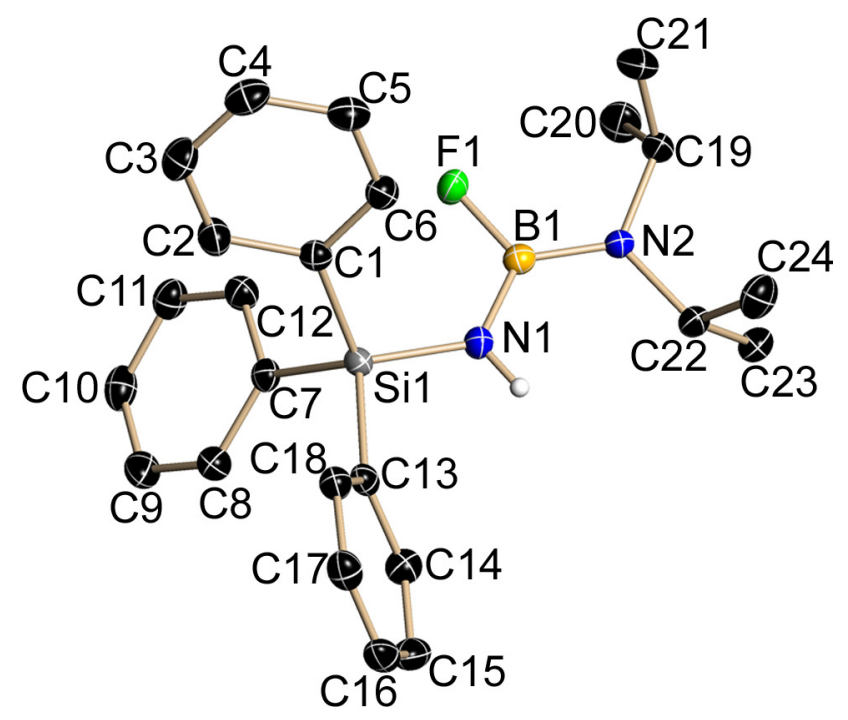

Figure 8-28. Asymmetric unit of $\mathrm{Ph}_{3} \mathrm{SiN}(\mathrm{H}) \mathrm{B}(\mathrm{F}) \mathrm{N}(i \mathrm{Pr})_{2}$ (only restrained hydrogen atom shown).

$\mathrm{Ph}_{3} \mathrm{SiN}(\mathrm{H}) \mathrm{B}(\mathrm{F}) \mathrm{N}(\mathrm{iPr})_{2}$ crystallises as colourless plates in the chiral monoclinic space group $P 2_{1}$. The refinement of the correct stereoisomer was proven by the Flack $\mathrm{x}$ parameter of $0.00(6)$.

Table 8-25. Crystallographic data for $\mathrm{Ph}_{3} \mathrm{SiN}(\mathrm{H}) \mathrm{B}(\mathrm{F}) \mathrm{N}(\mathrm{PPr})_{2}$.

\begin{tabular}{lc|lc}
\hline identification code & HOTTS019/st & $F(000)$ & 432 \\
empirical formula & $\mathrm{C}_{24} \mathrm{H}_{30} \mathrm{BFN}{ }_{2} \mathrm{Si}$ & min./max. transmission & $0.93 / 0.99$ \\
molar mass $\left[\mathrm{g} \mathrm{mol}^{-1}\right]$ & 404.40 & $\theta$ range $\left.{ }^{\circ}\right]$ & $2.35-26.40$ \\
crystal size $[\mathrm{mm}]$ & $0.50 \times 0.50 \times 0.15$ & completeness to $\theta_{\max }$ & 1.00 \\
temperature $[\mathrm{K}]$ & $100(2)$ & redundancy to $\theta_{\text {max }}$ & 3.29 \\
crystal system & monoclinic & reflections collected & 20537 \\
space group & $P 2_{1}$ & independent reflections & 4703 \\
$a[\AA]$ & $7.4661(7)$ & $R_{\text {int }} / R_{\sigma}$ & $0.0199 / 0.0131$ \\
$b[\AA]$ & $11.3214(11)$ & restraints/parameters & $2 / 269$ \\
$c[\AA]$ & $13.6868(13)$ & GoF & 1.054 \\
$\beta\left[{ }^{\circ}\right]$ & $98.6290(10)$ & $R 1($ all data $)$ & 0.0242 \\
$V\left[\AA^{3}\right]$ & $1143.80(19)$ & $R 1(I>2 \sigma(I))$ & 0.0239 \\
$\mathrm{Z}$ & 2 & $w R 2($ all data $)$ & 0.0649 \\
$\rho_{\text {calc }}\left[\mathrm{g} \mathrm{cm}^{-3}\right]$ & 1.174 & $w R 2(I>2 \sigma(I))$ & 0.0648 \\
$\mu\left[\mathrm{mm}^{-1}\right]$ & 0.123 & diff. peak/hole $\left[\mathrm{e} \AA^{-3}\right]$ & $0.207 /-0.146$ \\
\hline
\end{tabular}




\subsubsection{Inorganic salt containing an Al-O-Sn backbone}

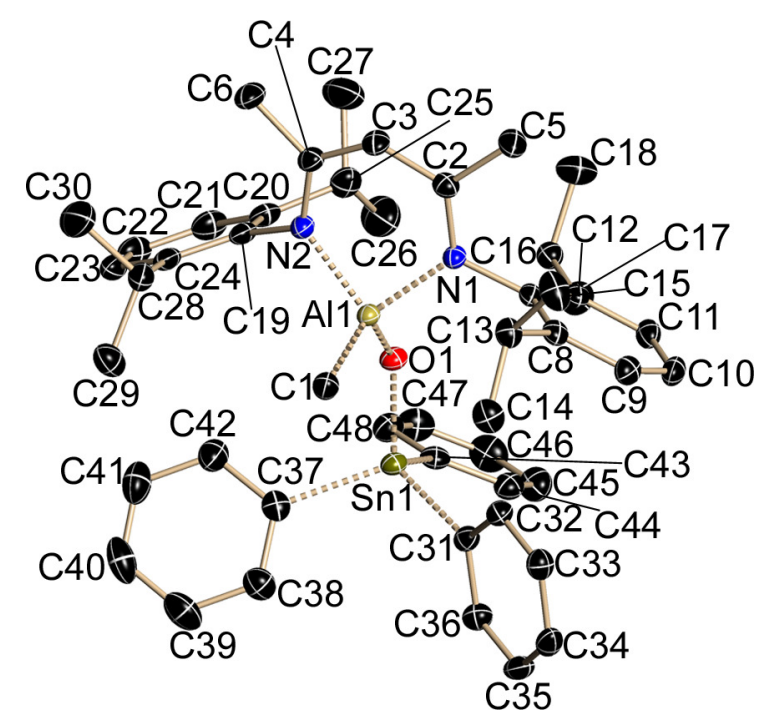

Figure 8-29. Asymmetric unit of HOTTS026 (hydrogen atoms are omitted).

The aluminium tin oxide crystallises in the triclinic space group $P \overline{1}$ with one formula unit in the asymmetric unit.

Table 8-26. Crystallographic data for HOTTS026.

\begin{tabular}{lc|lc}
\hline identification code & HOTTS026/st & $\mu\left[\mathrm{mm}^{-1}\right]$ & 0.645 \\
empirical formula & $\mathrm{C}_{48} \mathrm{H}_{59} \mathrm{AIN}_{2} \mathrm{OSn}$ & $F(000)$ & 864 \\
molar mass $\left[\mathrm{g} \mathrm{mol}^{-1}\right]$ & 825.64 & min./max. transmission & $0.89 / 0.99$ \\
crystal size $[\mathrm{mm}]$ & $0.13 \times 0.09 \times 0.04$ & $\theta$ range $\left[^{\circ}\right]$ & $2.25-25.36$ \\
temperature $[\mathrm{K}]$ & $100(2)$ & completeness to $\theta_{\text {max }}$ & 1.00 \\
crystal system & triclinic & redundancy to $\theta_{\text {max }}$ & 3.41 \\
space group & $P \overline{1}$ & reflections collected & 34307 \\
$a[\AA]$ & $11.4600(8)$ & independent reflections & 7966 \\
$b\left[^{\AA}\right]$ & $11.5128(8)$ & $R_{\text {int }} / R_{\sigma}$ & $0.0338 / 0.0262$ \\
$c\left[^{\AA}\right]$ & $16.7507(12)$ & restraints/parameters & $0 / 489$ \\
$\alpha\left[^{\circ}\right]$ & $84.5200(10)$ & GoF & 1.053 \\
$\beta\left[^{\circ}\right]$ & $84.9510(10)$ & $R 1($ all data $)$ & 0.0278 \\
$\gamma\left[^{\circ}\right]$ & $81.8840(10)$ & $R 1(I>2 \sigma(I))$ & 0.0238 \\
$V\left[^{3} \AA^{3}\right]$ & $2171.7(3)$ & $W R 2($ all data $)$ & 0.0558 \\
$Z$ & 2 & $W R 2(I>2 \sigma(I))$ & 0.0543 \\
$\rho_{\text {calc }}\left[\mathrm{g} \mathrm{cm}^{-3}\right]$ & 1.263 & diff. peak/hole $\left[\mathrm{e} \AA^{-3}\right]$ & $0.416 /-0.309$ \\
\hline
\end{tabular}




\subsubsection{2 (Cp* $)_{2} \mathrm{Zr}-\mathrm{O}-\mathrm{Mg}(\text { thf })_{2}-\mathrm{O}-\mathrm{Zr}(\mathrm{Cp} *)_{2}$}

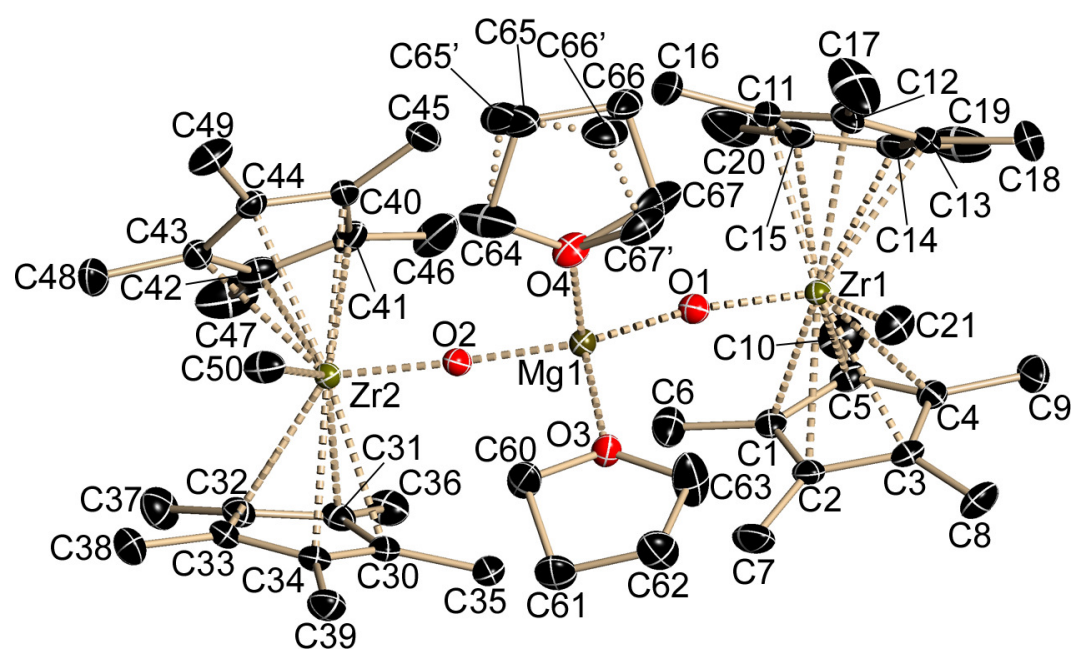

Figure 8-30. Asymmetric unit of HOTTS027 (hydrogen atoms are omitted for clarity).

The zirconium magnesium oxide crystallises in the monoclinic space group $P Z_{1} / C$ with one molecule in the asymmetric unit. One thf donor is disordered and the site occupation factor refined to 0.53 . The refinement was stabilised with DFIX commands.

Table 8-27. Crystallographic data for HOTTS027.

\begin{tabular}{lc|lc}
\hline identification code & HOTTS027/st & $F(000)$ & 2024 \\
empirical formula & $\mathrm{C}_{50} \mathrm{H}_{82} \mathrm{MgO}_{4} \mathrm{Zr}_{2}$ & min./max. transmission & $0.84 / 0.99$ \\
molar mass $\left[\mathrm{g} \mathrm{mol}{ }^{-1}\right]$ & 953.91 & $\theta$ range $\left.{ }^{\circ}\right]$ & $2.19-26.03$ \\
crystal size $[\mathrm{mm}]$ & $0.40 \times 0.30 \times 0.20$ & completeness to $\theta_{\max }$ & 0.97 \\
temperature $[\mathrm{K}]$ & $100(2)$ & redundancy to $\theta_{\max }$ & 3.81 \\
crystal system & monoclinic & reflections collected & 49392 \\
space group & $P 2_{1} / \mathrm{C}$ & independent reflections & 9669 \\
$a[\AA]$ & $12.3587(6)$ & $R_{\text {int }} / R_{\sigma}$ & $0.0252 / 0.0187$ \\
$b[\AA]$ & $17.7428(8)$ & restraints/parameters & $8 / 555$ \\
$c[\AA]$ & $21.8798(10)$ & GoF & 1.061 \\
$\beta\left[{ }^{\circ}\right]$ & $93.5650(10)$ & $R 1($ all data $)$ & 0.0298 \\
$V\left[\AA^{3}\right]$ & $4788.5(4)$ & $R 1(I>2 \sigma(I))$ & 0.0264 \\
$\mathrm{Z}$ & 4 & $w R 2($ all data $)$ & 0.0688 \\
$\rho_{\text {calc }}\left[\mathrm{g} \mathrm{cm}^{-3}\right]$ & 1.323 & $w R 2(I>2 \sigma(I))$ & 0.0668 \\
$\mu\left[\mathrm{mm}^{-1}\right]$ & 0.490 & diff. peak/hole $\left[\mathrm{e} \AA^{-3}\right]$ & $0.446 /-0.437$ \\
\hline
\end{tabular}




\subsubsection{3 $\left(\mathrm{Cp}^{*}\right)_{2} \mathrm{Zr}-\mathrm{O}-\mathrm{Mg}-\mathrm{O}-\mathrm{AIMe}\left(\mathrm{N}_{2} \mathrm{R}\right)$}
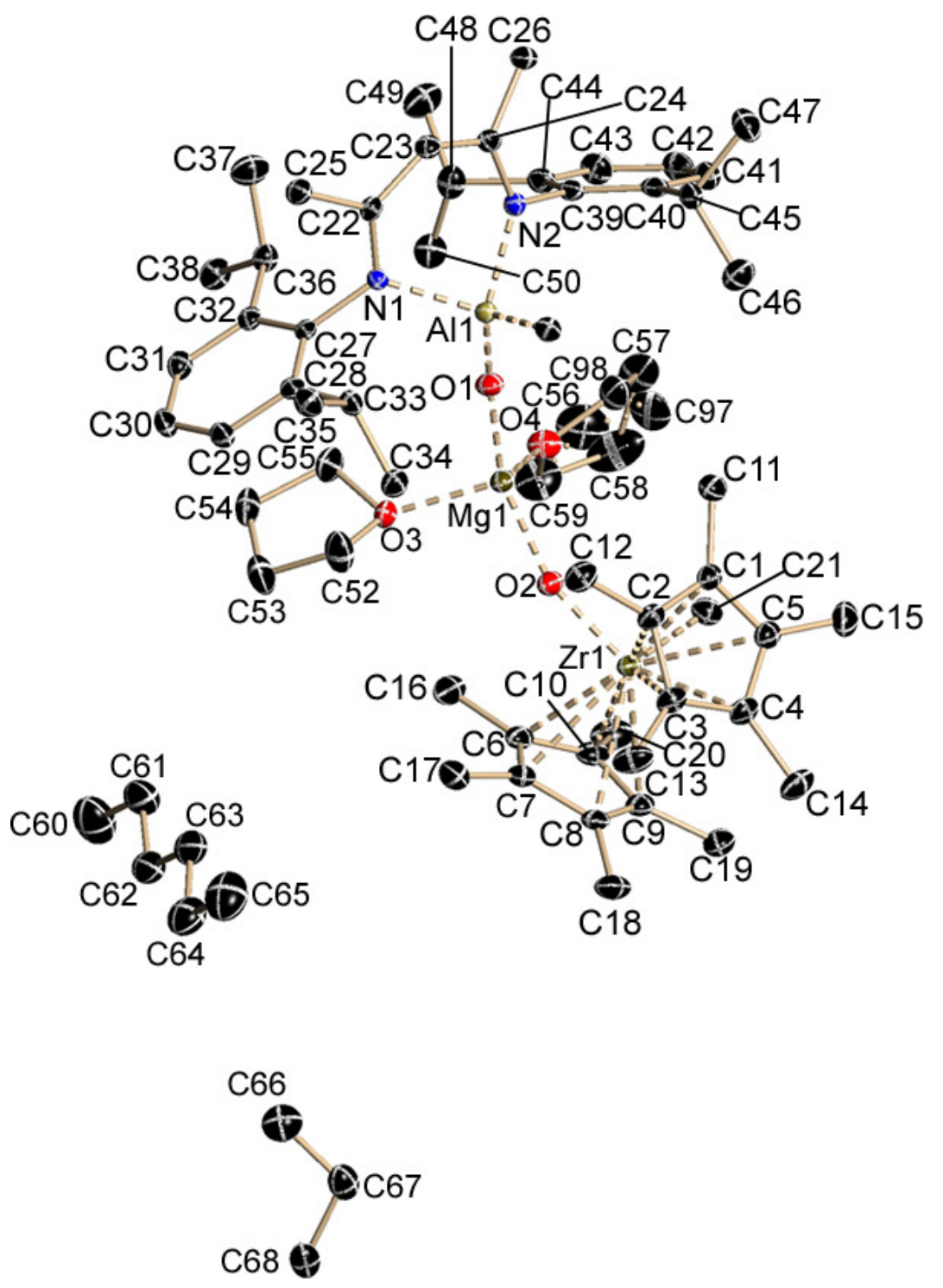

Figure 8-31. Asymmetric unit of HOTTS010 (hydrogen atom are excluded for clarity).

The mixed metal oxide compound HOTTS010, containing zirconium, magnesium, and aluminium, crystallises with one formula unit and 1.5 hexane molecules in the asymmetric unit (Figure 8-31). The second half of the hexane molecule is generated by an inversion at the origin and a translation by $(0,2,1)$. One thf donor molecule shows a standard disorder pattern of the 3/4-carbon atoms, which refined to a site occupation factor of 0.76 . The disorder as well as the refinement of the hexane molecule near the inversion centre was stabilised with DELU, SIMU, and DFIX commands. 
Table 8-28. Crystallographic data for HOTTS010.

\begin{tabular}{lc|lc}
\hline identification code & HOTTS010/st & $F(000)$ & 820 \\
empirical formula & $\mathrm{C}_{30} \mathrm{H}_{84} \mathrm{Li}_{2} \mathrm{~N}_{8} \mathrm{Si}_{6}$ & min./max. transmission & $0.93 / 0.99$ \\
molar mass $\left[\mathrm{g} \mathrm{mol}^{-1}\right]$ & 739.47 & $\theta$ range $\left.{ }^{\circ}\right]$ & $2.21-28.28$ \\
crystal size $[\mathrm{mm}]$ & $0.40 \times 0.30 \times 0.20$ & completeness to $\theta_{\max }$ & 0.99 \\
temperature $[\mathrm{K}]$ & $100(2)$ & redundancy to $\theta_{\max }$ & 9.69 \\
crystal system & monoclinic & reflections collected & 62481 \\
space group & $P 2_{1} / n$ & independent reflections & 5789 \\
$a[\AA]$ & $10.2284(2)$ & $R_{\text {int }} / R_{\sigma}$ & $0.0336 / 0.0159$ \\
$b[\AA]$ & $22.8681(4)$ & restraints/parameters & $0 / 220$ \\
$c[\AA]$ & $10.5095(2)$ & GoF & 1.066 \\
$\beta\left[{ }^{\circ}\right]$ & $106.6680(10)$ & $R 1($ all data $)$ & 0.0318 \\
$V\left[\AA^{3}\right]$ & $2354.93(8)$ & $R 1(I>2 \sigma(I))$ & 0.0280 \\
$\mathrm{Z}$ & 2 & $w R 2($ all data) & 0.0774 \\
$\rho_{\text {calc }}\left[\mathrm{g} \mathrm{cm}^{-3}\right]$ & 1.043 & $w R 2(I>2 \sigma(I))$ & 0.0750 \\
$\mu\left[\mathrm{mm}^{-1}\right]$ & 0.205 & diff. peak/hole $\left[\mathrm{e} \AA^{-3}\right]$ & $0.427 /-0.192$ \\
\hline
\end{tabular}

\subsubsection{4 $\left(\mathrm{RN}_{4}\right) \mathrm{MgCl}$}
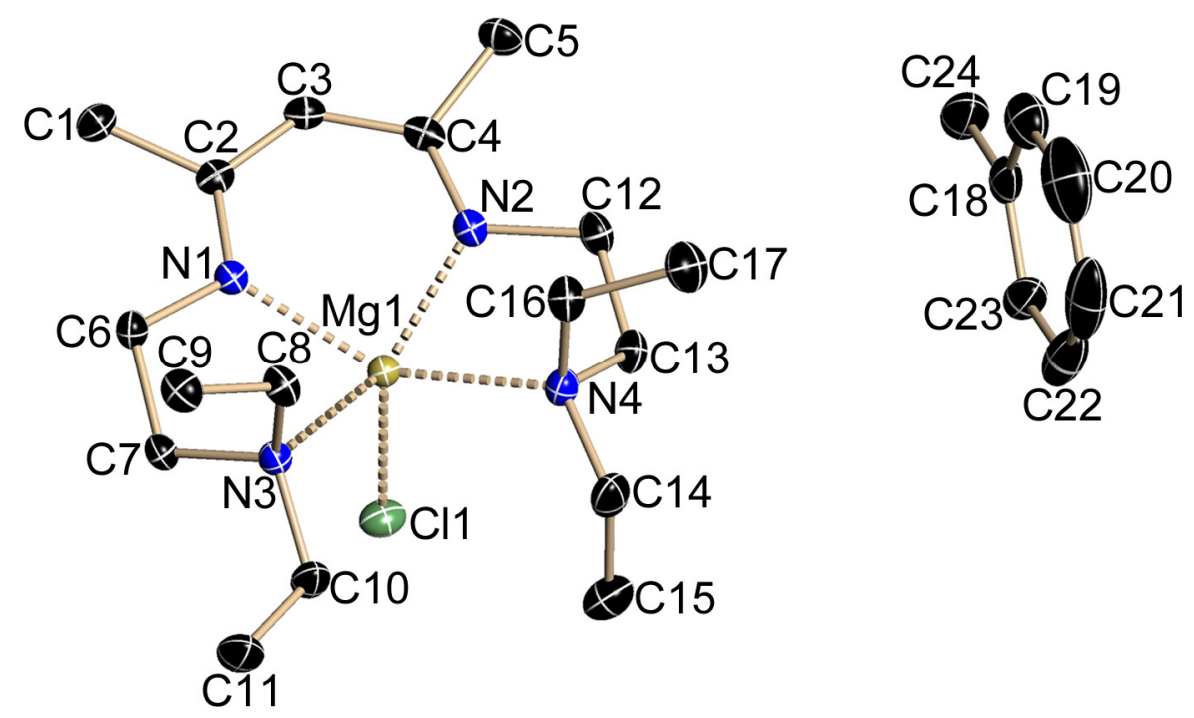

Figure 8-32. Asymmetric unit of $\left(\mathrm{RN}_{4}\right) \mathrm{MgCl}$. Hydrogen atoms are excluded for clarity.

The magnesium salt crystallises with one formula unit in the asymmetric unit, which also includes a toluene molecule positioned on an inversion centre (Figure 8-32). The occupancy was accordingly fixed to 0.5 . PART -1 was used to 
suppress the bond formation to the symmetry equivalent solvent molecule in the connectivity table. The execution of the inversion operation results in the known picked-fence disorder of the lattice solvent.

Table 8-29. Crystallographic data for $\left(\mathrm{RN}_{4}\right) \mathrm{MgCl}$

\begin{tabular}{lc|lc}
\hline identification code & HOTTS011/st & $F(000)$ & 876 \\
empirical formula & $\mathrm{C}_{20.5} \mathrm{H}_{39} \mathrm{MgClN}_{4}$ & min./max. transmission & $0.91 / 0.99$ \\
molar mass $\left[\mathrm{g} \mathrm{mol}^{-1}\right]$ & 401.32 & $\theta$ range $\left.{ }^{\circ}\right]$ & $2.12-25.69$ \\
crystal size $[\mathrm{mm}]$ & $0.40 \times 0.25 \times 0.10$ & completeness to $\theta_{\max }$ & 1.00 \\
temperature $[\mathrm{K}]$ & $100(2)$ & redundancy to $\theta_{\max }$ & 5.98 \\
crystal system & monoclinic & reflections collected & 36279 \\
space group & $P 2_{1} / \mathrm{c}$ & independent reflections & 4647 \\
$a[\AA]$ & $17.1111(8)$ & $R_{\text {int }} / R_{\sigma}$ & $0.0235 / 0.0125$ \\
$b[\AA]$ & $7.1331(3)$ & restraints/parameters & $0 / 266$ \\
$c[\AA]$ & $19.7089(9)$ & GoF & 1.049 \\
$\beta\left[{ }^{\circ}\right]$ & $110.7030(10)$ & $R 1$ (all data) & 0.0329 \\
$V\left[\AA^{3}\right]$ & $2250.23(17)$ & $R 1(I>2 \sigma(I))$ & 0.0303 \\
$\mathrm{Z}$ & 4 & $w R 2($ all data $)$ & 0.0812 \\
$\rho_{\text {calc }}\left[\mathrm{g} \mathrm{cm}^{-3}\right]$ & 1.185 & $w R 2(I>2 \sigma(I))$ & 0.0796 \\
$\mu\left[\mathrm{mm}^{-1}\right]$ & 0.210 & diff. peak/hole $\left[\mathrm{e} \AA^{-3}\right]$ & $0.575 /-0.257$ \\
\hline
\end{tabular}




\subsubsection{5 $\left[(t \mathrm{Bu})_{3} \mathrm{Ph}\right] \mathrm{B}(\mathrm{OH}) \mathrm{N}(\mathrm{H}) \mathrm{Si}(\mathrm{Me})_{2} t \mathrm{Bu}$}

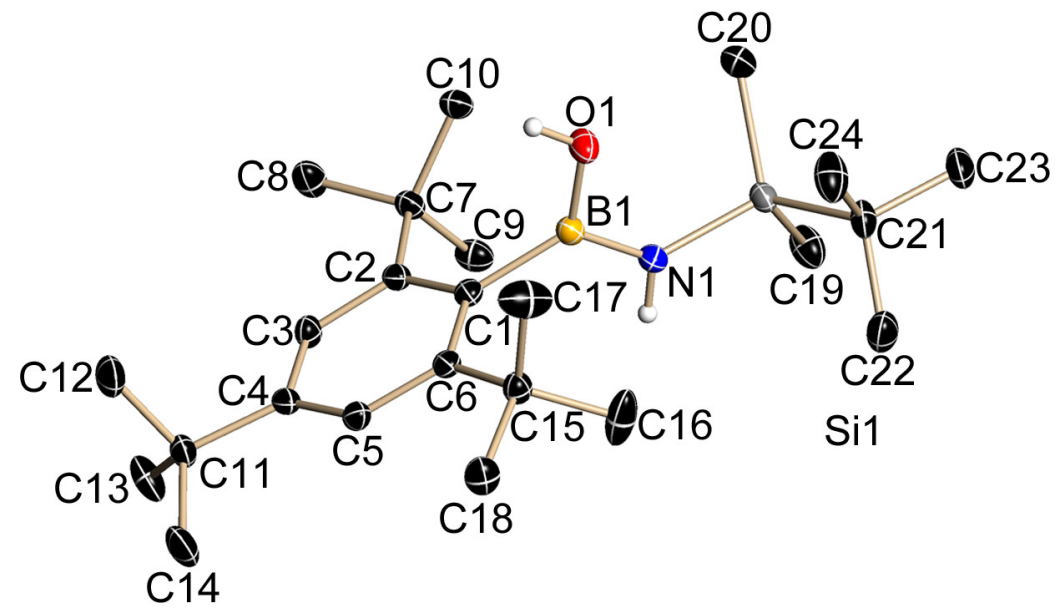

Figure 8-33. Asymmetric unit of $\left[(t \mathrm{Bu})_{3} \mathrm{Ph}\right] \mathrm{B}(\mathrm{OH}) \mathrm{N}(\mathrm{H}) \mathrm{Si}(\mathrm{Me})_{2} t \mathrm{Bu}$. Only freely refined hydrogen atoms shown.

$\left[(t \mathrm{Bu})_{3} \mathrm{Ph}\right] \mathrm{B}(\mathrm{OH}) \mathrm{N}(\mathrm{H}) \mathrm{Si}(\mathrm{Me})_{2} t \mathrm{Bu}$ crystallises in the non-centrosymmetric space group $P c a 2_{1}$ as monomers without detectable hydrogen bonds in the crystal. The absolute structure could not be determined (Flack $x=0.46(10)$ ).

Table 8-30. Crystallographic data for $\left[(t \mathrm{Bu})_{3} \mathrm{Ph}\right] \mathrm{B}(\mathrm{OH}) \mathrm{N}(\mathrm{H}) \mathrm{Si}(\mathrm{Me})_{2} t \mathrm{Bu}$.

\begin{tabular}{lc|lc}
\hline identification code & HOTTS016/st & min./max. transmission & $0.86 / 0.99$ \\
empirical formula & $\mathrm{C}_{24} \mathrm{H}_{46} \mathrm{BNOSi}$ & $\theta$ range $\left.{ }^{\circ}\right]$ & $2.72-25.35$ \\
molar mass $\left[\mathrm{g} \mathrm{mol}^{-1}\right]$ & 403.52 & completeness to $\theta_{\max }$ & 0.99 \\
crystal size $[\mathrm{mm}]$ & $0.40 \times 0.25 \times 0.20$ & redundancy to $\theta_{\text {max }}$ & 2.73 \\
temperature $[\mathrm{K}]$ & $100(2)$ & reflections collected & 18301 \\
crystal system & orthorhombic & independent reflections & 5154 \\
space group & $P c a 2_{1}$ & $R_{\text {int }} / R_{\sigma}$ & $0.0321 / 0.0268$ \\
$a[\AA]$ & $27.4263(16)$ & restraints/parameters & $1 / 273$ \\
$b[\AA]$ & $6.0748(4)$ & GoF & 1.052 \\
$c[\AA]$ & $14.9956(9)$ & $R 1$ (all data) & 0.0329 \\
$V\left[\AA^{3}\right]$ & $2498.4(3)$ & $R 1(I>2 \sigma(I))$ & 0.0308 \\
$Z$ & 4 & $w R 2($ all data $)$ & 0.0764 \\
$\rho_{\text {calc }}\left[\mathrm{g} \mathrm{cm}^{-3}\right]$ & 1.073 & $w R 2(I>2 \sigma(I))$ & 0.0752 \\
$\mu\left[\mathrm{mm}^{-1}\right]$ & 0.108 & diff. peak/hole $\left[\mathrm{e} \AA^{-3}\right]$ & $0.188 / 0.180$ \\
$F(000)$ & 896 & & \\
\hline
\end{tabular}




\subsubsection{6 $[t B u N(H) S i(C l) N(t B u)]_{2}$}
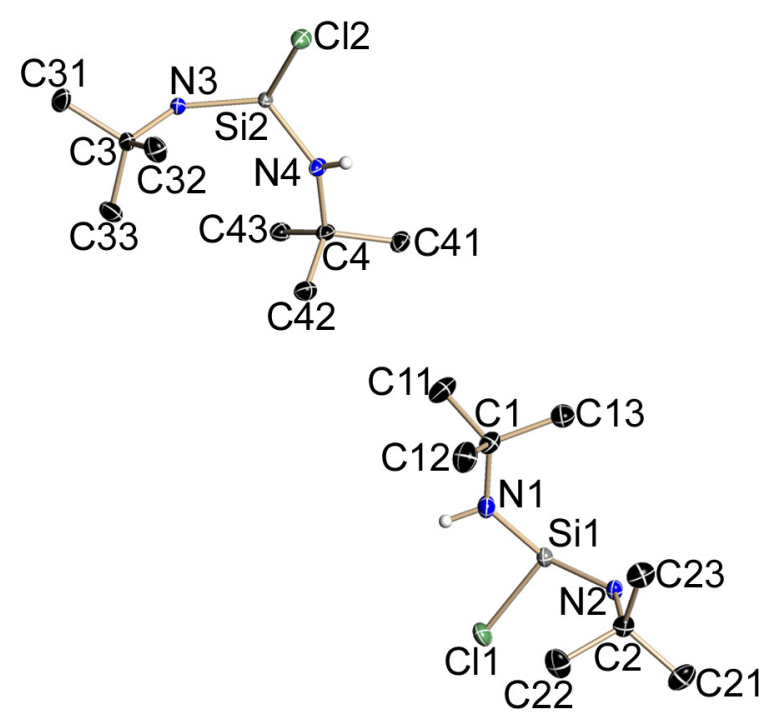

Figure 8-34. Asymmetric unit of $[t \mathrm{BuN}(\mathrm{H}) \mathrm{Si}(\mathrm{Cl}) \mathrm{N}(t \mathrm{Bu})]_{2}$. Constrained hydrogen atoms are omitted for clarity.

Two independent dimer halves are present in the asymmetric unit of $[t \mathrm{BuN}(\mathrm{H}) \mathrm{Si}(\mathrm{Cl}) \mathrm{N}(t \mathrm{Bu})]_{2}$. An inversion operation completes the dimers.

Table 8-31. Crystallographic data for $[t \mathrm{BuN}(\mathrm{H}) \mathrm{Si}(\mathrm{Cl}) \mathrm{N}(t \mathrm{Bu})]_{2}$.

\begin{tabular}{lc|lc}
\hline identification code & HOTTS018/st & $F(000)$ & 896 \\
empirical formula & $\mathrm{C}_{16} \mathrm{H}_{38} \mathrm{Cl}_{2} \mathrm{~N}_{4} \mathrm{Si}_{2}$ & min./max. transmission & $0.93 / 0.99$ \\
molar mass $\left[\mathrm{g} \mathrm{mol}^{-1}\right]$ & 413.58 & $\theta$ range $\left.{ }^{\circ}\right]$ & $2.17-26.02$ \\
crystal size $[\mathrm{mm}]$ & $0.20 \times 0.10 \times 0.04$ & completeness to $\theta_{\text {max }}$ & 1.00 \\
temperature $[\mathrm{K}]$ & $100(2)$ & redundancy to $\theta_{\max }$ & 2.96 \\
crystal system & monoclinic & reflections collected & 19261 \\
space group & $P 2_{1} / n$ & independent reflections & 4982 \\
$a[\AA]$ & $16.1553(6)$ & $R_{\text {int }} / R_{\sigma}$ & $0.0240 / 0.0207$ \\
$b[\AA]$ & $9.1740(4)$ & restraints/parameters & $2 / 235$ \\
$c[\AA]$ & $16.3306(6)$ & GoF & 1.074 \\
$\beta\left[{ }^{\circ}\right]$ & $109.4360(10)$ & $R 1$ (all data) & 0.0321 \\
$V\left[\AA^{3}\right]$ & $2282.41(16)$ & $R 1(I>2 \sigma(I))$ & 0.0279 \\
$Z$ & 4 & $w R 2($ all data) & 0.0755 \\
$\rho_{\text {calc }}\left[\mathrm{g} \mathrm{cm}^{-3}\right]$ & 1.204 & $w R 2(I>2 \sigma(I))$ & 0.0733 \\
$\mu\left[\mathrm{mm}^{-1}\right]$ & 0.396 & diff. peak/hole $\left[\mathrm{e} \AA^{-3}\right]$ & $0.447 /-0.221$ \\
\hline
\end{tabular}




\subsubsection{7 $\left[\left\{\mathrm{Si}(\mathrm{Bu})_{2} \mathrm{~N}(t \mathrm{Bu})_{2} \mathrm{Si}\right\}(\mathrm{NtBuLi})_{2}(\text { thf })_{3}\right]$}

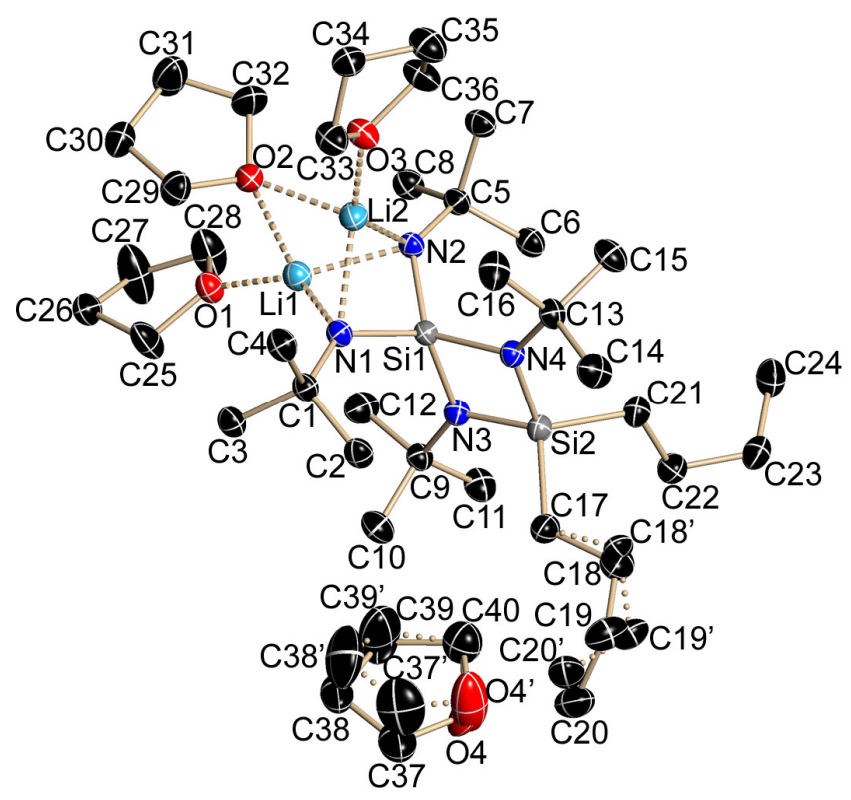

Figure 8-35. Asymmetric unit of $\left.\left[\left\{\mathrm{Si}(\mathrm{Bu})_{2} \mathrm{~N}(t \mathrm{Bu})_{2} \mathrm{Si}\right\}(\mathrm{N} t \mathrm{BuLi})_{2} \text { (thf }\right)_{3}\right]$. Hydrogen atoms excluded for clarity reasons.

The lithium salt shows slight disorder in one lattice thf molecule and one butyl side chain which could be stabilised with SADI, DELU, and SIMU commands.

Table 8-32. Crystallographic data for $\left[\left\{\mathrm{Si}(\mathrm{Bu})_{2} \mathrm{~N}(t \mathrm{Bu})_{2} \mathrm{Si}\right\}(\mathrm{N} t \mathrm{BuLi})_{2}(\text { thf })_{3}\right]$.

\begin{tabular}{lc|lc}
\hline identification code & HOTTS020/st & $F(000)$ & 1680 \\
empirical formula & $\mathrm{C}_{40} \mathrm{H}_{86} \mathrm{Li}_{2} \mathrm{~N}_{4} \mathrm{O}_{4} \mathrm{Si}_{2}$ & min./max. transmission & $0.81 / 0.99$ \\
molar mass $\left[\mathrm{g} \mathrm{mol}{ }^{-1}\right]$ & 757.19 & $\theta$ range $\left.{ }^{\circ}\right]$ & $2.10-25.36$ \\
crystal size $[\mathrm{mm}]$ & $0.20 \times 0.13 \times 0.10$ & completeness to $\theta_{\max }$ & 0.99 \\
temperature $[\mathrm{K}]$ & $100(2)$ & redundancy to $\theta_{\max }$ & 3.79 \\
crystal system & monoclinic & reflections collected & 44333 \\
space group & $P 2_{1} / n$ & independent reflections & 8958 \\
$a[\AA]$ & $18.073(6)$ & $R_{\text {int }} / R_{\sigma}$ & $0.0398 / 0.0305$ \\
$b[\AA]$ & $11.590(4)$ & restraints/parameters & $125 / 549$ \\
$c[\AA]$ & $22.485(7)$ & GoF & 1.054 \\
$\beta\left[{ }^{\circ}\right]$ & $99.126(4)$ & $R 1($ all data $)$ & 0.0617 \\
$V\left[\AA^{3}\right]$ & $4650(3)$ & $R 1(I>2 \sigma(I))$ & 0.0459 \\
$\mathrm{Z}$ & 4 & $w R 2($ all data $)$ & 0.1237 \\
$\rho_{\text {calc }}\left[\mathrm{g} \mathrm{cm}^{-3}\right]$ & 1.082 & $w R 2(I>2 \sigma(I))$ & 0.1160 \\
$\mu\left[\mathrm{mm}^{-1}\right]$ & 0.116 & diff. peak/hole $\left[\mathrm{e} \AA^{-3}\right]$ & $0.333 /-0.293$ \\
\hline
\end{tabular}




\subsubsection{8 $\left(\mathrm{Cl}_{3} \mathrm{Al}\right)\left(\mathrm{SiMe}_{3}\right) \mathrm{NBN}\left(\mathrm{SiMe}_{3}\right)_{2}$}

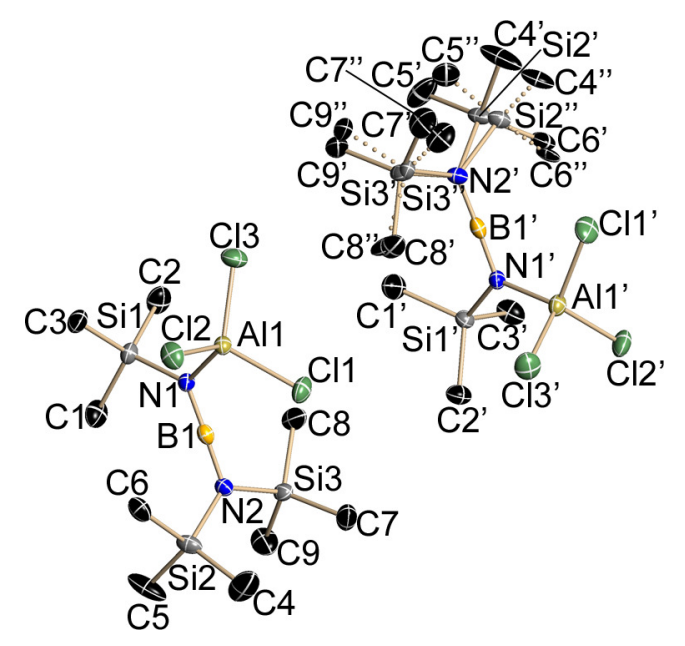

Figure 8-36. Asymmetric unit of $\left(\mathrm{Cl}_{3} \mathrm{Al}\right)\left(\mathrm{SiMe}_{3}\right) \mathrm{NBN}\left(\mathrm{SiMe}_{3}\right)_{2}$.

One molecule of the asymmetric unit is showing a disorder in the $\mathrm{N}\left(\mathrm{SiMe}_{3}\right)_{2}$ part, which could be refined with SAME, DELU, and SIMU commands, while the bonding situation of the second unit could be studied unambiguously.

Table 8-33. Crystallographic data for $\left(\mathrm{Cl}_{3} \mathrm{Al}\right)\left(\mathrm{SiMe}_{3}\right) \mathrm{NBN}\left(\mathrm{SiMe}_{3}\right)_{2}$.

\begin{tabular}{lc|lc}
\hline identification code & HOTTS021/st & $F(000)$ & 824 \\
empirical formula & $\mathrm{C}_{9} \mathrm{H}_{27} \mathrm{AlBCl}_{3} \mathrm{~N}_{2} \mathrm{Si}_{3}$ & min./max. transmission & $0.91 / 0.99$ \\
molar mass $\left[\mathrm{g} \mathrm{mol}^{-1}\right]$ & 391.74 & $\theta$ range $\left.{ }^{\circ}\right]$ & $2.29-25.68$ \\
crystal size $[\mathrm{mm}]$ & $0.20 \times 0.15 \times 0.05$ & completeness to $\theta_{\text {max }}$ & 0.99 \\
temperature $[\mathrm{K}]$ & $100(2)$ & redundancy to $\theta_{\text {max }}$ & 3.79 \\
crystal system & monoclinic & reflections collected & 40635 \\
space group & $P n$ & independent reflections & 8958 \\
$a[\AA]$ & $9.5289(4)$ & $R_{\text {int }} / R_{\sigma}$ & $0.0257 / 0.0197$ \\
$b[\AA]$ & $16.4713(7)$ & restraints/parameters & $189 / 416$ \\
$c[\AA]$ & $14.3882(6)$ & GoF & 1.036 \\
$\beta\left[{ }^{\circ}\right]$ & $107.5510(10)$ & $R 1$ (all data) & 0.0246 \\
$V\left[\AA^{3}\right]$ & $2153.15(16)$ & $R 1(I>2 \sigma(I))$ & 0.0232 \\
$\mathrm{Z}$ & 4 & $w R 2($ all data) & 0.0583 \\
$\rho_{\text {calc }}\left[\mathrm{g} \mathrm{cm}^{-3}\right]$ & 1.208 & $w R 2(I>2 \sigma(I))$ & 0.0575 \\
$\mu\left[\mathrm{mm}^{-1}\right]$ & 0.624 & diff. peak/hole $\left[\mathrm{e} \AA^{-3}\right]$ & $0.313 /-0.196$ \\
\hline
\end{tabular}




\subsubsection{9 $\left[\mathrm{Me}_{2} \mathrm{SiN}(t \mathrm{Bu}) \mathrm{N}(t \mathrm{Bu}) \mathrm{Si}\right]\left(\mathrm{N}(\mathrm{H}) \mathrm{Ph}(\mathrm{iPr})_{2}(t \mathrm{Bu})\right)_{2}$}

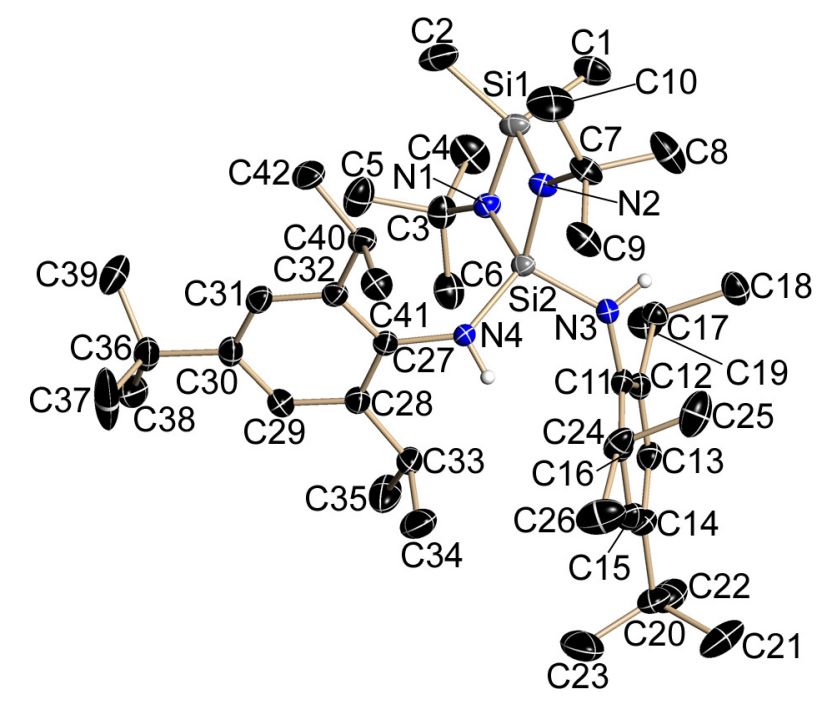

Figure 8-37. Asymmetric unit of $\left[\mathrm{Me}_{2} \mathrm{SiN}(t \mathrm{Bu}) \mathrm{N}(t \mathrm{Bu}) \mathrm{Si}\right]\left(\mathrm{N}(\mathrm{H}) \mathrm{Ph}(\mathrm{Pr})_{2}(t \mathrm{Bu})\right)_{2}$. Constrained hydrogen atoms are omitted for clarity.

The compound contains an $[\mathrm{SiN}]_{2}$ four-membered ring and crystallises in the monoclinic space group $P 2_{1} / c$ with one formula unit in the asymmetric unit.

Table 8-34. Crystallographic data for $\left[\mathrm{Me}_{2} \mathrm{SiN}(t \mathrm{Bu}) \mathrm{N}(t \mathrm{Bu}) \mathrm{Si}\right]\left(\mathrm{N}(\mathrm{H}) \mathrm{Ph}(i \mathrm{Pr})_{2}(t \mathrm{Bu})\right)_{2}$.

\begin{tabular}{lc|lc}
\hline identification code & HOTTS028/st & $F(000)$ & 1536 \\
empirical formula & $\mathrm{C}_{42} \mathrm{H}_{76} \mathrm{~N}_{4} \mathrm{Si}_{2}$ & min./max. transmission & $0.86 / 0.99$ \\
molar mass $\left[\mathrm{g} \mathrm{mol}{ }^{-1}\right]$ & 693.25 & $\theta$ range $\left[^{\circ}\right]$ & $2.17-25.03$ \\
crystal size $[\mathrm{mm}]$ & $0.20 \times 0.10 \times 0.05$ & completeness to $\theta_{\max }$ & 1.00 \\
temperature $[\mathrm{K}]$ & $100(2)$ & redundancy to $\theta_{\max }$ & 7.42 \\
crystal system & monoclinic & reflections collected & 79502 \\
space group & $P 2_{1} / \mathrm{C}$ & independent reflections & 8279 \\
$a[\AA]$ & $22.3056(14)$ & $R_{\text {int }} / R_{\sigma}$ & $0.0442 / 0.0220$ \\
$b[\AA]$ & $10.5520(7)$ & restraints/parameters & $2 / 461$ \\
$c[\AA]$ & $20.7085(13)$ & GoF & 1.067 \\
$\beta\left[{ }^{\circ}\right]$ & $115.2400(10)$ & $R 1($ all data $)$ & 0.0602 \\
$V\left[\AA^{3}\right]$ & $4408.8(5)$ & $R 1(I>2 \sigma(I))$ & 0.0535 \\
$Z$ & 4 & $w R 2($ all data $)$ & 0.1480 \\
$\rho_{\text {calc }}\left[\mathrm{g} \mathrm{cm}^{-3}\right]$ & 1.044 & $w R 2(I>2 \sigma(I))$ & 0.1430 \\
$\mu\left[\mathrm{mm}^{-1}\right]$ & 0.111 & diff. peak/hole $\left[\mathrm{e} \AA^{-3}\right]$ & $0.525 /-0.378$ \\
\hline
\end{tabular}




\subsubsection{0 (Cp* $)_{2} \mathrm{Zr}-\mathrm{O}-\mathrm{Ca}-\mathrm{N}\left(\mathrm{SiMe}_{3}\right)_{2}$}

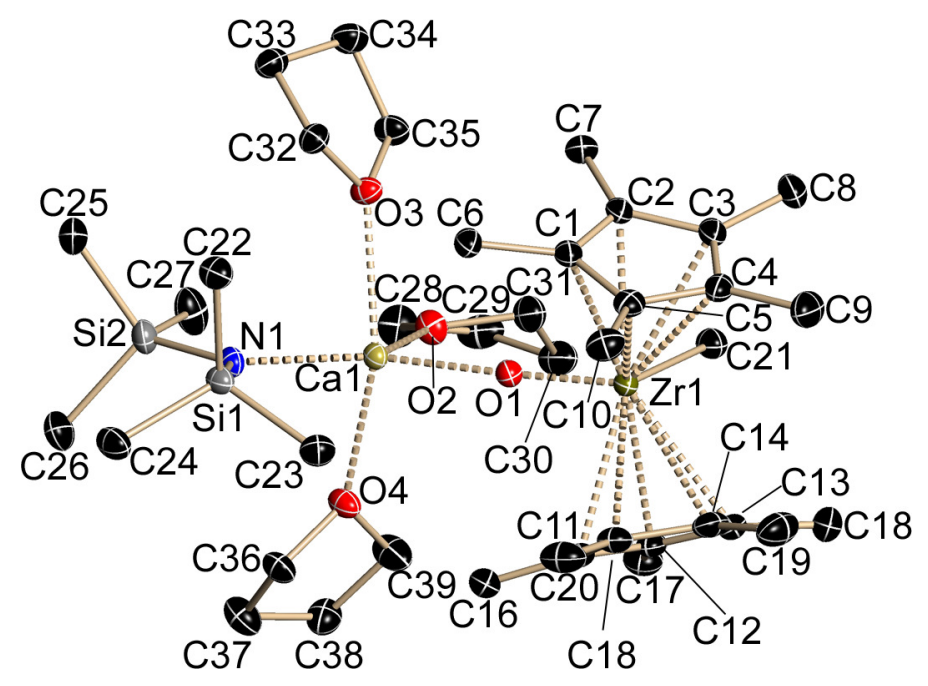

Figure 8-38. Asymmetric unit of $(\mathrm{Cp} *)_{2} \mathrm{Zr}-\mathrm{O}-\mathrm{Ca}-\mathrm{N}\left(\mathrm{SiMe}_{3}\right)_{2}$. All hydrogen atoms are omitted.

The zirconium calcium salt crystallises in the monoclinic space group $P 2_{1} / n$ with one formula unit in the asymmetric unit.

Table 8-35. Crystallographic data for $(\mathrm{Cp} *)_{2} \mathrm{Zr}-\mathrm{O}-\mathrm{Ca}-\mathrm{N}\left(\mathrm{SiMe}_{3}\right)_{2}$.

\begin{tabular}{lc|lc}
\hline identification code & HOTTS038/st & $F(000)$ & 1744 \\
empirical formula & $\mathrm{C}_{39} \mathrm{H}_{75} \mathrm{CaNO}_{4} \mathrm{Si}_{2} \mathrm{Zr}$ & min./max. transmission & $0.63 / 0.99$ \\
molar mass $\left[\mathrm{g} \mathrm{mol}^{-1}\right]$ & 809.48 & $\theta$ range $\left.{ }^{\circ}\right]$ & $3.02-26.03$ \\
crystal size $[\mathrm{mm}]$ & $0.40 \times 0.26 \times 0.20$ & completeness to $\theta_{\text {max }}$ & 0.99 \\
temperature $[\mathrm{K}]$ & $100(2)$ & redundancy to $\theta_{\text {max }}$ & 3.10 \\
crystal system & monoclinic & reflections collected & 37016 \\
space group & $P 2{ }_{1} / n$ & independent reflections & 8981 \\
$a[\AA]$ & $14.4608(12)$ & $R_{\text {int }} / R_{\sigma}$ & $0.0355 / 0.0317$ \\
$b[\AA]$ & $19.4611(16)$ & restraints/parameters & $0 / 450$ \\
$c[\AA]$ & $16.7106(15)$ & GoF & 1.048 \\
$\beta\left[{ }^{\circ}\right]$ & $109.4660(10)$ & $R 1$ (all data) & 0.0404 \\
$V\left[\AA^{3}\right]$ & $4433.9(7)$ & $R 1(I>2 \sigma(I))$ & 0.0306 \\
$Z$ & 4 & $w R 2($ all data) & 0.0715 \\
$\rho_{\text {calc }}\left[\mathrm{g} \mathrm{cm}^{-3}\right]$ & 1.213 & $w R 2(I>2 \sigma(I))$ & 0.0680 \\
$\mu\left[\mathrm{mm}^{-1}\right]$ & 0.453 & diff. peak/hole $\left[\mathrm{e} \AA^{-3}\right]$ & $0.320 /-0.320$ \\
\hline
\end{tabular}




\section{References}

[1] J. Deberitz, Lithium - Production and application of a fascinating and versatile element, Verlag Moderne Industrie, München, 2006.

[2] N. N. Greenwood, A. Earnshaw, Chemistry of the Elements, ButterworthHeinemann Ltd., 1984.

[3] W. Schlenk, J. Holtz, Ber. Dtsch. Chem. Ges. 1917, 50, 262.

[4] D. Seyferth, Organometallics 2009, 28, 2.

[5] R. G. Parr, R. G. Pearson, J. Am. Chem. Soc. 1983, 105, 7512.

[6] R. G. Pearson, J. Am. Chem. Soc. 1963, 85, 3533.

[7] W. Schlenk, A. Thal, Ber. Dtsch. Chem. Ges. 1913, 46, 2840.

[8] A. Maercker, Angew. Chem. 1987, 99, 1002; Angew. Chem. Int. Ed. Engl. 1987, 26, 972.

[9] R. L. Burwell, Jr., Chem. Rev. 1954, 54, 615.

[10] H. Gilman, F. K. Cartledge, J. Organomet. Chem. 1964, 2, 447.

[11] J. Suffert, J. Org. Chem. 1989, 54, 509.

[12] T. R. Hoye, B. M. Eklov, M. Voloshin, Org. Lett. 2004, 6, 2567.

[13] F. Leroux, M. Schlosser, E. Zohar, I. Marek, The preparation of organolithium reagents and intermediates in The chemistry of organolithium compounds (Eds.:

Z. Rappoport, I. Marek), John Wiley \& Sons, Chichester (GB), 2004, pp. 435.

[14] C. Elschenbroich, Organometallchemie, Teubner, Stuttgart, 2008.

[15] G. Wittig, U. Pockels, H. Dröge, Ber. Dtsch. Chem. Ges. 1938, 71, 1903.

[16] B. J. Wakefield, The Chemistry of Organolithium Compounds, Pergamon Press, Oxford, New York, 1974.

[17] M. Schlosser, Organoalkali Chemistry in Organometallics in Synthesis - A Manual (Ed. M. Schlosser), John Wiley \& Sons Ltd., Chichester, 2004, pp. 1.

[18] L. Brandsma, H. D. Verkruijsse, Preparative Polar Organometallic Chemistry 1, Springer, Berlin, 1987.

[19] L. Brandsma, Preparative Polar Organometallic Chemistry 2, Springer, Berlin, 1990, p. 195.

[20] V. Snieckus, Chem. Rev. 1990, 90, 879.

[21] J. Clayden, Directed metallation of aromatic compounds in The chemistry of organolithium compounds (Eds.: Z. Rappoport, I. Marek), John Wiley \& Sons, Chichester (GB), 2004, pp. 495.

[22] H. Yamataka, K. Yamada, K. Tomioka, Addition of organolithium reagents to double bonds in The chemistry of organolithium compounds (Eds.: Z. Rappoport, I. Marek), John Wiley \& Sons, Chichester (GB), 2004, pp. 901. 
[23] F. Chemla, E. Vrancken, Reactivity of oxiranes with organolithium reagents in The chemistry of organolithium compounds (Eds.: Z. Rappoport, I. Marek), John Wiley \& Sons, Chichester (GB), 2004, pp. 1165.

[24] G. Wittig, G. Geissler, Justus Liebigs Ann. Chem. 1953, 580, 44.

[25] G. Wu, M. Huang, Chem. Rev. 2006, 106, 2596.

[26] D. Hoppe, T. Hense, Angew. Chem. 1997, 109, 2376; Angew. Chem. Int. Ed. Engl. 1997, 36, 2282.

[27] D. Hoppe, G. Christoph, Asymmetric deprotonation with alkyllithium-(-)-sparteine in The chemistry of organolithium compounds (Eds.: Z. Rappoport, I. Marek), John Wiley \& Sons, Chichester (GB), 2004, pp. 1055.

[28] U. Schöllkopf, W. Hartwig, U. Groth, Angew. Chem. 1979, 91, 922; Angew. Chem. Int. Ed. Engl. 1979, 18, 863.

[29] B. H. Lipshutz, Organocopper Chemistry in Organometallics in Synthesis - A Manual (Ed. M. Schlosser), John Wiley \& Sons, Chichester, 2004, pp. 665.

[30] K. P. C. Vollhardt, N. E. Schore, Organische Chemie, Wiley VCH, Weinheim, 2005, p. 941.

[31] E. Kurras, J. Otto, J. Organomet. Chem. 1965, 4, 114.

[32] E. O. Fischer, A. Maasböl, Angew. Chem. 1964, 76, 645; Angew. Chem. Int. Ed. Engl. 1964, 3, 580.

[33] H. W. Gschwend, H. R. Rodriguez, Org. React. 1979, 26, 1.

[34] H. L. Hsieh, W. H. Glaze, Rubber Chem. Technol. 1970, 43, 22.

[35] M. Morton, L. J. Fetters, Rubber Chem. Technol. 1975, 48, 359.

[36] T. L. Brown, Adv. Organomet. Chem. 1965, 3, 365.

[37] T. L. Brown, Pure Appl. Chem. 1970, 23, 447.

[38] H. Dietrich, Acta Crystallogr., Sect. E. 1963, 16, 681.

[39] E. Weiss, E. A. C. Lucken, J. Organomet. Chem. 1964, 2, 197.

[40] H. L. Lewis, T. L. Brown, J. Am. Chem. Soc. 1970, 92, 4664.

[41] T. Kottke, D. Stalke, Angew. Chem. 1993, 105, 619; Angew. Chem. Int. Ed. Engl. 1993, 32, 580.

[42] E. Weiss, Angew. Chem. 1993, 105, 1565; Angew. Chem. Int. Ed. Engl. 1993, $32,1501$.

[43] H. Hope, Acta Crystallogr., Sect. B. 1988, 44, 22.

[44] T. Kottke, D. Stalke, J. Appl. Crystallogr. 1993, 26, 615.

[45] D. Stalke, Chem. Soc. Rev. 1998, 27, 171.

[46] Cambridge Structural Database, v5.29 (November 2007), Cambridge Crystallographic Data Centre, Cambridge, 2007.

[47] W. N. Setzer, P. v. R. Schleyer, Adv. Organomet. Chem. 1985, 24, 353. 
[48] T. Stey, D. Stalke, Lead structures in lithium organic chemistry in The chemistry of organolithium compounds (Eds.: Z. Rappoport, I. Marek), John Wiley \& Sons, Chichester (GB), 2004, pp. 47.

[49] E. Weiss, G. Hencken, J. Organomet. Chem. 1970, 21, 265.

[50] U. S. Siemeling, T. Redecker, B. Neumann, H.-G. Stammler, J. Am. Chem. Soc. 1994, 116, 5507.

[51] R. Zerger, W. Rhine, G. Stucky, J. Am. Chem. Soc. 1974, 96, 6048.

[52] W. Hiller, M. Layh, W. Uhl, Angew. Chem. 1991, 103, 339; Angew. Chem. Int. Ed. Engl. 1991, 30, 324.

[53] V. H. Gessner, C. Däschlein, C. Strohmann, Chem. Eur. J. 2009, DOI: 10.1002/chem.200900041.

[54] M. Schlosser, Struktur und Reaktivität polarer Organometalle, Springer, Berlin, 1973.

[55] C. D. Broaddus, J. Org. Chem. 1970, 35, 10.

[56] T. F. Bates, M. T. Clarke, R. D. Thomas, J. Am. Chem. Soc. 1988, 110, 5109.

[57] C. Strohmann, V. H. Gessner, Angew. Chem. 2007, 119, 4650; Angew. Chem. Int. Ed. 2007, 46, 4566.

[58] C. Strohmann, C. Däschlein, D. Auer, J. Am. Chem. Soc. 2006, 128, 704.

[59] A.-M. Sapse, D. C. Jain, K. Raghavachari, Theoretical Studies of Aggregates of Lithium Compounds in Lithium Chemistry - A Theoretical and Chemical Overview (Eds.: P. v. R. Schleyer, A.-M. Sapse), John Wiley \& Sons, New York, 1995, pp. 44.

[60] E. D. Jemmis, G. Gopakunar, Theoretical studies in organolithium chemistry in The chemistry of organolithium compounds (Eds.: Z. Rappoport, I. Marek), John Wiley \& Sons, Chichester (GB), 2004, pp. 1.

[61] R. F. W. Bader, Atoms in Molecules - A Quantum Theory, Oxford University Press, New York, 1990.

[62] P. Coppens, Angew. Chem. 2005, 117, 6970; Angew. Chem. Int. Ed. 2005, 44, 6810.

[63] C. Lecomte, M. Souhassou, S. Pillet, J. Mol. Struct. 2003, 647, 53.

[64] C. B. Hübschle, M. Messerschmidt, D. Lentz, P. Luger, Z. Anorg. Allg. Chem. 2004, 630, 1313.

[65] D. Förster, C. B. Hübschle, P. Luger, T. Hügle, D. Lentz, Inorg. Chem. 2008, 47, 1874.

[66] T. Haumann, R. Boese, S. I. Kozhushkov, K. Rauch, A. de Meijere, Liebigs Annalen - Recueil 1997, 2047.

[67] S. Grabowsky, T. Pfeuffer, L. Checinska, M. Weber, W. Morgenroth, P. Luger, T. Schirmeister, Eur. J. Org. Chem. 2007, 2759. 
[68] D. Lentz, M. Patzschke, A. Bach, S. Scheins, P. Luger, Org. Biomol. Chem. 2003, 1, 409.

[69] M. Messerschmidt, S. Scheins, L. Grubert, M. Pätzel, G. Szeimies, C. Paulmann, P. Luger, Angew. Chem. 2005, 117, 3993; Angew. Chem. Int. Ed. 2005, 44, 3925.

[70] D. Leusser, B. Walfort, D. Stalke, Angew. Chem. 2002, 114, 2183; Angew. Chem. Int. Ed. 2002, 41, 2079.

[71] N. Kocher, D. Leusser, A. Murso, D. Stalke, Chem. Eur. J. 2004, 10, 3622.

[72] D. Leusser, J. Henn, N. Kocher, B. Engels, D. Stalke, J. Am. Chem. Soc. 2004, 126, 1781.

[73] P. Macchi, A. Sironi, Coord. Chem. Rev. 2003, 238-239, 383.

[74] U. Flierler, M. Burzler, D. Leusser, J. Henn, H. Ott, H. Braunschweig, D. Stalke, Angew. Chem. 2008, 120, 4393; Angew. Chem. Int. Ed. 2008, 47, 4321.

[75] D. Stalke, H. Ott, Nachr. Chem. 2008, 56, 131.

[76] A. F. Holleman, N. Wiberg, Lehrbuch der Anorganischen Chemie, 102. Aufl., de Gruyter, Berlin, 2007.

[77] F. A. Cotton, G. Wilkinson, C. A. Murillo, M. Bochmann, Advanced inorganic chemistry, John Wiley \& Sons, Inc., New York, 1999.

[78] E. Riedel, Anorganische Chemie, Walter de Gruyter, Berlin, 2004.

[79] A. Streitwieser, S. M. Bachrach, A. Dorigo, P. v. R. Schleyer, Bonding, Structures and Energies in Organolithium Compounds in Lithium Chemistry - A Theoretical and Chemical Overview (Eds.: P. v. R. Schleyer, A.-M. Sapse), John Wiley \& Sons, New York, 1995, pp. 1.

[80] A. Streitwieser, J. Organomet. Chem. 1978, 156, 1.

[81] R. J. Bushby, H. L. Steel, J. Chem. Soc., Perkin Trans. 2 1990, 1143.

[82] H. Horn, R. Ahlrichs, J. Am. Chem. Soc. 1990, 112, 2121.

[83] T. Koizumi, O. Kikuchi, Organometallics 1995, 14, 987.

[84] F. M. Bickelhaupt, M. Solà, C. F. Guerra, J. Chem. Theory Comput. 2006, 2, 965.

[85] F. M. Bickelhaupt, N. J. R. van Eikema Hommes, C. F. Guerra, E. J. Baerends, Organometallics 1996, 15, 2923.

[86] W. Scherer, P. Sirsch, M. Grosche, M. Spiegler, S. A. Mason, M. G. Gardiner, Chem. Commun. 2001, 2072.

[87] W. Scherer, P. Sirsch, D. Shorokhov, G. S. McGrady, S. A. Mason, M. G. Gardiner, Chem. Eur. J. 2002, 8, 2324.

[88] S. Deuerlein, D. Leusser, U. Flierler, H. Ott, D. Stalke, Organometallics 2008, 27, 2306.

[89] J. A. Zoltewicz, L. S. Helmick, J. Org. Chem. 1973, 38, 658.

[90] R. R. Fraser, T. S. Mansour, S. Savard, J. Org. Chem. 1985, 50, 3232.

[91] A. Streitwieser, Jr., E. Juaristi, L. L. Nebenzahl, Equilibrium Carbon Acidities in Solution in Studies in Organic Chemistry 5 - Comprehensive Carbanion Chemistry 
Part A - Structure and Reactivity (Eds.: E. Buncel, T. Durst), Elsevier, Amsterdam, 1980, p. 323.

[92] K. Ziegler, H. Zeiser, Justus Liebigs Ann. Chem. 1931, 485, 174.

[93] F. W. Bergstrom, J. Am. Chem. Soc. 1931, 53, 4065.

[94] A. E. Chichibabin, Bull. Soc. Chim. Fr. 1936, 3, 1607.

[95] F. W. Bergstrom, T. R. Norton, R. A. Seibert, J. Org. Chem. 1945, 10, 452.

[96] H. C. Brown, W. A. Murphey, J. Am. Chem. Soc. 1951, 73, 3308.

[97] O. F. Beumel, W. N. Smith, B. Rybalka, Synthesis 1974, 1, 43.

[98] E. M. Kaiser, J. D. Petty, Synthesis 1975, 705.

[99] L. E. Tenenbaum, Alkylpyridines and Arylpyridines in The Chemistry of Heterocyclic Compounds - Pyridine and Its Derivatives - Part Two (Ed. E. Klingsberg), Interscience Publishers, New York, 1961, p. 155.

[100] H. L. Yale, Organometallic Compounds of Pyridine in The Chemistry of Heterocyclic Compounds - Pyridine and Its Derivatives - Part Two (Ed. E. Klingsberg), Interscience Publishers, New York, 1961, p. 421.

[101] H. L. Yale, Organometallic Compounds of Pyridine in The Chemistry of Heterocyclic Compounds - Pyridine and Its Derivatives - Part Two Supplement (Ed. R. A. Abramovitch), John Wiley \& Sons, New York, 1974, p. 489.

[102] R. G. Micetich, Alkylpyridines and Arylpyridines in The Chemistry of Heterocyclic Compounds - Pyridine and Its Derivatives - Part Two Supplement (Ed. R. A. Abramovitch), John Wiley \& Sons, New York, 1974, p. 263.

[103] S. W. Horgan, F. P. Palopoli, E. J. Schwoegler, (Richardson-Merrell Inc., USA), US 4069222, 1977.

[104] N. Vedernikov, R. Miftakhov, S. V. Borisoglebski, K. G. Caulton, B. N. Solomonov, Chem. Heterocycl. Compd. 2002, 38, 406.

[105] A. J. Canty, J. Patel, B. W. Skelton, A. H. White, J. Organomet. Chem. 2000, 607, 194.

[106] T. Kauffmann, M. Bisling, R. König, A. Rensing, F. Steinseifer, Chem. Ber. 1985, $118,4517$.

[107] U. Pieper, Dissertation, Göttingen, 1993.

[108] J. Verbeek, L. Brandsma, J. Org. Chem. 1984, 49, 3857.

[109] E. Profft, F. Schneider, J. Prakt. Chem. 1955, 2, 316.

[110] H. S. Mosher, J. E. Tessieri, J. Am. Chem. Soc. 1951, 73, 4925.

[111] A. Kermagoret, F. Tomicki, P. Braunstein, Dalton Trans. 2008, 22, 2901.

[112] K. K. Klausmeyer, F. Hung, Acta Crystallogr., Sect. E. 2006, 62, m2415.

[113] E. Lindner, H. Rauleder, P. Wegner, Z. Naturforsch. 1984, 39b, 1224.

[114] E. Lindner, H. Rauleder, W. Hiller, Z. Naturforsch. 1983, 38b, 417.

[115] E. Uhlig, M. Schäfer, Z. Anorg. Allg. Chem. 1968, 359, 67.

[116] A. Murso, D. Stalke, Dalton Trans. 2004, 2563. 
[117] A. Murso, M. Straka, M. Kaupp, R. Bertermann, D. Stalke, Organometallics 2005, $24,3576$.

[118] H. Staudinger, J. Meyer, Helv. Chim. Acta 1919, 2, 635.

[119] A. Murso, D. Stalke, Z. Anorg. Allg. Chem. 2004, 630, 1025.

[120] A. Murso, D. Stalke, Eur. J. Inorg. Chem. 2004, 4272.

[121] J. C. Jeffrey, T. B. Rauchfuss, Inorg. Chem. 1979, 18, 2658.

[122] I. Objartel, H. Ott, D. Stalke, Z. Anorg. Allg. Chem. 2008, 634, 2373.

[123] X.-B. Jiang, L. Lefort, P. E. Goudriaan, A. H. M. de Vries, P. W. N. M. van Leeuwen, J. G. de Vries, J. N. H. Reek, Angew. Chem. 2006, 118, 1245; Angew. Chem. Int. Ed. 2006, 45, 1223.

[124] G. Hilt, C. Walter, P. Bolze, Adv. Synth. Catal. 2006, 348, 1241.

[125] A. Aeby, G. Consiglio, Inorg. Chim. Acta 1999, 296, 45.

[126] M. Veith, S. Weidner, K. Kunze, D. Käfer, J. Hans, V. Huch, Coord. Chem. Rev. 1994, 137, 297.

[127] F. Baier, Z. Fei, H. Gornitzka, A. Murso, S. Neufeld, M. Pfeiffer, I. Rüdenauer, A. Steiner, T. Stey, D. Stalke, J. Organomet. Chem. 2002, 661, 111.

[128] T. Stey, J. Henn, D. Stalke, Chem. Commun. 2007, 413.

[129] Y. H. Kim, T. H. Kim, N. Y. Kim, E. S. Cho, B. Y. Lee, D. M. Shin, Y. K. Chung, Organometallics 2003, 22, 1503.

[130] R. Duchateau, E. A. C. Brussee, A. Meetsma, J. H. Teuben, Organometallics 1997, $16,5506$.

[131] T. v. d. Ancker, C. L. Raston, J. Organomet. Chem. 1995, 500, 289.

[132] A. Molter, F. Mohr, Z. Anorg. Allg. Chem. 2009, 635, 134.

[133] P. v. R. Schleyer, R. Hacker, H. Dietrich, W. Mahdi, J. Chem. Soc., Chem. Commun. 1985, 622.

[134] D. Colgan, R. I. Papasergio, C. L. Raston, A. H. White, J. Chem. Soc., Chem. Commun. 1984, 1708.

[135] R. I. Papasergio, B. W. Skelton, P. Twiss, A. H. White, C. L. Raston, J. Chem. Soc., Dalton Trans. 1990, 1161.

[136] K. Konishi, K. Takahashi, R. Asami, Bull. Chem. Soc. Jpn. 1971, 44, 2281.

[137] K. Takahashi, K. Konishi, M. Ushio, M. Takaki, R. Asami, J. Organomet. Chem. 1973, 50, 1.

[138] K. Konishi, K. Takahashi, Bull. Chem. Soc. Jpn. 1977, 50, 2512.

[139] K. Konishi, A. Yoshino, M. Katoh, K. Takahashi, Y. Kawada, T. Sugawara, H. Iwamura, Bull. Chem. Soc. Jpn. 1981, 54, 3117.

[140] K. Konishi, K. Takahashi, Bull. Chem. Soc. Jpn. 1983, 56, 1612.

[141] K. Konishi, H. Matsumoto, K. Saito, K. Takahashi, Bull. Chem. Soc. Jpn. 1985, 58, 2294. 
[142] R. I. Papasergio, C. L. Raston, A. H. White, J. Chem. Soc., Chem. Commun. 1983, 1419.

[143] W.-P. Leung, L.-H. Weng, R.-J. Wang, T. C. W. Mak, Organometallics 1995, 14, 4832.

[144] P. C. Andrews, D. R. Armstrong, C. L. Raston, B. A. Roberts, B. W. Skelton, A. H. White, J. Chem. Soc., Dalton Trans. 2001, 996.

[145] E. Anders, A. Opitz, R. Boese, Chem. Ber. 1992, 125, 1267.

[146] E. Anders, A. Opitz, N. J. R. v. Eikema Hommes, F. Hampel, J. Org. Chem. 1993, $58,4424$.

[147] T. S. Mansour, T. C. Wong, E. M. Kaiser, J. Chem. Soc., Perkin Trans. 2 1985, 2045.

[148] E. Anders, A. Opitz, W. Bauer, Synthesis 1991, 1221.

[149] H. Ott, Diplomarbeit, Würzburg, 2006.

[150] A. D. Bond, J. E. Davies, Acta Crystallogr., Sect. E. 2001, 57, 01089.

[151] P. v. R. Schleyer, T. Clark, A. J. Kos, G. W. Spitznagel, C. Rohde, D. Arad, K. N. Houk, N. G. Rondan, J. Am. Chem. Soc. 1984, 106, 6467.

[152] P. Rademacher, Strukturen organischer Moleküle, VCH, New York, 1987.

[153] P. v. R. Schleyer, A. J. Kos, Tetrahedron 1983, 39, 1141.

[154] A. E. Reed, C. Schade, P. v. R. Schleyer, P. V. Kamath, J. Chandrasekhar, J. Chem. Soc., Chem. Commun. 1988, 67.

[155] A. E. Reed, P. v. R. Schleyer, J. Am. Chem. Soc. 1990, 112, 1434.

[156] U. Salzner, P. v. R. Schleyer, J. Am. Chem. Soc. 1993, 115, 10231.

[157] A. F. Pozharskii, A. M. Simonov, V. N. Doron'kin, Russ. Chem. Rev. 1978, 47, 1042.

[158] K. Ziegler, H. Zeiser, Chem. Ber. 1930, 63, 1847.

[159] S. P. Patterman, I. L. Karle, G. D. Stucky, J. Am. Chem. Soc. 1970, 92, 1150.

[160] J. J. Brooks, W. Rhine, G. D. Stucky, J. Am. Chem. Soc. 1972, 94, 7339.

[161] N. Finkelmeier, Diplomarbeit, Göttingen, 2008.

[162] U. Pieper, D. Stalke, Organometallics 1993, 12, 1201.

[163] C. Strohmann, T. Seibel, K. Strohfeldt, Angew. Chem. 2003, 115, 4669; Angew. Chem. Int. Ed. 2003, 42, 4531.

[164] U. Schümann, J. Kopf, E. Weiss, Angew. Chem. 1985, 97, 222; Angew. Chem. Int. Ed. Engl. 1985, 24, 215.

[165] T. Tatic, H. Ott, D. Stalke, Eur. J. Inorg. Chem. 2008, 3765.

[166] T. Maetzke, D. Seebach, Helv. Chim. Acta 1989, 72, 624.

[167] W. Massa, Crystal Structure Determination, Springer, Berlin, 2004.

[168] W. H. Bragg, W. L. Bragg, Proc. Roy. Soc. A 1913, 88, 428. 
[169] B. K. Vainshtein, Fundamentals of Crystals - Symmetry, and Methods of Structural Crystallography in Modern Crystallography, Vol. 1 (Ed. B. K. Vainshtein), Springer, Berlin, 1996.

[170] P. Coppens, X-Ray Charge Densities and Chemical Bonding, Oxford University Press, Oxford, 1997.

[171] F. H. Allen, Acta Crystallogr., Sect. B. 1986, 42, 515.

[172] E. Keller, Chem. Unserer Zeit, 1982, 16, 71.

[173] E. Keller, Chem. Unserer Zeit, 1982, 16, 116.

[174] N. K. Hansen, P. Coppens, Acta Crystallogr., Sect. A. 1978, 34, 909.

[175] K. Meindl, J. Henn, Acta Crystallogr., Sect. A. 2008, 64404.

[176] D. Cremer, E. Kraka, Angew. Chem. 1984, 96, 612; Angew. Chem. Int. Ed. Engl. 1984, 23, 627.

[177] R. F. W. Bader, J. Phys. Chem. A 1998, 102, 7314.

[178] R. G. A. Bone, R. F. W. Bader, J. Phys. Chem. 1996, 100, 10892.

[179] C. F. Matta, J. Hernández-Trujillo, T.-H. Tang, R. F. W. Bader, Chem. Eur. J. 2003, 9, 1940.

[180] R. F. W. Bader, T. S. Slee, D. Cremer, E. Kraka, J. Am. Chem. Soc. 1983, 105, 5061.

[181] R. F. W. Bader, H. Essén, J. Chem. Phys. 1984, 80, 1943.

[182] R. F. W. Bader, P. M. Beddall, J. Chem. Phys. 1972, 56, 3320.

[183] N. Kocher, J. Henn, B. Gostevskii, D. Kost, I. Kalikhman, B. Engels, D. Stalke, J. Am. Chem. Soc. 2004, 136, 5563.

[184] J. Henn, D. Ilge, D. Leusser, D. Stalke, B. Engels, J. Phys. Chem. A 2004, 108, 9442.

[185] T. S. Koritsanszky, P. Coppens, Chem. Rev. 2001, 101, 1583.

[186] Bruker APEX v2.1-0, Bruker AXS Inst. Inc., Madison (WI, USA), 2007.

[187] SAINT v7.23A, Bruker AXS Inst. Inc., Madison (WI, USA), 2005.

[188] G. M. Sheldrick, SADABS 2008/1, Göttingen, 2008.

[189] G. M. Sheldrick, XPREP Version 2005/2 for Windows, Bruker AXS Inst. Inc., Madison (WI, USA), 2005.

[190] G. M. Sheldrick, Acta Crystallogr., Sect. A. 1990, 46, 467.

[191] G. M. Sheldrick, Acta Crystallogr., Sect. A. 2008, 64, 112.

[192] G. M. Sheldrick, XP in SHELXTL 6.10, Bruker AXS Inst. Inc., Madison (WI, USA), 2000.

[193] A. Volkov, P. Macchi, L. J. Farrugia, C. Gatti, P. R. Mallinson, T. Richter, T. Koritsanszky, XD2006, A Computer Program Package for Multipole Refinement, Topological Analysis of Charge Densities and Evaluation of Intermolecular Energies from Experimental or Theoretical Structure Factors, 2006.

[194] P. Macchi, P. Coppens, Acta Crystallogr., Sect. A. 2001, 57, 656. 
[195] Z. Su, P. Coppens, Acta Crystallogr., Sect. A. 1998, 54, 646.

[196] E. Clementi, D. L. Raimondi, J. Chem. Phys. 1963, 38, 2686.

[197] E. Clementi, C. Roetti, Atom. Data Nuc. Data Tab. 1974, 14, 177.

[198] A. Volkov, Y. A. Abramov, P. Coppens, Acta Crystallogr., Sect. A. 2001, 57, 272.

[199] F. L. Hirshfeld, Acta Crystallogr., Sect. A. 1976, 32, 239.

[200] C. Jones, C. H. L. Kennard, C. L. Raston, G. Smith, J. Organomet. Chem. 1990, 396, C39.

[201] C. Lee, W. Yang, R. G. Parr, Phys. Rev. 1988, B37, 785.

[202] A. D. Becke, J. Chem. Phys. 1993, 98, 5648.

[203] R. Krishnan, J. S. Binkley, R. Seeger, J. A. Pople, J. Chem. Phys. 1980, 72, 650.

[204] A. D. McLean, G. S. Chandler, J. Chem. Phys. 1980, 72, 5639.

[205] F. Biegler-König, J. Schönbohm, D. Bayles, J. Comput. Chem. 2001, 22, 545.

[206] H. Ott, U. Pieper, D. Leusser, U. Flierler, J. Henn, D. Stalke, Angew. Chem. 2009, DOI: 10.1002 /anie.200806221.

[207] A. Haaland, D. J. Shorokhov, N. V. Tverdova, Chem. Eur. J. 2003, 10, 4416.

[208] L. J. Farrugia, C. Evans, M. Tegel, J. Phys. Chem. A 2006, 110, 7952.

[209] R. Destro, F. Merati, Acta Crystallogr., Sect. B. 1995, 51, 559.

[210] R. F. W. Bader, J. Phys. Chem. 1998, A102, 7314.

[211] A. M. Pendás, E. Francisco, M. A. Blanco, C. Gatti, Chem. Eur. J. 2007, 13, 9362.

[212] J. Henn, D. Leusser, D. Stalke, J. Comput. Chem. 2007, 28, 2317.

[213] S. Dahaoui, V. Pichon-Pesme, J. A. K. Howard, C. Lecomte, J. Phys. Chem. A 1999, 103, 6240 .

[214] D. E. Hibbs, J. R. Hanrahan, M. B. Hursthouse, D. W. Knight, J. Overgaard, P. Turner, R. O. Piltz, M. P. Waller, Org. Biomol. Chem. 2003, 1, 1034.

[215] R. Destro, R. Soave, M. Barzaghi, L. Lo Presti, Chem. Eur. J. 2005, 11, 4621.

[216] I. V. Glukhov, K. A. Lyssenko, A. A. Korlyukov, M. Y. Antipin, Russ. Chem. Bull., Int. Ed. 2005, 54, 547.

[217] D. Chopra, T. S. Cameron, J. D. Ferrara, T. N. Guru Row, J. Phys. Chem. A 2006, 110, 10465.

[218] A. Volkov, Y. A. Abramov, P. Coppens, C. Gatti, Acta Crystallogr., Sect. A. 2000, $56,332$.

[219] A. Volkov, P. Coppens, Acta Crystallogr., Sect. A. 2001, 57, 395.

[220] N. Kocher, Dissertation, Würzburg, 2003.

[221] P. Sirsch, Dissertation, Augsburg, 2003.

[222] R. D. Shannon, Acta Crystallogr., Sect. A. 1976, 32, 751.

[223] C. B. Hübschle, P. Luger, J. Appl. Crystallogr. 2006, 39, 901.

[224] M. J. S. Dewar, W. Thiel, J. Am. Chem. Soc. 1977, 99, 4899.

[225] Series published in Bull. Chem. Soc. Jpn., Chem. Lett., and J. Organomet. Chem.

[226] H. E. Gottlieb, V. Kotlyar, A. Nudelman, J. Org. Chem. 1997, 62, 7512. 
[227] B. Wrackmeyer, G. Kehr, H. Zhou, S. Ali, Magn. Reson. Chem. 1996, 34, 921.

[228] G. Fraenkel, J. M. Geckle, J. Am. Chem. Soc. 1980, 102, 2869.

[229] G. Fraenkel, K. V. Martin, J. Am. Chem. Soc. 1995, 117, 10336.

[230] H. Barjat, G. A. Morris, S. Smart, A. G. Swanson, S. C. R. Williams, J. Magn. Reson. 1995, B108, 170.

[231] Y. Cohen, L. Avram, L. Frish, Angew. Chem. 2005, 117, 524; Angew. Chem. Int. Ed. 2005, 44, 520.

[232] I. Fernández, E. Martínez-Viviente, F. Breher, P. S. Pregosin, Chem. Eur. J. 2005, $11,1495$.

[233] P. W. Atkins, J. de Paula, Physikalische Chemie, 4. Auflage, Wiley-VCH, Weinheim, 2006.

[234] M. Karplus, J. Chem. Phys. 1959, 30, 11.

[235] M. Karplus, J. Am. Chem. Soc. 1963, 85, 2870.

[236] M. Hesse, H. Meier, B. Zeeh, Spektroskopische Methoden in der organischen Chemie, 7. Aufl. Thieme, Stuttgart, 2005.

[237] F. H. Köhler, N. Hertkorn, J. Blümel, Chem. Ber. 1987, 120, 2081.

[238] N. P. Lorenzen, J. Kopf, F. Olbrich, U. Schümann, E. Weiss, Angew. Chem. 1990, 102, 1481; Angew. Chem. Int. Ed. Engl. 1990, 29, 1441.

[239] R. Pi, W. Bauer, B. Brix, C. Schade, P. v. R. Schleyer, J. Organomet. Chem. 1986, 306, C1.

[240] L. Lochmann, Eur. J. Inorg. Chem. 2000, 1115.

[241] M. Schlosser, Pure Appl. Chem. 1988, 60, 1627.

[242] M. Schlosser, J. Organomet. Chem. 1967, 8, 9.

[243] L. Lochmann, J. Pospísil, D. Lím, Tetrahedron Lett. 1966, 2, 257.

[244] L. Lochmann, J. Trekoval, Collect. Czech. Chem. Commun. 1988, 53, 76.

[245] H. Gilman, A. L. Jacoby, H. Ludeman, J. Am. Chem. Soc. 1938, 60, 2336.

[246] N. Collignon, J. Organomet. Chem. 1975, 96, 139.

[247] L. Lochmann, J. Trekoval, J. Organomet. Chem. 1987, 326, 1.

[248] M. Schlosser, J. Hartmann, Angew. Chem. 1973, 85, 544; Angew. Chem. Int. Ed. Engl. 1973, 12, 439.

[249] I. R. Butler, W. R. Culen, J. Ni, S. J. Rettig, Organometallics 1985, 4, 2196.

[250] M. D. Rausch, G. A. Moser, C. F. Meade, J. Organomet. Chem. 1973, 51, 1.

[251] M. D. Rausch, D. J. Ciappenelli, J. Organomet. Chem. 1967, 10, 127.

[252] W. Clegg, K. W. Henderson, A. R. Kennedy, R. E. Mulvey, C. T. O'Hara, R. B. Rowlings, D. M. Tooke, Angew. Chem. 2001, 113, 4020; Angew. Chem. Int. Ed. 2001, 40, 3902.

[253] R. Tedesco, R. Fiaschi, E. Napolitano, Synthesis 1995, 1493.

[254] C. Schade, W. Bauer, P. v. R. Schleyer, J. Organomet. Chem. 1985, 295, C25.

[255] C. Schade, P. v. R. Schleyer, Adv. Organomet. Chem. 1987, 27, 169. 
[256] M. J. Weiss, C. R. Hauser, J. Am. Chem. Soc. 1949, 71, 2023.

[257] C. Schade, P. v. R. Schleyer, J. Am. Chem. Soc. 1986, 108, 2484.

[258] G. G. Eberhardt, W. A. Butte, J. Org. Chem. 1964, 29, 2928.

[259] D. R. Baker, W. Clegg, L. Horsburgh, R. E. Mulvey, Organometallics 1994, 13, 4170.

[260] D. Barr, W. Clegg, R. E. Mulvey, R. Snaith, J. Chem. Soc., Chem. Commun. 1989, 57.

[261] D. R. Baker, R. E. Mulvey, W. Clegg, P. A. O'Neil, J. Am. Chem. Soc. 1993, 115, 6472.

[262] F. Pauer, P. P. Power, Structures of Lihtium Salts of Heteroatom Compounds in Lithium Chemistry - A Theoretical and Chemical Overview (Eds.: P. v. R. Schleyer, A.-M. Sapse), John Wiley \& Sons, New York, 1995, pp. 295.

[263] R. E. Mulvey, Chem. Soc. Rev. 1991, 20, 167.

[264] X. Wei, Q. Dong, H. Tong, J. Chao, D. Liu, M. F. Lappert, Angew. Chem. 2008, 120, 4040; Angew. Chem. Int. Ed. 2008, 120, 4040.

[265] J. Knizek, I. Krossing, H. Nöth, H. Schwenk, T. Seifert, Chem. Ber. 1997, 130, 1053.

[266] F. Antolini, P. B. Hitchcock, A. V. Khvostov, M. F. Lappert, Eur. J. Inorg. Chem. 2003, 3391.

[267] H. Gilman, H. S. Broadbent, J. Am. Chem. Soc. 1948, 70, 2809.

[268] B. Prijs, A. H. Lutz, H. Erlenmeyer, Helv. Chim. Acta 1948, 31, 571.

[269] C. Osuch, R. Levine, J. Org. Chem. 1957, 22, 939.

[270] U. Ohms, H. Guth, W. Treutmann, G. Heger, J. Chem. Phys. 1985, 83, 273.

[271] P. Beak, A. I. Meyers, Acc. Chem. Res. 1986, 19, 356.

[272] K. J. Izod, P. Thornton, Polyhedron 1993, 12, 1613.

[273] E. Anders, U. Korn, A. Stankowiak, Chem. Ber. 1989, 122, 105.

[274] J. W. Connolly, G. Urry, Inorg. Chem. 1963, 2, 645.

[275] D. J. Peterson, J. Organomet. Chem. 1967, 9, 373.

[276] A. Doudouh, P. C. Gros, Y. Fort, C. Woltermann, Tetrahedron 2006, 62, 6166.

[277] P. C. Gros, A. Doudouh, C. Woltermann, Chem. Commun. 2006, 2673.

[278] A. Doudouh, C. Woltermann, P. C. Gros, J. Org. Chem. 2007, 72, 4978.

[279] P. C. Gros, A. Doudouh, C. Woltermann, Org. Biomol. Chem. 2006, 4, 4331.

[280] J. M. Yu, D. Teyssié, R. B. Khalifa, S. Bolleau, Polym. Bull. 1994, 32, 35.

[281] B. Tecle, A. F. M. M. Rahman, J. P. Oliver, J. Organomet. Chem. 1986, 317, 267.

[282] T. Tatic, Diplomarbeit, Göttingen, 2007.

[283] C. Strohmann, unpublished results.

[284] M. S. Medley, Master Thesis, Denton, Texas (US), 2004.

[285] Bruker AXS Inc., SAINT v7.46A, Bruker AXS Inst. Inc., Madison (WI, USA), 2007.

[286] G. M. Sheldrick, SADABS 2008/2, Göttingen, 2008. 
[287] D. Braga, F. Grepioni, K. Biradha, G. R. Desiraju, J. Chem. Soc., Dalton Trans. 1996, 3925.

[288] J. P. Ritchie, S. M. Bachrach, J. Am. Chem. Soc. 1987, 109, 5909.

[289] C. Gatti, P. Fantucci, G. Pacchioni, Theor. Chim. Acta 1987, 72, 433.

[290] R. J. Gillespie, Coord. Chem. Rev. 2008, 252, 1315.

[291] R. J. Gillespie, Coord. Chem. Rev. 2000, 197, 51.

[292] R. J. Gillespie, E. A. Robinson, Angew. Chem. 1996, 108, 539; Angew. Chem. Int. Ed. Engl. 1996, 35, 477.

[293] H. Ott, unpublished results.

[294] R. Ponec, J. Roithova, X. Gironés, L. Lain, A. Torre, R. Bochicchio, J. Phys. Chem. 2002, A106, 1019.

[295] R. F. W. Bader, P. J. MacDougall, J. Am. Chem. Soc. 1985, 107, 6788.

[296] D. B. Grotjahn, T. C. Pesch, J. Xin, L. M. Ziurys, J. Am. Chem. Soc. 1997, 119, 12368.

[297] D. L. Cooper, J. Gerratt, P. B. Karadakov, M. Raimondi, J. Chem. Soc., Faraday Trans. 1995, 91, 3363.

[298] A. E. Reed, R. B. Weinstock, F. Weinhold, J. Chem. Phys. 1985, 83, 735.

[299] N. Kocher, C. Selinka, D. Leusser, D. Kost, I. Kalikhman, D. Stalke, Z. Anorg. Allg. Chem. 2004, 630, 1777.

[300] P. Beak, D. R. Anderson, M. D. Curtis, J. M. Laumer, D. J. Pippel, G. A. Weisenburger, Acc. Chem. Res. 2000, 33, 715.

[301] A. Basu, S. Thayumanavan, Angew. Chem. 2002, 114, 740; Angew. Chem. Int. Ed. 2002, 41, 716.

[302] D. Hoppe, F. Marr, M. Brüggemann, Enantioselective Synthesis by Lithiation Adjacent to Oxygen and Electrophile Incorporation in Topics in Organometallic Chemistry, Vol. 5 (Ed. D. M. Hodgson), Springer, Berlin, 2003, pp. 61.

[303] T. H. Chan, P. J. Pellon, J. Am. Chem. Soc. 1989, 111, 8737.

[304] P. Beak, T. A. Johnson, D. D. Kim, S. H. Lim, Enantioselective Synthesis by Lithiation Adjacent to Nitrogen and Electrophile Incorporation in Topics in Organometallic Chemistry, Vol. 5 (Ed. D. M. Hodgson), Springer, Berlin, 2003, pp. 139.

[305] R. E. Gawley, I. Coldham, $\alpha$-Amino-organolithium compounds in The chemistry of organolithium compounds (Eds.: Z. Rappoport, I. Marek), John Wiley \& Sons, Chichester (GB), 2004, pp. 997.

[306] B. Kaiser, D. Hoppe, Angew. Chem. 1995, 107, 344; Angew. Chem. Int. Ed. Engl. 1995, 34, 323.

[307] D. Hoppe, B. Kaiser, O. Stratmann, R. Fröhlich, Angew. Chem. 1997, 109, 2872; Angew. Chem. Int. Ed. Engl. 1997, 36, 2784. 
[308] C. Strohmann, D. H. M. Buchhold, T. Seibel, K. Wild, D. Schildbach, Eur. J. Inorg. Chem. 2003, 3453.

[309] C. Strohmann, B. C. Abele, K. Lehmen, F. Villafane, L. Sierra, S. Martín-Barrios, D. Schildbach, J. Organomet. Chem. 2002, 661, 149.

[310] H. A. Bent, Chem. Rev. 1961, 61, 275.

[311] W. C. Still, C. Sreekumar, J. Am. Chem. Soc. 1980, 102, 1201.

[312] R. K. Dress, T. Rölle, R. W. Hoffmann, Chem. Ber. 1995, 128, 673.

[313] T. H. Chan, S. Lamonthe, Tetrahedron Lett. 1991, 32, 1847.

[314] C. Strohmann, B. C. Abele, K. Lehmen, D. Schildbach, Angew. Chem. 2005, 117, 3196; Angew. Chem. Int. Ed. 2005, 44, 3136.

[315] A. Carstens, D. Hoppe, Tetrahedron 1994, 50, 6097.

[316] G. Fraenkel, A. Chow, R. Fleischer, H. Liu, J. Am. Chem. Soc. 2004, 126, 3983.

[317] G. Fraenkel, J. Gallucci, H. Liu, J. Am. Chem. Soc. 2006, 128, 8211.

[318] C. Strohmann, K. Lehmen, K. Wild, D. Schildbach, Organometallics 2002, 21, 3079.

[319] G. Fraenkel, J. H. Duncan, K. Martin, J. Wang, J. Am. Chem. Soc. 1999, 121, 10538.

[320] in CRC Handbook of Chemistry and Physics (Ed. D. R. Lide), Taylor \& Francis, Boca Raton, 2006.

[321] H. D. Flack, Acta Crystallogr., Sect. A. 1983, 39, 876.

[322] G. Bernardinelli, H. D. Flack, Acta Crystallogr., Sect. A. 1985, 41, 500.

[323] G. M. Sheldrick, SADABS 2006/3, Göttingen, 2006.

[324] N. W. Mitzel, K. Vojinovic, R. Fröhlich, T. Foerster, H. E. Robertson, K. B. Borisenko, D. W. H. Rankin, J. Am. Chem. Soc. 2005, 127, 13705.

[325] J. R. Hwu, F. F. Wong, J.-J. Huang, S.-C. Tsay, J. Org. Chem. 1997, 62, 4097.

[326] S. Mebs, M. Messerschmidt, P. Luger, Z. Kristallogr. 2006, 221, 656.

[327] W. Scherer, G. Eickerling, M. Tafipolsky, G. S. McGrady, P. Sirsch, N. P. Chatterton, Chem. Commun. 2006, 2986.

[328] J. Henn, unpublished results.

[329] G. M. Sheldrick, SADABS 2007/4, Göttingen, 2007.

[330] G. M. Sheldrick, XPREP in SHELXTL v6.12, Bruker AXS Inst. Inc., Madison (WI, USA), 2000.

[331] X-AREA, Stoe \& Cie GmbH, Darmstadt, 2002.

[332] XSHELL v4.01 in SHELXTL v6.12, Bruker AXS Inst. Inc., Madison (WI, USA), 2000.

[333] R. W. W. Hooft, L. H. Straver, A. L. Spek, J. Appl. Crystallogr. 2008, 41, 96. 



\title{
APPENDIX
}

\section{Charge Density Study Of [2-PicLi-2-Pich $]_{2}(4)$}

\author{
PICLI
}

fractal dimension $\left(d^{f}\right)$ vs. residual density $\left(\rho_{0}\right)$

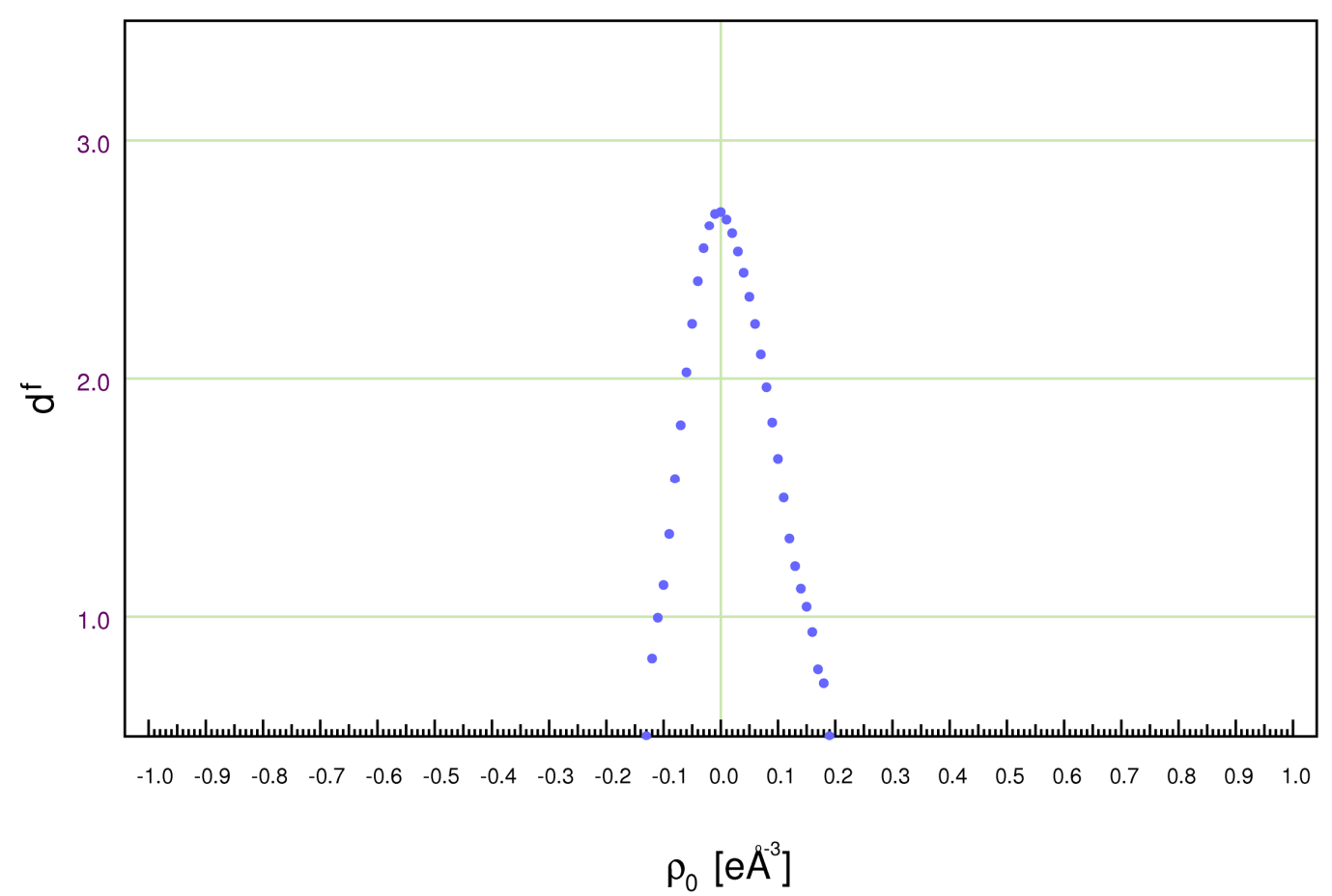

MODEL *model 42210

FOUR fmod1 422000 fmod2 42210

SELECT *fobs *fmod1 fmod2 print snlmin 0.000 snlmax 2.000

GRID 3-points perp *cryst

APPLY symm 3 trans 202 all

LIMITS xmin $0.0 \times \max 1.0 \mathrm{nx} 100$

LIMITS ymin 0.0 ymax $1.0 \mathrm{ny} 100$

LIMITS zmin 0.0 zmax $1.0 \mathrm{nz} 100$

$$
\begin{aligned}
& \mathrm{d}^{\prime}(0)=2.7001 \\
& \rho_{\min }(\mathrm{d}=2)=-0.0612 \mathrm{eA}^{\wedge}-3 \\
& \rho_{\max }(\mathrm{d}=2)=0.0773 \mathrm{eA}^{\wedge}-3 \\
& \mathrm{nx}=100 \quad \text { rho_min: }-0.1319 \mathrm{eA}^{\wedge}-3 \\
& \mathrm{ny}=100 \quad \text { rho_max: } 0.1937 \mathrm{eA}^{\wedge}-3 \\
& \mathrm{nz}=100 \quad \text { delta rho: } 0.3256 \mathrm{eA}^{\wedge}-3
\end{aligned}
$$

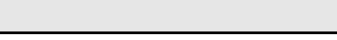

e_gross: $21.6800 \mathrm{e}$

e_net: -0.0000 e

jnk2RDA.exe

xd_fou.grd

17.12.2008, 19:03:19

Figure I. Output file generated with the programme jnk2RDA ${ }^{[175]}$ on a residual density grid (without a $\sin \theta \lambda^{-1}$ cut-off) of the unit cell of $[2-\mathrm{PicLi} \cdot \mathrm{PicH}]_{2}(4)$. The parabolic shape of the fractal dimension in relation to the residual density shows the excellent data quality and a successful multipole refinement. 
Table I. Differences of the mean-square displacement amplitudes (DMSDA) $\left(10^{-4} \mathrm{~A}^{2}\right)$ along interatomic vectors of $[2-\mathrm{PicLi} \cdot \mathrm{PicH}]_{2}(4)$.

\begin{tabular}{lccccccccc}
\hline \multicolumn{2}{c}{ Atom $\rightarrow$ Atom } & $\mathrm{d}[\AA]$ & DMSDA & Atom & $\mathrm{d}[\AA]$ & DMSDA & Atom & $\mathrm{d}[\AA]$ & DMSDA \\
\hline $\mathrm{N} 1$ & $\mathrm{C} 1$ & 1.3940 & 3 & $\mathrm{C} 5$ & 1.3578 & 6 & Li1 & 2.0210 & 20 \\
$\mathrm{~N} 2$ & $\mathrm{C} 7$ & 1.3466 & 2 & $\mathrm{C} 11$ & 1.3424 & 3 & Li1 & 2.0182 & 16 \\
$\mathrm{C} 1$ & $\mathrm{C} 2$ & 1.4477 & 2 & $\mathrm{C} 6$ & 1.3822 & 4 & & & \\
$\mathrm{C} 2$ & $\mathrm{C} 3$ & 1.3605 & -4 & & & & & & \\
$\mathrm{C} 3$ & $\mathrm{C} 4$ & 1.4226 & 2 & & & & & \\
$\mathrm{C} 4$ & $\mathrm{C} 5$ & 1.3720 & -1 & & & & & \\
$\mathrm{C} 7$ & $\mathrm{C} 8$ & 1.3952 & 2 & $\mathrm{C} 12$ & 1.4980 & 6 & & \\
$\mathrm{C} 8$ & $\mathrm{C} 9$ & 1.3898 & -1 & & & & & & \\
$\mathrm{C} 9$ & $\mathrm{C} 10$ & 1.3906 & 0 & & & & & & \\
$\mathrm{C} 10$ & $\mathrm{C} 11$ & 1.3889 & -1 & & & & & & \\
\hline
\end{tabular}

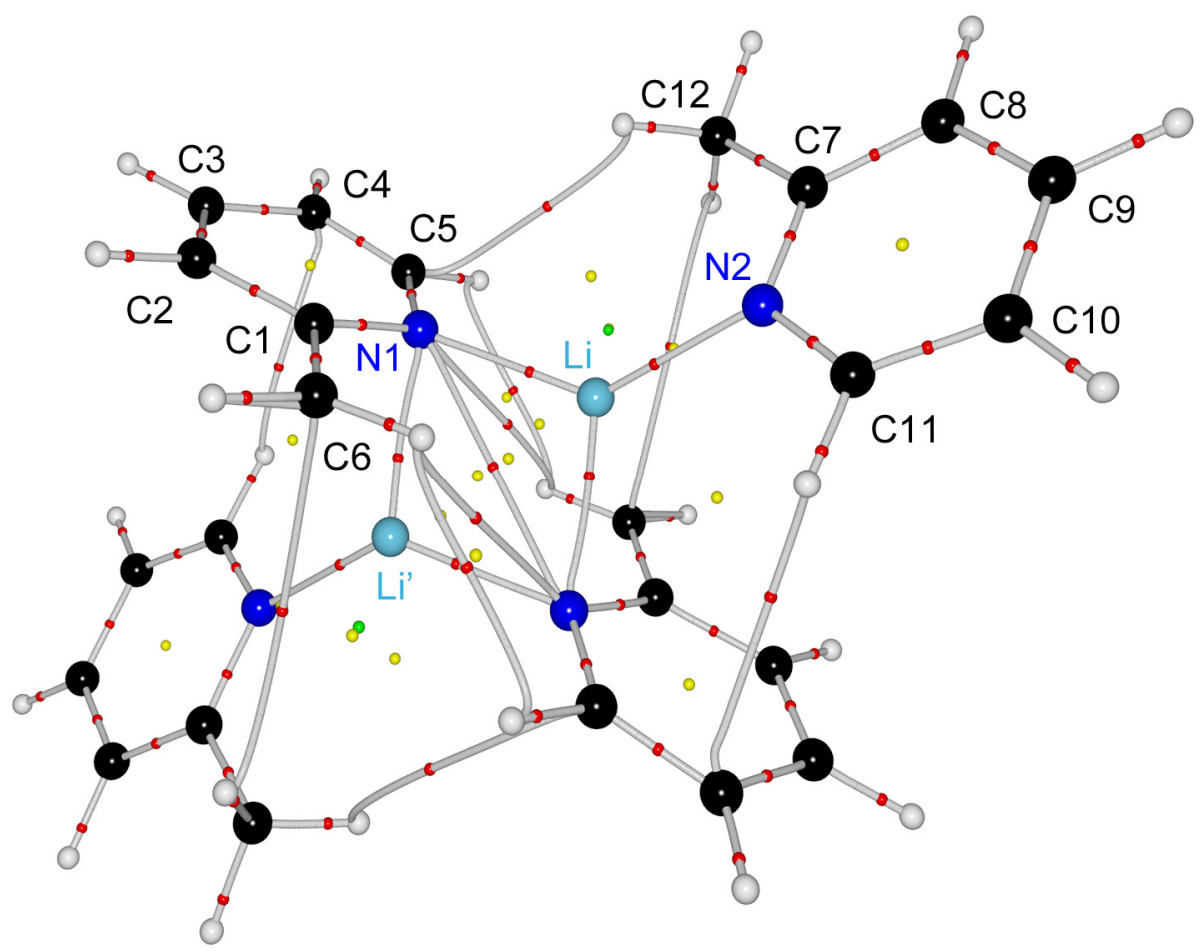

Figure II. Molecular graph of $[2-\mathrm{PicLi} \cdot \mathrm{PicH}]_{2}(4)$ including all determined bond paths, BCPs (red dots), RCPs (yellow dots), and CCPs (green dots). In summary 56 atoms, 71 BCPs, 18 RCPs, and 2 CCPs were found. Accordingly the Poincaré-Hopf equation (Eq. $2-13$ ) is fulfilled. 


\title{
Charge Density Study OF [TMSCH 2 LI $_{6}$ (14)
}

\author{
MELI
}

fractal dimension $\left(d^{f}\right) v s$. residual density $\left(\rho_{0}\right)$

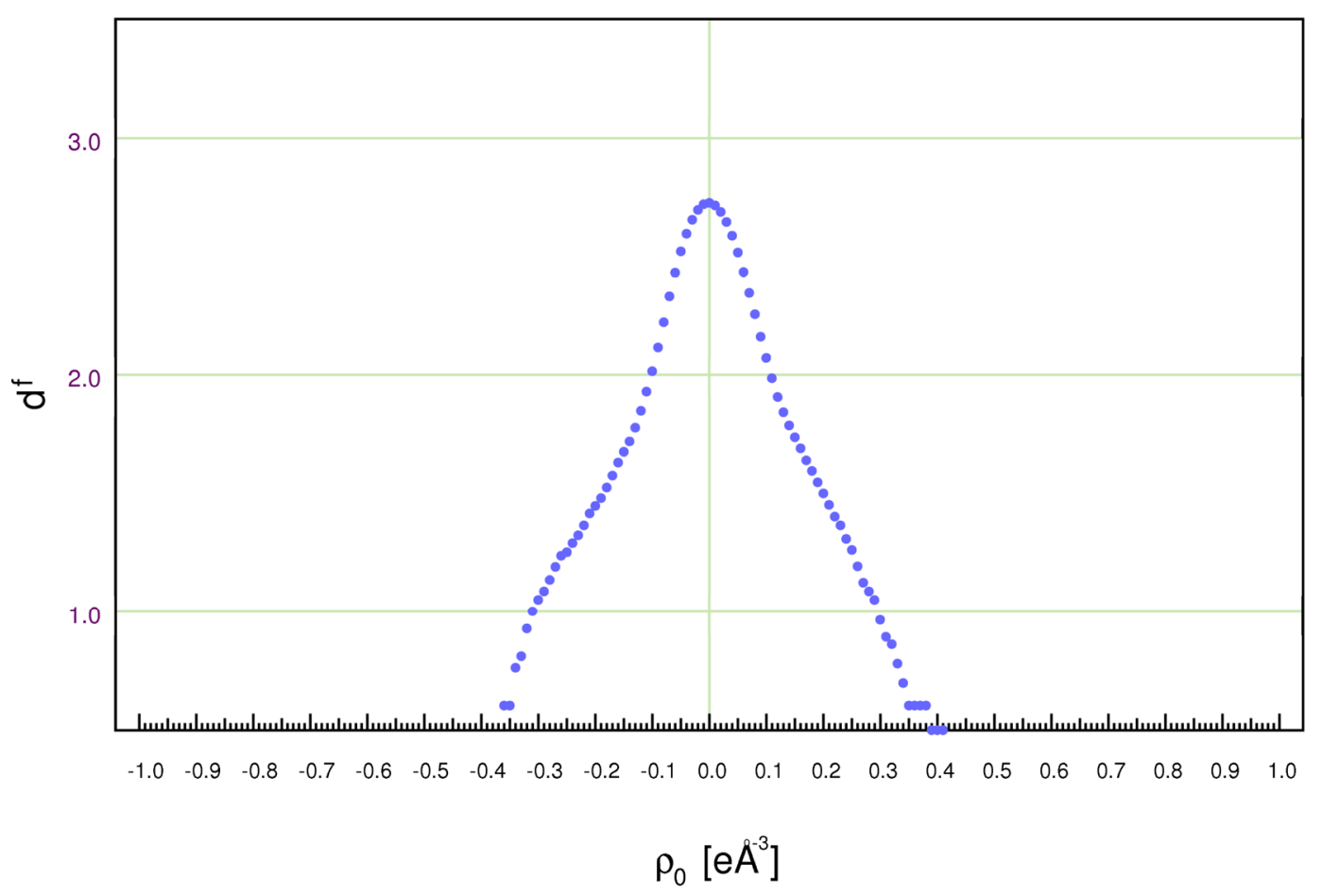

MODEL *model 4210

FOUR fmod1 4200 fmod2 4210

SELECT * fobs *fmod1 fmod2 print snlmin 0. snlmax 2.

GRID 3-points perp *cryst

LIMITS $x \min 0.0 \times \max 1.0 \mathrm{nx} 100$

LIMITS ymin 0.0 ymax 1.0 ny 100

LIMITS zmin $0.0 \mathrm{zmax} 1.0 \mathrm{nz} 100$

jnk2RDA.exe

$x d \_$fou_cell.grd $d^{\prime}(0)=2.7235$

$\rho_{\min }(d=2)=-0.1017 e A^{\wedge}-3$

$\rho_{\max }(\mathrm{d}=2)=0.1083 \mathrm{eA}^{\wedge}-3$

$n x=100$ rho_min: $-0.3651 \in A^{\wedge}-3$

ny $=100$ rho_max: $0.4123 \mathrm{eA}^{\wedge}-3$

$\mathrm{nz}=100$ delta rho: $0.7774 \mathrm{eA}^{\wedge}-3$

e gross: $61.1103 \mathrm{e}$

e_net: $0.0000 \mathrm{e}$

Figure III. Output file generated with the programme jnk2RDA ${ }^{[175]}$ on a residual density grid of the unit cell (without $\sin \theta \lambda^{-1}$ cut-off) of $\left[\mathrm{TMSCH}_{2} \mathrm{Li}\right]_{6}(\mathbf{1 4})$. The fractal dimension plot displays shoulders, which means that the residual density map shows features other than random noise. 
Table II. Differences of the mean-square displacement amplitudes (DMSDA) $\left(10^{-4} \mathrm{~A}^{2}\right.$ ) along interatomic vectors of $\left[\mathrm{TMSCH}_{2} \mathrm{Li}\right]_{6}(\mathbf{1 4})$.

\begin{tabular}{lccccccccc}
\hline Atom $\rightarrow$ Atom & $\mathrm{d}[\AA]$ & DMSDA & Atom & $\mathrm{d}[\AA]$ & DMSDA & Atom & $\mathrm{d}[\AA]$ & DMSDA \\
\hline Si1 & C1 & 1.8452 & 8 & $\mathrm{C} 2$ & 1.8722 & 26 & $\mathrm{C} 3$ & 1.8700 & 13 \\
& $\mathrm{C} 4$ & 1.8878 & 7 & & & & & & \\
Si2 & $\mathrm{C} 5$ & 1.8543 & 8 & $\mathrm{C} 6$ & 1.8727 & 4 & $\mathrm{C} 7$ & 1.8684 & 3 \\
& $\mathrm{C} 8$ & 1.8879 & 11 & & & & & & \\
Si3 & $\mathrm{C} 9$ & 1.8538 & 4 & $\mathrm{C} 10$ & 1.8792 & 9 & $\mathrm{C} 11$ & 1.8680 & 21 \\
& $\mathrm{C} 12$ & 1.8830 & 10 & & & & & & \\
Si21 & $\mathrm{C} 21$ & 1.8466 & 9 & $\mathrm{C} 22$ & 1.8766 & 16 & $\mathrm{C} 23$ & 1.8719 & 6 \\
& $\mathrm{C} 24$ & 1.8894 & 10 & & & & & & \\
Si22 & $\mathrm{C} 25$ & 1.8567 & 4 & $\mathrm{C} 26$ & 1.8744 & 6 & $\mathrm{C} 27$ & 1.8740 & 14 \\
& $\mathrm{C} 28$ & 1.8896 & 11 & & & & & & \\
Si23 & $\mathrm{C} 29$ & 1.8486 & 8 & $\mathrm{C} 30$ & 1.8717 & 16 & $\mathrm{C} 31$ & 1.8671 & 17 \\
& $\mathrm{C} 32$ & 1.8762 & 13 & & & & & & \\
\hline
\end{tabular}




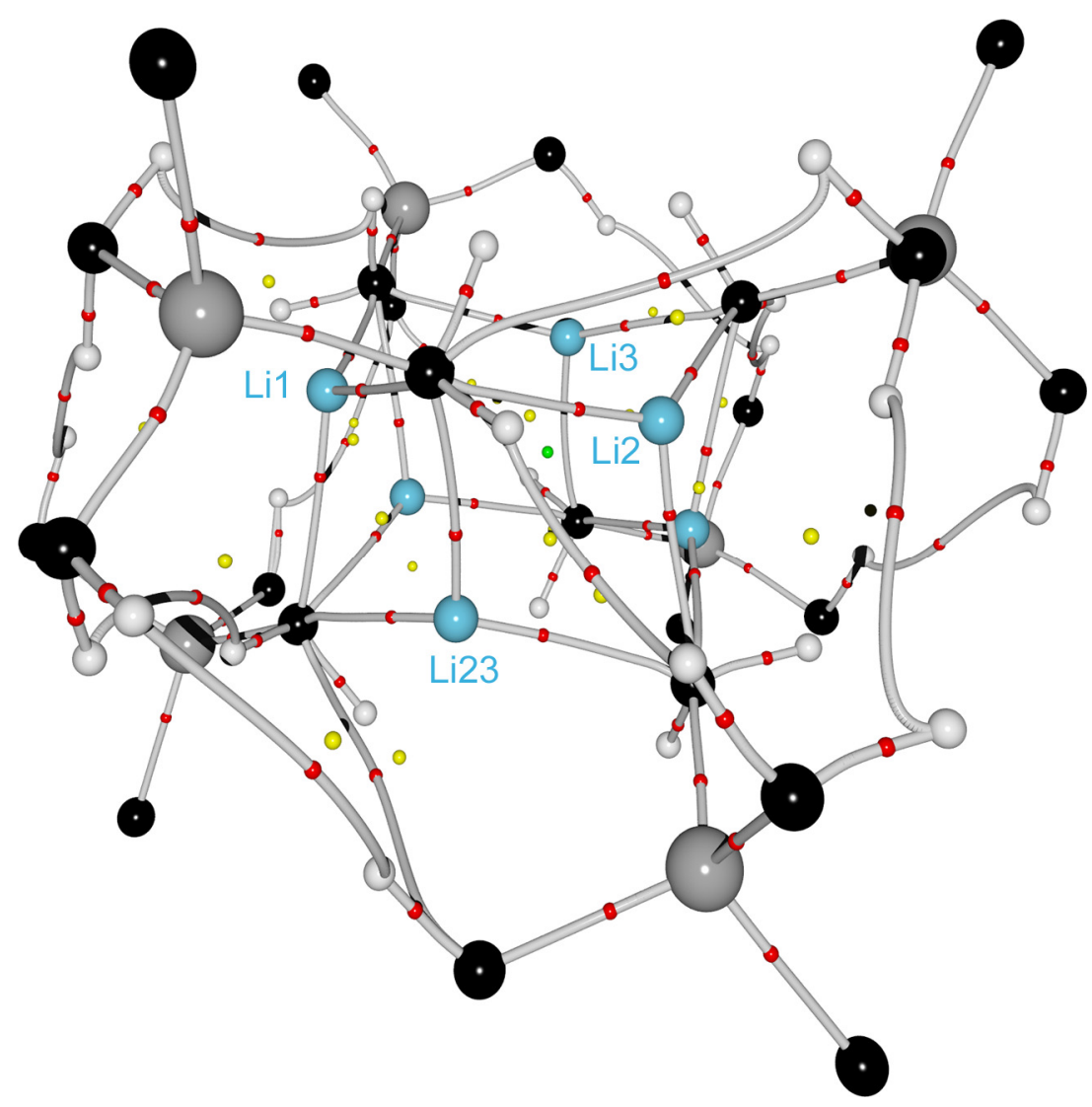

Figure IV. Molecular graph of $\left[\mathrm{TMSCH}_{2} \mathrm{Li}\right]_{6}$ (14) including all determined bond paths, except methyl hydrogen bonds that are only involved in one $\mathrm{C}-\mathrm{H}$ bond, BCPs (red dots), RCPs (yellow dots), and CCPs (green dots). In total (not all could be shown due to limitations of the XD programme), 102 atoms, 122 BCPs, 22 RCPs, and 1 CCP were found. Hence the Poincaré-Hopf equation (Eq. 2-13) is fulfilled.

However, the molecular graph in Figure IV does not include hydrogen atoms and hydrogen bond paths of the methyl groups due to software limitations (only 99 critical points allowed). 


\section{Charge Density Study of a Chiral Benzyllithium DeRIVATIVE (17)}

Table III. Differences of the mean-square displacement amplitudes (DMSDA) $\left(10^{-4} A^{2}\right.$ ) along interatomic vectors in $\mathbf{1 7}$.

\begin{tabular}{|c|c|c|c|c|c|c|c|c|c|}
\hline \multicolumn{2}{|c|}{ Atom $\rightarrow$ Atom } & \multirow{2}{*}{$\begin{array}{c}\mathrm{d}[\AA] \\
1.8817\end{array}$} & \multirow{2}{*}{$\frac{\text { DMSDA }}{11}$} & \multirow{2}{*}{$\begin{array}{c}\text { Atom } \\
\mathrm{C} 2\end{array}$} & \multirow{2}{*}{$\begin{array}{c}\mathrm{d}[\AA] \\
1.8860\end{array}$} & \multirow{2}{*}{$\frac{\text { DMSDA }}{13}$} & \multirow{2}{*}{$\frac{\text { Atom }}{\text { C3 }}$} & \multirow{2}{*}{$\begin{array}{c}\mathrm{d}[\AA] \\
1.8131\end{array}$} & \multirow{2}{*}{$\frac{\text { DMSDA }}{5}$} \\
\hline Si1 & $\mathrm{C} 1$ & & & & & & & & \\
\hline & C10 & 1.9070 & 6 & & & & & & \\
\hline $\mathrm{O} 1$ & $\mathrm{C} 15$ & 1.4202 & 10 & $\mathrm{C} 16$ & 1.4208 & 9 & & & \\
\hline $\mathrm{N} 1$ & $\mathrm{C} 10$ & 1.4811 & 6 & $\mathrm{C} 11$ & 1.4695 & 10 & $\mathrm{C} 14$ & 1.4762 & 3 \\
\hline $\mathrm{N} 2$ & $\mathrm{C} 17$ & 1.4772 & 6 & C19 & 1.4754 & 6 & $\mathrm{C} 21$ & 1.4776 & -2 \\
\hline $\mathrm{C} 3$ & $\mathrm{C} 4$ & 1.4343 & 5 & & & & & & \\
\hline $\mathrm{C} 4$ & C5 & 1.4272 & 2 & C9 & 1.4266 & 2 & & & \\
\hline $\mathrm{C} 5$ & $\mathrm{C} 6$ & 1.3859 & 4 & & & & & & \\
\hline $\mathrm{C} 6$ & $\mathrm{C7}$ & 1.3947 & 0 & & & & & & \\
\hline $\mathrm{C7}$ & $\mathrm{C} 8$ & 1.3992 & 4 & & & & & & \\
\hline $\mathrm{C} 8$ & C9 & 1.3902 & -4 & & & & & & \\
\hline $\mathrm{C} 11$ & $\mathrm{C} 12$ & 1.5226 & 0 & & & & & & \\
\hline $\mathrm{C} 12$ & $\mathrm{C} 13$ & 1.5382 & -3 & & & & & & \\
\hline $\mathrm{C} 13$ & $\mathrm{C} 14$ & 1.5399 & 3 & & & & & & \\
\hline $\mathrm{C} 14$ & $\mathrm{C} 15$ & 1.5143 & 1 & & & & & & \\
\hline $\mathrm{C} 17$ & $\mathrm{C} 18$ & 1.5403 & 1 & & & & & & \\
\hline $\mathrm{C} 18$ & $\mathrm{C} 23$ & 1.5235 & 2 & & & & & & \\
\hline C19 & $\mathrm{C} 20$ & 1.5435 & 7 & & & & & & \\
\hline $\mathrm{C} 20$ & $\mathrm{C} 23$ & 1.5278 & -3 & & & & & & \\
\hline $\mathrm{C} 21$ & $\mathrm{C} 22$ & 1.5367 & 2 & & & & & & \\
\hline $\mathrm{C} 22$ & $\mathrm{C} 23$ & 1.5251 & 3 & & & & & & \\
\hline
\end{tabular}




\section{STROH1B}

fractal dimension $\left(d^{f}\right) v s$. residual density $\left(\rho_{0}\right)$

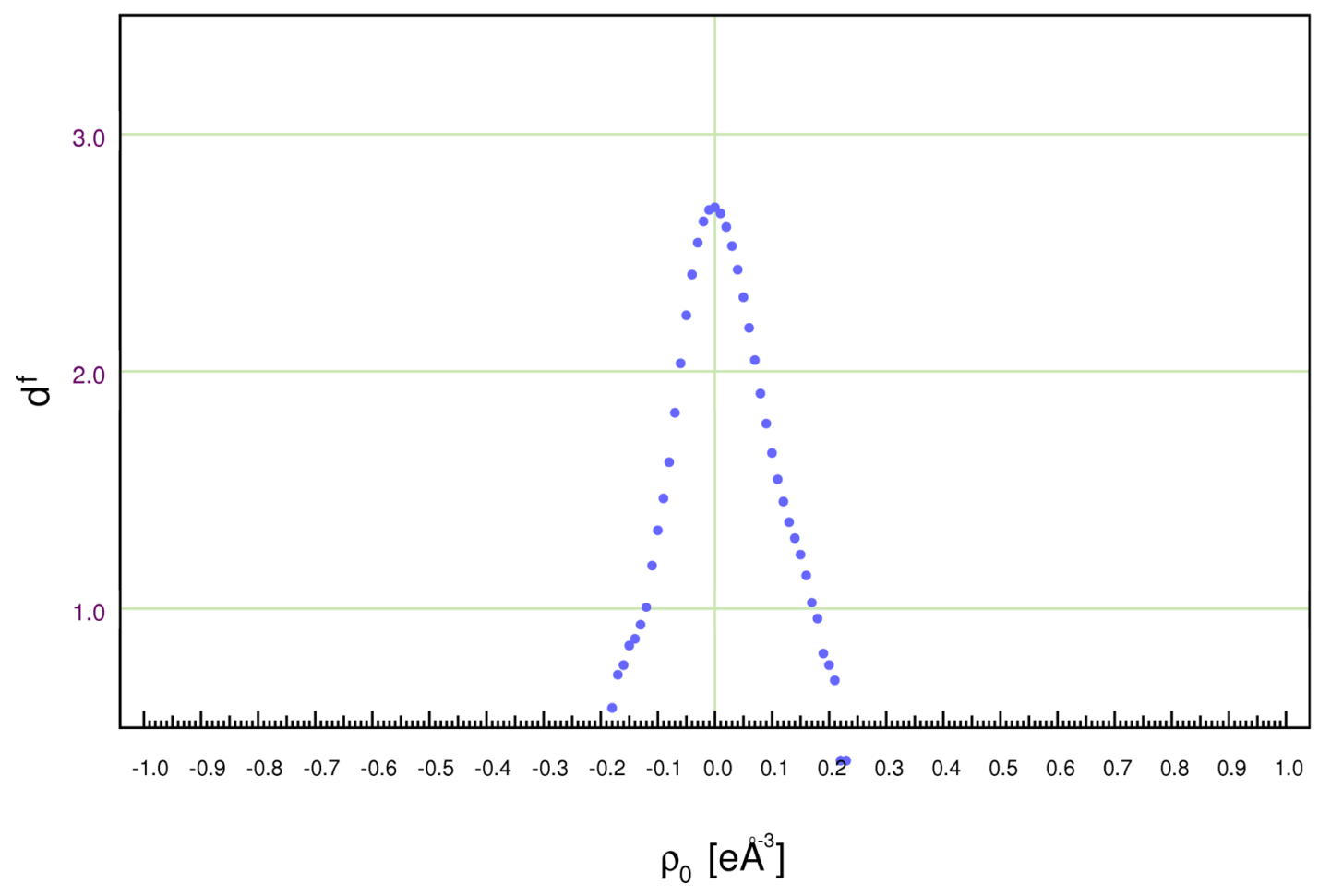

MODEL model 4210

FOUR fmod1 42000 fmod2 4200

SELECT *fobs * fmod1 fmod2 print snlmin 0.000 snlmax 2.000

GRID 3-points perp * ${ }^{*}$ cryst

LIMITS xmin $0.0 \times \max 1.0 \mathrm{nx} 100$

LIMITS ymin 0.0 ymax 1.0 ny 100

LIMITS zmin $0.0 \mathrm{zmax} 1.0 \mathrm{nz} 100$

$\mathrm{d}^{\mathrm{t}}(0)=2.6885$

$\rho_{\min }(d=2)=-0.0616 e^{\wedge}-3$

$\rho_{\max }(\mathrm{d}=2)=0.0734 \mathrm{eA}^{\wedge}-3$

$\mathrm{nx}=100$ rho_min: $-0.1835 \mathrm{eA^{ \wedge } - 3}$

ny $=100$ ro_max: $0.2326 \mathrm{eA}^{\wedge}-3$

$\mathrm{nz}=100$ delta rho: $0.4161 \mathrm{eA}^{\wedge}-3$

e_gross: $23.4501 \mathrm{e}$

e_net: -0.0000 e

13.03.2009, 14:25:27

Figure $\mathrm{V}$. Output file generated with the programme jnk2RDA ${ }^{[175]}$ on a residual density grid of the unit cell (without $\sin \theta \lambda^{-1}$ cut-off) of $\mathbf{1 7}$. The parabolic shape of the fractal dimension in relation to the residual density illustrates the excellent data quality and a successful multipole refinement. 


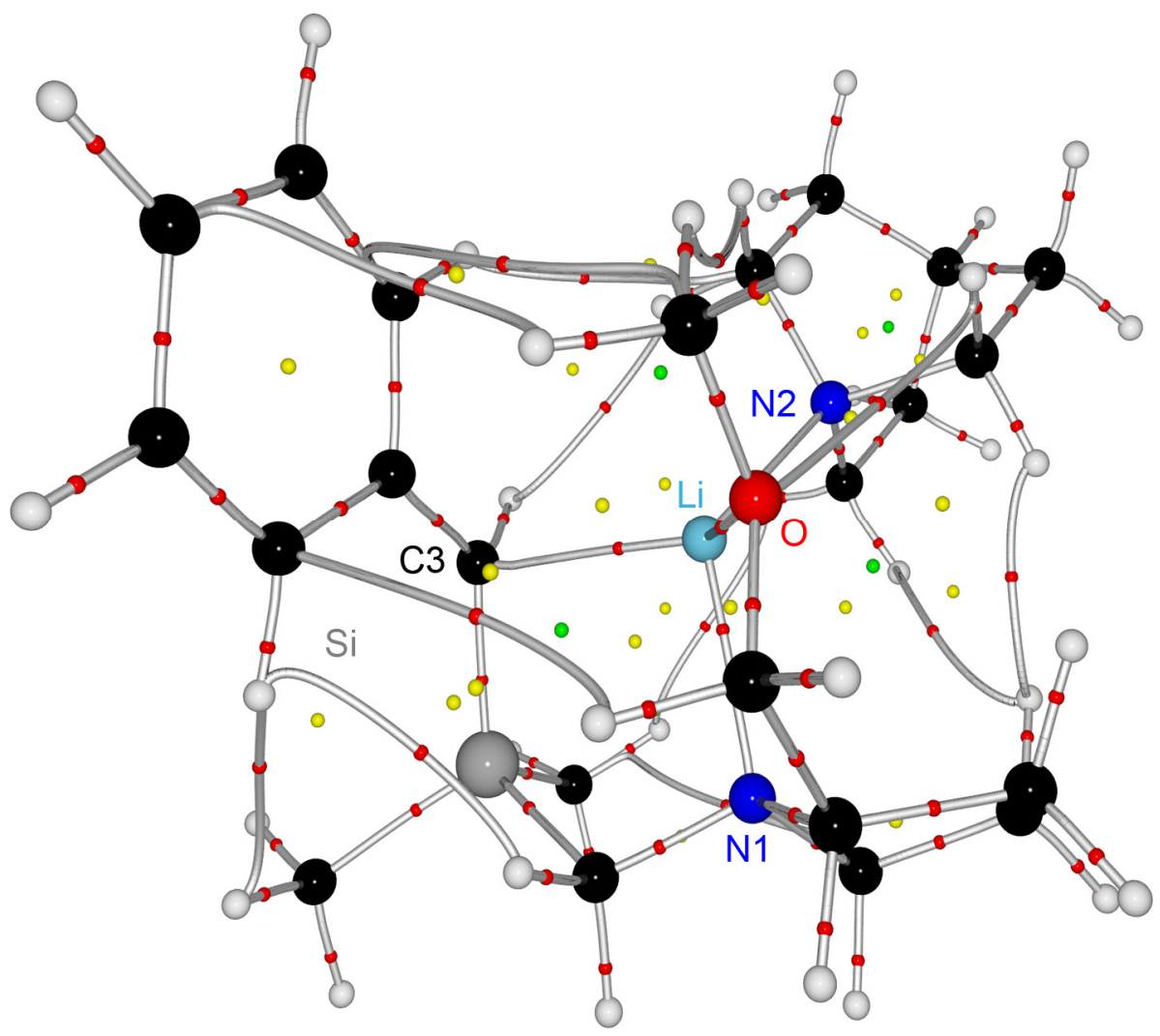

Figure VI. Molecular graph of $\mathbf{1 7}$ including all determined bond paths, BCPs (red dots), RCPs (yellow dots), and CCPs (green dots). In total 67 atoms, 85 BCPs, 24 RCPs, and 5 CCPs were found. Accordingly the Poincaré-Hopf equation (Eq. 2-13) is fulfilled. 


\section{Curriculum Vitae}

\section{Persönliche Daten}

Name:

Holger Ott

Geburtsdatum/-ort: 15. April 1981, München

Staatsangehörigkeit:

Familienstand:

deutsch

verheiratet, keine Kinder

\section{Schulische Ausbildung}

09/1987-07/1991

Besuch der Grundschule Pleinfeld

09/1991-06/2000

Besuch des Werner-von-Siemens Gymnasiums in Weißenburg und Abschluss mit der Allgemeinen Hochschulreife (Note: 1.7)

Leistungskurse: Mathematik und Chemie

\section{Wehrdienst}

11/2000-10/2001 Wehrdienst als Versorgungsdienstfeldwebel-Gehilfe in der 4.Kompanie/PzBtl. 304, Hahnenkammkaserne, Heidenheim am Hahnenkamm

Ausgeschieden als Hauptgefreiter der Reserve Ausbildung zum LKW-Fahrer

\section{Universitäre Ausbildung}

10/2001-05/2006 Studium der Chemie (Diplom) an der Bayerischen

$10 / 2003$

$06 / 2005$

$05 / 2006$

$06 / 2006$ Julius-Maximilians-Universität Würzburg Mündliche Diplom-Vorprüfung (Note: sehr gut) Mündliche Diplomprüfung (Note: sehr gut) Abschluss des Studiums mit Abgabe der Diplomarbeit über "Abstraktion benzylischer Protonen durch s-BlockBasen" (Betreuer: Prof. Dr. H. Braunschweig, Note: sehr gut)

Beginn der Promotion an der Georg-AugustUniversität Göttingen (Betreuer: Prof. Dr. D. Stalke)

\section{Letzter Abschluss}

05/2006

Studienabschluss als Dipl.-Chem. Univ. (Note: sehr gut) und Verleihung des Fakultätspreises der Chemie und Pharmazie im Juli 2007 


\section{Beruflicher Werdegang/Forschungspraktika}

$06 / 2000-10 / 2000$

$07 / 2003-08 / 2003$

$03 / 2004-04 / 2004$

$07 / 2005-05 / 2006$

$06 / 2006-01 / 2007$

$02 / 2007$
Arbeit bei der Firma W. L. Gore Pleinfeld

Praktikum im Zentrallabor für Polymerwerkstoffe der Firma Dynamit Nobel Pappenheim

Forschungspraktikum bei Prof. M. G. Davidson an der University of Bath, UK

Wissenschaftliche Hilfskraft

Wissenschaftlicher Mitarbeiter

Kekulé Stipendiat des Fonds der chemischen Industrie

\section{Universitäre Tätigkeiten}

- Sachkunde im Umgang mit Gefahrstoffen

- Fach- und Sachkunde im Strahlenschutz nach StISchV und RöV

- Stellv. Vorsitzender des JungChemikerForums Göttingen

- Schwerpunktpraktikum in Technischer und Makromolekularer Chemie

- Vorträge auf Einladung:

- Chemical Interpretation of Experimental Charge Density III - Study on an $\alpha$-Lithiated Benzylsilane, Bruker User's Meeting 2007, Göttingen.

- Charge Density Distributions in Lithium Organic Compounds, $1^{\text {st }}$ Indo German Symposium 2007, Indian Institute of Technology Kanpur (Indien). 


\section{LIST Of PUBLICATIONS}

[1] L. E. Turner, M. G. Davidson, M. D. Jones, H. Ott, V. S. Schulz, P. J. Wilson, Bis(bismuth)toluene Inverted-Sandwich Complex Supported by Aminetris(phenoxide) Ligands, Inorg. Chem. 2006, 45, 6123-6125.

[2] U. Flierler, M. Burzler, D. Leusser, J. Henn, H. Ott, H. Braunschweig, D. Stalke, Electron-Density Investigation of Metal-Metal Bonding in the Dinuclear "Borylene" Complex $\left[\left\{\mathrm{Cp}(\mathrm{CO})_{2} \mathrm{Mn}\right\}_{2}(\mu-B t B u)\right]$, Angew. Chem. Int. Ed. 2008, 47, 4321-4325.

[3] T. Tatic, H. Ott, D. Stalke, Deaggregation of Trimethylsilylmethyllithium, Eur. J. Inorg. Chem. 2008, 3765-3768.

[4] H. Ott, C. Däschlein, D. Leusser, D. Schildbach, T. Seibel, D. Stalke, C. Strohmann, Structure/Reactivity Studies on an $\alpha$-Lithiated Benzylsilane: Chemical Interpretation of Experimental Charge Density, J. Amer. Chem. Soc. 2008, 130, 11901-11911.

[5] D. Stalke, H. Ott, Was Chemiker aus der Elektronendichte lernen, Nachr. Chem. 2008, 2, 131-135.

[6] S. Deuerlein, D. Leusser, U. Flierler, H. Ott, D. Stalke, [(thf $\left.\mathrm{Li}_{2}\left\{\mathrm{H}_{2} \mathrm{CS}(\mathrm{NtBu})_{2}\right\}\right]_{2}$ : Synthesis, Polymorphism, and Experimental Charge Density to Elucidate the Bonding Properties of a Lithium Sulfur Ylide, Organometallics 2008, 27, 2306-2315.

[7] C. Kling, H. Ott, G. Schwab, D. Stalke, Heteroaromatic Substituted Phosphoranes with Enhanced Hemilabile Character, Organometallics 2008, 27, 5038-5042.

[8] I. Objartel, H. Ott, D. Stalke, Low-Temperature NMR and Crystal Structure Analyses of a Hemilabile Tin Complex, Z. Anorg. Allg. Chem. 2008, 634, 2373-2379.

[9] C. Matthes, U. Klingebiel, S. Deuerlein, H. Ott, D. Stalke, Von $N, N^{\prime}$-Bis(1,1,3-trisila-2-aza)-ethylen-diaminen $\quad z u \quad N, N^{\prime}$-Bis(1,3-disila2-lithiumamido)-ethylensilylaminen - Synthesen, Kristallstrukturen, Reaktionen, Z. Anorg. Allg. Chem. 2008, 634, 2402-2410.

[10] H. Ott, C. Matthes, S. Schmatz, U. Klingebiel, D. Stalke, First Trichloroaluminum Adducts of Silyliminoborenes, Z. Naturforsch. 2008, B9, 1023-1026. 
[11] H. Ott, U. Pieper, D. Leusser, U. Flierler, J. Henn, D. Stalke, Carbanion or Amide? First Charge Density Study of Parent 2-Picolyllithium, Angew. Chem. Int. Ed. 2009, 48, 2978-2982.

[12] U. Flierler, D. Leusser, H. Ott, G. Kehr, G. Erker, S. Grimme, D. Stalke, Catalytic abilities of $\left[\left(\mathrm{C}_{6} F_{5}\right)_{2} \mathrm{BR}\right], \mathrm{R}=\mathrm{NC}_{4} \mathrm{H}_{4}$ and $\mathrm{NC}_{4} \mathrm{H}_{8}$, deduced from experimental and theoretical charge density investigations, Chem. Eur. J. 2009, DOI: $10.1002 /$ chem.200802344.

[13] H. Ott, C. Matthes, D. Stalke, U. Klingebiel, On the Track of Novel Triel Stabilised Silylaminoiminoborenes, Chem. Eur. J. 2009, in press.

[14] S. Nembenna, S. Singh, A. Jana, H. W. Roesky, Y. Yang, H. Ye, H. Ott, D. Stalke, Preparation and Structural Characterization of Molecular Al-OSn(II) and Al-O-Sn(IV) Compounds, Inorg. Chem. 2009, 48, 2273-2276. 